\title{
The Dependence of the Evolution of Early-Type Galaxies on their Environment
}

\author{
Dissertation \\ zur Erlangung des Doktorgrades \\ der Mathematisch-Naturwissenschaftlichen Fakultäten \\ der Georg-August-Universität zu Göttingen
}

vorgelegt von

Alexander Fritz

aus Wien, Österreich

Göttingen 2006 
D7

Referent: Prof. Dr. K. J. Fricke

Korreferent: Prof. Dr. W. Lauterborn

Tag der mündlichen Prüfung: 17. Mai 2006 
TO MY PARENTS 



\section{Contents}

1 Introduction $\quad 1$

1.1 Galaxy Formation Theory . . . . . . . . . . . . . . . . . 2

1.2 Types of Galaxies . . . . . . . . . . . . . . . . . . . . . 4

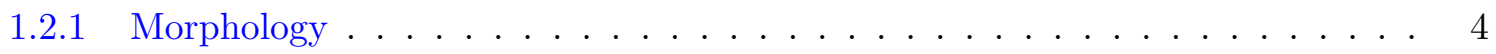

1.2 .2 Three-Dimensional Shape . . . . . . . . . . . . . . . . . . . . 6

1.2.3 Spectral Energy Distribution _... . . . . . . . . . . . . 7

1.2 .4 Kinematics . . . . . . . . . . . . . . . . . . . . . . . . . . . . . . . . .

1.3 Scaling Relations . . . . . . . . . . . . . . . . . . . 10

1.3.1 The Colour-Magnitude Relation . . . . . . . . . . . . . . . . 10

1.3.2 The Kormendy Relation . . . . . . . . . . . . . . . . . . . . 11

1.3.3 The Fundamental Plane . . . . . . . . . . . . . . . . . . . . . . . 12

1.4 Galaxy Evolution . . . . . . . . . . . . . . . . . . . . . . . . . . . . . . . . .

1.4.1 Theory meets Observations . . . . . . . . . . . . . . . 14

1.4 .2 High versus Low Densities . . . . . . . . . . . . . . . . . . . . . . . . . . . . . . . . . .

1.4 .3 Elliptical and S0 Galaxies . . . . . . . . . . . . . . . . . . . . . . . . . .

1.5 Motivation and Overview . . . . . . . . . . . . . . . . 18

2 Sample Selection and Observations 21

2.1 Cluster Samples . . . . . . . . . . . . . . . . . . . . . . . 22

2.1 Calar Alto Observatory . . . . . . . . . . . . . . . . . . . . . . . . . . . . . 22

2.1 .2 MOSCA Configuration . . . . . . . . . . . . . . . . . . 22

2.1.3 Abell 2390 Cluster . . . . . . . . . . . . . . . . . . . . . 23

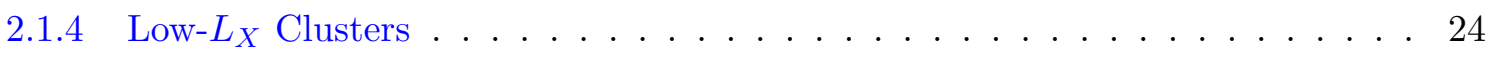

2.1.5 Selection Criteria . . . . . . . . . . . . . . . . 24

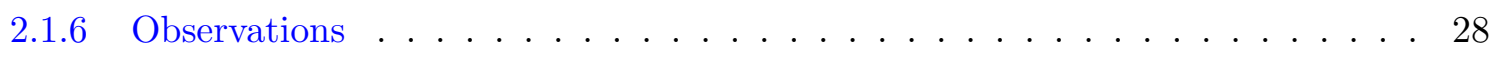

2.2 Field Sample . . . . . . . . . . . . . . . . . . . . . . . . . . 31

2.2 .1 The FORS Deep Field . . . . . . . . . . . . . . . . . . 31

2.2 .2 William Herschel Deep Field . . . . . . . . . . . . . . . . . . . . . . . . . . . . . . . . .

2.2 .3 FORS Configuration . . . . . . . . . . . . . . . . . . . . . . . . . . . . . . . . . . .

2.2 .4 Selection Criteria . . . . . . . . . . . . . . . . . . . . . . . . . . . . . . . . .

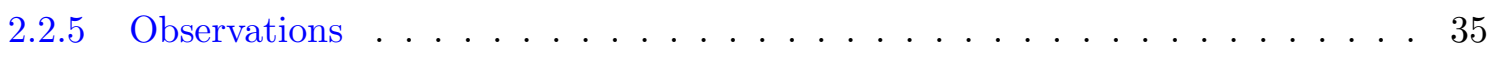


3 Data Reduction $\quad 39$

3.1 Bias Correction . . . . . . . . . . . . . . . . . . . 39

3.2 Cosmic Rays . . . . . . . . . . . . . . . . . . . . . . . . . . . . . . . . . . . . . . . .

3.3 Distortion Correction . . . . . . . . . . . . . . . . . . . . 40

3.4 Flat-fielding . . . . . . . . . . . . . . . . . . . . . 42

3.5 Sky Subtraction . . . . . . . . . . . . . . . . . . . . . 42

3.6 Wavelength Calibration . . . . . . . . . . . . . . . . . 43

3.7 Template Spectra . . . . . . . . . . . . . . . . . . . . . 44

4 Photometric Analysis $\quad 47$

4.1 Photometry of Abell $2390 \ldots \ldots$. . . . . . . . . . . . . . . . 49

4.1 .1 Ground-based $U B I$ Imaging . . . . . . . . . . . . . . . . . . . . . . . . . . 49

4.1 .2 HST Photometry . . . . . . . . . . . . . . . . . . 51

4.2 Photometry of Low- $L_{X}$ Clusters . . . . . . . . . . . . . . . . . 53

4.2 .1 Low $L_{X}$ Sample . . . . . . . . . . . . . . . . . . . 53

4.2 .2 Ground-based Photometry . . . . . . . . . . . . . . 54

4.2 .3 HST Photometry . . . . . . . . . . . . . . . . . 60

4.3 Surface Brightness Profile Fitting . . . . . . . . . . . . . . . . . . . 60

4.3.1 Choice of Luminosity Profile . . . . . . . . . . . . . . . . 60

4.3.2 The Surface Brightness Models . . . . . . . . . . . . . . . . 62

4.3 .3 Error Evaluation . . . . . . . . . . . . . . . . . . 66

4.3.4 Rest-Frame Properties . . . . . . . . . . . . . . . . . . . . 69

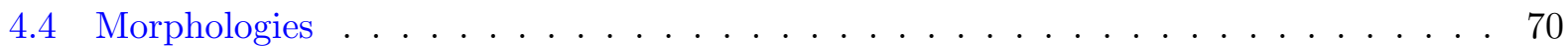

4.4 .1 Visual Classification ....................... 70

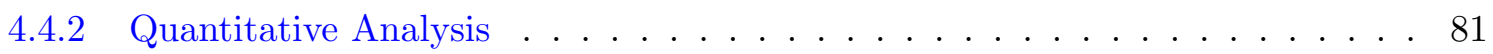

4.5 Luminosity Derivation . . . . . . . . . . . . . . . . . . . 84

4.5.1 Apparent Magnitudes . . . . . . . . . . . . . . . . . 85

4.5.2 Galactic Absorption . . . . . . . . . . . . . . . . 85

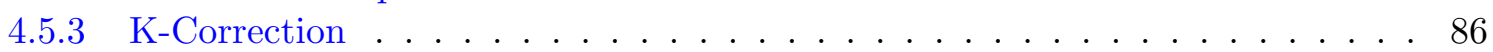

4.6 Luminosity Distribution and Errors . . . . . . . . . . . . . . . . 89

5 Kinematic Analysis 93

5.1 Galaxy Kinematics . . . . . . . . . . . . . . . . . . . . . 93

5.1 Kinematic Extraction Methods . . . . . . . . . . . . . . . 93

5.2 The FCQ Algorithm . . . . . . . . . . . . . . . . . . . . . . 97

5.2 .1 Error evaluation . . . . . . . . . . . . . . . . . . . . 99

5.3 Velocity Dispersions . . . . . . . . . . . . . . . . . . . . . 99

5.3 .1 Visual Inspection of $\sigma \ldots \ldots \ldots \ldots \ldots \ldots$

5.3 .2 Aperture Correction . . . . . . . . . . . . . . . . . 106

5.3.3 Comparison between Different Absorption Features . . . . . . . . . . . . . 107

5.3.4 Comparison between Repeat Observations . . . . . . . . . . . . . . . 111

5.3.5 Comparison between Different Extraction Procedures . . . . . . . . . . . . 112

5.3.6 Comparison between Different Stellar Templates . . . . . . . . . . . . . . 113

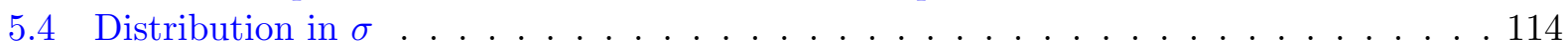

5.4 Cluster Samples . . . . . . . . . . . . . . . . . 114 
5.4 .2 Field Sample . . . . . . . . . . . . . . . . . . . . . 117

5.5 Redshift Distribution . . . . . . . . . . . . . . . . . . . 118

5.6 Comparison between Rich Clusters . . . . . . . . . . . . . . . . . 122

$6 \quad$ Galaxy Scaling Relations at $\mathrm{z} \sim 0.2 \quad 127$

6.1 The Local Reference . . . . . . . . . . . . . . . . . . . . . . 127

6.2 The Faber-Jackson Relation . . . . . . . . . . . . . . . . . . . . 129

6.3 The Kormendy Relation . . . . . . . . . . . . . . . . . . . . . . . . . . . . . 132

6.4 The Fundamental Plane . . . . . . . . . . . . . . . . . . . . . . . . . . . . . . . . . . . . . . . . . . . . . . .

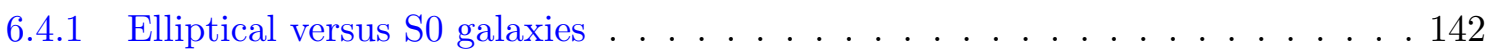

$6.5 \quad M / L$ Evolution . . . . . . . . . . . . . . . . . . . 147

6.6 Comparison with Previous Studies . . . . . . . . . . . . . . . 152

7 Environmental Effects on Galaxy Properties 155

7.1 The Faber-Jackson Relation . . . . . . . . . . . . . . . . . . . . . 156

7.1.1 Luminosity Dependence . . . . . . . . . . . . . . . . 156

7.1 .2 Mass Dependence . . . . . . . . . . . . . . . . . . . . 159

7.1 .3 Radial Dependence . . . . . . . . . . . . . . . . . . . 161

7.1.4 A Dependence on Galaxy Colours? . . . . . . . . . . . . . . . . . . . . . . . . . . . . . . . . . . . .

7.2 Stellar Population Ages . . . . . . . . . . . . . . . . . . . 168

7.3 Further Discussion and Conclusions . . . . . . . . . . . . . . 173

8 Summary and Outlook $\quad 175$

$\begin{array}{lr}\text { A Stellar Templates } & 179\end{array}$

$\begin{array}{ll}\text { Bibliography } & 185\end{array}$ 



\section{Chapter 1}

\section{Introduction}

Since the last twenty years extragalactic astrophysics and cosmology have experienced an enormous progress. The scientific knowledge and understanding of structure formation and the chemical evolution within the cosmological framework is steadily growing. Thanks to the development of high-speed parallel computer architectures, high-resolution cosmological simulations on various spatial scales of the Universe can be carried out. As a powerful complement, the construction of new $10 \mathrm{~m}$-class telescopes such as the four ESO Very Large Telescopes (VLT, Chile) and the twin telescopes at W. M. Keck Observatory (Hawaii) provide new light on astrophysical issues from the observational side.

Even so, several topics remain puzzling. Within the standard paradigm of modern cosmology today, a hierarchical structure formation using Cold Dark Matter particles, a wealth of observational evidences on large scales of $>1 \mathrm{Mpc}^{1}$ from the early cosmic beginning up to the Universe as seen today can be modelled very successfully. On smaller scales in the order of kpc and below, smaller galaxies with lower-masses are formed first at early epochs, whereas larger high-mass galaxies are assembled through merging and accretion events of smaller sub-units over longer timescales up to the recent past. In this picture, different evolutionary paths are predicted for the densest environments of clusters of galaxies and the lowest densities of isolated individual

\footnotetext{
${ }^{1}$ One parsec (pc) corresponds to a distance of approximately 3.26 light years or $3.086 \cdot 10^{16} \mathrm{~m}$.
}

galaxies. The environment is suggested to play an important role to quantify and characterise the formation and evolution of galaxies as well as their overall properties.

Clusters of galaxies offer a unique laboratory to study the physical properties of galaxies and their formation and evolution. The environment of rich galaxy clusters, where degree of richness refers to the overall mass density of a cluster (typically between $10^{14}$ to $10^{15} \mathrm{M}_{\odot}$ ), provides an ideal location to explore how the physical processes of galaxies are related to varying local number density, interactions with other galaxies and exposure to a hot intra-cluster medium, in which the galaxies are embedded. In particular for the most massive galaxies known, the early-type galaxies, the effects of environment on their formation and evolution can be revealed. Most previous studies concentrated on the environments of galaxy clusters as they provide the opportunity to observe a large number of early-type galaxies with less amount of telescope time simultaneously using the technique of multi-object spectroscopy.

Detailed investigations of galaxies in the nearby Universe $(z<0.05)$ have revealed very complex formation and evolution histories of galaxies, thereby involving merging events, bursts of star formation, and morphological diversifications. Nevertheless, the global properties of the galaxies, which encompass luminosities, rotation velocities, velocity dispersions, sizes, and absorption line-strengths, obey very tight em- 
pirical scaling relations, such as the Fundamental Plane for early-type galaxies and the TullyFisher relation for spiral galaxies. Such scaling laws put strong constraints on theoretical galaxy formation models and on the evolution of galaxies. Both high signal-to-noise spectroscopy and deep multi-colour photometry is required to construct these powerful tools.

Local early-type galaxies from the Sloan-DigitalSky-Survey (SDSS) show a weak trend in their kinematics and structure parameters with galaxy density (Bernardi et al. 2003). Galaxies residing in the dense environments of cluster cores have slightly different properties to their counterparts in lower density regions. Such a dependence on environment can only be discovered with sufficient number statistics but was not yet studied at higher redshift.

It was one of the basic motivations of this study to investigate a large sample of early-type galaxies at $z \sim 0.2$, thereby covering the densest environments of galaxy clusters down to the lowest densities of isolated field galaxies in order to test a possible dependence of their galaxy properties on environment. A detailed analysis of the internal kinematics, structure and stellar content of these galaxies and their involved physical and chemical processes is therefore essential. Before the strategy of this thesis will be given in more detail, the two basic galaxy formation scenarios will be opposed to each other (next section) and the main properties of galaxies in the local Universe discussed (section 1.2). Empirical scaling relations which are also well understood from the theoretical point of view, are described in section 1.3. An application of these tools to studies of galaxy evolution is one of the main topics in section 1.4, where the current state of theory and observations as well as the role of environment and stellar populations of early-type galaxies will be addressed. Finally, the motivation of this study and a brief overview of the thesis will be the content of section 1.5.

\subsection{Galaxy Formation Theory}

Ever since galaxies have been classified into different morphological types substantial effort was undertaken to understand their formation and evolution. Although a number of models have been introduced, a complete consensus between theoretical approach and observational evidence on the individual mechanisms and involved timescales has not yet been reached. In the current adopted "concordance" cosmology, two main scenarios have been suggested: the monolithic collapse and the hierarchical scenario. Fig. 1.1 gives a brief overview of the two different theories. The wealth of empirical constraints supports parts of both these predictions. However, a full understanding of the formation of early-type galaxies as a function of morphology, redshift and environment is still pending. The truth probably is a combination of both theories. In the following the two alternative scenarios of monolithic collapse and hierarchical paradigm will be introduced.

In the monolithic collapse which is also called the classical model because historically first suggested, galaxies form out of individual supergiant gas clouds (Eggen et al. 1962; Larson, 1974). A collapse of a cool proto gas cloud (or also several gas clumps, see Fig. 1.1) where gas is falling to the centre in radial orbits generates rapidly old stars with low metallicity. The stars collapse quickly from a halo to a thin rotating disc and star formation is initially high and enriches the disc with heavy elements. The morphology of a galaxy is determined by the angular momentum of the gas cloud and the timescale of transforming gas into stars. If the gas is turned into stars on longer timescales than the timescale of the gas cloud (high angular momentum) a disc galaxy is generated. Via dissipation the gas is settled in a disc plane where star formation continues until the remaining gas is completely used up. For timescales shorter than the dynamical friction timescale of the cloud (low angular momentum) a spheroidal system is formed 
through processes of violent relaxation (LyndenBell 1967). A critical point in Fig. 1.1 is when the first massive stars evolve to the supernova (SN) phase. The gas is expelled from the system by the SN-driven wind and star formation can only continue if the dark matter halo is massive enough. If all gas is removed a spheroidal star system remains and the subsequent evolution depends on the environment. Cluster galaxies loose their material to the intra-cluster medium (ICM), while for galaxies in low density environments a subsequent accretion of gas onto the disc with a quiescent star formation over an extended period is possible (Larson 1975; Arimoto \& Yoshii 1987). In this traditional picture, the bulk of stars in early-type galaxies are created in a short intense burst of star formation at high redshift $z \gtrsim 2$, where the most massive structures form first in the primordial density fluctuations (top-down scenario). The modern version of the classical scenario is not necessarily assuming that galaxies form by a collapse of a single gas cloud but rather that ellipticals are generated at high redshift and on shorter timescales relative to spirals and that they are assembled out of gas and not of preexisting stars.

By contrast, in the hierarchical scenario earlytype galaxies are formed via mergers of two disc galaxies at relatively recent times (Toomre \& Toomre 1972), White \& Rees 1978). Mass is accreted in a continuous process over an extended time period through multiple major and minor mergers as well as accretion events of smaller subunits up to the recent past. In a first step a bulge is formed in the assembled structure, then accretion of baryonic gas sets in and finally a thin disc is build up where star formation is induced. Thick discs could be created through heating of the thin disc by companions. During a lifetime of a galaxy this process is repeated several times and thus a significant fraction of stars is formed below redshift of unity. As a result, massive galaxies should have more extended star formation histories (bottom-up scenario). How-

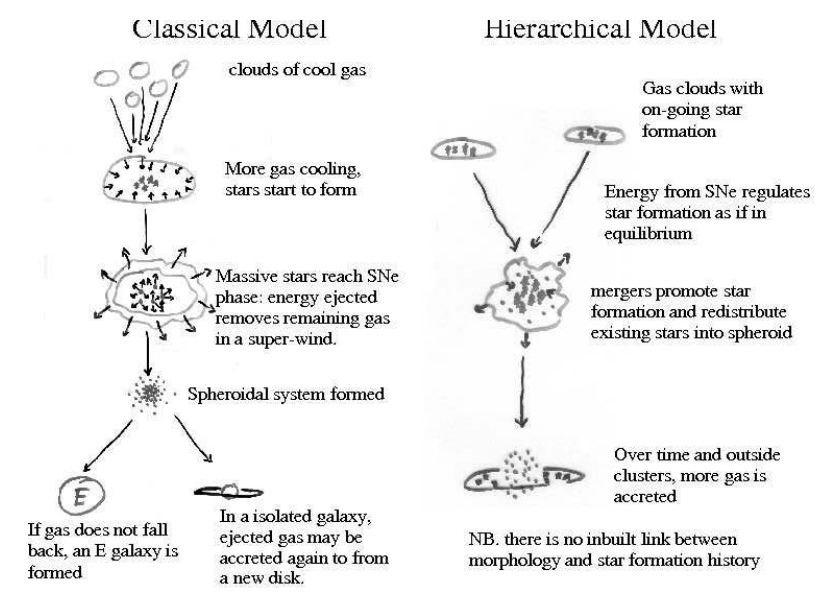

Figure 1.1: A sketch of galaxy formation in the Classical and Hierarchical scenarios from Bower et al. (1999). In the Classical model the spheroidal component is formed in a rapid burst of star formation at high redshift and a subsequent passive evolution (E) or a continuous gas accretion onto the disc over a long period (isolated galaxy in a low density environment). In the Hierarchical model, galaxies can change between different morphological types depending on the number and strength of mergers and interactions.

ever, the latter prediction is no longer supported by recent observations (down-sizing theory).

The hierarchical assembly of galaxies is nowadays implemented in semi-analytic simulations within the framework of Cold-Dark-Matter (CDM) haloes (Kauffmann 1996; Baugh et al. 1996; Cole et al. 2000). In these models, environmental processes such as galaxy interactions or starbursts at high redshift can be successfully simulated and tested against the observations. Contrary to the classical approach, there is an ongoing not variable star formation occurring at different epochs. The equilibrium is regulated between the inflow of gas and the ejection rate by a SN-driven wind. A replenishment of the gas for the continuous star formation processes is warranted through the haloes of the galaxies. The mergers promote star formation and rearrange the stars in spheroids and over time accretion of further gas in low densities is possible thereby changing the morphological appearance 


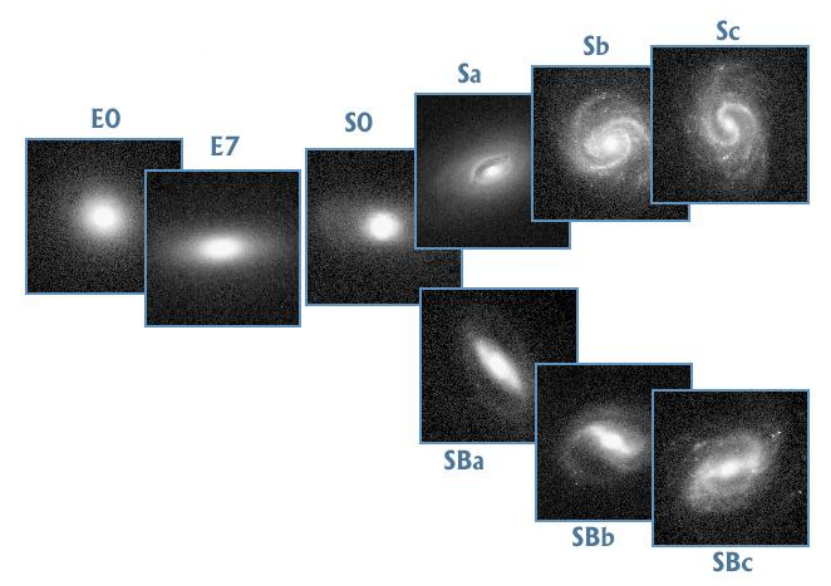

Figure 1.2: The Hubble "tuning fork" classification for morphological galaxy types. A range from early-type galaxies (ellipticals and S0 galaxies) on the left to late-type galaxies (spirals) on the right hand side is shown. Ellipticals are divided according to their axial ratio, normal and barred spirals are subclassified according to the fractional size of the central bulge component and the size of the spiral arms. From left to right the disc (nonexistent in ellipticals) together with the amount of gas and young stars becomes more prominent. The presence of a central bar is denoted by a "B" in the lower panel. (C) Z. Frei and J. E. Gunn, Princeton University Press 1999).

of the system.

A key difference between the scenarios is galaxy morphology. In the classical model this parameter is established at an early time, whereas in the hierarchical formation galaxies have no fixed morphology but can vary between different morphological types depending on the number and strength of interactions and merger processes. This segregation between the two scenarios can be interpreted as a division between the roles of nature (monolithic) and nurture (hierarchical) in forming the morphology of the galaxies.

While some predictions of the hierarchical model for the evolution of individual galaxies are supported by empirical evidences, other observational results favour a classical galaxy formation. A comparison between the theory and observation in the picture of galaxy evolution to- gether with their pros and cons is presented in section 1.4.

\subsection{Types of Galaxies}

Nearby galaxies can be separated into three principal categories: Ellipticals, spirals and irregular galaxies. Spirals comprise two classes, normal spirals and barred spirals, both can be further divided according to the fractional size of the central bulge component. The fundamental properties of ellipticals and spirals as inferred from their morphological shape, spectral characteristics and kinematics are distinct from each other. As a third group, irregular galaxies, which are in many cases low-mass gas-rich systems, cannot be assimilated in either of the two classes formed by ellipticals or spirals. However, research during the last two decades yielded a more complex picture than the above separation into three distinct types of galaxies.

In the following, three criteria will be employed to construct a classification scheme for galaxies. Emphasis will be given to elliptical and S0 galaxies and their general shape. The kinematics and dynamics of these systems are presented in section 1.2.4.

\subsubsection{Morphology}

In 1926, Edwin Hubble introduced his famous "tuning fork" diagram, see Fig. 1.2, through a classification of galaxies in terms of their morphology (Hubble 1926). This scheme represents also a sequence of decreasing luminous matter and increasing dark matter from early-type to late-type galaxies.

Elliptical galaxies, also know as spheroidal galaxies, basically comprise a one-component structure of a bulge and are sub-classified into E0 and E7 ellipticals according to their ellipticity $\epsilon$. The ellipticity is defined over the axial ratio $b / a$ (varying from 1 to 3 ) as $\epsilon=1-b / a$ where $a$ and $b$ being the major and minor axis, respectively. 
Values of $\epsilon$ range from 0 to 0.7 . The radial surface brightness distribution of ellipticals is very smooth with a high central concentration and follows closely a $r^{1 / 4}$-law, where $r$ denotes the galactocentric radius, which is know as the "de Vaucouleurs"-profile (de Vaucouleurs 1948).

Spirals basically consist of two components, a central bulge characterised by a $r^{1 / 4}$-law and an additional disc component with an exponential luminosity profile proportional to $e^{-r / r_{\mathrm{d}}}$. The characteristic size of the disc is given by the disc scale length $r_{\mathrm{d}}$. Normal spiral galaxies are subclassified from Sa ("early") to Sd ("late") according to the fractional size of the central bulge component (the disc-to-bulge $D / B$ ratio) and the size and form of the spiral arms. The bulge fraction decreases from Sa to Sd whereas at the same time the dominance and open loose form of spiral arm as well as the presence of gas, dust and ionised regions of young stars increases from early to late spirals, which can be seen from left to right in Fig. 1.2. For barred spirals which exhibit a central bar, the nomenclature is changed to SBa to SBd.

Lenticular galaxies (S0) form a transition zone between elliptical and early-type spirals. They have a bulge and a clearly visible disc with no spiral arm structure but only a little gas fraction and dust. Their luminosity profile along the major axis is well characterised by an $r^{1 / 4}$ law, which is overlayed by an exponential disc component. With respect to the ellipticals the differences in the shape of S0s are small (cf. section 1.2.2). The ellipticity has a uniform radial distribution along the major axis with values up to $\epsilon=0.7$. Similar to ellipticals, generally the position angle is constant out to large radii.

Irregular galaxies (Im) and peculiar galaxies (abbreviated with an additional "pec") do not fit in any of these schemes. Together with the group of very late-type $\mathrm{Sd}$ spirals these objects are not displayed in Fig. 1.2. Galaxies of this class are isolated systems with an irregular or peculiar isophotal shape which are neither described

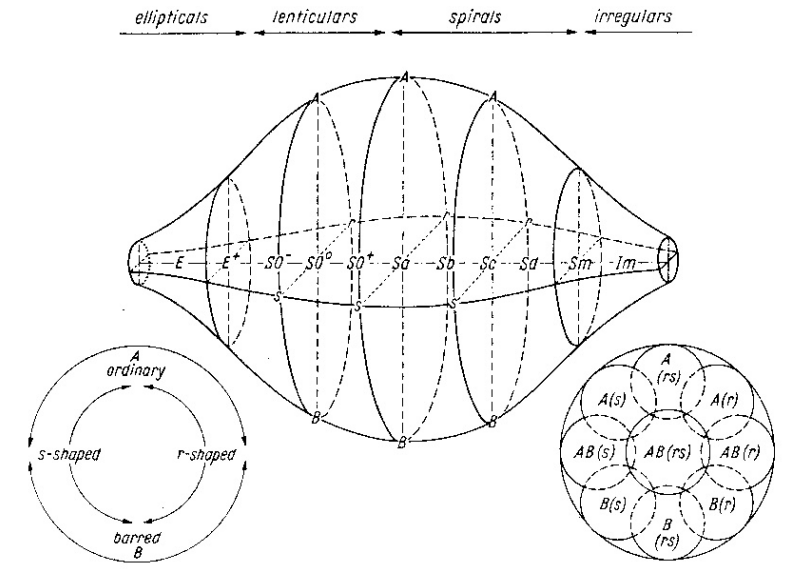

Figure 1.3: Schematic drawing of de Vaucouleurs 3-D classification volume (de Vaucouleurs 1959).

via a de Vaucouleurs nor an exponential luminosity profile. Systems which clearly show signs of interactions with objects in the close neighbourhood (e.g., distortions via tidal forces) or even undergo a merging event also fall into this regime.

Dwarf galaxies are a separate class of their own. These low-mass systems are usually classified on basis of their absolute $B$-band magnitude of $M_{B}>-18$ or their total (virial) mass with a limit of $M_{\text {vir }}<10^{9} M_{\odot}$ (e.g., Babul \& Rees 1992). Sub-classes of dwarf galaxies comprise e.g., dwarf irregulars, dwarf spheroidals, blue compact dwarf or tidal dwarf galaxies.

Another morphological classification by de Vaucouleurs (de Vaucouleurs 1959) uses a revised version of the Hubble system of galaxies which is shown in Table 1.1 and in Fig. 1.3. Along the Hubble sequence the stage parameter $T$ (on a numerical scale $-5 \leq t \leq+10$ ) correlates well with several fundamental parameters of a galaxy, such as photometric structure, colour index or hydrogen content et cetera. The long axis relates to the basic physics of the galaxy, while the crosssection displays details on the dynamics. The presence or absence of varieties within a class (e.g., bar or ring in spirals) should have only minor influence on the dynamics rather than basic 
Table 1.1: The revised Hubble sequence of galaxies according to de Vaucouleurs (de Vaucouleurs 1959).

\begin{tabular}{ccccccccccccc}
\hline Stage & $t$ & -6 & -5 & -4 & -3 & -2 & -1 & 0 & 1 & 2 & 3 & 4 \\
Type & $T$ & $\mathrm{E}^{-}$ & $\mathrm{E}^{0}$ & $\mathrm{E}^{+}$ & $\mathrm{S}^{-}$ & $\mathrm{S}^{0}$ & $\mathrm{~S}^{+}$ & $\mathrm{S} 0 \mathrm{a}$ & $\mathrm{Sa}$ & $\mathrm{Sab}$ & $\mathrm{Sb}$ & $\mathrm{Sbc}$ \\
Stage & $t$ & 5 & 6 & 7 & 8 & 9 & 10 & 11 & & & & \\
Type & $T$ & $\mathrm{Sc}$ & $\mathrm{Scd}$ & $\mathrm{Sd}$ & $\mathrm{Sdm}$ & $\mathrm{Sm}$ & $\mathrm{Im}$ & $\mathrm{Im}^{+}$ & & & & \\
\hline
\end{tabular}

differences in the physical properties of the galaxy. For example, a compact elliptical galaxy is represented as $\mathrm{E}^{-}$, a normal elliptical described as $\mathrm{E}^{0}$ and an $\mathrm{cD}$ galaxy (which is the central galaxy of a cluster) is denoted as $\mathrm{E}^{+}$. Irregular galaxies are specified in this scheme as Im and $\mathrm{Im}^{+}$, which include compact irregular systems $(\mathrm{cIm})$ and dwarf irregular galaxies (dIm). Furthermore, the classification allows to distinguish between transition stages, e.g. S0/a, which indicates a type between lenticulars and spirals. This classification system has been used in this thesis too, see chapter 4.4 on page 70 for details.

One striking discovery was that the fraction of various types of galaxies to the overall galaxy population depends on the environment. The number of galaxies changes as a function of mean projected density in clusters of galaxies. Beyond a critical density of $\sim 1$ galaxy per $\mathrm{Mpc}^{3}$ the fraction of elliptical and S0 galaxies increased dramatically and exceeds that of gas-rich spiral and irregular galaxies (Dressler 1980). At the highest densities in the centres of clusters of galaxies ellipticals and S0s contribute the dominant part of galaxy population whereas in the lowest densities of the field isolated spirals are the most frequent galaxy type. This is known as the morphologydensity relation.

A more quantitative classification of morphological types provides the bulge-to-total ratio $B / T$, which gives the contribution of the bulge component to the total luminosity of a galaxy. This ratio decreases from early to late-type galaxies, with the highest values for bulge dominated ellipticals and the lowest values for spiral $\mathrm{Sd}$ and irregular galaxies. In the case of a non-detectable bulge $B / T=0$. For a more detailed description and an application of this morphological classification the reader is referred to chapter 4.4.2.

\subsubsection{Three-Dimensional Shape}

Since the first observations in the 18 century up to the end of 1970s, elliptical galaxies were interpreted as galaxies without any substructure. The effects of projection prohibited insights into the intrinsic shape of ellipticals. For a long time, therefore, they were thought of axialsymmetric ellipsoids or triaxial systems, which are flattened by rotation and consisting entirely of (metal rich) population II stars and devoid of cold gas. Shortly after the beginning of the Universe, these old stellar systems should have been formed via a single collapse of a huge gas cloud. In the late eighties detailed high-signal-to noise investigations of the intrinsic isophote shapes of local elliptical galaxies were carried out, revealing substantial substructure (Carter 1987; Bender 1988). The isophotes which represent contours of constant surface brightness of an observed galaxy can be modelled by ellipses to derive the variations of the ellipticity from the center to the edge. Many ellipticals do not appear to feature a perfectly elliptical shape when projected onto the sky. Usually, the deviations from a perfect ellipsoid symmetric along both major axes are with $\lesssim 4 \%$ very small, described by the parameter $a_{4}$ which defines a separation into boxy or discy isophotes. Typical values for the ratio of $a_{4}$ to mean radius $a_{0}$ are in the range of $-0.02 \leq a_{4} / a_{0} \leq 0.04$. Discy ellipticals have in 
addition a parameter $a_{6} \neq 0$.

The shape of the isophotes is very important as it correlates with numerous properties of elliptical galaxies. For this reason, Kormendy \& Bender (1996) proposed a revision of the Hubble scheme for the elliptical galaxies and suggested that they can be divided basically into two classes according to their isophotal shape:

- Boxy E(b): Generally these are luminous galaxies $\left(M_{V} \lesssim-22.0\right)$ which are supported by anisotropic velocity dispersions $(\sigma)$. These almost isothermal objects show a slow or zero rotation rate of $\left(v_{\text {rot }} / \sigma\right)^{*}<1$ along the major or minor axis. Deviations from perfect ellipses are negative $\left(a_{4}<0\right)$ and they often feature hot $\mathrm{X}$-ray gas $\left(9.8 \geq \log \left(L_{B}\right) \geq 11.2 \mathrm{~L}_{\odot}\right)$ or radio emission $(\sim 1020-1025 \mathrm{~W} / \mathrm{Hz})$. Their centres often show signs of kinematically peculiar/decoupled cores, which is believed to be a by-product of mainly stellar mergers. Deep HST imaging detected shallow (cuspy) core profiles (Faber et al. 1997).

- Discy E(d): Usually these are fainter galaxies $\left(M_{V} \gtrsim-20.5\right)$ which are sustained by ordered rotation along the minor axis $\left(v_{\text {rot }} / \sigma\right)^{*} \sim 0.2-1.5$. Variations of the isophotes are specified as $a_{4}>0$. Within the resolution of the Hubble Space Telescope (HST), high density power-law profiles in the galaxy centres were found but no signs of a distinct core was revealed. Kinematical peculiar cores and $\mathrm{X}$-ray emission from hot gas are quite rare $\left(\log \left(L_{B}\right) \lesssim 9.2 \mathrm{~L}_{\odot}\right)$. This together with the presence of a faint disc may suggested that dissipation was an essential ingredient during their formation, which includes also mergers of two spirals with dissipation involved.

Besides these two groups also intermediate types exist. Elliptical galaxies with luminosities between $-22.0 \lesssim M_{V} \lesssim-20.5$ are represented in both classes. Furthermore, many discy $L^{*}$ el-

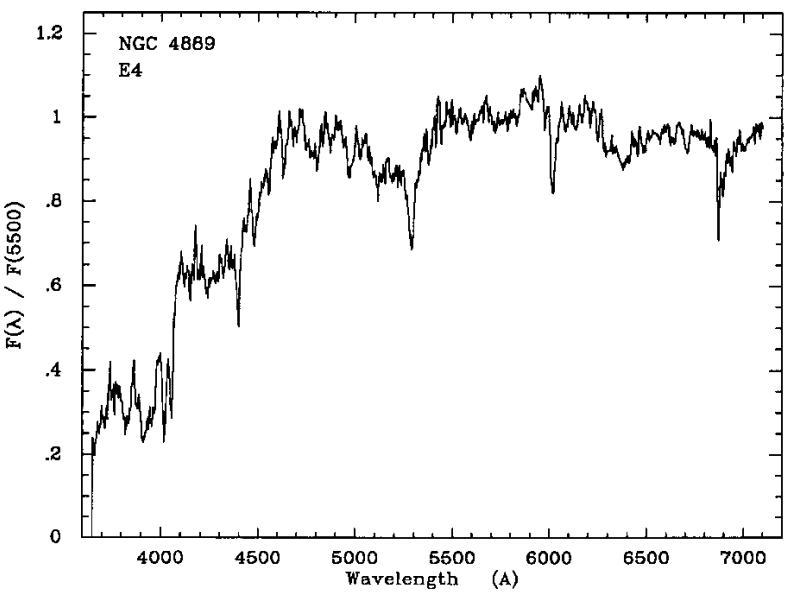

Figure 1.4: Spectrum of an elliptical galaxy from Kennicutt (1992).

lipticals contain faint discs which usually contribute a few per cent but sometimes up to approximately $30 \%$ of the total luminosity of the galaxy (Rix \& White 1990). Note that the sequence from boxy to discy ellipticals is not necessarily a continuous sequence (Kormendy \& Bender 1996).

Being aware of these different physical properties of discy and boxy ellipticals, several authors proposed different formation scenarios. By definition lenticular galaxies comprise a bulge and a clearly visible disc component. As a consequence of the disc for most cases the disciness parameter $a_{4}$ takes values $a_{4}>0$ and $a_{6} \neq 0$. Therefore, from a morphological point of view, S0 galaxies are very close to discy ellipticals. In this context it seems obvious to consider the Hubble tuning fork from discy $\mathrm{E}(\mathrm{d})$ over S0 to spiral galaxies as a continuous sequence of decreasing bulge-todisc ratios (Bender et al. 1992).

\subsubsection{Spectral Energy Distribution}

The morphological galaxy types along the Hubble sequence correlate strongly with the overall properties of the Spectral Energy Distribution (SED). From early to late-types the broad band colours become on average bluer. Generally, the 


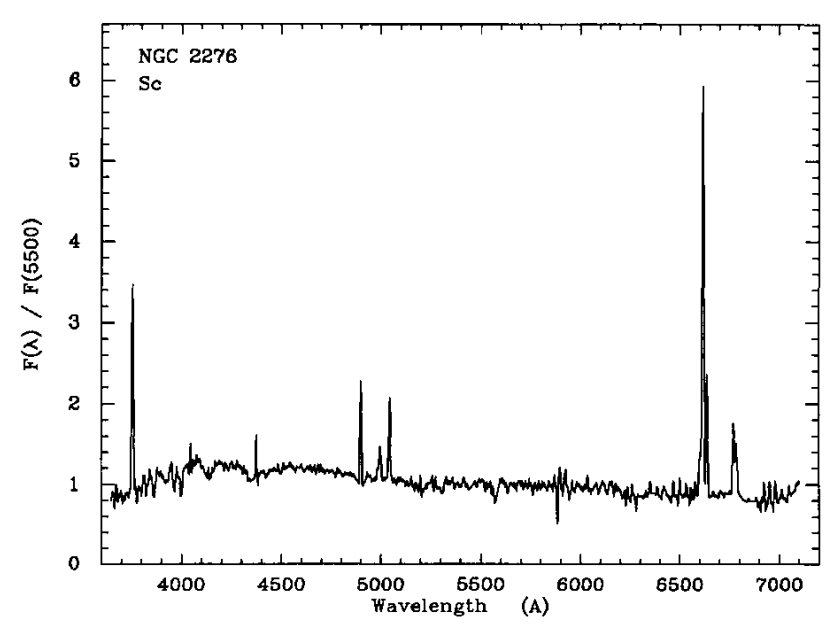

Figure 1.5: Spectrum of an Sc spiral from Kennicutt (1992).

nucleus of elliptical galaxies is redder than the outer regions. This colour gradient is considered being solely due to metallicity variations as for a given age a stellar population appears to be bluer when the metal abundance is lower. The colours of spirals show a very uniform radial distribution in the disc. Globally, their disc component becomes bluer with later-types, whereas the red central bulges of spiral galaxies feature similar colours to the ellipticals and S0 galaxies.

A similar view is gained from the properties of integrated optical spectra at rest-frame wavelengths. Early-type galaxies comprise strong absorption lines, in particular $\mathrm{CN}, \mathrm{Ca}$ $\mathrm{H}+\mathrm{K}, \mathrm{G}-$-band, $\mathrm{Mg}_{b}$ and the Balmer series $(\mathrm{H} \delta, \mathrm{H} \gamma \mathrm{H} \beta)$, with a characteristic break in their spectra, called the $4000 \AA$ break D4000. Emission lines are rare and only weak if present. The strength of emission lines, typically apparent via [OII] $3727, \mathrm{H} \beta[\mathrm{OIII}] 5007$ and $\mathrm{H} \alpha(+[\mathrm{NII}] 6548 / 6584)$, increases from early to late-type spiral galaxies. While a combination of age and metallicity in the stellar content of $\mathrm{E}+\mathrm{S} 0$ galaxies is responsible for the depths of absorption lines, the fraction of hot, high-massive stars in the spiral galaxy population correlates with the emission line strengths. Examples of

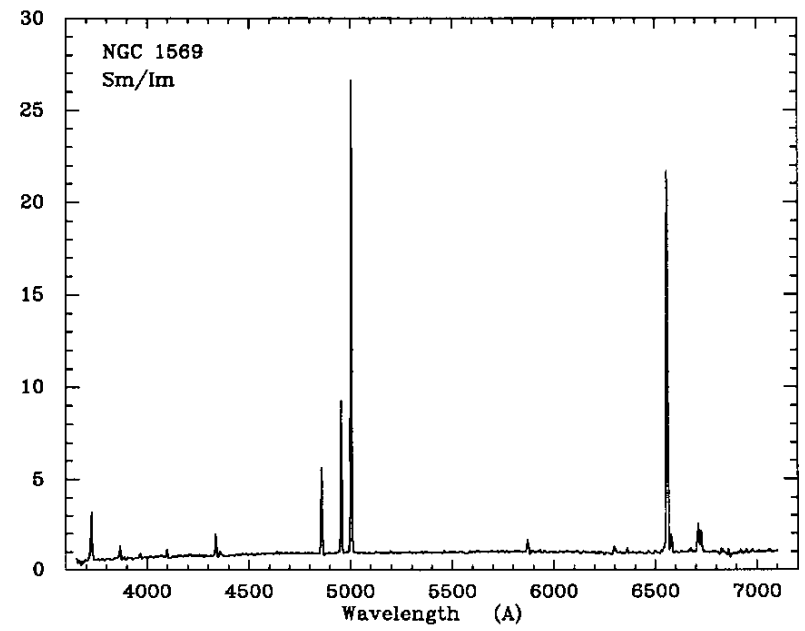

Figure 1.6: Spectrum of a very late-type galaxy (Sm/Im) from Kennicutt (1992).

a typical elliptical, an Sc and a very late-type $\mathrm{Sm} / \mathrm{Im}$ spiral taken from the Kennicutt (1992) catalog are shown in Figs. 1.4 to 1.6. Note that these spectra have been normalised to the flux at $5000 \AA$.

The colour trend seen for local galaxies along the Hubble sequence can be explained by looking at the Star Formation Rate (SFR) $\psi(t)$. If the Star Formation History (SFH) of $\psi(t)$ for a specific galaxy type is approximated by an exponential law of $\psi(t) \propto \mathrm{e}^{-t / \tau}$, where $\tau$ is the characteristic $e$-folding time (i.e. the timescale to turn a mass $M / e$ into stars, typically $\tau \approx 1-5 \mathrm{Gyr}$ ), then $\tau$ increases from early to late-types (Larson \& Tinsley 1978). The stellar populations of earlytype galaxies indicate a short intense star burst at high redshift, which is followed by a slow quiescent evolution. During this period, the populations become mainly the red, cool, very old population II giant stars, whereas the blue, very hot, high-mass stars are rapidly destroyed in supernovae explosions of Type Ia. The smooth radial colour distribution of spiral galaxies in the disc suggest that their star formation rate is constant independent on the amount of cold gas. Indeed, the SFR of spiral galaxies shows a shallower decrease or may be even a constant rate, which 
yields to hot, massive and young supergiant stars of population I and therefore blue colours.

However, today there is no doubt that at least some elliptical galaxies comprise young stellar populations. The situation gets even more complex as at higher redshifts post-starbust galaxies which are devoid of emission lines and therefore without any signs of star formation could be miss-classified as S0 galaxies. These socalled $\mathrm{E}+\mathrm{A}$ (or $\mathrm{k}+\mathrm{a})$ galaxies show very strong Balmer absorption lines (preferably in $\mathrm{H} \delta$ ) and contribute approximately $20 \%$ to the population in clusters at $z \sim 0.5$ (Poggianti et al. 1999).

\subsubsection{Kinematics}

With respect to the internal kinematics of galaxies, i.e. the motion of their stars and gas, two main classes can be distinguished. Early-type galaxies which comprise the hot, pressure supported spheroidal elliptical and lenticular galaxies, are stabilised by the random motion of the stars as expressed in their velocity dispersion $(\sigma)$. This regime is also valid for the dominant bulges of early spiral galaxies. For this reason, these systems are referred as Dynamically Hot Galaxies (DHGs, e.g. Dressler et al. 1987), where the velocity dispersion exceeds their rotation velocity $\left(V_{\text {rot }}\right)$ at all radii. In contrast, for spiral galaxy discs the movement of their stars and gas are stabilised by ordered rotation. Therefore, they are dynamically cold systems where the source of their angular momentum could be the result of tidal torques created during the process of disc formation (e.g., Silk 2000). Both quantities, the velocity dispersion and the rotation velocity, are a function of galactocentric radius.

Detailed studies of the kinematics of elliptical galaxies in measuring the velocity $V_{\text {rot }}$ and dispersion $\sigma$ as a function of radius from the galaxy centre exist only for a minority of nearby galaxies. In most cases, the central galaxy profile with the systemic radial velocity $v_{\text {rad }}$ defined by the position of lines and the profile width giving the dispersion at the centre $\sigma_{0}$ can be derived.
These difficulties are based on the fact that the measurements of rotation curves in early-type galaxies rely solely on absorption lines which are usually relatively weak. As the luminosity decreases very rapidly from the centre, the derivation of rotation curves are limited to radial distances of approximately $r<10 \mathrm{kpc}$ (Davies et al. 1983). Nevertheless, these measurements are sufficient to detect the maximal rotation velocity $V_{\max }$ which is generally located at $r \sim 2 \mathrm{kpc}$. Beyond this characteristic radius the rotation curve declines slowly. Furthermore, as projection effects are impossible to correct for elliptical and S0 galaxies, the true rotation velocity remains inaccessible.

The internal velocity dispersions of early-type galaxies give insight into two basic properties, their galaxy mass and their formation. A considerable fraction of the kinetic energy in these systems is distributed in random motions of the stars, which is expressed via the (stellar) velocity dispersion. Applying the virial theorem as

$$
M \bar{v}^{2}=G M^{2}\left(3 R_{\mathrm{e}}\right)^{-1}
$$

where $\bar{v}^{2}$ is the mean square of velocities weighted by the mass of the stars and $R_{\mathrm{e}}$ being the radius containing half of the light (or mass) of a system, the galaxy mass $M$ can be derived using this "chaotic" distribution of velocities. Moreover, as the velocity dispersion should have not been changed after the initial collapse of the galaxy, it provides information about the processes which played a role during the formation of the stellar system. The stellar kinetic energy is a measure of the binding energy per particle and hence gives the rate of dissipative processes which occurred during the collapse phase (Fish 1964). For this reason, the velocity dispersion is a key parameter which must be accounted for in any galaxy formation theory.

Faber \& Jackson measured the internal velocity dispersions of 25 elliptical galaxies (Faber \& Jackson 1976) and discovered that the luminosities of these galaxies are tightly correlated with 


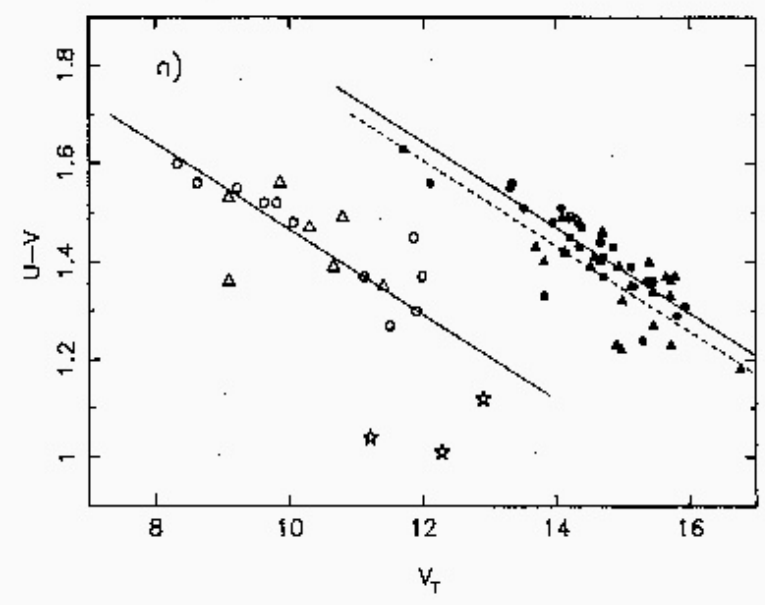

Figure 1.7: Colour-Magnitude Relation for Virgo and Coma cluster galaxies from Bower et al. (1992). Open and filled symbols are Virgo and Coma galaxies, respectively. Elliptical and S0 are denoted by circles and triangles, S0/a and later types by stars. Solid lines represent the median fits to the relation and the scatter around the fit for early-type galaxies amounts to only $0.05 \mathrm{mag}$. The dashed line shows the expected Coma cluster relation predicted from the Virgo zeropoint plus a relative distance modulus.

their spread in velocities according to

$$
L \propto \sigma_{0}^{\alpha},
$$

where $L$ is the luminosity and $\sigma_{0}$ is the central stellar velocity dispersion of an early-type galaxy. In the $B$-band the power-law exponent in the so-called Faber-Jackson Relation (hereafter FJR) yields $\alpha \approx 4$, which can be easily explained if galaxies are not enveloped by darkmatter haloes. Usually, the velocity dispersions of elliptical and S0 galaxies increase with absolute magnitude as $L_{B} \propto \sigma_{0}^{4}$. The central velocity dispersion of the bulges of spirals also follows this relation. However, the slope is not valid for all luminosities, e.g., the bright $\mathrm{cD}$ galaxies located in the centres of galaxy clusters have a $\sigma_{0}$ which is slightly less than the $\sim L^{4}$ curve. Due to the dependence of $L$ and $\sigma$, small errors in the velocity dispersions propagate into large errors in absolute magnitude. For this reason, both the velocity dispersions and the luminosities have to be measured with high accuracy. As the FJR is a strong correlation between the two observables it provides a powerful test on the formation and evolution of elliptical and lenticular galaxies. As for distant galaxies the apparent sizes decrease, a measurement of the velocity dispersion is not restricted to the central parts of the galaxy but contains a larger area or maybe the whole size of the system. For this reason, when distant galaxies are compared to galaxies in the local Universe with central velocity dispersions $\left(\sigma_{0}\right)$, the measured velocity dispersions have to be corrected for aperture size. More details on the kinematics, in particular the derivation of velocity dispersions, will be discussed in chapter 5 on page 93 .

\subsection{Scaling Relations}

Since a long time it is well known that several observable properties of early-type galaxies which characterise the dynamical state, size or chemical composition correlate with their luminosity. Typical parameters include the effective radius $R_{e}$ (Fish 1964), the central velocity dispersion $\sigma_{0}$ (Faber \& Jackson 1976), the integrated colours (Bower et al. 1992) or metal absorption line indices such as the $\mathrm{Mg}_{2}$ index (Bender et al. 1993). Apart from the Faber-Jackson Relation which was already presented in the previous section, in the following, a short review of the basic empirical relationships together with their involved quantities will be given.

\subsubsection{The Colour-Magnitude Relation}

Early-type galaxies in clusters at low-redshift $(z<0.05)$ form an extremely homogeneous class of galaxies. It has been known for a long time that for early-type galaxies exists a fundamental relation between their colour and magnitude, referred to as the colour-magnitude relation (e.g., Sandage \& Visvanathan 1978). The relation, which is often also called red-sequence, implies 
a link between the mass of the stellar population and its age or metallicity. Presently, the latter parameter is being favoured because highredshift clusters have bluer colour-magnitude relations with a small scatter than in local clusters (e.g., Kodama \& Arimoto 1997).

In the local Universe, early-type galaxies obey a tight colour-magnitude relation (CMR) with a homogeneity across various clusters. Brighter early-type galaxies are redder than less luminous ones. At given absolute magnitude, the dispersion in optical and infrared colours is small with less than $0.05 \mathrm{mag}$ (Bower et al. 1992). Since the colours of the stellar populations evolve with age, the width of the CMR puts strong constraints on the age scatter at a given absolute magnitude. For the local Coma and Virgo clusters the rms scatter in age was less than $15 \%$, which implies that these galaxies have most likely formed at high-redshift of $z>2$. A widely supported interpretation suggests that early-type galaxies are more metal rich and that the star formation processes in cluster early-types have taken place and already ceased at an early cosmic time that the resulting effects of a possible spread in their formation epoch are not visible through their broad-band colours. Evidence for this is accumulated by the redshift evolution of the CMR. The almost constant slope of the CMR with redshift indicates that this scaling relation is not an age-mass sequence (e.g., van Dokkum et al. 2000). Moreover, the small scatter of the CMR in massive clusters at high-redshift out to $z=1.2$ suggest that the stellar populations of early-type galaxies are uniformly old $z_{\mathrm{f}}>2$ and quiescent (Ellis et al. 1997; Lidman et al. 2004). However, there are some hints for a flatter colour-magnitude sequence at high-z (van Dokkum et al. 2001a) and a possible evolution in the scatter of the colours (Holden et al. 2004). This picture gets further support by a modest evolution of the colour-gradients in cluster galaxies from $z \sim 0.4$ to $z=0$ in the VRI HST filter passbands (Saglia et al. 2000). This homogene- ity of early-type galaxies in clusters needs to be considered with some considerations. The population of early-type galaxies in distant clusters at high-redshift does not only comprise possible progenitors of present-day early-type galaxies. Some progenitors at $z \sim 1$ might not have yet been accreted onto the cluster or might not be visually detectable due to ongoing morphological transformations from late-type star forming to early-type passive galaxies. For this reason, the tightness of the CM sequence in clusters at high$z$ could partly represent a selection effect (the so-called progenitor bias; van Dokkum \& Franx 2001b). Most dramatic scenarios are rather unlikely due to the evolution of the morphologydensity relation (Dressler 1980; Dressler et al. 1997).

Up to now, there are only a few studies which focus on the CMR for distant early-type field galaxies (Schade et al. 1999; Bell et al. 2004). Results suggests that there is a CM sequence in the field out to $z \sim 1$, albeit with a considerably larger scatter than in clusters.

\subsubsection{The Kormendy Relation}

In 1977, John Kormendy published a pioneering study on the structural parameters of 35 elliptical galaxies (Kormendy 1977). The surface brightness and the size of an elliptical galaxy are related as

$$
\left\langle\mu_{\mathrm{e}}\right\rangle_{X}=\alpha+\beta \log R_{\mathrm{e}}
$$

where $\left\langle\mu_{\mathrm{e}}\right\rangle_{X}$ is the surface brightness in the passband $X$ in units of mag/ $\operatorname{arcsec}^{2}$ and $R_{\mathrm{e}}$ denotes the effective radius, given in kiloparsecs. $R_{\mathrm{e}}$ is also referred as the radius which contains half of the total galaxy light distribution. Because of the colours and colour gradients in the stellar populations of early-type galaxies the quantities of $\left\langle\mu_{\mathrm{e}}\right\rangle$ and $R_{\mathrm{e}}$ depend on the observed wavelength. Kormendy measured for the zeropoint $\alpha=19.74$ and for the slope $\beta=3.02$ in the Johnson $B$-band. The Kormendy Relation 
(hereafter KR) holds for both types of earlytype galaxies, elliptical as well as S0 galaxies. In general, brighter, i.e. more luminous galaxies, have larger effective radii and fainter surface brightnesses. With a relatively large dispersion of $0.28 \mathrm{mag}$ in the $B$-band for normal ellipticals the measurements of both structural parameters have to be necessarily performed in a very accurate way. Subsequent studies demonstrated that the magnitude-size relation holds for bright early-type galaxies in different passbands from the optical to the near-infrared (Hoessel et al. 1987; Lubin \& Sandage 2001; La Barbera 2003). Based on Virgo cluster galaxies, Capaccioli, Caon \& D'Onofrio (1992) found that the ellipticals, early-type dwarfs, and bulges of S0s and of spirals form two distinct families in the plane of the effective parameters: Bright early-type galaxies follow the KR which may have undergone a merger phenomenon, whereas a so-called 'ordinary' family comprising galaxies fainter than approximately $M_{B}=-19.3$ are more diverse and heterogenous. This effect is also well-known as the "dichotomy" between early-type galaxies. To solve this issue, Graham \& Guzmán 2003 suggested that the dichotomy is only valid between bright and dwarf early-types with a continuous transition plane of structural parameters between the sub-classes. Differences in their properties and in the slope of their relations can be explained by a change in surface brightness profile slope $\left(\gamma^{\prime}\right)$ with their luminosity.

The magnitude-size relation is the projection of the Fundamental Plane (FP) along the velocity dispersion onto the photometric plane and a very useful probe to study the luminosity evolution of early-type galaxies. As this relation does not comprise galaxy kinematics, it can be studied to fainter magnitudes and therefore higher redshifts. A number of studies have utilised the $\mathrm{KR}$ to investigate the luminosity evolution of $\mathrm{E}+\mathrm{S} 0$ galaxies up to redshifts of one (Ziegler et al. 1999; Lubin \& Sandage 2001; Treu et al. 2001b; La Barbera 2003; Holden et al. 2005). Most previous works suggested that the evolution of the zero-point of the KR can be explained by a pure Tolman signal which is superposed to the luminosity evolution of stellar populations having a high formation redshift $\left(z_{\mathrm{f}}>2\right)$. The Tolman test predicts that the bolometric surface brightness magnitudes decrease as $(1+z)^{4}$ and it has been applied as an observational verification that redshifts really correspond to an expansion of the Universe, and not to an once assumed phenomena of light dimming (Sandage \& Perelmuter 1990). A drawback of the Kormendy relation is that the samples have larger scatter than in the FP because they rely only on the photometric measurements and thus are more affected by selection biases (Ziegler et al. 1999). At $z=1.2$ the observed scatter of cluster early-type galaxies is $0.76 \mathrm{mag}$ around a fixed size (Holden et al. 2005). Nevertheless, the amount of luminosity evolution found for the Kormendy relations are in good agreement with results obtained by the evolution of colours or from the FP of early-type galaxies.

\subsubsection{The Fundamental Plane}

In the search of a "second parameter" to explain the relatively large scatter of the correlations between different parameters mentioned in the beginning of this section, large spectrophotometrical surveys during the 1980s discovered a relationship, called the Fundamental Plane. This correlation represents a refinement of the FaberJackson Relation and is a powerful tool to measure the star formation history of early-type galaxies at a given mass by studying the evolution of the Fundamental Plane with redshift.

In a three dimensional parameter space, defined by three observables, the effective radius $R_{\mathrm{e}}$, effective surface brightness within $R_{\mathrm{e}},\left\langle\mu_{\mathrm{e}}\right\rangle$, and velocity dispersion $\sigma$, the Fundamental Plane (FP) establishes a tight correlation (Djorgovski \& Davis 1987; Dressler et al. 1987) in the fol- 
lowing form:

$$
\log R_{\mathrm{e}}=\alpha \log \sigma+\beta\left\langle\mu_{\mathrm{e}}\right\rangle+\gamma,
$$

where $\alpha$ and $\beta$ are the slopes and $\gamma$ denotes the intercept. The half-light radius $R_{\mathrm{e}}$ is given in $\mathrm{kpc}, \sigma$ is in $\mathrm{km} \mathrm{s}^{-1}$ and $\left\langle\mu_{\mathrm{e}}\right\rangle$ is in units of mag $\operatorname{arcsec}^{-2}$. This empirical relationship relates galaxy structure and size $\left(R_{\mathrm{e}}\right.$ and $\left.\left\langle\mu_{\mathrm{e}}\right\rangle\right)$ to kinematics $(\sigma)$. As the Hubble constant enters the relation via the effective radius in $\mathrm{kpc}$, the value of $\gamma$ depends on $H_{0}$. In the following, the physical origin of the FP will be outlined as well as its connection to the mass-to-light ratio and application to constrain the formation and evolution of elliptical and S0 galaxies.

The actual existence of the FP is a remarkable matter of fact. Any galaxy formation and evolution theory must account for the tightness and universality of the FP, which amounts along the edge-on projection to only $0.08 \mathrm{rms}$ in $\log R_{e}$ (Bernardi et al. 2003). Thanks to this small intrinsic scatter of the local FP, the formation and evolution of $\mathrm{E}+\mathrm{S} 0$ galaxies can be constrained with high precision.

In general, FP studies presume that $\mathrm{E}+\mathrm{S} 0$ galaxies are a homologous group, i.e., that they exhibit a similar structure. Under this assumption the total galaxy mass $\mathcal{M}$ (including dark matter if present) is proportional to its virial mass $M$ as (Treu et al. 2001b):

$$
M \equiv \sigma^{2} R_{\mathrm{e}} G^{-1} \cdot C=\mathcal{M}
$$

where the constant $C$ depends on the mass, i.e., weak homology holds. Let us further define an effective luminosity $L$

$$
\log L=-0.4\left\langle\mu_{\mathrm{e}}\right\rangle+2 \log R_{\mathrm{e}}+\log 2 \pi .
$$

Based on the definitions of the equations 1.5 and 1.6, the (effective) mass-to-light $(M / L)$ ratio can be easily deduced in terms of the FP as $M / L \propto 10^{0.4\left\langle\mu_{\mathrm{e}}\right\rangle} \sigma^{2} R_{\mathrm{e}}^{-1}$. By comparing the $M / L$ ratio to the value as predicted by the $\mathrm{FP}$ at a given effective radius and velocity dispersion,

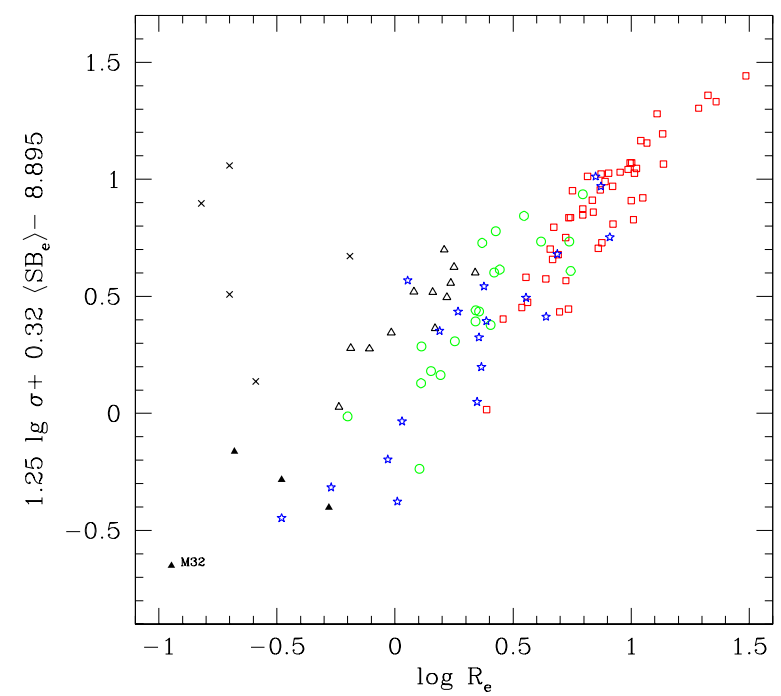

Figure 1.8: Fundamental Plane relation for dynamically hot galaxies from Bender et al. (1992). Elliptical galaxies are ordered according to their $B$ band luminosity, giant $\mathrm{E}$ (red squares), intermediate $\mathrm{E}$ (green circles), bright dwarf $\mathrm{E}$ (open triangles), spiral bulges (blue stars), compact E (filled triangles, e.g., M32) and dwarf spheroidals (crosses). Note that $M_{B}$ encompasses from giant to compact ellipticals approximately 8 orders of magnitudes and thus is not an intrinsic property of a few specific objects.

the effective surface brightness can be eliminated which yields

$$
M / L \propto 10^{-\frac{\gamma}{2.5 \beta}} \sigma^{\frac{10 \beta-2 \alpha}{5 \beta}} R_{\mathrm{e}^{\frac{2-5 \beta}{5 \beta}}} .
$$

As the scatter of the FP can be translated into a scatter in $M / L$ the tightness of the FP implicates a very low scatter in $M / L$ for $\mathrm{E}+\mathrm{S} 0$ galaxies, too. The evolution of the Fundamental Plane can be expressed in terms of its zero-point $\gamma$ that varies as a function of redshift $z$. So far, no dramatic change in the slopes with redshift were reported. However, an accurate measurement is tough as large samples are required and intrinsic effects such as completeness corrections and fitting methods have to be accounted for (Kelson et al. 2000b). If a galaxy sample at $z>0$ which observables are characterised by a superscript $i$ is considered, the offset of the mass-to-light ratio 
from the local value can be addressed via

$$
\begin{gathered}
\Delta \log (M / L)^{i}=\Delta\left(\frac{10 \beta-2 \alpha}{5 \beta}\right) \log \sigma^{i} \\
+\Delta\left(\frac{2-5 \beta}{5 \beta}\right) \log R_{\mathrm{e}}^{i}-\Delta\left(\frac{\gamma^{i}}{2.5 \beta}\right),
\end{gathered}
$$

where $\Delta$ describes the difference of the quantities at two different redshifts and $\gamma^{i}$ is $\log R_{\mathrm{e}}^{i}-$ $\alpha \log \sigma^{i}-\beta\left\langle\mu_{\mathrm{e}}\right\rangle^{i}$ (Treu et al. 2001b). Under assumption that the FP coefficients $\alpha$ and $\beta$ are constant the evolution of the $M / L$ is independent on the mass $M$, and by adopting that there is no structural and dynamical evolution (i.e. the effective radius $R_{\mathrm{e}}$ and the velocity dispersion $\sigma$ are constant), the evolution of $\Delta \log (M / L)^{i}$ depends only on the evolution of the parameter $\gamma^{i}$, hence it follows

$$
\Delta \log (M / L)^{i}=-\frac{\Delta \gamma^{i}}{2.5 \beta} .
$$

By measuring $\gamma^{i}$ for a number of galaxies at higher redshift and by comparing it to the intercept of the local Universe, the average evolution of $\Delta \log (M / L)$ can be derived as

$$
\langle\Delta \log (M / L)\rangle=-\frac{\langle\Delta \gamma\rangle}{2.5 \beta} .
$$

As the evolution of this effective $M / L$ ratio corresponds to an evolution of the dynamical (stellar and dark matter) $M / L$ ratio, the application of the FP becomes a powerful diagnostic tool to assess the evolution of the stellar populations in the galaxies under consideration. A practical realisation to observational data will be demonstrated in chapter 7.2 on page 168 .

\subsection{Galaxy Evolution}

\subsubsection{Theory meets Observations}

Theoretical galaxy formation models based on a hierarchical structure growth use numerical $N$ body computations to simulate the gravitational clustering of DM haloes from the initial density perturbations up to the present-day Universe. These CDM simulations can reproduce the large scale structure which range from cluster of galaxies and superclusters up to $\sim 100 \mathrm{Mpc}$, such as the Great Wall (Geller \& Huchra 1989) but also account for the voids in between. The hierarchical scenario predicts that early-type galaxies in galaxy clusters were assembled at an early epoch at high redshift $(z \gtrsim 2)$ because the redshift of a collapse on galaxy scales is heavily driven by the presence of the surrounding overdensity. Therefore, galaxies forming out of the highest peaks of primordial density fluctuations which collapse at $z \gtrsim 2$ quickly merge together within groups and their star formation gets truncated when they have used up their gas. However, only for the dense environments of rich clusters, cluster galaxies experienced their last major merger at $z \sim 1-2$ (Kauffmann 1996). Because of ongoing merging events, the population of early-type galaxies in lower-densities should be more diverse. For individual galaxies, the hierarchical model predicts that low-mass galaxies were formed first in the early cosmos and larger massive galaxies subsequently build up in merging or accretion of smaller systems at a later stage. Semianalytical CDM models derive explicit age variations for early-type cluster galaxies and $\mathrm{E}+\mathrm{S} 0$ s in low-density regions. For clusters, models predict ellipticals to have a mean luminosity-weighted age of 9.6 Gyrs and lenticular galaxies to be younger by $~ 1$ Gyr (Baugh et al. 1996; Cole et al. 2000). Both types, ellipticals and S0 galaxies, indicate a weak trend that fainter galaxies $\left(-17.5>M_{B}>-20.1\right)$ are older. On the contrary, for early-type galaxies in low-density regions the hierarchical cluster models predict a considerably broader age spread over a larger luminosity range and mean luminosity-weighted ages of $~ 5.5$ Gyrs. In addition, brighter field galaxies should feature on average younger ages and comprise more solar element abundance ratios than their cluster representatives (Thomas, Maraston \& Bender 2002). 
A wide range of formation histories is suggested for galaxy clusters of similar present-day richness class (Kauffmann \& Charlot 1998). For the timescale of the cluster accumulation strong radial age gradients are predicted, whereas the spread in metallicities between cluster and field galaxies should be similar. This would imply that scaling relations such as the CMR and FP should be more diverse with a larger scatter for the field, which is not endorsed by observations (Schade et al. 1999; van der Wel 2005). However, in support of the hierarchical scheme comes the observed large increase of the merger fraction with redshift (van Dokkum et al. 1999). Further empirical evidence from a dynamical perspective is accumulated by interacting and ongoing merging events of galaxies at low redshift and the detection of ellipticals with disturbed morphologies, such as kinematically peculiar (decoupled or counter-rotating) cores, dust lanes, ripples et cetera (e.g., Franx \& Illingworth 1988; Kormendy \& Djorgovski 1989), or the excess of blue galaxies with redshift regarding to the coloursequence of passive elliptical galaxies (ButcherOemler effect, Butcher \& Oemler 1984). On the other hand, models have still problems with the high angular momentum of spiral galaxies (Cole et al. 2000) and too low predicted $[\alpha / \mathrm{Fe}]$ ratios for early-type galaxies (Thomas, Maraston \& Bender 2002). In particular, the observations find that the $[\alpha / \mathrm{Fe}]$ enhancements are increasing with velocity dispersions whereas the models suggest the opposite trend.

In the classical galaxy formation scenario earlytype galaxies are formed dissipationally in a rapid, single burst of star formation at high redshift $(z \gtrsim 2)$. After this initial period, galaxies evolve passively without merging and yield a population of old ellipticals where their mean metallicity scales with galaxy mass (Eggen et al. 1962; Larson 1975). As already outlined in section 1.1, SN feedback and the chemical enrichment of the ICM are crucial ingredients in the current monolithic formation scenarios. Based on the stellar population properties of local ellipticals, the estimated timescales required to develop a wind are relatively short with $\leq 1 \mathrm{Gyr}$ (Pipino \& Matteucci 2004). These models can explain the majority of empirical constraints relative to the stellar populations of early-type galaxies, such as $\mathrm{CMR}, \mathrm{Mg}-\sigma$ relation, $\mathrm{FP}$ and the increase of the $[\alpha / \mathrm{Fe}]$ ratio with galactic mass, the existence of metallicity gradients and the characteristic $r^{1 / 4}$ surface brightness profile.

Qualitatively the two scenarios seem to predict opposite trends. The monolithic collapse model suggests that ellipticals form on shorter timescales than spirals and the hierarchical scheme predicts that spirals form before ellipticals which continue to assemble until recent times.

\subsubsection{High versus Low Densities}

In the nearby Universe, inconsistent results have been acquired regarding any possible difference between early-type galaxies in high densities (galaxy clusters) and $\mathrm{E}+\mathrm{S} 0$ galaxies in low density environments (isolated field objects). For example, de Carvalho \& Djorgovski (1992) derived from a subset of cluster and field early-type galaxies taken from the "Seven Samurai" group (Faber et al. 1989) and a second sample drawn from Djorgovski \& Davis (1987) that field ellipticals show a larger scatter in their properties indicating that they consist of younger stellar populations than cluster galaxies. Bernardi et al. (1998) analysed a large sample of ENEAR field and cluster galaxies and found slight zeropoint changes in the $\mathrm{Mg}_{2}-\sigma$ relation. They explain this as an age difference, with field objects being younger by $\sim 1$ Gyr. However, they conclude that the bulk of stellar populations of $\mathrm{E}+\mathrm{S} 0$ in both environments has been formed at high redshifts $(z \gtrsim 3)$. James \& Mobasher (1999) investigated near-infrared (NIR) spectra of 50 ellipticals in three nearby clusters and in the field, using the $\mathrm{CO}(2.3 \mu \mathrm{m})$ absorption feature to explore the presence of an intermediate- 
age population. They detected no stronger CO absorption for the field ellipticals. Very isolated field ellipticals show a very homogenous population and a small range of metallicity with no sign of recent star formation (SF). In groups, ellipticals have a wide range in metallicity, mostly showing evidence for an intermediate-age population, whereas in rich clusters they exhibit intermediate properties in metallicity and $\mathrm{CO}$ absorption. Kuntschner et al. (2002) detected in a sample of nine local early-type galaxies (five morphologically disturbed) in low-density environments (LDR) no strong ongoing SF. The results were compared to cluster E+S0s in Fornax. The ages of the LDR galaxies are spread over a broad distribution, similar to that of Fornax S0 galaxies and being on average by 2-3 Gyrs younger than the E+S0s in Fornax. These LDR galaxies indicate 0.2 dex higher metallicities and super solar $\mathrm{Mg} / \mathrm{Fe}$ ratios (in conflict with semianalytical models), which suggests that the formation of $\mathrm{E}+\mathrm{S} 0$ galaxies in low-densities continues to $z \lesssim 1$, whereas in clusters most stars have already been generated at $z \gtrsim 2$. Recently, Sánchez-Blázquez et al. (2003) studied 98 E+S0 galaxies in the field and in clusters and found higher $\mathrm{C} 4668$ and $\mathrm{CN}_{2}$ absorption line strengths for the field population. They interpret this as a difference in abundance ratios arising from different star formation histories. However, both field and cluster E+S0s show similar relations in $\mathrm{Mg}_{b}-\sigma$ and $\langle\mathrm{Fe}\rangle-\sigma$.

At higher redshift differences between field and cluster galaxies should become more apparent. Recent results from studies. based on the Fundamental Plane at intermediate redshift $(z \leq 0.5)$, indicate no significant variations between the cluster and field early-type populations (van Dokkum et al. 2001c; Treu et al. 2001b; Rusin \& Kochanek 2005). With respect to the mean age of these populations, field galaxies seem to comprise slightly younger stars than the cluster population, whereas the majority of stars must have formed at a much higher redshift of $z_{\mathrm{f}}>2$. How- ever, at higher redshift $(z \sim 0.7)$, some investigations derive a significant offset between field and cluster galaxies (Treu et al. 2002; Gebhardt et al. 2003). Differences might mainly arise due to the low number of analysed galaxies. As the selection criteria differ strongly among these studies, the samples might also be affected by the progenitor bias (van Dokkum \& Franx 2001b).

\subsubsection{Elliptical and S0 Galaxies}

Over the last years a multiplicity of investigations of distant rich clusters have been performed (Ellis et al. 1997; Dressler et al. 1997; Kelson et al. 2000b; van Dokkum et al. 2000; Ziegler et al. 2001a; Wuyts et al. 2004). Most of these studies can be reconciled with the picture of a monolithic collapse with a high redshift formation of the stellar populations of E+S0 galaxies. Results from these distant clusters have not found any differences in the properties of $\mathrm{E}$ and S0 galaxies (e.g., Kelson et al. 2000b). Recently, in a re-analysis of two high redshift clusters at $z=0.58$ and $z=0.83$ no environmental dependence of the FP residuals was detected (Wuyts et al. 2004). When looking at the residuals of the $\mathrm{FP}$, and suggesting that the residuals correlate with environment, it is difficult to distinguish if this effect is due to changes in velocity dispersion, size or luminosity of the galaxies. Selection effects have strong influence on the parameters and can also mimic possible correlations. In a study of $\sim 9000$ early-type galaxies from the SDSS (Bernardi et al. 2003), a weak correlation between the local density and the residuals from the FP was revealed, in the sense that the residuals in the direction of the effective radii increase slightly as local density increases. However, the offset is quite small and subject to selection and evolutionary effects. The open question still to address is, how this dependence occurs.

Looking at the morphology, the formation and evolution of lenticular galaxies is different and stands in contrast to elliptical galaxies. Deep studies of galaxies in distant rich clusters using 
the WFPC2 camera onboard the Hubble Space Telescope revealed that $\mathrm{S} 0$ galaxies show a rigorous evolution with redshift in these dense environments (e.g., Dressler et al. 1997). Although S0 galaxies form the dominant population in local rich clusters of $\sim 60 \%$, at intermediate redshift $(z \sim 0.5)$ spiral and disturbed galaxies compose the major part of the luminous galaxies, whereas S0 galaxies are less abundant (1020\%). Schade et al. (1999) studied early-type field galaxies at intermediate redshifts $(z \sim 0.5)$ and detected $[\mathrm{OII}] \lambda 3727$ emission lines in about $1 / 3$ of these galaxies, which indicates ongoing star formation. Furthermore, in about the same fraction of faint spheroidal Hubble Deep Field galaxies significant variations of internal colours were found, frequently showing objects with blue cores (Menanteau et al. 2001). The authors conclude that at $z \sim 1$ about half of the field S0 galaxies show clear signs of star formation activity. Some evidence for young populations in local S0 galaxies was recently found by Mehlert et al. (2003). Based on high signal-to-noise spectroscopy of early-type galaxies in the Coma cluster, two families of S0 galaxies were detected, one group with old ( 10 Gyrs) stellar populations comparable to ellipticals and a second one with very young average ages ( $\sim 2$ Gyrs) and weaker metallic lines.

These results seem to imply that galaxy transformation via interaction is an important phenomenon in clusters. Due to the large velocity dispersion mergers are less frequent in rich clusters, whereas effects such as ram-pressure stripping by the hot intra cluster medium or tidal interactions between the galaxies are more likely. A unique mechanism for the transformation into S0 galaxies is still missing to explain the strong decrease in the frequency of S0's since the last 5 Gyrs $(z \sim 0.5)$. A possible scenario is that field spiral galaxies falling into the cluster centre experience a starburst phase, resulting in the Butcher-Oemler effect. Ram-pressure stripping by the ICM (also maybe through tidal stripping) over a short time-scale of less than one Gyr, could cause the wide-spread and rapid decline in star formation leading to post-starburst galaxies and red passive spiral galaxies (Barnes \& Hernquist 1992). Harassment by the tidal field of the galaxy cluster and high speed encounters have a non negligible effect on the following passive evolution of a galaxy by removing stars from the disc which may end up in an S0 galaxy (Moore et al. 1996; Poggianti et al. 1999).

In terms of structural parameters, elliptical galaxies comprise not a single homogenous group of galaxies but encompass two different groups, discy and boxy ellipticals (see section 1.2.2 for details). The shape of these galaxies is very important since it correlates with other physical properties, such as luminosity, shape, rotation (axis) and core profile. Recently, the origin of discy and boxy ellipticals was investigated (Naab \& Burkert 2003). Equal-mass mergers result in an anisotropic system with slow major axis rotation and a large amount of minoraxis rotation (boxy elliptical), whereas unequalmass merger of mass ratio $3: 1$ and $4: 1$ lead to a rotationally supported system with only a small rotation along the minor-axis (discy elliptical). Generally, giant high-luminosity ellipticals preferably contain boxy isophotes, whereas lowluminosity ellipticals comprise a discy structure, which might be a hint for different evolutionary paths. At intermediate redshift it is impossible to distinguish between discy and boxy galaxies. However, with respect to the large sample in this thesis low-luminosity elliptical galaxies can be separated from high-luminosity ones and possible differences in their evolution can be explored. Results of such a comparison would give conclusions if the two types of ellipticals might undergo different formation scenarios. 
Table 1.2: Overview of samples in this thesis.

\begin{tabular}{rllcrrr}
\hline $\begin{array}{r}\text { Density } \\
{\left[\mathrm{N} / \mathrm{Mpc}^{2}\right]}\end{array}$ & Locus & Tel./Instrument & Sample & $\langle z\rangle$ & $\begin{array}{r}N_{\text {obj }} \\
\text { E+S0 }\end{array}$ & $\begin{array}{r}N_{\text {obj }} \\
\text { FP }\end{array}$ \\
\hline$\sim 100-200$ & Rich Cluster & CA 3.5/MOSCA & A 2390 & 0.23 & 48 & 14 \\
$\sim 100-200$ & Rich Cluster & WHT/LDSS2 & A 2218 & 0.18 & 48 & 20 \\
$\sim 50-100$ & Poor Cluster & CA 3.5/MOSCA & Cl 0849/Cl 1701/Cl 1702 & 0.23 & 27 & 10 \\
$\sim 1$ & Field & VLT/FORS & FDF/WHDF & 0.40 & 24 & 21 \\
\hline
\end{tabular}

${ }^{\star}$ From Ziegler et al. (2001a).

\subsection{Motivation and Overview}

Scaling relations are powerful tools to analyse the formation and evolution of early-type galaxies. A combination between kinematic and stellar population properties is an even superior approach to investigate in great detail the dynamics and stellar content of these galaxy types as well as to constrain their predicted evolution scenarios. With increasing look-back time, theoretical galaxy formation models suggest a variety of evolutionary paths depending on the environment of a galaxy. To give more insight into the formation epoch of early-type galaxies, the study described in this thesis is based on three homogenous data sets looking into different environmental galaxy densities. For each sample several criteria have to be fulfilled:

- High signal-to-noise spectroscopy to study the internal kinematics and stellar populations of early-type galaxies over a large range of masses.

- Detailed morphological and structural information based on HST/WFPC2 and HST/ACS images for $25 \%$ of all galaxies.

- A large coverage of various environments to reveal differences within the samples.

- Wide field-of-view to explore any radial dependence of the galaxy properties in clusters complemented by a wide range in luminosity to distinguish between brighter and fainter galaxies.

- Large number of galaxies for each project at a redshift of $z \sim 0.2$, corresponding to a look-back time of $\sim 3$ Gyrs, equivalent to $20 \%$ the age of the Universe ( 14 Gyrs according to recent achievements (e.g., Spergel et al. 2003).

Using the Calar Alto $3.5 \mathrm{~m}$ and the ESO/VLT $8.2 \mathrm{~m}$ telescopes in Multi Object Spectroscopy mode, spectra of 121 early-type galaxies and 28 kinematic stellar templates were taken in total over a period of time between September 1999 to October 2002. Table 1.2 gives an overview of the samples which are the subject of this thesis. Besides the density and environment specifications, the instrumentation, median redshift and the total number of early-type galaxies for each data set are listed. In the last column, galaxies within the HST field-of-view are shown, which have kinematic plus structural characteristics and enter the Fundamental Plane relation. Note that only spectroscopic confirmed cluster members are considered. The Abell 2218 cluster analysis was already outlined in Ziegler et al. (2001a), but is also shown as the data will be combined at a later stage with the Abell 2390 cluster to study a total of $\sim 100$ early-type galaxies in the densest environments of rich galaxy clusters. The basic aims of this project were the following: 
- Derivation of velocity dispersions and radial velocities of the early-type galaxies.

- Construction of robust scaling relations at redshift of $z \sim 0.2$. Quantifying the evolution in luminosity and comparison to theoretical evolutionary model predictions.

- To distinguish between the sub-populations of early-type galaxies, the spherical elliptical and discy S0 galaxies.

- Test of environmental effects on the galaxy properties.

- Comparison of the observations with model predictions for the evolution of single-age stellar populations to constrain the formation redshift of the galaxies and the simulations of hierarchical structure formation.

These topics will be addressed in the chapters 4 to 7 . Prior, chapter 2 describes the construction of the individual samples for the Calar Alto and VLT spectroscopy used in this work. Details on the telescope and instrumental configurations and the observation strategies are given.

Chapter 3 describes the spectroscopic data reduction steps for the target galaxies and stellar templates. The photometric analysis is presented in chapter 4 . This includes the groundbased and HST photometry, analysing the surface brightness profile distributions for the galaxies based on HST imaging and computation of the rest-frame properties, measurements of structural parameters, visual and quantitative morphological classification and derivation of luminosities from the ground-based photometry. In chapter 5 the kinematic analysis is discussed. Radial velocities and internal velocity dispersions are measured for each galaxy. A number of simulations of velocity dispersions are performed to ensure a reliable and accurate treatment of uncertainties on the velocity dispersions at high redshift. Various tests for the kinematics of the galaxies are carried out and the redshift distributions for the galaxy clusters and the field ob- jects are determined. To extend the rich cluster sample by a factor of two, a detailed comparison of the overall properties of cluster galaxies in Abell 2390 and Abell 2218 was performed.

Galaxy scaling relations are the main topic in chapter 6. After defining an appropriate local reference, the Fundamental Plane and its projections, the Faber-Jackson and Kormendy relations will be constructed. It will be demonstrated that there is mass dependent evolution with a faster evolution for lower-mass galaxies. Differences between the populations of elliptical and lenticular galaxies will be explored via the Fundamental Plane and the evolution of the mass-to-light ratios.

Possible dependences of the galaxy properties on the environment will be addressed in chapter 7 . In this part, the stellar populations of early-type galaxies will be compared to stellar population models which provide constraints on their evolutionary histories. The impact of these results on the formation scenarios will be discussed.

A summary of the thesis is given in chapter 8 , which also comprises a brief outlook of the continuation of this project.

Unless otherwise stated, the concordance cosmology for a flat universe will be assumed throughout this thesis, with $\Omega_{\mathrm{m}}=0.3, \Omega_{\Lambda}=0.7$ and $H_{0}=70 \mathrm{~km} \mathrm{~s}^{-1} \mathrm{Mpc}^{-1}$. These values are in compliance with the most recent analysis of the power spectrum of the Cosmic Microwave Background observed by the WMAP satellite (Wilkinson Microwave Anisotropy Probe, Spergel et al. 2003). 


\section{Chapter 2}

\section{Sample Selection and Observations}

Cluster environments provide the opportunity to observe a larger number of early-type galaxies at the same redshift with multi-object spectroscopy simultaneously. Therefore, the main goal for the cluster sample was to acquire spectroscopically a large and representative sample of $\sim 50$ earlytype galaxies. At a redshift of $z \sim 0.2$, the cluster galaxies are bright enough to observe even sub- $L^{*}$ systems with $4 \mathrm{~m}$ class telescopes, while still representing a look-back time of $\sim 3$ Gyrs, adequate to address evolutionary questions. In particular, for the rich cluster Abell 2390 (section 2.1.3) it was proposed to investigate galaxy sub-populations, like Balmer-line enhanced galaxies or morphologically classified S0 galaxies, with sufficient numbers that their individual FPs can be constructed. With a large sample of early-type galaxies the evolution of the luminosity in the Faber-Jackson relation (FJR) can be explored accurately by comparing the distant galaxies directly with the relationship of local samples like Coma and Virgo (Davies et al. 1987; Lucey et al. 1991). The low number of observed cluster galaxies at higher redshift $(z \gtrsim 0.4)$ with instruments like the TWIN spectrograph prohibited the measurement of the scatter and possible slope change amongst the clusters (Ziegler \& Bender 1997). In targeting 50 cluster members, an order of about one magnitude improvement, the evolution within the FJR can be studied for different sub-populations as well as subsamples, such as low- and high-luminosity or low- and high-mass galaxies. Furthermore, in combining the A 2390 sample with a previous study of the rich cluster A 2218 at redshift of $z=0.18$ (Ziegler et al. 2001a), the sample would be large enough to explore possible radial dependences in the luminosity evolution of the FJR. Moreover, the investigation of a second cluster at a similar redshift is essential to not only understand possible systematic effects arising from instrument characteristics but to investigate the variance of cluster galaxy populations both within and between different rich clusters. Both clusters may well serve in the future as suitable benchmarks for the comparison to rich, high redshift clusters, since aperture corrections are less crucial than, for example, to the Coma cluster.

To look for the environmental dependence within the cluster, it is desirable to investigate possible radial dependences in relations between the absorption line-strengths of early-type galaxies and stellar parameters (age and metallicity). In particular, the relationship between the kinematics and stellar population (e.g., $\mathrm{Mg}_{b}-\sigma$ relation) which was found to be very tight for local galaxies, can be used to probe a large number of early-type galaxies in different clusters at higher redshifts. Furthermore, if, as suggested by some local studies (Mehlert et al. 2003), elliptical or S0 galaxies do have young populations these should be increasingly apparent at $z=0.2$. For a sub-sample of E+S0 galaxies with structural parameters based on HST/WFPC2 imaging, the FP could be explored. At the begin- 
ning of this project, there were only few previous studies at higher redshift $(z \geq 0.33)$ and none within $0.05<z<0.33$. Therefore, this FP investigation at a redshift of $z=0.228$ would be very important to cover this interval in redshift space. By observing two clusters at $z \sim 0.2$ in detail, changes in the form of the FP with redshift can be revealed and tested if the change in mass-tolight ratio $(M / L)$ is confirmed. Moreover, it is possible to look for evidence of a variation in the tilt and scatter of the FP with redshift and environment.

But all these observational approaches apply only to the special environment of dense rich clusters. Therefore, the constraints are biased towards the densest peaks with the largest amplitudes of the initial density fluctuation spectrum. In general, the semi-analytic Cold Dark Matter paradigm predicts a hierarchical galaxy evolution with a continuous assembling and merging of galaxies and sub-units. Only in the dense environments of rich clusters galaxies did experience their last major merger at $z \sim 1-2$. The age distribution and thus the populations of earlytype galaxies are however believed to be more diverse in lower density regions. For this reason, a project was proposed to analyse the evolutionary status of early-type galaxies in poor clusters (section 2.1.4) at a look-back time of $\approx 3$ Gyrs with the same quantitative methods as was extensively done in rich clusters. In particular, the $\mathrm{Mg}-\sigma$ relation will reveal whether there is more spread in the age/metallicity distribution than in the rich clusters Abell 2218 and Abell 2390 at similar epochs. Results on the FP will give insights whether the mass/structure evolution is more consistent with the monolithic or the hierarchical formation scenario.

To compare the different cluster samples with even lower-densities of individual galaxies, two field samples of early-type galaxies were selected from the FORS Deep Field (section 2.2.1) and the William Herschel Deep Field (section 2.2.2). Based on deep VLT/FORS spectroscopy comple- mented with HST/ACS imaging, the kinematic status of these field elliptical galaxies will serve as an additional comparison sample to test environmental effects in detail.

\subsection{Cluster Samples}

\subsubsection{Calar Alto Observatory}

The Centro Astronómico Hispano Alemán $(\mathrm{CAHA})^{1}$ at Calar Alto is located at an altitude of $2168 \mathrm{~m}$ in the Sierra de Los Filabres, Andalucia, Spain (longitude $02^{\circ} 32^{\prime} 48^{\prime \prime}$ west; latitude $37^{\circ} 13^{\prime} 16^{\prime \prime}$ north). The observatory is operated jointly by the Max-Planck-Institut für Astronomie, Heidelberg, and the Instituto de Astrofísica de Andalucía in Granada (CSIC) and provides three telescopes with apertures of $1.23 \mathrm{~m}, 2.2 \mathrm{~m}$ and $3.5 \mathrm{~m}$. In addition, an $1.5 \mathrm{~m}$ telescope is operated under the control of the Observatory of Madrid.

The CAHA observatory is a powerful counterpart to the ESO observatories at La Silla in targeting clusters of galaxies in the northern hemisphere. In particular, faint cluster galaxies at $z \sim 0.2$ are still within the reach of the $3.5 \mathrm{~m}$ telescope with reasonable exposure times. However, to measure line-strengths in the observed galaxy spectra as well, observations have to be splitted into multiple exposures in order to increase the final signal-to-noise $(S / N)$ in the spectra. This requires a very stable and reliable combination between telescope and spectrograph instrument, which in the latter case is perfectly fulfilled with the MOSCA spectrograph.

\subsubsection{MOSCA Configuration}

The focal reducer MOSCA $^{2}$, mounted at the Richey-Chrétien (RC) Focus of the 3.5-m telescope on Calar Alto, is a powerful instrument

\footnotetext{
${ }^{1}$ Local page: http: $\backslash \backslash$ www.caha.es $\backslash$ CAHA $\backslash$ index.html.

${ }^{2}$ The MOSCA Multi Object Spectrograph for Calar Alto instrument had first light in September 1996. (http: \\www.mpia-hd.mpg.de \CAHA \index.html).
} 
because it provides the possibilty of both direct imaging and spectroscopy of low- and intermediate resolution. Furthermore, due to MOSCA's large field-of-view (FOV) of $11^{\prime} \times 11^{\prime}$, valuable exposure time can be reduced in using a MultiObject-Mask of up to 25 slits on different object targets. The following chapter gives a brief summary of the MOSCA configuration and capabilities when used in spectroscopic mode, in particular for executing Multi-Object Spectroscopy (MOS) with user-supplied masks.

The design of MOSCA itself is quite simple. Depending on the observational mode, various apertures, such as mask, long-slit or multislit can be chosen to be mounted in the focal plane. A collimator optic sends a parallel light beam which bypasses one or two analysers (filter, grism, Fabry-Pérot Instrument (FPI), and/or polarizer) and is then focussed by a camera optics onto the detector. A second filter wheel is situated in the convergent beam. The calibration unit of MOSCA has three different spectral lamps for wavelength calibration ( $\mathrm{Ne}, \mathrm{Ar}$, and $\mathrm{HgAr}$ ) and one halogen-continuum source with variable intensity $(40 \%, 60 \%, 80 \%$, and $100 \%)$.

In order to gain a dispersion function with high precision for the wavelength calibration, the spectral lamps have a different wavelength sensibility. The Ar lamp samples strong lines with large intensity at $\sim 7200 \AA$ to $\sim 8500 \AA$, the Ne lamp numerous lines within a range of $\sim 5500 \AA$ to $\sim 7000 \AA$, whereas the combined HgAr lamp is mainly sensible to the strong lines between $\sim 3500 \AA$ to $\sim 4100 \AA$. A detailed discussion on the derivation of an accurate dispersion function for the wavelength calibration in presented in section 3.6.

Table 2.1 gives a brief overview of the general properties of MOSCA. The optical system has a reduction ratio of 3.67 with an effective focal ratio of $\mathrm{f} / 2.7$. This yields an image scale of 3 pixel per arcsec and a total FOV of $11^{\prime} \times 11^{\prime}$ (effectively of $\sim 10^{\prime} \times 10^{\prime}$ ) with a pixel size of $15 \mu \mathrm{m}$. A thinned CCD (SITE \# 16a) with 2048×4096 pixels is used as a detector.

Based on laboratory tests, the grism GREEN 1000 gives a wavelength coverage of $\lambda \lambda=4200$ $6500 \AA$, with a central wavelength of $\lambda_{0}=5300 \AA$ and a dispersion of $5.85 \AA$ /arcsec for a typical MOSCA slit width of $1.5^{\prime \prime}$.

Table 2.1: Overview of basic MOSCA facts.

\begin{tabular}{l}
\hline Instrument properties: \\
\hline effective focal ratio: $\mathrm{f} / 2.7$ \\
reduction ratio: 3.67 \\
CCD chip: SITE \# $16 \mathrm{a}: 2048 \times 4096$ pixel \\
\hline Image scale in focal plane: \\
\hline $1 \mathrm{~mm}=5.88^{\prime \prime}, 1^{\prime \prime}=169.7$ micron \\
mean magnification factor: 0.2755 \\
1 pixel $(15$ micron pixel size $)=0.3208^{\prime \prime}$ \\
FOV: $660^{\prime \prime} \times 660^{\prime \prime}=11^{\prime} \times 11^{\prime}$ \\
\hline
\end{tabular}

The reproduction scale of an optical system is defined as the ratio between the image size and the object size. The variation of the reproduction scale along the image area is called distortion. This means that an image is deformed. A square pattern of straight lines will give an image as a pattern of convex curves when there is a barrel-shaped distortion and an image of concave curves when there is pincushion or pillowshaped distortion. Due to the fact that MOSCA is a focal reducer an original image is displayed with concave lines, i.e. it gets distorted in pillowshaped form. For this reason, a special correction method was applied to the MOS frames (see section 3.3 for a further discussion).

\subsubsection{Abell 2390 Cluster}

With the up-coming of the MOSCA instrument at CAHA with its multi-object capability and its big field-of-view in 1998, it was feasible and reasonable to carry out the proposed observation of a large number of galaxies in the cluster A 2390. Using the TWIN spectrograph, only a 
few brighter galaxies would be possible to acquire thereby providing only a limited picture of the whole early-type galaxy population of a cluster (e.g., Ziegler \& Bender 1997). Furthermore, a similar investigation of 48 different early-type cluster galaxies in Abell 2218 at $z=0.175$ using LDSS2 at the WHT already proved the extremely high efficiency of the multi-object spectroscopy technique. At a cluster redshift of $z=0.23$, high $S / N$ spectra of early-type galaxies covering a wide wavelength range $(\approx 2000 \AA)$ and spanning a wide range of luminosity (down to $M_{B}=M^{*}+1$ ) could still be acquired with a $4 \mathrm{~m}$ class telescope and reasonable exposure times of $\sim 8-10$ hours. Given the definition of the Abell radius of $R_{\mathrm{A}}=\frac{1.72^{\prime}}{z}$, the $10^{\prime} \times 10^{\prime} \mathrm{FOV}$ of MOSCA matches the typical angular size of a rich cluster at $z \sim 0.2$ quite well. The physical FOV for the A 2390 cluster at $z=0.23$ is $\sim 1.4$ Abell radii $(\sim 2.7 \mathrm{Mpc})$. Therefore, combining the high quality spectra for a large fraction of cluster members and addressing a significant look-back time, detailed tests of the stellar population evolution are possible.

Besides the MOS spectroscopy with MOSCA at the $3.5 \mathrm{~m}$ telescope, the study of the earlytype galaxy population in A 2390 is based upon optical photometry with the $5.1 \mathrm{~m}$ Hale telescope at Mount Palomar Observatory. In addition, HST/WFPC2 images were exploited to provide high-quality morphological information for a subset of the sample. In section 2.1.6 on page 30 , Table 2.2 gives a summary of the acquired observations for the A 2390 project.

\subsubsection{Low- $L_{X}$ Clusters}

This project is an extensive observational campaign to study the evolutionary status of galaxies in 10 poor clusters in the northern hemisphere $^{3}$ at intermediate redshifts of $0.2<z<0.3$ selected to have very low $\mathrm{X}$-ray luminosities

\footnotetext{
${ }^{3}$ The cluster $\mathrm{Cl} 1444+63$ turns out to be two clusters aligned along the line of sight (see section 4.2), and thus the two clusters are considered separately.
}

$\left(L_{X}<5 \times 10^{43} \mathrm{erg} / \mathrm{s}\right)^{4}, 100$ times lower than the rich $\mathrm{X}$-ray bright CNOC clusters, and poor optical richness class. It comprises ground-based optical and NIR photometry, medium-resolution multi-object spectroscopy, and HST/F702W imaging. From the previously analysed lowresolution MOSCA spectra (Balogh et al. 2002b) the location of cluster members with little or no ongoing star formation is known. Therefore, early-type cluster members have been selected based on the low-resolution spectra and their HST morphology (see next section 2.1.5 for details).

HST/F702W imaging during Cycle 8 (P.I. Prof. R. L. Davies (Oxford), Proposal ID 8325) was completed in July 2000 for 8 of the 9 clusters. The cluster $\mathrm{Cl} 1633+57$ was observed in Cycle 7 by another group (see section 4.2 .3 for further details). With an integration time of $7.500 \mathrm{sec}$, these images are perfectly suited to derive the structural and photometric parameters of those galaxy candidates targeted for the FP study. Outside the HST field additional $~ 10$ $\mathrm{E}+\mathrm{S} 0$ galaxies in each cluster will provide a look into the $\mathrm{Mg}-\sigma$ and Faber-Jackson relations in detail. For these scaling relations the number of observed galaxies is sufficient to explore possible variations between the different poor clusters as a function of their velocity dispersion $\sigma$ or X-ray luminosity $L_{X}$.

\subsubsection{Selection Criteria}

A pre-selection of possible targets for spectroscopy for all data sets was performed within the ESO-MIDAS (Munich Image Data Analysis System) environment, whereas the final construction of the setups for the field galaxies (section 2.2) was carried out with the FORS Instrumental Mask Simulator (FIMS). For constructing the MOSCA masks, special MIDAS programs, kindly provided by Prof. J. W. Fried, were utilised. Different catalogues containing

\footnotetext{
${ }^{4} L_{X}$ between $0.1-2.4 \mathrm{keV}, H_{0}=100 \mathrm{~km} \mathrm{~s}^{-1} \mathrm{Mpc}^{-1}$, see Table 2.3.
} 
the positions, magnitudes, structural parameters, morphologies et cetera of the target galaxies were created within MIDAS and used as input for setting up of the masks and visualising the slits.

The aim of the A 2390 project is to investigate the stellar populations of a large sample of earlytype cluster galaxies spanning a wide range in luminosity. However, in order to enable a good sky subtraction which requires long slit lengths (with an average length of $22^{\prime \prime}$ ), each mask was constrained to only about 20 galaxies (with typical galaxy sizes of $\left.4^{\prime \prime}\right)$ in total. For this reason, we were very careful to select only galaxies which were likely to be cluster members based upon their $U B I$ broad-band colours. Target objects were selected on the basis of the ground-based $I$ band images and a combination of defined colour regions. The selection procedure was very similar to the cluster galaxies in A 2218 (Ziegler et al. 2001a). Using the existing catalogue for the field (Smail et al. 1998), the distribution of galaxies on the $(U-B)-(B-I)$ colour-colour plane was investigated, after a correction for blue field galaxy contamination by $\sim 28 \%$ at $I=20^{m}$ with a deep $U$ exposure in a high-latitude blank field was performed. The colour-colour diagram was compared to the locus of colours expected for non-evolved Spectral Energy Distributions (SEDs) representative for the spectral types of galaxies with E/S0, Sab, Sbc, Scd and Sdm morphologies in the local Universe, as they would be observed at $z=0.23$ (see Smail et al. 1998 for further details). A region in $U B I$ colour space was defined which was most likely occupied by cluster members. Galaxies falling outside a colour region, defined as $2.60<(B-I)<3.50$ and $-0.2<(U-B)<0.40$, were rejected. The width of this colour range puts negligible restrictions on the stellar populations of the selected objects, but eliminates the majority of background galaxies. Combined with the richness of the cluster this approach ensured a high rate of success in targeting cluster members in our spec-

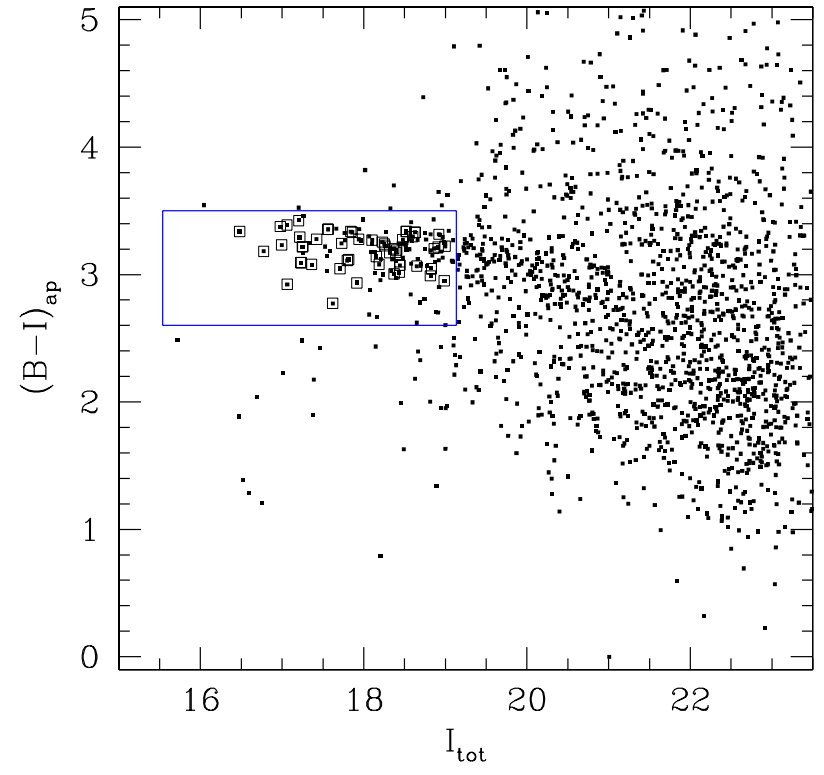

Figure 2.1: $(B-I)-I$ colour magnitude diagram from the Hale imaging for bright galaxies lying in a $9.7^{\prime} \times 9.7^{\prime}(2.12 \mathrm{Mpc})$ region centered on A 2390. The sequence of red cluster members is readily seen extending down to $I \sim 21^{m}\left(M_{B} \sim-19^{m}\right)$. The box encompasses galaxies matching the selection criteria, while squares denote those galaxies which were eventually observed spectroscopically.

troscopic sample (4 non-members out of 52 targets). The colour-selected sample of galaxies includes 122 galaxies with $15.53^{m}<I_{\text {tot }}<19.14^{m}$ within the $9.7^{\prime} \times 9.7^{\prime}$ area covered by the Hale images. Fig. 2.1 illustrates the selection in the $(B-I)-I$ colour-magnitude diagram for galaxies brighter than $I=23.5$ mag within the Hale field-of-view. The box encompasses all galaxies matching the selection criteria, while those galaxies which were actually observed are indicated as squares. The magnitude limit for the selection of spectroscopic targets was therefore adopted as $I_{\text {tot }}=19.14^{m}$.

A catalogue of the colour-selected galaxy sample was utilised as an input for the mask design preparation. Two masks contained galaxies with an average $I$-band surface brightness within the spectroscopic slit brighter than $\mu_{I}=21.0 \mathrm{mag} \operatorname{arcsec}^{-2}$. In a third mask fainter 
galaxies with surface brightnesses as faint as $\mu_{I}=21.5 \mathrm{mag} \operatorname{arcsec}^{-2}$ were included. In splitting the galaxies between the individual masks in this way, enabled to vary the cumulative exposure times of the masks to ensure that a similar signal-to-noise of about 30 per $\AA$ (in the continuum at $\lambda_{\text {obs }}=6300 \AA$ ) was obtained in each galaxy spectrum. Particular care was taken to maximise the number of galaxies with early-type morphologies (E-S0-S0/Sa) within the HST field. For this reason, the position angles of the masks were aligned accordingly to fulfill this requirement. With a total of 56 selected galaxies, a sub-population of $25 \%$ will have sufficient members to construct an independent $\mathrm{FP}$ and $\mathrm{Mg}-\sigma$ relation for different galaxy populations. This required number of galaxies was fulfilled by selecting galaxies down to $V=20.1^{m}$ (0.5 mag fainter than $\left.L^{*}\right)$ which show typical average $R$-band surface brightnesses of $\left\langle\mu_{R}\right\rangle \approx 20.6 \mathrm{mag} \operatorname{arcsec}^{-2}$, assuming an integration over a spatial region of $1.5^{\prime \prime}$. In order to study the evolution of early-type galaxies out to large clustercentric distances, target galaxies were selected covering the whole MOSCA fieldof-view of $\sim 10^{\prime} \times 10^{\prime}$ (see Fig. 4.1). Each slit was allocated to a galaxy of interest manually. An average slit length of $22^{\prime \prime}$ was used (for a few exceptions it was set to a minimum length of 15 arcsec) and the position of the slit was also chosen so that fainter companion galaxies did not obscure the sky and an accurate sky subtraction was possible.

A first target selection and determination of world coordinates of the sample of poor clusters was performed using the ESO skycat tool. In a second step, the MOS masks for the MOSCA instrument were constructed with special MIDAS programs. The central coordinates of the field of interest (in $\alpha$ and $\delta$ ), target positions with precision $<0.3$ arcsec, the slit positions and the length of slits were computed. The slit widths were set to 1.5 arcsec. Afterwards these definitions were transformed to $\alpha$ and $\delta$ coordinates of the same equinox on the plane sky (including a rotation of the coordinates according to a given position angle) and for a spectral coverage of the used grism and visualised via a graphic version of the mask. Examples of final MOSCA masks are illustrated in Fig. 2.2.

Finally, individual configuration setups for computing a mechanical MOS plate mask for MOSCA were created. These program files were sent to the mechanical shop at the MPIA (Heidelberg) which manufactured the final MOSCA plates. For the construction of configuration files for the mechanical plates special CNC machine programs were used. Before the observations, these masks are supplied by each observer and put into the aperture unit. A maximum of two masks are accessible via the GUI interface but one plate can be changed during the night if necessary. The orientation of the mask in the MOSCA instrument is unique and thus fixed.

To align the mask and targets, relatively bright stars $\left(V \approx 19^{m}\right)$ were selected and distributed over the whole MOSCA field. Their positions can be measured precisely even on short exposures ( $\sim 10 \mathrm{sec})$. A small but important improvement in the programs was the use of three (instead of usually at least two) reference stars for the mask alignment. An additional star warrants an even higher accurate positioning and alignment also for lower horizontal distances. The holes for the stars have a radius of 5 arcsec (see Figs. 2.2 and 2.3). In taking short exposures through these holes and measuring the positions of the stars, Cassegrain flange angle and telescope position can be corrected which results in an improvement of the mask alignment. This tricky alignment of the MOS mask is visualised in Fig. 2.3. For each mask and exposure, the position of the galaxies and alignment stars had to be verified to avoid loss of galaxy fluxes. Since the positions of holes with 10 arcsec diameter can not be measured precisely enough on images, additional reference holes with 1 arcsec radius are slightly offset put from the large holes in the 


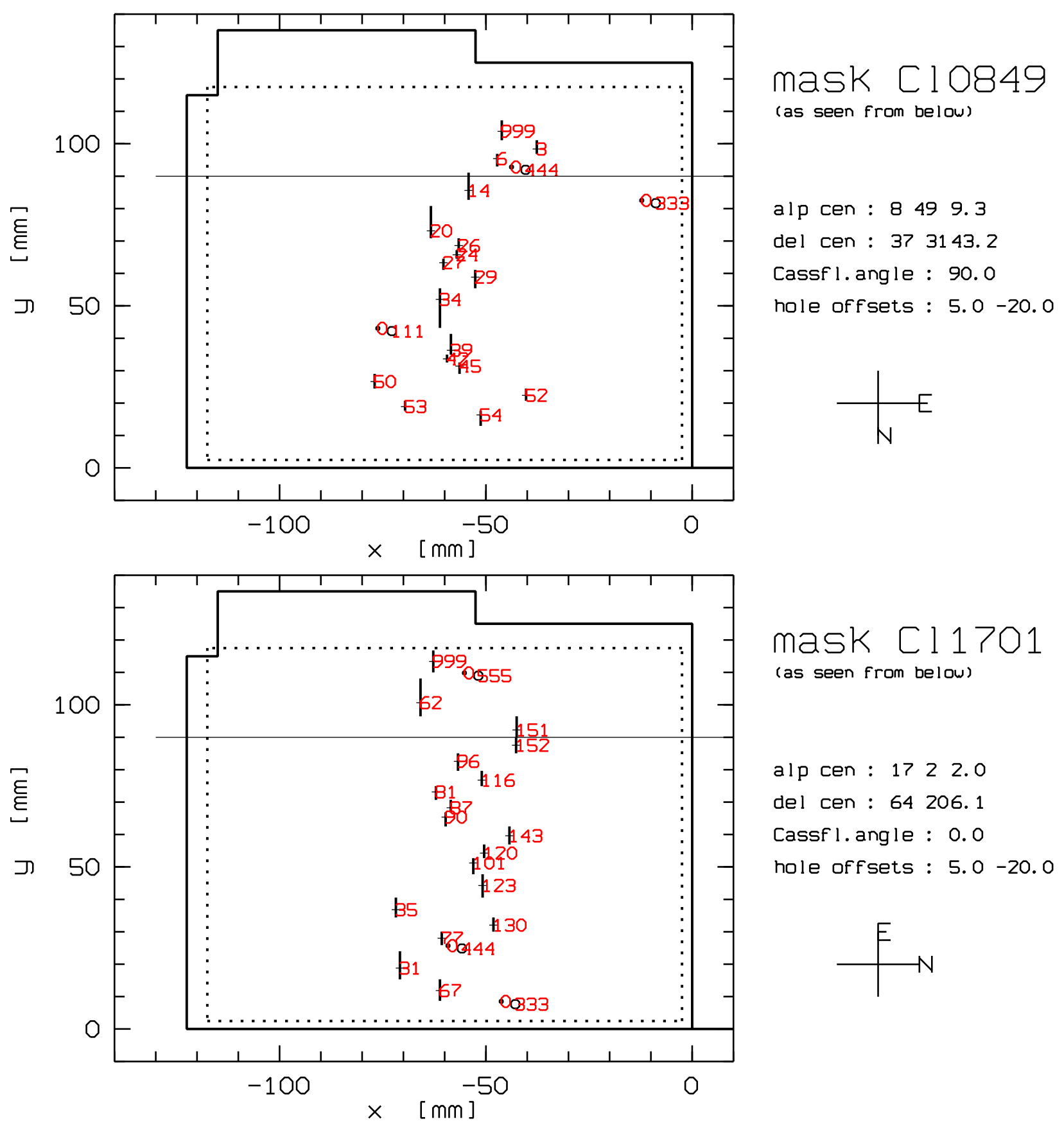

Figure 2.2: MOSCA masks for the poor clusters Cl0849 and Cl1701/Cl1702 seen from below (i.e., the upper surface is the focal plane). The images visualise the whole MOS mask (solid line) with all slits and the field of MOSCA is indicated as a dotted square. The position angle PA of MOSCA is defined in the usual way (i.e. zero degrees, which is normally used for direct imaging, gives direct images in the standard orientation. The values of Cassegrain flange angle have to be rotated by $90^{\circ}$, i.e. Cl0849 has an angle of $\mathrm{PA}=0^{\circ}$ and $\mathrm{Cl} 1701$ has $\mathrm{PA}=+90^{\circ}$. Note that for Cl1701 the slits are in East-West direction. 


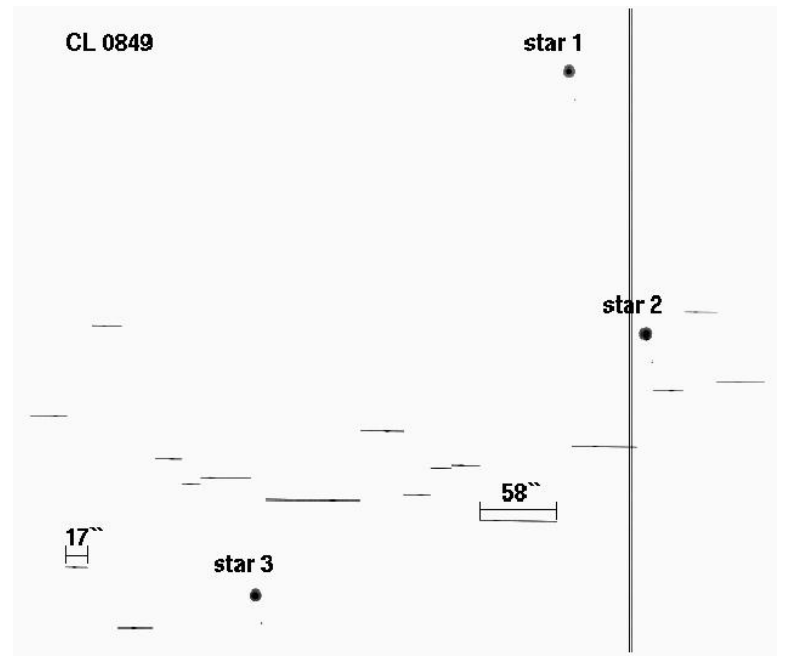

Figure 2.3: Mask alignment image for Cl0849. An exposure of $\sim 90 \mathrm{sec}$ was taken through the slits to verify that all galaxies are accurately positioned onto their slits and the stars positioned in their holes. The lengths for two slits are indicated. The PSF of this image is $1.1^{\prime \prime}$ FWHM. The vertical line are six bad columns located on the CCD chip between 1540-1551 pixel.

mask. The positions of these smaller reference holes can be measured precisely.

The basic selection criteria for the poor clusters was different to the rich clusters. Thanks to our previous study of the poor clusters based on MOSCA and LDSS-2 low-resolution spectroscopy using the grism GREEN 500 and the medium-blue grism (Balogh et al. 2002b), for a total of 317 galaxies spectroscopic redshifts were available. By using these measurements and the catalog of 172 Low $-L_{X}$ cluster members, the target selection for the follow-up spectroscopy could be performed very efficiently.

In order to fill each mask, several catalogues of early-type candidates were constructed. One catalogue contained all objects (spiral and earlytype galaxies) with low-resolution spectroscopy, another one held galaxies with absorption lines at cluster redshift (i.e. passive early-type spectra) and another list only galaxies with structural information (i.e. residing in the HST field). Furthermore, galaxy catalogues suitable for fill- up objects (at different redshifts than the cluster or without any spectral information) were created. All catalogues were loaded into the ESO skycat software simultaneously and marked with different symbols (see Figs 2.4 and 2.5). For this reason, the final object selection could be performed time-efficiently. As the clusters comprise relatively few members in comparison to a rich cluster and only one mask was constructed for each cluster, particular care was taken to select only cluster members which show early-type spectral features in their low-resolution spectra. Furthermore, the position angles of the masks were chosen in such a way that the number of galaxies with early-type morphologies (E-S0$\mathrm{S} 0 / \mathrm{Sa}$ ) selected within the HST field was maximum. For the outermost slits located at the mask edges, the coverage of the catalogues was poor or even null. Therefore, anonymous objects had to be selected as fill-up objects. In this situation, preference was given to galaxies which featured similar apparent luminosities as the cluster galaxies. However, for all setups the number of anonymous galaxies was not larger than three.

\subsubsection{Observations}

The observations were carried out during two observing runs in four nights of September 7.11., 1999 and five nights of July 26.-31., 2000. To achieve intermediate-resolution spectra for the galaxies in A 2390 at $z=0.23$, the grism GREEN 1000 with a typical wavelength range of $\lambda \lambda=4500-7500 \AA$ was chosen, encompassing important absorption lines such as $\mathrm{H} \gamma, \mathrm{H} \beta$, $\mathrm{Mg}_{b}, \mathrm{Fe} 5270$ and Fe5335 at the cluster redshift of $z=0.228$. The slit widths were set to $1.5^{\prime \prime}$ and the instrumental resolution around $\mathrm{H} \beta$ and $\mathrm{Mg}_{b}\left(5900 \lesssim \lambda_{\text {obs }} \lesssim 6400 \AA\right)$ was $5.0 \AA$ FWHM, corresponding to $\sigma_{\text {inst }} \sim 100 \mathrm{~km} \mathrm{~s}^{-1}$ around $\lambda_{\text {obs }}=6360 \AA$ (see section 5.3 for a discussion). The spatial scale was $0.33^{\prime \prime}$ per pixel and seeing conditions varied between $1.2^{\prime \prime} \leq \mathrm{FWHM} \leq 1.7^{\prime \prime}$.

Three masks were observed with total exposure 


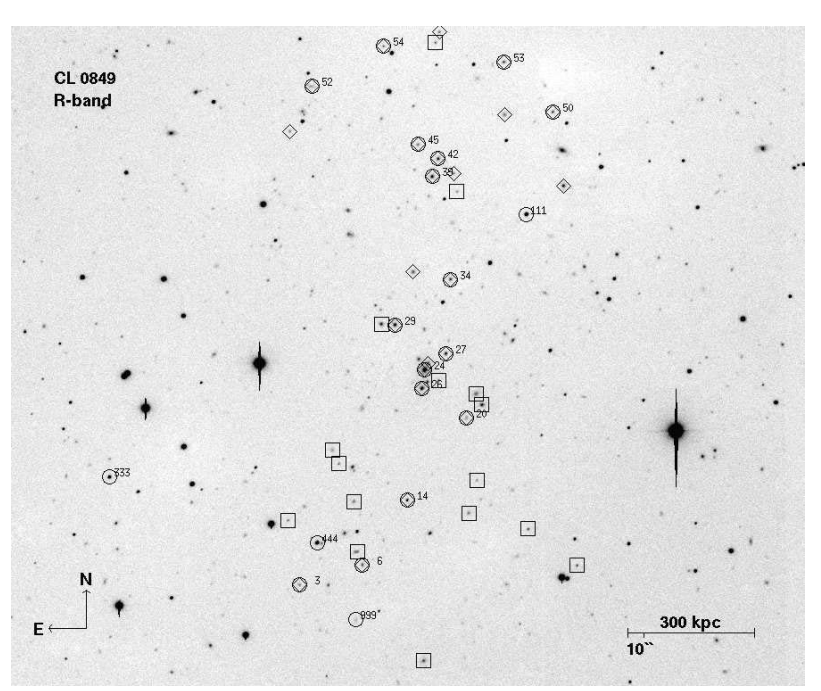

Figure 2.4: Hale R-band image of CL 0849 $(\mathrm{z}=0.2343)$ used for the mask preparation. The image size is $8.4 \times 7 ! 0$. Galaxies with low-resolution spectra are indicated as diamonds. Possible spiral galaxies or objects without any absorption lines and/or different redshifts are marked as squares. Early-type cluster candidates are denoted as circles. Stars used for the mask alignment are also shown (\#111, \#333 and \# 444). The bar corresponds to $300 \mathrm{kpc}$ in projection at the clusters redshift of $z=0.2343$ and the small bar to $10^{\prime \prime}(38.1 \mathrm{kpc})$.

times between $29880 \mathrm{sec}(8.3 \mathrm{hrs})$ and $42480 \mathrm{sec}$ (11.8 hrs) each (see Table 2.2). In total, 63 highsignal-to noise spectra of 55 different galaxies were obtained, of which 15 are situated within the HST field. Three objects, \#1507, \# 1639 and \# 2933, turned out to be background galaxies at $z=0.3275, z=0.3249$ and $z=0.3981$, respectively; one galaxy (\# 3038) is located in the foreground at $z=0.1798$. Four other galaxies fell into the slits by coincidence (\# 2222, \# 5552, \# 5553 and \# 6666) which are all cluster members. However, apart from \# 6666, their spectra are too faint $(S / N \lesssim 8)$ to determine accurate velocity dispersions and no photometry is available for the other objects (cf. section 5.5). Therefore, these galaxies were also discarded from the sample yielding a total of 48 different early-type cluster members, proving that

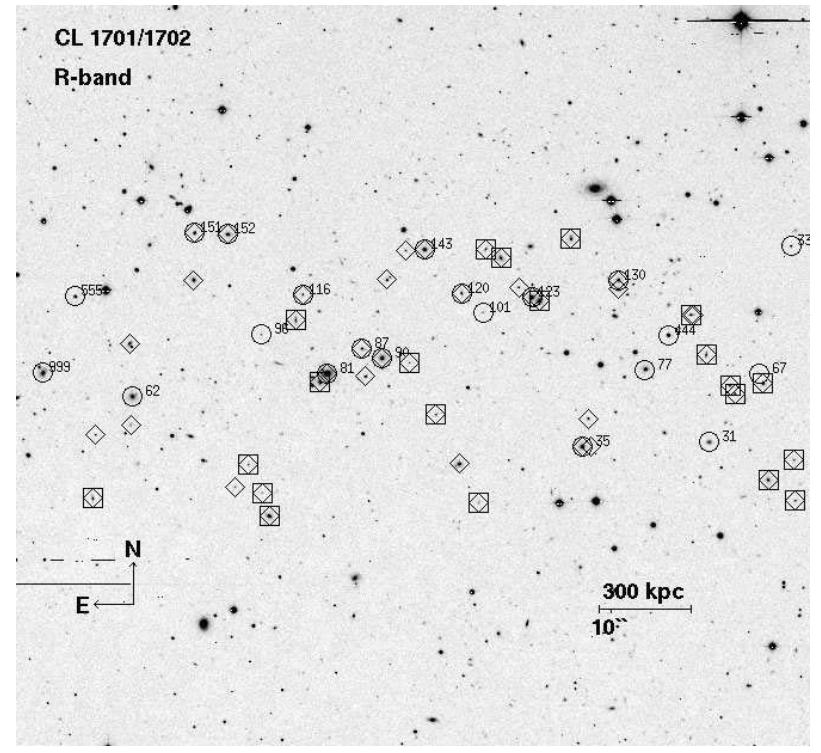

Figure 2.5: INT R-band image of CL 1701 $(\mathrm{z}=0.2458)$ and CL $1702(\mathrm{z}=0.2233)$ used for the mask preparation. The image size is $11^{\prime} \cdot 11 \times 10 ! 82$. Galaxies with low-resolution spectra are indicated as diamonds. Possible spiral galaxies or objects without any absorption lines and/or different redshifts are marked as squares. Early-type cluster candidates are denoted as circles. Stars used for the mask alignment are also shown (\# 333, \# 444 and \# 555). The bar corresponds to $300 \mathrm{kpc}$ in projection at the clusters redshift of $z=0.2458$ and the small bar to $10^{\prime \prime}$ $(38.9 \mathrm{kpc})$.

our sample selection was highly efficient.

For the Low $-L_{\mathrm{X}}$ clusters, spectroscopic MOSCA observations were carried out during six nights in April 17.-22., 2001 and six nights during February 4.-9., 2002. A summary of the cluster sample with the mean redshifts, number of objects on a mask and total exposure times for spectroscopy is given in Table 2.3. In total, five masks (one per cluster) were observed. The only exception is the mask for the clusters with $\alpha=17^{\mathrm{h}}(\mathrm{Cl} 1701$ and $\mathrm{Cl} 1702$ ), which were acquired with the same mask due to resons of telescope time-efficiency. For the study within the FP, spectra of 5-7 galaxies lying within the HST fields in a single MOS mask were obtained. Based on the whole cluster sample, a combined FP sample of $\sim 30$ early- 
Table 2.2: Observations of Abell 2390

\begin{tabular}{lllcc}
\hline Tel. & Instrument & Date & $\begin{array}{c}\text { Band (phot) } \\
\text { Mask / } N_{\text {spec }}\end{array}$ & $\begin{array}{c}T_{\text {exp }} \\
{[\mathrm{ksec}]}\end{array}$ \\
\hline HALE & COSMIC & $09-12 / 06 / 94$ & $U$ & 3.00 \\
& COSMIC & $09-12 / 06 / 94$ & $B$ & 0.50 \\
& COSMIC & $09-12 / 06 / 94$ & $I$ & 0.50 \\
HST & WFPC2 & $10 / 12 / 94$ & F555W & 8.40 \\
& WFPC2 & $10 / 12 / 94$ & F814W & 10.50 \\
CA 3.5 & MOSCA & $07-10 / 09 / 99$ & $1 / 17$ & 29.88 \\
& MOSCA & $07-10 / 09 / 99$ & $2 / 22$ & 42.12 \\
& MOSCA & $26-28 / 07 / 00$ & $3 / 24$ & 42.48 \\
\hline
\end{tabular}

type galaxies can be constructed, which is comparable to that of a single rich cluster. Outside the HST field additional spectra of $\sim 10 \mathrm{E}+\mathrm{S} 0$ galaxies in each cluster serve for the investigation of the $\mathrm{Mg}-\sigma$ and Faber-Jackson relation. In total, 76 high $S / N$ galaxy spectra of poor cluster candidates were acquired.

In this thesis the analysis concentrates on the observations from 2001 of the three clusters $\mathrm{Cl} 0849+37(\mathrm{z}=0.2343), \mathrm{Cl} 1701+64(\mathrm{z}=0.2458)$ and $\mathrm{Cl} 1702+64(\mathrm{z}=0.2233)$. Note, that $\mathrm{Cl} 1701$ and $\mathrm{Cl} 1702$ were only observed with one mask and the number of galaxies and the observing time is not splitted for into each cluster in Table 2.3 .

During the observations at Calar Alto, the alignment of the MOSCA masks was conducted with the special program alimask. First, the Cassegrain flange was changed to the required position angle and the telescope moved to the center coordinates of the mask. Second, the alignment process with alimask was initiated. Usually, this procedure has four steps which have to be performed consecutively (1) exposure of the mask with the installed calibration lamp to measure the positions of the reference holes, (2) short exposure of the whole field to measure the alignment stars for improvement of telescope position and Cassegrain flange angle, (3) two short exposures to measure the positions of the stars in two large holes in the mask to compute flange angle and telescope position corrections. This step may be iterated. (4) Exposure through the slits to verify that all objects are located in their slits. This step is optional (see Fig. 2.3). Together with the previous settings the whole procedure takes about 25 minutes. However, as two observers (A. Fritz \& K. Jäger in 2001, A. Fritz \& B. Ziegler in 2002) could work simultanously (one with the telescope settings and one with the mask alignment) and due to an optimised scheduling (e.g., skipping step 2 when executed once for a night, changing cuts for image display) the alignment process could be shortened to $\approx 15$ minutes. In particular, the verification of the mask alignment (step 4) was done after starting the $2700 \mathrm{sec}$ science exposure and thus valuable minutes of dark time could be gained. This was a non-negligible factor as a high amount of dark time was lost due to bad weather (60\% in 2001, $50 \%$ in 2002) and due to a forest fire (sixth night of 2001) and an earthquake (first night of 2002). 
Table 2.3: Observations of six poor clusters

\begin{tabular}{|c|c|c|c|c|c|c|c|c|}
\hline Cluster & R.A. & $\begin{array}{l}\text { DEC. } \\
000)\end{array}$ & $<z>$ & $\begin{array}{c}\sigma_{c} \\
{[\mathrm{~km} / \mathrm{s}]}\end{array}$ & $L_{X}{ }^{1}$ & Date & $\begin{array}{l}N_{\text {obj }} \\
\text { mask }\end{array}$ & $\begin{array}{l}T_{\exp } \\
{[\mathrm{ksec}]}\end{array}$ \\
\hline $\mathrm{Cl} 0818+56$ & 081904 & +565449 & 0.2670 & $651 \pm 165$ & 1.50 & 04-09/02/02 & 14 & 29.40 \\
\hline $\mathrm{Cl} 0841+70$ & 084144 & +704653 & 0.2397 & $399 \pm 170$ & 1.22 & $04-09 / 02 / 02$ & 13 & 24.54 \\
\hline $\mathrm{Cl} 0849+37$ & 084911 & +373109 & 0.2343 & $764 \pm 090$ & 1.93 & $17-22 / 04 / 01$ & 17 & 18.00 \\
\hline $\mathrm{Cl} 1633+57$ & 163342 & +571412 & 0.2402 & $582 \pm 360$ & 0.49 & $04-09 / 02 / 02$ & 14 & 30.97 \\
\hline $\mathrm{Cl} 1701+64$ & 170147 & +642057 & 0.2458 & $834 \pm 647$ & 0.40 & $17-22 / 04 / 01$ & 18 & 27.90 \\
\hline $\mathrm{Cl} 1702+64$ & 170214 & +641953 & 0.2233 & $386 \pm 426$ & 0.74 & $17-22 / 04 / 01$ & & \\
\hline
\end{tabular}

${ }^{1} L_{X}(0.1-2.4 \mathrm{keV}) 10^{43} h_{100}^{-2} \operatorname{erg~s}^{-1}$.

\section{$2.2 \quad$ Field Sample}

\subsubsection{The FORS Deep Field}

The FORS Deep Field (FDF) is a very deep, multi-band photometric study of a sky region located near the south galactic pole. The primary aim of the FDF project was to perform a multicolour imaging investigation in the optical and near-infrared with visible limiting magnitudes comparable to the Hubble Deep Fields (HDFs, Williams et al. 1996, 2000) but with a substantially larger field-of-view of $\sim 7^{\prime} \times 7^{\prime}$ (about 4 times the combined HDFs). Its main scientific driver was to improve the understanding of the formation and evolution of galaxies in the young Universe using a combination of deep photometry and extensive follow-up spectroscopy (see Appenzeller et al. 2000 for an overview).

In the selection of a suitable field, the sky area had to fulfill several selection criteria. The cutoff limit on the galactic extinction was chosen to be $E(B-V)<0.02^{m}$ and large, nearby galaxies, bright stars $\left(V<18^{m}\right)$ within the FOV and very bright stars $\left(V<5^{m}\right)$ within $5^{\circ}$ should be absent in the field in order to allow reasonably long exposure times and to avoid ghost images etc. The two latter constraints ruled out the option to use the HDF-S region. Moreover, the field had to be devoid of strong radio or X-ray sources to avoid potentially present galaxy clusters at medium redshifts. The field which was finally chosen is located near the south galactic pole with center coordinates of $\alpha_{2000}=01^{\mathrm{h}} 06^{\mathrm{m}} 03.6^{\mathrm{s}}$, $\delta_{2000}=-25^{\circ} 45^{\prime} 46^{\prime \prime}$.

The optical observations were carried out with the Focal Reducer / Low Dispersion Spectrograph (FORS, see Appenzeller et al. 1998 or www.eso.org/instruments/fors1/ for an overview) which was designed and constructed in a collaboration of the Universitäts-Sternwarte Göttingen, the Landessternwarte Heidelberg and the Universitätssternwarte München. Since 1999, two versions of the FORS instrument (called FORS1 and FORS2) are available, mounted on two different units of the Very Large Telescope (VLT) which is operated by the European Southern Observatory (ESO) at Cerro Paranal, Chile. In return for the instrumental contributions, the three institutes above were given a pool of guaranteed observing time (socalled Guaranteed Time Observations, GTOs) by ESO. A high fraction of this GTO was reserved for the FDF imaging in the optical $(\approx 10$ GTO nights), the rest of the allocated GTO was shared between several follow-up studies of the FDF project (e.g., the Tully-Fisher relation project, see Ziegler et al. 2002, Böhm et al. 2004; or a study of high-redshift galaxies, see Mehlert et al. 2002; Noll et al. 2004). In the next sec- 
tions 2.2.3 and 2.2.4, the instrumental setup and the target selection procedures for the field elliptical study are outlined.

\subsubsection{William Herschel Deep Field}

The William Herschel Deep Field (WHDF, Metcalfe et al. 2001) is a deep optical and nearinfrared $U B R I H K$ photometric survey performed over a $7 \times 7$ arcmin square sky area with the William Herschel Telescope (WHT) at Canary Islands, and the United Kingdom Infrared Telescope (UKIRT) at Mauna Kea and the $3.5 \mathrm{~m}$ telescope on Calar Alto. The WHDF is located in the northern hemisphere with center coordinates of $\alpha_{2000}=00^{\mathrm{h}} 22^{\mathrm{m}} 33.3^{\mathrm{s}}, \delta_{2000}=+00^{\circ} 20^{\prime} 57^{\prime \prime}$ (centre of B-band image). Compared to the FDF, the WHDF is not as deep and has much worse seeing conditions varying between $1.2^{\prime \prime} \leq$ $\mathrm{FWHM} \leq 1.5^{\prime \prime}$ (mean seeing $1.3^{\prime \prime} \mathrm{FWHM}$ ). The main scientific driver for our study was to perform spectroscopy within the WHDF to enlarge the FDF sub-samples of field spiral and earlytype galaxies by a factor of two in order to gain large data set with statistical significance.

The WHDF sample of elliptical galaxy candidates were drawn from the deep $U B R I H K$ photometry of the WHDF. UBRI imaging is based on the WHT, the infrared $K$ and $K_{\text {deep }}$ (only $1.8 \times 1.8 \operatorname{arcmin}^{2}$ ) imaging was obtained with the UKIRT. After our observations in 2002, the WHDF was observed in the H-band (whole field) using the $\Omega$ Prime camera at the $3.5 \mathrm{~m}$ telescope on CAHA. The $3 \sigma$ magnitude limits for point sources as derived from images are 26.8, 27.9, $26.3,25.6,22.8,20.5,22.8$ in the U, B, R, I, $\mathrm{H}, \mathrm{K}, \mathrm{K}_{\text {deep }}$ filters (2 arcmin diameter sub-area), respectively. All magnitudes are in the Vegasystem.

\subsubsection{FORS Configuration}

The spectroscopic observations of the sample of FDF field ellipticals were performed simultaneously with those of the late-type galaxies in the FDF which were subject to the investigation of the evolution of the Tully-Fisher relation (Ziegler et al. 2002; Böhm et al. 2004). A full description of the instrumental setup is given in Böhm (2003). Here, only the setup for the investigation of the field elliptical galaxies is described. In addition, a second sample of field early-type galaxies selected from the WHDF was observed together with a project studying the Tully-Fisher relation of late-type galaxies (Böhm \& Ziegler 2006) under similar conditions with the VLT at Cerro Paranal. As the sample selections and instrument configurations were very similar the two individual data sets are discussed in combination.

In order to construct a large spectroscopic sample with less amount of observing time, the Multi Object Spectroscopy (MOS) Mode was chosen to be the best option. The Mask eXchangeable Unit (MXU) mode of FORS2 was non existent and thus the FDF observations in 2001 were restricted to the usage of FORS1. For an effective filling of the MOS masks, the early-type galaxies were observed simultaneously with the spiral galaxies. Both versions of FORS instruments offer 19 slitlets in the MOS configuration, 9 with slit lengths of $22 \operatorname{arcsec}, 8$ with slit lengths of 20 arcsec, whereas the uppermost and lowermost slits have lengths of $\sim 11$ arcsec.

FORS was operated at standard resolution setup, i.e. for the configuration of the CCDs (read out in one-port mode) and the collimator the default values of the MOS mode were used. The spatial scale was $0.2 \mathrm{arcsec} / \mathrm{pixel}$ and in low gain mode (using port A) FORS1 offers a gain of $3.51 e^{-} / \mathrm{ADU}$ and a read-out-noise $(\mathrm{RON})$ of $\mathrm{RON}=7.21 e^{-}$. The grism $600 \mathrm{R}$ was an optimal choice to achieve a medium resolution within the resulting wavelength range appropriate for spiral galaxies at $z \leq 1.0$ that covers the spectral ranges of the [O II] 3727 doublet or the $\mathrm{H} \beta$ and the [O III] 5007 emission lines. The default order separation filter GG435+81 was used. A slit positioned in the center of the CCD chip covers a 
wavelength range between $5200 \AA \leq \lambda \leq 7400 \AA$. However, as the starting and the ending wavelength depend on the position of the slit on the $\mathrm{X}$ axis, the wavelength range can vary between two extremes of the MOS mask $6300 \AA \leq \lambda \leq 8500 \AA$ (extreme left hand side) or $4100 \AA \leq \lambda \leq 6300 \AA$ (extreme right hand side).

Each MOS slit can be set individually to slit widths between $0.3 \operatorname{arcsec}$ and 60 arcsec. For all observations a fixed value of one arcsecond was chosen, yielding a spectral resolution of $\mathrm{R} \approx 1200$ with the $600 \mathrm{R}$ grism. Although a smaller slit width would have increased the instrumental resolution, a significant loss of flux would have occurred for seeing conditions above $\mathrm{FWHM} \approx 0.8$ arcsec, which roughly corresponds to the median seeing at Paranal since 1998 according to the Differential Image Motion Monitor (DIMM).

Between October and November 2001 an upgrade of the FORS2 CCD system was installed at UT4 (Yepun) for testing purposes, which was available for science use in March $2002^{5}$. The new FORS2 upgrade detector system consists of two $2048 \times 4096$ pixel MIT/LL CCID-20 CCD chips $(15 \mu \mathrm{m}$ pixel size). The MIT CCDs provide much higher response in the red wavelength range $(>8000 \AA)$, with impressively low fringe amplitudes. At default (standard) setup, the new chip has a spatial scale of $0.25 \mathrm{arcsec} /$ pixel with a gain of $0.70 e^{-} / \mathrm{ADU}$ (in high gain $100 \mathrm{kHz}$ mode). The RON differs slightly between the chips, for the "master" chip ("Thor") $\mathrm{RON}=2.7 e^{-}$and the "slave" chip ("Belenos") $\mathrm{RON}=3.0 e^{-}$. Moreover, the volume phased holographic grism 600RI for FORS2 turned out to be even more efficient than the grism 600R, particularly at redder wavelengths. Again, the default order separation filter GG435+81 was used.

In order to get an $S / N \sim 16$ over an area of $1^{\prime \prime} \times 0.25^{\prime \prime}$ at $\lambda_{c} \approx 6552 \AA$ in the continuum

\footnotetext{
${ }^{5}$ FORS2 upgrade: www.eso.org/projects/odt/Fors2/Fors2u.html
}

for a typical early-type galaxy with brightness $R \leq 20.5^{m}$, the on-line ESO Exposure Time Calculator (www.eso.org/observing/etc/), suggested an integration time of $\sim 2.5 \mathrm{hrs}$ with the instrumental configuration as given above.

\subsubsection{Selection Criteria}

After a pre-selection of possible spectroscopic field target objects, different galaxy catalogues were generated within the ESO-MIDAS environment and used as input for setting up and constructing the MOS masks with the FIMS tool. The FIMS package output consisted of three files per mask. For example, these give definitions on the position, angle and orientation of the mask on the plane of the sky, the positions of the individual slits, coordinates of reference stars in the field-of-view (for positioning of the mask during the observations with an accuracy $<0.1$ arcsec), et cetera. In turn, these output files were used for the construction of the so-called Observing Blocks (OBs) which act as input to the operating system of the VLT telescope in the execution of the observations at Paranal for the field sample. For a more extensive description of the operation environment, the reader is referred to the "FORS1+2 User's Manual" released by ESO (www.eso.org/instruments/fors/doc/).

The object selection for the FDF sub-sample was based on the deep $U B g R I$ photometry of the FDF (Heidt et al. 2003). The $50 \%$ completeness limits for point sources as derived from the co-added images are 25.64, 27.69, 26.86, 26.68, 26.37 in the U, B, g, R, I filters (Vega-system), respectively. The candidates were selected according to their spectrophotometric type, their elongated structureless appearance, their luminosity and photometric redshift. Main criterion was the apparent brightness. The other parameters were taken into account to put additional constraints on the selection. For the early-type targets, this limit in total apparent magnitude was $R \leq 22.0^{m}$ as derived with the Mag_auto algorithm of the Source Extractor package (Bertin 
\& Arnouts 1996). This constraint was set to ensure sufficient signal-to-noise of $S / N \geq 10$ in the absorption lines which is mandatory for a robust determination of velocity dispersions and line strengths measurements. For this reason, faint early-type candidates with apparent magnitudes $R>20.5^{m}$ were included in more than one MOS setup and the individual spectra combined after data reduction.

Spectrophotometric types and estimated redshifts were selected from the FDF photometric redshifts catalogue of more than 3800 objects in the August 2000 release (Bender et al. 2001). Only respective candidates with an early (E/S0) model Spectral Energy Distribution (SED) were considered and the photometric redshifts were restricted to $z_{p} \leq 0.6$. Based on the photometric redshifts, the elliptical candidates were spread out on different CCD positions among the spirals for each MOS setup in the observed spectral wavelength range such that either the $\mathrm{Mg}_{b^{-}}$ feature passband $\left(\lambda_{0} \approx 5170 \AA\right)$ or the G-band $\left(\lambda_{0} \approx 4300 \AA\right)$ was included. Both features of interest could not be observed for all candidates because of the correlation of the slit position with the available wavelength window (see also section 2.2.3 before). Moreover, for an additional star/galaxy separation criterion, targets with insignificant photometric redshift and large uncertainty (i.e., $z_{\text {phot }}-d z_{\text {phot }} \leq 0$ ) were rejected.

In contrast to spiral galaxies which require a selection upon inclinations and position angles for the derivation of rotational velocities, for the MOS targets with an early-type SED, neither inclinations nor position angles have been taken into account as selection criteria. However, as a small fraction of early-type galaxies in the photometric redshift catalogues could be misclassified $\mathrm{M}$-dwarf stars, it was mandatory to use structural parameters for a robust star/galaxy separation. Detections of the Source Extractor on the I-band image with $0.49^{\prime \prime}$ FWHM which were most likely bonafide stellar objects exhibited a star classification parameter star $\geq 0.9$ and $a \approx b \approx 2.5$ pixel. Therefore, to avoid misclassifications targets with star $\geq 0.9$ or $a \approx b \leq 3$ pixel have been rejected.

Although the two-dimensional distribution of FDF objects indicated a possible cluster of galaxies with $z_{p} \approx 0.33$ and the primary goal was to target field elliptical galaxies, no preselection against such candidates was performed. In the spectral analysis (see section 5.5), it was confirmed that 15 out of 32 observed elliptical galaxy candidates are most probably members of a cluster. Based on the the radial velocity measurements for these galaxies, the lower limit for the velocity dispersion of the cluster is $\sigma_{c} \gtrsim 430 \mathrm{~km} \mathrm{~s}^{-1}$. Since the cluster centre is not located on the FDF but only its outskirts, the true $\sigma_{c}$ is probably larger. At a redshift of $z=0.33$, the $\mathrm{Mg} 5170$ absorption line is unfortunately corrupted due to the terrestrial absorption of the B band. As an accurate measurement of the internal galaxy velocity dispersions is impossible due to this effect these cluster galaxies were discarded from the analysis.

Apart from photometric redshifts, the target selection for the WHDF field early-type galaxies was performed with the same constraints as for the FDF field elliptical galaxies. As no photometric redshifts were available, the redshifts of field early-type candidates were estimated using a combination of colour-colour diagrams and apparent magnitudes. Fig. 2.6 illustrates the target selection of the WHDF field elliptical candidates in the $(B-R)-(R-I)$ colourcolour diagram. The WHDF data and the final WHDF field ellipticals are compared to evolutionary tracks for E/S0 with different formation redshifts of $z_{\mathrm{f}}=2$ (solid line) and $z_{\mathrm{f}}=4$ (dotted line) as predicted by the passive evolution models of Bruzual \& Charlot 1993; GISSEL96 version, hereafter BC96). Note, that the BC96 models have been computed for a slightly different cosmology with $H_{0}=60 \mathrm{~km} \mathrm{~s}^{-1} \mathrm{Mpc}^{-1}$ and $q_{0}=0.1$. In the $(B-R)-(R-I)$ colour-colour diagram elliptical and spiral galaxies are well sep- 


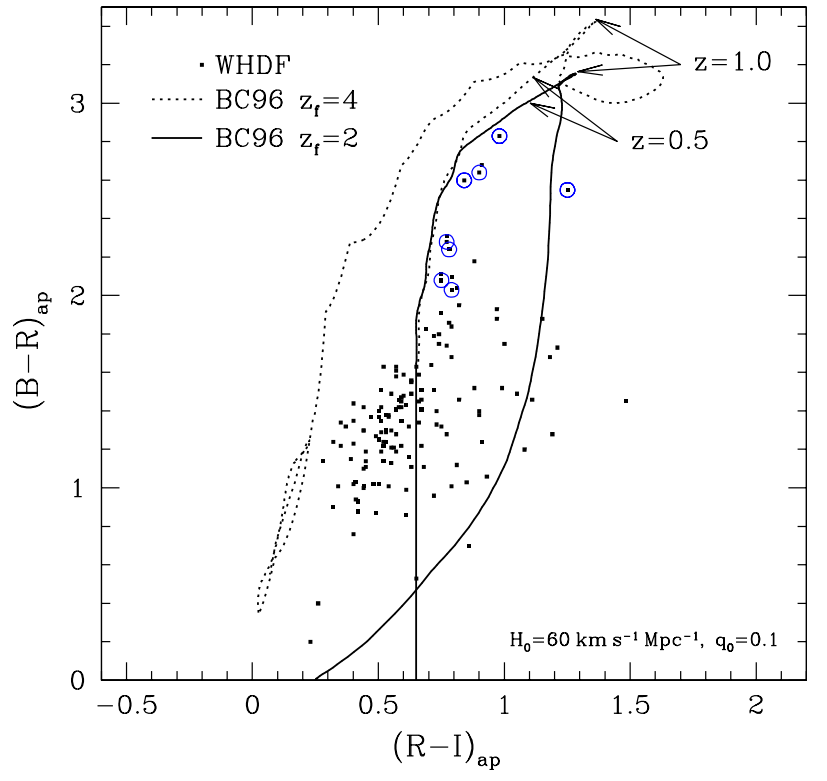

Figure 2.6: $(B-R)-(R-I)$ colour-colour diagram for the WHDF field elliptical galaxies. The WHDF spiral candidates (squares) are displayed with the final WHDF field ellipticals (circles) and compared to evolutionary tracks for E/S0 with different formation redshifts of $z_{\mathrm{f}}=2$ (solid line) and $z_{\mathrm{f}}=4$ (dotted line) predicted by the BC96 models. For the BC96 models a cosmology with $H_{0}=60 \mathrm{~km} \mathrm{~s}^{-1} \mathrm{Mpc}^{-1}$ and $q_{0}=0.1$ was adopted.

arated until $z \approx 0.5$ (indicated by the arrows) and follow the predictions of the evolutionary models quite well. The apparent $R$ magnitude was used as an additional constraint to avoid a selection of high redshift elliptical galaxies with $z>1$. As shown in Fig. 2.6, galaxies populating a narrow range in the colour-colour diagram of $2 \gtrsim(B-R) \gtrsim 2.9$ and $0.7 \gtrsim(R-I) \gtrsim 1.1$ have been selected as bona fide field ellipticals and successfully verified. One object (ID \# 14) with $(R-I)=0.79,(B-R)=1.78$ and $R=19.04^{m}$ $(z=0.1060)$ is an intermediate-type Sc spiral galaxy and was therefore discarded as an earlytype candidate. In fact, on the ACS images it turned out that this galaxy is a spiral with a clearly visible disc.

\subsubsection{Observations}

To construct a distant field elliptical galaxy sample with $N_{\text {obj }} \approx 30$ within the FDF, roughly $30 \%$ of the reserved observing time for the TullyFisher project were used. However, as half of these galaxies turned out to be possible members of a cluster at $z=0.33$, an additional sample of ten elliptical galaxies was selected from the WHDF. The FDF and WHDF target selection for the observations was done by A. Böhm and A. Fritz. An outline of the observations of the field early-type galaxies is given in Table 2.4 for the FDF sample and in Table 2.5 for the WHDF galaxies.

The first FDF spectroscopic observations were carried out with FORS1 in December 1999 in Visitor Mode by C. Möllenhoff (Heidelberg) and K. Reinsch (Göttingen) with a mean DIMM seeing of 0.66 arcsec. However, only one MOS setup was observed and two major drawbacks occurred. At this time the FDF imaging data reduction was not fully completed yet and the target selection was therefore based on a coadded 1200 sec $R$-band image (Point-Spread-Function (PSF) of $0.72^{\prime \prime}$ FWHM) gained during commissioning time of FORS1. Furthermore, for the selection only a preliminary version of the photometric catalog was available. In total, 3 earlytype galaxies, 13 spiral galaxies and 3 objects without any catalog information (without SED and $\left.z_{\text {phot }}\right)$ have been selected. The galaxies classified as "anonymous" turned out to be earlytype galaxies with $R$-band magnitudes of $19.1^{m}$, $19.4^{m}$ and $20.8^{m}$. Unfortunately, after the redshift analysis 3 early-type galaxies were identified to be members of a cluster at $z=0.33$. Therefore, these galaxies were rejected for this investigation.

In September and October 2000 a total of 6 MOS setups were observed by S. Noll and D. Mehlert (both Heidelberg) with FORS2. Each setup was splitted into three single exposures of $3000 \mathrm{sec}$ with a total integration time of 2.5 hours. The targets were selected from a deep FORS2 I- 
Table 2.4: Observations of FDF early-type galaxies

\begin{tabular}{|c|c|c|c|c|c|c|c|}
\hline Tel./Instrument & Date & $\theta$ range & airm. & DIMM & $\mathrm{E}+\mathrm{S} 0$ & anon. & $\begin{array}{c}T_{\text {exp }} \\
{[\mathrm{ksec}]}\end{array}$ \\
\hline VLT/FORS1 & $\sum^{12 / 99}$ & $-30^{\circ} / 0^{\circ}$ & 1.08 & $0.66^{\prime \prime}$ & $\begin{array}{l}3 \\
6\end{array}$ & $\begin{array}{l}3 \\
-\end{array}$ & 9.0 \\
\hline VLT/FORS2 & $\begin{array}{c}09 / 27 / 00 \\
09 / 27 / 00 \\
10 / 04 / 00 \\
10 / 05 / 00 \\
10 / 05 / 00 \\
10 / 06 / 00 \\
\sum\end{array}$ & $\begin{array}{r}-90^{\circ} /-60^{\circ} \\
-60^{\circ} /-30^{\circ} \\
-30^{\circ} / 0^{\circ} \\
0^{\circ} /+30^{\circ} \\
+30^{\circ} /+60^{\circ} \\
+60^{\circ} /+90^{\circ}\end{array}$ & $\begin{array}{l}1.21 \\
1.33 \\
1.40 \\
1.36 \\
1.28 \\
1.15\end{array}$ & $\begin{array}{l}0.51^{\prime \prime} \\
0.43^{\prime \prime} \\
0.81^{\prime \prime} \\
0.80^{\prime \prime} \\
0.74^{\prime \prime} \\
0.66^{\prime \prime}\end{array}$ & $\begin{array}{r}5 \\
5 \\
6 \\
5 \\
8 \\
7 \\
28\end{array}$ & $\begin{array}{l}1 \\
- \\
1 \\
2 \\
- \\
2 \\
6\end{array}$ & $\begin{array}{l}9.0 \\
9.0 \\
9.0 \\
9.0 \\
9.0 \\
9.0\end{array}$ \\
\hline VLT/FORS1 & $\begin{array}{c}10 / 12 / 01 \\
10 / 14 / 01 \\
10 / 12 / 01 \\
\sum\end{array}$ & $\begin{array}{r}0^{\circ} /+30^{\circ} \\
+30^{\circ} /+60^{\circ} \\
+60^{\circ} /+90^{\circ}\end{array}$ & $\begin{array}{l}1.43 \\
1.07 \\
1.38\end{array}$ & $\begin{array}{l}0.76^{\prime \prime} \\
0.89^{\prime \prime} \\
0.82^{\prime \prime}\end{array}$ & $\begin{array}{r}6 \\
5 \\
5 \\
10\end{array}$ & $\begin{array}{l}1 \\
0 \\
3 \\
4\end{array}$ & $\begin{array}{l}9.0 \\
9.0 \\
9.0\end{array}$ \\
\hline
\end{tabular}

band reference image consisting of 10 seeing averaged $I$-band images (between $0.47-0.50$ arcsec FWHM) with a final integration time of $3000 \mathrm{sec}$ and a PSF of 0.49 arcsec FWHM (see Böhm 2003 for the construction of the FORS1/FORS2 reference images). All in all $28 \mathrm{E}+\mathrm{S} 0$ galaxies were observed from a list of 32 candidates with available spectrophotometric information (see previous section). Eight faint early-type candidates with apparent magnitudes $R>20.5^{\mathrm{m}}$ were observed with two MOS setups to increase the respective exposure time and the $S / N$ in the final spectra. Two galaxies were already observed in 1999. Typically, each setup contained 2-4 elliptical candidates. Using slit widths of $1^{\prime \prime}$, with the $600 \mathrm{R}$ grism a spectral resolution of $R=1160$ was achieved. The seeing conditions (DIMM seeing values) were varying between $0.43^{\prime \prime}$ and $0.81^{\prime \prime}$ FWHM (median of $0.66^{\prime \prime}$ FWHM) and sufficient to meet the Nyquist theorem to allow a perfect reconstruction of the signal from the samples (see section 5.1.1). The constraint on the airmass was $A \leq 2.0$ in order to limit the corrections of atmospheric absorption.
Early in 2001, an additional amount of GTO time was granted by ESO for the FDF collaboration to compensate the high amount of time loss during the imaging phases of the FDF which was caused by the El Niño phenomenon. From this GTO pool, the P.I. of the FDF collaboration, Prof. I. Appenzeller (Heidelberg), thankworthy allocated one night of dark time for the field galaxy project of spiral and elliptical galaxies.

In October 2001 additional three different setups were acquired with with FORS1 at the VLT in Visitor Mode by J. Heidt (Heidelberg). The target selection was based on a FORS1 $I$-band reference image with a PSF of 0.52 arcsec FWHM. In the search of the photometric redshifts catalogues for new field elliptical candidates with $R \leq 22^{m}$ and $z_{\text {phot }}<0.6$ only two objects were detected which had not been observed previously. Again the setups were splitted into three separate exposures $\left(T_{\text {tot }}=9000 \mathrm{sec}\right)$ with an integration time of 2.5 hours for each setup. 15 out of 29 galaxies located in the southwestern corner of the FDF were neglected to avoid the outskirts of the cluster at $z=0.33$ (see chap- 
Table 2.5: Observations of WHDF early-type galaxies

\begin{tabular}{llrrrrrr}
\hline Tel./Instrument & Date & $\theta$ range & airm. & DIMM & E+S0 & anon. & $\begin{array}{c}T_{\exp } \\
{[\mathrm{ksec}]}\end{array}$ \\
\hline VLT/FORS2 & $07 / 10,10 / 03 / 02$ & $-90^{\circ} /-60^{\circ}$ & 1.18 & $0.64^{\prime \prime}$ & 1 & 1 & 9.0 \\
& $08 / 07,09 / 11 / 02$ & $-60^{\circ} /-30^{\circ}$ & 1.19 & $0.73^{\prime \prime}$ & 2 & - & 9.0 \\
& $08 / 04,08 / 07 / 02$ & $-30^{\circ} / 0^{\circ}$ & 1.51 & $0.92^{\prime \prime}$ & 3 & - & 9.0 \\
& $08 / 04,10 / 02 / 02$ & $0^{\circ} /+30^{\circ}$ & 1.13 & $0.89^{\prime \prime}$ & 3 & 1 & 9.0 \\
& $09 / 10,09 / 12 / 02$ & $+30^{\circ} /+60^{\circ}$ & 1.18 & $0.91^{\prime \prime}$ & 2 & - & 9.0 \\
& $09 / 12,10 / 4-5 / 02$ & $+60^{\circ} /+90^{\circ}$ & 1.14 & $0.85^{\prime \prime}$ & 2 & - & 9.0 \\
$\sum$ & & & & 11 & 1 & \\
\hline
\end{tabular}

ters 5.5 for a further discussion of this topic). In addition, eight bonafide field $\mathrm{E}+\mathrm{S} 0$ galaxies with $R>20.5^{m}$ which already were observed in 2000 were selected to increase the $S / N$ in the combined spectra. A total of 10 early-type galaxies was targeted and thus except one bright elliptical with $R=19.0^{m}$, all early-type candidates were observed two or three times including the spectroscopy in 1999 and 2000. The seeing conditions were slightly poorer than during autumn 2000 , but still in the sub-arcsecond regime. The median DIMM seeing was $0.82^{\prime \prime}$ FWHM.

Between July and October 2002 spectroscopic observations for 10 WHDF ellipticals were carried out with the FORS2 instrument attached to the ESO/VLT at Cerro Paranal, Chile. This observing time was not part of any GTO programme but additionally granted. A total of six different setups were observed, each splitted into three separate exposures of $3000 \mathrm{sec}$ with a total integration time of 2.5 hours. Targets were selected through a combination of colourcolour diagrams and apparent magnitudes (see section 2.2.4). For the MOS mask construction with FIMS, a deep WHDF I-band reference image consisting of 5 seeing averaged $I$-band images with a final integration time of $1500 \mathrm{sec}$ and a PSF of 0.6 arcsec FWHM was used. The slit width was set to $1^{\prime \prime}$, and with the 600RI grism a spectral resolution of $R=1000$ was achieved. The DIMM seeing values were be- tween $0.73^{\prime \prime}$ and $0.92^{\prime \prime}$ FWHM with a median of $0.89^{\prime \prime}$ FWHM. Two additional objects, one in the setup $0^{\circ} \leq \theta \leq+30^{\circ}$ and one in the setup $-90^{\circ} \leq \theta \leq-60^{\circ}$, fell by coincidence into the slit of the galaxy \#810 and \#92, respectively. The second object in slit \# 810 turned out to be a field S0 galaxy at $z=0.2118$ and was named \# 810b. The other object in slit \# 92 is a background spiral galaxy at $z=0.5569$ and thus was discarded. Therefore, the total sample of WHDF elliptical galaxies comprises eleven field early-type galaxies. 


\section{Chapter 3}

\section{Data Reduction}

This thesis comprises a large amount of data from three different projects, the rich Abell 2390 cluster, the three Low $-L_{X}$ clusters and two field galaxy samples from the FDF and WHDF, all aiming to investigate the population of earlytype galaxies in various environments. The reduction of the optical ground-based multi-band imaging data for the two cluster samples, from which the apparent magnitudes of the early-type galaxies were derived, was performed mainly by M. L. Balogh and I. R. Smail. Nevertheless, some aspects for this study had to be re-analysed (see chapter 4). For the FDF and WHDF field elliptical galaxies A. Böhm was kindly providing the measurement of ground-based magnitudes. As a full review of the imaging reduction is beyond the topic of this thesis, it therefore will not be included. A summary of the observations and imaging for Abell 2390 is provided in the Table 2.2 on page 30 and for the three poor clusters in Table 2.3 (page 31) and Table 4.2 (page 56).

All the subsequent sections will focus on the special aspects of the MOSCA spectra reduction. As the reduction of the FDF and WHDF VLT/FORS spectra was performed in an analogous manner it thus will not be described here separately. The reduction procedure was undertaken using the ESO-MIDAS ${ }^{1}$ environment with own FORTRAN program routines and the IRAF ${ }^{2}$

\footnotetext{
${ }^{1}$ ESO-MIDAS, the European Southern Observatory Munich Image Data Analysis System is developed and maintained by the European Southern Observatory.

${ }^{2}$ IRAF (Image Reduction and Analysis Facility) is dis-
}

data analysis software and followed the standard procedure.

The ESO-MIDAS package provides a variety of algorithms for data processing for applications of long-slit reduction, such as wavelength calibration and rebinning of two-dimensional spectra. Nevertheless, special routines had to be developed, for example, to rectify the distortions of the spectra due to the focal reducer (see section $3.3)$.

A theoretical review of data reduction will not be given here, see, e.g., Wagner (1992) or Volume B of the ESO-MIDAS User's Guide, Chapter 6 (available for download at www.eso.org/projects/esomidas/doc/). For a discussion of the derivation of HST structural parameters, see section 4.3). In the following, the individual stages of the reduction procedure will be described in the same order as they were applied to the data.

\subsection{Bias Correction}

For each observing run, bias frames were taken at the beginning and end of the night. All frames showed a very stable two-dimensional structure with spatial variations of $\leq 3 \mathrm{ADU}$. Thus, all bias frames from the individual nights

tributed by the National Optical Astronomy Observatories (NOAO) in Tucson, Arizona, which is operated by the Association of Universities for Research in Astronomy (AURA), Inc., under cooperative agreement with the National Science Foundation. 
were used to generate master biases, one for each observing run. An averaged super bias image was constructed of median-scaled individual bias frames. After calculating the median in the overscan region of the super bias ( $\mathrm{X}>2022$ pixel), which is $\sim 271 \mathrm{ADU}$, the super bias frame was scaled to the median of each individual bias image. A total of six bad columns were cleaned by interpolating from non affected adjacent columns. Finally, this scaled master bias frame was subtracted from each frame. Variations in the bias level were $\leq 5 \%$.

Several dark exposures were gained at the beginning and the end of one night for each observing run. Since the dark current was very uniform across the CCD chips and amounted to less then 5 ADU per 45 minutes, an additional dark frame subtraction has been neglected.

\subsection{Cosmic Rays}

Although the observation of each MOS mask setup was split into at least five individual exposures, a cosmic ray rejection by a simply medianaveraging of the frames was not adequate because the CCD positions of the individual galaxy spectra were not time-independent. If during one observation of a mask the zenithal distance was significantly varying, the spatial position of a spectrum differed by up to two pixel (0.66 arcsec) between the first and last exposure. Therefore, 2D images of each slit spectrum were extracted individually from the MOS frame (after bias subtraction) and reduced separately as long-slit data. In total, for each sample a number of $N_{\text {exp. }} \times N_{\text {slits }}$ science spectra per MOS setup and $N_{\text {mask }} \times N_{\text {MOS }}$ science spectra were reduced, in numbers 735 science spectra for A 2390 (3 masks) and 264 science spectra for the Low$L_{X}$ clusters (one mask each). Table 3.1 gives a summary of the analysed science spectra.

Cosmic ray events were removed very careful using the MIDAS algorithm FILTER/COSMIC to each individual slit spectrum. The parameters of
Table 3.1: Summary of MOSCA science spectra.

\begin{tabular}{lrccc}
\hline Sample & Exp. & Slits/Obj. & MOS & $\sum$ \\
\hline A 2390 & 11 & $16 / 17$ & 176 & \\
& 13 & $20 / 22$ & 260 & \\
& 13 & $23 / 24$ & 299 & 735 \\
Cl 0849 & 6 & $17 / 17$ & 102 & \\
Cl 1701/Cl 1702 & 9 & $18 / 18$ & 162 & 264 \\
\hline
\end{tabular}

this $\kappa-\sigma$ clipping which tune the discrimination between physical objects and artificial artefacts were set to moderate values in order not to affect possible emission features from the galaxies. In addition, after the filtering process each slit frame and the map of rejected pixels were visually inspected and compared to each other. A second cosmic ray inspection was done at the stage of the sky subtraction. However, this final correction was performed by a purely manual removal of any contamined pixels, if artificial features were identified in a visual investigation of the completely reduced 1D spectra.

Furthermore, between one and up to five bad columns per slitlet were cleaned by interpolating from unaffected adjacent columns.

\subsection{Distortion Correction}

Normally, the step of rectification is applied after the correction of the science spectra using flatfield exposures. However, due to the fact that the MOSCA focal reducer causes very strong geometric distortions and an approximation of the CCD response function is impossible, the rectification has to be performed before the flatfield correction.

For the slits at the top and bottom of the CCD chip which are lying closest to the edges of the MOS field, the distortions were strongest and the curvature of spectral features corresponds to a displacement of up to 20-22 pixel in Y direction, 


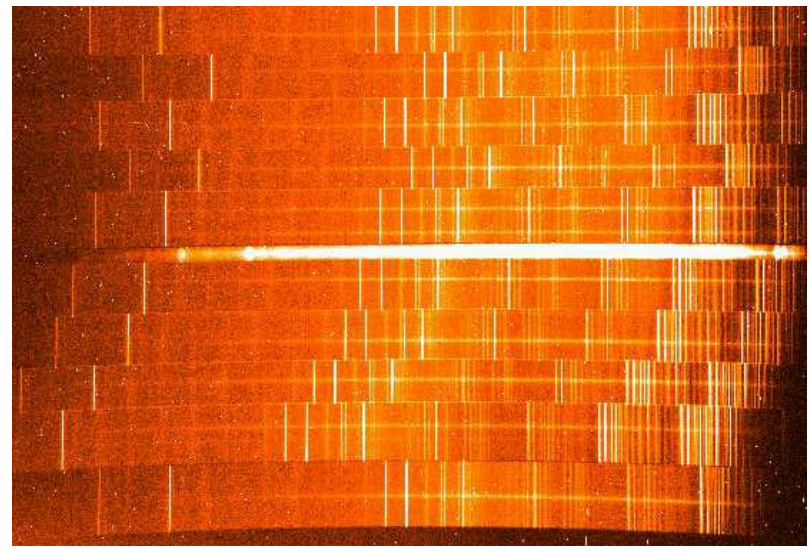

Figure 3.1: Lower part of a raw MOS frame of mask 1 for Abell 2390 with 3000 seconds exposure time showing the slits \# 1 to \# 10. The magnification scale along the $\mathrm{X}$ axis corresponds to $\sim 680$ pixel, and along the $\mathrm{Y}$ axis to $\sim 720$ pixel. The bright spectrum in the middle belongs to a star which was used for the alignment of the MOS mask. The wavelength is increasing parallel to the $\mathrm{X}$ axis from left to right. The curvature of the spectra is clearly visible for the lower slits, whereas slit \# 7 in the center of the CCD chip is nearly undistorted.

whereas slits \# 7 and \#8 situated in the middle of the chip were least undistorted (9 pixel). In the worst case, the outermost slit \# 17 was distorted by 35 pixel (Low- $L_{X}$ April 2001 run). Figs. 3.1 and 3.2 visualise the curvature of the raw MOS spectra. Note, that the slits are numbered from bottom to top by convention.

For the purpose of correcting the S-distortions (curvature) of the spectra, an IRAF program was upgraded which fits a user-defined Legendre polynomial (in most cases of third order) to the galaxy spectra in each slit to derive the parameters for the curvature correction along the spatial axis. The same set of coefficients were then applied to the science, flatfield and corresponding wavelength calibration frames, respectively. The rectification was performed by rebinning the respective spectra in $\mathrm{Y}$ direction to a scale of 0.033 arcsec and shifting each column according to the fit either in the $+\mathrm{Y}$ direction for the slits \# 1 to \# 8 or $-\mathrm{Y}$ direction

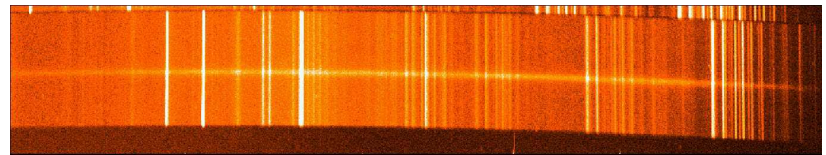

a. original

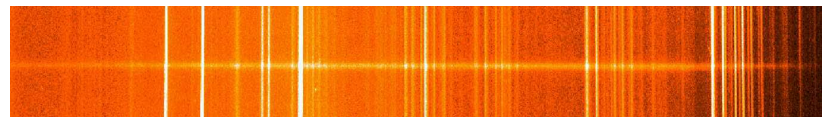

b. corrected

Figure 3.2: $\quad$ S-distortion correction for the A 2390 galaxy \# 2453 ( $I=17.27 \mathrm{mag})$ of slit \# 1 . The upper panel a. shows the uncorrected $2 \mathrm{D}$ spectrum and in the lower panel $\mathbf{b}$. the corrected $2 \mathrm{D}$ spectrum is plotted. The $\mathrm{X}$ axis corresponds to $\sim 2000$ pixel, the $\mathrm{Y}$ axis to $\sim 180$ pixel. The positions of the skylines, which showed no distortion in spatial direction were used to judge the quality of the curvature correction.

in case of slits \# 9 to \# 17 for mask 1 and 2 of the Low- $L_{X}$ April 2001 runs. The number of slits varied for different MOS setups (see e.g., Table 2.2 of Observations for A 2390), with a maximum of 24 slits for mask 3 of the A 2390 Sep. 2000 run only. This procedure is shown as an example for galaxy \#2453 in Fig. 3.2, where the original, uncorrected $2 \mathrm{D}$ spectrum is compared to the spectrum after the S-distortion correction. Tests with other rectification algorithms confirmed that this procedure leads to flux-conserving results to within a few percent. In addition, the routine features also a step for the removal of cosmic ray hits (the "clean" task). However, this rejection was not applied as no cosmics were detected.

Possible artifical tilts of the emission lines (i.e. such visible in FORS spectra) were tested for different wavelength calibration frames. However, as no distortions in spatial direction were detected, no further rectification along the $\mathrm{X}$ axis was necessary. For this reason, the positions of the skylines were applied to judge the quality of the curvature correction. 


\subsection{Flat-fielding}

In this step two features are addressed, the correction of the pixel-to-pixel variations due to intrinsic sensitivity differences of the detector and to account for the illumination function of the slit. Dome flat-field (FF) exposures through the masks were taken by illuminating the telescope dome with the external (within the dome installed) FF lamp. With exposure times of $100 \mathrm{sec}$, spectra have typically $\sim 4000$ ADU. For both Low- $L_{X}$ runs all three lamps 2,3 and 4 were switched on together, yielding in $\sim 40000$ ADU for an exposure time of $30 \mathrm{sec}$. Due to the relatively short exposure times, none of these calibration frames were contaminated by cosmic ray artefacts, which was additionally confirmed by a cosmic ray removal test and a visual inspection.

A minimum number of five dome flat-fields were used to construct a master flatfield through a combination of median-averaged single flatfield exposures. After the rectification, the individual flatfield slit exposures were approximated separately by a spline function of third degree to the continuum of each spectrum in dispersion direction (X axis) to account for the CCD response function. The smoothed spline fit was afterwards applied for normalisation of the original flatfield frame in order to correct for pixel-to-pixel variations.

Several tests with different setups using the internal tungsten continuum source (at $60 \%$ illumination) resulted in $\mathrm{FF}$ images with too low counts. In addition, there are two main problems in using the tungsten lamp. Firstly, the continuum lamp is hot and therefore generates a blackbody spectrum different from the night sky, which has to be removed before it can be applied for the flatfielding correction. Secondly, as this lamp is located relatively near the CCD chip, the slits get illuminated in a different way than when pointed at the sky. However, after a removal of the blackbody spectrum of the tungsten source, the counts in the FF frames towards the end of the spectrum are quite low.
This might affect blue absorption indices such as $\mathrm{CN}\left(\lambda_{\mathrm{c}} \sim 3830 \AA\right)$ or $\mathrm{CaH}+\mathrm{K}\left(\lambda_{\mathrm{c}} \sim 3980 \AA\right.$ and $\lambda_{\mathrm{c}} \sim 3950 \AA$, respectively) for a galaxy at a redshift of $z \sim 0.1$, which fall near to the blue end of the spectrum. A FF calibration exposure with low $S / N$ will only add additional noise to these blue features of interest.

Skyflats offer maybe the best results in correcting the illumination of the CCD chips. However, due to varying weather conditions only a few dusk sky FF calibration frames per night could be taken. As a median over at least five skyflats is needed for a master sky FF, a flatfield correction with skyflats was neglected.

\subsection{Sky Subtraction}

The sky background was subtracted using the FORTRAN routine hisupoly, kindly provided by Prof. R. Bender. This algorithm approximates the night sky by iteratively fitting each CCD column separately through a $\kappa-\sigma$ clipping algorithm. The order of the polynomial fit is a free parameter. For most cases a first order was used, if the night sky was relatively bright or varied within the slit frame, a fit of zero order was applied. The quality of sky subtraction was warranted through a generated verification frame containing all fitted and extracted night sky lines. If this image comprised only few sky lines or some artefacts were still within the sky subtracted spectrum the procedure was repeated again.

To ensure consistent profile centers to within at least half a pixel, corresponding to $0.17^{\prime \prime}$, the one-dimensional spectra were extracted for each exposure separately using the Horne-algorithm (Horne 1986). Therefore, possible shifts of the spectra which may have occurred during different nights are accounted for in the final onedimensional summed-up spectra. The Horneprocedure optimally weights the rows of the extracted spectrum profile to maximise the signalto-noise in the resulting one-dimensional spec- 
trum. In addition, this algorithm removes cosmic ray events by analysing the profile perpendicular to the dispersion (along the $\mathrm{Y}$ axis).

However, the method of optimal weighting by Horne (1986) might have an effect on the velocity dispersion $(\sigma)$ measurements of the galaxies. For this reason, a second independent extraction method was performed but the impact on the velocity dispersions is smaller than their typical measurement uncertainty (see section 5.3.5 for a more detailed discussion).

\subsection{Wavelength Calibration}

For the wavelength calibration exposures, the $\mathrm{HgAr}$ and Ne lamps were switched on together in order to gain a sufficient number of emission lines over a large range of $\lambda \lambda \approx 5450-7450 \AA$. After each series of two science mask observations, while the telescope still pointing at the target object, an additional $\mathrm{HgAr} / \mathrm{Ne}$ exposure was taken. This procedure allows to account for any possible flexure or deflection in the instrument due to a varying telescope pointing position which might affect the wavelength calibration frames if they were gained while the telescope was still in parking mode and pointing at zenith. Repeat observations were unacceptable because of a too large overhead time. However, the combination of $\mathrm{HgAr} / \mathrm{Ne}$ lamps has the disadvantage of almost total lack of calibration lines below $5461 \AA$ : only two stronger $\mathrm{Hg}$ emission lines at $4358 \AA$ and $4047 \AA$ are visible. In order to overcome this inconvenience, a combination between the Ar lamp and the standard MOSCA BV-filter $\left(\lambda_{0}=4720 \AA\right.$, with a $\approx 785 \AA$ bandwidth over a wavelength region of $\lambda \lambda \approx 3930-5510 \AA$ ) was used to increase the useable wavelength lines within the blue spectral region. During the CAHA observations of the Low- $L_{X}$ run (April 2001), different setups of the Ar lamp with the BV-filter $(472 / 78)$ were observed. Table 3.2 gives an overview of wavelength calibration setups with and without the
Table 3.2: Calibration setups using the BV-filter.

\begin{tabular}{llcr}
\hline Case & Lamp & Filter & $T_{\text {exp }}[\mathrm{sec}]$ \\
\hline & & & \\
$\mathrm{A}$ & $\mathrm{HgAr}$ & - & 5 \\
$\mathrm{~B}$ & $\mathrm{HgAr}$ & $\mathrm{BV}$ & 15 \\
$\mathrm{C}$ & $\mathrm{HgAr} / \mathrm{Ne}$ & - & 15 \\
$\mathrm{D}$ & $\mathrm{Ar}$ & $\mathrm{BV}$ & 120 \\
$\mathrm{E}$ & $\mathrm{Ar}$ & - & 3000 \\
& & & \\
\hline
\end{tabular}

BV-filter.

Different combinations between a wavelength calibration frame and the BV-filter were tested. Overall, wavelength calibration setups with exposure times of $5 \mathrm{sec}$ (case A) or without using the Ne lamp (case B) resulted in too few emission lines. For case E, only a long exposure of the Ar lamp, too many lines were overexposured and thus were not useable for measuring the line-widths. The most promising test was a standard wavelength calibration frame of $15 \mathrm{sec}$ of all three $\mathrm{HgAr} / \mathrm{Ne}$ lamps, augmented by a $120 \mathrm{sec}$ exposure of the Ar lamp using the $\mathrm{BV}$-filter (case $\mathrm{C}+\mathrm{D})$. This test wavelength calibration spectrum is shown in Fig. 3.3. The long exposure with the single Ar lamp was used in order to uncover weak emission lines within the blue wavelength region and the BV-filter to suppress lines at the blue and especially at the red end of the spectrum, which would otherwise become due to the long integration time saturated. As a result, a couple of weaker emission lines are gained below $5461 \AA$ and a handful of lines within the blue region of interest below $4358 \AA$. However, despite the BV-filter all of these blue lines are overexposured and therefore "smeared-out", making them impossible for a reliable wavelength calibration (see "blue region" in Fig. 3.3). In addition, the important line at $4047 \AA$ gets totally corrupted by these very broad blue lines, thereby becoming nearly invis- 


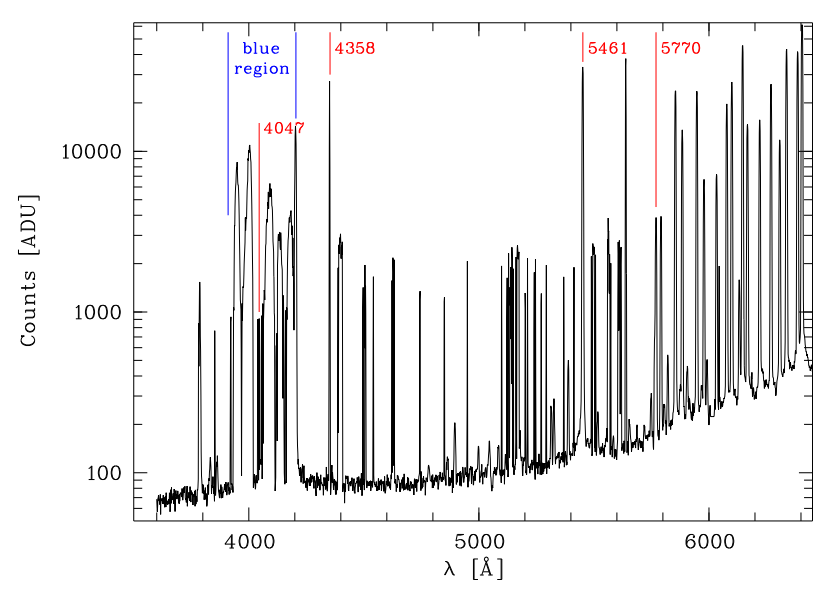

Figure 3.3: Wavelength calibration spectrum with a supplementary BV-filter. The dotted lines indicate calibration lines of the standard $\mathrm{HgAr} / \mathrm{Ne}$ wavelength spectrum, whereas the 'blue region' marks a region of saturated, broad emission lines below $\sim 4358 \AA$. The usage of these blue lines revealed no improvements for the wavelength calibration. Since the displayed lines cover strengths over a broad range, the counts are given in logarithmic scale.

ible and not clearly detectable in the final wavelength calibration image. Nevertheless, several tests of wavelength calibrations with the BVfilter were performed. None of these resulted in an improved calibration of the spectra. Therefore, an usage of the combined $\mathrm{HgAr} / \mathrm{Ne}$ and $\mathrm{Ar}+\mathrm{BV}$ calibration frame for the wavelength calibration was discarded.

For the wavelength calibration the input catalogue which is scanned by the MIDAS routines for the line search was thoroughly edited to ensure that it only contained emission features which are clearly detectable in the MOSCA spectra. This was carried out by comparing two calibration spectra (one with very low starting and one with very high ending wavelengths, respectively) to the list of calibration lamp lines as presented in the MOSCA User Manual which was released by the PI of MOSCA Prof. J. W. Fried in May $2000^{3}$. A total of 53 lines were included in the final catalogue.

\footnotetext{
${ }^{3}$ http://www . mpia-hd.mpg.de/MOSCA/index.html
}

For the performance of the wavelength calibration, two of the most crucial parameters are the tilt of the emission lines along the $\mathrm{Y}$ axis and the degrees of the polynomial fits to the dispersion relation along the $\mathrm{X}$ axis, respectively. Firstly, different spectra were examined and neither a tilt of emission lines as a function of exposure time or wavelength nor of slit position on the MOS mask was found. Secondly, the fit degrees were fixed for the entire spectrum (using the MIDAS algorithm REBIN/LONG), whereas the fit coefficients are defined via a linear rebinning row-by-row.

Various degrees of fits along the spatial axis were compared for individual spectra. It turned out that a two-dimensional dispersion relation using polynomial fit functions of third and second degree in dispersion and in spatial direction, respectively, yielded the best results. As a rough guide, one should aim for an r.m.s. value of less than $10 \%$ in the dispersion for the calibration process (e.g., Wagner 1992), which is in case of MOSCA $<0.1 \AA$. The typical mean r.m.s. (error) that was achieved with the dispersion fit was 0.05-0.06 $\AA$ at a dispersion of $\sim 1.28 \AA /$ pixel.

After the wavelength calibration, individual exposures were summed up. Fig. 3.4 shows typical final one-dimensional spectra in the A 2390 sample. These fully reduced $1 \mathrm{D}$ spectra were rebinned to logarithmic wavelength steps in preparation for the measurement of the velocity dispersions (chapter 5).

\subsection{Template Spectra}

In a similar manner to the spectra of the galaxies, the spectra of standard stars were reduced. Therefore, only a summary is presented but no explicit description will be given here. During each observing run at least two spectrophotometric flux standards (HZ2 and HZ4) were observed through an acquisition star hole in one mask. Moreover, a multiplicity of kinematic template stars were gained during each observation period for all described projects. In total, 12 
templates in Sep. 1999, ten in July 2000, six in April 2001 and three stars in February 2002. The stars were observed through different longslit widths between $0.5-1.5^{\prime \prime}$. However, only the stars of the last run (Feb. 2002) had a spectral resolution which was sufficiently high to utilise them as templates for even resolving velocity dispersions with $\sigma_{\text {gal }} \approx 90 \mathrm{~km} \mathrm{~s}^{-1}$. A more detailed description of the data for these kinematic templates with an application to the Lick/IDS system is illustrated in appen. A. For the kinematic templates of the Feb. 2002 run, three K giant stars (SAO 32042 (K3III), SAO 80333 (K0III), SAO 98087 (K0III)) were observed through a $0.5^{\prime \prime}$ longslit using the same grism GREEN 1000 as for the galaxies. These stars had a spectral resolution at $\mathrm{H} \beta$ and $\mathrm{Mg}_{b}$ of $\sim 2.2 \AA \mathrm{FWHM}$, corresponding to $\sigma_{*}=55 \mathrm{~km} \mathrm{~s}^{-1}$ ). A stellar template spectrum was split into several positions along the $\mathrm{Y}$ axis, which were slightly shifted from each other by $<3 \times 10^{-3 \prime \prime}$ via a manually controlled zig-zag movement. To minimise the effect of any possible variation (e.g., of instrumental line profile, see also section 5.2) in slit width, the star spectra were averaged over a small number of rows only. 

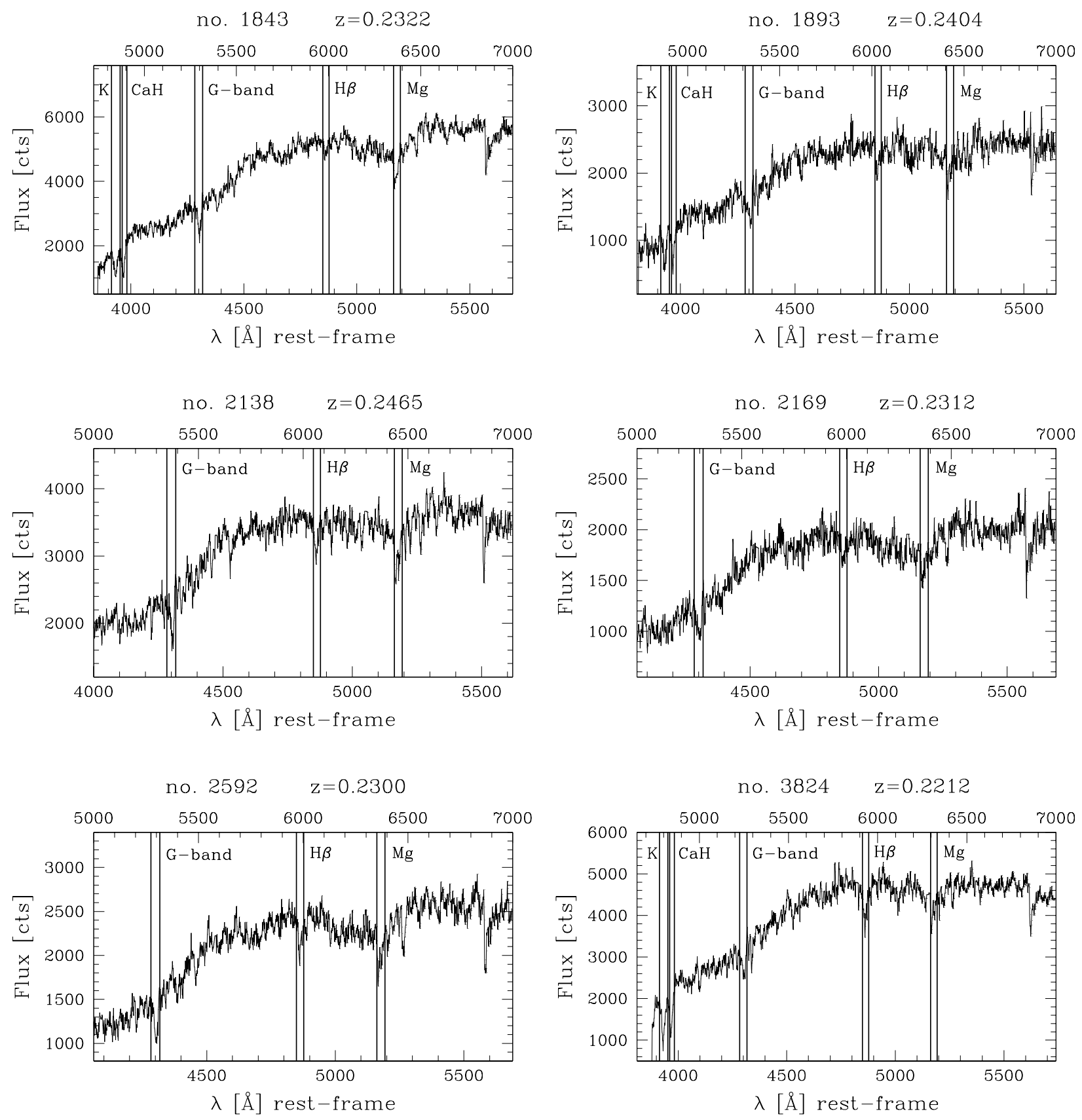

Figure 3.4: Example spectra (not flux-calibrated) of early-type galaxies in the A 2390 sample. The lower $\mathrm{X}$ axis represents the rest frame wavelengths, the upper one the observed wavelengths (both in $\AA$ ). The ordinate gives the flux in counts $\left(1 \mathrm{ADU}=1.1 e^{-1}\right)$. Prominent absorption features are marked and the ID and the determined redshift are given above each panel. 


\section{Chapter 4}

\section{Photometric Analysis}

Apart from the VLT/FORS and MOSCA intermediate-resolution spectroscopy, this study uses multi-band imaging data from different sources. The ground-based photometry provides the basis for the derivation of the luminosities of the early-type galaxies which are utilised in the scaling laws of the Faber-Jackson relation. Hubble Space Telescope (HST) imaging with the Wide Field and Planetary Camera 2 (WFPC2) offers a spatial resolution of $\sim 0.15$ arcsec FWHM and the Advanced Camera for Surveys (ACS) which is operational since July 2002 even $\sim 0.05$ arcsec FWHM. Both photometric instruments are perfectly suited to analyse the surface brightness distribution of the distant galaxies and to perform a detailed morphological classification into the sub-classes of early-type galaxies. Photometry in different passbands ( $U, B$ and $I$ for Abell 2390 and $B$, $V, R$ and $I$ for the Low $-L_{X}$ clusters) for both cluster samples was acquired from ground-based telescopes. Additionally, each cluster centre was observed with the HST/WFPC2 instrument either in the $V I$ (A 2390) or the $R$ (Low- $L_{X}$ clusters) filters. Thanks to the very deep multi-band imaging of the FDF and WHDF, the basis for the derivation of the luminosities of the field earlytype galaxies is more robust than most of other distant field early-type samples. For the FDF, photometry in four filters $(B, g, R$ and $I)$ is available, whereas for the WHDF sample three filter passbands ( $B, R$ and $I$ ) could be used.

In the next two sections 4.1 and 4.2 , details will be given on the ground-based and HST photometry of the Abell 2390 cluster and of the three Low- $L_{X}$ clusters, respectively. Besides a description of the imaging data, the analysis will concentrate on the derivation of absolute magnitudes for the galaxies. The reduction and calibration of this large amount of photometric data is described elsewhere (for A 2390: Smail et al. 1998; for the Low $-L_{X}$ clusters: Balogh et al. 2002b) and was not part of this thesis. The measurement of structural parameters by modelling the surface brightness distribution for all early-type galaxy candidates located on the HST/WFPC2 and HST/ACS images is presented in section 4.3. To distinguish between the main classes of early-type galaxies, elliptical and S0 galaxies, section 4.4 will focus on the morphological classification. Two independent approaches will be presented, a visual type classification according to the original Hubble scheme and a quantitative analysis following the de Vaucouleurs' classification of the revised Hubble sequence using the bulge-to-total fraction. A possible correlation of the measured bulge fraction with visual morphological Hubble type is investigated and performance tests between different filter passbands are discussed. The analysis steps to derive the absolute magnitudes, one basic parameter for the construction of the Faber-Jackson scaling relations, will be presented in section 4.5. Results on the ground-based luminosities will be outlined in section 4.6. In the following analysis, it is assumed that the spectroscopic redshifts of 


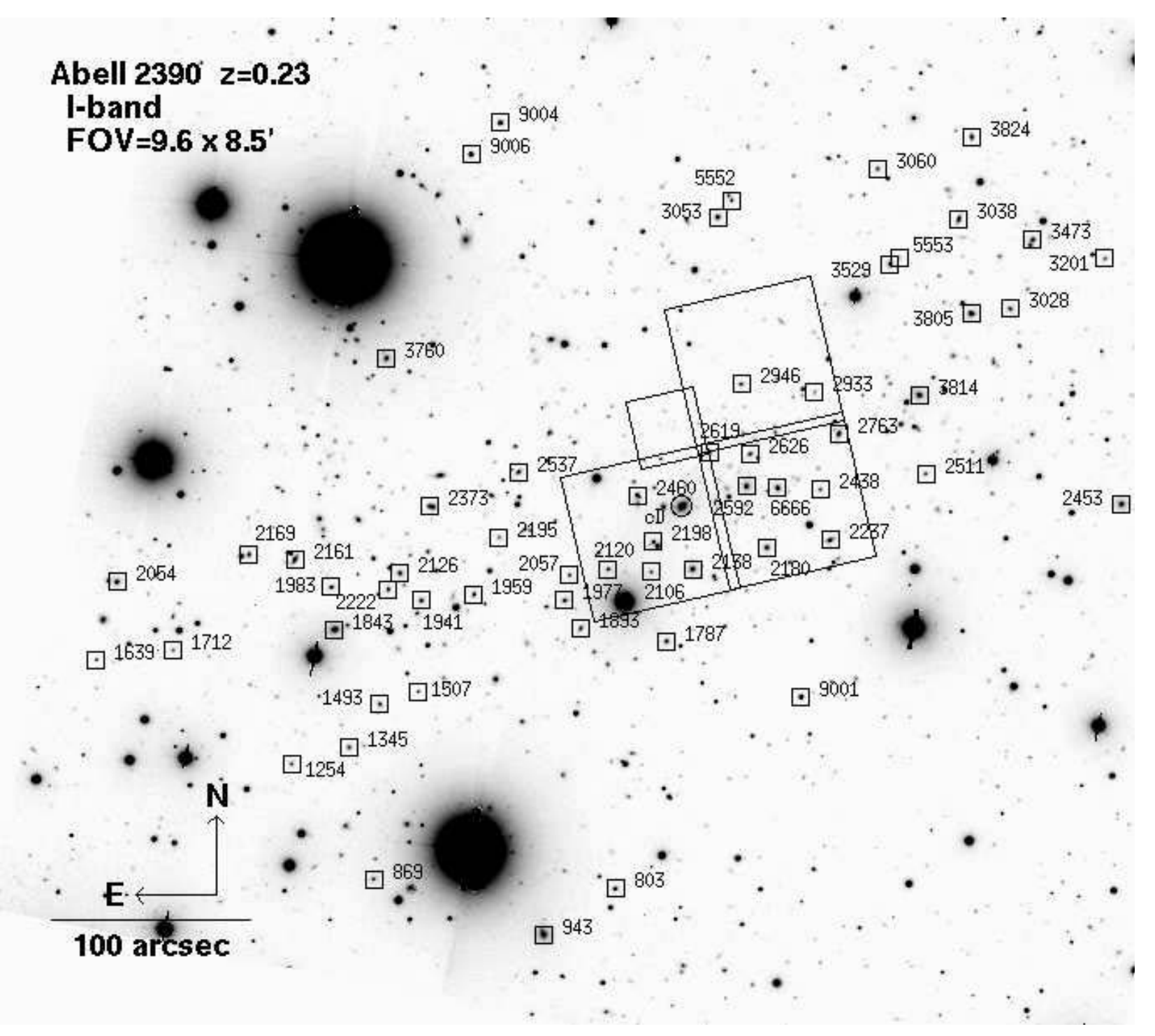

Figure 4.1: $I$-band image of the cluster Abell 2390 at $z=0.23$, taken with the 5.1-m Hale telescope on Mt. Palomar. The total FOV is $9 ! 6 \times 8 \cdot 5$. North is up, east to the left. The length of the scale bar in the left corner corresponds to $100^{\prime \prime}$, or $365 \mathrm{kpc}$ at the distance of A 2390. Galaxies with spectra are indicated by squares together with their ID number. The $\mathrm{cD}$ galaxy is marked with a circle. Stars used for the mask alignment are labelled as ID $=900 \mathrm{X}$. An overlay of the HST/WFPC2 field-of-view is also shown. 
the galaxies have already been determined. The measurements are presented in chapter 5 .

\subsection{Photometry of Abell 2390}

The cluster Abell $2390\left(\alpha_{2000}=21^{\mathrm{h}} 53^{\mathrm{m}} 34^{\prime \prime} \cdot 6\right.$, $\left.\delta_{2000}=+17^{\circ} 40^{\prime} 10^{\prime \prime} 9\right)$ at $z=0.228$, richness class 1, has a large velocity dispersion of $\sigma=1100 \pm 63 \mathrm{~km} \mathrm{~s}^{-1}$ (Carlberg et al. 1996) and a high X-ray luminosity, $L_{\mathrm{X}}(0.7-3.5 \mathrm{keV})=4.7 \times 10^{44} \mathrm{erg} \mathrm{s}^{-1}$ (Le Borgne et al. 1991). Carlberg et al. (1996) analysed the dynamical state of the cluster and its mass distribution and found a virial radius of $R_{v}=3.156 h_{100}^{-1} \mathrm{Mpc}$ and virial mass of $M_{v}=2.6 \times 10^{15} h_{100}^{-1} M_{\odot}$, which makes A 2390 more massive than Coma $\left(M_{v}=2.1 \times 10^{15} h_{100}^{-1} M_{\odot}\right)$. At a mean interior density of $200 \rho_{c}$, A 2390 and Coma have $M_{200^{-}}$ masses of 1.2 and $1.3 \times 10^{15} h_{100}^{-1} M_{\odot}{ }^{1}$, respectively.

The study of the early-type galaxy population in A 2390 is based upon Multi-Object Spectroscopy (MOS) using MOSCA at the Calar Alto 3.5-m telescope on Calar Alto Observatory in Spain. Section 2.1.6 describes these spectroscopic observations. In addition, optical photometry from the 5.1-m Hale telescope at Palomar Observatory is available (section 4.1.1) and the WFPC2 images taken with $H S T$ providing high-quality morphological information for a subset of the sample have been exploited (section 4.1.2). Table 2.2 on page 30 gives a summary of the photometric and spectroscopic observations for this project.

Since the galaxies were distributed over the whole field-of-view (FOV) of $\sim 10^{\prime} \times 10^{\prime}$, corresponding to $1.53 \times 1.53 h_{70}^{-2} \mathrm{Mpc}^{2}$, the evolution of early-type galaxies out to large clustercentric distances of $\sim 0.5$ virial radii can be studied.

\footnotetext{
${ }^{1}$ For the virial masses a cosmology with $H_{0}=100 \mathrm{~km} \mathrm{~s}^{-1} \mathrm{Mpc}^{-1}, q_{0}=0.1$ and $\Lambda=0$ was assumed.
}

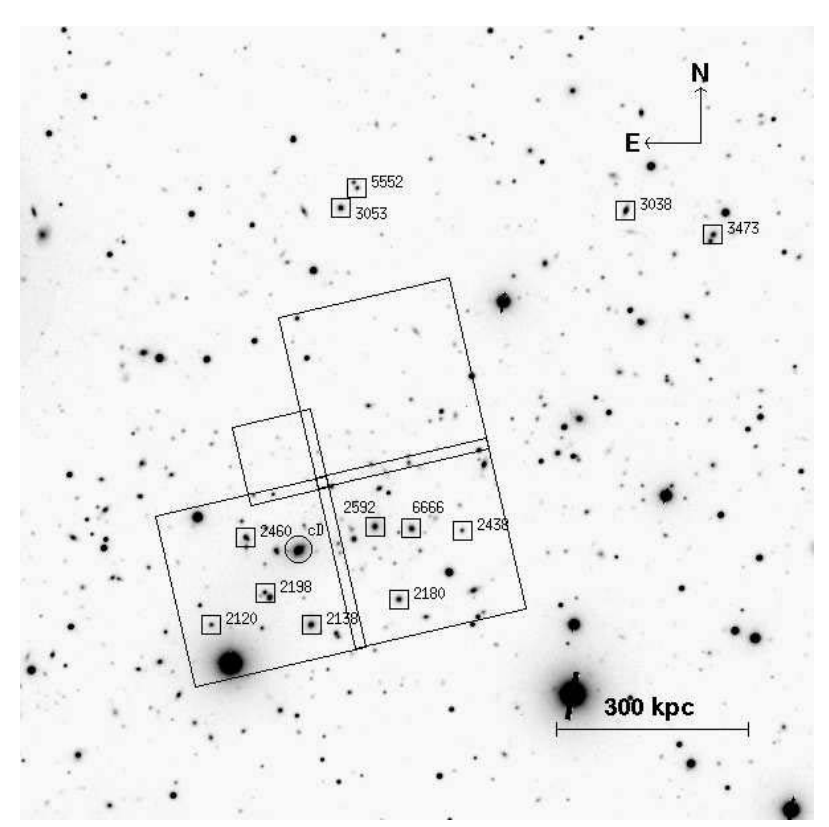

Figure 4.2: Zoom of the Hale $I$-band image of the cluster Abell 2390 at $z=0.23$. Galaxies with spectra are denoted with squares and the $\mathrm{cD}$ galaxy is marked with a circle. The HST/WFPC2 field-of-view is overlayed, with the WF chip corresponding to $114^{\prime \prime}$ along one side and the PC chip with a size of $52^{\prime \prime}$.

\subsubsection{Ground-based $U B I$ Imaging}

Abell 2390 was observed at the 5.1-m Hale telescope on Mount Palomar using COSMIC (Carnegie Observatories Spectroscopic Multislit and Imaging Camera) in the $U$ (3000 sec), $B$ $(500 \mathrm{sec})$ and $I$-band $(500 \mathrm{sec})$, allowing to select early-type galaxies in the full field-of-view of MOSCA due to the nearly equally large field-ofview of COSMIC of $9.7^{\prime} \times 9.7^{\prime}$. Seeing conditions ranged from $1.4^{\prime \prime}$ in the $U$-band, $1.3^{\prime \prime}$ in the $B$ to $1.1^{\prime \prime}$ in the Cousins $I_{C}$-band (Smail et al. 1998). Hereafter the Cousins $I_{C}$-band is referred to as $I$-band. At $I=22.5 \mathrm{mag}$ a completeness level of $80 \%$ from a comparison with deeper field counts is warranted. All frames from the ground-based imaging data were reduced in a standard manner with IRAF using standard reduction packages.

Fig. 4.1 shows the $I$-band image of the cluster Abell 2390 at $z=0.23$, gained with the 5.1-m 

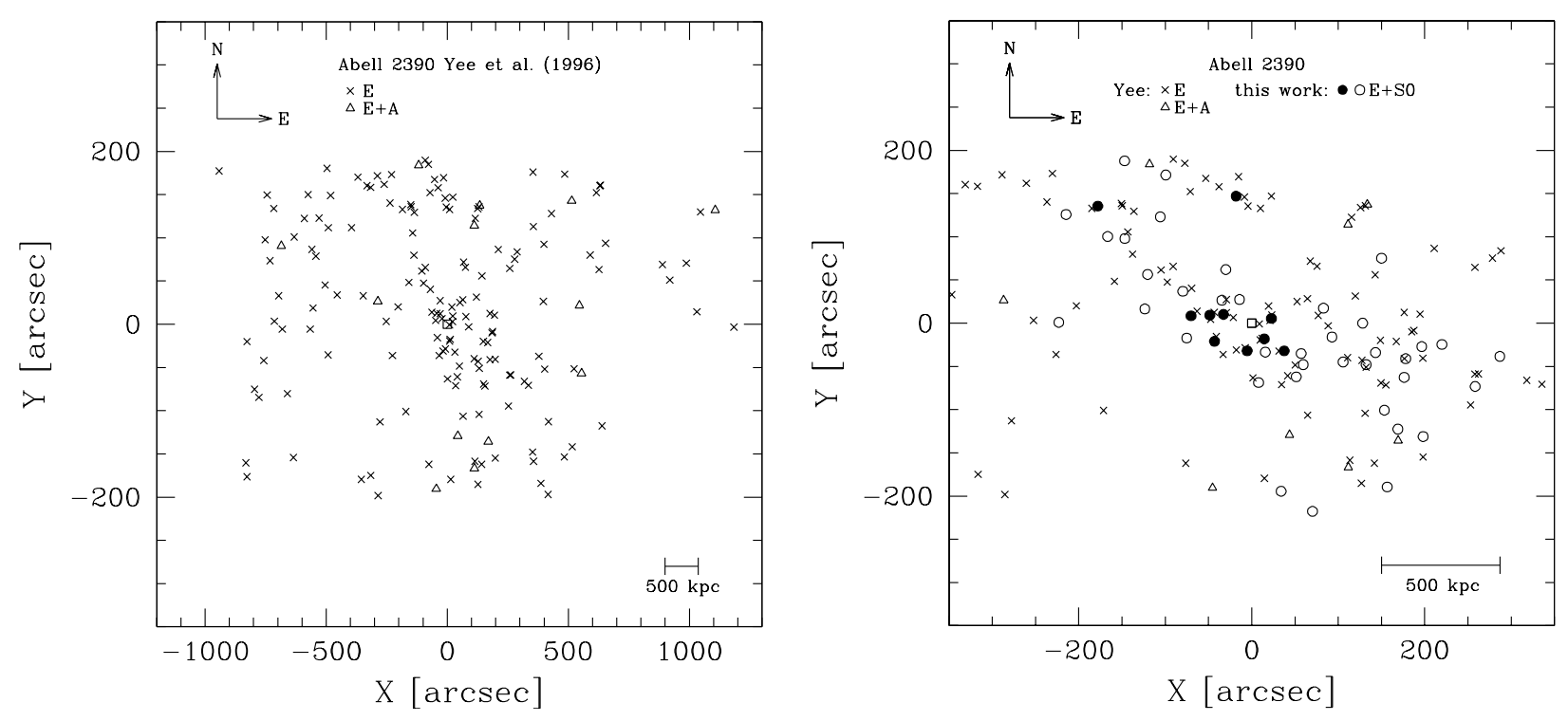

Figure 4.3: Left: Distribution of Abell 2390 cluster members at $z=0.228$ by Yee et al. (1996). In total, 159 early-type galaxies (E) and $13 \mathrm{E}+\mathrm{A}$ galaxies are distributed over a strip of $6.47^{\prime} \times 35.43^{\prime}$, centered on the cluster $\mathrm{cD}$ galaxy (square). Only early-type galaxies (asterisks) and $\mathrm{E}+\mathrm{A}$ galaxies (triangles) within a redshift interval of $z_{\text {clus }} \pm 2 \sigma$ are shown. Right: Comparison between Yee et al. (1996) and the catalogued A 2390 galaxies. Spectroscopically observed galaxies of this work are indicated with open circles. Ten galaxies with spectra which are included in both data sets are marked as solid circles.

Hale telescope on Mount Palomar. The total FOV is $9.6 \times 8.5$, north is up and east to the left. Galaxies with available MOSCA spectra are denoted by squares and the central $\mathrm{cD}$ galaxy (no spectroscopic information) is indicated with a circle. The central $150^{\prime \prime} \times 150^{\prime \prime}$ of the cluster are covered by the HST/WFPC2 mosaic, which provides a detailed morphological and structural analysis.

As a consistency check of the ground-based photometry the photometric data was compared with the results for the cluster A 2390 by Yee et al. (1996), which were derived as part of the CNOC cluster redshift survey. Through a crosscorrelation, 12 galaxies with spectra were identified which are included in both data sets. The selection process for these galaxies is visualised in Fig. 4.3. The left panel shows the distribution of 159 early-type cluster members and 13 $\mathrm{E}+\mathrm{A}$ galaxies in A 2390 by Yee et al. (1996) over a large strip of the sky of $6.47^{\prime} \times 35.43^{\prime}$ cen- tered on the galaxy cluster. On the right hand plot, a comparison between Yee et al. (1996) and the A 2390 galaxies in this study is illustrated. Galaxies with available spectra are indicated with open circles, whereas the galaxies by Yee are indicated by asterisks. In total, ten spectroscopic cluster members which were found in both samples are marked as solid circles. One object (\# 5552), fell by coincidence into the slit and only a redshift was derived but no magnitude could be measured. The galaxy (\# 3038) shown with an open circle is a foreground galaxy at $z=0.18$ (cf. Table 4.1). Fig. 4.2 shows a zoom of the ground-based Hale $I$-band image containing these 12 early-type galaxies which are in common. After the transformation of the $I$ to Gunn $r$ observed magnitudes using $r-I=1.14$ based on Kinney E/S0 spectra, no significant difference between the magnitudes was found, $\Delta\left(\left|r-r_{\text {Yee }}\right|\right)=0.04 \pm 0.12$ mag. Figure 4.4 and Table 4.1 give a comparison of the Gunn $r$ mag- 
nitudes for the 11 galaxies which are in common. In addition, the redshift determinations of all these objects show a very good agreement $\left(\left|z-z_{\text {Yee }}\right|=0.3^{-3} \pm 0.2^{-3}\right)$. Note that the transformation from $I_{\mathrm{obs}}$ to $r_{\mathrm{obs}}$ for the $I$-band magnitudes are very large at a redshift of $z=0.23$. For this reason, in the subsequent analysis of the scaling relations the magnitudes were transformed to Gunn $r$ rest-frame magnitudes which involves much smaller $k$-corrections and therefore less uncertainties. A detailed description on the transformation of magnitudes from observed to rest-frame wavelength range can be found in section 4.5.3.

In Fig. 4.5 a) the colour-magnitude diagram (CMD) $(B-I)$ versus $I$ from the Hale imaging is shown for all galaxies brighter than $I=23.5^{\mathrm{m}}$ lying in a $9.7^{\prime} \times 9.7^{\prime}(2.12 \mathrm{Mpc})$ region centered on A 2390. Bona fide stellar objects selected by a SExtractor based star classification parameter of star $\geq 0.9$ have been discarded. The 48 early-type cluster members of A 2390 with available spectroscopic information are indicated with open squares. The red sequence of earlytype cluster members is readily seen extending down to $I \sim 21^{m}\left(M_{B} \sim-19^{m}\right)$. A leastsquares fit to seven ellipticals in A 2390 gives $(B-I)_{\mathrm{E}}=-0.011\left(I_{\text {tot }}\right)+3.405$ which is shown by the solid line in Fig. 4.5. One elliptical E/S0 galaxy (\# 6666) is not included in the CMDs as this galaxy was not selected as a spectroscopic target but fell by coincidence into the slit during the observations. The outlier object \# 2237 with the bluest colour of $(B-I)=2.77$ was classified a spiral Sa galaxy. Fig. $4.5 \mathrm{~b}$ ) displays the $(U-B)-I$ colour magnitude relation for the same galaxies as in Fig. 4.5 a. Again, a least-squares fit to seven A 2390 cluster ellipticals yields $(U-B)_{\mathrm{E}}=-0.020\left(I_{\text {tot }}\right)+1.092$.

The $(B-I)-(U-B)$ colour-colour plane for galaxies brighter than $I=23.5^{m}$ is shown in Fig. 4.6. At first glance a "clump" of red galaxies at $(B-I) \sim 3.2$ and $(U-B) \sim 0.6$ is clearly visible. This region is occupied by all $\mathrm{E}+\mathrm{S} 0$ clus-

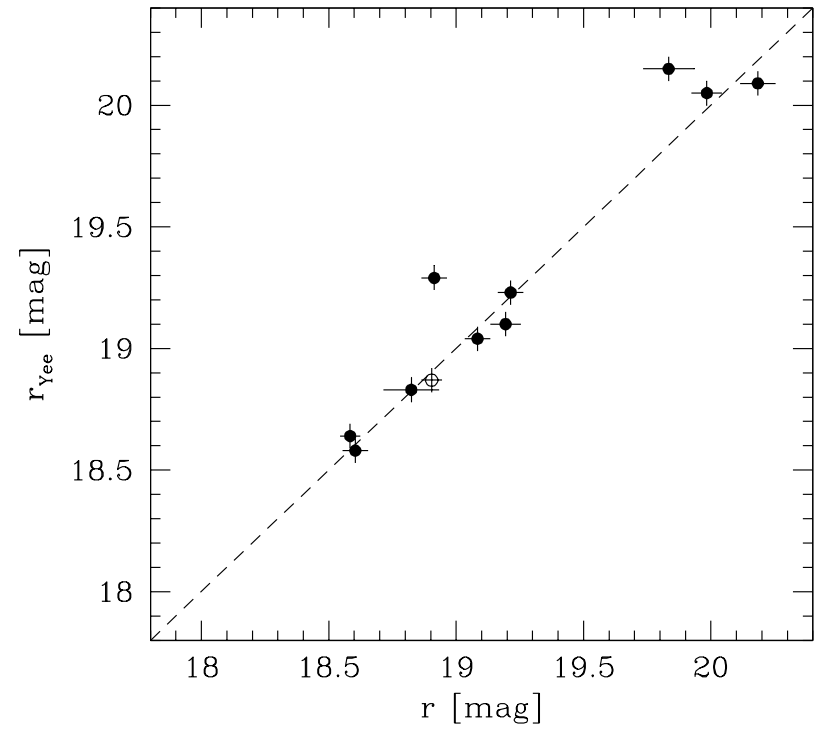

Figure 4.4: Comparison between ground-based Gunn $r$ magnitudes by Yee et al. (1996) and this study for A 2390 cluster members (solid circles). Gunn $r$ magnitudes were derived from the $I_{\text {tot }}$ magnitudes. The galaxy denoted with the open circle is a foreground galaxy at $z=0.18$ (see text for details).

ter members of A 2390 which are indicated with open squares. The observed galaxies are compared to evolutionary tracks for E/S0 galaxies using the BC96 models assuming a formation redshift of $z_{\mathrm{f}}=2$. The predicted BC96 colour for a passively evolved galaxy with an old stellar population at redshift $z=0.23$ suggests colours of $(B-I)=3.06$ and $(U-B)=0.57$, which are shown as dashed lines in Fig. 4.6. Note that for the computation of the BC96 models a slightly different cosmology with $H_{0}=60 \mathrm{~km} \mathrm{~s}^{-1} \mathrm{Mpc}^{-1}$ and $q_{0}=0.1$ was adopted.

\subsubsection{HST Photometry}

Apart from the deep multi-colour ground-based imaging, detailed morphological information is provided via two HST/WFPC2 images of A 2390 for a subset of the galaxies in the rich cluster sample.

During Cycle 4 A 2390 was observed on Decem- 
Table 4.1: Comparison between ground-based Gunn $r$ magnitudes by Yee et al. (1996) and the Gunn $r$ magnitudes of this study. The mean errors in Gunn $r$ and $(g-r)$ colour by Yee et al. (1996) are $0.05^{m}$ and $0.07^{m}$, respectively. The uncertainties in the redshifts are listed in units of $10^{-5}$.

\begin{tabular}{ccccccccc}
\hline ID & $z$ & $\begin{array}{c}r \\
{[\mathrm{mag}]}\end{array}$ & ID & $\begin{array}{c}r_{\text {Yee }} \\
{[\mathrm{mag}]}\end{array}$ & $\begin{array}{c}(g-r) \\
{[\mathrm{mag}]}\end{array}$ & $z_{\text {Yee }}$ & $\begin{array}{c}\delta z_{\text {Yee }} \\
10^{-5}\end{array}$ & $\begin{array}{c}\Delta\left(r-r_{\text {Yee }}\right) \\
{[\mathrm{mag}]}\end{array}$ \\
\hline 2120 & 0.2280 & $19.83 \pm 0.10$ & 100873 & 20.15 & 0.78 & 0.2286 & 23 & -0.316 \\
2138 & 0.2465 & $18.58 \pm 0.04$ & 100908 & 18.64 & 0.86 & 0.2470 & 28 & -0.056 \\
2180 & 0.2283 & $19.21 \pm 0.05$ & 100995 & 19.23 & 0.91 & 0.2290 & 24 & -0.016 \\
2198 & 0.2335 & $19.98 \pm 0.06$ & 100975 & 20.05 & 0.87 & 0.2332 & 29 & -0.066 \\
2438 & 0.2372 & $20.18 \pm 0.07$ & 101197 & 20.09 & 0.85 & 0.2371 & 37 & 0.094 \\
2460 & 0.2319 & $18.91 \pm 0.05$ & 101106 & 19.29 & 0.94 & 0.2318 & 25 & -0.376 \\
2592 & 0.2300 & $18.60 \pm 0.05$ & 101183 & 18.58 & 0.87 & 0.2304 & 22 & 0.024 \\
3038 & 0.1798 & $18.90 \pm 0.04$ & 101992 & 18.87 & 0.78 & 0.1796 & 22 & 0.034 \\
3053 & 0.2282 & $19.08 \pm 0.05$ & 101930 & 19.04 & 0.91 & 0.2281 & 28 & 0.044 \\
3473 & 0.2228 & $19.19 \pm 0.06$ & 101961 & 19.1 & 0.94 & 0.2221 & 26 & 0.094 \\
5552 & 0.2231 & & 101987 & 20.25 & 0.94 & 0.2233 & 29 & \\
6666 & 0.2240 & $18.82 \pm 0.11$ & 101190 & 18.83 & 0.88 & 0.2244 & 24 & -0.006 \\
\hline
\end{tabular}

ber 10, 1994 with the $H S T^{2}$ in the F555W $\left(V_{555}\right)$ and in the $\mathrm{F} 814 \mathrm{~W}\left(I_{814}\right)$ filters as part of a large gravitational lensing survey (P.I. Prof. B. Fort (CNRS, Paris), Proposal ID 5352). In total, 5 exposures in the $I_{814}$-filter and 4 in the $V_{555^{-}}$ filter each with $2100 \mathrm{sec}$ resulting in a total exposure time of $T_{\text {tot }}=10.5 \mathrm{ks}$ and $T_{\text {tot }}=8.4 \mathrm{ks}$ for the $I_{814}$ and $V_{555}$, respectively. These exposure times are deep enough to determine structural parameters down to rest-frame $M_{B} \sim 23^{m}$ (Ziegler et al. 1999). The final mosaic covers a field of approximately $2.5^{\prime} \times 2.5^{\prime}$ at $\sim 0.15^{\prime \prime}$ resolution in the core of the cluster. All single standard WFPC2 pipeline reduced frames were further reduced and afterwards combined to a final mosaic image by I. Smail. Here only a brief overview is given to the reader. Further details about the general reduction process and the mosaic align-

\footnotetext{
${ }^{2}$ Based on observations made with the NASA/ESA Hubble Space Telescope, obtained at the Space Telescope Science Institute, which is operated by the Association of Universities for Research in Astronomy, Inc., under NASA contract NAS 5-26555. These observations are associated with program \# 5352 .
}

ment are given in Smail et al. (1997). In oder to allow for hot pixel rejection, individual exposures were grouped in sets of four single-orbit exposures each offset by 2 !'0. After standard pipeline reduction, the images were aligned using integer pixel shifts and then combined using the IRAF/STSDAS ${ }^{3}$ task CRREJ. In the final mosaic the PC chip has the same linear scale as the WF chips and the sky background levels are equalised for all four chips. Details on the calibration will be outlined in section 4.3. The WFPC2 image of A 2390 has a $1 \sigma$ surface brightness limit of $\mu_{I} \sim 28 \mathrm{mag} / \mathrm{pixel}^{2}$, which is more than adequate to provide high-quality morphological information on the brighter cluster members. A two colour VI HST image of the cluster A 2390 is shown in Fig. 4.7. Thumbnail images of the 14 galaxies for which MOSCA spectra have been acquired are displayed in Fig. 4.12 and the

\footnotetext{
${ }^{3}$ The Space Telescope Science Data Analysis System (STSDAS) is a data processing software pipeline for calibrating and analysing data from the HST using IRAF, operated by the STScI. (http: \\www.stsci.edu $\backslash$ resources $\backslash$ software_hardware $\backslash$ stsdas).
} 

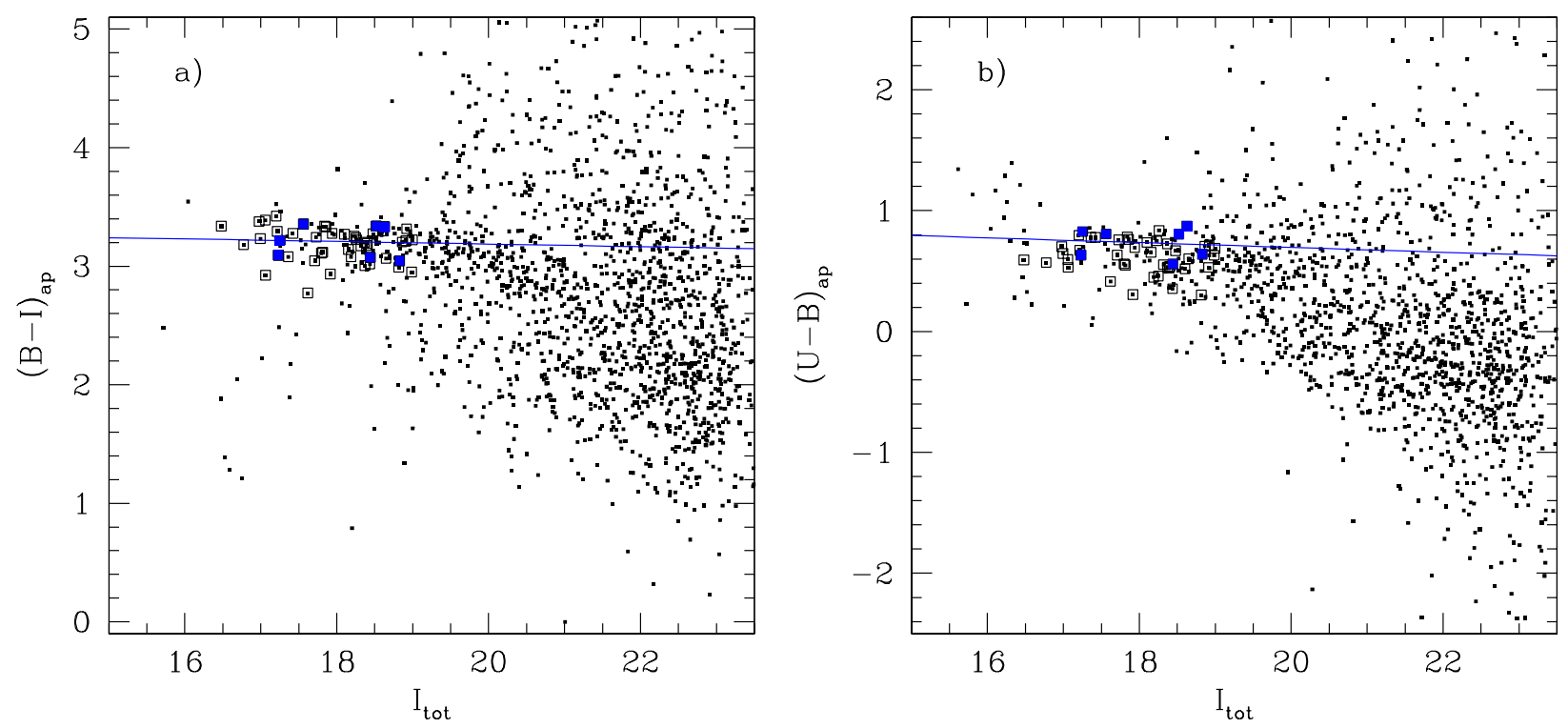

Figure 4.5: Left: $(B-I)-I$ colour magnitude diagram from the Hale imaging for galaxies brighter than $I=23.5^{m}$ lying in a $9.7^{\prime} \times 9.7^{\prime}(2.12 \mathrm{Mpc})$ region centered on A 2390 . The sequence of red cluster members is readily seen extending down to $I \sim 21^{m}\left(M_{B} \sim-19^{m}\right)$. The 48 early-type cluster members of A 2390 with available spectra are denoted with squares. Filled squares represent the ellipticals in A 2390 which enter the Fundamental Plane. The solid line is a least-square fit to seven ellipticals. Right: $(U-B)-I$ colour magnitude diagram from the Hale photometry for bright galaxies lying in a $9.7^{\prime} \times 9.7^{\prime}(2.12 \mathrm{Mpc})$ region centered on A 2390. Symbol notations as in the left Figure.

position of the HST field is indicated on Fig. 4.1. The $\mathrm{cD}$ was spectroscopically not observed and thus is not shown. Several strong and faint gravitational lensed (cuspy and folded) arcs are visible next to numerous galaxies (e.g., cD, \# 2592, \# 6666). Remarks on special features for individual HST objects of Abell 2390 are summarised in Table 4.9. Two arcs features (a cusp arc H3 and a fold arc H5) associated with the early-type galaxy \# 6666 were analysed through lens modelling by Pelló et al. (1999) and identified as multiple images of a high-redshift source at $z=4.05$, which is in rest-frame wavelength a $\operatorname{Ly} \alpha$ emitter behind the cluster A 2390. The "straight arc" associated in the western part of the galaxy \# 2592 was analysed and can be splitted into two components, where component " $\mathrm{A}$ " is located in the southern part with $z_{A}=1.033$ and object "C" in the northern part with $z_{C}=0.913$ (Bunker, Moustakas \& Davis 2000).
The analysis of structural parameters and morphologies as well as the derivation of rest-frame properties for the sub-sample of $\mathrm{E}+\mathrm{S} 0$ galaxies falling into the HST field is presented in section 4.3.

\subsection{Photometry of Low- $L_{X}$ Clusters}

\subsubsection{Low- $L_{X}$ Sample}

One of the most comprehensive X-ray-selected galaxy cluster samples is the Vikhlinin catalogue (Vikhlinin et al. 1998). This catalog consists of 203 clusters of galaxies serendipitously detected as spatially extended X-ray emission sources in the inner 17!.5 of 647 ROSAT Position Sensitive Proportional Counter (PSPC) pointings. The ROSAT (ROentgen SATellit) PSPC has a fieldof-view with a diameter of $2^{\circ}$. For the cata- 


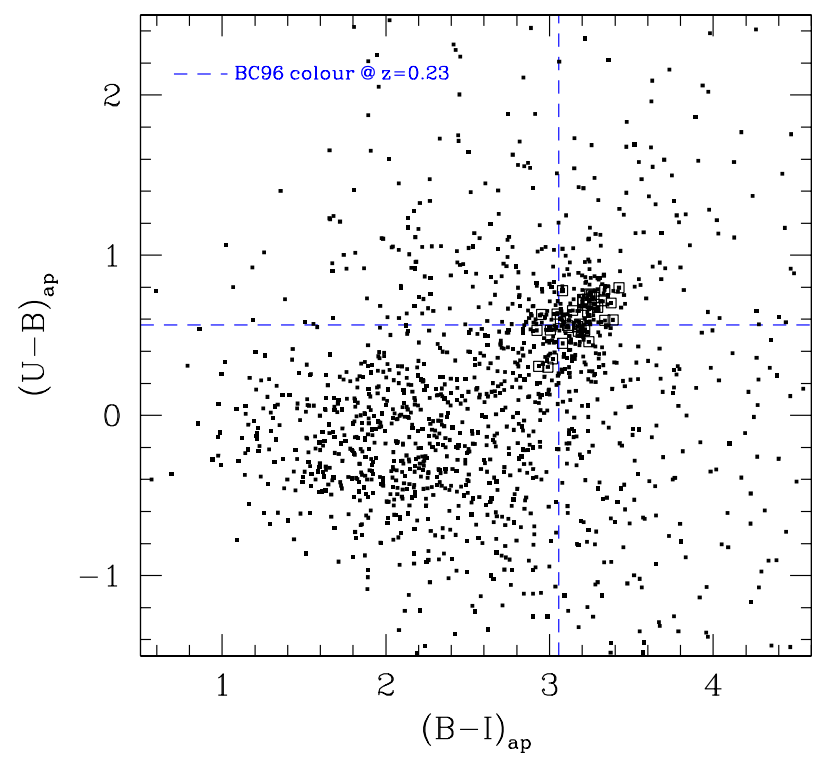

Figure 4.6: $(B-I)-(U-B)$ colour-colour plane from the Hale imaging for galaxies brighter than $I=23.5^{\mathrm{m}}$ lying in a $9.7^{\prime} \times 9.7^{\prime}(2.12 \mathrm{Mpc})$ region centered on A 2390. A $2390 \mathrm{E}+\mathrm{S} 0$ cluster members with available spectroscopic information are represented with open squares. The predicted BC96 colour for a passively evolved galaxy with old stellar populations $\left(z_{\mathrm{f}}=2\right)$ at $z=0.23$ takes values $(B-I)=3.06$ and $(U-B)=$ 0.57 and is indicated with the dashed lines.

logue, only high Galactic latitude pointings covering 158 square degree were included. Out of $203(91 \%)$ optically confirmed galaxy clusters, 73 feature spectroscopic redshifts (based on 2-3 galaxies per cluster) while photometric redshifts are given for all galaxy clusters. Galaxy cluster redshifts range from $z=0.015$ to $z>0.5$. The $\mathrm{X}$-ray luminosities $L_{X}$ derived from the observed fluxes in the $0.5-2.0 \mathrm{keV}$ energy band vary from $10^{42}$ to $\sim 5 \times 10^{44} \mathrm{erg} \mathrm{s}^{-1}$, corresponding to very poor groups up to rich clusters respectively.

The Low $-L_{X}$ clusters of galaxies comprising ten $\mathrm{X}$-ray faint clusters in the northern hemisphere were selected from the Vikhlinin et al. (1998) cluster sample. The target clusters were restricted to a relatively narrow redshift range, $0.22 \leq z_{\text {spec }} \leq 0.29$ and a mean redshift of $\bar{z}=0.25$, in oder to reduce the effects of differential distance moduli and $k$-correction ef- fects on the comparison between the systems. In total, using these redshift limits and the restriction of accessibility from the northern hemisphere, a sample of 9 galaxy cluster were selected with an upper constraint on the $X$-ray flux of $L_{X}<2-20 \times 10^{44} \mathrm{erg} \mathrm{s}^{-1}$. The $X$-ray luminosities of these selected systems cover a range of $0.15 \leq L_{X} \leq 1.5 \times 10^{44} h_{50}^{-2} \mathrm{erg} \mathrm{s}^{-1}$. Based on the follow-up spectroscopic investigation, the cluster CL 1444+63 turned out to be two separate structures aligned along the line of sight (Balogh et al. 2002b). This yields to a final sample of 10 $\mathrm{X}$-ray faint clusters. In contrast to typical Xray luminosities of X-ray selected clusters studied with the HST so far $\left(L_{X} \sim 5 \times 10^{44} \mathrm{erg} \mathrm{s}^{-1}\right)$, these galaxy clusters exhibit 1.0 dex lower X-ray luminosities.

The X-ray luminosities $L_{X}$ were computed in the $0.1-2.4 \mathrm{keV}$ band from the observed fluxes in the $0.5-2.0 \mathrm{keV}$ band and were corrected for galactic Hi absorption and transformed to restframe (Balogh et al. 2002a). These luminosities are listed in Table 4.4, and range from 0.40 to $4.0 \times 10^{43} h_{100}^{-2} \operatorname{erg~s}^{-1}[0.1-2.4 \mathrm{keV}]$. Although CL $1444+63$ is treated as two separate clusters, the X-ray luminosity in Table 4.4 is that of the combined clusters.

\subsubsection{Ground-based Photometry}

The ground-based imaging for the clusters is based on three different observatories (i) Calar Alto CAFOS (2.2 m telescope) and MOSCA (3.5 m telescope) imaging mode observations, (ii) Isaac Newton telescope (INT) Wide-Field Camera (WFC) imaging and (iii) Palomar 200inch (P200) telescope observations at Palomar Observatory. Table 4.2 gives a summary of the ground-based imaging observations of all ten poor clusters. The Calar Alto CAFOS observations were carried out by R. G. Bower during the 28th of March 1999 and the MOSCA observations by B. L. Ziegler between the 3.-6. March 2000. The COSMIC imaging of the Palomar 200inch telescope was performed by I. Smail dur- 


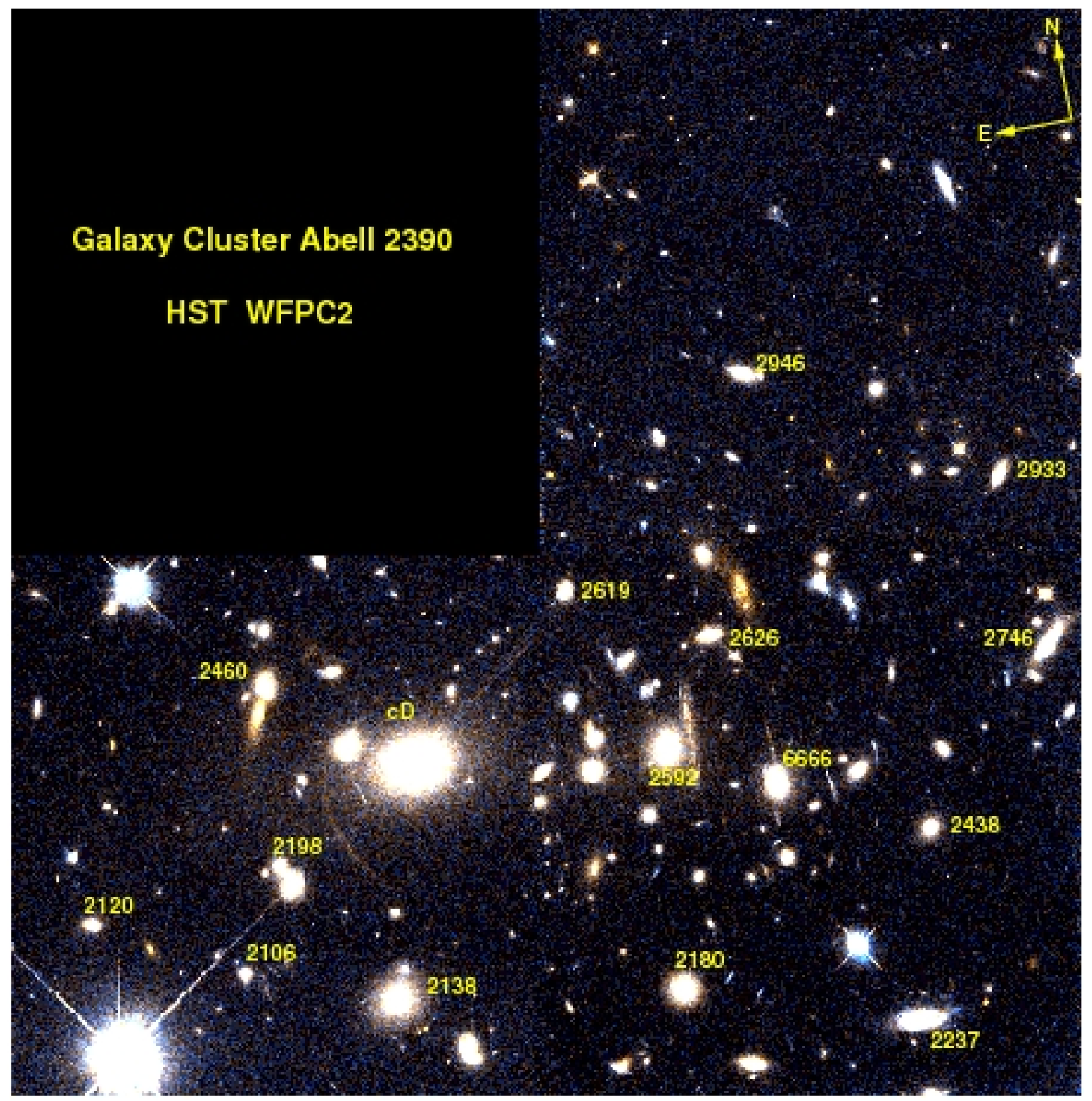

Figure 4.7: VI colour composite HST WFPC2 image of the cluster Abell 2390, The total FOV is $2.5 \times 2.5$. Galaxies with MOSCA spectra are indicated with their ID numbers. The cD galaxy is also marked, but was not observed. The PC chip is not shown as no galaxy was selected from it. All candidates display red colours. Object \# 2933 is an Sbc background galaxy at $z=0.398$. 
Table 4.2: Ground-based Observations of Low- $L_{X}$ clusters. Total exposure times are given in [ksec].

\begin{tabular}{|c|c|c|c|c|c|c|}
\hline Name & Instrument & Date & $B$ & $V$ & $R$ & $I$ \\
\hline \multirow[t]{2}{*}{ CL $0818+56$} & COSMIC & $26 / 11 / 98$ & 0.60 & & 0.30 & \\
\hline & CAFOS & $28 / 03 / 99$ & & & & 1.20 \\
\hline \multirow[t]{3}{*}{ CL 0819+70 } & COSMIC & $26 / 11 / 98$ & 0.60 & & 0.25 & \\
\hline & CAFOS & $28 / 03 / 99$ & 0.30 & & 0.60 & 1.80 \\
\hline & MOSCA & 03/03/00 & 1.20 & & & \\
\hline \multirow[t]{3}{*}{ CL $0841+70$} & COSMIC & $26 / 11 / 98$ & 0.60 & & 0.25 & \\
\hline & CAFOS & $28 / 03 / 99$ & 0.30 & & 0.60 & 1.80 \\
\hline & MOSCA & 03/03/00 & 1.20 & & & \\
\hline \multirow[t]{5}{*}{ CL $0849+37$} & COSMIC & $26 / 11 / 98$ & 0.60 & & 0.25 & \\
\hline & CAFOS & $28 / 03 / 99$ & 0.30 & & 0.60 & 1.20 \\
\hline & MOSCA & $04 / 03 / 00$ & 1.20 & & & \\
\hline & MOSCA & $05 / 03 / 00$ & 0.60 & & & \\
\hline & MOSCA & 05/03/00 & 0.30 & & & \\
\hline \multirow[t]{3}{*}{ CL $1309+32$} & INT/WFC & $19 / 06 / 98$ & & 1.80 & 2.40 & 0.60 \\
\hline & CAFOS & $28 / 03 / 99$ & & 0.30 & 0.60 & 0.60 \\
\hline & MOSCA & $06 / 03 / 00$ & 5.40 & & & \\
\hline \multirow[t]{3}{*}{ CL $1444+63$} & INT/WFC & $18 / 01 / 99$ & & 1.10 & 2.40 & 0.72 \\
\hline & CAFOS & $28 / 03 / 99$ & & 0.30 & 0.30 & 0.30 \\
\hline & MOSCA & $06 / 03 / 00$ & 5.40 & & & \\
\hline \multirow[t]{4}{*}{ CL $1633+57$} & INT/WFC & $10 / 02 / 99$ & & 1.80 & 0.60 & 1.20 \\
\hline & CAFOS & 28/03/99 & 0.30 & 0.30 & 0.60 & 0.30 \\
\hline & MOSCA & $04 / 03 / 00$ & 1.20 & & & \\
\hline & MOSCA & $05 / 03 / 00$ & 1.20 & & & \\
\hline \multirow{2}{*}{$\begin{array}{l}\text { CL } 1701+64 / \\
\text { CL } 1702+64\end{array}$} & INT/WFC & $10 / 02 / 99$ & 2.40 & & 0.60 & 0.90 \\
\hline & CAFOS & $28 / 03 / 99$ & 0.30 & 0.30 & 0.30 & \\
\hline
\end{tabular}


Table 4.3: Overview of the observing conditions for ground-based photometry of four Low $-L_{X}$ clusters. The mean seeing FWHM values for a night are given in each column.

\begin{tabular}{lccc}
\hline Name & CAFOS & MOSCA & P200 \\
& $R$ & $\begin{array}{c}B \\
\mathrm{n} 2 / \mathrm{n} 3\end{array}$ & $R$ \\
\hline CL 0819+70 & $1.9^{\prime \prime}$ & $1.1^{\prime \prime}$ \\
CL 0841+70 & $1.9^{\prime \prime}$ & $1.5^{\prime \prime}$ & \\
CL 0849+37 & $2.5^{\prime \prime}$ & $\sim 3.8^{\prime \prime} / 1.4^{\prime \prime}$ & $1.7^{\prime \prime}$ \\
CL 1633+57 & $1.6^{\prime \prime}$ & $\sim 3.8^{\prime \prime} / 1.0^{\prime \prime}-1.4^{\prime \prime}$ & \\
\hline
\end{tabular}

ing two nights in November 26.-27. 1998. The galaxy clusters CL 1701 and CL 1702 cover the same field on the sky. For this reason, only one pointing was needed and thus are shown in combination in Table 4.2. Seeing conditions were not photometric and varied between values of $1^{\prime \prime} .0 \leq \mathrm{FWHM}_{B} \leq 1$ 1". 1 1". $\leq \mathrm{FWHM}_{R} \leq 1$ 1".9 for the $B$ and $R$-band images, respectively. Exposures with seeing conditions worse than 2 .'5 were replaced by repeat observations or by images of other telescopes. Table 4.3 lists the observing conditions for ground-based photometry of the CAHA $B$ and $R$-band images as well as the P200 $R$-band exposures of the CL $0849+37$ cluster. The $I$-band images are not included because they were not used for this study. First results of the Calar Alto $B$ and $R$-band images for four clusters of the sample, CL 0819, CL 0841, CL 0849 and CL 1633 were presented by Gaztelu (2000) and Ziegler et al. (2001b). As the $R$ band data from Calar Alto of the cluster CL 0849 had poor seeing $\left(2.5^{\prime \prime}\right)$, the Palomar $R$ image was used instead. The remaining imaging data was reduced and analysed by M. L. Balogh and I. R. Smail.

During the imaging observations the conditions were not photometric. Therefore, the images were calibrated by comparing aperture magnitudes of several (usually 2-3) relatively isolated,

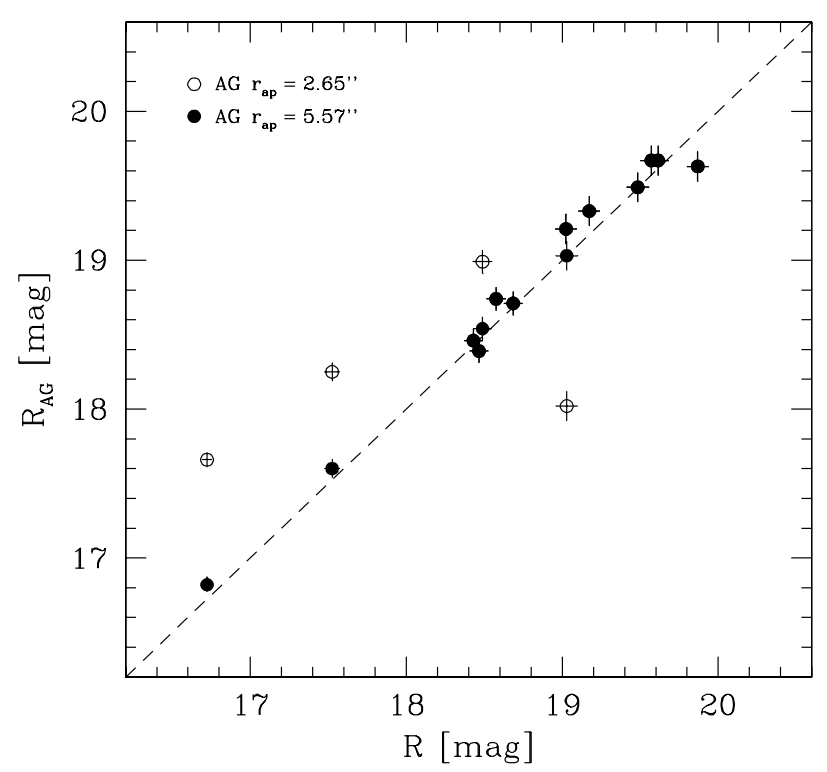

Figure 4.8: Comparison between ground-based $R$ magnitudes by Gaztelu (2000) and the $R$ magnitudes for CL 0849 cluster members. Open circles denote measured $R$ magnitudes by Gaztelu $\left(R_{\mathrm{AG}}\right)$ with an aperture radius of $r_{a p}=2.65^{\prime \prime}$, solid circles represent re-measured $R_{\mathrm{AG}}$ magnitudes with an aperture of $r_{a p}=5.57^{\prime \prime}$ (see text for details).

early-type galaxies with the F702W photometry on the WFPC2 images (see next section 4.2.3), and converted this to standard $R$ magnitudes, assuming $R_{\mathrm{F} 702 \mathrm{~W}}-R_{c}=-0.2$ (Fukugita et al. 1995). The photometric calibration is accurate to approximately $0.1^{m}$, including blue cluster members (but see discussion in the following paragraph).

Based on ground-based imaging from the Palomar 200-inch telescope and the INT, a sample of 581 galaxies was selected for low-resolution follow-up spectroscopy of all 10 clusters (Balogh et al. 2002b). The sample selection was performed on the single INT WFC chip covering $11.4^{\prime}$ at $0^{\prime \prime} 33$ arcsec/pixel. The Palomar COSMIC images have a field-of-view of $13.7^{\prime}$ with a pixel scale of $0.44 \mathrm{arcsec} /$ pixel. Galaxies were selected for spectroscopic follow-up from the $R$-band images, except for two clusters (CL 1309+32 and CL 1444+63), where the selec- 
tion was done on the $I$-band images because no $R$-band images were available. Reliable redshifts could be derived for a total of 317 galaxies, of which 172 are cluster members (see section 5.5 for more details). From this final catalog of 172 Low $-L_{X}$ cluster members, galaxies for follow-up spectroscopy with intermediate-resolution for a detailed kinematic analysis presented here were selected.

To check the consistency between the different data sets, the ground-based $R$ photometry of the CL 0849 cluster galaxies was compared with the results from Gaztelu (2000). Fig. 4.8 gives a comparison between the ground-based $R$ magnitudes by Gaztelu (2000) and the $R$ magnitudes used in this work for CL 0849 cluster members at $z=0.234$. On average the differences between the magnitudes of $\langle\Delta R\rangle=0.06^{m}$ are satisfactory for fainter galaxies with $R \gtrsim 18.4^{\mathrm{m}}$. However, for the brightest galaxies significant deviations arise. Note that the work of Gaztelu concentrated only on the fainter galaxy population and thus chose a fixed aperture radius of $r_{a p}=2.65^{\prime \prime}$ (5 pixel) to measure the $R$-band magnitudes. Furthermore, the Palomar $R$ band image was analysed with a wrong seeing value and calibrated using the CAHA CAFOS $R$ image which introduced an additional calibration offset of $0.05^{m}$ to the magnitudes. Therefore, the calibrated magnitudes by Gaztelu have an accuracy of $0.08^{m}$ only. The measured $R$ magnitudes by Gaztelu are indicated as open circles. The choice of this fixed aperture size has a dramatic effect on the brighter galaxies, showing fainter magnitudes with differences of about $\Delta R \approx 0.45^{m}$, for the brightest object even up to $0.9 \mathrm{mag}$. Due to the inconsistent values and to check the SExtractor analysis of the galaxies in this study, the $R$ magnitudes of the galaxies by Gaztelu (2000) were re-measured using a nominal aperture of $r_{a p}=5.57^{\prime \prime}$. The size of the aperture was examined for the whole set of brightest galaxies and yielded the best approximation. The re-measured $R$ magnitudes of Gaztelu derived with an aperture diameter of $d_{a p}=11.14^{\prime \prime}$ are represented as solid circles. Results of these re-measurements were compared to the aperture magnitudes derived with variable elliptical apertures using the SExtractor and GIM2D algorithms on both ground-based and HST images (section 4.5.1). All three independent approaches resulted in very similar $R$ magnitudes. The differences were less than their scatter, e.g., $\Delta\left\langle R_{\mathrm{SEx}}-R_{\mathrm{GIM} 2 \mathrm{D}}\right\rangle=0.001^{m}$. For the subsequent analysis the SExtractor magnitudes were applied. These total apparent magnitudes showed mean uncertainties of $\delta \bar{R}=0.029 \pm 0.013^{m}$ (median value 0.03 ).

Fig. 4.9 shows the $(B-R)-R$ CMD from the Hale imaging for all galaxies brighter than $R=22.5^{m}$ lying in a $9.7^{\prime} \times 9.7^{\prime}(2.17 \mathrm{Mpc})$ region centered on the cluster CL 0849. The 14 early-type cluster members with available intermediate-resolution spectroscopic information are represented with open squares. Galaxy with ID \#54 has no $(B-R)$ colour and is therefore not included. One galaxy (object \#3), which was previously not targeted in Balogh et al. (2002b), was revealed to be a cluster member based on its intermediate-resolution spectra. Circles denote 13 early-type cluster members, which were verified by low-resolution spectroscopy (Balogh et al. 2002b). Rejecting one galaxy which is included in both setups, this yields a total sample of 26 spectroscopic confirmed cluster members for CL 0849. At $R \sim 17.7^{m}, R \sim 19.3^{m}$ and $R \sim 20.6^{m}$ the mean errors in $R$ are $0.01^{m}$, $0.03^{m}$ and $0.07^{m}$ and in $(B-R) 0.05^{m}, 0.08^{m}$ and $0.18^{m}$, respectively. These typical uncertainties are shown in the top of Fig. 4.9 as error bars. A least-squares fit to three ellipticals gives $(B-R)_{\mathrm{E}}=-0.0667\left(R_{\mathrm{tot}}\right)+3.6889$ which is shown by the solid line in Fig. 4.9. The CM sequence for the CL 0849 cluster is less well established than for the colour-magnitude relation of A 2390 (cp. Fig. 4.5) and shows a larger scatter compared to the early-type galaxies in massive clusters. To verify these results, the ob- 
servations can be compared to theoretical SSP model predictions by Kodama \& Arimoto (1997, hereafter KA97). These authors calibrate their models using the CM zero-point and slope of the observed $(V-K)$ vs. $M_{V}$ colour-magnitude relation (CMR) for Coma cluster ellipticals (Bower et al. 1992). The population synthesis code uses as main characteristic parameters an IMF slope of $x=1.20$, a mass range $0.10 \leq m / M_{\odot} \leq 60.0$, a metallicity range of $0.1^{-3} \leq Z \leq 0.05$ and a burst duration of $\tau=0.1$ Gyr. The KA97 models assume that elliptical galaxies have been formed in a single burst event at a high formation redshift of $z_{\mathrm{f}} \sim 2$ with a following passive evolution of their stellar populations. KA97 demonstrate that for sub- $L^{*}$ galaxies the CM slope changes are mainly caused by fainter galaxies due to variations in their mean stellar metallicity. The timescales for the loss of metals via galactic winds is shorter for less massive galaxies, which results on average in lower metallicities for these systems. The scatter around the CM relation is rather influenced by age effects. Applying the KA97 models, the evolution of the slope and the zero-point for any given red sequence of galaxies as a function of redshift can be derived. Comparing our observed red sequence with SSP KA97 model assumptions, for a redshift of $z=0.234$ a red sequence of $(B-R)_{\mathrm{E}}=-0.0668\left(R_{\mathrm{tot}}\right)+3.6500$ is predicted, which is indicated in Fig. 4.9 by the dashed line. This "theoretical" red cluster sequence is in extremely good agreement with the observed red cluster galaxies spanned by the elliptical galaxies of CL 0849. The model CMR has almost identical slope and zero-point as the observed CMR defined by the red cluster elliptical galaxies.

For the spectroscopic confirmed cluster member galaxies of CL 0849 the fraction of blue galaxies $f_{b}$ within the CM plane can be derived (Butcher \& Oemler 1984). Galaxy clusters at low redshift have a very homogeneous group of galaxies with the cluster cores essentially devoid of blue galaxies, whereas at $z>0.1$ the blue galaxy

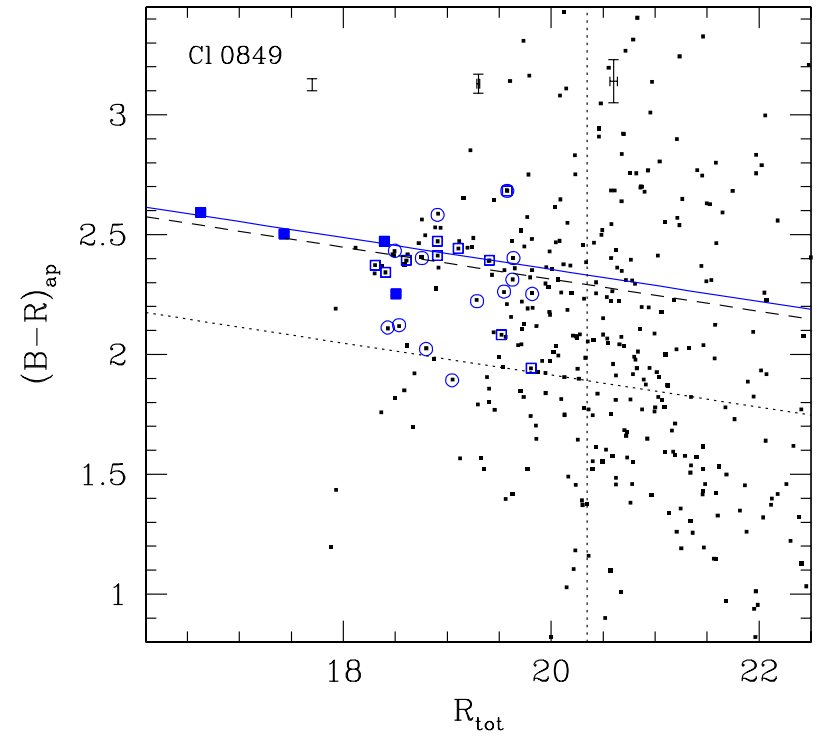

Figure 4.9: $(B-R)-R$ colour magnitude diagram from the P200 ( $R$-band) and CAHA ( $B$-band) imaging for galaxies brighter than $R=22.5^{m}$ lying in a $9.7^{\prime} \times 9.7^{\prime}(2.17 \mathrm{Mpc})$ region centered on CL 0849 . 14 early-type cluster members with available spectra are denoted with squares. Filled squares represent ellipticals which enter the Fundamental Plane with the solid line being a least-square fit to three ellipticals. Circles indicate 13 early-type cluster members verified through low-resolution spectroscopy. The dashed line is the predicted CMDs using SSP models by Kodama \& Arimoto (1997). The vertical dotted line, which denotes $M_{V}<-20^{m}$, and the horizontal dotted line are used to define the blue galaxy fraction.

fraction increases with redshift and the number of $f_{b}$ becomes significantly higher at $z \sim 0.5$. Only galaxies brighter than $M_{V}<-20^{m}$ in the rest-frame (indicated as the vertical dotted line in Fig. 4.9) and those objects whose rest-frame $(B-V)$ colors are at least 0.2 mag bluer than the ridge line of the early-type galaxies at that magnitude cut are considered. For the cluster redshift of CL $0849 M_{V}<-20^{m}$ corresponds to $R=20.34^{m}$ and at $z=0 \Delta(B-V)=-0.2$ is approximately the difference in colour of an elliptical and Sab-type spiral galaxy (Fukugita et al. 1995). At $z=0.2$, the $\Delta(B-V)$ colour criterium translates into $(B-R)_{\mathrm{E}}=-0.0668\left(R_{\text {tot }}\right)+$ 
3.2500 , which is indicated as the horizontal dotted line in Fig. 4.9. The blue galaxy fraction for CL 0849 gives $f_{b}=0.077 \pm 0.052$. To put probability limits on the galaxy fractions, the error of $f_{b}$ was evaluated assuming Poisson statistics.

\subsubsection{HST Photometry}

HST/F702W ( $\left.R_{702}\right)$ imaging during Cycle 8 (P.I. Prof. R. L. Davies (Oxford), Proposal ID 8325) was acquired in July 2000 for 8 of the 9 clusters between $0.2<z<0.30$ and with a mean redshift of $\bar{z}=0.25$. Each cluster was observed with three single orbit exposures in the F702W filter with exposure times ranging from 2100 to $2600 \mathrm{sec}$ per orbit. Total integration times of $7.500 \mathrm{sec}$ warrant an accurate measurement of the structural and photometric parameters of those galaxy candidates located within the HST fields. The three pointing positions for the exposures were offset by 10 pixels (Wide Field Camera (WFC) pixel size) from one another but during the reduction procedure the exposures were aligned and coadded in oder to remove cosmic rays artefacts and hot pixels. The images were not drizzled or regridded because this does not accurately preserve the noise characteristics of the pixels. After coadding the single exposures, each WFC chip image was trimmed to the area of full sensitivity (see Balogh et al. 2002a for details). The photometry is calibrated on the Vega system and updated zero-points were taken from the current instrument manual. The final images reach a $3 \sigma$ point source sensitivity of $R_{702} \sim 25.5^{m}$, and cover a field of $2.5^{\prime} \times 2.5^{\prime}$ (or $0.57 h_{70}^{-1} \mathrm{Mpc}$ at $z=0.25$ ) with an angular resolution of $0.17^{\prime \prime}\left(\sim 0.71 h_{70}^{-1} \mathrm{kpc}\right)$. The cluster CL 1633+57 was observed in Cycle 7 (P.I. Prof. P. Rosati (ESO), Proposal ID 7374). The data of cluster CL $1633+57$ were retrieved from the $\mathrm{CADC}^{4} \mathrm{HST}$ archive. These data are WFPC2/F702W observations, with four expo-

\footnotetext{
${ }^{4}$ Canadian Astronomy Data Centre, which is operated by the Herzberg Institute of Astrophysics, National Research Council of Canada.
}

sures of 1200 seconds. The total exposure time of 4800 seconds is therefore less than that of the other nine clusters. Table 4.4 gives a summary of the HST imaging and the X-ray properties for the rich and poor cluster samples.

For the Low $-L_{X}$ clusters a nominal magnitude limit of $R_{702}=23.0^{m}$ at $z=0.25$ was adopted, which corresponds to $M_{702}=-17.6+5 \log h_{70}$ or approximately $M_{R} \sim-17.4+5 \log h_{70}$ (assuming a $\Lambda \mathrm{CDM}$ cosmology and including small $k$ corrections of $\lesssim 0.1^{m}$ ), which is 4.2 magnitudes below $M_{R}^{*} \approx-21.60^{m}$ (Blanton et al. 2001). Galaxies brighter than this limit are luminous enough and have large enough sizes in oder to warrant a reliable and accurate classification.

\subsection{Surface Brightness Profile Fitting}

\subsubsection{Choice of Luminosity Profile}

A fundamental question when determining the structural parameters of galaxies is what kind of surface brightness profile type is the most suitable. Generally, derivation of total magnitudes involves an extrapolation of curves of growth to infinity, thus relying on fits to the luminosity profiles of the galaxies. For example, Caon et al. (1993) and La Barbera et al. (2002) used Sérsic profiles to fit the surface brightness profile of early-type galaxies. Another approach is to use the classical de Vaucouleurs $\left(r^{1 / 4}\right)$ law with an exponential disc component (e.g., Simard et al. 2002; Tran et al. 2003).

Tran et al. (2003) explored the results of three different profile models (double exponential, Sérsic bulge+exponential disc and an $r^{1 / 4}$ bulge+exponential disc) using the GIM2D package (Simard et al. 2002, see section 4.4.2 for details) on a sample of 155 cluster galaxies in CL $1358+62$ at $z=0.33$. Based on their extensive profile tests the authors conclude that the de Vaucouleurs bulge with exponential disc profile is the most appropriate way to investigate 
Table 4.4: HST observations of the cluster samples.

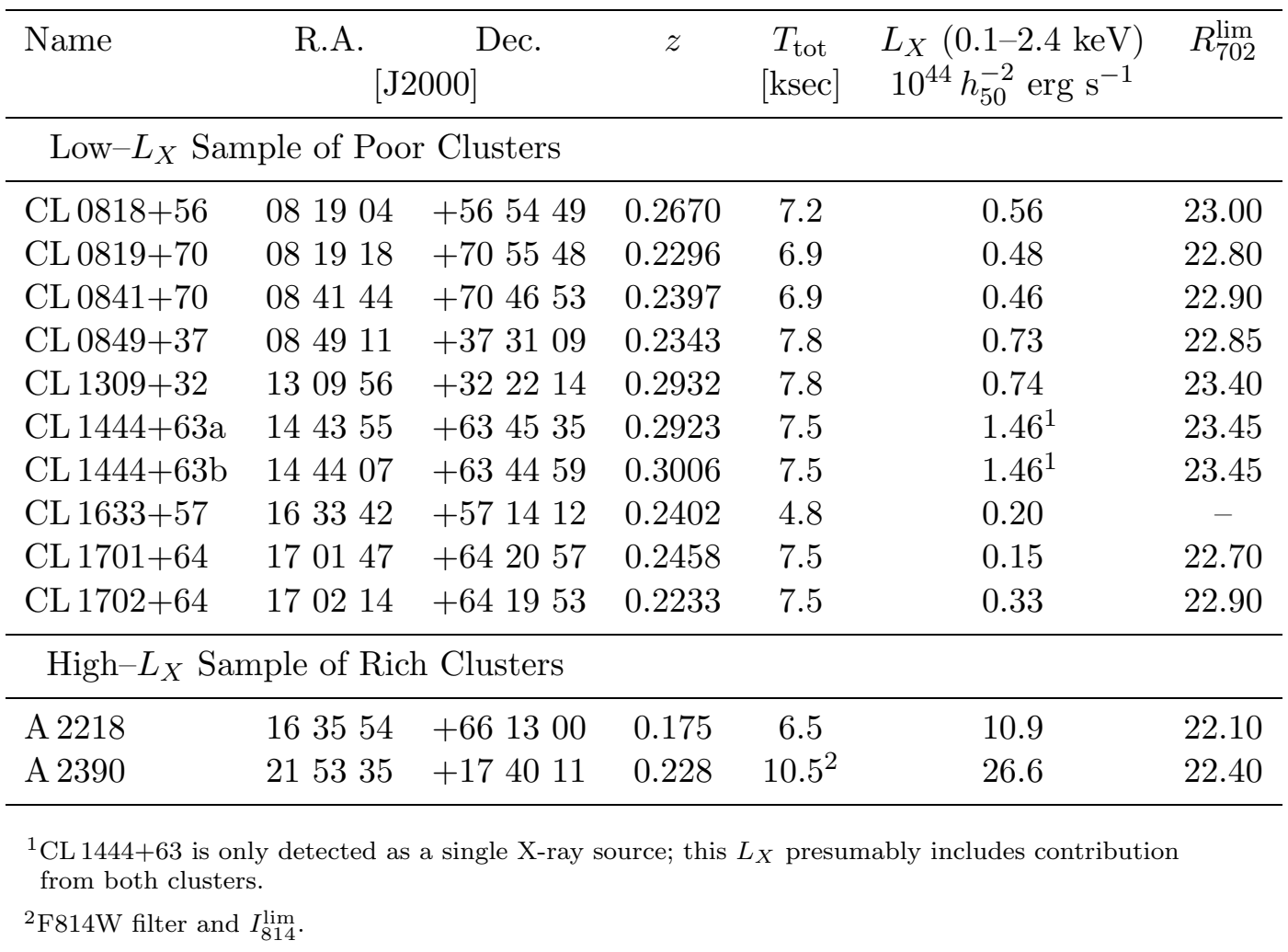

the structural properties. As the cluster samples in the present study comprise only early-type galaxies a similar approach is used, namely a combination of an $r^{1 / 4}$ plus exponential disc profile by Saglia et al. (1997a). These authors demonstrated that the Sérsic $r^{1 / n}$ profiles can be understood as a "subset" of $r^{1 / 4}+$ exponential models. This algorithm also explores the effect of an efficient sky-subtraction. For example, for $n>4$ large extrapolations are needed which could lead to large errors in the sky-subtraction of up to $\pm 3 \%$ and, therefore, to additional uncertainties in the absolute magnitudes and half-light-radii. The two-dimensional surface brightness distribution of the early-type field galaxies was fitted with a combination of $r^{1 / n}$ plus exponential disc profile using the GALFIT package (Peng et al. 2002). The Sérsic exponent $n$ was restricted to variable only within a range between $n=1-4$. For an internal consistency, the field galaxies in the FDF were modelled with both algorithms and the parameters compared to each other. It turned out, that a combination of de Vaucouleurs and exponential disc surface brightness profiles as used for the cluster galaxies is an equivalent approach to the GALFIT measurements and yields within the uncertainties to similar results.

To test the effects on the structural parameters a second pure $r^{1 / 4}$ profile model was applied to fit the surface brightness distribution of the galaxies. Results were extensively tested and their variations and imprints will be discussed together with the scaling relations in sections 6.3 and 6.4 . 


\subsubsection{The Surface Brightness Models plied:}

Structural parameters were analysed using the fitting algorithm developed by Saglia et al. (1997a, 1997b). The galaxies' parameters are derived searching for the best combination of seeing-convolved, sky-corrected $r^{1 / 4}$ and exponential laws. This approach accounts for various types of observed luminosity profiles, i.e. it models the extended luminosity profiles of $\mathrm{cD}$ galaxies and provides fits to the range of profile shapes of early-type galaxies (ellipticals featuring a flat core to $\mathrm{S} 0$ galaxies with the presence of a prominent disc).

Subframes of all early-type cluster galaxies both with spectroscopic and $H S T$ information were extracted and each galaxy was analysed individually. In a first step stars and artifacts around the galaxies were masked in order not to cause any problems in the fitting process. In the next step the circularly averaged surface brightness profile of the galaxy was fitted with PSFconvolved $r^{1 / 4}$ and exponential components, both simultaneously and separately. The individual PSFs for the profiles were computed using the TinyTim program by considering the Fourier transform of the PSF as $\exp \left[(-k b)^{\gamma}\right]$ and adopting $\gamma=1.6$. The $\gamma$ exponent defines the exponential decrease of the PSF function (hereafter referred as $\gamma$ PSF) and for $\gamma=2$ the PSF is a standard Gaussian. The assumption of $\gamma=1.6$ was extensively tested by Saglia et al. (1997b) using $\gamma$ PSFs of $\gamma=1.5-1.7$ with a pixel size of $0.8^{\prime \prime}$. The choice of a different PSF introduced a small effect. If the true PSF is $\gamma=1.5$, then the total luminosity, half-luminosity radius and scale length of the bulge will be slightly overestimated and the disc-to-bulge $(D / B)$ ratio slightly underestimated. Opposite trends are found if the true $\gamma$ is 1.7. Systematic differences become negligible when $R_{\mathrm{e}}>2$ FWHM.

For the $r^{1 / 4}$ bulge profile a special form of the Sérsic profile (Sérsic 1968), the classical de Vaucouleurs profile, in the following form was ap-

$$
\mu(R)=\mu_{\mathrm{e}} \exp \left\{-7.67\left[\left(\frac{R}{R_{\mathrm{e}}}\right)^{1 / 4}-1\right]\right\},
$$

where $\mu(R)$ denotes the surface brightness at $R$ along the semi-major axis radius and $\mu_{\mathrm{e}}$ the effective surface brightness at the effective radius $R_{\mathrm{e}}$, which refers to half of the total light of the galaxy. The effective surface brightness at the effective radius is given as $\mu_{\mathrm{e}}=-2.5 \times \log I_{\mathrm{e}}$, where $I_{\mathrm{e}}$ is the intensity at the effective radius $R_{\mathrm{e}}$. The disc profile is well represented by an exponential law and defined as:

$$
\mu(R)=\mu_{0} \exp \left(-\frac{R}{h}\right)
$$

where $\mu_{0}$ is the central (face-on) surface brightness of the disc and $h$ indicates the exponential disc scale length. For an de Vaucouleurs profile, the central surface brightness of the disc $\mu_{0}$ can be computed as $\mu(R=0) \equiv \mu_{0}=\mu_{\mathrm{e}}-8.327$. This method allowed to derive the effective (halflight) radius $R_{\mathrm{e}}$ (in arcsec), the total $\mathrm{F} 702 \mathrm{~W}$ band magnitude $R_{702}\left(R_{\text {tot }}\right)$ or total $\mathrm{F} 814 \mathrm{~W}$ band magnitude $I_{814}\left(I_{\text {tot }}\right)$, the central surface brightness $\mu_{0}$ and the mean surface brightness within $R_{\mathrm{e}},\left\langle\mu_{\mathrm{e}}\right\rangle$, for the entire galaxy as well as the luminosity and scale of the bulge $\left(m_{b}\right.$ and $\left.R_{\mathrm{e}, b}\right)$ and disc $\left(m_{d}\right.$ and $\left.h\right)$ component separately, within the limitations described by Saglia et al. (1997a). An extensive description of the uncertainties and errors is discussed in the next section 4.3.3.

In total, structural parameters could be determined for 29 early-type galaxies, 14 in the rich cluster A 2390 and 15 in the three poor clusters CL 0849, CL 1701 and CL 1702, which are all spectroscopic confirmed cluster members. In numbers, 14 out of 15 (one is a background galaxy) in A 2390 (see Table 4.5), 6/8 in CL 0849, $7 / 12$ in CL 1701 and 2/8 CL 1702. In case of the Low $-L_{X}$ clusters, all brighter cluster candidates down to $R_{702} \sim 20.75^{m}$ were fitted because at 

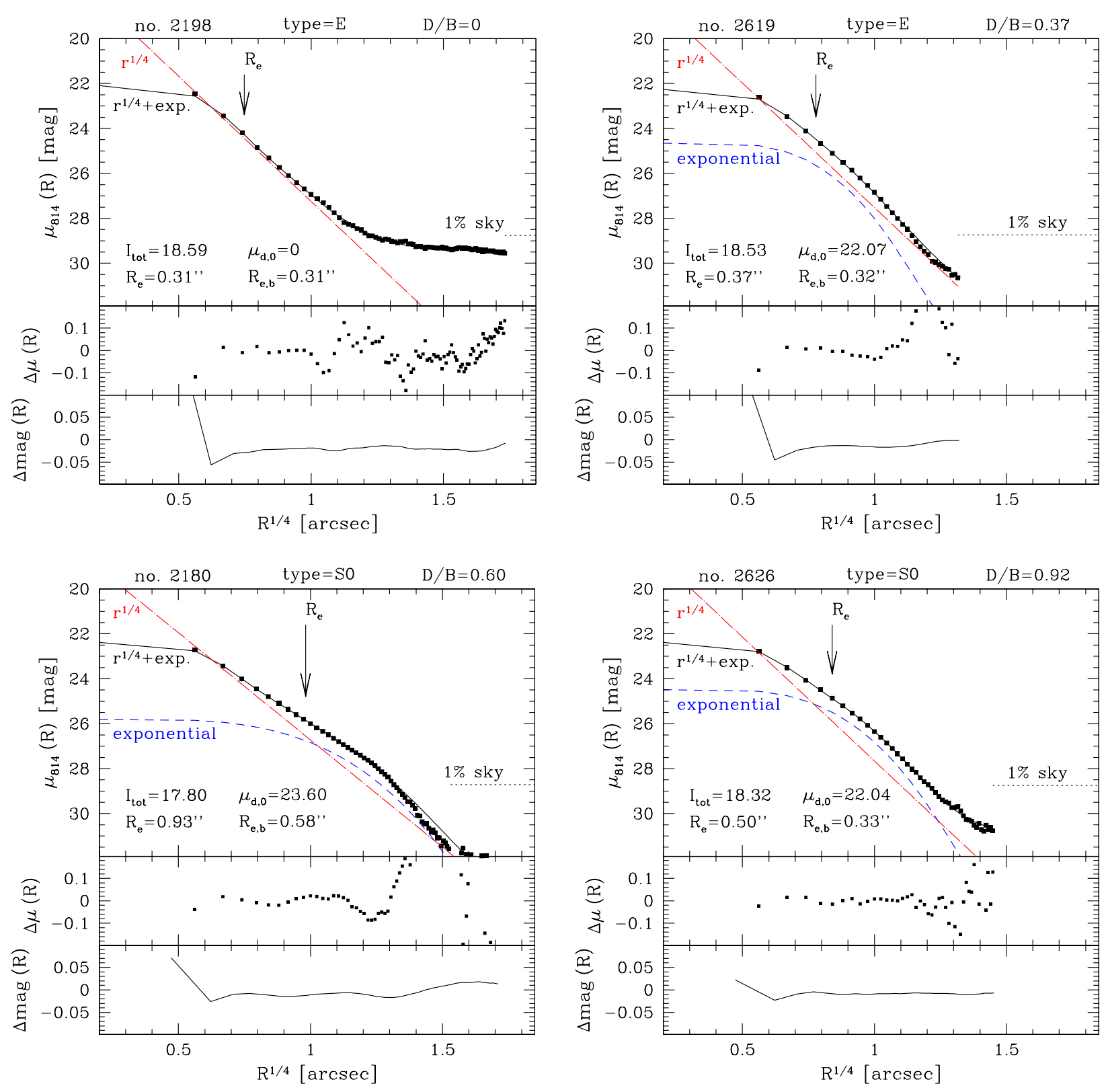

Figure 4.10: Circularly averaged $I_{814}$ surface brightness profiles of four early-type galaxies of A 2390 with available spectral information. The calibrated surface brightness profile (squares) is shown together with the fitted bulge $\left(r^{1 / 4}\right)$ and/or disc (exponential) profiles as a function of the $1 / 4$ power of the distance $R$ from the galaxy center in units of arcsec. The horizontal dotted line denotes the position corresponding to 1 per cent of the sky background level. The arrow indicates the position of the effective radius $R_{\mathrm{e}}$. Values for the total magnitude $I_{\text {tot }}$, the surface brightness of the disc $\mu_{\mathrm{d}, 0}\left(\forall \mu_{\mathrm{d}, 0}=0 \rightarrow \nexists \mu_{\mathrm{d}, 0}\right)$, the effective radius $R_{\mathrm{e}}$ and the effective radius of the bulge $R_{\mathrm{e}, b}$, and the disc-to-bulge ratio $D / B$ are listed. Galaxies are ordered with increasing $D / B$ ratio. Below each surface brightness profile the residuals of the fits, $\Delta \mu(R)=$ $\mu_{\text {obs }}(R)-\mu_{\text {fit }}(R)$ (dots) and the integrated magnitude difference $\Delta \operatorname{mag}(R)=\operatorname{mag}_{\text {obs }}(<R)-\operatorname{mag}_{\text {fit }}(<R)$ (full lines) are indicated. Note that $\Delta \operatorname{mag}(R)<0.06$, even if large deviations of $\Delta \mu$ are present. 
the time of this analysis no spectra were available to select galaxies based upon their redshifts. For this reason, the first number represents the final poor cluster members (including the lowresolution spectroscopy) and the second the total number of analysed galaxies within the HST FOV, i.e., members plus "anonymous" galaxies (see section 4.4.1). Fig. 4.10 and Fig. 4.11 show typical examples of the surface brightness profile fits for early-type galaxies in A 2390. In most cases, the observed profile is best described by a combination of a bulge (de Vaucouleurs law) and a disc component (exponential law), indicated in the figures as (sum: $r^{1 / 4}+$ exp.-law). Note that for an $r^{1 / 4}$ profile the combination $\left(r^{1 / 4}+\right.$ exp. $)$ represents the seeing convolved $r^{1 / 4}$ fit only. Fig. 4.10 illustrates in detail the residual errors on the structural parameters for different profile fits. For this reason, below each surface brightness profile the residuals of the fits, $\Delta \mu(R)=\mu_{\mathrm{obs}}(R)-\mu_{\mathrm{fit}}(R)$ (dots), in units of $\mathrm{mag} / \operatorname{arcsec}^{2}$, and the integrated magnitude difference $\Delta \operatorname{mag}(R)=\operatorname{mag}_{\text {obs }}(<R)-\operatorname{mag}_{\text {fit }}(<R)$ (full lines), in units of magnitudes, are shown. Note that for all cases $\Delta \operatorname{mag}(R)<0.06$, even if large deviations of $\Delta \mu$ are present. Note that if the surface brightness of the disc $\mu_{\mathrm{d}, 0}$ is shown as $\mu_{\mathrm{d}, 0}=0$ it has no value but is indefinite. Typical residuals $\Delta \mu_{814}$ between the observed profile and the fit as a function of $R^{1 / 4}$ are in the order of $0.01^{m} \leq \Delta \mu_{I} \leq 0.10^{m}$. Comparing the differences between elliptical and lenticular (S0) galaxies, a small disc component for the S0 exhibiting a lens-like structure is detected. For example, the elliptical galaxy \# 2438 of the A 2390 cluster is well represented by a pure de Vaucouleurs profile. In the case of the S0 galaxy \# 2946 a combined model of an $r^{1 / 4}$-law and an additional disc component results in the best luminosity profile. In order to explore the impact of an additional exponential component to the observed surface brightness profile, Fig. 4.10 lists the early-type galaxies ordered with increasing $D / B$ ratio, ranging from $0<D / B<0.93$. The different panels clearly show how the exponential component becomes stronger as a function of $D / B$ ratio with a simultaneous weakening of the bulge component. For large $D / B$ values of $D / B>0.6$ the exponential component dominates over the $r^{1 / 4}$ component. In case of the galaxy \#2198, the weak flattening of the surface brightness profile at $R \approx 1.2^{\prime \prime}$ is caused by a close neighbour. However, this effect starts at faint magnitudes $\mu_{814} \approx 28.7 \mathrm{mag} / \operatorname{arcsec}^{2}$ at large radii $R \approx 1.2^{\prime \prime}$ and thus not influence the measurement of structural parameters.

For some cases (e.g., \# 2198, \# 2438 and \# 2763 in the A 2390 sample) no additional disc component is detected, i.e. a pure classical de Vaucouleurs law profile $(D / B=0)$ results in the best profile for the galaxy's light distribution. Therefore, this approach also allows for the possibility that dynamically hot galaxies may all have $r^{1 / 4}$ profiles. But most of the galaxies are well described by the superposition of $r^{1 / 4}$ bulge and exponential disc profiles. Bulge+disc models provide better fits than a pure $r^{1 / 4}$ law, and the addition of an exponential disc component improves the fit for many of the galaxies. The surface brightness at the half-light radius is a strong function of the chosen fitting profile and the halflight radius is dependent on the assumed surface brightness model. However, the product $R_{\mathrm{e}}\left\langle I_{\mathrm{e}}\right\rangle^{\beta}$ (with $\beta \simeq 0.8$ ) provides a Fundamental Plane parameter which is stable for all applied profile types, from $r^{1 / 4}$ to exponential laws (Saglia et al. 1993b; Kelson et al. 2000a). Under the constraint of this coupling, galaxies can only move parallel to the edge-on view of the Fundamental Plane.

Some previous Fundamental Plane studies derived their structural parameters with a pure $r^{1 / 4}$ profile. Therefore, the results were also tested using surface brightness models based on a pure de Vaucouleurs law only. Only systems featuring a dominant disc (Sa bulges) show some deviations. As expected, these galaxies move only along the edge-on projection of the Fundamental 
Table 4.5: Galaxy properties of the A $2390 H S T$ sub-sample. (1) galaxy ID, (2) velocity dispersion $\sigma_{c}$ (aperture corrected) with errors [in $\left.\mathrm{km} \mathrm{s}^{-1}\right],(3)$ extinction-corrected, Vega-based total magnitude in WFPC2 F814W filter, (4) mean surface brightness magnitude $\left\langle\mu_{\mathrm{e}}\right\rangle$ in Gunn $r$ corrected for cosmic expansion, (5) rest-frame Gunn $r$ magnitude [in mag], (6) effective radius [in arcsec] and (7) effective radius [in kpc], (8) effective radius of the bulge [in arcsec], (9) FP parameter $R_{\mathrm{e}}\left\langle I_{\mathrm{e}}\right\rangle^{0.8}$, (10) disc scale-length [in arcsec], (11) disc-to-bulge ratio $(D / B),(12)$ morphology $T$ of the galaxy.

\begin{tabular}{rccccccccccc}
\hline ID & $\begin{array}{c}\sigma_{c} \\
{\left[\mathrm{~km} \mathrm{~s}^{-1}\right]}\end{array}$ & $\begin{array}{c}I_{814} \\
{[\mathrm{mag}]}\end{array}$ & $\begin{array}{c}\left\langle\mu_{\mathrm{e}}\right\rangle_{r} \\
{\left[\mathrm{mag} / \square^{\prime \prime}\right]}\end{array}$ & $\begin{array}{c}M_{r} \\
{[\mathrm{mag}]}\end{array}$ & $\begin{array}{c}R_{\mathrm{e}} \\
{\left[{ }^{\prime \prime}\right]}\end{array}$ & $\begin{array}{r}\log R_{\mathrm{e}} \\
{[\mathrm{kpc}]}\end{array}$ & $\begin{array}{c}R_{\mathrm{e}, \mathrm{b}} \\
{\left[{ }^{\prime \prime}\right]}\end{array}$ & $\mathrm{FP}$ & $\begin{array}{c}\mathrm{h} \\
{\left[{ }^{\prime \prime}\right]}\end{array}$ & $D / B$ & $T$ \\
$(1)$ & $(2)$ & $(3)$ & $(4)$ & $(5)$ & $(6)$ & $(7)$ & $(8)$ & $(9)$ & $(10)$ & $(11)$ & $(12)$ \\
\hline 2106 & $144.5 \pm 16.8$ & 19.18 & 18.28 & 19.93 & 0.282 & 0.013 & 0.250 & 0.033 & 0.20 & 0.36 & $\mathrm{E}$ \\
2120 & $140.3 \pm 15.3$ & 18.88 & 18.22 & 19.63 & 0.314 & 0.060 & 0.202 & 0.154 & 0.34 & 0.46 & $\mathrm{~S} 0$ \\
2138 & $165.4 \pm 08.7$ & 16.97 & 20.60 & 17.72 & 2.264 & 0.918 & 1.324 & 1.800 & 4.48 & 0.36 & $\mathrm{E}$ \\
2180 & $161.2 \pm 10.6$ & 17.80 & 19.49 & 18.55 & 0.928 & 0.530 & 0.580 & 1.195 & 0.88 & 0.60 & $\mathrm{~S} 0$ \\
2198 & $148.9 \pm 15.6$ & 18.59 & 17.92 & 19.34 & 0.313 & 0.058 & 0.313 & 0.155 & 0.00 & 0.00 & $\mathrm{E}$ \\
2237 & $162.2 \pm 12.3$ & 17.66 & 19.94 & 18.41 & 1.217 & 0.648 & 1.195 & 1.385 & 0.73 & 0.81 & $\mathrm{Sa}$ \\
2438 & $119.8 \pm 15.9$ & 18.74 & 19.59 & 19.49 & 0.631 & 0.363 & 0.631 & 0.808 & 0.00 & 0.00 & $\mathrm{E}$ \\
2460 & $258.6 \pm 10.2$ & 18.00 & 18.90 & 18.75 & 0.642 & 0.370 & 0.509 & 0.892 & 1.09 & 0.17 & $\mathrm{E}$ \\
2592 & $192.0 \pm 11.4$ & 17.20 & 20.11 & 17.95 & 1.625 & 0.774 & 1.104 & 1.618 & 2.55 & 0.29 & $\mathrm{E}$ \\
2619 & $229.3 \pm 14.3$ & 18.53 & 18.20 & 19.28 & 0.366 & 0.126 & 0.320 & 0.326 & 0.27 & 0.37 & $\mathrm{E}$ \\
2626 & $195.6 \pm 12.7$ & 18.31 & 18.65 & 19.06 & 0.497 & 0.259 & 0.325 & 0.640 & 0.38 & 0.92 & $\mathrm{~S} 0$ \\
2763 & $300.5 \pm 12.2$ & 17.55 & 18.89 & 18.30 & 0.789 & 0.460 & 0.789 & 1.108 & 0.00 & 0.00 & $\mathrm{E}$ \\
2946 & $131.9 \pm 15.2$ & 18.69 & 19.26 & 19.44 & 0.554 & 0.306 & 0.405 & 0.708 & 0.60 & 0.34 & $\mathrm{~S} 0$ \\
6666 & $211.0 \pm 13.5$ & 17.65 & 18.79 & 18.40 & 0.721 & 0.421 & 0.488 & 1.024 & 1.00 & 0.32 & $\mathrm{E} / \mathrm{S} 0$ \\
\hline
\end{tabular}

Plane. Note, that the errors of the FP parameters of effective radius $\left(R_{\mathrm{e}}\right)$ and surface brightness $\left(\mu_{\mathrm{e}}\right)$ are highly correlated. However, the rms uncertainty in the product $R_{\mathrm{e}}\left\langle I_{\mathrm{e}}\right\rangle^{0.8}$ which propagates into the $\mathrm{FP}$ is only $\approx 4 \%$ for all galaxies in this study, corresponding to $\Delta \mu_{\mathrm{e}}=\beta \Delta \log R_{\mathrm{e}}$ with $\beta=0.328$ and a scatter of the latter relation of $0.05 \mathrm{mag} \operatorname{arcsec}^{-2}$. The results of the $r^{1 / 4}$ structural measurements are presented in Table 4.6. In chapter 6, the impacts of different surface brightness models on the Fundamental Plane will be discussed. In particular, Fig. 6.14 on page 144 shows the Fundamental Plane for A 2218 and A 2390 constructed based on a pure $r^{1 / 4}$-law profile.

Surface brightness profiles of those galaxies situated within the HST field of Abell 2390 with available spectroscopic information are shown in Fig. 4.11. The surface brightnesses were corrected for galactic extinction. In most cases the observed profile is best fitted by a combination of a bulge (de Vaucouleurs law) and a disc component (exponential law). Values for the total magnitude $I_{\text {tot }}$, the surface brightness of the disc $\mu_{\mathrm{d}, 0}$, the effective radius $R_{\mathrm{e}}$ and the effective radius of the bulge $R_{\mathrm{e}, b}$, and the disc-to-bulge ratio $D / B$ are listed. The arrow in each diagram indicates the position of the effective radius $R_{\mathrm{e}}$. Typical residuals $\Delta \mu_{I}$ between the observed profile and the modelled fit are in the order of \pm 0.10 mag at $R^{1 / 4} \approx 1.3$ arcsec. Note that the disc fit indicated in Fig. 4.11 represents the disc component of the combined best fit for the corresponding galaxies. The single galaxy which 
Table 4.6: Galaxy parameters of the A 2390 HST sub-sample based on pure de Vaucouleurs surface brightness fits. Columns are analogous tabulated as in Table 4.5. The velocity dispersions $\sigma$ with corresponding errors [in $\left.\mathrm{km} \mathrm{s}^{-1}\right]$ are not aperture corrected.

\begin{tabular}{rcccccccc}
\hline ID & $\begin{array}{c}\sigma \\
{\left[\mathrm{km} \mathrm{s}^{-1}\right]}\end{array}$ & $\begin{array}{c}I_{814} \\
{[\mathrm{mag}]} \\
(3)\end{array}$ & $\begin{array}{c}\left\langle\mu_{\mathrm{e}}\right\rangle_{r} \\
{\left[\mathrm{mag} / \square^{\prime \prime}\right]}\end{array}$ & $\begin{array}{c}M_{r} \\
{[\mathrm{mag}]}\end{array}$ & $\begin{array}{r}R_{\mathrm{e}} \\
{\left[{ }^{\prime \prime}\right]}\end{array}$ & $\begin{array}{r}\log R_{\mathrm{e}} \\
{[\mathrm{kpc}]}\end{array}$ & $\begin{array}{r}R_{\mathrm{e}, \mathrm{b}} \\
{\left[{ }^{\prime \prime}\right]}\end{array}$ & $\mathrm{FP}$ \\
\hline 2106 & $131.0 \pm 16.8$ & 19.07 & 18.55 & 19.82 & 0.335 & 0.088 & 0.335 & 0.219 \\
2120 & $127.2 \pm 15.3$ & 18.75 & 18.51 & 19.50 & 0.382 & 0.145 & 0.382 & 0.363 \\
2138 & $150.0 \pm 08.7$ & 17.14 & 20.04 & 17.89 & 1.621 & 0.772 & 1.621 & 1.630 \\
2180 & $146.2 \pm 10.6$ & 17.67 & 19.79 & 18.42 & 1.133 & 0.617 & 1.133 & 1.342 \\
2198 & $135.0 \pm 15.6$ & 18.59 & 17.92 & 19.34 & 0.313 & 0.058 & 0.313 & 0.155 \\
2237 & $147.1 \pm 12.3$ & 17.12 & 21.10 & 17.87 & 2.667 & 0.989 & 2.667 & 1.802 \\
2438 & $108.6 \pm 15.9$ & 18.74 & 19.59 & 19.49 & 0.631 & 0.363 & 0.631 & 0.808 \\
2460 & $234.5 \pm 10.2$ & 18.01 & 18.86 & 18.76 & 0.628 & 0.361 & 0.628 & 0.872 \\
2592 & $174.1 \pm 11.4$ & 17.27 & 19.87 & 18.02 & 1.408 & 0.711 & 1.408 & 1.533 \\
2619 & $207.9 \pm 14.3$ & 18.40 & 18.55 & 19.15 & 0.456 & 0.222 & 0.456 & 0.553 \\
2626 & $177.4 \pm 12.7$ & 18.01 & 19.29 & 18.76 & 0.768 & 0.448 & 0.768 & 1.033 \\
2763 & $272.5 \pm 12.2$ & 17.55 & 18.89 & 18.30 & 0.789 & 0.460 & 0.789 & 1.108 \\
2946 & $119.6 \pm 15.2$ & 18.62 & 19.36 & 19.37 & 0.599 & 0.340 & 0.599 & 0.778 \\
6666 & $191.3 \pm 13.5$ & 17.65 & 18.74 & 18.40 & 0.701 & 0.408 & 0.701 & 1.000 \\
\hline
\end{tabular}

could not be fitted (\# 2933) is a late-type spiral galaxy $(\mathrm{Sbc})$ with clear signs of spiral pattern at $z=0.398$. Thumbnail images for the 14 galaxies in A 2390 are provided in Fig. 4.12. A detailed description of the morphological classification of galaxies residing in the HST field is outlined in the section 4.4. The final classification is listed in Table 4.5 and is also given on top of each galaxy surface brightness plot in Fig. 4.11.

As an additional comparison, an isophote analysis based on the procedure introduced by Bender \& Möllenhoff (1987) was performed. Deviations from the elliptical isophotes were recorded as a function of radius by a Fourier decomposition algorithm. The presence and strength of the $a_{4}$ coefficient, which represents the signature of discyness, is in good agreement with the visually classified morphologies of S0 and spiral bulges.

\subsubsection{Error Evaluation}

The errors of the photometric parameters of effective radius and surface brightness are correlated and enter the FP in combination. The errors in $R_{\mathrm{e}}$ (in arcseconds) and $\mu_{\mathrm{e}}$ (in magnitudes) propagate into the FP relationship in the following form (Treu et al. 2001a):

$$
\delta \mathrm{FP}_{\mathrm{phot}}=\log \delta R_{\mathrm{e}}-\beta \delta \mu_{\mathrm{e}}
$$

An error calculation with this formula is not an approximation but a particularly robust computation, as in three-dimensional space defined by the FP it is the only natural way to provide an accurate error estimation (Saglia et al. 1997a; Kelson et al. 2000a). For this reason, the errors in the FP are only shown in the edge-on projection along the short axis that separates kinematic measurements and photometric parameters (see upper right panel in Fig. 6.11). The errors are calculated for the $\mathrm{F} 814 \mathrm{~W}$ filter with a 

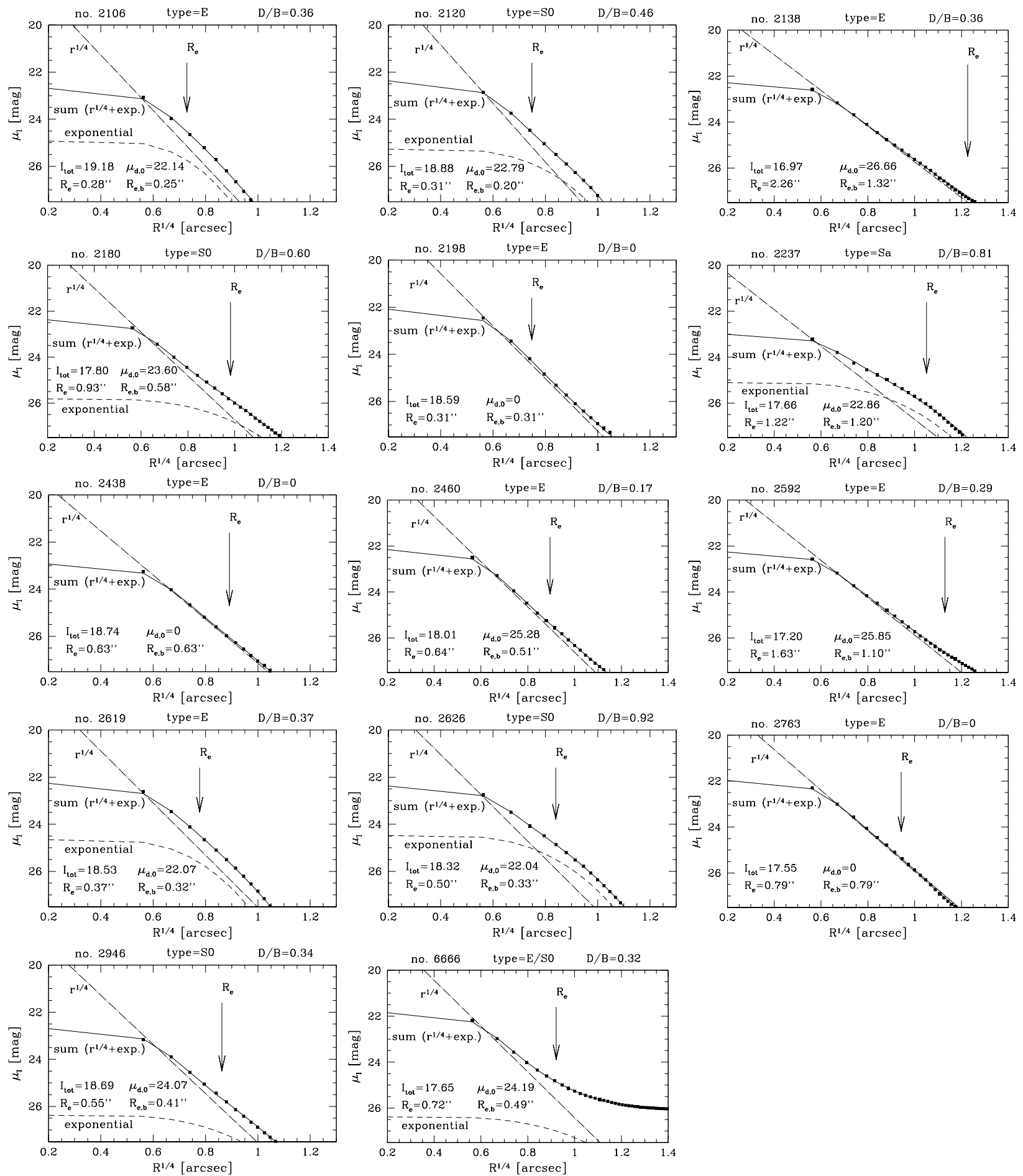

Figure 4.11: $I_{814}$ Surface brightness profiles of A 2390 galaxies lying within the $H S T$ image with available spectral information. The $I_{814}$ surface brightness magnitude (extinction $\mathrm{A}_{\mathrm{F} 814 \mathrm{~W}}$ corrected) is plotted against the radius $R^{1 / 4}$ (in arcsec). Filled squares show the observed profile, lines the different best models for the bulge (de Vaucouleurs law), disc (exponential law) component fit and for a combination of bulge and disc components (sum: $r^{1 / 4}+$ exp.-law). The arrow indicates the position of the effective radius $R_{\mathrm{e}}$. Some structural parameters are given (see text for details). 
Table 4.7: Error assessment via the parameter $Q$.

\begin{tabular}{lccc}
\hline Name & $Q=1$ & $Q=2$ & $Q=3$ \\
\hline A 2390 & 6 & 8 & 0 \\
CL 0849 & 2 & 3 & 1 \\
CL 1701 & 0 & 7 & 0 \\
CL 1702 & 2 & 0 & 0 \\
\hline
\end{tabular}

parameter value of $\beta=0.328$. Since the slope $\beta$ is very well defined with only a small variation with wavelength, the error estimations change negligibly within the observed range of $\beta$ values (Pahre et al. 1998).

The errors on the derived structural parameters are assigned through a combination of individual quality parameters, including the effects of sky subtraction errors, seeing and pixel sampling, average galaxy surface brightness relative to the sky, $S / N$ variations, extrapolation involved to derive $M_{\text {tot }}$, radial extent of the profile and reduced $\chi^{2}$ of the fit resulting in a final global quality parameter. The total quality parameter $Q$ is defined by the maximum of the quality parameters of individual errors $Q=\operatorname{Max}\left(\mathrm{Q}_{\max }\right.$, $\mathrm{Q}_{\Gamma}, \mathrm{Q}_{S / N}, \mathrm{Q}_{\mathrm{Sky}}, \mathrm{Q}_{\delta \mathrm{Sky}}, \mathrm{Q}_{\mathrm{E}}, \mathrm{Q}_{\chi^{2}}$ ) (Saglia et al. 1997a). The accuracy of the quality parameter $Q$ is given by a combination of several factors, such as a maximal radius ratio which should be larger than the effective radius $\left(\mathrm{Q}_{\max }\right)$, the seeing conditions and/or possible inadequate sampling effects $\left(\mathrm{Q}_{\Gamma}\right)$, the signal-to-noise $\left(\mathrm{Q}_{S / N}\right)$, the difference between the sky surface brightness and the average effective surface brightness of the models ( $\left.Q_{\text {Sky }}\right)$, sky subtraction errors $\left(\mathrm{Q}_{\delta \mathrm{Sky}}\right)$, the fraction of light extrapolated beyond $R_{\max }$ used in the determination of $M_{\text {tot }}\left(\mathrm{Q}_{\mathrm{E}}\right)$ and the reduced $\chi^{2}$ of the fit to the luminosity profile of the galaxy $\left(\mathrm{Q}_{\chi^{2}}\right)$. For $Q=1$ the typical uncertainty in total magnitude is $\Delta M_{\text {tot }}=0.05$ and in effective radius $\Delta \log R_{\mathrm{e}}=0.04(<10 \%)$. A value of $Q=2$ leads to errors of $\Delta M_{\text {tot }}=0.15$ and $\Delta \log R_{\mathrm{e}}=0.1(<25 \%)$, respectively. A
Table 4.8: Error analysis for the FP of A 2390. The total error on the combination that enters the FP, $\delta \mathrm{FP}_{\text {phot }}=\log \delta R_{\mathrm{e}}-\beta \delta \mu_{\mathrm{e}}$ (see equation 4.3), is listed as $\delta \mathrm{FP}_{\text {phot }}$, adopting $\beta=0.328$. The final $\mathrm{FP}$ error in the rest-frame properties is given in the column $\delta \mathrm{FP}_{r}$.

\begin{tabular}{ccrcc}
\hline ID & $\delta \mu_{\mathrm{e}}$ & $\log \delta R_{\mathrm{e}}$ & $\delta \mathrm{FP}_{\mathrm{phot}}$ & $\delta \mathrm{FP}_{r}$ \\
\hline 2106 & 0.078 & -0.055 & 0.081 & 0.082 \\
2120 & 0.071 & -0.020 & 0.044 & 0.045 \\
2138 & 0.136 & 0.124 & 0.080 & 0.081 \\
2180 & 0.049 & -0.001 & 0.017 & 0.019 \\
2198 & 0.073 & -0.020 & 0.044 & 0.046 \\
2237 & 0.076 & 0.050 & 0.025 & 0.027 \\
2438 & 0.027 & -0.040 & 0.049 & 0.051 \\
2460 & 0.030 & -0.019 & 0.029 & 0.031 \\
2592 & 0.105 & 0.094 & 0.059 & 0.061 \\
2619 & 0.054 & -0.018 & 0.035 & 0.037 \\
2626 & 0.029 & -0.030 & 0.040 & 0.041 \\
2763 & 0.032 & -0.004 & 0.015 & 0.016 \\
2946 & 0.021 & -0.026 & 0.033 & 0.034 \\
6666 & 0.028 & -0.014 & 0.023 & 0.025 \\
\hline
\end{tabular}

quality parameter of $Q=3$ ensues errors of $\Delta M_{\text {tot }}=0.4$ and $\Delta \log R_{\mathrm{e}}=0.3$. Table 4.7 gives an overview of the quality parameter $Q$ measurements for the galaxies in A 2390, CL 0849, CL 1701 and CL 1702 which were revealed to be cluster members. For the galaxy in CL 0849 (ID \# 25) which has $Q=3$ and for the galaxies in CL 1701 \# 118, \# 124, \# 141 and \# 149 which have all $Q=2$, only low-resolution spectroscopy is available. For A 2390 it was found that $43 \%$ (6) of the galaxies have $Q=1,57 \%$ (8) galaxies have $Q=2$ and no galaxy yield a quality parameter of $Q=3$. The average error in $I_{814}$ was estimated to be $\approx 10 \%$ and the error in $R_{\mathrm{e}}$ of $<25 \%$.

Furthermore, for the error evaluation variations in the zero-point calibration of $\delta \mathrm{ZP}=0.03$ were accounted for (Saglia et al. 1997b), an uncertainty in galactic extinction $\delta \mathrm{E}(\mathrm{B}-\mathrm{V})=$ 0.010 , as well as errors in the $k$-correction of $\delta m_{r}=0.02^{m}$. The combination of the uncer- 
tainties on the measured parameters $\delta \mu_{\mathrm{e}}$ [in mag] and $\log \delta R_{\mathrm{e}}$ [in arcsec] according to equation 4.3 are listed as $\delta \mathrm{FP}_{\text {phot }}$. Adding the errors of galactic extinction correction, the k-correction and the errors in zero-point in quadrature ensues the final total error of the FP in the rest-frame Gunn $r$-band, $\delta \mathrm{FP}_{\mathrm{r}}$. A summary of the error analysis for the galaxies in A 2390 is given in Table 4.8.

\subsubsection{Rest-Frame Properties}

In the following, a description of the transformation of the measured surface brightnesses and effective radii on the HST images to rest-frame is given. Here, only a summary of the synthetic photometry is presented as it will be explained in more detail for the ground-based photometry in section 4.5.3.

At the cluster redshift of A 2390, the observed F $814 \mathrm{~W}$ passband is close to rest-frame Gunn $r_{\text {rest }}$. Therefore, it is more promising to use a single $k$-correction term of the observed $I_{814}$ to Gunn $r$ in rest-frame rather than to take a more complicated way in converting to $R_{C}$ first and then using a colour transformation. The latter procedure would increase the overall uncertainties.

Analysis of the rest-frame luminosities of the galaxies, was performed in the following way. According to Holtzman et al. (1995), the instrumental magnitude in a filter $X$ is given by:

$$
I_{X}=-2.5 \log (\mathrm{DN}) / t_{\mathrm{exp}}+Z P+2.5 \log (\mathrm{GR})
$$

where $X$ denotes the observed HST filter passband, either the F814W or F702W filter. GR represents the respective gain ratios for the $\mathrm{WF}$ chips and $Z P$ the zero-point for an exposure time $t_{\exp }=1 \mathrm{sec}$ with $Z P=20.986\left(I_{814}\right)$, $Z P=22.581\left(R_{702}\right)$, both with consideration of the difference between "short" and "long" exposures of $0.05 \mathrm{mag}$ (Hill et al. 1998) and an aperture correction of 0.0983 for the $I_{814}$ filter and of 0.1087 for the $R_{702}$ passband (Holtzman et al. 1995), respectively.
Since no ground-based $(V-I)$ colours are available for the cluster A 2390, a transformation from Cousins $I$ to Gunn $r$ as published in the literature (e.g., Ziegler et al. 2001a) can not be conducted. Instead, the transformation was performed in the following way. A spectral template for typical E/S0 galaxy by Kinney et al. (1996) was selected and was redshifted to the cluster redshift of $z=0.23$ to determine the flux through the $I_{814}$ filter and afterwards at $z=0$ through the Thuan \& Gunn (1976) $r$ filter (see section 4.5.3 for details). From this procedure the translation $I_{814 \text { obs }}-$ Gunn $r_{\text {rest }}=-0.75$ was derived. The result was checked by transforming the synthetic SEDs by Möller et al. (2001) and comparing the results to each other. Both approaches gave very similar results with the latter yielding only a slightly higher value of 0.02 mag. Typical transformations from the $R_{702}$ filter to Gunn $r$ for the Low $-L_{X}$ clusters using the E/S0 spectral template by Kinney et al. (1996) gave $R_{702 \text { obs }}-$ Gunn $r_{\text {rest }} \approx-0.25$.

In a next step, the rest-frame properties of the galaxies were derived. The mean surface brightness within $R_{\mathrm{e}}$ is defined as:

$\left\langle\mu_{r}\right\rangle_{\mathrm{e}}=r_{\text {rest }}+2.5 \log (2 \pi)+5 \log \left(R_{\mathrm{e}}\right)-10 \log (1+z)$,

where the parameter $r_{\text {rest }}$ denotes rest frame Gunn $r$ magnitude. The dimming due to the expansion of the Universe is corrected by the last term of this equation. The mean surface brightness $\langle I\rangle_{\mathrm{e}}$ in units of $\mathrm{L}_{\odot} / \mathrm{pc}^{2}$ is defined as

$$
\log \langle I\rangle_{\mathrm{e}}=-0.4\left(\left\langle\mu_{r}\right\rangle_{\mathrm{e}}-26.4\right)
$$

For each cluster, the angular distance $d(z)$ at a specific cluster redshift $z$ was calculated individually. This yields for A $2390(z=0.2280)$ $d=753.41 \mathrm{Mpc}$ and for CL $0849(z=0.2343)$ $d=769.06 \mathrm{Mpc}, \quad$ CL $1701 \quad(z=0.2458)$ $d=797.04 \mathrm{Mpc}, \quad$ CL $1702 \quad(z=0.2233)$ $d=741.60 \mathrm{Mpc}$, respectively. Using the angular distance, the angular scale as in units of $\mathrm{kpc} / \operatorname{arcsec}$ was determined and the effective ra- 
dius in kpc was computed for each galaxy as

$$
\log \left(R_{\mathrm{e}}\right)=\log \left(r_{\mathrm{e}}\right)+\log (a s),
$$

where $r_{\mathrm{e}}$ is the measured effective radius in arcseconds assuming the concordance cosmology. For Coma $(z=0.024)$ the angular distance is $d=99.90 \mathrm{Mpc}$.

\subsection{Morphologies}

One main aim of this work was to investigate possible differences in the properties of the two major morphological sub-classes of early-type galaxies, elliptical and S0 galaxies. Unfortunately, the resolution provided by the ground-based photometry was not sufficient for a morphological classification. However, for a sub-sample of early-type galaxies the acquired HST/WFPC2 and HST/ACS images offer a powerful alternative to perform a detailed investigation of the visual structure of individual galaxies.

In Edwin Hubble's original galaxy classification scheme (Hubble 1926), one of the main qualitative selection criteria was the bulge-to-disc $(B / D)$ ratio. As a combination of bulge and disc models measure a basic extension of the $B / D$ parameter, the bulge-to-total fraction $(B / T)$, it should be possible to correlate the bulge fraction with Hubble type. However, depending on the adopted bulge profile type (classical de Vaucouleurs law or Sérsic $r^{1 / n}$ model), the bulge fraction varies. A fundamental question still remains: Which profile gives the best correlation for galaxies spanning a range of different morphologies in the Hubble sequence?

Morphological studies of distant cluster galaxies with HST introduced a visual classification of galaxies onto the revised Hubble system (Abraham et al. 1996b; Smail et al. 1997; van Dokkum et al. 2000). Another approach is the application of automated techniques which allow a measurement of a quantitative morphology related to but distinct from the Hubble classification (Schade et al. 1995; Marleau \& Simard 1998; Simard et al. 2002). For the purpose of this work, both methods were chosen to yield the most reliable results.

Elliptical and S0 galaxies were morphologically classified in three independent ways. First, a visual classification was performed for a smaller sub-set of elliptical and lenticular galaxies (section 4.4.1). In addition, the results of the surface brightness profile fitting (section 4.3) provided a second verification of the visual structure and appearance for the galaxies. Thirdly, a general quantitative analysis using an automatic software algorithm (section 4.4.2) was conducted over the whole field covered by the HST images for a large sample of early-type and late-type galaxies. The information of all three independent approaches was combined to yield the best and most reliable decision on the final morphologies.

\subsubsection{Visual Classification}

A visual classification of the early-type galaxies was performed by four people separately but for different sample sizes. All galaxies were inspected by A. Fritz who additionally classified solely the field galaxies in the WHDF. For comparison, morphologies of the galaxies in the rich cluster A 2390 were obtained by I. Smail and for the Low $-L_{X}$ cluster galaxies by M. L. Balogh, whereas the field galaxies in the FDF were visually analysed by M. Pannella. The results were compared to each other and yielded a very good agreement with very similar morphologies. In all cases, differences were only between transition stages of one galaxy class, e.g. S0 or S0/Sa. But there were never discrepancies between a general galaxy type, i.e. an elliptical was never classified as an S0 or vice versa.

Thumbnail images of the cluster members and field galaxies are presented in Figs. 4.12 to 4.19. Objects were morphologically classified in two independent ways, based on visual inspection as well as fitting routine output. The findings from 
Table 4.9: Noteworthy remarks on individual HST galaxies in Abell 2390.

\begin{tabular}{lcl}
\hline ID & morp & comment \\
\hline 2120 & S0 & signs of isophotal twists out to outer isophote \\
2138 & E & very extended low-surface brightness (LSB) environment \\
2198 & E & close to a bright neighbour \\
2237 & Sa & [O III] 5007 and H $\beta$ emission, asymmetric LSB extension \\
2460 & E & lensed?, very close to S0 \\
2592 & E & next to giant arc \\
2763 & E & slight twisted low SB \\
2946 & S0 & small companion \\
2933 & Sbc & no member, no structural analysis, weird spiral structure, no interaction \\
\hline
\end{tabular}

the luminosity profile fitting provided a consistency check, which resulted in the same classification scheme except for four objects. In the cluster A 2390, \#2180: E (visual), S0 (fit) and \# 2763: S0/Sa (visual), E (fit), in CL 0849, \# A9: S0 (visual), E (fit) and for one field galaxy in the WHDF, \# 810b: S0 (visual), E (fit). Objects which are best described with an $r^{1 / 4}$-law are classified as an elliptical (E). Those galaxies for which an additional exponential component yields in a slightly improved fit (without a dramatic change in structural properties) are classified as E/S0. S0 galaxies are best approximated by a combination of an $r^{1 / 4}$-law plus an exponential profile.

Remarks on special features on individual HST objects of Abell 2390 are listed in Table 4.9. Comments on the Low $-L_{X}$ cluster CL 0849 can be found in Table 4.10, on the cluster CL 1701 in Table 4.11 and on the cluster CL 1702 in Table 4.12. Morphologies and notes for all field galaxies in the FDF and in the WHDF are listed in Table 4.13 and in Table 4.14, respectively. The first column of each table shows the galaxy ID, the second indicates the morphology of the objects and the last one the respective comments. The central $\mathrm{cD}$ galaxy of a galaxy cluster (i.e. the the Brightest Cluster Galaxy BCG) is denoted as EcD. In the third column, additional information about special features regarding to the galaxy is given. Some notations and abbreviations which will be used in the following Figures 4.12 to 4.19 and Tables 4.9 to 4.14 are: background (bg), interaction (int), merger (m), object (obj) and weird (w). Anonymous galaxies, which are either no cluster members or without any spectroscopic information, are classified with an additional letter "A". For some anonymous galaxies intermediate-resolution spectra have been acquired but they are field galaxies, in CL 1701 the galaxies \# A77 and \# A101 and in CL 1702 the object \# A96. 

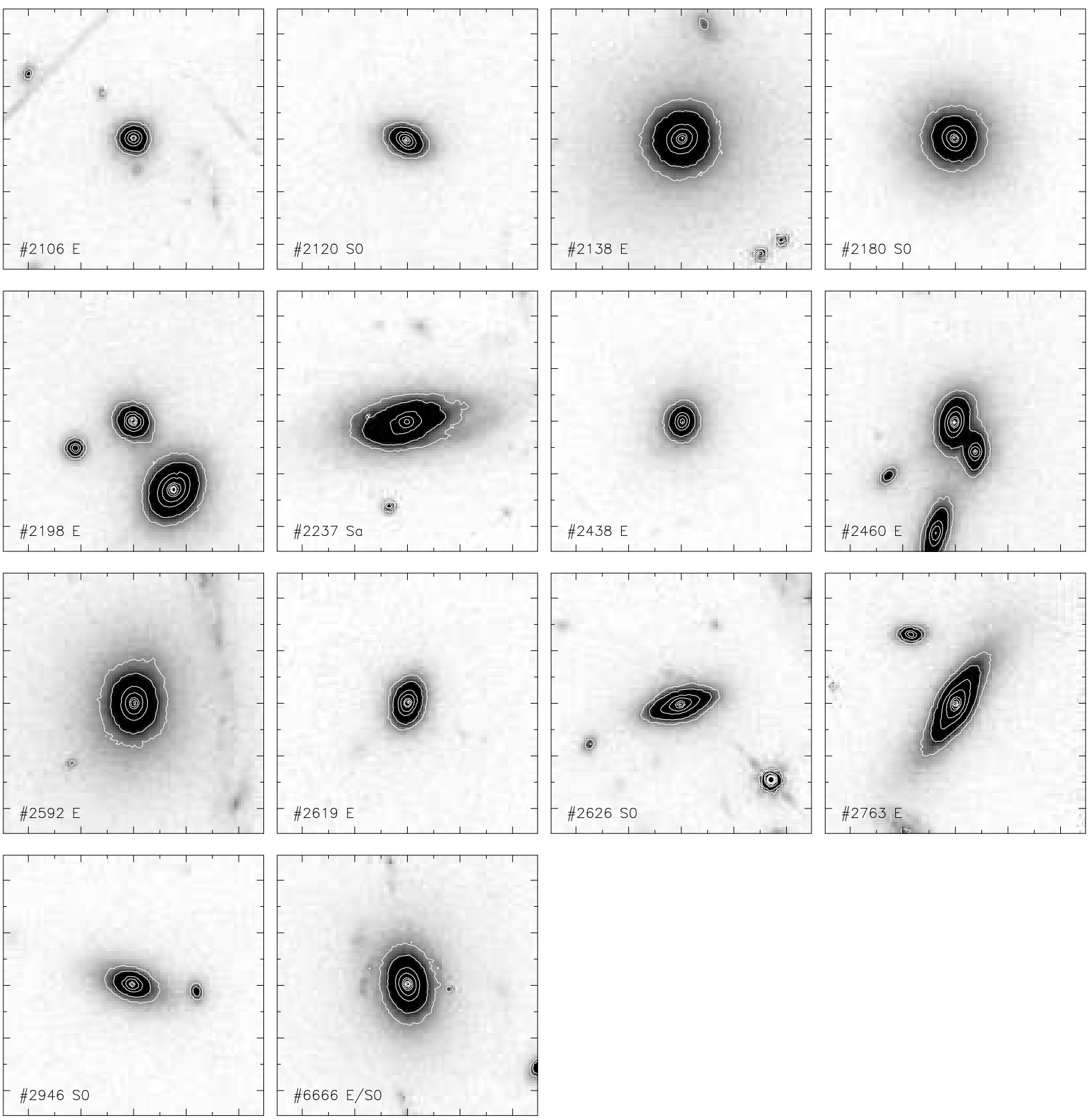

Figure 4.12: $10^{\prime \prime} \times 10^{\prime \prime} \mathrm{HST} / \mathrm{WFPC} 2$ images of 14 galaxies with available spectroscopic information which fall within the WFPC2 mosaic of A 2390. The labels give the galaxy ID and the visual morphology as listed in Table 4.9. North is up and East to the left. The colour coding ranges from white (sky-background at $\left.\sim 26.30 \mathrm{mag} \operatorname{arcsec}^{-2}\right)$ to black $\left(\leq 23.11 \mathrm{mag} \operatorname{arcsec}^{-2}\right)$. Isophotal contours are indicated between the range $22.00 \leq C_{i} \leq 24.50 \mathrm{mag} \operatorname{arcsec}^{-2}$, in increments of 0.5 magnitudes. 

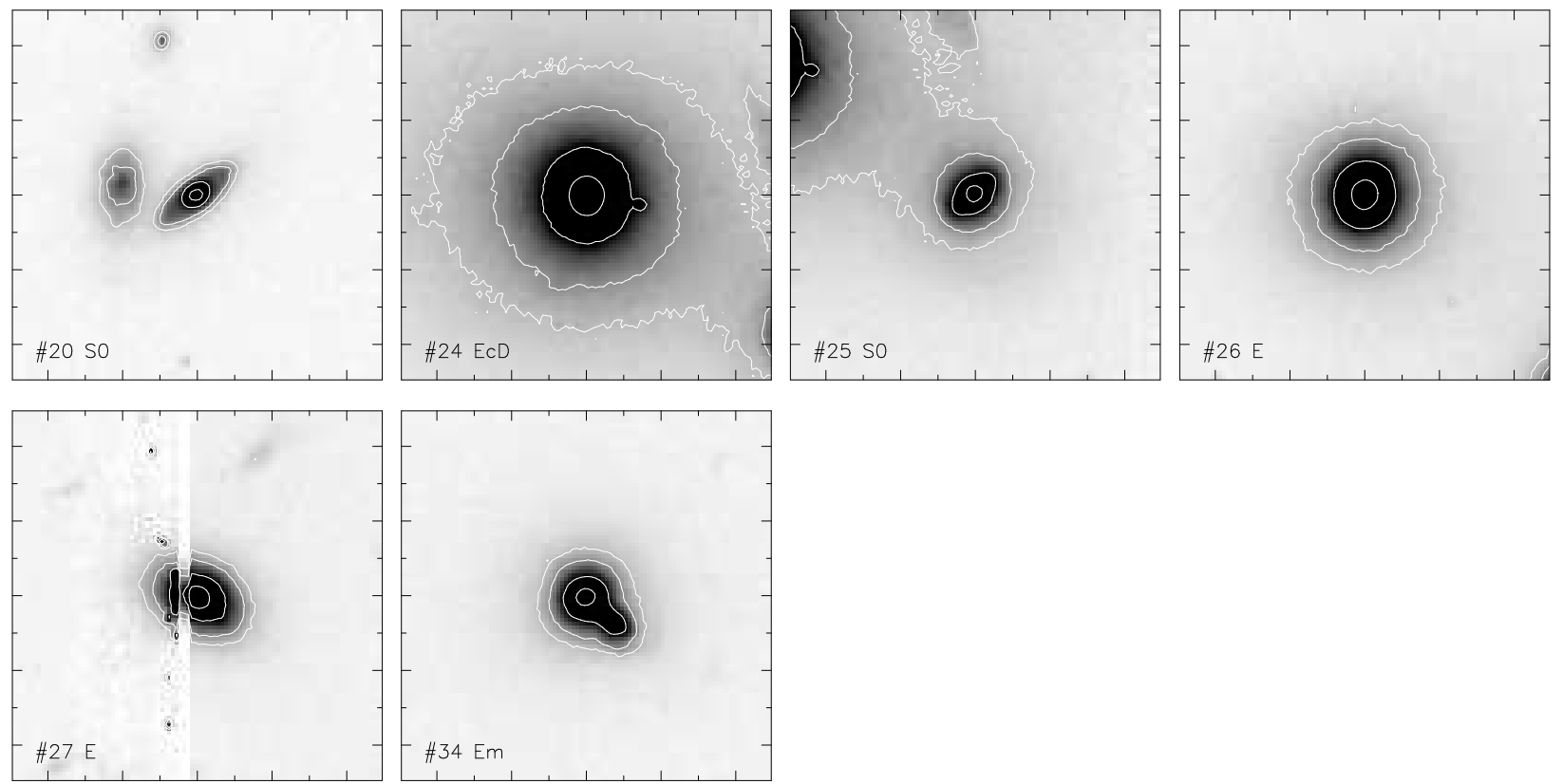

Figure 4.13: $10^{\prime \prime} \times 10^{\prime \prime} \mathrm{HST} / \mathrm{WFPC} 2 \mathrm{~F} 702 \mathrm{~W}$ images of spectroscopic confirmed cluster galaxies of CL 0849 which fall within the WFPC2 mosaic. The labels give the galaxy ID and the visual morphology as listed in Table 4.10. Galaxy \# 25 is a cluster member based on its low-resolution spectrum and galaxy \# 27 falls between two chips. East is up and north to the right.
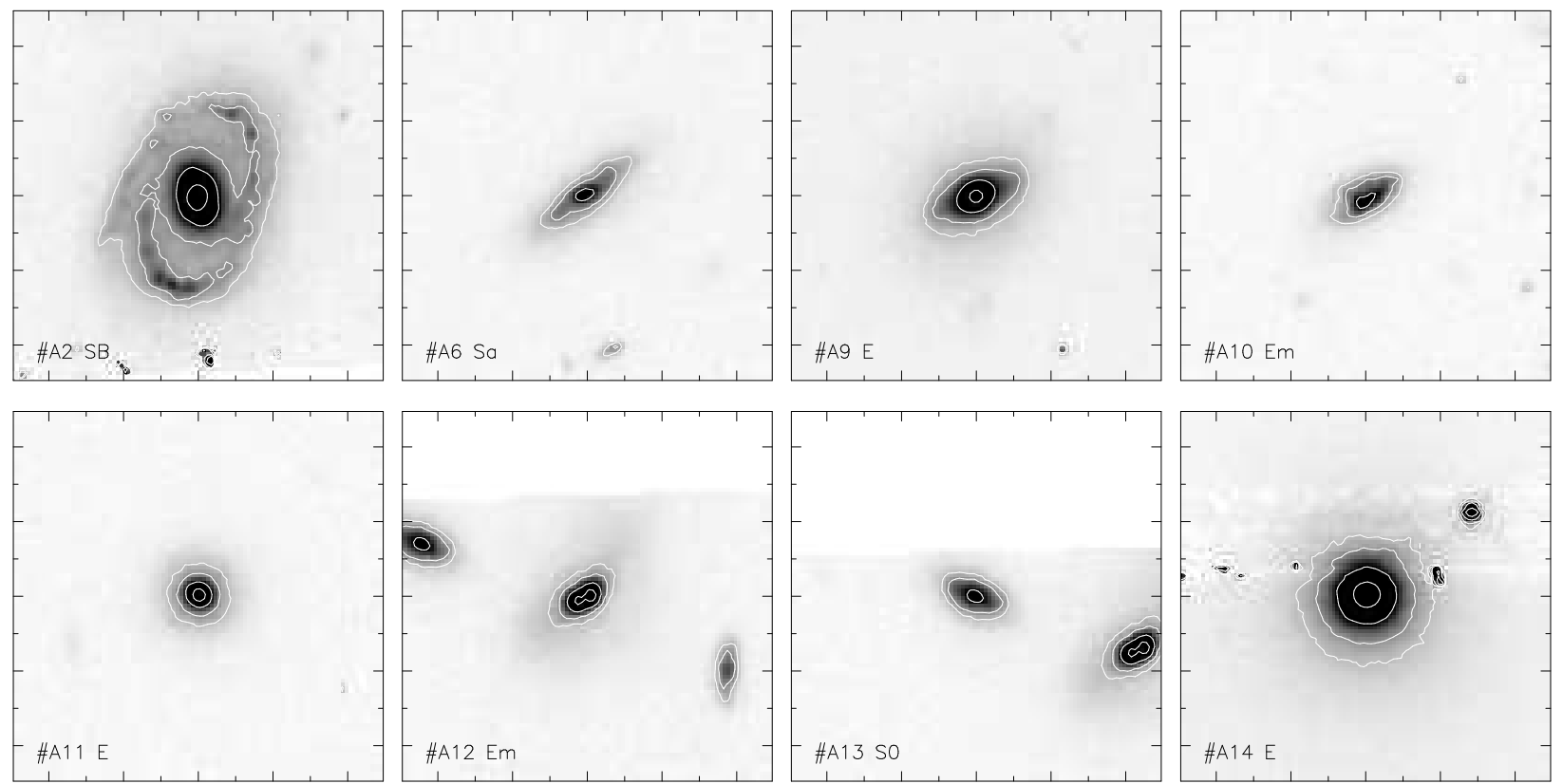

Figure 4.14: $10^{\prime \prime} \times 10^{\prime \prime} \mathrm{HST} / \mathrm{WFPC} 2 \mathrm{~F} 702 \mathrm{~W}$ images of 8 anonymous galaxies classified as "A" which fall within the WFPC2 mosaic of CL 0849. The labels give the galaxy ID and the visual morphology as listed in Table 4.10. East is up and north to the right. 
Table 4.10: Noteworthy remarks on individual HST galaxies in CL 0849.

\begin{tabular}{lcl}
\hline ID & morp & comment \\
\hline \# A2 & SB & 2 stars at top \\
\# A10 & Em & merger \\
$\#$ A12 & Em & merger \\
$\#$ A14 & E & between WF3 \& WF4 chip \\
$\# 24$ & EcD & star left \\
$\# 27$ & E & between WF2 \& WF3 chip \\
$\# 34$ & Em & merger, no SF in spectrum \\
\hline
\end{tabular}

Table 4.11: Noteworthy remarks on individual $H S T$ galaxies in CL 1701.

\begin{tabular}{lcl}
\hline ID & morp & comment \\
\hline \# A6 & Ew & isophotal twists, merger?, or bg spiral, faint \\
\# A7 & S0 & close to chip edge, between WF2 \& WF3 chip \\
\# A17 & E & between WF3 \& WF4 chip \\
$\# 62$ & E & small companion, LSB environment \\
$\# 120$ & S0 & LSB environment \\
$\# 123$ & EcDm & double nucleus and star close by \\
$\# 124$ & SB0 & lensed obj in lower right corner?, spiral arms, no int with \#123 \\
$\# 141$ & SBa & 2. bg gal at top, no int \\
$\# 149$ & SBm & SB merger with E \\
\hline
\end{tabular}

Table 4.12: Noteworthy remarks on individual HST galaxies in CL 1702.

\begin{tabular}{lcl}
\hline ID & morp & comment \\
\hline$\#$ A2 & E & star close by \\
$\#$ A4 & Sm & merger, weird structure \\
$\#$ A10 & Sa & close to chip edge \\
$\# 81$ & EcD & star close by \\
$\# 96$ & S0 & merger? \\
\hline
\end{tabular}



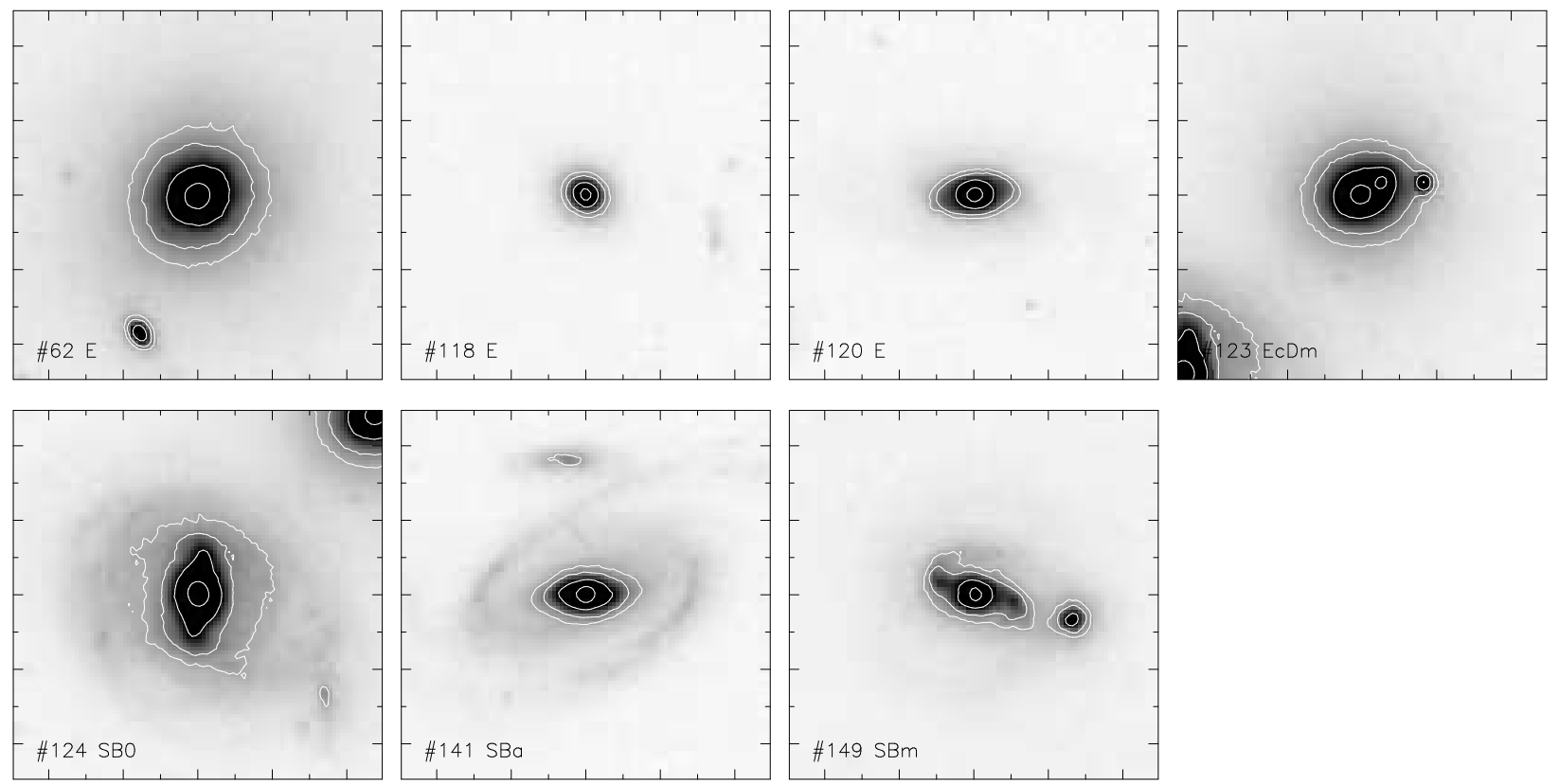

Figure 4.15: $10^{\prime \prime} \times 10^{\prime \prime}$ HST/WFPC2 F702W images of 7 spectroscopic confirmed cluster galaxies of CL 1701 which fall within the WFPC2 mosaic. The labels give the galaxy ID and the visual morphology as listed in Table 4.11. For the galaxies \# 118, \# 124, \# 141 and \# 149 cluster membership is based on low-resolution spectra. East is up and north to the right. 

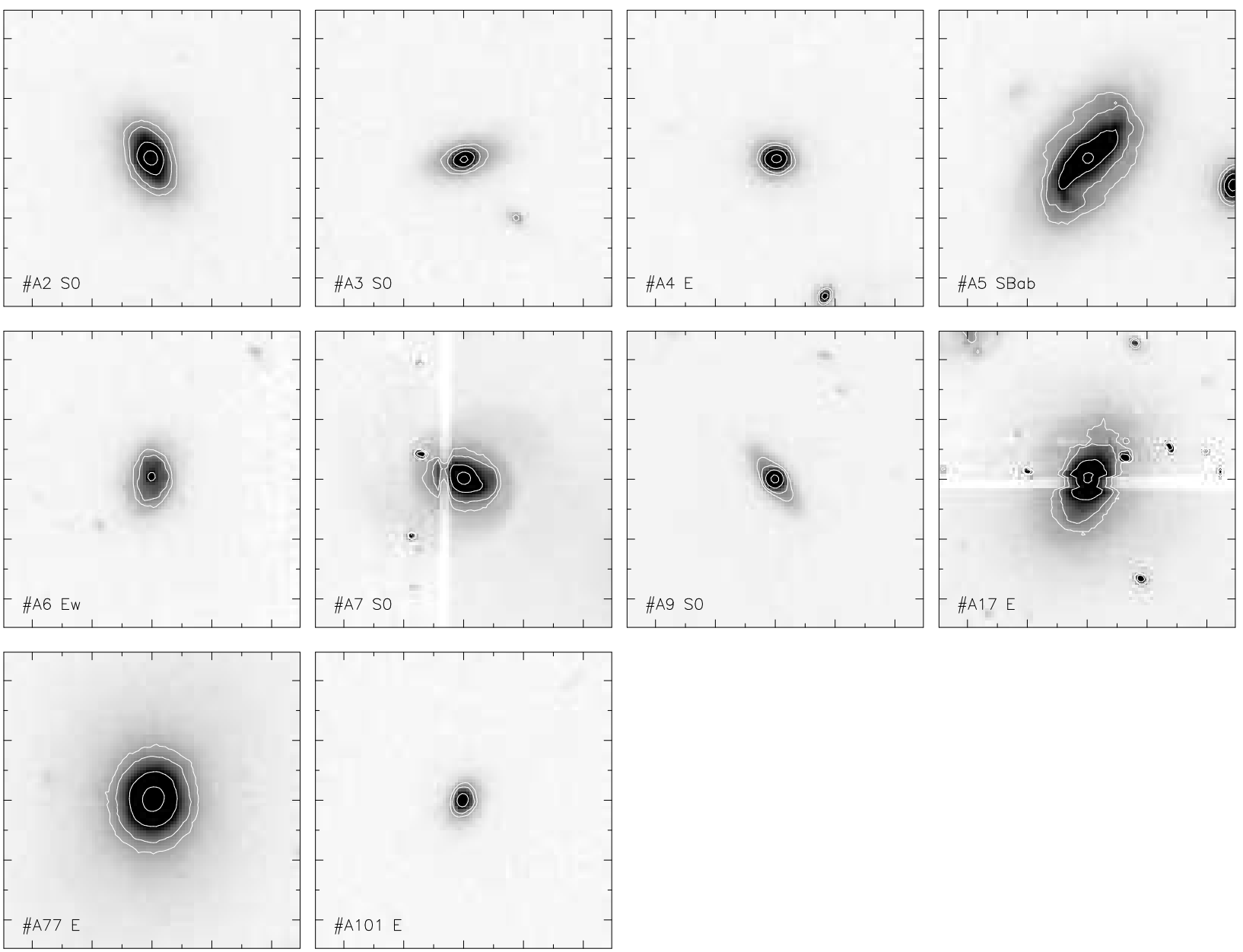

Figure 4.16: $10^{\prime \prime} \times 10^{\prime \prime} \mathrm{HST} / \mathrm{WFPC} 2 \mathrm{~F} 702 \mathrm{~W}$ images of 10 anonymous galaxies classified as "A" which fall within the WFPC2 mosaic of CL 1701. The labels give the galaxy ID and the visual morphology as listed in Table 4.11. For the galaxies \# A77 and \# A101 spectra are available but they are not cluster members. East is up and north to the right. 

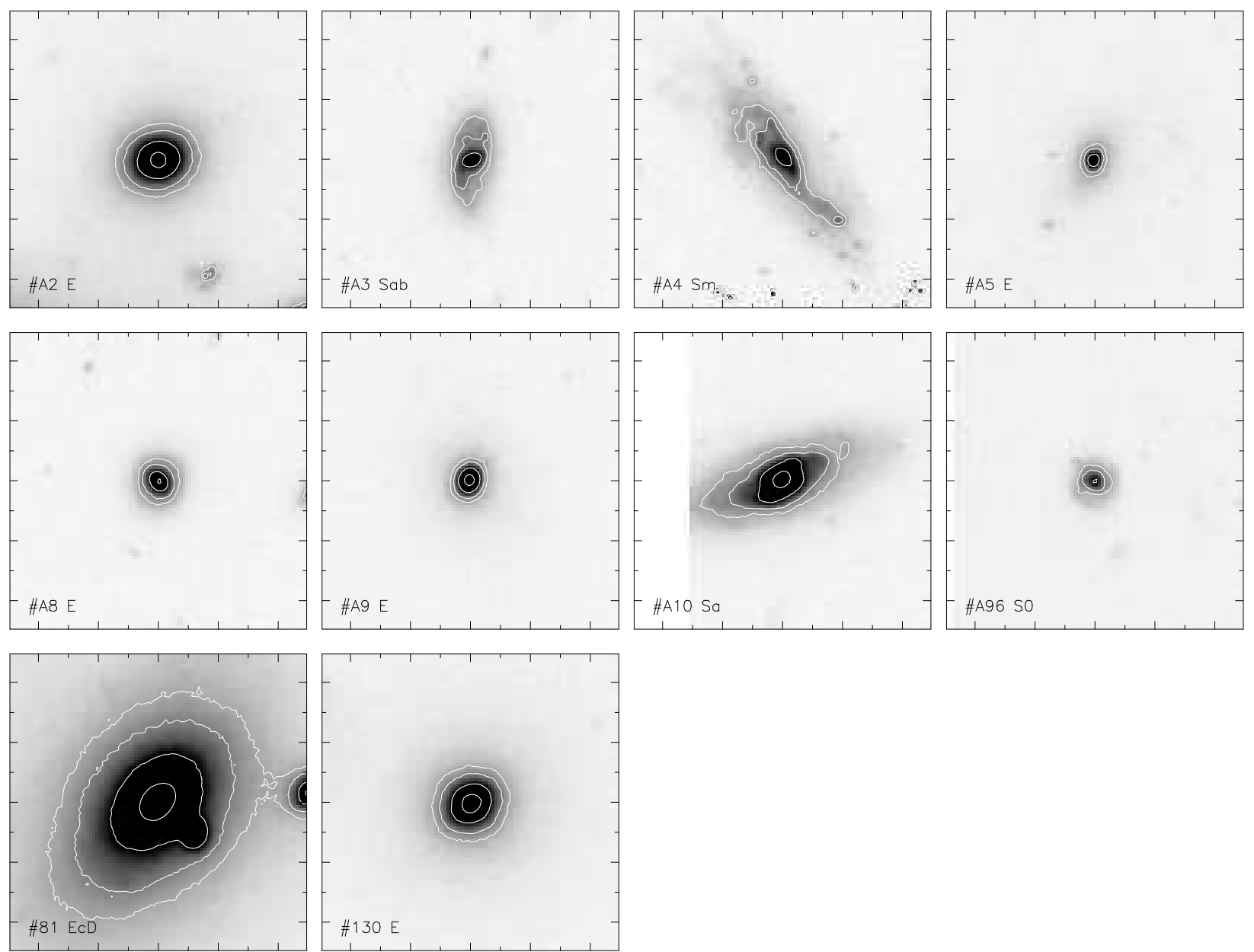

Figure 4.17: $10^{\prime \prime} \times 10^{\prime \prime}$ HST/WFPC2 F702W images of 2 spectroscopic confirmed cluster galaxies of CL 1702 . Galaxy \# A96 is not a cluster member based on its intermediate-resolution spectrum. The labels give the galaxy ID and the visual morphology as listed in Table 4.12. Anonymous galaxies are classified as "A". East is up and north to the right. 

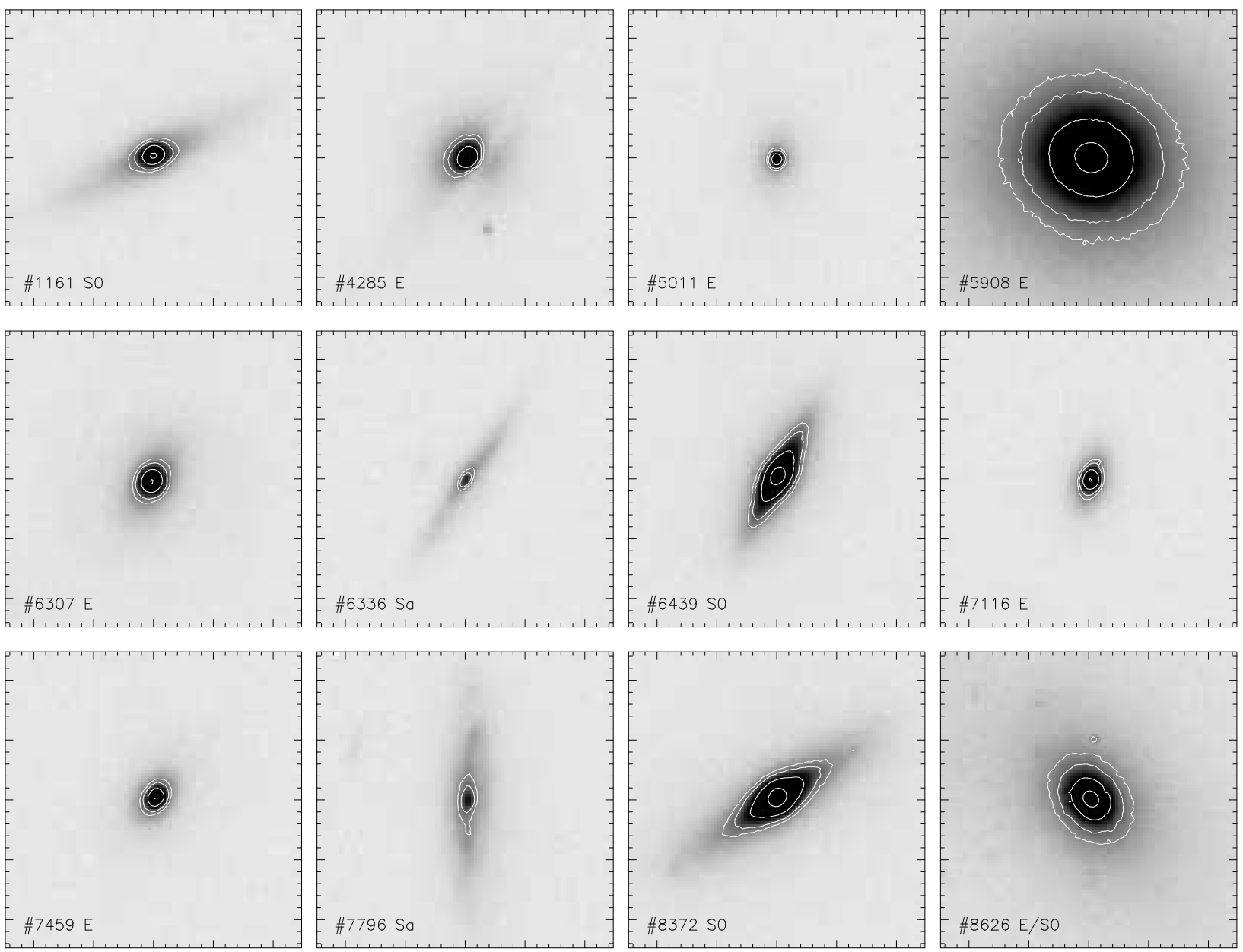

Figure 4.18: $5^{\prime \prime} \times 5^{\prime \prime}$ HST/ACS F814W images of 12 FDF galaxies with available spectroscopic information. The labels give the galaxy ID and the visual morphology as listed in Table 4.13. 

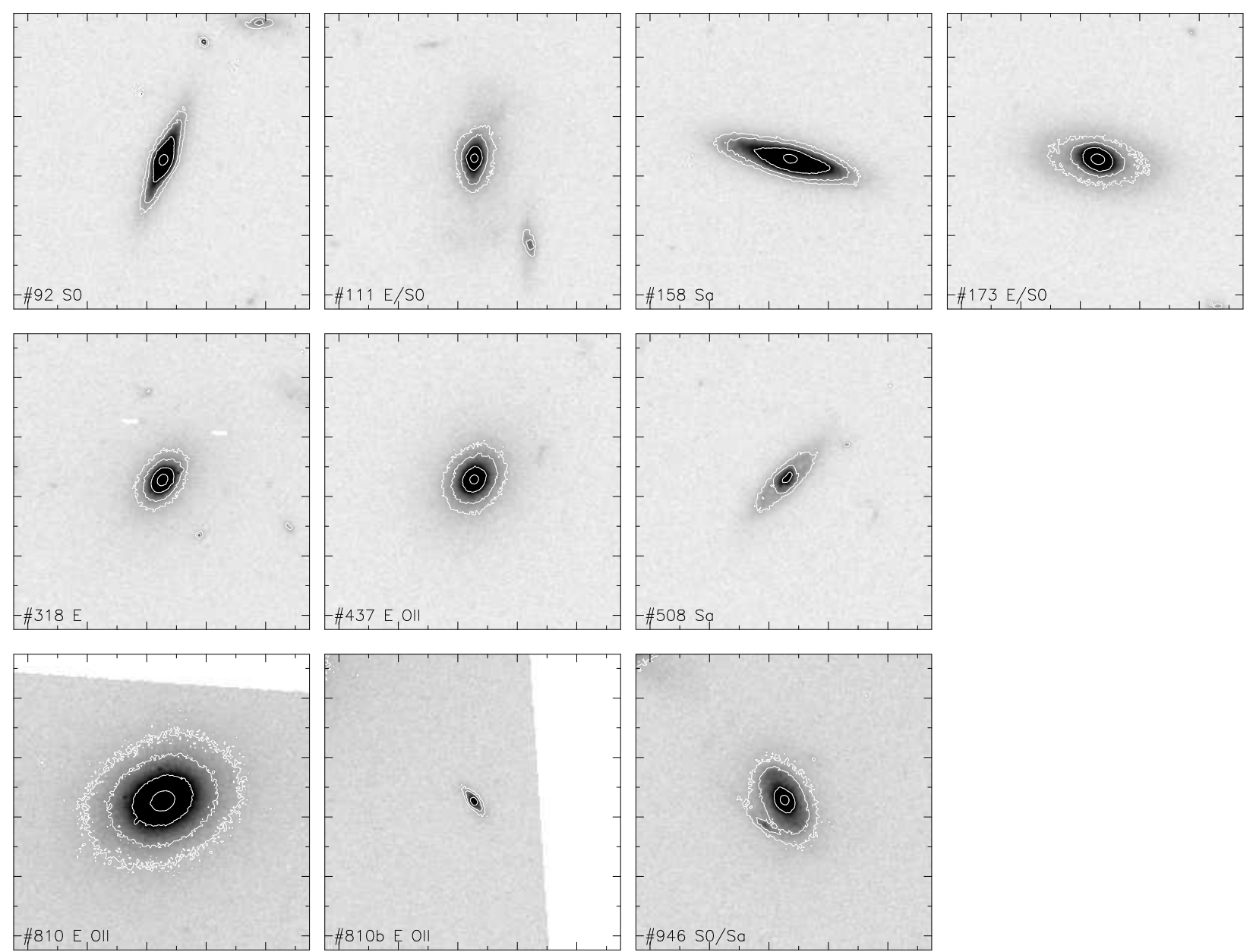

Figure 4.19: $10^{\prime \prime} \times 10^{\prime \prime} \mathrm{HST} / \mathrm{ACS}$ F814W images of $10 \mathrm{WHDF}$ galaxies with available spectroscopic information. South is up and east is to the left. The labels give the galaxy ID and the visual morphology as listed in Table 4.14 . 
Table 4.13: Morphologies and noteworthy remarks on individual HST/ACS FDF galaxies.

\begin{tabular}{lcl}
\hline ID & morp & comment \\
\hline 1161 & S0 & \\
4285 & E & gal with dust lane, (merger of S0 \& S with dust lane?) \\
5011 & E & \\
5908 & E & \\
6307 & E & model too boxy, residuum at core \\
6336 & Sa & Sb?, weird structure, warps \\
6338 & S & no ACS image, no analysis, morph based on spec \\
6439 & S0 & \\
7116 & E & hot pixel \\
7459 & E & no analysis \\
7796 & Sa & weird structure, SF in upper spiral arm?, warps \\
8372 & S0 & hot pixel \\
8626 & E/S0 & close to edge of chip \\
\hline
\end{tabular}

Table 4.14: Morphologies and noteworthy remarks on individual HST/ACS WHDF galaxies.

\begin{tabular}{ccl}
\hline ID & morp & comment \\
\hline 92 & S0 & 2 bg? obj in north \\
111 & E/S0 & external halo in upper north part, 2 obj in north-west \\
158 & Sa & \\
173 & S0 & \\
318 & E & close to star, star in center \\
437 & $\mathrm{E}$ & face on, faint star in north-east, [O II] 3727 emission \\
508 & Sa & weird, dust pattern in south-west?, rotation?, [O II] 3727 emission?, star in west \\
749 & E/S0 & no ACS image, no analysis, morph based on spec \\
810 & E & boxy isophotes, stars in center, [O II] 3727 emission \\
$810 b$ & E & discy structure, faint, [O II] 3727 emission \\
946 & S0/Sa & SB0?, spiral arm in north-east, int with obj in south-east? \\
\hline
\end{tabular}




\subsubsection{Quantitative Analysis}

A very popular and often used algorithm for analysing two-dimensional surface brightness profiles of galaxies is the Galaxy IMage TwoDimensional (GIM2D, see Simard et al. 2002) package which operates within the IRAF environment ${ }^{5}$. GIM2D is a software package in Subset Pre-Processor (SPP) language using various procedure calls of the IRAF operating system to perform detailed bulge and disc decompositions of low signal-to-noise images of distant galaxies. The program derives a maximum of up to 12 different structural parameters for each input galaxy.

A detailed morphological analysis for the Low$L_{X}$ cluster sample down to $R_{702}=23.0^{m}$ was performed with the GIM2D software algorithm on the HST/F702W images within our collaboration (Balogh et al. 2002a). For the central $\sim 0.6 \mathrm{Mpc}$ of these clusters which are covered by the HST imaging, a large fraction of discdominated galaxies (36\%) with low $B / T \sim 0$ was found, whereas high X-ray-luminous galaxy clusters are characterised by galaxies with intermediate bulge-to-total fractions of $B / T \sim 0.4$. However, only $18 \pm 5 \%$ of these Low $-L_{X}$ galaxy populations were revealed to be spectroscopically confirmed cluster members brighter than $R_{702}=20.0^{m}$.

To ensure a uniform measurement of visual appearances and to quantify the results of the morphological visual classification between rich and poor clusters, two verifications have been employed. A first consistency check of $B / T$ measurements between different filters was performed. In addition, the correlation between $B / T$ and visual morphological type was established. In the following, these verifications are discussed in detail.

In November 2004, the author initiated a project with M. Pannella and Dr. R. P. Saglia (both MPE/Munich) to investigate in detail galaxy

\footnotetext{
${ }^{5}$ GIM2D: http://www.hia-iha.nrc-cnrc.gc.cal $\mathrm{STAFF} \backslash 1 \mathrm{sd} \backslash$ gim $2 \mathrm{~d} \backslash$
}

structural parameters using various combinations of surface brightness profiles and different fitting techniques. A comparison of these independent methods will reveal how the colour gradients depend on the computed structural parameters. Structural properties of an initial sample of 16 early-type galaxies in A 2218 in the HST/ACS BVI filters were analysed using a combination between a classical de Vaucouleurs $\left(r^{1 / 4}\right)$ bulge with an exponential disc component in GIM2D. In addition, the same galaxies were investigated with the algorithm by Saglia et al. (1997a) on the HST/WFPC2 BVI images. Both approaches utilised the same luminosity profile type $\left(r^{1 / 4}+\right.$ exp. disc $)$ which was applied to model the surface brightness distribution of the galaxies (see section 4.3.2). For this reason, the GIM2D and Saglia et al. (1997a) parameters could be directly compared to the results of surface brightness modelling. Through a cross-correlation of all three filter passbands, the $B / T$ ratios for these galaxies in the cluster A 2218 were derived. In Fig. 4.20, the bulgeto-total ratios for the 16 early-type cluster galaxies of A 2218 down to a magnitude limit of $I_{814}=21.20^{m}$ are shown. Note that all earlytype galaxies are spectroscopically verified cluster members, which were selected based upon the study of Ziegler et al. (2001a). The E+S0 cluster galaxies of A 2218 cover a range in apparent magnitudes of $15.68<R_{702}<18.68$ with a median value of $\left\langle R_{702}\right\rangle=17.67$. The dotted line in Fig. 4.20 indicates the adopted break between bulge $B / T \geq 0.4$ and disc $B / T<0.4$ dominated galaxies.

From Fig. 4.20 it is clearly visible that these galaxies are bulge-dominated $(B / T \geq 0.4)$ systems with a median in the $I_{814}$ filter of $\left\langle B / T_{814}\right\rangle=0.69$. Early-type galaxies encompass sub-classes from elliptical over S0 to Sa, splitted into $8 \mathrm{E}, 1 \mathrm{E} / \mathrm{S} 0,4 \mathrm{~S} 0,2 \mathrm{SB} 0 / \mathrm{a}$ and $1 \mathrm{Sa}$. Apart from one galaxy which was not included on the WFPC2 frames (\# 2738 which is an elliptical galaxy based on ground-based Hale $I$ image 


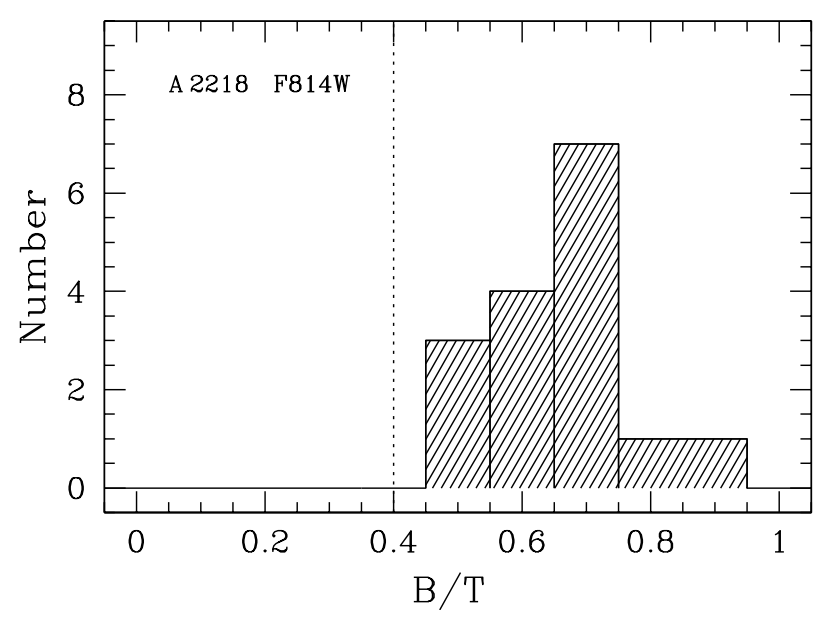

Figure 4.20: Bulge-to-total ratios of 16 earlytype cluster galaxies brighter than $R_{702}=18.68^{m}$ in A 2218. The dotted line denotes the adopted break between bulge $B / T \geq 0.4$ and disc $B / T<0.4$ dominated galaxies. All early-type cluster galaxies are bulge-dominated systems.

of A 2218), all galaxies were analysed via the FP, see discussion in section 6.4. For the $V_{606}$ and $B_{450}$ similar values to the $I_{814}$ filter are derived $\left\langle B / T_{606}\right\rangle=0.74$ and $\left\langle B / T_{450}\right\rangle=0.61$. Comparing the $B / T$ measurements and other structural parameters in all three filter passbands, only slight differences in their general distributions are found. In particular, the results in $V_{606}$ and $I_{814}$ are in very good agreement and the distributions do not dependent strongly on the assumed filter. The uncertainties in the $B / T$ measurements were assessed through Monte Carlo simulations in the GIM2D software. Typical mean errors in the $B / T$ ratios are between 0.04 to 0.07 with a median value of only 0.02 between all $B V I$ filter passbands. The $B / T$ ratios for the A 2218 galaxies are also in good agreement with the GIM2D $B / T$ determinations of A 2218 in the $R_{702}$ passband by Balogh et al. (2002a). For galaxies down to a magnitude limit of $R_{702}=22.1^{\mathrm{m}}$ within the HST image, these authors derive a mean of $\langle B / T\rangle \sim 0.60$. To account for contamination by field galaxies, this study performed a statistical background correction, very simi-

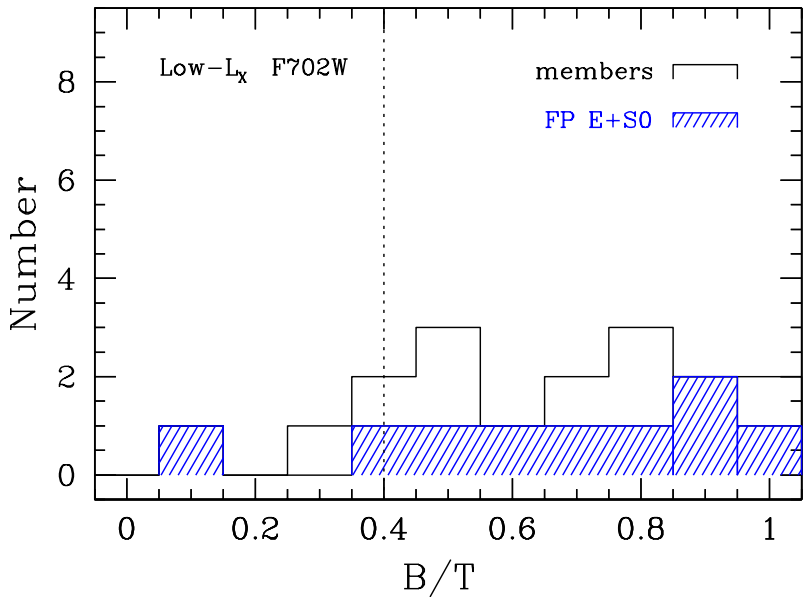

Figure 4.21: Bulge-to-total ratios of 26 earlytype cluster members brighter than $R_{702}=19.48^{m}$ in three Low $-L_{X}$ clusters. The shaded histogram gives the distribution of early-type cluster members which were selected for the FP study.

lar to the method as described in section 2.1.5. Although the galaxies were not selected upon photometric or spectroscopic membership, the $B / T$ distribution is very similar to the one in Fig. 4.20.

Fig. 4.21 displays the bulge-to-total ratios of early-type cluster members brighter than $R_{702}=19.48^{m}$ in the three Low $-L_{X}$ clusters CL 0849, CL 1701 and CL 1702. This analysis is restricted to spectroscopic verified cluster galaxies with available $B / T$ measurements in the $R_{702}$ filter only. One sample contains 17 cluster members based on the low-resolution study by Balogh et al. (2002b), which are indicated by the open $B / T$ distribution in Fig. 4.21. A sample of 9 Low $-L_{X}$ cluster galaxies (for the galaxy \# 130 no $B / T$ could be derived), which were selected for an investigation within the FP, are denoted by the shaded $B / T$ histogram. The Low $-L_{X}$ FP galaxies show a spread in apparent magnitudes of $16.12<R_{702}<19.48\left(\left\langle R_{702}\right\rangle=18.15\right)$. The $B / T$ distribution of these galaxies is characterised by higher $B / T$ ratios with a median of $\langle B / T\rangle=0.71$ than the low-resolution sample with $\langle B / T\rangle=0.65$. This is to be expected, as 


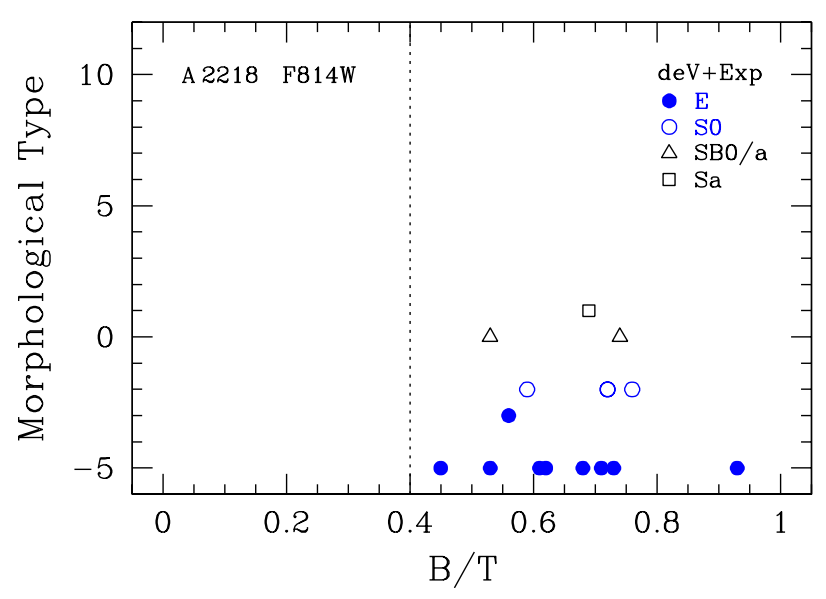

Figure 4.22: Bulge-to-total fractions as a function of visual morphological Hubble type of 16 earlytype cluster members brighter than $R_{702}=18.68^{\mathrm{m}}$ in A 2218. $B / T$ is derived using a de Vaucouleurs bulge plus an exponential disc. The dotted line denotes the adopted break between bulge $B / T \geq 0.4$ and disc $B / T<0.4$ dominated galaxies. The derived $B / T$ corresponds well to visual morphologies.

the FP sample was selected to have early-type morphologies yielding on average to higher $B / T$ ratios. The outlier galaxy \# 923 has the lowest $B / T$ ratio with $B / T=0.12$, which is the secondary galaxy of an ongoing merger in the $\mathrm{cD}$ galaxy \# 123 of the cluster CL 1701 . As the $B / T$ values and their distributions in different filters $\left(B_{450}, V_{606}\right.$ and $\left.I_{814}\right)$ yield consistent results and no strong variations were found, a comparison with the $B / T$ ratios for the Low $-L_{X}$ clusters in the $R_{702}$ filter is allowed.

In comparison to the distribution of rich cluster galaxies of A 2218 in Fig. 4.20, the B/T measurements of the Low $-L_{X}$ clusters are slightly differently distributed. The poor cluster galaxies show a broader distribution and more disc-dominated galaxies with lower $B / T$ ratios in the $R_{702}$ band. This first analysis confirms that the Low $-L_{X} \mathrm{FP}$ sample is restricted to bulge-dominated galaxies and that the same class of early-type galaxies will be compared when analysing the FP for rich and poor clusters.

To test in even greater detail if the $B / T$ distri-

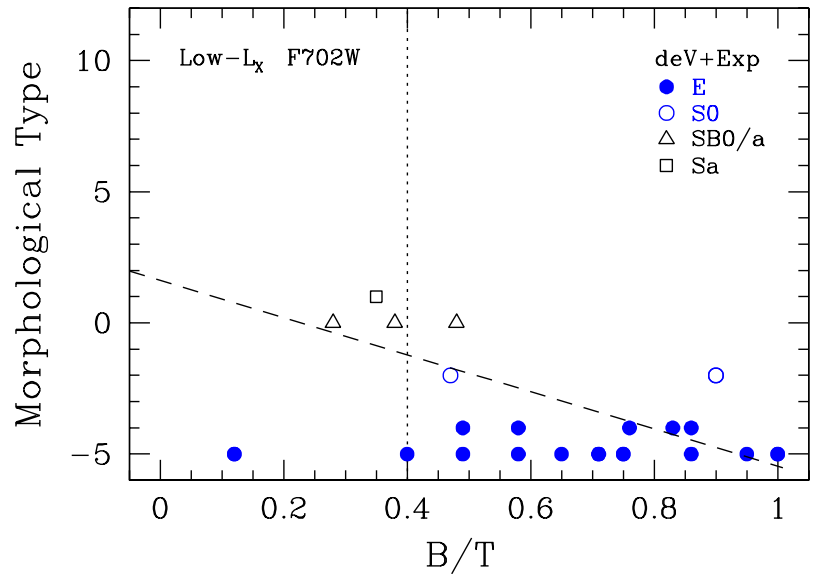

Figure 4.23: Bulge-to-total fractions as a function of visual morphological Hubble type of 21 earlytype cluster members brighter than $R_{702}=19.48^{m}$ in three Low $-L_{X}$ clusters. $B / T$ is derived using a de Vaucouleurs bulge plus an exponential disc. The dotted line denotes the adopted break between bulge $B / T \geq 0.4$ and disc $B / T<0.4$ dominated galaxies, the dashed line is a least-squares fit to all galaxies with $B / T>0.2$. The derived $B / T$ corresponds well to visual morphologies.

butions comprise the same populations of bulgedominated galaxies and to separate between the classes of elliptical and S0 galaxies, a correlation between measured $B / T$ and visual classified morphology was derived. Fig. 4.22 shows the $B / T$ fractions as a function of visual morphological type for 16 early-type cluster members brighter than $R_{702}=18.68^{m}$ in A 2218. In Fig. $4.23, B / T$ versus visual morphological type is displayed for the Low $-L_{X}$ sample. All galaxies were classified according to the de Vaucouleurs' classification of the revised Hubble scheme (de Vaucouleurs 1959). Visual morphological types are represented as $\mathrm{E}(-5), \mathrm{cD}(-4), \mathrm{E} / \mathrm{S} 0(-3)$, S0 (-2), SB0/a or SB (0) and Sa (1). The dashed line is a least-squares fit to all galaxies with $B / T>0.2, T=-7.09 \cdot B / T+1.63$, with $T$ being the morphological Hubble type. In Figs. 4.22 and 4.23, elliptical and S0s galaxies are denoted as filled and open circles, while early-type spiral galaxies of SB0/a and Sa are indicated as trian- 
gles and squares. The adopted break between bulge $(B / T \geq 0.4)$ and disc $(B / T<0.4)$ dominated galaxies is visualised by the dotted line. There is a large scatter and only a weak trend between the $B / T$ and visual type for the de Vaucouleurs bulge+exponential disc. An explanation is that the SB0/a and Sa types have a strong bulge component and therefore a high $B / T$ ratio. With the de Vaucouleurs bulge+exponential disc profile, for the Low $-L_{X} \mathrm{E}+\mathrm{S} 0$ galaxies a weak correlation between the $B / T$ and and visual morphological type is found, albeit with a large scatter. The trend between de Vaucouleurs bulge+exponential disc and type is not surprising. First, the surface brightness distribution of bright ellipticals and the bulges of early-type spirals are well-fitted by a de Vaucouleurs profile (Andredakis et al. 1995). Second, the majority of cluster galaxies are bulge-dominated systems. The lack of a stronger correlation between $B / T$ with visual classified morphology in Figs. 4.22 and 4.23 is partly due to a low number of spiral galaxies in both galaxy samples. However, apart from the found trend, there is a large scatter in the distribution of $B / T$ ratio versus visual type and it is difficult to distinguish between earlytype spirals from elliptical and S0 galaxies using the $B / T$ parameter alone.

In the next section details on the derivation of the absolute magnitudes will be given.

\subsection{Luminosity Derivation}

Rest-frame absolute magnitudes were calculated from the deep ground-based multi-band imaging data. For each project, ground-based imaging in various filter passbands was acquired which turned out to be especially beneficial for the field studies in the FDF and WHDF. The apparent brightness of a given field galaxy was derived from the filter $X$ which best matched the $B$-band response curve in rest-frame. According to the redshift of the respective galaxy, this filter $X$ was either the $B-, g_{-}, R$ - or $I$-band (see section 4.5.3).
For the E+S0 galaxies the adopted equation for the computation of absolute $Y$ magnitudes was

$$
\begin{aligned}
M_{Y}= & m_{X}-A_{X}-D M_{\Lambda}\left(z, H_{0}\right) \\
& -k_{Y}(X, T, z)
\end{aligned}
$$

where $M_{Y}$ denotes either the absolute $B$ magnitude (field galaxies) or absolute Gunn $r$ magnitude (cluster galaxies).

$m_{X}$ is the apparent total brightness in the passband $X$ as derived with the Source Extractor (see section 4.5.1).

$A_{X}$ is the Galactic absorption in the wavelength regime of filter $X$ (see section 4.5.2).

$D M_{\Lambda}\left(z, H_{0}\right)$ is the distance modulus for the respective redshift of a cluster or field object. As already stated earlier, throughout this thesis the so-called concordance cosmology (e.g., Spergel et al. 2003) is adopted, i.e. a flat universe with a matter density $\Omega_{m}=0.3$, a cosmological constant corresponding to an energy density of $\Omega_{\Lambda}=0.7$ and a Hubble constant of $H_{0}=70 \mathrm{~km} \mathrm{~s}^{-1} \mathrm{Mpc}^{-1}$. In most previous studies also a non-zero cosmological constant was assumed. Possible deviations caused due to different values of $H_{0}$ were accounted for when a comparison with the literature was performed.

$k_{Y}(X, T, z)$ is the $k$-correction for the transformation from filter $X$ to the rest-frame $Y$-band which accounts for three effects: (i) the different response curves of filter $X$ in observer's frame and filter $Y$ (either $B$ or Gunn $r$ ) in rest-frame, (ii) for the redshifted and "stretched" SED in observer's frame and (iii) the SED type $T$. For all cases, a SED type $T=-5 /-2$, i.e. an earlytype E/S0 galaxy was adopted. The individual correction factors $k_{Y}$ have been derived via synthetic photometry, which is described in detail in section 4.5.3.

In a second independent approach, for the subsample of galaxies with HST structural information, the rest-frame absolute magnitudes were computed using the transformation procedure as discussed in section 4.3.4. 
Table 4.15: Galactic extinction coefficients for the poor cluster fields.

\begin{tabular}{lcccccccc}
\hline Name & R.A. & Dec. & $\begin{array}{c}A_{B \text { BH }} \\
{[\mathrm{mag}]}\end{array}$ & $\begin{array}{c}A_{B \text { SFD }} \\
{[\mathrm{mag}]}\end{array}$ & $\begin{array}{c}E(B-V) \\
{[\mathrm{mag}]}\end{array}$ & $\begin{array}{c}A_{\mathrm{F} 702} \\
{[\mathrm{mag}]}\end{array}$ & $\begin{array}{c}A_{R} \\
{[\mathrm{mag}]}\end{array}$ & $\begin{array}{c}A_{I} \\
{[\mathrm{mag}]}\end{array}$ \\
\hline CL 0849+37 & 084911 & +373109 & 0.090 & 0.151 & 0.035 & 0.085 & 0.094 & 0.068 \\
CL 1701+64 & 170147 & +642057 & 0.060 & 0.112 & 0.026 & 0.063 & 0.070 & 0.050 \\
CL 1702+64 & 170214 & +641953 & 0.060 & 0.110 & 0.026 & 0.063 & 0.068 & 0.050 \\
\hline
\end{tabular}

\subsubsection{Apparent Magnitudes}

Total apparent magnitudes were derived with the Source Extractor package (SExtractor, Bertin \& Arnouts 1996). This program offers different algorithms for photometry of stellar objects and extended sources and a choice between circular or elliptical apertures. Apparent magnitudes measured within fixed circular apertures are the best approach for the derivation of galaxy colours. Total apparent magnitudes are not reliable to compute galaxy colour indices as the apparent diameter of the galaxies could deviate between different passband filters. For the derivation of absolute magnitudes it is mandatory to prevent flux losses. For this purpose, variable elliptical apertures yield the best results.

SExtractor offers three algorithms for the usage of elliptical apertures, called Mag_iso, Mag_best and Mag_auto. Tests showed that the latter two routines are consistent to within a few $0.01 \mathrm{mag}$ for early-type galaxies in all bandpasses and for different field and cluster environments. However, in particular for faint detections, the Mag_iso algorithm systematically resulted in underestimated brightnesses. The Mag_auto automatic aperture routine is based on the "first moment" algorithm by Kron (1980) and designed to best reproduce the total magnitudes of extended sources. This algorithm has been used for both field studies whereas for the cluster galaxies the Mag_best routine was applied.

\subsubsection{Galactic Absorption}

The correction for the extinction due to Galactic absorption was performed in using a special program written by the author, called SFDextc. This program calculates automatically the extinction coefficients for a specific $E(B-V)$ input value based on the $C O B E$ dust maps by Schlegel et al. (1998, hereafter SFD). As a consistency check, results were compared to the Galactic extinction values by Burstein \& Heiles (1982, hereafter $\mathrm{BH})$ and $A_{V}=3.1 \cdot E(B-V)$. As the dust maps provide a more reliable measurement only the SFD extinction values were adopted. For comparison, for the Low $-L_{X}$ clusters also the $A_{B}$ coefficients by $\mathrm{BH}$ are shown (see Table 4.15). The uncertainties in the extinction values are $\mathrm{E}(\mathrm{B}-\mathrm{V})=0.010^{m}$.

For the cluster A 2390 an $E(B-V)=0.110^{m}$ was assumed, resulting in extinction coefficients for the Johnson-Cousins filters of $\mathrm{A}_{U}=0.600^{m}$, $\mathrm{A}_{B}=0.476^{m}$ and $\mathrm{A}_{I}=0.214^{m}$, respectively. For the HST/WFPC2 F814W filter $A_{814}=0.214^{m}$ was derived.

The Galactic extinction coefficients for the Johnson-Cousins and the HST F702W filter passbands of the Low $-L_{X}$ cluster fields are summarised in Table 4.15.

For the Galactic absorption at the coordinates of the FDF, the values which are given in Heidt et al. (2003) were adopted. These coefficients were derived with the formulae given in Cardelli et al. (1989) under the assumption of $E(B-V)=0.018^{m}$ (derived from $\mathrm{BH}$ ) and $A_{V}=3.1 \cdot E(B-V)$. For the individual 
broadband filters used for the FDF imaging, the resulting respective absorption factors are $A_{U}=0.087^{m}, A_{B}=0.076^{m}, A_{g}=0.062^{m}$, $A_{R}=0.041^{m}$ and $A_{I}=0.035^{m}$. For the HST /ACS F $814 \mathrm{~W}$ filter $A_{814}=0.035^{m}$ was computed. At the central coordinates of the WHDF of $\quad \alpha_{2000}=00^{\mathrm{h}} 19^{\mathrm{m}} 59.6^{\mathrm{s}}, \quad \delta_{2000}=+00^{\circ} 04^{\prime} 18^{\prime \prime}$ (B1950.0), an $E(B-V)=0.025^{m}$ was adopted, yielding to Galactic extinction coefficients of $A_{U}=0.137^{m}, A_{B}=0.108^{m}, A_{R}=0.067^{m}$, and $A_{I}=0.049^{m}, A_{H}=0.014^{m}$, and $A_{K}=0.009^{m}$. The absorption coefficient for the HST/ACS F814W filter is $A_{814}=0.049^{m}$.

\subsubsection{K-Correction}

The $k$-correction accounts for the fact that sources which are observed at different redshifts are compared with each other at different restframe wavelengths. The transformation between an observed apparent magnitude in a photometric broad passband $X$ to a corresponding restframe magnitude in another broad-band photometric passband $Y$ involves the term known as the " $k$-correction". Such a transformation is defined by

$$
k_{Y}(X, T, z)=X(T, z)_{\mathrm{obs}}-Y(T, 0)_{\text {rest }}
$$

If photometric broad-band information is limited to a few filters, the different wavelength ranges covered by a passband in observer's frame and rest-frame introduce a strong dependence of the achievable $k$-correction accuracy on SED type. At redshift $z=0.2$, e.g., the difference between SED types E/S0 and Sbc corresponds to a change of $\Delta k_{B}=0.4^{m}$ in the transformation from $B_{\text {obs }}$ to $B_{\text {rest }}$ (e.g., Frei \& Gunn 1994). For this reason, even a slight misclassification can introduce a substantial offset in the derived luminosity if observations are limited to one or two filters only. In contrast to this, the field galaxy projects of the FDF and WHDF greatly benefitted from the multi-band imaging data. With the FDF photometry in $B$, $g, R$ and $I$, and the WHDF photometry in $B$,

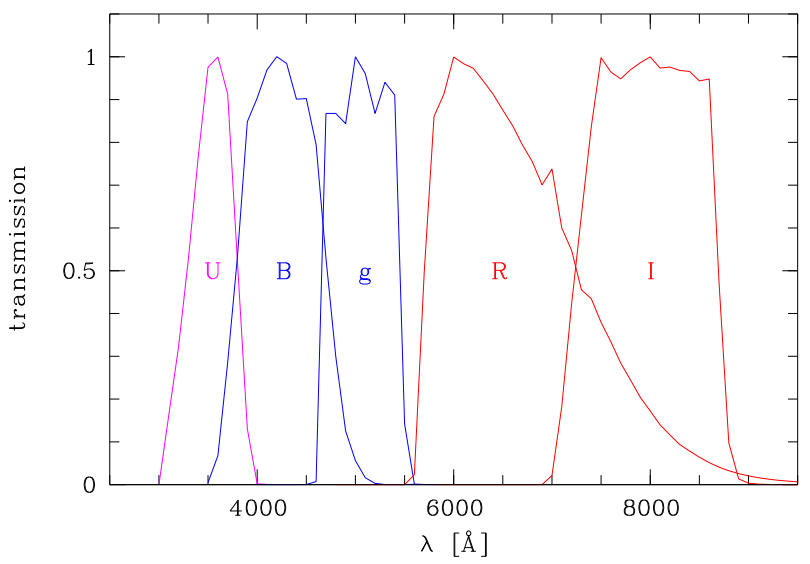

Figure 4.24: Transmission curves of the FORS $U$, $B, g, R$ and $I$ filters which were used for the imaging of the FDF.

$R$ and $I$, the filter that best matched the restframe $B$-band could be used to transform an apparent magnitude $X$ into absolute magnitudes $M_{B}$. For both, rich and poor early-type cluster samples, observations were carried out in the $R$ or $I$ filter bands. At $z=0.2$, the observed $R$, $I$ and the $R_{702}, I_{814}$ HST filter passbands (cf. section 4.3.4) are very close to rest-frame Gunn $r$. For the clusters CL $0849(z=0.234)$, CL 1701 $(z=0.246)$ and CL $1702(z=0.223)$ the transformation $R_{\text {obs }} \rightarrow r_{\text {rest }}$ amounts to $0.02^{m}$ only. Note that these numbers are less than the correction term for Galactic extinction. Therefore, the advantages of using the Gunn $r$-band as restframe passband instead of the bluer Johnson $V$ or $B$ bands are the smaller $k$-corrections and the lower corrections of galactic absorption.

In order to ensure the highest possible accuracy in the transformation between observedframe and rest-frame, the $k_{Y}$-corrections were not taken from the literature but computed via synthetic photometry as outlined in Böhm (2003). For a given SED of type $T$ at redshift $z$ with wavelength-dependent flux $F(T, \lambda)$, the magnitude in a passband $X$ was defined as

$$
m_{X}(T, z)=-2.5 \log \frac{\int F(T, z, \lambda) S_{X}(\lambda) d \lambda}{\int S_{X}(\lambda) d \lambda}
$$




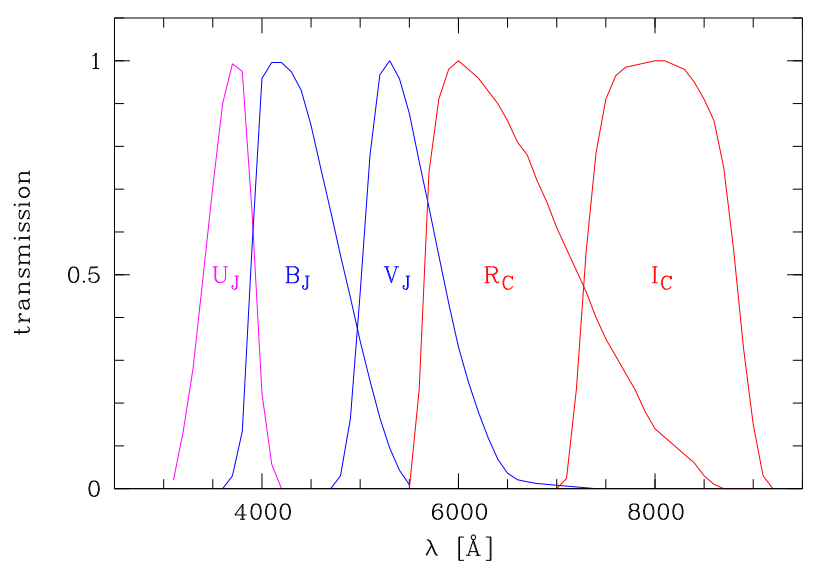

Figure 4.25: Transmission curves of the Johnson $U_{J}$, $B_{J}, V_{J}$ and Cousins $R_{C}, I_{C}$ filters.

$$
+C_{X}+C_{\text {sys }}
$$

(e.g., Fritze-v. Alvensleben 1989). The parameter $S_{X}(\lambda)$ is the response function of filter $X . C_{X}$ is a constant for the calibration of the respective filter within the corresponding filter system (e.g, the Johnson, Cousins or Gunn filter system). $C_{\text {sys }}$ is an optional constant to calibrate between different filter systems. The derivation of the calibration factors $C_{X}$ and $C_{\text {sys }}$ is discussed below. Note that Eq. 4.10 yields relative magnitudes which are appropriate for the transformation between different filters or different systems, but is not reliable for the computation of physical apparent brightnesses in the form given above. For the latter purpose, an additional calibration constant is needed, which can be derived via synthetic photometry with an observed SED of a galaxy with known apparent brightness. However, since the synthetic photometry only was executed to derive the $k$ corrections, i.e., for relative photometry, this calibration was not needed.

In Fig. 4.24, the FORS filters $U, B, R, I$ and Gunn $g$ are shown. This set of filters was used for the imaging of the FDF in the optical regime. In Fig. 4.25, the filter response curves of the standard Johnson-Cousins system $\left(U_{J}, B_{J}, V_{J}\right.$, $\left.R_{C}, I_{C}\right)$ are displayed. These curves were re-

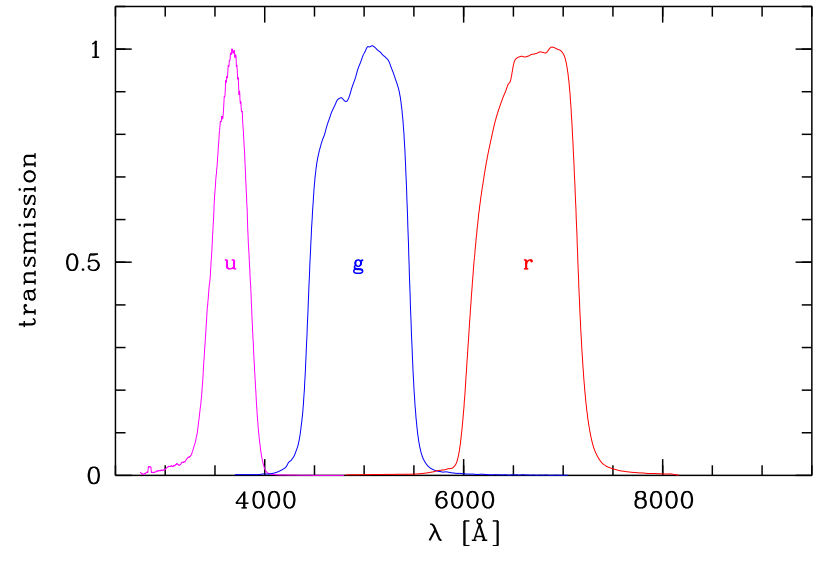

Figure 4.26: Transmission curves of the ThuanGunn $u, g$ and $r$ filters.

trieved as ascii tables from the ESO webpages. Fig. 4.26 shows the filter response curves of the Thuan-Gunn filter system $(u, g, r)$ which were retrieved as ascii tables from the CAHA instruments webpages. All these filter curves were used for the definition of the respective response functions $S_{X}$.

For the Johnson system, the constants $C_{X}$ were taken from Allen (1973). They are defined in such a way to yield zero colour terms for an A0V star spectrum in all passbands. To calibrate the FORS filter system, the A0V spectrum from Pickles (1998) was used and the finetuning of the respective factors $C_{X}$ was performed with the equations given in Sinachopoulos \& van Dessel (1998). Finally, the constant $C_{\text {sys }}$ for transformations between the FORS and the Johnson system was calculated from the colour ( $\left.V_{\text {fors }}-V_{J}\right)$ (ibid.). For the Cousins $R_{C}$ and $I_{C}$ filters, the factors $C_{R}$ and $C_{I}$ of the FORS calibration were adopted as initial values. The calibration factors were verified through a comparison of the $\left(R_{J}-R_{C}\right)$ and $\left(I_{J}-I_{C}\right)$ colours, assessed for various SED types, to the numbers published in Fukugita et al. (1995), which yielded consistent values within $<0.03$ mag.

Since the FORS $g$ filter has a slightly different shape from the original Thuan-Gunn $g$ filter def- 


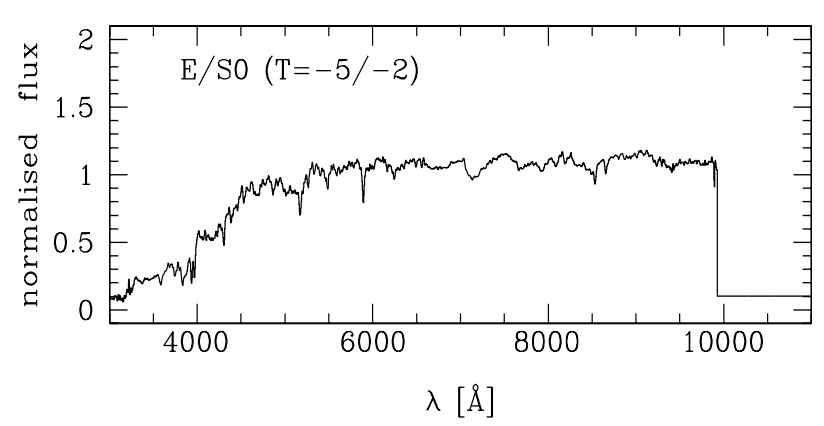

Figure 4.27: SED template used for the computation of the $k$-corrections via synthetic photometry. This is an observed spectrum of an E/S0 galaxy with an age of 12 Gyrs (Kinney et al. 1996). For this plot, the template has been normalised to the flux at $5500 \AA$.

inition, the following more complicated calibration procedure has to be performed. The FORS $g$ filter is broader by $\sim 90 \AA$ and has $\mathrm{a} \sim 130 \AA$ higher central wavelength than the Thuan-Gunn $g$ filter. A first calibration was conducted adopting the transformation by Jørgensen (1994)

$$
g_{\text {gunn }}-V_{J}=0.503\left(B_{J}-V_{J}\right)-0.226 .
$$

Based on the A0V spectrum, the colour ( $g_{\text {fors }}-$ $\left.g_{\text {gunn }}\right)$ then was computed, which yielded a value of $0.10 \mathrm{mag}$ and thus a difference to the Johnson system of $\left(g_{\text {fors }}(A 0 V)-V_{J}(A 0 V)\right)=-0.13^{m}$. Again, the colours were compared through a cross-correlation with the $g$ passband definitions given by Fukugita et al.

As SED templates for the computation of the $k$ corrections, the UV/optical spectra published by Kinney et al. (1996) were used. These observed template spectra comprise different morphological galaxy types of elliptical, bulge, S0, Sa, Sb, and Sc and starburst galaxies and cover a wavelength range of $\sim 1200-9800 \AA$ with a resolution between $\sim 8$ to $10 \AA$. For the synthetic photometry described here, only elliptical galaxy templates with an age of 12 Gyrs were used solely. One of these templates is shown in Fig. 4.27. For the purpose of this graph, the spectrum was normalised to the flux at $5500 \AA$. No separation

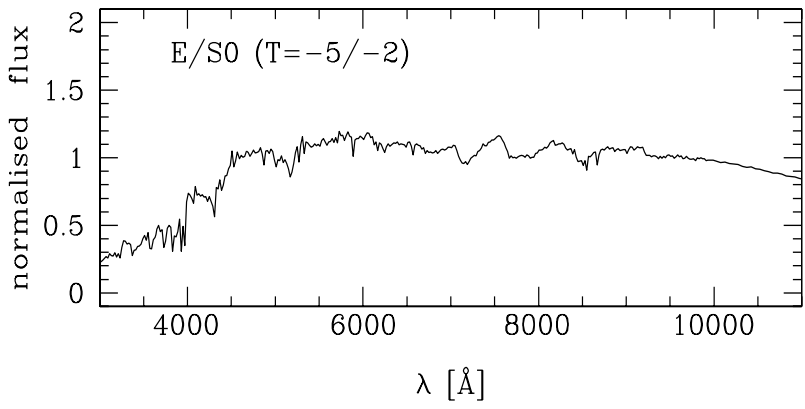

Figure 4.28: SED template used for the computation of the $k$-corrections via synthetic photometry. This spectrum was generated via chemically consistent evolutionary synthesis models (Möller et al. 2001). For this plot, the template has been normalised to the flux at $5500 \AA$.

between elliptical and S0 types was done because both offer similar (optical and UV) spectral shapes, Balmer discontinuities (4000 $\AA$ ) and absorption features for $\lambda>5000 \AA$ which are indistinguishable from their broadband optical and near-IR colours.

For internal consistency, the synthetic spectral SED templates by Möller et al. (2001) were also applied to compute the $k$-corrections. These templates were generated with evolutionary synthesis models and the authors provide two versions of each template, one including dust reddening and one without dust. For the synthetic photometry performed here, templates without dust were used solely, since no intrinsic absorption has to be accounted for early-type galaxies in this wavelength regime. The basic advantage of synthetic spectra with respect to observed spectra is of course that no noise is introduced in the photometry. Elliptical galaxy templates with an age of 12 Gyrs were used in order to give a good reproduction of the observed colors of present-day early-type galaxies. One E/S0 template is shown in Fig. 4.28, which was normalised to the flux at $5500 \AA$ for plotting purposes. Gas emission was not incorporated in the models, but this has a negligible effect on broad band photometry for very late-type SEDs $(T \geq 8)$ only. To derive the flux $F$ at wavelength $\lambda$, the SEDs 
were redshifted according to

$$
F(T, z, \lambda)=\frac{F_{0}(T, \lambda /(1+z))}{(1+z)}
$$

(e.g., Contardo, Steinmetz \& Fritze-v. Alvensleben 1998), where $F_{0}$ is the flux of the unredshifted spectrum. Finally, the transformation from the apparent magnitude of a spectrum of type $T(\mathrm{E} / \mathrm{S} 0)$ at redshift $z$ observed with a FORS/Johnson-Cousins filter $X$ to the Johnson $B$ or Gunn $r$ magnitude $m_{Y}$ in rest-frame was performed via

$$
k_{Y}(X, T, z)=m_{X}(T, z)-m_{Y}(T, z=0),
$$

where the two terms on the right hand side were derived according to Eq. 4.10. $k$-corrections were computed with the equation given above for all source filters (FORS and Johnson-Cousins filter system), redshifts (cluster redshift or redshift of the field galaxy) and environments covered by the early-type galaxies. Typical deviations in the $k$-corrections between the templates of Kinney et al. (1996) and Möller et al. (2001) are very small, e.g. $\Delta \bar{k}_{r}=0.03^{m}$ in the Gunn $r$ filter, for the SEDs of both ellipticals and S0 galaxies.

To transform to Johnson $B$ rest-frame (cf. Eq. 4.8), the following filters were used depending on the redshift: $B_{\text {fors }}$ for $z<0.25, g_{\text {fors }}$ for $0.25 \leq z<0.55$ and $R_{\text {fors }}$ for $0.55 \leq z<0.7$ (only one object). For this reason, the $k$ correction was much less sensitive to spectral type than using a global transformation of $B_{\text {obs }} \rightarrow B_{\text {rest }}$, which was particularly important at higher redshifts $z \gtrsim 0.6$ in the case of spiral galaxies with late-type $\operatorname{SEDs}(T \geq 1)$ in the FDF (Böhm 2003). An analogous approach was performed for the WHDF elliptical galaxies. For field objects with $z>0.25$ a transformation to $B$ rest-frame was conducted based on the $B$ passband and for $0.55 \leq z<0.75$ the $R_{C}$ filter was utilised. Nevertheless, for internal consistency the rest-frame magnitudes derived with the FORS filter systems were checked by transforming the observed F814W-magnitudes into rest-frame Johnson- $B$. The deviations between magnitudes were small (differences were less than their errors).

For testing purposes, the colors of the templates were derived purely within the Johnson-Cousins Filter system and afterwards compared to the values given by Fukugita et al. The deviations were very small, with absolute colors $(X-Y)$ within the range $0.03^{m} \leq|\Delta(X-Y)| \leq 0.08^{m}$.

\subsection{Luminosity Distribution and Errors}

The distribution of the early-type galaxies in the cluster A 2390 in absolute Gunn $r$-band magnitude is shown in Fig. 4.29. A range of $-22.99 \leq M_{r} \leq-20.47\left(\left\langle M_{r}\right\rangle=-21.31\right)$ is covered by the $48 \mathrm{E}+\mathrm{S} 0$ cluster members of A 2390. The dashed arrow gives the mean value $\left(\bar{M}_{r}=-21.48\right)$ of the distribution and the median is displayed by the solid arrow. The FP sub-sample of 14 galaxies with high-resolution structural HST information, shows a spread $-22.56 \leq M_{r} \leq-20.35\left(\left\langle M_{r}\right\rangle=-21.21\right)$. Note that the central cD galaxy of A 2390 (i.e. the Brightest Cluster Galaxy BCG) was not spectroscopically observed. Including a set of 4 non-cluster members with determined redshifts which were either identified as foreground or as background objects (see section 5.5 for a discussion) to the cluster sample of A 2390 yields a distribution with a range in absolute Gunn $r$ magnitudes of $-22.99 \leq M_{r} \leq-20.16$ $\left(\left\langle M_{r}\right\rangle=-21.28\right)$. These non-cluster galaxies affect only the fainter end of the distribution of absolute magnitudes.

Note that the distributions of the complete cluster samples are taken from the ground-based imaging, whereas the FP sub-sample of galaxies are based on the HST magnitudes. In general, the HST photometry using the techniques of curves of growth fitting is deeper and therefore fainter magnitudes can be derived than if measurements are restricted to a circularly aper- 


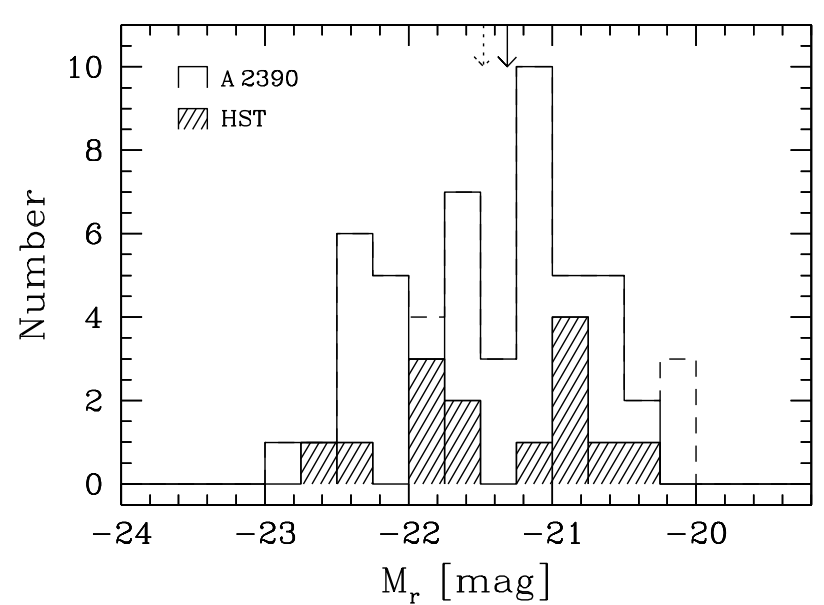

Figure 4.29: Absolute Gunn $r$-band magnitude distribution of all 52 cluster candidates in A 2390 with determined redshifts (dashed line), the sub-sample of 48 early-type cluster members of A 2390 from which velocity dispersions could be derived (solid line) and the subset of $14 \mathrm{E}+\mathrm{S} 0$ galaxies with HST/WFPC2 information (shaded region). The dashed and the solid arrow give the mean $\left(\bar{M}_{r}=-21.48\right)$ and the median $\left(\left\langle M_{r}\right\rangle=-21.31\right)$ values of the cluster member distribution, respectively.

ture. For this reason, small differences between the histograms in the order of $\sim 0.05$ mag might arise and cause a slightly different distribution.

In Fig. 4.30 the distribution of the early-type galaxies in the three Low- $L_{X}$ clusters CL 0849, CL 1701 and CL 1702 in absolute Gunn $r$-band magnitude is displayed. The $27 \mathrm{E}+\mathrm{S} 0$ cluster members (combined for all Low $-L_{X}$ clusters), cover a range of $-23.74 \leq M_{r} \leq-20.08$ $\left(\left\langle M_{r}\right\rangle=-21.77\right)$. Again, the dashed arrow indicates the mean value $\left(\bar{M}_{r}=-21.69\right)$ of the distribution and the median is displayed by the solid arrow. An FP sub-sample of ten galaxies is distributed over a range of $-23.88 \leq M_{r} \leq-20.68\left(\left\langle M_{r}\right\rangle=-21.85\right)$. Two cD galaxies (of the clusters CL 0849 and CL 1702) are included in both samples of cluster members and FP galaxies.

A set of additional 9 non-cluster members with determined redshifts which revealed to be either foreground or background objects (cf. sec-

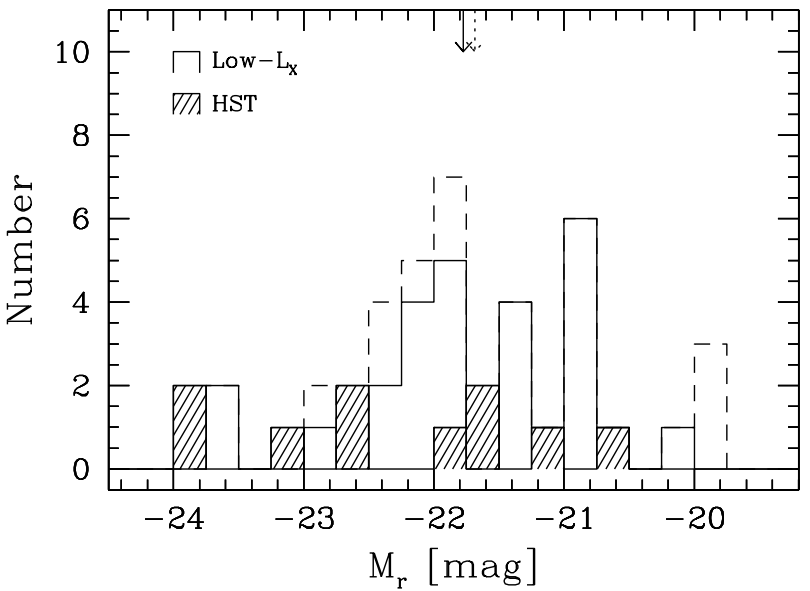

Figure 4.30: Absolute Gunn $r$-band magnitude distribution of all 36 cluster candidates in the Low- $L_{X}$ clusters CL 0849, CL 1701 and CL 1702 with determined redshifts (dashed line), the sub-sample of 27 early-type cluster members for these three clusters with available velocity dispersions (solid line) and the subset of $10 \mathrm{E}+\mathrm{S} 0$ galaxies with HST/WFPC2 information (shaded region). The dashed and the solid arrow give the mean $\left(\bar{M}_{r}=-21.69\right)$ and the median $\left(\left\langle M_{r}\right\rangle=-21.77\right)$ values of the cluster member distribution, respectively.

tion 5.5) is included for the distribution of cluster candidates for the Low $-L_{X}$ galaxies (dashed line). These 36 galaxies cover a range in Gunn $r$ magnitudes of $-23.74 \leq M_{r} \leq-19.83$ $\left(\left\langle M_{r}\right\rangle=-21.85\right)$.

In Fig. 4.31 the distribution of the early-type field galaxies in the FDF and WHDF in absolute Johnson $B$-band magnitude are shown. Only the rest-frame magnitudes derived with the FDF and WHDF photometry are shown (see also discussion in section 4.5.3). The $23 \mathrm{E}+\mathrm{S} 0$ field galaxies cover a range in absolute $B$-band magnitude of $-22.78 \leq M_{B} \leq-19.40$ $\left(\left\langle M_{B}\right\rangle=-20.60\right)$. The dashed arrow corresponds to the mean value $\left(\bar{M}_{B}=-20.74\right)$, whereas the median is denoted by the solid arrow.

Total errors on the absolute Gunn $r$ and $B$-band magnitudes for all galaxies were computed via 


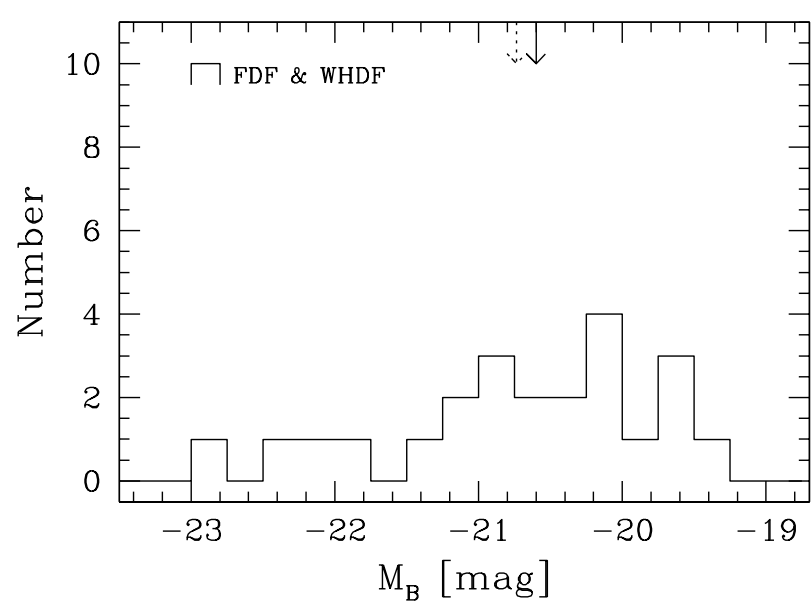

Figure 4.31: Absolute B-band magnitude distribution of 23 early-type field galaxies in the FDF and WHDF. Rest-frame magnitudes were derived based on the FDF and WHDF photometry. The dashed and the solid arrow give the mean $\left(\bar{M}_{B}=-20.74\right)$ and the median $\left(\left\langle M_{B}\right\rangle=-20.60\right)$ values of the distribution, respectively.

the relation

$$
\delta M_{Y}^{2}=\delta X^{2}+\delta k^{2}+\delta A_{X}^{2} .
$$

where $\delta M_{Y}$ is the total error in the absolute restframe of Gunn $r$ passband $\delta M_{r}$ or in the absolute Johnson $B$ passband $\delta M_{B}$.

$\delta X$ indicates the photometric error of groundbased photometry in the respective filter passbands, either $B, g, R$ or $I$-band. For the cluster galaxies in A 2390 the total $I$-band magnitude was adopted and for the Low $-L_{X}$ clusters the total $R$-band magnitude was utilised. The errors in total $I$-band magnitudes $\delta I$ cover a range between $0.02^{m} \leq \delta I \leq 0.09^{m}$, with a median uncertainty of $\langle\delta I\rangle=0.04^{m}$. Errors in total $R$-band magnitudes $\delta R$ take values of $0.01^{m} \leq \delta I \leq 0.05^{m}$, with a median of $\langle\delta I\rangle=0.03^{m}$. Thanks to the very deep imaging of the FDF and deep WHDF, the uncertainties $\delta B$ for the field $\mathrm{E}+\mathrm{S} 0$ galaxies are very small for all filter passbands. The median values are $\langle\delta X\rangle<0.015^{m}$ in $B, g, R$ and $I$, respectively. $\delta k$ denotes the $k$-correction error. The uncertainty between the SED templates is very small, a typical error of $\delta \bar{k}_{r}=0.03^{m}$ was found. For all morphological types (E, E/S0, S0, Sa bulge) and redshifts of the field galaxies, the error in the $k$-correction is $\delta k_{B} \leq 0.1^{m}$. No error due to SED type misclassification was assumed as the SEDs of both ellipticals and S0 galaxies are very similar (section 4.5.3).

Finally, $\delta A_{X}$ in Eq. 4.14 gives the uncertainty of the galactic absorption correction.

Contributions of the distance modulus to $\delta M_{Y}$ were assumed to be negligible. For the A 2390 sample, the total error in $\delta M_{r}$ falls into the range $0.07^{m} \leq \delta M_{r} \leq 0.14^{m}$, with a median of $\delta \overline{M_{r}}=0.09^{m}$. For the Low $-L_{X}$ sample, the total uncertainty in $\delta M_{r}$ covers a range between $0.06^{m} \leq \delta M_{r} \leq 0.12^{m}$, with a median error of only $\delta \overline{M_{r}}=0.07^{m}$. For the FDF and WHDF field sample, the total error falls into the range $0.08^{m} \leq \delta M_{B} \leq 0.20^{m}$ with a median of $0.15 \mathrm{mag}$. 


\section{Chapter 5}

\section{Kinematic Analysis}

\subsection{Galaxy Kinematics}

An extracted galaxy spectrum is the superposition of each individual star spectrum over this selected region of the galaxy along the line-ofsight. Because of the random motions of the stars in early-type galaxies, the stellar spectra are slightly shifted to each other in wavelength range. The resulting net effect is a broadening in the galaxy spectrum owing to these shifts. In other words, the galaxy spectrum is the convolution of the star spectra with an appropriate velocity distribution along the line-of-sight.

In order to quantify this broadening, the LineOf-Sight-Velocity-Distribution (LOSVD) F( $\left.v_{\text {los }}\right)$ is characterised as the fraction of stars contributing to the spectrum with a line-of-sight between $v_{\text {los }}$ and $\left(v_{\text {los }}+d v_{\text {los }}\right)$. Under the assumption that all the stellar spectra are identical $(S(u))$, with $u$ being the stellar velocity, the galaxy spectrum is given as:

$$
G(u) \approx \int F\left(v_{\mathrm{los}}\right) \cdot S\left(u-v_{\mathrm{los}}\right) \cdot d v_{\mathrm{los}}
$$

This is one of the basic equations in the field of galaxy kinematics. To derive the kinematics of one particular galaxy a spectrum of a stellar template is needed, which is required to give a good approximation of the galaxy's spectrum and its dominant stellar population. The better the selection of the template star, the more accurate the fit to the galaxy spectrum will get and thus the more precise the kinematics of the gal- axy under consideration. Two main properties of the LOSVD are its mean velocity $\bar{v}$ :

$$
\bar{v}=\int F\left(v_{\mathrm{los}}\right) \cdot v_{\mathrm{los}} \cdot d v_{\mathrm{los}}
$$

and its velocity dispersion $\sigma_{\text {los }}^{2}$ :

$$
\sigma_{\mathrm{los}}^{2}=\int F\left(v_{\mathrm{los}}\right) \cdot\left(v_{\mathrm{los}}-\bar{v}_{\mathrm{los}}\right)^{2} \cdot d v_{\mathrm{los}}
$$

As an initial approximation, the LOSVD of the stars is assumed to be Gaussian. However, due to improvements of the detectors and new developed methods it is possible to measure the deviations from the Gaussian shape. In the following paragraph, a review of the most relevant algorithms from the literature is given.

\subsubsection{Kinematic Extraction Methods}

All techniques described here benefit from the circumstance that the convolution show in Eq. 5.1 becomes a multiplication in Fourier domain. The deconvolution therefore involves dividing the Fourier transform of the galaxy spectra by the Fourier transform of the stellar template. However, noise makes such a direct computation impractical. Noise dominates the Fourier transformed spectra at high frequencies, hence the ratio of the transforms at these frequency components will consist mainly of very amplified noise. Generally, the noise is white, i.e. its transform is essentially a constant out to high frequencies. To derive robust results, it 
is therefore necessary to suppress the noise at high frequencies through filtering. For example, "Wiener" or "optimal filtering" (Brault \& White 1971) provides one of the best balancing between filtering insufficiently, resulting in more residual noise than necessary and filtering too severely, thereby losing important information. Fig. 5.1 illustrates the effect of Wiener filtering of the Fourier transform. The Wiener filter is constructed by a least-square fitting to the computed power spectrum $P_{\text {obs }}$. The complex signal and noise power spectra are approximated by a model of the signal power $P_{\text {signal }}^{\prime}$ and a model of the noise power $P_{\text {noise }}^{\prime}$ as $P_{\text {obs }}(\kappa)=$ $P_{\text {signal }}^{\prime}(\kappa)+P_{\text {noise }}^{\prime}(\kappa)$ which represents a good approximation of the observed power spectrum $P_{\text {obs }}$. The modelled signal and noise components are used to constructed the filter, which is indicated as the solid line in Fig. 5.1. Working in 'Fourier space' offers two great advantages. On the one hand, the LOSVD is easier to extract and to analyse. On the other hand, a filtering of residual low-order and high-order frequency components in the original spectrum is possible which arise from an imperfect continuum subtraction and Poisson noise. An alternative approach to fit the LOSVD by a direct leastsquares solution in 'Real space' and describing the velocity dispersion by a set of delta-functions in logarithmic wavelength space has been developed by Rix \& White (1992). To overcome the amplification of high frequency noise in the LOSVD, a method of weighting the eigenvalues is used. However, such computer codes (Rix \& White 1992; van der Marel \& Franx 1993) will not be discussed here in greater detail.

\section{- Classical Fourier Quotient Method} (Sargent et al. 1977): The algorithm adopts that the LOSVD $F\left(v_{\text {los }}\right)$ has a Gaussian shape. Under this assumption, a Gaussian function is fitted to the quotient of the galaxy spectra and the template spectra in
Fourier space as:

$\tilde{F}(\kappa)=\frac{\tilde{G}(\kappa)}{\tilde{S}(\kappa)} \approx a \cdot \exp \left[-\frac{1}{2} \frac{2 \pi \kappa \sigma}{N}+\frac{2 \pi i \kappa v}{N}\right]$

with $\tilde{F}(\kappa), \tilde{G}(\kappa)$ and $\tilde{S}(\kappa)$ being the discrete Fourier transforms of $F\left(v_{\text {los }}\right), G(v)$ and $S(v)$, respectively. $a$ is a normalisation factor which measures the strength of the galaxy lines with respect to stellar lines (i.e., the mean relative line strength), $\sigma$ denotes the velocity dispersion and $v$ the mean radial velocity, which is derived through the logarithmic redshift in the channels. The parameter $N$ gives the number of pixels in the input spectra.

\section{- Cross-Correlation Method}

(Simkin 1974; Tonry \& Davies 1979; Franx \& Illingworth 1988): Initiated by Simkin (1974) and improved by Tonry \& Davies (1979), the Cross-Correlation approach is based on the fitting of a smooth symmetric function (quadratic polynomial) to the cross-correlation peak defined by the functions $\tilde{G}(\kappa)$ and $\tilde{S}(\kappa)$ :

$$
\tilde{C}(\kappa)=\frac{1}{N \sigma_{g} \sigma_{t}} \tilde{G}(\kappa) \cdot \tilde{S}^{*}(\kappa)
$$

where $\sigma_{g}$ is the rms of the galaxy spectra with $\sigma_{g}^{2}=\frac{1}{N} \sum_{u} G(u)^{2}$ and $\sigma_{t}$ the rms of the template spectrum, respectively. The star * denotes the complex conjugation. Cross-correlating the galaxy spectrum with the template spectrum produces a function $\tilde{C}(\kappa)$, which has a peak at the redshift of the galaxy and a width related to the dispersion of the galaxy. The resulting function is then fitted with a Gaussian in Fourier space.

\section{- Fourier Correlation Quotient (FCQ)}

(Bender 1990): In contrast to the two previous methods, which assume a Gaussian broadening function, the FCQ algorithm is an improvement of the former and a generalisation of the problem because it provides 


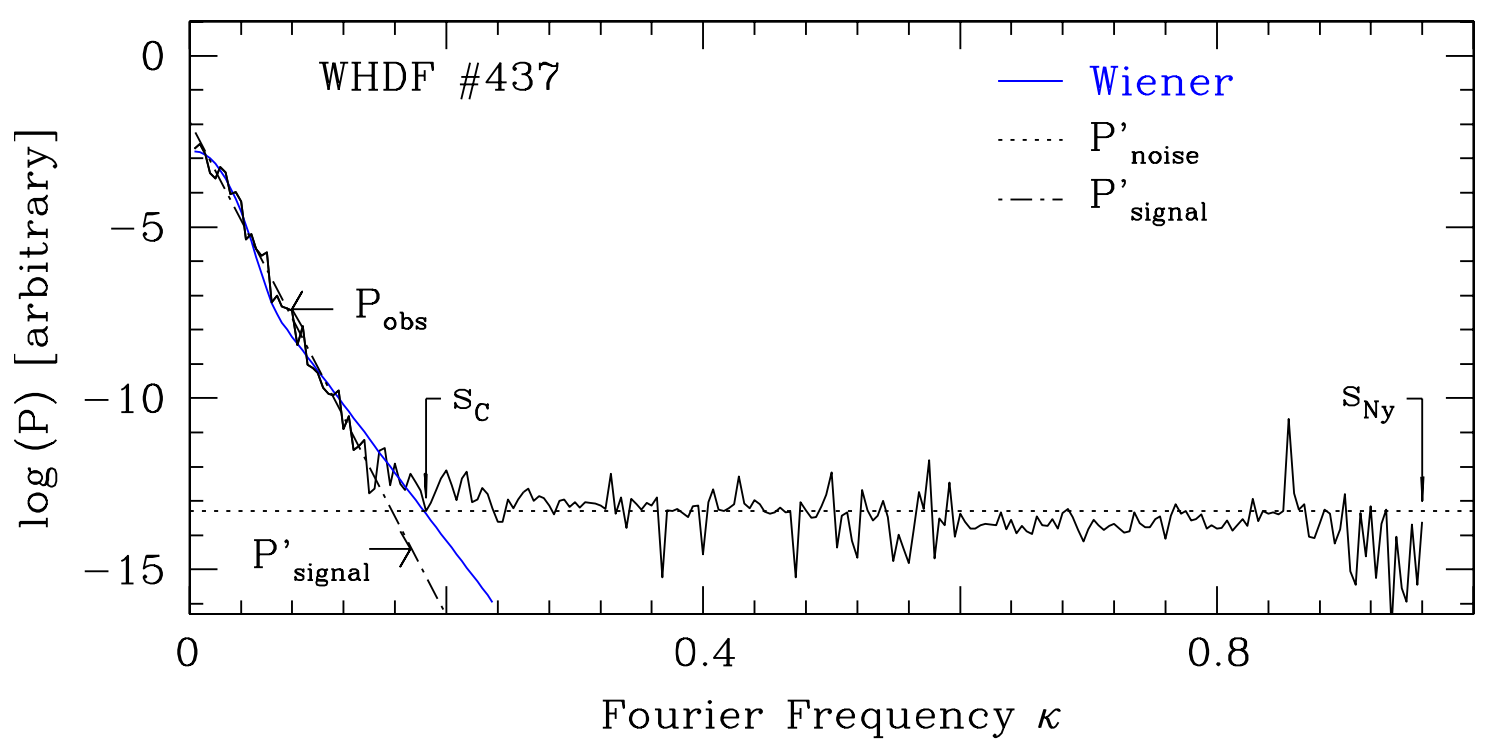

Figure 5.1: Illustration of the optimum (Wiener) filtering technique for the power spectrum of the galaxy WHDF \# 437. The definition of the smooth power models of the signal $P_{\text {signal }}^{\prime}$ and the noise $P_{\text {noise }}^{\prime}$ are constructed by a least-square fitting (dot-dashed line) of the computed power spectrum $P_{\text {obs }}$. The cutoff frequency $s_{\mathrm{c}}$ of the measurement, which is beyond the band-limit of the basic signal, and the characteristic frequency due to sampling $s_{\mathrm{Ny}}$ are also indicated. The Nyquist frequency or critical frequency $s_{\mathrm{Ny}}$, is half the sampling rate for a signal $\left(s_{\mathrm{Ny}}=0.5 / \Delta \lambda\right)$. The sampling theorem is satisfied as long as the band-width of the measurement is less than the Nyquist frequency. However as noise is broadband, an band-limiting analog filter must be employed prior to sampling to avoid aliasing (i.e., contributions from undersampled high-frequencies) by the transform of the random noise signal in the spectrum.

the full information of the LOSVD function. The method is based on the deconvolution of the peak of the template-galaxy correlation function with the peak of the autocorrelation function of a template star as:

$$
\tilde{F}(\kappa)=\frac{\tilde{G}(\kappa) \cdot \tilde{S}^{*}(\kappa)}{\tilde{S}(\kappa) \cdot \tilde{S}^{*}(\kappa)}
$$

In comparison to other procedures, the main advantage of the FCQ is that the most appropriate function to fit the LOSVD can be performed a posteriori rather than a priori. Furthermore, the method is less sensitive to template mismatch due to the fact that the LOSVD is determined from the ratio of the template-galaxy correlation and template autocorrelation functions, which are both smooth functions, instead of the division of the galaxy and the stellar spec- tra (see next section 5.2 for details).

- Unresolved Gaussian Decomposition (UGD) (Kuijken \& Merrifield 1993): This technique adopts that the deviations from a pure Gaussian are due to the composition of several Gaussians which are uniformly distributed in mean velocity and velocity dispersion. However, the Gaussians are not homogeneously distributed in amplitude:

$$
\tilde{F}(\kappa) \approx \sum_{\kappa=1}^{N} a_{\kappa} \cdot \exp \left[-\frac{\left(v_{\mathrm{los}}-v_{\kappa}\right)^{2}}{2 \sigma_{\kappa}}\right]
$$

where $a_{\kappa}$ indicates the amplitude, $v_{\kappa}$ the mean velocity and $\sigma_{\kappa}$ the velocity dispersion of each Gaussian. The UGD method computes the different amplitudes $a_{\kappa}$ for each component that provide the best fit to the galaxy spectrum in the least-square sense. 

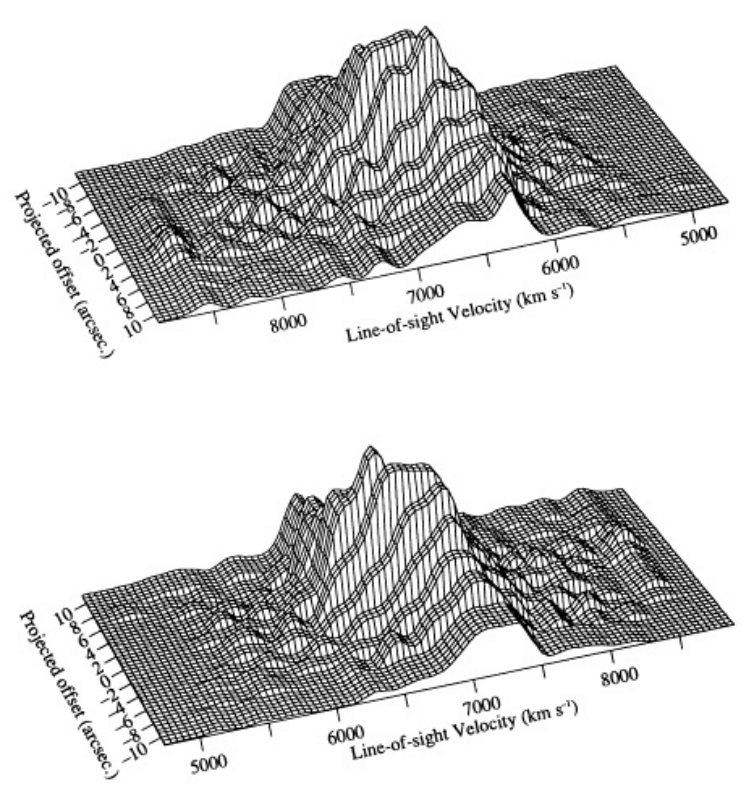

Figure 5.2: LOSVDs $F\left(R, v_{\text {los }}\right)$ in the galaxy UGC 12591. The surface height corresponds to the stellar density as a function of major axis radius and line-of-sight velocity. The surface is displayed from two opposite perspectives, demonstrating the symmetry between the LOSVDs from both sides of the galaxy. It is clearly evident that the line profiles are not Gaussian distributed (Kuijken \& Merrifield 1993).

However, this can only be achieved under certain physical constraints (i) The LOSVD must be non-negative everywhere, (ii) The LOSVD is expected to vary smoothly on small scales and (iii) The LOSVD is assumed to be non-zero over a fairly small range in velocity only. In addition, the Rayleigh criterion is required in order to be able to define the separation between two adjacent peaks. To avoid dips between two peaks, the separation should be less than $2 \sigma$.

Fig. 5.2 shows two different viewpoints of the LOSVDs for the local galaxy UGC 12591 (S0/Sa). This galaxy is moderately inclined to the line-of-sight $\left(\approx 67^{\circ}\right)$, with the azimuthal component being the main contribution to the 3D velocity dis- persion along the line-of-sight on the major axis. UGC 12591 has a large rotation velocity $v_{\text {rot }} \approx 500 \mathrm{~km} \mathrm{~s}^{-1}$ (Giovanelli et al. 1986) and a central velocity dispersion of $\sigma_{0} \approx 780 \mathrm{~km} \mathrm{~s}^{-1}$ (Kuijken \& Merrifield 1993). Fig. 5.2 demonstrates the symmetry between the LOSVDs from two different perspectives of the galaxy and clearly shows that the line profiles are not Gaussian.

\section{- Gauss-Hermite Expansion}

(van der Marel \& Franx 1993; Gerhard 1993): The approach of this method assumes the line profile $F\left(v_{\text {los }}\right)$ to be a Gaussian with free parameters of line strength $a$, mean radial velocity $v$ and velocity dispersion $\sigma$ as:

$$
F\left(v_{\mathrm{los}}\right)=\frac{a \alpha(\omega)}{\sigma}, \quad \omega \equiv \frac{\left(v_{\mathrm{los}}-v\right)}{\sigma}
$$

where $\alpha(y)$ defines the standard Gaussian function:

$$
\alpha(y)=\frac{1}{\sqrt{2 \pi}} e^{-(1 / 2) y^{2}}
$$

The LOSVD is derived via the product of this normal Gaussian and a truncated expansion of the Hermite polynomials in the form:

$$
F\left(v_{\mathrm{los}}\right)=\left[\frac{a \alpha(\omega)}{\sigma}\right] \cdot\left\{1+\sum_{j=3}^{N} h_{j} H_{j}(\omega)\right\}
$$

The Hermite coefficients $h_{j}$ describe deviations from the Gaussian shape of the LOSVD, $h_{3}$ measures asymmetries (mostly taking values of $\left.h_{3} \lesssim 0\right)$ and $h_{4}$ symmetries $\left(h_{4} \geq 0\right)$ and $H_{j}$ characterise the Hermite polynomials. Results for the parameters of $\left(a, v, \sigma, h_{3}, \ldots, h_{N}\right)$ are found using a simultaneous $N+1$ parameter fit that are based on a $\chi^{2}$ residual minimisation of the galaxy spectrum and the convolution of the LOSVD (broadening function) with the template spectrum in the Fourier domain. 
In practice, the fits are restricted to the case $N=4$, but extension to higher orders is straightforward. Note, that although physically unmotivated, negative LOSVD are allowed (e.g., resulting from a non-zero $h_{3}$ ).

\subsection{The FCQ Algorithm}

In the next paragraphs, a general description of the Fourier Correlation Quotient technique will be given. A specific application to the data of this work with slight modifications and well adapted parameter settings is discussed in section 5.3.

The absorption lines of early-type galaxies get broadened due to a combination of intrinsic velocity dispersion and integration along the lineof-sight. For this reason, an analysis of the broadening function gives more detailed insights into the internal kinematics than, for example, using the mean velocity integrated along the lineof-sight and the velocity dispersion. Algorithms such as the Fourier quotient technique (e.g., Sargent et al. 1977) or the correlation method (e.g., Franx \& Illingworth 1988) provide no information about the velocity dispersion along the lineof-sight.

The Fourier Correlation Quotient (FCQ) method (Bender 1990) measures the kinematical broadening function of early-type galaxies based on the deconvolution of correlation peaks. In particular, the shape of the broadening function (see also Fig. 5.7) is recovered in great detail by deconvolving the peak of the template-galaxy correlation function with the peak of the autocorrelation function of a template star. One major advantage is a high insensitivity due to template mismatching than the standard (classical) Fourier quotient method. In the following a brief overview of the FCQ technique is presented. For a more detailed description as well as different calibration tests and error treatments the reader is referred to Bender (1990).
As a first step the input galaxy and template spectra must have been reduced in the same way (see chapter 3) and afterwards linear rebinned on a logarithmic wavelength scale. The reason for going to logarithmic space is manifold. Firstly, the LOSVD is easier to extract and to analyse there and secondly, even more important, an unique and therefore reliable solution is derived as a specific logarithmic wavelength region corresponds to a well, unique defined space in velocity space (along X-axis). Thirdly, the logarithmic technique shows more clearly in the power spectrum the subtle power variations at the high frequencies. In addition, following requirements have to be fulfilled: (i) the absorption line widths in a template star and in a typical star of the galaxy are negligible in comparison to the instrumental broadening. In general, early-type galaxies comprise mostly of K0 III/K3 III stars which have a small intrinsic line width $\left(\approx 20 \mathrm{~km} \mathrm{~s}^{-1}\right)$. Thus, this assumption should not induce any problems. (ii) the continuum level in the spectra has been subtracted by dividing a low-order polynomial (zero or first order) from the spectra. (iii) the instrumental line profile is constrained not to vary strongly along the wavelength range covered by the spectra and can be characterised as a function $L(x)$, with $x$ being the location of a specific line. However, variations of the instrumental line profile along the slit are allowed, which can be easily accounted for by taking stellar template spectra at several positions, slightly shifted from each other along the slit (see section 3.7).

As for a template star the line width profile $L(x)$ is identical to the instrumental profile, a template spectra can be defined as line profile $L(x)$ :

$$
\begin{aligned}
& S(x)=\sum_{m} a_{m} L\left(x-x_{m}\right) \\
& S(\lambda)=\sum_{m} a_{m} L\left(\lambda-\lambda_{m}\right)
\end{aligned}
$$

where $x_{m}$ is again the location of the $m$ th absorption line and $a_{m}$ indicates the line strength of the $m$ th absorption line. As the location $x$ 
corresponds to the wavelength range, the equation can be also expressed as a function of $\ln \lambda$ (see equation 5.11). For reasons of simpleness, only $\lambda$ is written although strictly speaking it is $\ln (\lambda)$ as the analysis is in logarithmic space. The line profile $G(\lambda)$ of galaxy spectrum is described by the convolution of the instrumental line profile with the velocity dispersion integrated along the line-of-sight, or also the broadening function $B(\lambda)$.

$$
G(\lambda)=\int B(\lambda-y)\left\{\sum_{m} b_{m} L\left(y-\lambda_{m}\right)\right\} d y
$$

The parameter $b_{m}$ gives the line strength of the $m$ th absorption line and the redshift of a spectrum is represented by the shift of the center of $B(\lambda)$ in the $\lambda$-domain.

Based on the theorem of the classical Fourier quotient method that a convolution is a multiplication in Fourier space, a transformation of the galaxy spectrum $G(\lambda)$ results in:

$$
\tilde{G}(\kappa)=\int G(\lambda) e^{i \kappa \lambda} d \lambda=\sum_{m} b_{m} e^{i \kappa \lambda_{m}} \tilde{B}(\kappa) \tilde{L}(\kappa)
$$

with the superscript tilde denoting the transformed functions. The parameter $i$ is the imaginary unit, and the exponential form of a complex number $z$ in polar coordinates is $z=\rho e^{i \kappa}$, where $\rho$ is defined as the modulus and $\kappa$ the argument. Therefore, are $G(\lambda)$ and $e^{i \kappa \lambda}$ the real and the imaginary part of the function $\tilde{G}(\kappa)$, respectively. In an analogous way the Fourier transformation of the template spectrum $S(\lambda)$ yields the transformed template function $\tilde{S}(\kappa)$, but without the broadening function $\tilde{B}(\kappa)$. A reliable broadening function can only be derived if $a_{m}=b_{m}$ for all $m$, in other words if the instrumental line profiles of the star and the galaxy are identical. A slight mismatching would introduce wave patterns and thus fail to deduce a correct broadening function. The wave patterns $\omega$ affect the broadening function as

$$
\frac{\tilde{G}(\kappa)}{\tilde{S}(\kappa)}=\omega \tilde{B}(\kappa)
$$

with $\omega=\frac{\sum_{m} b_{m} e^{i \kappa \lambda_{m}}}{\sum_{n} a_{n} e^{i \kappa \lambda_{n}}}$. At this point the FCQ method comes into play. It uses an analogous way as the classical Fourier quotient method but with the difference of deconvolving the correlation peak of the template-galaxy correlation function $\left(K_{S, G}(z)\right)$ with the peak of the autocorrelation function of the star $\left(K_{S, S}(z)\right)$. As a consequence the Fourier transformations of $K_{S, G}(z)$ and $K_{S, S}(z)$ yield the same result as in equation 5.14 (see Bender 1990 for details). However, in contrast to the classical Fourier quotient technique, solely the correlation peaks are deconvolved (instead of the complete correlation functions). The resulting formulae are a combination of two terms, between the correlation peak plus the correlation function outside the correlation peak. Using a minimisation of the absorption lines of the spectra, the distance between the correlation peak and the nearest secondary peak can be derived. This distance is independent of the broadening of the spectra. Assuming that the width of the broadening function $B(\lambda)$ and the width of instrumental line profile $L(\lambda)$ are described by velocity dispersions $\sigma_{B}$ and $\sigma_{L}$, the correlation peak will not affect the closest secondary peak if the two peaks are separated larger than approximately two times of their FWHM. For this case, the deconvolution can be restricted to a relatively narrow (wavelength) interval around the correlation peaks and the second terms can be neglected. The resulting correlation functions of the peaks are defined as:

$$
\frac{\tilde{K}_{S, G, \text { peak }}(\kappa)}{\tilde{K}_{S, S, \text { peak }}(\kappa)}=\frac{\sum_{m} a_{m} b_{m}}{\sum_{n} a_{n} a_{n}} \tilde{B}(\kappa)
$$

In other words, the correlation functions of the peaks are described by a constant factor $C$ times 

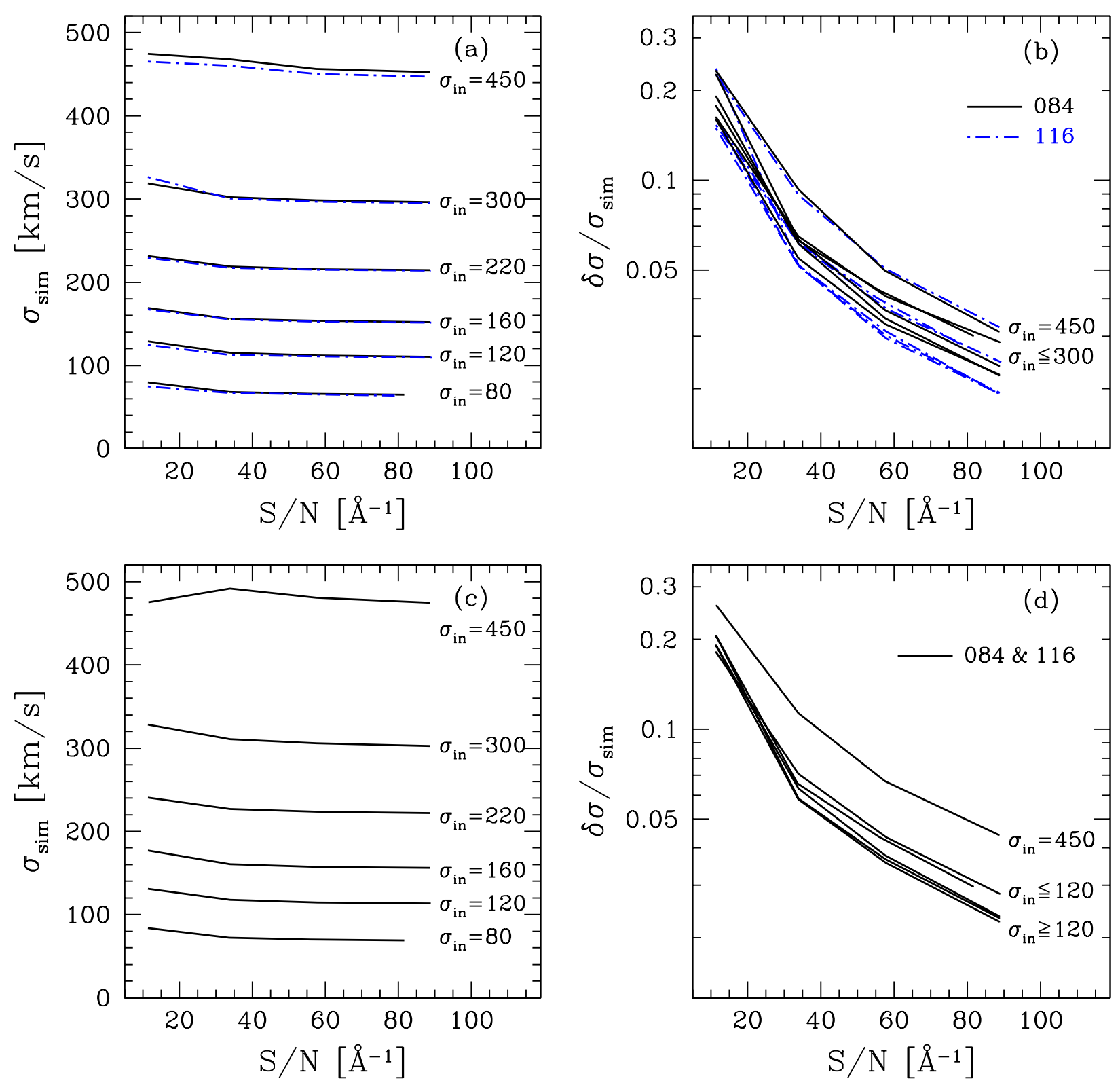

Figure 5.4: Monte Carlo simulations for the MOSCA setup. Left: For a range of input velocity dispersions $\left(\sigma_{\text {in }}\right)$ the simulated velocity dispersion $\left(\sigma_{\text {sim }}\right)$ is displayed as a function of $\mathrm{S} / \mathrm{N}$ per $\AA$. Panel (a) using the same star as "galaxy" and template and panel (c) using a different star as template. Right: Scatter of the distribution as a function of S/N per Å. Panel (b) same star as template and galaxy, Panel (d) different star as template and galaxy. Velocity dispersions are systematically underestimated, on average by $4 \%$ (a,b) and $5 \%$ (c,d). For $\sigma_{\text {in }} \geq 300$ and $\mathrm{S} / \mathrm{N}<10$ the $\sigma_{\text {sim }}$ are systematically overestimated by $\sim 6 \%$. Different lines indicate the results for different stars: Panel (a,b) 084 (solid), 116 (dot-dashed) and panel (c,d) 084 \& 116 (solid). 
mite coefficients $h_{3}$ and $h_{4}$ which characterise the deviations from the Gaussian shape of the LOSVD, cannot be recovered. Therefore, the Hermite coefficients are set to zero $h_{3,4}=0$ and only a Gaussian function is fitted to the quotient of the galaxy spectra and the template spectra in Fourier space. (ii) Subtraction of continuum level. Usually, the continuum in the spectra is subtracted by dividing a low-order polynomial of zero or first order. However, several test in fitting orthogonal polynomials along the dispersion of the 1D stellar templates and the galaxy spectra revealed that a polynomial of fourth-order is the best approximation for both continuae. For this reason, the continuum level in the template and galaxy spectra was subtracted by dividing a fourth-order polynomial from the spectra.

(iii) Calibration of errors. The errors derived with the FCQ method are based on the $\mathrm{S} / \mathrm{N}$ in the continuum of the spectra. In particular, the uncertainties depend on the $\mathrm{S} / \mathrm{N}$, the wavelength region and the number of absorption lines. As for each set of templates, the wavelength region and number of absorption lines varies, adjustments to the variables which define the error terms have to be made. Using 100 Monte Carlo simulations for each stellar template the errors of FCQ were calibrated on the basis of a set of input S/N. In particular, for a range of values of $\mathrm{S} / \mathrm{N}$ of 10,30 , 50 , and 80 per $\AA$ and velocity dispersions $\sigma_{\text {in }}$ of 80, 120, 160, 220, 300, and $450 \mathrm{~km} \mathrm{~s}^{-1}$ Monte Carlo simulations for synthetic generated galaxy spectra were performed, thereby adding artificial noise and accounting for the instrumental sampling and resolution. In the next step, the velocity dispersions $\sigma_{\text {sim }}$ were recovered by the FCQ software. The results of the Monte Carlo simulations for two different templates are shown in Fig. 5.4.

For each input velocity dispersion $\sigma_{\text {in }}$ the recovered $\sigma_{\text {sim }}$ as a function of $\mathrm{S} / \mathrm{N}$ is displayed. In panel (a) two examples using the same stellar spectrum as "galaxy" and template are shown (\#084 (K0III) and \#116 (K3III)), and in panel (c) a different star as template (K3III) is adopted. For all values of between $80 \leq \sigma_{\text {in }} \leq 300 \mathrm{~km} \mathrm{~s}^{-1}$ the velocity dispersions are systematically underestimated, on average by $4 \%$. At $\sigma_{\text {in }} \geq 300$ and $\mathrm{S} / \mathrm{N}<10$ the $\sigma_{\text {sim }}$ are systematically overestimated by $\sim 6 \%$. For the smallest values of $\sigma_{\text {in }}=80 \mathrm{~km} \mathrm{~s}^{-1}$ the fit values are on average lower by $14 \%$. But with increasing velocity dispersion the deviations drop quickly: $4 \%$ for $\sigma_{\text {in }} \leq 100 \mathrm{~km} \mathrm{~s}^{-1}$ for a given $\mathrm{S} / \mathrm{N}$ and only $2 \%$ for $\sigma_{\text {in }} \leq 100 \mathrm{~km} \mathrm{~s}^{-1}$ and $\mathrm{S} / \mathrm{N}>10$. For panel (c) the situation is similar. On average, the velocity dispersions are systematically underestimated by $\sim 5 \%$. However, for $\sigma_{\text {in }}=80 \mathrm{~km} \mathrm{~s}^{-1} \sigma_{\text {sim }}$ are underestimated by $10 \%$, for $\mathrm{S} / \mathrm{N}=10$ by $4 \%$. Again for higher velocity dispersions the uncertainties are lower by $4 \%$ for $\sigma_{\text {in }} \leq 100 \mathrm{~km} \mathrm{~s}^{-1}$ at each $\mathrm{S} / \mathrm{N}$ and $3 \%$ for $\mathrm{S} / \mathrm{N}=10$. The conclusion from the simulations is that the systematic uncertainty on small values of $\mathrm{S} / \mathrm{N}$ is hard to correct. For $\mathrm{S} / \mathrm{N} \geq 15$ the effect becomes negligible but data with $\mathrm{S} / \mathrm{N}<10$ should be rejected. In Fig. 5.4 also the relative scatter around the mean for the same configurations is displayed (template \# 084 and \# 116, panel (c); template \#084 \& \#116 panel (d)). For a given $\mathrm{S} / \mathrm{N}$, the uncertainty on the velocity dispersions is even small for the smallest $\sigma_{\text {in }}$ values. Only for the largest input velocity dispersions of $\sigma_{\text {in }}=450 \mathrm{~km} \mathrm{~s}^{-1}$, the error is slightly larger for lower $\mathrm{S} / \mathrm{N}$. However, as usually these bright galaxies offer high $\mathrm{S} / \mathrm{N}$ values of $\mathrm{S} / \mathrm{N}>40$, the systematic uncertainty becomes negligible.

In general, for $\mathrm{S} / \mathrm{N}$ of the same value, the error estimated by FCQ were a factor 1.3 smaller than the rms of the error values in the Monte Carlo simulation, which corresponds to $\sigma_{\text {sim }}$ values that are $5 \%$ too low. To account for this effect, this derived error parameter was fixed for all subsequent velocity dispersion measurements to ensure a reliable error treatment.

Before feeding the FCQ algorithm, a number of important input parameters $\left(\sigma_{\text {inst }}\right.$, scale, row, redshift and broad) were gained in a semi- 


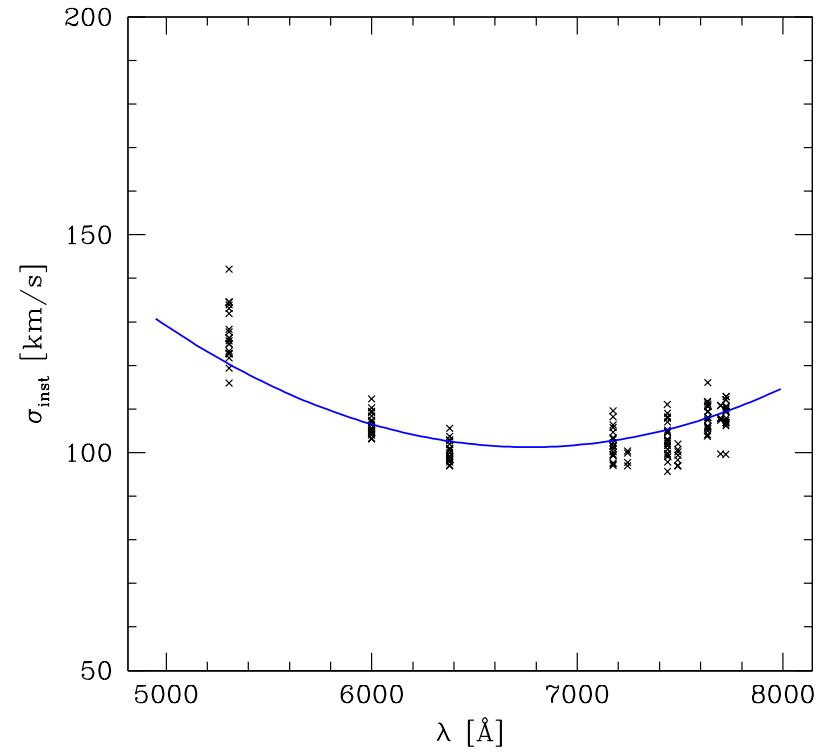

Figure 5.5: Instrumental resolution $\sigma_{\text {inst }}$ as a function of wavelength as determined from the width of $\mathrm{HgAr} / \mathrm{Ne}$ lines for the MOSCA setup. Symbols represent individual measurements, the solid line the best fitting parabola.

automatic manner using a special program. In the following a short summary of the required analysis steps for each galaxy is given:

- Measurement of the instrumental resolution of the galaxy spectra. For this purpose, a set of 12-14 unblended, isolated and notsaturated $\mathrm{HgAr} / \mathrm{Ne}$ lines in the wavelength calibration frames were selected and their FWHM width were measured for ten different positions over the whole wavelength region (5300 $\AA \lesssim \lambda_{\text {obs }} \lesssim 7725 \AA$ corresponding to $4300 \AA \lesssim \lambda_{\text {rest }} \lesssim 6260 \AA$ ). This was performed on frames in pixel space, thereby covering the same wavelength region as for the galaxy spectra under consideration. The results were stable from frame to frame and for different zenithal positions of the telescope as well as for different nights. Fig. 5.5 shows the instrumental resolution $\sigma_{\text {inst }}$ as a function of wavelength as determined from the width of $\mathrm{HgAr} / \mathrm{Ne}$ lines for the MOSCA setup. Each point corresponds to a single
Table 5.1: Instrumental resolution for MOSCA.

\begin{tabular}{|c|c|c|c|c|c|}
\hline$\lambda_{\mathrm{obs}}$ & $N$ & $\bar{\sigma}_{\text {inst }}$ & $\left\langle\sigma_{\text {inst }}\right\rangle$ & $\bar{\sigma}_{\text {inst }}$ & $\left\langle\sigma_{\text {inst }}\right\rangle$ \\
\hline \multicolumn{2}{|l|}{$\AA$} & \multicolumn{2}{|c|}{$[\AA \mathrm{FWHM}]$} & \multicolumn{2}{|c|}{$\left[\mathrm{km} \mathrm{s}^{-1}\right]$} \\
\hline 5306 & 20 & $5.28 \pm 0.25$ & 5.24 & $127.1 \pm 6.3$ & 126.0 \\
\hline 5998 & 17 & $5.06 \pm 0.19$ & 5.00 & $106.5 \pm 2.6$ & 105.6 \\
\hline 6380 & 17 & $5.02 \pm 0.19$ & 4.99 & $100.2 \pm 2.5$ & 99.2 \\
\hline 7174 & 17 & $5.75 \pm 0.21$ & 5.71 & $102.3 \pm 3.8$ & 101.5 \\
\hline 7245 & 4 & $5.61 \pm 0.09$ & 5.67 & $98.7 \pm 1.7$ & 99.9 \\
\hline 7439 & 24 & $6.03 \pm 0.22$ & 5.99 & $103.4 \pm 3.9$ & 102.7 \\
\hline 7489 & 8 & $5.81 \pm 0.11$ & 5.84 & $99.0 \pm 2.0$ & 99.4 \\
\hline 7634 & 19 & $6.48 \pm 0.19$ & 6.47 & $108.3 \pm 3.2$ & 108.0 \\
\hline 7697 & 6 & $6.48 \pm 0.25$ & 6.51 & $107.4 \pm 4.2$ & 108.0 \\
\hline 7723 & 17 & $6.59 \pm 0.20$ & 6.62 & $108.9 \pm 3.3$ & 109.4 \\
\hline
\end{tabular}

measurement of an unblended $\mathrm{HgAr} / \mathrm{Ne}$ line in one calibration frame. For testing purposes, in case of the G4300-band (G-band hereafter), $\mathrm{H} \beta$ and $\mathrm{Mg}_{b}$ feature, additional measurements were performed within these wavelength ranges and the mean resolution of 3-5 calibration lines computed to verify that during one night $\sigma_{\text {inst }}$ remains at the same resolution for each single slit observation. Results of the two approaches were compared to each other and are consistent within the errors. For this reason, the entire set of single measurements was used to establish a relation for the resolution of the spectra as a function of wavelength. As shown in Fig. 5.5, the measurements for the resolution $\left(\mathrm{km} \mathrm{s}^{-1}\right)$ are well described by a parabola.

All measurements in Fig. 5.5 are listed in Table 5.1. For each wavelength the mean $\left(\bar{\sigma}_{\text {inst }}\right)$ and median $\left(\left\langle\sigma_{\text {inst }}\right\rangle\right)$ values of the instrumental resolution in units of $\AA$ FWHM and $\mathrm{km} \mathrm{s}^{-1}$ are given. $N$ shows the number of individual measurements, for the Gband, $\mathrm{H} \beta$ and $\mathrm{Mg}_{b}$ band the average values. Typical numbers for the instrumental resolution of MOSCA spectra are between $97 \leq \sigma_{\text {inst }} \leq 130 \mathrm{~km} \mathrm{~s}^{-1}$. The wavelength range around the $\mathrm{H} \beta$ and $\mathrm{Mg}_{b}$ feature 
$(\approx 6119 \AA)$ has a $\sigma_{\text {inst }}$ of $\sim 3.84$ pixel $(4.99 \AA$ FWHM) corresponding to $104 \mathrm{~km} \mathrm{~s}^{-1}$ and around the G-band $(\approx 5290 \AA) \sigma_{\text {inst }}$ takes values of $\sim 4.03$ pixel $(5.24 \AA$ FWHM) corresponding to $126 \mathrm{~km} \mathrm{~s}^{-1}$. The instrumental resolution of the FORS spectra were derived in the same way as for the MOSCA spectra. For example, the galaxy WHDF \# 173 $(z=0.4507)$, has a $\sigma_{\text {inst }}$ at the $\mathrm{Mg}_{b}$ band of $\sim 3.61$ pixel (5.91 $\AA$ FWHM) or $100 \mathrm{~km} \mathrm{~s}^{-1}$ and around the G-band $\sim 3.75$ pixel $(6.14 \AA$ FWHM), which corresponds to $126 \mathrm{~km} \mathrm{~s}^{-1}$.

- Calculation of the number of extracted rows (row) over which the two-dimensional spectra was summed up (see also section 5.3.2).

- Determination of the instrumental resolution of the template star spectra. The kinematic templates which were observed through a $0.5^{\prime \prime}$ longslit aperture have typical instrumental resolutions at the $\mathrm{H} \beta$ and $\mathrm{Mg}_{b}$ feature of $\sim 2.3 \AA \mathrm{FWHM}$, corresponding to $\sigma_{*}=58 \mathrm{~km} \mathrm{~s}^{-1}$ and around the Gband $\sim 2.3 \AA$ FWHM, which corresponds to $\sigma_{*}=71 \mathrm{~km} \mathrm{~s}^{-1}$. A complete list for all stellar templates together with their measured resolution can be found in Table A.1 on page 181.

- Since the slitlets of the galaxies had small variations in width size and were not identical in width to the longslit of the stars, a simple comparison and interpretation of the calculated velocity dispersions would be wrong. To correct for this effect, the following correction procedure to the stars was applied before using them as templates in the FCQ algorithm to compute the velocity dispersion for each galaxy. First, the stars and galaxies were rebinned to a fixed logarithmic wavelength scale. Afterwards the spectra of the stars are broadened onto the same instrumental resolution (in $\mathrm{km} \mathrm{s}^{-1}$ ) as the spectra of the target galaxy using the relation

$$
\text { broad }=\sqrt{\sigma_{\text {inst Gal }}^{2}-\sigma_{\text {inst Star }}^{2}}
$$

where broad denotes the factor of broadening for the stellar spectra. This procedure has the advantage that the galaxy velocity dispersion results directly and no additional correction of the velocity dispersion of the template star $\sigma_{\text {star }}$ is needed.

- Extraction of a spectral wavelength range of the broadened stars which is used as an input for FCQ. Typical wavelength ranges for analysing the G-band, the $\mathrm{Mg}_{b}$ and the $\mathrm{H} \beta$ feature cover a region between $\lambda \lambda 4142-4798 \AA$ (G4300-Fe4668), $\lambda \lambda$ 5000$5500 \AA$ ([O III] - Fe 5335) and $\lambda \lambda 4606-5116 \AA$ (around $\mathrm{H} \beta$ ), respectively.

- Extraction of an equivalent spectral range in the target galaxy spectra as for the templates and analysis of this region via the FCQ method. Residuals of night sky lines within this wavelength range are masked out in order to not affect the fitting process of the broadened stars to the galaxy spectra. In particular, the masking of telluric lines was performed in setting the flux value to a fixed mean value which was derived over the adjacent continuum level of the spectrum.

- Derivation of velocity dispersions $(\sigma)$ and radial velocities $\left(v_{\text {rad }}\right)$ via the FCQ analysis by deconvolving the peak of the templategalaxy correlation function with the peak of the autocorrelation function of the template star (see previous section 5.2 for the theoretical description). A minimum of at least three templates was used for determining $\sigma$, $v_{\text {rad }}$ and $S / N$ for each galaxy. All individual parameter measurements were compared to each other (see next section 5.3.1). If the single $\sigma$ and $v_{\text {rad }}$ values varied only within their errors the mean value of the individual determinations was calculated. Velocity 


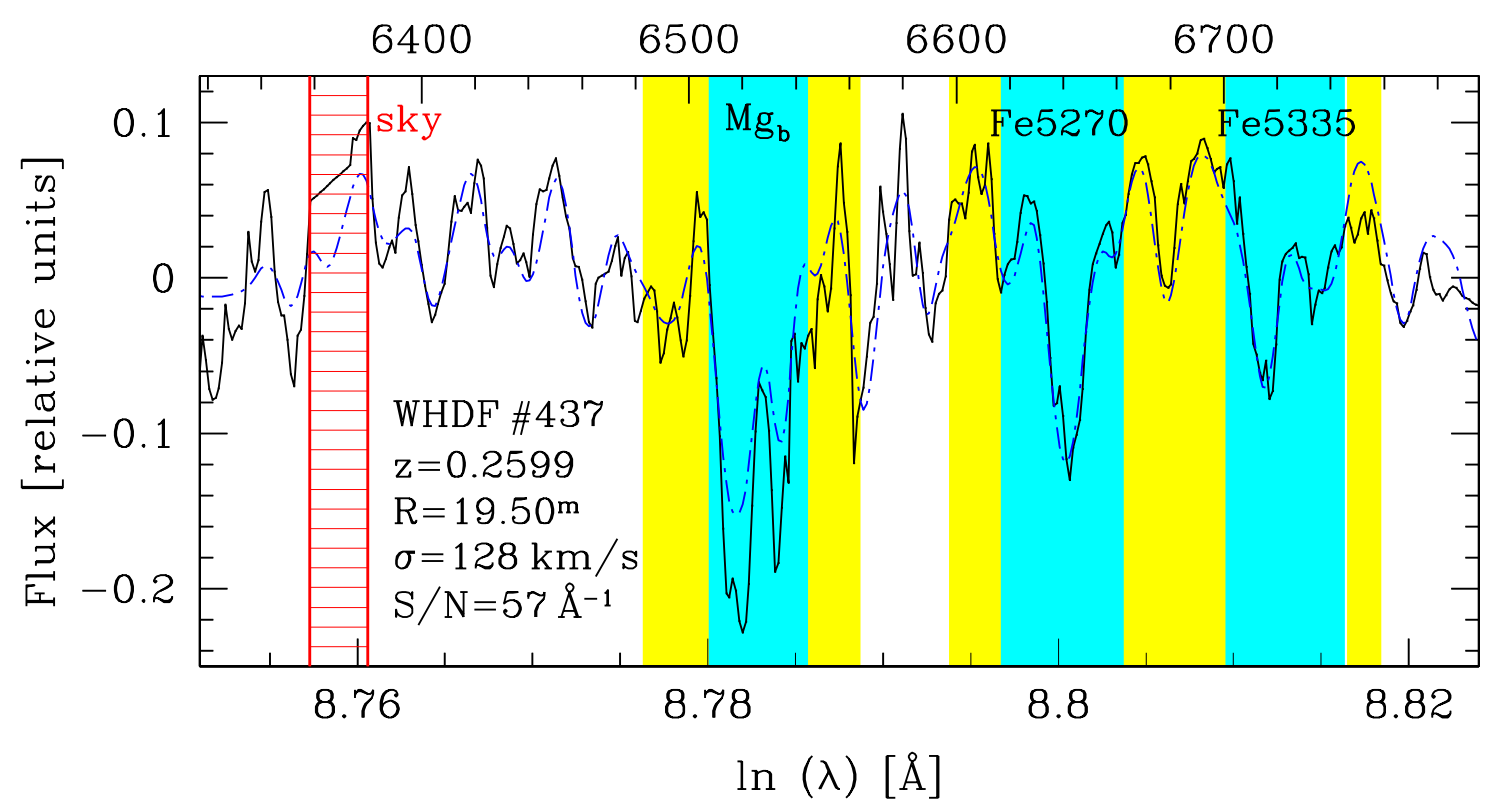

Figure 5.6: Visual inspection of the velocity dispersion measurement using the $\mathrm{Mg}_{b}$ feature for the field elliptical galaxy WHDF \# 437 with $R=19.50^{\mathrm{m}}$ at $z=0.260$. The galaxy spectrum (solid line) is compared to the star spectrum (dot-dashed) which is broadened to the derived $\sigma$ of the galaxy. Absorption lines of $\mathrm{Mg}_{b}$ Fe5270 and Fe5335 are shown, splitted into their defined feature passbands (blue and red continuum and central passband). The central and continuum bands are indicated as shaded regions. The spectral region labelled as 'sky' is affected by the telluric line at $\lambda=6367 \AA$. The lower $\mathrm{X}$-axis displays the spectrum in logarithmic units, the upper X-axis in linear units (both observed wavelengths in $\AA$ ).

dispersions with too large errors resulting from low $\mathrm{S} / \mathrm{N}$ spectra were rejected from the averaging process. To test possible variations in observing conditions and to identify spectra with low $\mathrm{S} / \mathrm{N}$, the sum of individual spectra for each night was compared to the combined spectra of all nights. With this method, exposures with very low $\mathrm{S} / \mathrm{N}$ could easily be identified and excluded from the final summarisation procedure. For example, for the A 2390 galaxies the $\sigma$ determinations of the summarised spectra of five different nights were compared to the final combined spectrum of all nights. For the poor cluster galaxies, the sum of individual spectra for three different nights and the summarised spectrum of all three nights were compared to each other. In general, the agreement was very good and for a few cases up to two sin- gle spectra had to be rejected in the final averaging process. For six out of 98 cluster galaxies, no reliable measurement was possible. These objects have very low signal ( $\mathrm{S} / \mathrm{N} \lesssim 8$ per $\AA$ ) and three are faint background spiral galaxies.

In case of the Low $-L_{X}$ clusters and the two field galaxy samples, the $\mathrm{S} / \mathrm{N}$ in all galaxy spectra was sufficient to derive velocity dispersions. A complete summary of the checks on the velocity dispersions determinations will be presented in the next section.

\subsubsection{Visual Inspection of $\sigma$}

To verify the quality and reliability of the velocity dispersion measurements, the individual results for each broadened star spectra have to be compared to the target galaxy of interest. The inspection of the $\sigma$ determinations was per- 


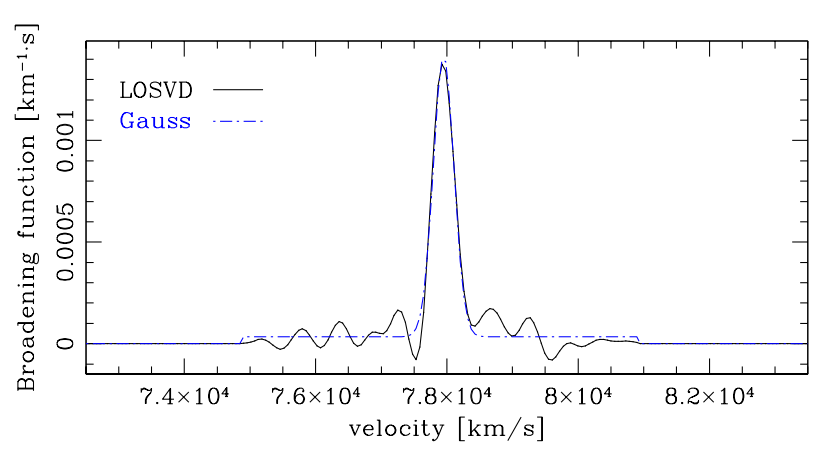

Figure 5.7: Comparison of the Wiener filtered LOSVD (solid line) derived with the FCQ method using the $\mathrm{Mg}_{b}$ feature with the broadening function resulted from a fit by a Gauss-Hermite series (dotdashed line) with $h_{j}=0$ for the field elliptical galaxy WHDF \# 437. The broadening function gained from the FCQ method reproduces the LOSVD extremely well and is hardly distinguishable from it.

formed in Fourier space, which provides a direct comparison between the LOSVD and the FCQ results. For each galaxy, following checks on $\sigma$ have been executed:

- Comparison of the broadened template star spectra with the galaxy spectrum. Fig. 5.6 shows an example for the broadened template star \# 157 (dot-dashed line) compared to the spectrum of the field elliptical galaxy WHDF \# 437 at $z=0.2599$ (solid line) with an apparent total brightness of $R=19.50^{\mathrm{m}}$. As only a narrow wavelength range of the spectrum is displayed, the logarithmic axis $\ln (\lambda)$ appears to be linear but actually it is a weak increasing function towards longer wavelengths. The final velocity dispersion for this galaxy is $\sigma=128 \pm 8 \mathrm{~km} \mathrm{~s}^{-1}$ with a signal-to-noise level of $S / N=57$ per $\AA$ derived within the extracted spectral region around the $\mathrm{Mg}_{b}$ feature. This value was averaged over three individual measurements $\sigma_{084}=127 \pm 8 \mathrm{~km} \mathrm{~s}^{-1}, \sigma_{116}=134 \pm 8 \mathrm{~km} \mathrm{~s}^{-1}$ and $\sigma_{157}=124 \pm 10 \mathrm{~km} \mathrm{~s}^{-1}$ which are in very good agreement as the scatter of the values are within their uncertainty.
- A comparison of the line-of-sight-velocitydistribution with broadening function is performed. In particular, the Wiener filtered Fourier transform (LOSVD) is the output of the FCQ algorithm and the broadening function is characterised by a pure Gaussian. As the Gauss-Hermitepolynomial coefficients $h_{j}$ were set to zero, only a Gaussian was fitted to the LOSVD of the galaxy. Fig. 5.7 compares the filtered LOSVD to the broadening function $B(\lambda)$. The Gaussian function recovers the LOSVD extremely well and is nearly indistinguishable from it. By contrast, the standard Fourier quotient method becomes strongly affected even by slight mismatching of the template star. For example, using a K0 III star instead of a K3 III star underestimates the velocity dispersion of the recovered broadening function by $\approx 5 \%$ (corresponding to $10 \mathrm{~km} \mathrm{~s}^{-1}$ for a typical galaxy with $\sigma_{\text {Gal }}=200 \mathrm{~km} \mathrm{~s}^{-1}$ ). However, this small mismatch is hardly detectable through a visual inspection of the template's spectra (see also Fig. 3 of Bender 1990). The width of the Gaussian function (broadening function) in Fig. 5.7 represents the velocity dispersion of the object and the position on the $\mathrm{X}$-axis indicates the distance to the target galaxy. If the position of the broadening function is slightly shifted, the radial velocity of the galaxy was not exactly measured. On the other hand, if the broadening function cannot completely recover the width of the LOSVD, the absorption line might be affected by weak sky line residuals and the total flux of the absorption feature cannot be measured, which results in an uncertain velocity dispersion of the galaxy.

- Comparison of $B(\lambda)$ with the peak of the correlation function, see Fig. 5.8.

At a redshift of $z=0.231$ the $\mathrm{Mg}_{b}$ line is strongly affected by the telluric emission line at $\lambda=6367 \AA$. In particular, for the clusters A 2390 


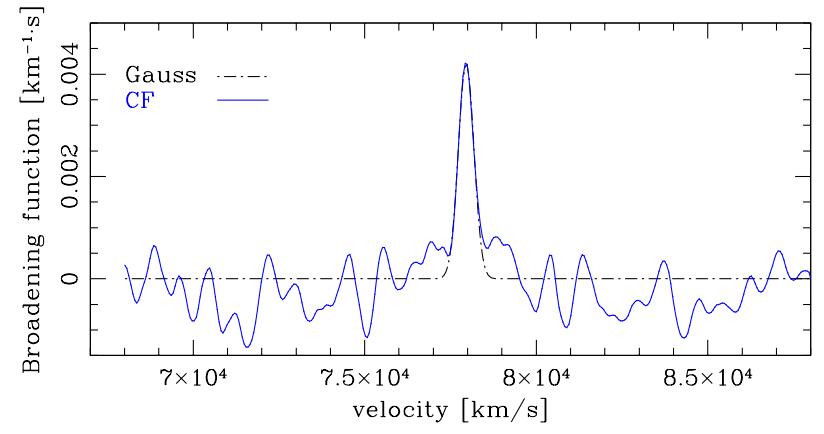

Figure 5.8: Comparison of the LOSVD (dot-dashed line) derived by a simple Fourier transformation $(\tilde{G})$ assuming the region around the $\mathrm{Mg}_{b}$ band with the correlation function ( $\mathrm{CF}$, solid line) for the galaxy WHDF \# 437. The wings of the correlation function vary strongly because no filter was applied.

$(z=0.228)$ and the Low- $L_{X}$ clusters $\mathrm{Cl} 0849+37$ $(z=0.2343)$ and $\mathrm{Cl} 1702+64(z=0.2233)$ the $\mathrm{Mg}_{b}$ feature falls around the mean cluster redshift, thereby causing problems when the derivation of velocity dispersions is based solely on this wavelength region. For this reason, three separate wavelength regimes were analysed. The first wavelength range between $\lambda \lambda_{\text {obs }}=6204-$ $6748 \AA$ was centered on the $\mathrm{Mg}_{b}$ feature. In those cases where the $\mathrm{Mg}_{b}$ line was affected, either in the central or in the adjacent continuum windows (see Fig. 5.6), a reliable measurement of the velocity dispersion could not be ensured so that a second calculation in the range around the G4300 band between $\lambda \lambda_{\text {obs }}=4964-5541 \AA$ was performed. In addition, for two masks of the A 2390 sample a wavelength range around the $\mathrm{H} \beta$ feature $\lambda \lambda_{\text {obs }}=5598-6248 \AA$ was analysed.

\subsubsection{Aperture Correction}

Early-type galaxies feature radial gradients in their velocity dispersion, radial velocity and in the $\mathrm{Mg}_{2}$ and $\mathrm{Fe}$ indices. For this reason, depend the central measurements of these parameters on the distance of the galaxies and the size of the aperture. The apparent sizes of distant galaxies are so small that a slitlet of width $1-1.5^{\prime \prime}$ covers a large fraction of the galaxy's light distribution, typically $1-2$ half-light radii $R_{\mathrm{e}}$. The derived velocity dispersions and line strengths are only luminosity-weighted average measurements, but are not equivalent to the central values of local galaxies if a radial gradient across the galaxy exists. To take this effect into account, an aperture correction was applied to the observed velocity dispersions based on the logarithmic gradients as outlined by Mehlert et al. (2003), who measured central velocity dispersions and absorption line strengths in high- $S / N$ spectra of 35 earlytype Coma cluster galaxies. At a later stage, the A 2390 cluster sample will be combined with the a sample of the cluster A 2218. Therefore, for reasons of consistency, the velocity dispersions of the A 2218 galaxies were re-analysed using this aperture correction. Velocity dispersions of cluster and field galaxies were aperture corrected via the relation

$$
\Delta(\log \sigma)=0.06 \cdot \log \left(\frac{d_{\text {Clus }}}{d_{\text {Coma }}} \cdot \frac{a_{\text {Clus }}}{a_{\text {Coma }}}\right)
$$

where $d_{X}$ is the angular distance of the target cluster under consideration, $d_{\text {Clus }}$ or the reference cluster $d_{\text {Coma }}$, and $a_{X}$ is the aperture radius of the target or the local counterpart, respectively. For each galaxy, the aperture $a$ during the observations is given by:

$$
a_{\text {Clus }}=\sqrt{\frac{r \cdot \text { row } \cdot \text { scale }}{\pi}},
$$

where $r$ is the slit width (for A 2218 and A 2390 and the three poor clusters $1.5^{\prime \prime}$, for the FDF and WHDF ellipticals 1.0" was used), row denotes the number of extracted rows over which the spectrum was averaged by the Horne algorithm, which differs from galaxy to galaxy and was measured for each object individually and scale represents the scale in arcsec/pixel. Median values of row are 4.7 pixels, corresponding to $2.8^{\prime \prime}$ for A 2218, 8.4 pixels (2.8") for A 2390, 12.28 pixels $\left(4.1^{\prime \prime}\right)$ for $\mathrm{Cl} 0849,10.89$ pixels $\left(3.6^{\prime \prime}\right)$ for $\mathrm{Cl} 1701,10.49$ pixels $\left(3.5^{\prime \prime}\right)$ for $\mathrm{Cl} 1701,6.46$ 
pixels $\left(1.3^{\prime \prime}\right)$ for the FDF and 7.61 pixels $\left(1.9^{\prime \prime}\right)$ for the WHDF galaxies, respectively. This logarithmic gradient is slightly steeper than the one proposed by Jørgensen, Franx \& Kjærgaard (1995) and Jørgensen (1999):

$$
\Delta(\log \sigma)=0.04 \cdot \log \left(\frac{d_{\text {Clus }}}{d_{\text {Coma }}} \cdot \frac{a_{\text {Clus }}}{a_{\text {Coma }}}\right)
$$

However, aperture corrections based on either gradient are consistent within their errors. To match the circular aperture with a radius of $1.7^{\prime \prime}$ of the Coma cluster galaxies by Jørgensen et al. $(1995,1999)$, the spectroscopic data was corrected following the correction formula as given by equation 5.18. For the $\sigma$ measurements this results in median corrections for the cluster A 2218 of $\Delta\left(\log \sigma_{\mathrm{A} 2218}\right)=+0.039$, and the clusters A 2390 of $\Delta\left(\log \sigma_{\mathrm{A} 2390}\right)=+0.042$, $\mathrm{Cl} 0849$ of $\Delta\left(\log \sigma_{0849}\right)=+0.048, \mathrm{Cl} 1701$ of $\Delta\left(\log \sigma_{1701}\right)=+0.047$ and for $\mathrm{Cl} 1702$ of $\Delta\left(\log \sigma_{1702}\right)=+0.045$, and for the field galaxies in the FDF of $\Delta\left(\log \sigma_{\mathrm{FDF}}\right)=+0.034$ and in the WHDF of $\Delta\left(\log \sigma_{\mathrm{WHDF}}\right)=+0.040$, respectively.

\subsubsection{Comparison between Different Absorption Features}

For the majority of the early-type galaxies, two different spectral bands could be used for the derivation of $\sigma$. However, for some clusters with a mean cluster redshift around $\left\langle z_{\text {Clus }}\right\rangle \approx 0.231$ the $\mathrm{Mg}_{b}$ absorption line is impractical due to strong sky lines in this wavelength region. Therefore, an additional second region around the G4300 index band was analysed in the case of A 2390, $\mathrm{Cl} 0849$ and $\mathrm{Cl} 1702$. Moreover, for A 2390 a third wavelength range around $\mathrm{H} \beta$ was used as a further verification. For the field galaxies the situation changed for each object because different absorption passbands get affected due to sky lines. Nevertheless, at least one $\sigma$ measurement around the $\mathrm{Mg}_{b}$ or G-band was possible. In four WHDF field galaxy spectra, both features were free of telluric emission lines and two independent $\sigma$ results could be derived.
For each galaxy, the fiducial value of the velocity dispersion is based on the comparisons of the broadened template star spectra with the galaxy spectrum, the line-of-sight-velocity-distribution with the Gauss-Hermite (GH) polynomial fit and the Fourier output results with the correlation function (cf. section 5.3.1). As a further consistency check, it is worthwhile to compare the velocity dispersion measured from different absorption feature passbands. For the following tests, the aperture corrected velocity dispersions of distant samples are used.

The reference velocity dispersion measurements are indicated as $\sigma_{1}$, whereas velocity dispersions derived from other features are denoted with the corresponding absorption line. As for the reference $\sigma$ values, the $\sigma$ determinations of the comparison feature were verified with the tests as outlined in section 5.3.1. Based on these verifications, velocity dispersions were classified according to a scheme from 1 to 5 based on their overall quality and accuracy. Galaxies with a quality parameter $Q_{\sigma}=1$ are very good measurements without artifacts or variations in their LOSVD and GH fit and free from any contamination by night sky emission lines. $Q_{\sigma}=2$ are good measurements with slight variations in LOSVD and GH fit and $Q_{\sigma}=3$ corresponds to intermediate quality with some variations in LOSVD and/or slight influences of residual sky lines near the feature of interest. $Q_{\sigma}=4$ represent bad $\sigma$ determinations with strong variations in LOSVD and clear signs of sky lines which deplete and affect parts or the whole absorption line. In cases of $Q_{\sigma}=5$, no measurement was possible.

As very closely to the mean redshift of the A 2390 cluster $(z=0.231)$ the $\mathrm{Mg}_{b}$ line is strongly affected by a telluric emission line $(\lambda=6367 \AA)$, it has to be verified how strong the effect reflects on the velocity dispersion determinations based on the $\mathrm{Mg}_{b}$ feature. In Fig. 5.9 the velocity dispersions based on the $\mathrm{Mg}_{b}$ feature are shown as a function of the $\sigma$ reference values for the A 2390 galaxies. A total of 36 member gal- 


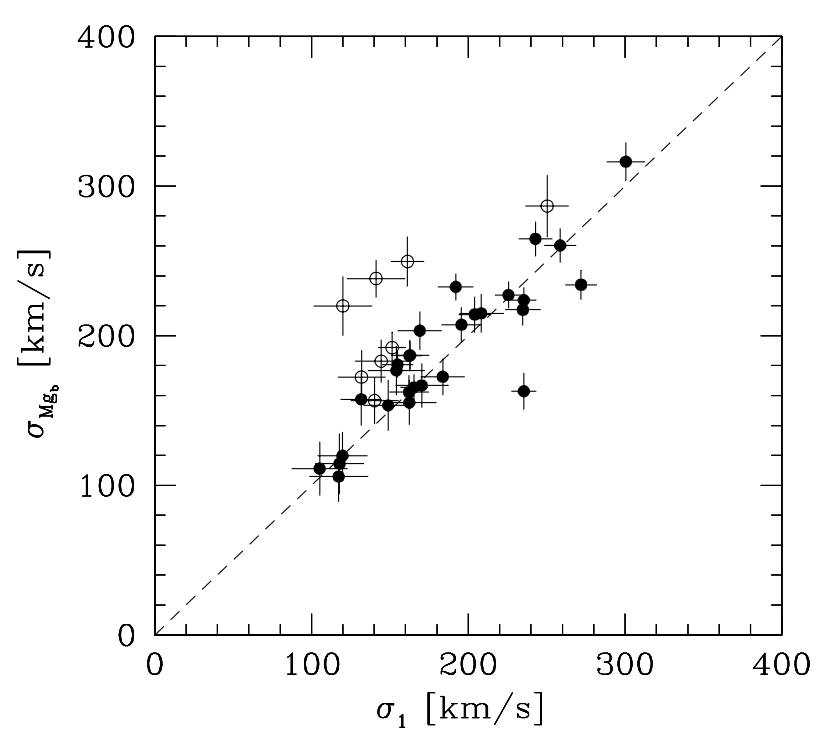

Figure 5.9: Comparison of velocity dispersion measurements derived from different absorption features. The values taken as reference are displayed on the $\mathrm{x}$-axis, velocity dispersions based on the $\mathrm{Mg}_{b}$ feature are shown on the y-axis. Filled symbols denote high quality $\sigma$ measurements, open symbols the low quality $\sigma$ determinations.

axies, splitted into 28 galaxies with $Q_{\sigma}=1$ and $Q_{\sigma}=2$ and 8 objects with $Q_{\sigma}=3$ are compared to each other. 11 galaxies with a quality parameter of $Q_{\sigma}=4$ were discarded and for one object (\# 2106) no $\sigma$ based on the $\mathrm{Mg}_{b}$ feature could be derived. On average, the 28 galaxies with $1 \leq Q_{\sigma} \leq 2$ show a difference of $\Delta\left(\sigma_{1}-\sigma_{\mathrm{mg}}\right)=-6 \pm 17 \mathrm{~km} \mathrm{~s}^{-1}$ with a median $\left\langle\Delta\left(\sigma_{1}-\sigma_{\mathrm{mg}}\right)\right\rangle=-2 \mathrm{~km} \mathrm{~s}^{-1}$. Both numbers are less than the typical internal uncertainty of the velocity dispersions, which is $\delta \sigma>6 \mathrm{~km} \mathrm{~s}^{-1}$. The 8 galaxies with $Q_{\sigma}=3$ have an average difference of $\Delta\left(\sigma_{1}-\sigma_{\mathrm{mg}}\right)=-43 \pm 39 \mathrm{~km} \mathrm{~s}^{-1}$ and a median of $\left\langle\Delta\left(\sigma_{1}-\sigma_{\mathrm{mg}}\right)\right\rangle=-41 \mathrm{~km} \mathrm{~s}^{-1}$. Although a careful analysis of the $\sigma$ determinations was performed, the secondary $\sigma$ determinations based on the $\mathrm{Mg}_{b}$ absorption line yield on average to $1 \%$ and to $27 \%$ higher $\sigma$ values than the reference values for $Q_{\sigma}=1,2$ and for $Q_{\sigma}=3$, respectively. A possible dependence on the mask setup for the galaxies with quality par-
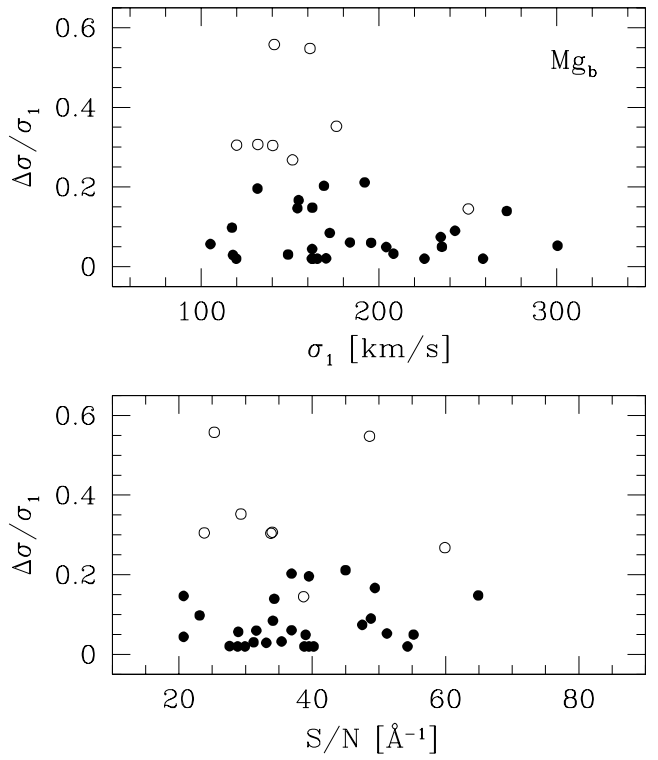

Figure 5.10: Comparison of velocity dispersion measurements derived from different absorption features. Upper panel: relative uncertainty of velocity dispersions with the $\mathrm{Mg}_{b}$ values as a function of velocity dispersions $\sigma_{1}$. Lower panel: $\Delta \sigma / \sigma_{1}$ versus the $S / N$ per Å. Symbol notations as in Fig. 5.9.

ameter of 1 and 2 was not detected, all three sub-samples feature the similar scatter and uncertainties. The velocity dispersions for galaxies with intermediate quality $\left(Q_{\sigma}=3\right)$, are clearly offset. A probably reason is that a weak residual sky lines in the spectra close to the absorption line are present which affect strongly the final velocity dispersion measurement. In particular, all velocity dispersions with $Q_{\sigma} \geq 3$ show higher $\sigma$ values in comparison to their reference value, which gives support to the assumption that a contamination by residual sky lines in the spectra is even existent for the intermediate quality determinations. From this comparison it seems already clearly evident that the telluric emission line around the $\mathrm{Mg}_{b}$ has dramatic effect on the derived velocity dispersions. Before the conclusions are drawn from this test, the influence of the signal-to-noise ratio on the accuracy of the velocity dispersion determinations is discussed. 
The quality of the velocity dispersion measurement and therefore its formal uncertainty depends on the $\mathrm{S} / \mathrm{N}$ in the spectrum, the comparison of the galaxy velocity dispersion with the instrumental resolution and to a lesser extent the chosen wavelength interval used for the determination. Fig. 5.10 displays the formal relative uncertainty $\Delta \sigma / \sigma_{1}$ as a function of velocity dispersion (upper panel) and as a function of the $\mathrm{S} / \mathrm{N}$ (lower panel) per $\AA . \Delta \sigma / \sigma_{1}$ was derived as the relative difference in velocity dispersion values of the G-band with the $\mathrm{Mg}_{b}$ line, normalised to the reference velocity dispersions $\sigma_{1}$. As expected, the deviations of velocity dispersions increase with decreasing $\mathrm{S} / \mathrm{N}$ ratios. The relative uncertainty for galaxies with $Q_{\sigma}=3$ falls into the range $0.14 \leq \Delta \sigma / \sigma_{1} \leq 0.56$ with a median of $\left\langle\Delta \sigma / \sigma_{1}\right\rangle=0.31$. For galaxies with high quality measurements $\left(Q_{\sigma}=1\right)$, the relative errors cover the range $0.02 \leq \Delta \sigma / \sigma_{1} \leq 0.21$ with a median of $\left\langle\Delta \sigma / \sigma_{1}\right\rangle=0.06$. Two effects of the uncertainties can be seen in Fig. 5.10. First, the errors of velocity dispersions with intermediate quality are large even for high $\mathrm{S} / \mathrm{N}$. This means that the $\sigma$ determination for the $Q_{\sigma}=3$ is not reliable and a different absorption line should be used. Second, the uncertainties of the high quality measurements depend only weak on the $\mathrm{S} / \mathrm{N}$, which suggests that the $\sigma$ values are in fairly good agreement. However, as even for the high quality $\sigma \mathrm{Mg}_{b}$ determinations a slight offset to higher $\sigma$ values is found and a possible (not visual detectable) interference due to sky line residuals can not completely be excluded, for the majority of cluster galaxies in A 2390 and the two poor clusters the G-band was used for the derivation of velocity dispersions. For this reason, 45 out of $47 \sigma_{1}$ values for the $\mathrm{A} 2390$ galaxies are based on the G4300 feature, which showed in all cases no contamination of any residual sky lines. Moreover, the G-band was appropriate feature for the poor clusters and was solely used for the $\sigma$ measurements of 15 and 7 cluster members in $\mathrm{Cl} 0849$ and $\mathrm{Cl} 1702$, respectively.
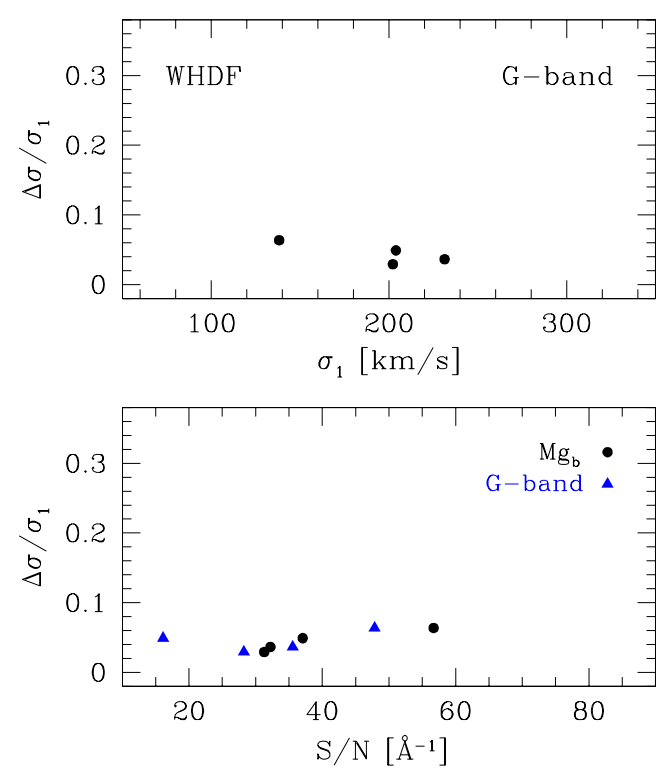

Figure 5.11: Comparison of velocity dispersion measurements derived from different absorption features. Upper panel: relative uncertainty of velocity dispersions with the $\mathrm{G}$-band values as a function of velocity dispersions $\sigma_{1}$ of the WHDF field galaxies. All galaxies are high quality $\sigma$ measurements. Lower panel: $\Delta \sigma / \sigma_{1}$ versus the $S / N$ per $\AA$. Measurements are splitted according to the $S / N$ of the feature $\left(\mathrm{Mg}_{b}\right.$ circles, G-band triangles) to visualise possible systematic errors of low $S / N$ on $\sigma$. For $S / N \gtrsim 15$, effects of systematic uncertainties become negligible.

To test if the $\mathrm{Mg}_{b}$ absorption lines yield accurate velocity dispersion measurements and not any bias is introduced with the restriction to the G-band absorption feature, $\mathrm{Mg}_{b} \sigma$ determinations for four WHDF field early-type galaxies are compared to the $\sigma$ values derived based on the G-band feature. Fig 5.11 shows the formal relative uncertainty $\Delta \sigma / \sigma_{1}$ as a function of velocity dispersion (upper panel) and as a function of the S/N (lower panel) per $\AA$ for the WHDF early-type galaxies where both measurements are available. The lower panel is divided according to the $S / N$ of the feature of interest, $\mathrm{S} / \mathrm{N}$ values from the $\mathrm{Mg}_{b}$ lines are denoted as circles, whereas the $\mathrm{S} / \mathrm{N}$ based on the $\mathrm{G}$-band is indicated as triangles. Again, $\Delta \sigma / \sigma_{1}$ was computed 


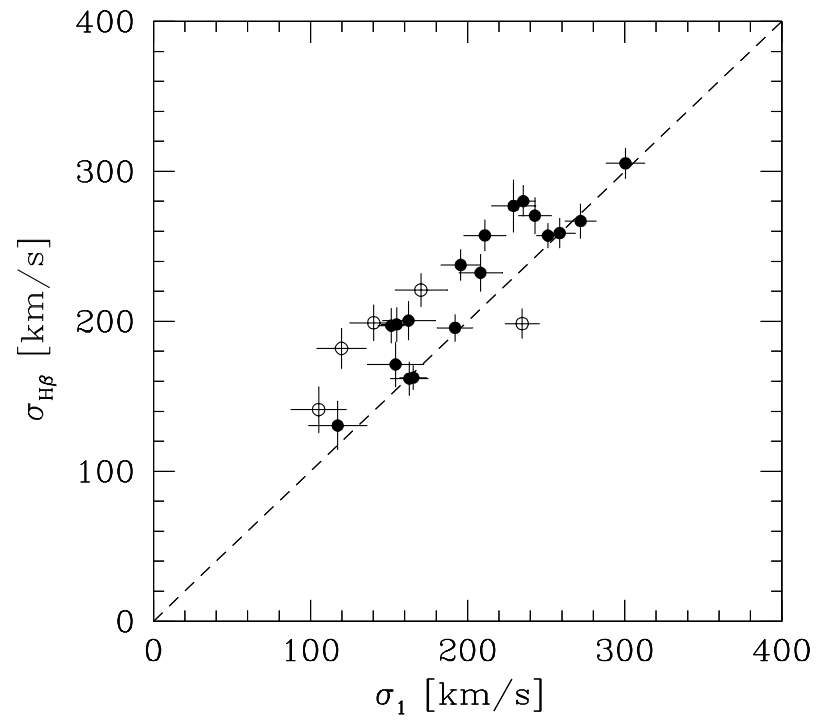

Figure 5.12: Comparison of velocity dispersion measurements derived from different absorption features. The values taken as reference are displayed on the $\mathrm{x}$ axis, velocity dispersions based on the $\mathrm{H} \beta$ feature are shown on the y-axis. Symbol notations as in Fig. 5.9.

as the relative difference in velocity dispersion values of the $\mathrm{Mg}_{b}$ with the G-band line, normalised to the reference velocity dispersions $\sigma_{1}$. Both features were free from any contamination due to sky lines. The relative uncertainties cover the range $0.03 \leq \Delta \sigma / \sigma_{1} \leq 0.06$ with a median of $\left\langle\Delta \sigma / \sigma_{1}\right\rangle=0.05$. The $\sigma$ determinations are in very good agreement and show similar absolute errors between 8 to $18 \mathrm{~km} \mathrm{~s}^{-1}$. Even for the lowest $\mathrm{S} / \mathrm{N}$ ratios the formal uncertainty of $5 \%$ is less than the typical individual error in the $\sigma$ measurement. This limit is also the smallest formal uncertainty that can be reached with the instrumental resolution and the template stars. Although this comparison is based on a small number, no trend of increasing systematic errors on the $\sigma$ values with low $\mathrm{S} / \mathrm{N}$ can be found. In addition, velocity dispersions derived for galaxies in the other clusters and field samples show similar results and a good agreement between $\sigma_{\mathrm{Mg}_{b}}$ and $\sigma_{\mathrm{G}-\text { band }}$. For all galaxy samples in this thesis, regardless of their environment loci, the $\mathrm{S} / \mathrm{N}$ was at least 13 per $\AA$. Results from the simula-
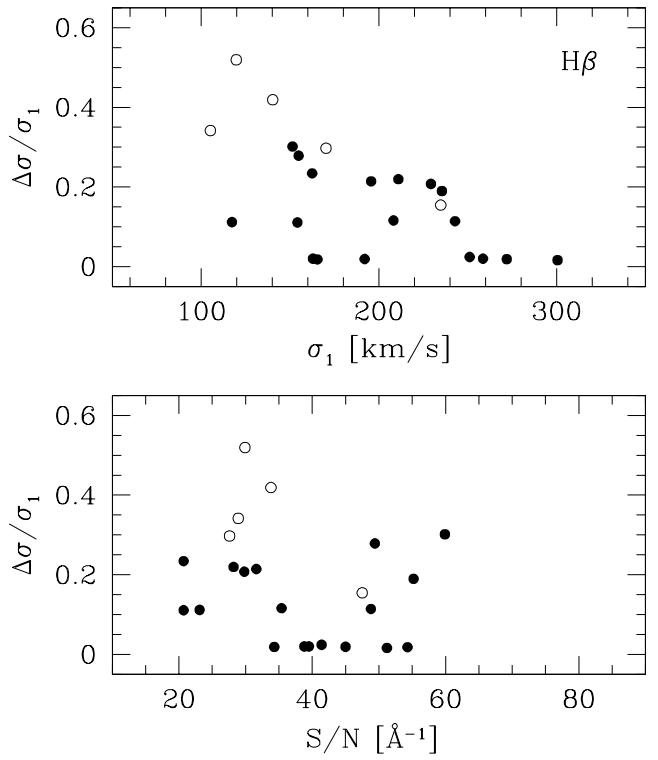

Figure 5.13: Comparison of velocity dispersion measurements derived from different absorption features. Upper panel: relative uncertainty of velocity dispersions with the $\mathrm{H} \beta$ values as a function of velocity dispersions $\sigma_{1}$. Lower panel: $\Delta \sigma / \sigma_{1}$ versus the $S / N$ per Å. Symbol notations as in Fig. 5.9.

tions yielded that the effects of systematic errors on $\sigma$ become important at $S / N \lesssim 10$ (cf. section 5.3). As this limit is lower than the average $\mathrm{S} / \mathrm{N}$ in the spectra of low-velocity dispersion galaxies, the formal uncertainty therefore becomes negligible even for the lowest derived velocity dispersions in the galaxy samples. A comparison of the absolute velocity dispersions for the WHDF ellipticals is presented in Fig 5.14.

A third comparison of velocity dispersions was performed for a sub-sample of 23 galaxies in A 2390 by analysing a wavelength range around the $\mathrm{H} \beta$ feature. In contrast to metallicitysensitive indices, the $\mathrm{H} \beta$ index (commonly referred as an age-sensitive index) varies considerably among elliptical galaxies (González 1993). In most local early-type galaxies nebular emission is present at a measurable level, which readily can fill in the $\mathrm{H} \beta$ absorption, thus making the accurate measurement of the true absorption 
a difficult business. As particularly lower order Balmer lines $(\mathrm{H} \alpha$ and $\mathrm{H} \beta)$ are stronger affected by emission from ionised gas (Osterbrock 1989), higher-order Balmer lines $(\mathrm{H} \gamma$ and $\mathrm{H} \delta)$ are preferred for the age determinations of galaxies at higher redshift, because lower order Balmer lines possibly get contaminated by night sky emission lines. In addition, for galaxies at higher redshift a possible weak nebular emission might not be clearly detectable and overlayed onto the absorption feature. For this reason, the agreement between the reference velocity dispersions and the measurements of velocity dispersions based on the $\mathrm{H} \beta$ index are less confident and the $\sigma$ values derived from the $\mathrm{H} \beta$ feature are only regarded as an additional consistency check.

In a first step, the spectra of the template stars and the galaxies were inspected if the $\mathrm{H} \beta$ indices might be subjected to weak emission. Neither for the stars nor for the galaxies any $\mathrm{H} \beta$ emission was detected. However, as just mentioned above, one must be aware of possible variations of the $\mathrm{H} \beta$ index. In Fig. 5.12 the $\sigma$ reference values for the A 2390 galaxies are compared to the velocity dispersions derived via the $\mathrm{H} \beta$ absorption line. Again, spectra were divided with respect to their quality. 18 early-type spectra were classified with a $Q_{\sigma}$ parameter of 1 and 2 , five galaxies have $Q_{\sigma}=3$. On average, similar results are found as for the velocity dispersions based on the $\mathrm{Mg}_{b}$ line, although with a larger scatter. Galaxies with quality parameter $Q_{\sigma}=1,2$ are shown as filled circles, objects of lower reliability with $Q_{\sigma}=3$ are indicated as open circles. in Fig. 5.12. Again, the deviations of velocity dispersions decrease with increasing $S / N$ ratios. With respect to the reference values, the 18 galaxies with $1 \leq Q_{\sigma} \leq 2$ have a difference of $\Delta\left(\sigma_{1}-\sigma_{\mathrm{H} \beta}\right)=-22 \pm 20 \mathrm{~km} \mathrm{~s}^{-1}$ with a median of $\left\langle\Delta\left(\sigma_{1}-\sigma_{\mathrm{H} \beta}\right)\right\rangle=-17 \mathrm{~km} \mathrm{~s}^{-1}$, corresponding to $8 \%$ higher $\sigma$ values than the G-band. This result is in fair agreement to the $\sigma$ reference values. The 5 galaxies of lower quality with $Q_{\sigma}=3$ have a difference of $\Delta\left(\sigma_{1}-\sigma_{\mathrm{H} \beta}\right)=-34 \pm 41 \mathrm{~km} \mathrm{~s}^{-1}$ and a median of $\left\langle\Delta\left(\sigma_{1}-\sigma_{\mathrm{H} \beta}\right)\right\rangle=-51 \mathrm{~km} \mathrm{~s}^{-1}$, which are higher in $\sigma$ by $\sim 30 \%$. Both $\sigma$ measurements based on the $\mathrm{H} \beta$ line are larger than the typical internal uncertainty of the velocity dispersions.

Fig. 5.13 shows the relative difference in velocity dispersions derived from the $\mathrm{H} \beta$ feature plotted against the reference velocity dispersions $\sigma_{1}$. The lower panel displays $\delta \sigma / \sigma_{1}$ as a function of the $S / N$ per $\AA$. The relative uncertainties for the 18 galaxies with $Q_{\sigma}=1,2$ cover the range $0.02 \leq \Delta \sigma / \sigma_{1} \leq 0.30$ with a median of $\left\langle\Delta \sigma / \sigma_{1}\right\rangle=0.11$. For galaxies with low quality velocity dispersions $\left(Q_{\sigma}=3\right)$ the relative errors fall in the range $0.15 \leq \Delta \sigma / \sigma_{1} \leq 0.52$ with a median of $\left\langle\Delta \sigma / \sigma_{1}\right\rangle=0.34$.

The agreement between the $\mathrm{H} \beta$ and the G-band measurements is less confident because of the following two reasons. Besides the presence of weak $\mathrm{H} \beta$ emission superimposed over the spectrum, another effect may be small variations of the $\mathrm{H} \beta$ line itself. The strength of the $\mathrm{H} \beta$ absorption line is assumed to be sensible due to changes in the (mean) age of the underlying stellar population. Both effects can modify the shape of the absorption line which has influence on the derived measure of the velocity dispersion. A combination of both effects is therefore most likely.

\subsubsection{Comparison between Repeat Observations}

For a total of eight galaxies in the A 2390 sample and five field galaxies in the WHDF sample which are included in two or more different MOS masks, it is possible to confirm the internal reliability of the spectral analysis. Galaxy \# 2933 was discarded as it is a background galaxy located behind the A 2390 cluster and no velocity dispersion based on the $\mathrm{Mg}_{b}$ feature could be derived. A total of nine repeat observations was acquired for the WHDF ellipticals. Two galaxies were observed thrice (\#92 and \#111) and \# 173 four times. The velocity dispersions have been measured for both individual setups and 


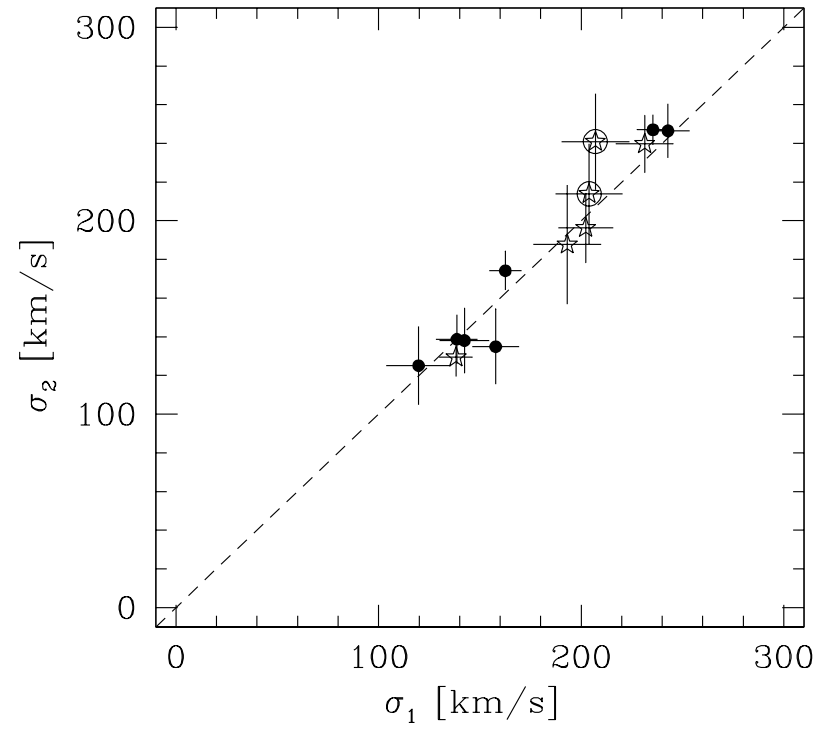

Figure 5.14: Comparison of velocity dispersion measurements for spectra which have been observed with two different MOS mask setups. Solid symbols are galaxies in A 2390, open symbols are WHDF field galaxies. One galaxy with four measurements is denoted with two additional large circles.

the agreement between these two determinations is very good with a median offset in $\sigma$ at the $4 \%$ level. Fig. 5.14 compares the velocity dispersion measurements for the eight A 2390 spectra which were observed with two different mask setups and five WHDF field galaxy spectra with two or up to four repeat observations. The A 2390 and WHDF galaxies are denoted as solid dots and open stars, respectively. In general, velocity dispersions of the secondary measurements are based on lower $S / N$ spectra and therefore show larger errors. The $\sigma$ values of galaxy \# 173 based on four individual spectra are shown as two separate symbols which are indicated with additional circles. The final measurement with the smallest error yielded $\sigma_{173}=204 \pm 16 \mathrm{~km} \mathrm{~s}^{-1}(S / N=37)$, which represents also the smaller deviation from the one-to-one line. The relative difference between reference and secondary measurements of the A 2390 galaxies is $\delta \sigma / \sigma_{1}=0.051 \pm 0.048$ with $26 \leq S / N \leq 65$. For the WHDF field ellipticals the relative difference between reference and sec- ondary measurements is $\delta \sigma / \sigma_{1}=0.041 \pm 0.015$ with $8 \leq S / N \leq 57$. In order to increase the signal-to-noise in the final spectra, these repeat observations have been co-added. For example, the $S / N$ in the final spectra of the A 2390 sample varies between 21 and 65 with a median value of $S / N \sim 34$ per $\AA$ and a mean value of $S / N \sim 37$ per $\AA$.

\subsubsection{Comparison between Different Extraction Procedures}

As described in section 3.5, for each exposure one-dimensional spectra were extracted separately using the Horne-algorithm (Horne 1986), which optimally weights the rows of the extracted spectrum profile to maximise the signalto-noise in the resulting one-dimensional spectrum. Therefore, possible shifts of the spectra which may have occurred during different nights are accounted for in the final one-dimensional summed-up spectra.

However, it is possible that the method of optimal weighting by Horne (1986) might have an effect on the velocity dispersion measurements of the galaxies. The reason is that the aperture correction assumes equal weight in the extraction aperture. This means, that a certain contribution comes from radii outside the very galaxy center, where the velocity dispersion is lower. With optimal weighting, rows away from the galaxy center get lower weight, which means the measured velocity dispersion might be higher. The following aperture correction then overcorrects the velocity dispersions, as it assumes a significant contribution from rows away from the center. In order to test this effect, the velocity dispersion measurements on spectra derived using optimally-weighted extractions and equalweight extractions have been compared.

For the following test, ten galaxies of the A 2390 sample (thereby encompassing both faint and bright spectra) on different mask and slitlet configurations have been selected. In particular, the galaxies of slitnumber \# 01 to \# 05 of mask 1 and 

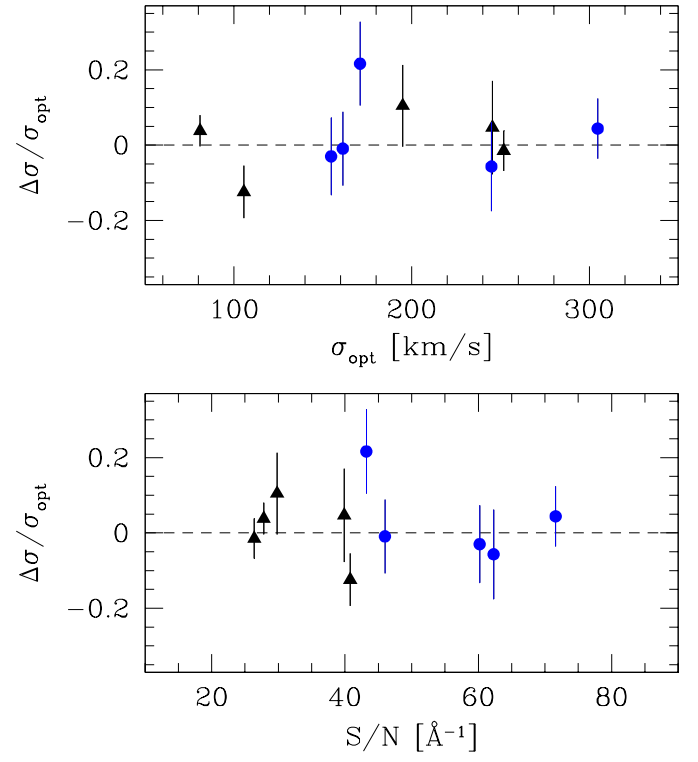

Figure 5.15: Comparison of velocity dispersion measurements on spectra derived using optimallyweighted and equal-weight extractions. Objects were observed on different mask and slitlet configurations, mask 1 (circles), mask 3 (triangles). $U p$ per panel: relative difference of velocity dispersions $\Delta \sigma / \sigma_{\mathrm{opt}}$ as a function of velocity dispersion using the method of optimally-weighted extractions. Lower panel: $\Delta \sigma / \sigma_{\text {opt }}$ versus the $S / N$ per $\AA$.

the galaxies with slitnumbers \# 06 and \# 20 to \# 23 of the mask 3 setup. All galaxies have been reduced and analysed following the same procedures (wavelength calibration and velocity dispersion measurements), except the different slit extraction procedure. Fig. 5.15 displays the relative differences of the velocity dispersions using optimally-weighted extractions and equal-weight extractions as a function of velocity dispersion and $S / N$ per $\AA$. The aim of this Figure is to verify whether the different extraction procedures introduce a systematic error in the velocity dispersions of as a function of $S / N$, hence a systematic under- or overestimation has to be tested. For this purpose, the median of the relative difference $\Delta \sigma / \sigma_{\mathrm{opt}}$ is the appropriate quantity in Fig. 5.15. It is demonstrated that the varia- tions between the velocity dispersion measurements are small, the relative difference between optimally-weighted and equal-weight extractions is on average $\left(\sigma_{\mathrm{opt}}-\sigma_{\mathrm{eq}}\right) / \sigma_{\mathrm{opt}}=0.022 \pm 0.093$ with a median of $\Delta \sigma / \sigma_{\text {opt }}=0.038$. The effect can be regarded as negligible, as the mean uncertainty in the velocity dispersion $\delta \sigma$ for a galaxy is larger than its difference in $\sigma$. Furthermore, for the aperture corrections the full range of extracted rows was not used but the rows corresponding to the "FWHM" of the spatial profile only.

\subsubsection{Comparison between Different Stellar Templates}

In general, a homogenous library of stellar spectra is a key ingredient to measure the internal kinematics of a galaxy. To be able to investigate stellar population effects it should cover the largest possible range of spectral types encompassed by early-type galaxies, covering giant stars with spectral types A, F, G and K. In order to assess possible effects of the template stars on the derivation of velocity dispersions, a different set of templates taken from the Toulouse stellar library STELIB was used (A stellar Library for stellar population synthesis models ${ }^{1}$ ). This library contains 249 different stellar spectra within the visible wavelength range of 3200 to $9500 \AA$ with intermediate resolution of $\lesssim 3 \AA$ FWHM and a sampling of $1 \AA$ thereby spanning a wide range in metallicities (Le Borgne et al. 2003). Stars of the same spectral type, thereby covering spectral types from A0 to K5, were compared. Overall, the measurements are in good agreement $\left(<20 \mathrm{~km} \mathrm{~s}^{-1}\right)$ regardless of comparing brighter or fainter objects. Moreover, as discussed in the previous sections, the FCQ method has a low sensitivity on template mismatching and for $S / N>10$ per pixel velocity dispersions can be measured with a high degree of accuracy.

\footnotetext{
${ }^{1}$ STELIB $\quad$ http: $\backslash \backslash$ webast.ast.obs-mip.fr $\backslash$ stelib $\backslash$ index.html
} 


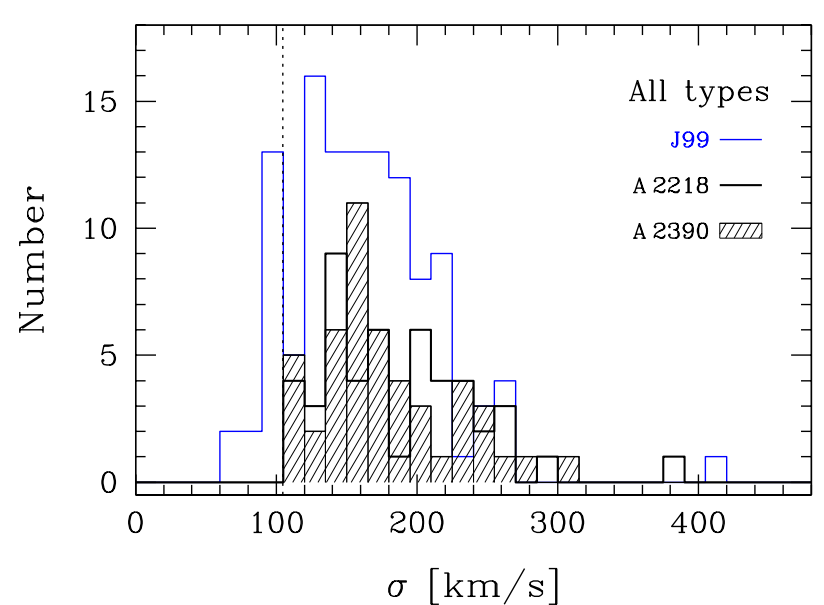

Figure 5.16: Distribution of velocity dispersions for the two rich clusters A 2218 and A 2390 in comparison to the local samples of Jørgensen, Franx \& Kjærgaard 1995 and Jørgensen 1999 (J99). The complete histograms comprise $48 \mathrm{E}+\mathrm{S} 0$ galaxies in A 2218 (thick solid line) and $48 \mathrm{E}+\mathrm{S} 0$ s in A 2390 (shaded histogram) and 115 early-type galaxies in Coma (J99). The vertical dotted line indicates the completeness limit of the combined distant cluster samples.

\subsection{Distribution in $\sigma$}

Local data samples of early-type galaxies cover a broad range in velocity dispersions as the kinematics of high- and low-luminosity galaxies can be resolved. Going to higher redshifts, a magnitude-limited sample of galaxies gets affected by the so-called "Malmquist bias of the 2nd kind" (Malmquist 1920; Teerikorpi 1997), where the magnitude limit cuts away parts of the fainter wing of the luminosity function. Due to this "selection effect", the detection rate of lowluminous galaxies becomes significantly lower and therefore the overall data set relys only on the most luminous objects, thereby resulting in a higher average luminosity for an intermediate redshift sample than for the local counterpart sample.

For this reason, it is important to check whether the velocity dispersions of the distant $\mathrm{E}+\mathrm{S} 0$ galaxies show a distribution different from that of local elliptical and S0 galaxies. If indications

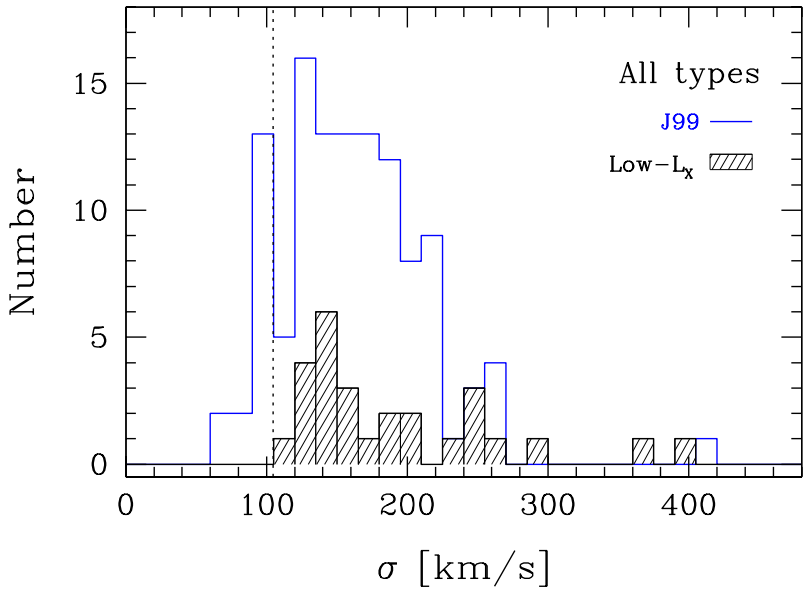

Figure 5.17: Distribution of velocity dispersions for the combined Low- $L_{X}$ clusters CL 0849, CL 1701 and CL 1702 in comparison to the local samples of Jørgensen, Franx \& Kjærgaard 1995 and Jørgensen 1999 (J99). The complete histograms comprise 15 $\mathrm{E}+\mathrm{S} 0$ galaxies in CL 0849, five E+S0s in CL 1701, seven E+S0s in CL 1702 and $115 \mathrm{E}+\mathrm{S} 0$ s in Coma (J99). The vertical dotted line indicates the completeness limit of the combined distant cluster samples.

of an evolution in the galaxy kinematics are detected, than these would have to be taken into account in the attempts to quantify the evolution in luminosity within the scaling relation of the FP and its projections (FJR and KR).

Two different large sets of local comparison samples were used for this purpose. For the clusters, a sample of local early-type Coma galaxies in the Gunn $r$-band was chosen, whereas for the field galaxies a reference of Coma galaxies and a data set comprising of Virgo and Coma galaxies in Johnson $B$-band was used.

\subsubsection{Cluster Samples}

Jørgensen, Franx \& Kjærgaard 1995 and Jørgensen 1999 (hereafter collectively J99) performed a detailed study of 115 early-type Coma galaxies in the Gunn $r$-band. The combined sample is divided into subclasses of $35 \mathrm{E}, 55$ S0 and 25 intermediate types (E/S0). Absolute 

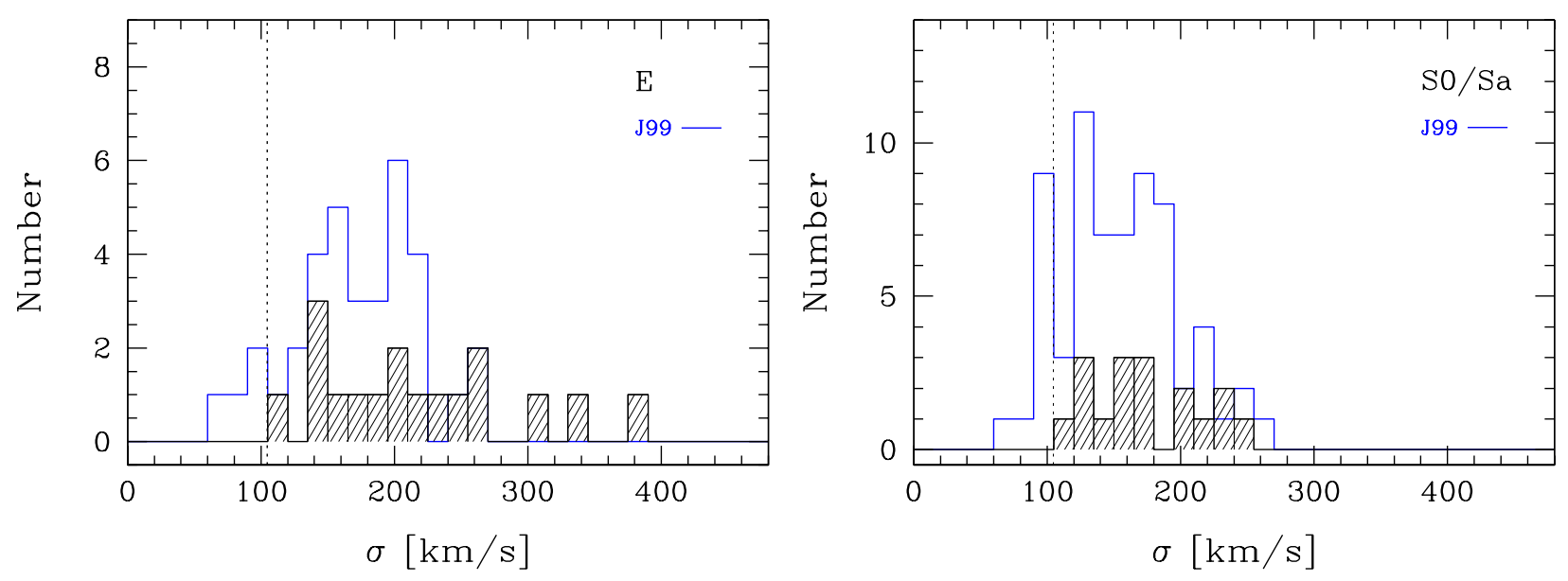

Figure 5.18: Left panel: Distribution of velocity dispersions for the two rich clusters A 2218 and A 2390 in comparison to the local samples of Jørgensen, Franx \& Kjærgaard 1995 and Jørgensen 1999 (J99). Subsample of 17 ellipticals (E) in A 2218 and A 2390 (shaded histogram) and 35 Coma ellipticals (J99). Right panel: Distribution of velocity dispersions for the two rich clusters A 2218 and A 2390 in comparison to the local samples of Jørgensen, Franx \& Kjærgaard 1995 and Jørgensen 1999 (J99). Sub-sample of 17 lenticular (S0/Sa) galaxies in A 2218 and A 2390 (shaded histogram) and 66 Coma S0/Sa galaxies (J99). The vertical dotted line indicates the completeness limit of the combined distant cluster samples.

magnitudes cover a range down to $M_{r}<-20.02$, which corresponds according to the authors to a completeness level of $93 \%$. In oder to match the local J99 sample, the parameters of the distant clusters were aperture corrected (cf. section 5.3.2). In the intermediate redshift range, observations of early-type galaxies are preferably in the $R$ or $I$ filters. At $z=0.2$, the observed $I$ and $I_{814}$ passbands are very close to rest-frame Gunn $r$. Therefore, the advantages of using the Gunn $r$-band instead of the bluer Johnson $V$ or $B$ bands are the smaller $\mathrm{k}$-corrections and the lower galactic extinction corrections.

The distribution of velocity dispersions for the complete cluster samples of A 2218 and A 2390 is compared to the local reference in Fig. 5.16. Fig. 5.18 shows the distributions in $\sigma$ for the sub-samples of ellipticals (E) and lenticular (S0) galaxies in comparison to the local J99 samples of E and S0 galaxies. The binning was set to $15 \mathrm{~km} \mathrm{~s}^{-1}$ in order to give a representative view for both data sets. Overall, both distributions show a similar spread in $\sigma$. However, the distant samples failed to reproduce the gal- axies with low velocity dispersions due to the selection upon apparent magnitudes. For this reason, the Coma galaxies were restricted to $M_{r}<-20.42$ and $\log \sigma>2.02\left(105 \mathrm{~km} \mathrm{~s}^{-1}\right)$, which represent the completeness limit of the combined A 2218 and A 2390 data. Both the distant clusters and the local sample are fitted only within this region shown by a vertical dotted line in Fig. 5.16 and the subsequent plots. The velocity dispersions for the galaxies are equally distributed. In particular, the $\sigma$ distribution of the unrestricted 115 local galaxies has a median value of $\langle\sigma\rangle_{\mathrm{J} 99 \mathrm{u}}=154 \mathrm{~km} \mathrm{~s}^{-1}$ and the restricted 97 early-type galaxies a median of $\langle\sigma\rangle_{\mathrm{J} 99 \mathrm{r}}=168 \mathrm{~km} \mathrm{~s}^{-1}$. The distant galaxies for A 2218 and for A 2390 show medians of $\langle\sigma\rangle_{\mathrm{A} 2218}=179 \mathrm{~km} \mathrm{~s}^{-1}$ and $\langle\sigma\rangle_{\mathrm{A} 2390}=165 \mathrm{~km} \mathrm{~s}^{-1}$, respectively. The combined cluster sample of 96 galaxies indicates a median in $\sigma$ of $\langle\sigma\rangle_{\text {Rich }}=170 \mathrm{~km} \mathrm{~s}^{-1}$, which is in very good agreement (differs by only $1 \%$ ) with the restricted local comparison data. As the velocity dispersion is an indicator for the mass of an object and the measured $\sigma$ values 
exhibit similar ranges, it can also be concluded that there are no significant differences in mean galaxy masses between the two samples.

A one-dimensional Kolmogorov-Smirnov (KS) test (Press et al. 1992) gives a maximum deviation between the cumulative distribution functions of the combined distant and the local J99 sample of $D=0.14$ with a probability of $P=0.34$ for a larger value. The KS result yields a fairly good agreement, although the probability value is quite high.

In Fig. 5.17 the distribution of velocity dispersions for the complete Low $-L_{X}$ cluster samples of CL 0849, CL 1701 and CL 1702 is shown. As a local comparison, again the J99 data set is applied. To account for the completeness limit of the combined poor cluster galaxies, the Coma galaxies were again restricted to $M_{r}<-20.42$ and $\sigma>105 \mathrm{~km} \mathrm{~s}^{-1}$. Spectroscopically confirmed cluster members comprise 15, five and seven galaxies for CL 0849, CL 1701 and CL 1702, respectively. Due to the low number of galaxies per cluster, a separation into elliptical and S0 is not provided for each individual cluster. The velocity dispersions for the poor clusters show a very homogenous distribution. In particular, the $\sigma$ distribution for CL 0849, CL 1701 and CL 1702 feature median values of $\langle\sigma\rangle_{\mathrm{CL} 0849}=195 \mathrm{~km} \mathrm{~s}^{-1}$, $\langle\sigma\rangle_{\mathrm{CL} 1701}=155 \quad \mathrm{~km} \mathrm{~s}^{-1}$ and $\langle\sigma\rangle_{\mathrm{CL} 1702}=164 \mathrm{~km} \mathrm{~s}^{-1}$, respectively. The combined cluster sample of 27 galaxies indicates a median in $\sigma$ of $\langle\sigma\rangle_{\text {Poor }}=164 \mathrm{~km} \mathrm{~s}^{-1}$, which is lower by $4 \mathrm{~km} \mathrm{~s}^{-1}$ than the restricted J99 sample and differs only by $2 \%$ from the local counterpart. This is in very good agreement although the Low- $L_{X}$ clusters show a lack of galaxies around $\sim 200 \mathrm{~km} \mathrm{~s}^{-1}$. In addition, the $\sigma$ distribution of the Low $-L_{X}$ cluster galaxies is similar to the distribution covered by the rich cluster sample. Their median $\sigma$ values differ by $6 \mathrm{~km} \mathrm{~s}^{-1}$ or by $4 \%$.

For a comparison of the Low- $L_{X}$ sample with the J99 data set in terms of statistics, the J99 was reduced to the same number of 27 poor cluster galaxies, thereby accounting for the original J99 coverage. A KS test yields a maximum deviation between the cumulative distribution functions of the combined poor cluster and the local J99 sample of $D=0.15$ with a probability of $P=0.93$ for a larger value. This result is of less confidence as the distant sample contains only 27 objects and depends on the selection of the local counterpart data set. Due to the small numbers for the sub-samples of elliptical and lenticular galaxies, a quantitative interpretation of these distributions in terms of a KS test is not appropriate.

The sub-samples of ellipticals (E) and lenticular (S0) galaxies for the two rich clusters are similar distributed to the local E and S0 galaxies of J99, which is shown in Fig. 5.18. In total, the local sample comprises $35 \mathrm{E}, 5 \mathrm{E} / \mathrm{S} 0,55 \mathrm{~S} 0,6 \mathrm{~S} 0 / \mathrm{Sa}$. The distant sub-sample with accurate structural parameters provided by HST comprises in total $34 \mathrm{E}+\mathrm{S} 0$ cluster galaxies. The clusters of A 2218 and A 2390 are divided into sub-classes of $9 \mathrm{E}$, 1 E/S0, 5 S0, 3 SB0/a, 1 Sa and 1 Sab spiral bulge and $8 \mathrm{E}, 1 \mathrm{E} / \mathrm{S} 0,4 \mathrm{~S} 0,1 \mathrm{Sa}$, respectively. A detailed morphological classification is presented in section 4.4. For the ellipticals, only morphologically classified galaxies as $\mathrm{E}$ have been included. Intermediate types of $\mathrm{E} / \mathrm{S} 0, \mathrm{~S} 0 / \mathrm{Sa}$ and Sa galaxies are accounted for in the discy subsample of S0/Sa types. In particular, five E/S0 are within the local J99 sample and only two in the distant sample which have a negligible effect on the overall distributions if the they are considered as elliptical types. Moreover, the histograms are not biased when excluding the subclasses of S0/Sa galaxies. There are only six local S0/a galaxies and $3 \mathrm{Sa}(\mathrm{b})$ types in the distant clusters. This is due to the fact, that actually the bulge of Sa galaxies is measured which is dominated by the chaotic random motion of the stars (i.e. by $\sigma$ ) rather than their rotational velocity. Therefore, the notation of Sa bulge is a much more appropriate term and will be used in the 


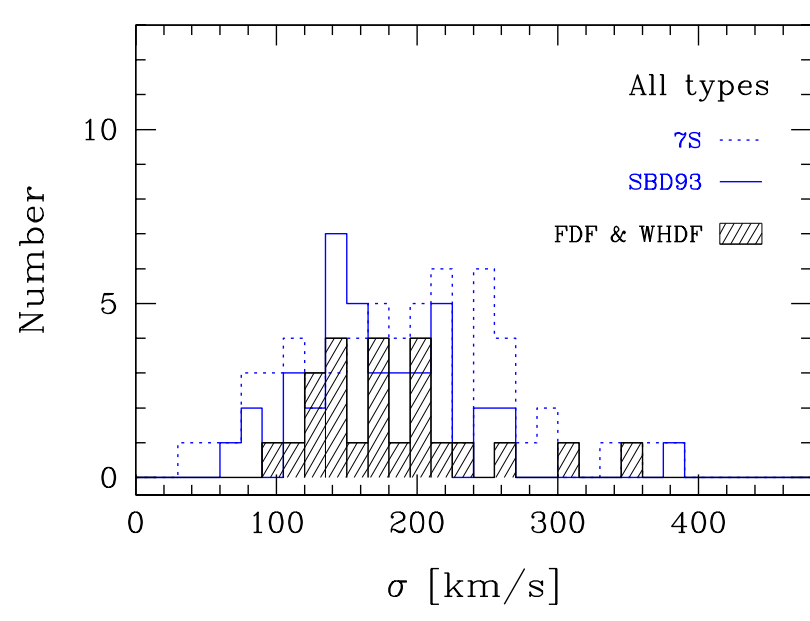

Figure 5.19: Distribution of velocity dispersions for the FDF and WHDF early-type galaxies in comparison to the local sample of Saglia, Bender \& Dressler 1993 (SBD93) and Dressler et al. 1987 (7S). The complete histograms comprise $24 \mathrm{E}+\mathrm{S} 0$ field galaxies in the FDF, WHDF (shaded histogram) and 39 Coma E+S0 galaxies of SBD93 (solid line) and 59 Virgo and Coma $\mathrm{E}+\mathrm{S} 0$ galaxies of $7 \mathrm{~S}$ (dotted histogram).

following. The poor cluster sample comprises $9 \mathrm{E}$ but only one S0 galaxy. Therefore, a comparison of sub-types cannot be conducted.

Median $\sigma$ values of the $17 \mathrm{E}$ and $17 \mathrm{~S} 0$ galaxies in the two rich clusters and the $35 \mathrm{E}$ and $66 \mathrm{~S} 0$ Coma galaxies are $\langle\sigma\rangle_{\text {Rich } \mathrm{E}}=208 \mathrm{~km} \mathrm{~s}^{-1}$, $\langle\sigma\rangle_{\text {Rich S0 }}=170 \mathrm{~km} \mathrm{~s}^{-1},\langle\sigma\rangle_{\mathrm{J} 99 \mathrm{E}}=170 \mathrm{~km} \mathrm{~s}^{-1}$, $\langle\sigma\rangle_{\mathrm{J} 99 \mathrm{~S} 0}=154 \mathrm{~km} \mathrm{~s}^{-1}$, respectively. Overall, there is a good agreement between the distant and local sub-classes of elliptical and lenticular galaxies. Elliptical galaxies in the rich clusters appear to have slightly larger velocity dispersions. However, the J99 sample comprises no elliptical galaxies with $\sigma>260 \mathrm{~km} \mathrm{~s}^{-1}$.

\subsubsection{Field Sample}

For the 24 FDF and WHDF early-type field galaxies encompassing on average higher redshifts over a broader range in redshift than the clusters, the rest-frame Gunn $r$-band is not an appropriate choice and thus the magnitudes were transformed to rest-frame Johnson $B$. For a local reference, a well studied sample of 39 early-type galaxies (splitted into $25 \mathrm{E}$ and $14 \mathrm{~S} 0 \mathrm{~s}$ ) in the Coma cluster (Saglia, Bender \& Dressler 1993, hereafter SBD93) of the "7 Samurai" data set (Faber et al. 1989), and the original "7 Samurai" sample (Dressler et al. 1987, hereafter 7S) comprising 59 early-type galaxies in the Virgo and Coma cluster were chosen. No morphological information is available for the latter.

Fig. 5.19 displays the distribution of velocity dispersions for the complete FDF and WHDF field ellipticals compared to the local reference of SBD93 and 7S, respectively. Overall, there is a good agreement between the two data set. The 13 FDF ellipticals feature a median in $\sigma$ of $\langle\sigma\rangle_{\mathrm{FDF}}=178 \mathrm{~km} \mathrm{~s}^{-1}$, the 11 WHDF ellipticals exhibit a median of $\langle\sigma\rangle_{\mathrm{WHDF}}=150 \mathrm{~km} \mathrm{~s}^{-1}$, whereas the combined field sample shows a median in $\sigma$ of $\langle\sigma\rangle_{\mathrm{FDF}+\mathrm{WHDF}}=178 \mathrm{~km} \mathrm{~s}^{-1}$. The median velocity dispersion in the local SBD93 sample is $\langle\sigma\rangle_{\mathrm{SBD} 93}=162 \mathrm{~km} \mathrm{~s}^{-1}$ and for the $7 \mathrm{~S}$ sample $\langle\sigma\rangle_{7 \mathrm{~S}}=191 \mathrm{~km} \mathrm{~s}^{-1}$.

The 24 FDF and WHDF early-type field galaxies are divided into $10 \mathrm{E}$ and $12 \mathrm{~S} 0$. Two galaxies are not visible on the ACS images and therefore not included in either group. Analogous as for the cluster galaxies, only morphologically classified field elliptical galaxies were regarded as E type (cf. section 4.4). Fig. 5.20 gives a detailed look on the sub-samples of ellipticals and lenticular field galaxies compared to the local E and S0 sample of SBD93, which comprises $25 \mathrm{E}$ and $14 \mathrm{~S} 0 / \mathrm{SB} 0$ galaxies. Both, the field elliptical and lenticular FDF and WHDF galaxies show a similar distribution in $\sigma$ to their local counterparts. Median values for the distant field $\mathrm{E}$ and $\mathrm{S} 0$ galaxies and the E and S0s of the SBD93 samples are $\langle\sigma\rangle_{\text {Field } \mathrm{E}}=200 \mathrm{~km} \mathrm{~s}^{-1}$, $\langle\sigma\rangle_{\text {Field S0 }}=170 \quad \mathrm{~km} \quad \mathrm{~s}^{-1}$, $\langle\sigma\rangle_{\mathrm{SBD} 93 \mathrm{E}}=198 \mathrm{~km} \mathrm{~s}^{-1}$, $\langle\sigma\rangle_{\mathrm{SBD} 93 \mathrm{~S} 0}=150 \mathrm{~km} \mathrm{~s}^{-1}$, respectively. The agreement between the sub-classes is good. Moreover, differences between the FDF and WHDF ellipticals and S0s are negligible. 

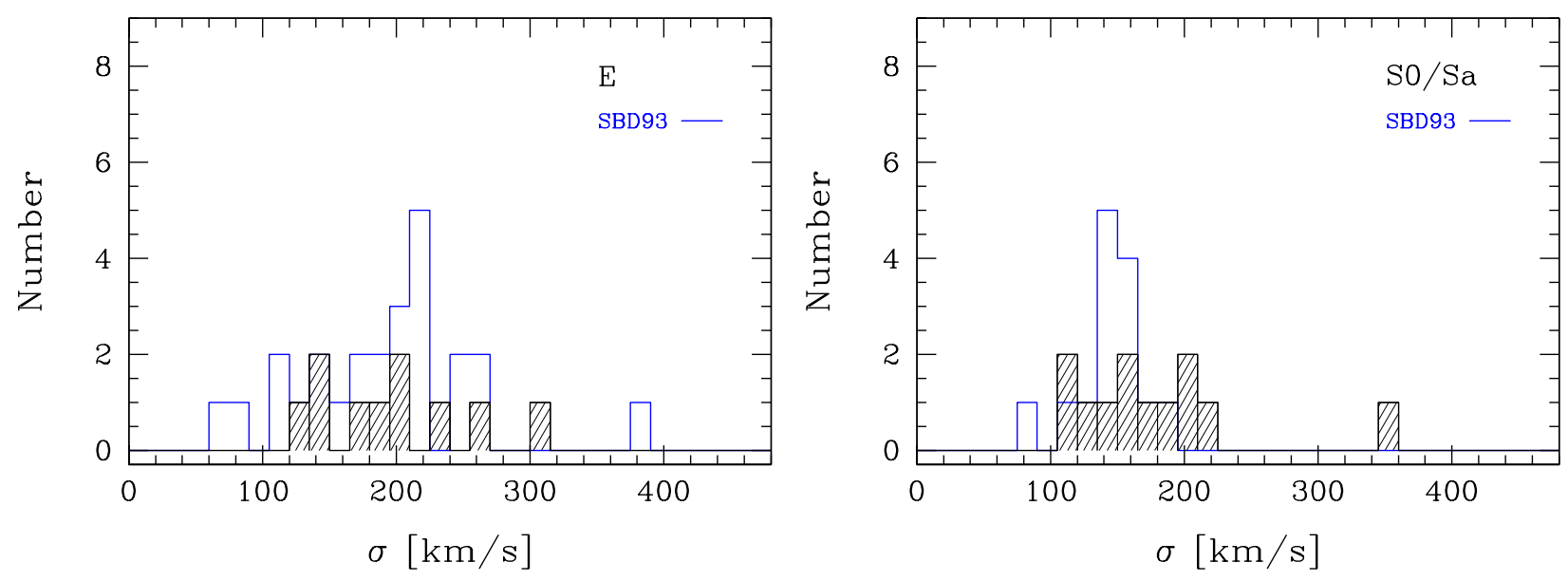

Figure 5.20: Left panel: Distribution of velocity dispersions for the FDF and WHDF early-type galaxies in comparison to the local sample of Saglia, Bender \& Dressler 1993 (SBD93). Sub-sample of 10 ellipticals (E) in the FDF and WHDF (shaded histogram) and 25 Coma ellipticals (SBD93). Right panel: Distribution of velocity dispersions for the FDF and WHDF early-type galaxies in comparison to the local sample of Saglia, Bender \& Dressler 1993 (SBD93). Sub-sample of 12 lenticular (S0/Sa) galaxies in the FDF and WHDF (shaded histogram) and 14 Coma S0/SB0 galaxies (SBD93).

However, for this comparison it has to be taken
into account that observations of distant field galaxies at higher $z$ tend to miss low-luminous galaxies which lower velocity dispersions due to limitations in luminosity. Therefore, part of slightly different $\sigma$ distributions is not attributed to deviations between the samples but due to a selection effect.

On the basis of the complete cluster and field samples as well as the sub-samples for ellipticals and lenticulars it therefore can be concluded that the distant early-type galaxies do not indicate an evolution in the velocity dispersions. Note that it is particularly important when comparing the evolution of the $M / L$ ratios for distant and local early-type galaxies, to reduce differences in the overall mass distribution of the samples. This point will be re-addressed in section 6.5. From the comparison given above it is strengthened that the distant cluster and field galaxies constitute representative early-type galaxy samples and deviations between local and distant scaling relations will mainly have to be attributed to an evolution in the luminosity, but not to differences in the kinematics.

\subsection{Redshift Distribution}

Galaxies' redshifts were determined by two independent methods. A first measurement was performed in fitting Gaussian profiles to prominent absorption line features of a typical early-type galaxy. In a second approach, the redshift determinations were refined and improved using the FCQ method.

After the wavelength calibration of the 2-D spectra and extraction of one-dimensional spectra, which were constructed by averaging all rows within the FWHM of the galaxies' profile along the spatial axis ( $\mathrm{Y}$-axis), a search of absorption line-strengths and possible emission lines was performed in a semi-automatic manner. As a first redshift estimate, either the mean redshift of a galaxy cluster or a by-eye identification of characteristic absorption features was used. A program displayed the most prominent absorption lines of a typical elliptical galaxy simultaneously with the galaxy spectrum to verify the 
estimated value of the redshift $z$. For the derivation of the exact redshift, Gaussian profiles were fitted to the identified absorption lines to compute the central wavelength of each feature. The catalogue of lines which was used herein encompassed the Balmer series, [O II] 3727, CaII H+K, CN, G4300-band, [O III] 4959, [O III] 5007, $\mathrm{Mg}_{b}$ and Fe5015. For a few cases with very low $\mathrm{S} / \mathrm{N}$ spectra $(S / N \lesssim 8)$, a slight filtering of the averaged spectra in the order of the seeing of $1.5^{\prime \prime}$ FWHM for the line search was performed to suppress the strong sky lines which dominated the spectral continuum. Typical uncertainties were in the range of $0.0005 \lesssim z \lesssim 0.0015$, corresponding to 150 to $450 \mathrm{~km} \mathrm{~s}^{-1}$.

This redshift method was extensively tested on low-resolution spectra of the 10 Low $-L_{X}$ clusters (Balogh et al. 2002b). Out of 581 galaxy spectra in total, reliable redshifts could been measured for 317 galaxies, of which 172 are cluster members. Results were compared to two independent methods, the routine FXCOR within the IRAF environment which cross-correlates the spectra with high $\mathrm{S} / \mathrm{N} z=0$ galaxy spectral templates of similar resolution and to the FCQ method which was applied to spectra with higher $\mathrm{S} / \mathrm{N}$. The agreement between the three measurements was good and generally within the uncertainty (typically $\sim 100 \mathrm{~km} \mathrm{~s}^{-1}$ ). The independent estimates allowed to identify galaxies where one method failed (e.g., because of low $\mathrm{S} / \mathrm{N}$ ratio around a critical line). Spectra for which the discrepancy between different redshift estimates could not be resolved were always of low $\mathrm{S} / \mathrm{N}$ ratio $(\mathrm{S} / \mathrm{N} \$ 5 \%)$, and were rejected from further analysis.

For the second approach, the FCQ technique was applied. As an input for an initial estimate, the determinations from the Gaussian profile measurements were adopted. Typical uncertainties for the FCQ measurements were in the order of 8 to $15 \mathrm{~km} \mathrm{~s}^{-1}$, thereby improving the first computation by a factor of 10 or even higher. These final FCQ output values were used for the sub- sequent analysis. Both independent approaches were consistent within the errors for all galaxies without any dependency on the environment of the sub-sample.

In total, redshifts of 121 early-type galaxies and 9 spiral galaxies could be determined. Additional spectra of 12 secondary objects were obtained, which were covered by a MOS slit by chance during the observations. The whole early-type galaxy sample is splitted into $52 \mathrm{E}+\mathrm{S} 0$ galaxies for the A 2390 MOSCA setup, $30 \mathrm{E}+\mathrm{S} 0 \mathrm{~s}$ for the three Low $-L_{X}$ clusters and 39 elliptical field galaxy candidates for the FDF and WHDF. No galaxy spectra of the targets had too low $S / N$ to derive a value of $z$. Three of the fill-up objects for the poor cluster setup were bona fide spiral galaxies which featured a clear disc structure on the ground-based images. These galaxies showed similar apparent luminosities as to the cluster galaxies and were therefore assumed to be likely members of the targeted cluster which was the primary goal if it was not possible to select any E+S0 galaxy as a MOS target based on the low-resolution spectra. However, unfortunately non of these objects turned out to be a cluster member.

Figure 5.21 shows the redshift distribution of all 51 early-type rich cluster galaxies of A 2390 with determined redshifts and one foreground galaxy. The cluster members are indicated with the shaded histogram and the foreground object (\# 3038) is an E/S0 galaxy located at $z=0.1798$. From the 55 different observed galaxies, three objects, \# 1507, \# 1639 and \# 2933, turned out to be background spiral galaxies at $z=0.3275, z=0.3249$ and $z=0.3981$, respectively. The spiral \# 2933 shows clear signs of spiral arms on the HST/WFPC2 image of the A 2390 cluster. As this work concentrates on $\mathrm{E}+\mathrm{S} 0$ galaxies all these spirals were discarded from the plot. During the observations of the target galaxies, four other galaxies fell into the slits by coincidence (\# 2222, \# 5552, \# 5553 and \# 6666). However, apart from \# 6666, their 


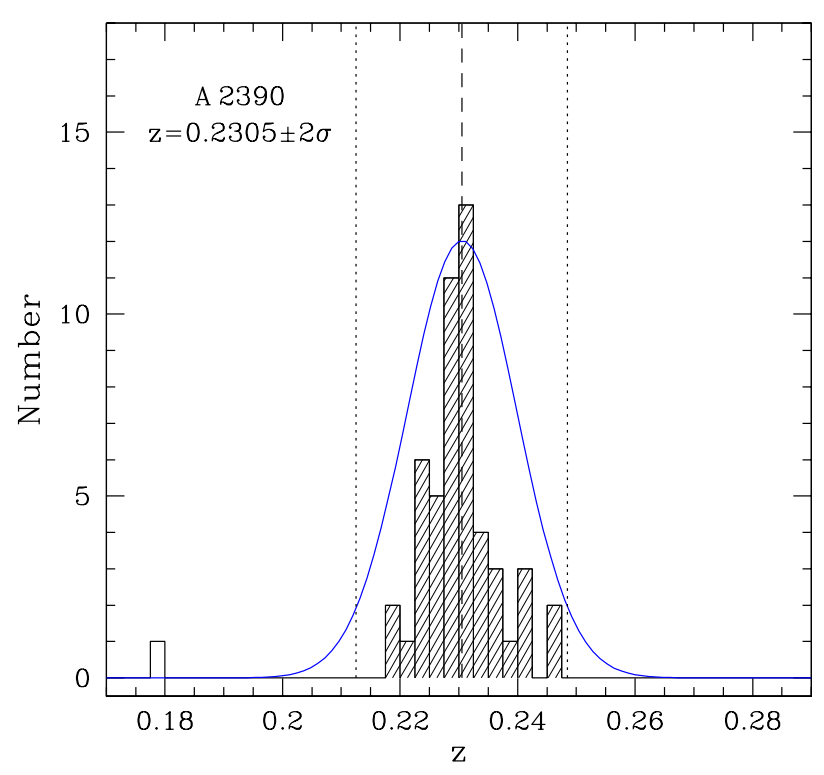

Figure 5.21: Redshift distributions of the full sample of 51 A 2390 cluster galaxies (shaded histogram) and one foreground galaxy at $z=0.1798$. Three background objects at $z \geq 0.32$ have been neglected. The mean redshift of the cluster and the $2 \sigma$ scatter are indicated as dashed and dotted lines, respectively. A Gaussian of width as the $1 \sigma$ deviation is overlayed.

spectra are too faint to measure accurate velocity dispersions and no photometry is available for the first three objects. Although these galaxies are included for the redshift distribution of A 2390 in Figure 5.21, they were rejected from the final sample. The mean redshift of the cluster $\bar{z}_{\mathrm{A} 2390}=0.2305 \pm 0.0094$ (median $\left.\left\langle z_{\mathrm{A} 2390}\right\rangle=0.2300\right)$ and the $2 \sigma$ deviation derived from the A 2390 sample are indicated as dashed and dotted lines, respectively. The cluster redshift is in good agreement with the value of $\bar{z}_{\mathrm{A} 2390}=0.2280$ derived from the CNOC study by Yee et al. (1996). For the further analysis always the CNOC redshift is adopted as this value is based on a larger cluster sample of 217 galaxies (within $z_{\text {clus }} \pm 2 \sigma$ ), splitted into $159 \mathrm{E} / \mathrm{S} 0,13$ $\mathrm{E}+\mathrm{A}, 31 \mathrm{Sa}-\mathrm{Sbc}$ and 14 emission line (Irr) galaxies, and thus encompasses also the spiral galaxy population of A 2390. In Fig. 5.21 a Gaussian of width as large as the $1 \sigma$ deviation is overlayed.

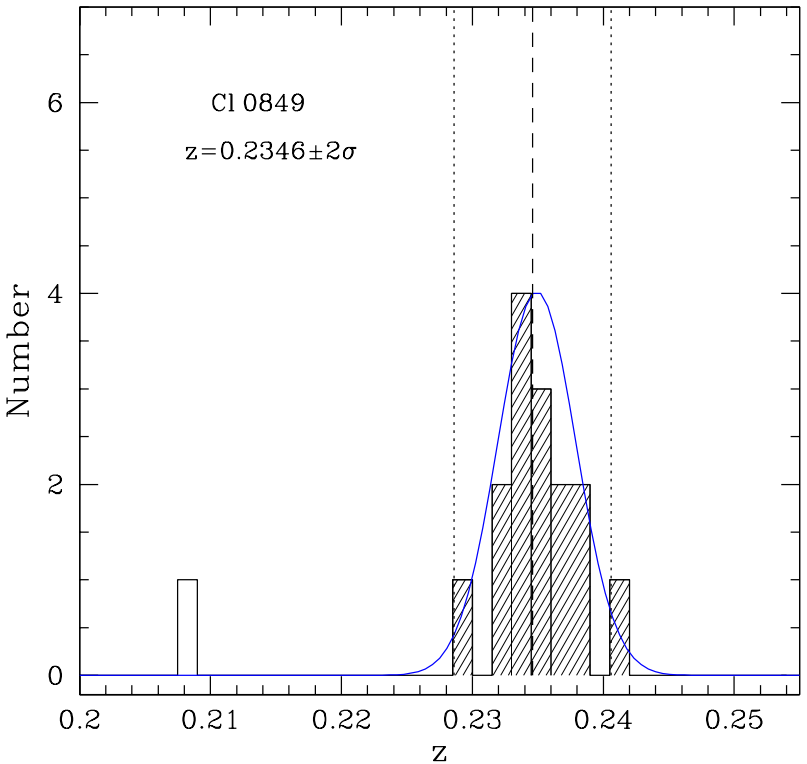

Figure 5.22: Redshift distributions of the full sample of $15 \mathrm{Cl} 0849$ cluster galaxies (shaded histogram) and one foreground galaxy at $\mathrm{z}=0.2090$. The median redshift of the cluster and the $2 \sigma$ scatter are indicated as dashed and dotted lines, respectively. A Gaussian of width as the $1 \sigma$ deviation is overlayed.

The redshift distributions of the Low $-L_{X}$ galaxy clusters are shown in the Figs. 5.22, 5.23 and 5.24. Shaded histograms denote the distributions of cluster members for 15 early-type galaxies in $\mathrm{Cl} 0849,5 \mathrm{E}+\mathrm{S} 0$ galaxies in $\mathrm{Cl} 1701$ and seven E+S0s in the cluster $\mathrm{Cl} 1702$. Non cluster members are indicated as the open histogram. Again, the mean cluster redshifts are labelled with $\bar{z}_{0849}=0.2346 \pm 0.0030, \bar{z}_{1701}=0.2452 \pm 0.0015$ and $\bar{z}_{1702}=0.2228 \pm 0.0025$. The $2 \sigma$ deviations of the mean cluster redshift values are indicated as dotted lines. In Fig. 5.24 the selection of cluster members for the Low $-L_{X}$ cluster data set is illustrated. The redshift distribution of the complete sample of 18 galaxies which were targeted with a single MOS mask for the clusters Cl 1701 and $\mathrm{Cl} 1702$ are shown. Again, the median redshift of both clusters are denoted as dashed lines. These values together with their $2 \sigma$ deviations (dotted lines) are based on the larger samples of 


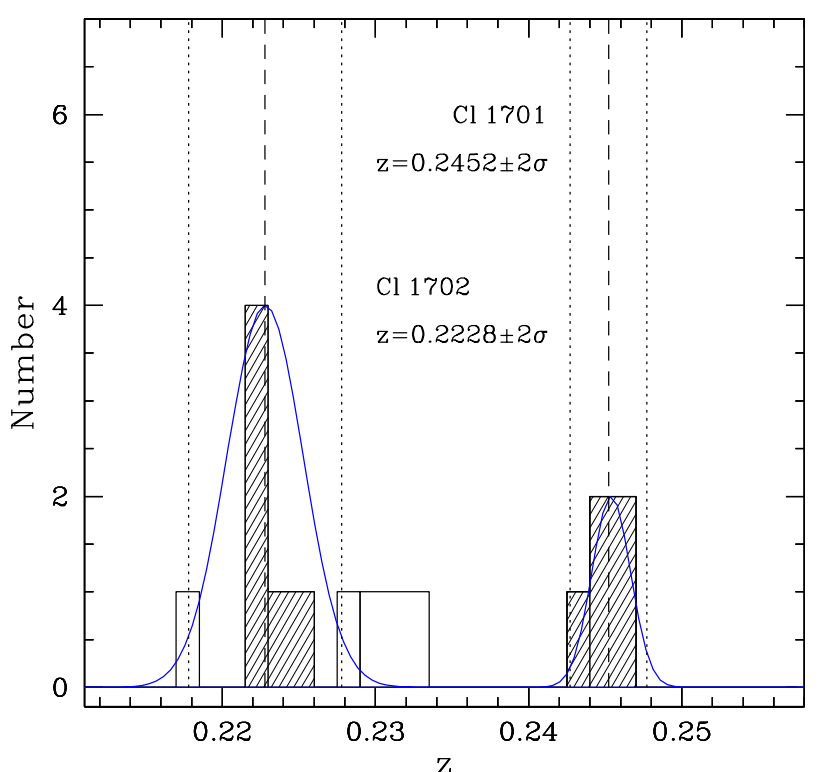

Figure 5.23: Redshift distributions of the full sample of $5 \mathrm{Cl} 1701$ and $7 \mathrm{Cl} 1702$ cluster galaxies (shaded histogram) and non cluster members (open histogram). The median redshift of the clusters and their $2 \sigma$ scatter are indicated as dashed and dotted lines, respectively. A Gaussian of width as the $1 \sigma$ deviation is overlayed.

galaxies with low-resolution spectra which also account for cluster spiral galaxies.

In Fig. 5.25 the redshift distribution of the FDF and WHDF field early-type galaxies is displayed. Spectroscopic confirmed elliptical and S0 galaxies are shown as shaded histogram, whereas one background spiral galaxy at $z=0.5569$ is marked with an open histogram. The 13 FDF $\mathrm{E}+\mathrm{S} 0$ field galaxies encompass a range in redshifts of $0.22 \leq z_{\mathrm{FDF}} \leq 0.65$ with a mean of $\bar{z}_{\mathrm{FDF}}=0.42 \pm 0.11\left(\left\langle z_{\mathrm{FDF}}\right\rangle=0.41\right)$ and the 11 WHDF field galaxies cover a redshift space of $0.21 \leq z_{\mathrm{WHDF}} \leq 0.74$ with a mean of $\bar{z}_{\mathrm{WHDF}}=0.36 \pm 0.16\left(\left\langle z_{\mathrm{WHDF}}\right\rangle=0.40\right)$. The total field sample of $24 \mathrm{E}+\mathrm{S} 0$ galaxies exhibits a mean redshift of $\bar{z}_{\text {Field } \mathrm{E}}=0.39 \pm 0.14$ and a median of $\left\langle z_{\text {Field } \mathrm{E}}\right\rangle=0.40$.

It turned out that the southwestern corner of the FDF most probably covers the outskirts of a galaxy cluster at $z=0.33$. Based on the radial ve-

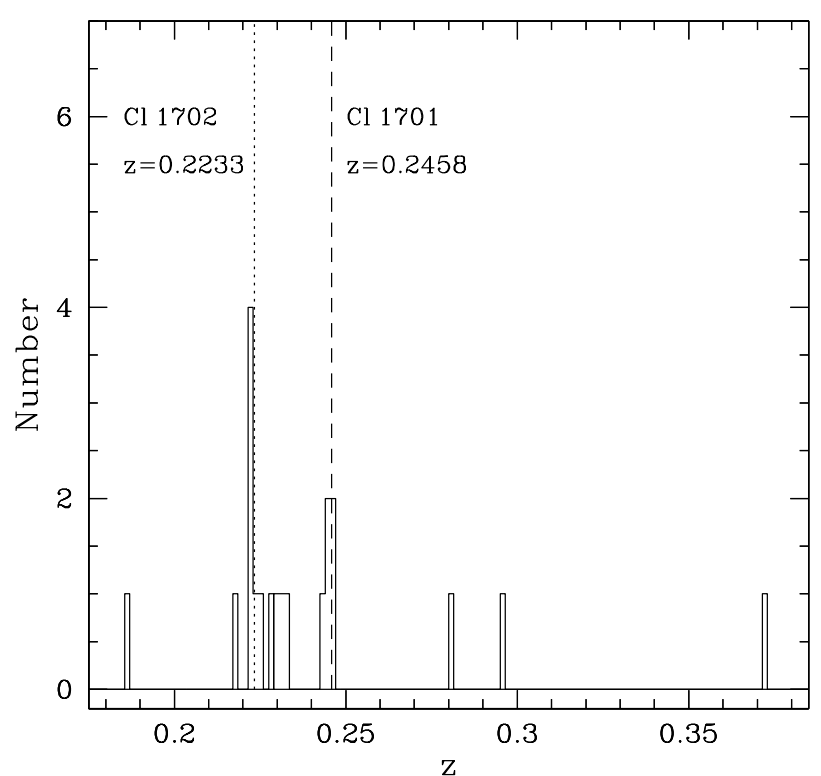

Figure 5.24: Redshift distribution of the complete sample of 18 galaxies which where targeted with a MOS mask for the $\mathrm{Cl} 1701$ and $\mathrm{Cl} 1702$ cluster. The median redshift of $\mathrm{Cl} 1701$ and $\mathrm{Cl} 1702$ are indicated as dashed and dotted lines, respectively.

locity measurements for the elliptical galaxy candidates in the FDF, the lower limit for the velocity dispersion of the cluster is $\sigma_{\mathrm{c}} \gtrsim 430 \mathrm{~km} \mathrm{~s}^{-1}$. The cluster velocity dispersion $\sigma_{\mathrm{c}}$ is only a lower limit as the cluster centre is not located on the FDF but only its outskirts. Assuming a spread in redshift space of $\Delta z=0.01$, which corresponds to $\sim 1.5$ times the typical velocity dispersion of a rich galaxy cluster $\left(\sigma_{\mathrm{c}} \approx 1000 \mathrm{~km} \mathrm{~s}^{-1}\right)$, it is found that a total of 13 spiral galaxies and 15 early-type galaxies based on their spectroscopic redshifts are likely to be members of this cluster, which is also visible through a small "gap" in redshift space at $z \approx 0.3$.

The spatial distribution of all early-type field galaxies in the FDF is shown in Fig. 5.26. Potential cluster members are additionally denoted by squares. Adding the possible spiral cluster candidates, it is suggestive that the cluster has an elongated shape. If the center indeed is positioned to the southwestern corner of the FDF, the distribution of the galaxies will be a 


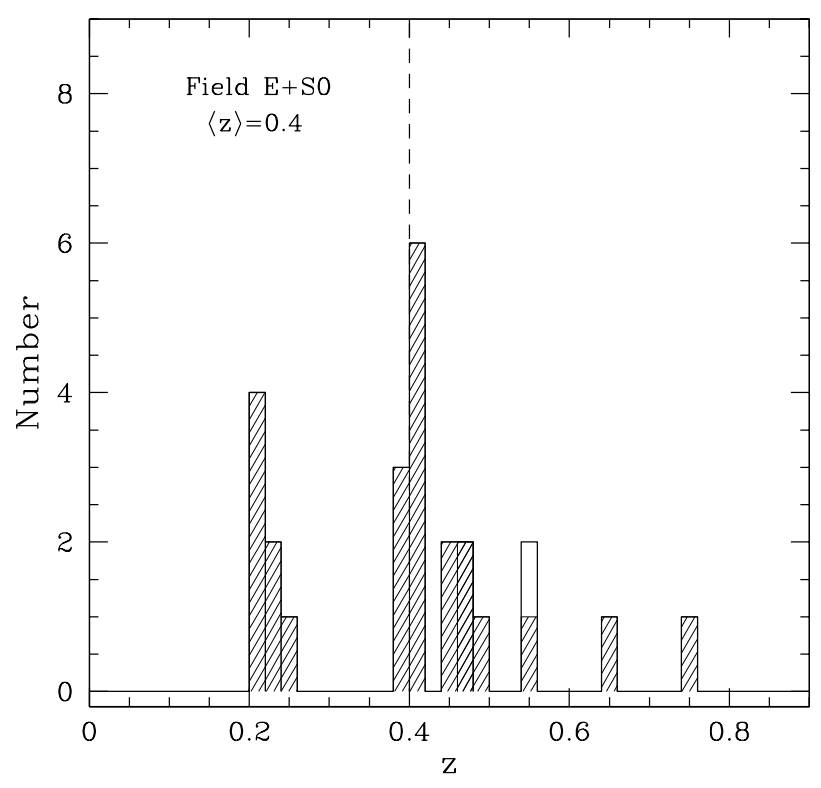

Figure 5.25: Redshift distribution of the 24 field early-type galaxies within the FDF and WHDF. Spectroscopic confirmed E and S0 field galaxies are denoted with a shaded histogram. One background spiral galaxy at $z=0.5569$ is plotted as an open histogram. The median redshift of for the combined field sample $\langle z\rangle=0.4$ is indicated as a dashed line.

striking example of the morphology-density relation (Dressler 1980) with the early-type galaxies mainly populating the dense, inner region and the late-type galaxies at larger clustercentric radii. Unfortunately at a redshift of $z=0.33$, the Mg 5170 absorption line is strongly affected due to the terrestrial absorption of the B band, which makes an accurate measurement of the internal galaxy velocity dispersions impossible. For this reason, the early-type galaxies which are possible members of a cluster at $z \approx 0.33$ will be discarded from the further analysis.

Fig. 5.27 illustrates the spatial distribution of the early-type field galaxies in the WHDF. Apart from three galaxies which are located in the southern (upper middle) part of the image, all field ellipticals are homogenous distributed over the field. Based on the radial velocity measurements for the three galaxies it turned out that these galaxies exhibit a dispersion in radial veloc-

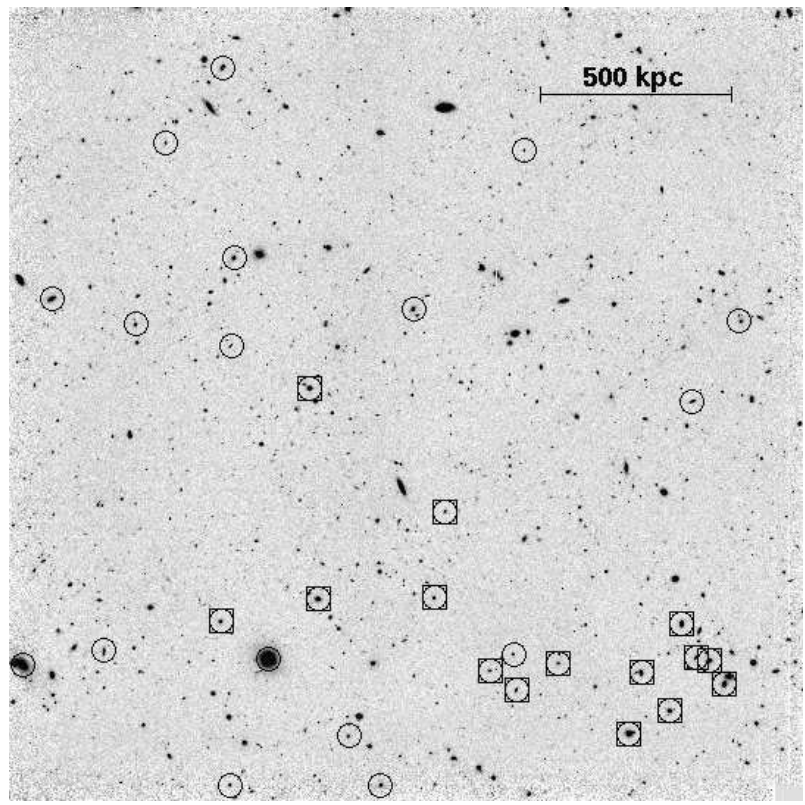

Figure 5.26: Distribution of the field early-type galaxies within the FDF and possible members of a cluster in the southwestern (lower right) corner. 15 early-type cluster candidates are denoted additionally by squares, the 17 early-type field galaxies solely by circles. The bar corresponds to $500 \mathrm{kpc}$ in projection at the clusters redshift of $z=0.33$.

ities of $\Delta v_{\mathrm{rad}} \approx 450 \mathrm{~km} \mathrm{~s}^{-1}$. Furthermore they are not visually associated to each other through tidal arms or interaction signatures on the ACS images. For this reason, the three objects are are not likely members of a group at $z \approx 0.21$ but isolated early-type field galaxies.

\subsection{Comparison between Rich Clusters}

To investigate possible environmental effects in the galaxy properties and differences in the stellar populations of elliptical and lenticular galaxies, a large sample of more than 50 early-type galaxies is required. In a recent study, Ziegler et al. (2001a) performed a detailed analysis of a sample of 48 early-type galaxies in the rich cluster A 2218 at a redshift of $z=0.18$. If this work 


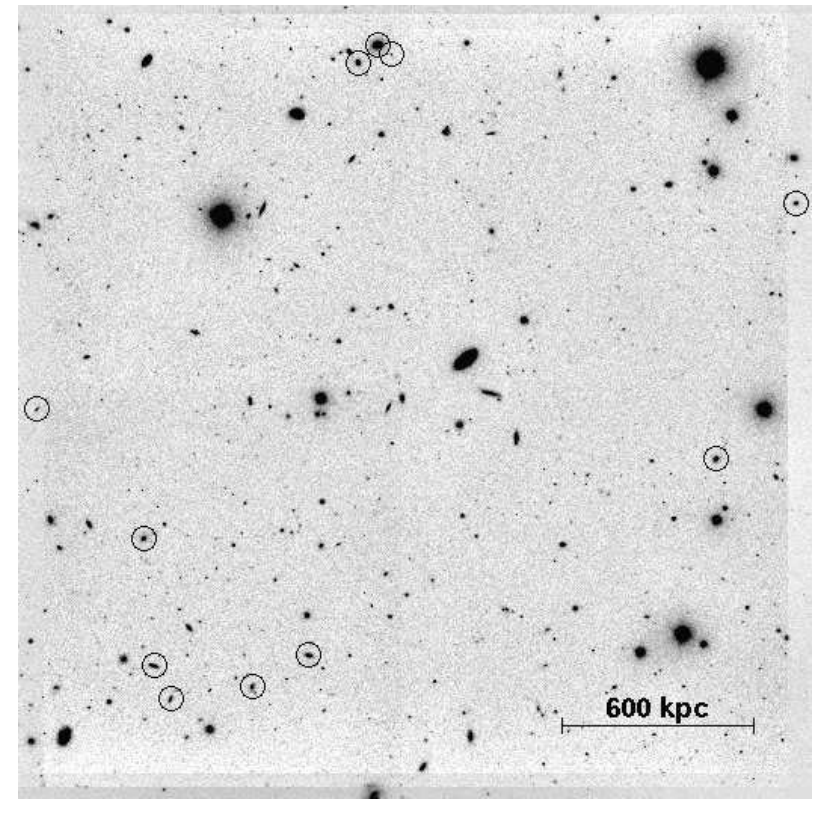

Figure 5.27: Distribution of the field early-type galaxies within the WHDF. The bar corresponds to $600 \mathrm{kpc}$ in projection at a redshift of $z=0.33$. The three galaxies located in the southern (upper middle) part of the WHDF are not members of a group. North is down and east is to the left.

could be combined with the sample of $N=48$ in A 2390, it would offer the possibility to explore the evolution of $\sim 100$ early-type galaxies over a large luminosity range and wide field-of-view at similar cosmic epochs.

Before the individual galaxies of the two clusters can be combined into one large sample, it has to be proven that both data sets are characterised by very similar properties and that possible differences within the sub-samples were accounted for. For this reason, the cluster galaxies were compared with respect to their luminosity, colour, size and velocity dispersion distributions. Both clusters feature similar global cluster properties (e.g., richness class, X-ray luminosity). Furthermore, the sample selection and all observations, especially the spectroscopy, have been carried out in very similar manner. Therefore, it is not expected that significant differences between the galaxy samples exist.
In the subsequent analysis the cluster galaxies are investigated over the same dynamical range in their properties. Therefore, the two cD galaxies of the cluster A 2218 were excluded as no cD galaxy is included in the A 2390 sub-sample. A comparison of the cluster properties in absolute rest-frame Gunn $r$ magnitude, effective radius, $(B-I)$ rest-frame colour and velocity dispersion (aperture corrected) is presented in Fig. 5.28. For each set of parameters, the mean values with the resp. $\pm 1 \sigma$ scatter are indicated as overlayed Gaussian curves. Overall, the galaxies are similarly distributed in all plots. For the galaxies in A 2218 the range of absolute Gunn $r$ magnitudes is $-20.50 \geq M_{r} \geq-23.42$, for the objects in A $2390-20.47 \geq M_{r} \geq-22.99$ (upper left panel in Fig. 5.28) The median value for A 2390 is $\left\langle M_{r}\right\rangle=-21.31^{m}, 0.17$ mag fainter than the median luminosity for the galaxies in A 2218. For the size distribution only the 32 objects within the HST fields are considered. In the distributions of galaxy size (upper right panel) small (but not significant) differences are visible. The sizes of the A 2218 galaxies cover a range between 0.22 and 0.82 in $\log R_{\mathrm{e}}(\mathrm{kpc})$, with a median of $\log \left\langle R_{\mathrm{e}}\right\rangle=0.46$. The A 2390 sample contains more galaxies with smaller effective radii $0.01 \leq \log R_{\mathrm{e}} \leq 0.92$, with a mean of $\log \overline{R_{\mathrm{e}}}=0.38 \pm 0.27$ (median $\log \left\langle R_{\mathrm{e}}\right\rangle=0.37$ ), resulting in a broader distribution than for A 2218. These galaxies are low-luminosity galaxies. This issue will be addressed in more detail in the forthcoming section 6.4. The rest-frame colour distributions show no significant differences between the clusters. The galaxies' colours in A 2390 cover a range of $1.86<(B-I)<2.51$, in A $22181.95<(B-I)<2.44$. For the A 2390 galaxies a median value of $\langle(B-I)\rangle=2.29$ is derived, for the A 2218 objects $\langle(B-I)\rangle=2.28$, respectively. Both are in very good agreement with the typical colour of $(B-I)=2.27$ for ellipticals at $z=0$, given by Fukugita et al. (1995). The velocity dispersions for the galaxies are equally distributed (lower right panel), 
with a median value of $165 \mathrm{~km} \mathrm{~s}^{-1}$ and a mean of $\log \bar{\sigma}=2.238 \pm 0.11$ for A 2390 and $178 \mathrm{~km} \mathrm{~s}^{-1}$ $(\log \bar{\sigma}=2.253 \pm 0.12)$ for A 2218. As the velocity dispersion is an indicator for the mass of an object and the measured $\sigma$ values exhibit similar ranges, it can also be concluded that there are no significant differences in mean galaxy masses between the two samples (see section 6.5 for a further discussion). In addition, the scale lengths $(h)$, disc-to-bulge ratios $(D / B)$ and the surface brightnesses of the member galaxies were compared to each other and again negligible differences between the distributions were found.

As a conclusion no significant offset in the distribution of any galaxy parameters between the two samples was detected. The comparison asserted that properties of the galaxies within the two clusters are very homogeneous and therefore a combination of the two data sets is adequate and warranted, which results in a final sample of 96 early-type galaxies.

The combined sample allows an extensive investigation of the evolutionary status of galaxies in rich clusters at $z \sim 0.2$. A sub-sample with accurate structural parameters provided by HST comprises $34 \mathrm{E}+\mathrm{S} 0$ galaxies, splitted into 17 ellipticals (E), 2 E/S0, 9 S0, 3 SB0/a, 2 Sa bulges and 1 Sab spiral bulge that can be investigated in the FP. With this large sample, possible radial and environmental dependences can be explored in detail for the galaxy properties from the cluster centre to the outskirts using the Faber-Jackson relation and for different sub-populations (E/S0 and S0/Sa bulges). For the subsequent analysis, the sample of early-type galaxies in A 2218 and A 2390 is referred to as the rich cluster sample. 

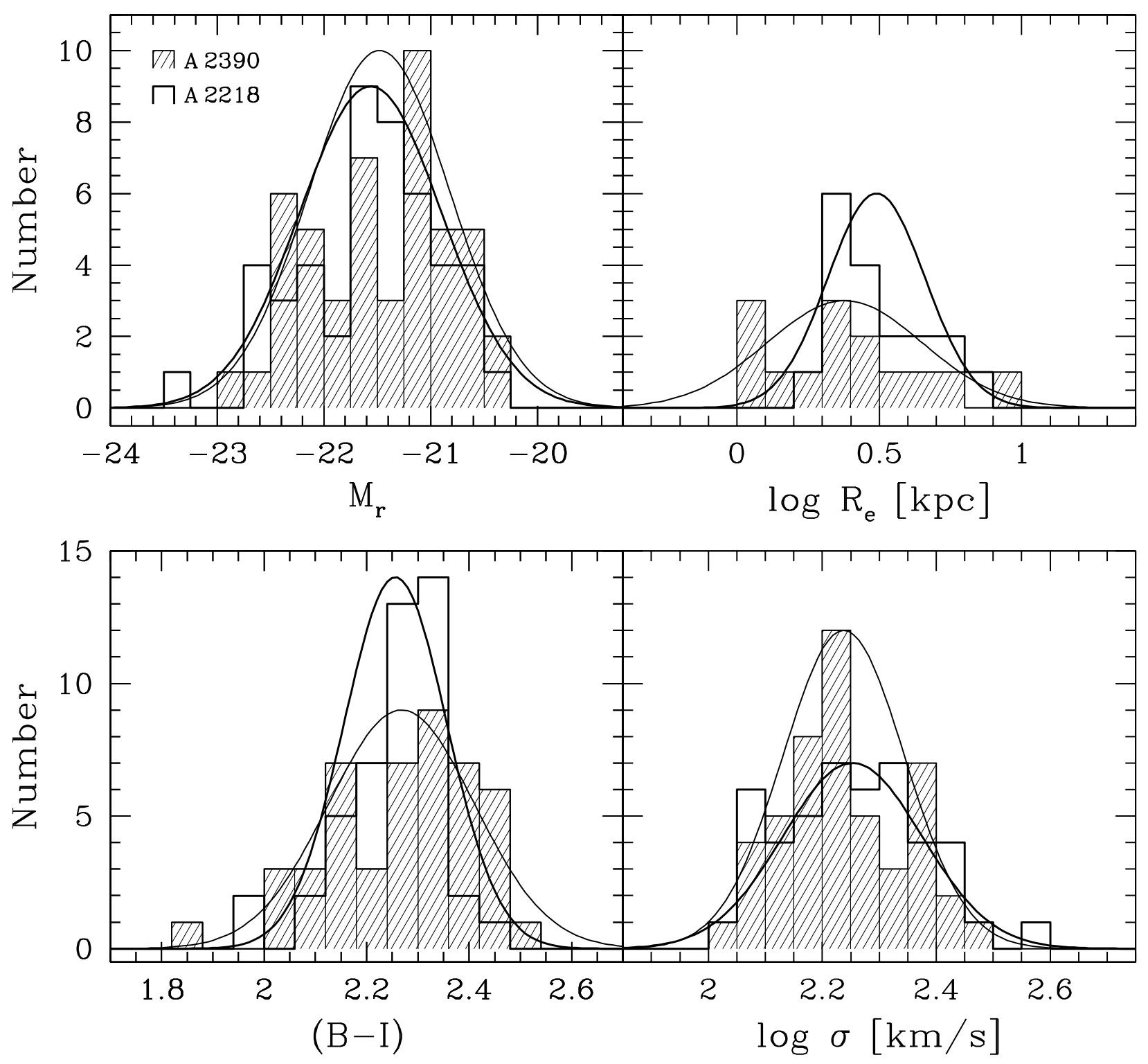

Figure 5.28: Comparison of galaxy properties. Thin lines and hashed areas represent the distribution for the members in A 2390, solid thick lines the characteristics of the galaxies in A 2218. Gaussian fits showing the mean values with $\pm 1 \sigma$ scatter are overlayed. Top left: Absolute Gunn $r$ magnitudes for the whole sample. Top right: Size distribution of the HST sub-sample. Bottom left: $(B-I)$ rest-frame colours of member galaxies. Bottom right: Distribution of velocity dispersions. 


\section{Chapter 6}

\section{Galaxy Scaling Relations at $\mathrm{z} \sim 0.2$}

After the descriptions of the sample construction and the derivations of photometric properties, structural parameters and the measurement of velocity dispersions, the analysis and results of the distant scaling relations of early-type galaxies will be presented.

In a first step, a sample of nearby early-type galaxies has to be chosen which acts as a local comparison reference (section 6.1). Afterwards the scaling relations of the projections of the Fundamental Plane, the Faber-Jackson (section 6.2) and Kormendy relation (section 6.3) of the distant cluster and field early-type galaxies will be compared to the local ones in the present-day universe. In a next step, the Fundamental Plane (FP) is outlined in section 6.4. After an investigation of the whole early-type galaxy population in the FP, the discussion concentrates on possible differences between the morphological types of early-type galaxies, elliptical and S0 galaxies. To rule out systematic errors in the interpretation of the evolution within the FP, the mass ranges of the galaxies are analysed and the evolution of the mean mass-to-light $(M / L)$ ratios are explored (section 6.5). A comparison with previous studies of the FP is given in section 6.6. Detailed tests on possible environmental effects on the properties of $\mathrm{E}+\mathrm{S} 0$ galaxies will be described in the next chapter 7 .

Some aspects of the analysis in this chapter have been presented in Fritz et al. (2005a, 2005b) and Ziegler et al. (2005).

\subsection{The Local Reference}

To derive the kinematic and/or the spectrophotometric evolution of early-type galaxies at intermediate redshift, it is crucial to carefully choose a sample of early-type galaxies at low redshift which can be utilised as a reference for the purpose of comparison. As already discussed in chapter 1, a large number of local samples have been constructed based on the Virgo and Coma clusters. Recent results on the SDSS Data Release 1 database encompass maybe one of the largest sample of early-type galaxies located at different redshifts and in various environments. However, unfortunately at the time of this study, the SDSS sample was not yet electronically available.

The Coma cluster at $z=0.024$ is one of the best studied local rich clusters. The cluster redshift of $z=0.024$ corresponds to a look-back time of only $\sim 0.3$ Gyrs, or $2 \%$ of the age of the Universe, and therefore galaxies within this limit can be considered to represent the same cosmic epoch, i.e., the present-day Universe. A number of previous works in the literature (Saglia et al. 1993b; van Dokkum \& Franx 1996; Jørgensen et al. 1999; Kelson et al. 2000b; Treu et al. 2001b; Ziegler et al. 2001a; Holden et al. 2005) used this cluster for the purpose of comparison of the Fundamental Plane at $z>0.1$ and it therefore provides a reliable and widely accepted local reference when addressing evolutionary questions. To achieve a direct comparability with 
the results of these authors, the Coma data by Jørgensen 1999 and Jørgensen et al. 1995 (hereafter collectively J99) will be used as a local comparison sample for the following analysis of the early-type cluster galaxies. These authors performed a detailed study of a large number of early-type Coma galaxies in Gunn $v, g$ and $r$ bands and Johnson $U$ and $B$ filters. The combined sample comprises 115 early-type galaxies, divided into subclasses of $35 \mathrm{E}, 55 \mathrm{~S} 0$ and 25 intermediate types (E/S0). Note that J99 did not find any differences between elliptical and $\mathrm{S} 0$ or Sa bulge galaxies. For this reason, the local early-type galaxies are not splitted into different sub-classes based on their morphologies. Absolute magnitudes cover a range down to $M_{r}<-19.58$ and the sample is $93 \%$ complete at absolute magnitudes $M_{r}<-20.02$, corresponding to $r=15.08^{m}$. In oder to match the local J99 sample, the parameters of the distant clusters were aperture corrected (cf. section 5.3.2). In the intermediate redshift range, observations of early-type galaxies are preferably in the $R$ or $I$ filters. At $z=0.2$, the observed $I$ and $I_{814}$ passbands are very close to rest-frame Gunn $r$. For this reason, the advantages of using the Gunn $r$-band instead of the bluer Johnson $V$ or $B$ bands are the smaller $k$-corrections and the lower Galactic extinction corrections.

In all subsequent figures, large symbols denote the distant early-type galaxies, either of the two rich clusters Abell 2218 and Abell 2390 or the three Low $-L_{X}$ clusters $\mathrm{Cl} 0849, \mathrm{Cl} 1701$ and $\mathrm{Cl} 1702$, whereas small boxes represent the local reference sample. Morphologically classified lenticular galaxies ( $\mathrm{S} 0$ ) or bulges of early-type spiral galaxies within the HST field of the clusters are indicated by open symbols, elliptical galaxies are denoted by filled symbols. Under assumption of a non changing slope, the residuals from the local relation are computed and derive a mean evolution for the distant galaxies. For a comparison of the slope of the local and distant galaxies, the bisector method is used (e.g.,

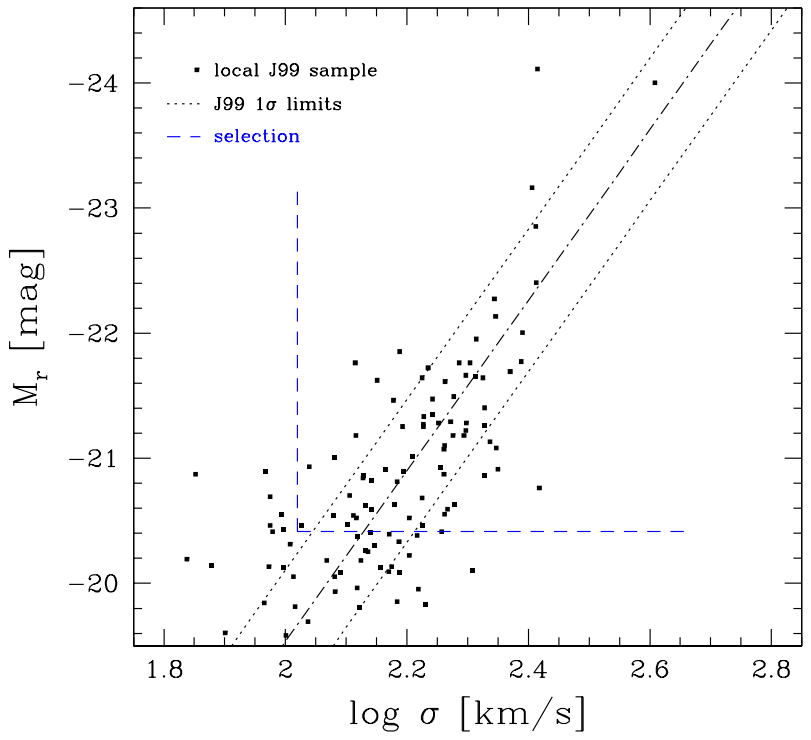

Figure 6.1: The local Faber-Jackson relation in Gunn $r$ for 115 early-type galaxies in the Coma cluster from Jørgensen (1999, J99) and Jørgensen et al. 1995, J99). The individual objects of the J99 sample are denoted by the small squares. The dot-dashed line is a bisector fit to the local FJR within the selection boundaries (dashed lines) as defined by the distant cluster samples, together with the $1 \sigma$ errors.

Ziegler et al. 2001a), which is a combination of two least-square fits with the dependent and independent variables interchanged. The errors on the bisector fits were evaluated through a bootstrap re-sampling of the data 100 times.

The early-type field galaxies in the FDF and WHDF cover a broader redshift range than the clusters and on average higher redshifts. A transformation of the measured magnitudes to the Gunn $r$ rest-frame band is not appropriate as it would involve large $k$-corrections (cf. section 4.5.3). For this reason, as a local reference the well studied Coma sample in the Johnson B-band by Saglia, Bender \& Dressler (1993, hereafter SBD93) comprising 39 early-type cluster galaxies (splitted into $25 \mathrm{E}$ and $14 \mathrm{~S} 0 \mathrm{~s}$ ) was chosen. This data compilation is a sub-set of the original "7 Samurai" sample (Faber et al. 1989; Dressler et al. 1987) which contains 59 earlytype galaxies in the Virgo and Coma cluster (cf. 


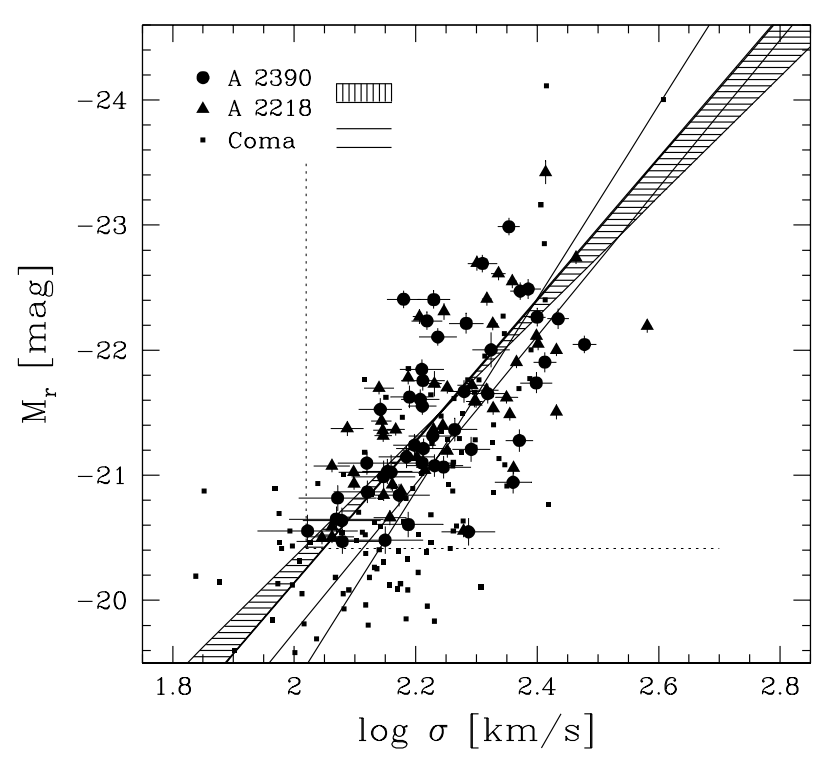

Figure 6.2: Faber-Jackson relation in Gunn $r$ for 96 early-type galaxies in A 2390 and A 2218, compared to the local Coma sample of J99. The solid lines show the $\pm 1 \sigma$ errors of the 100 iteration bootstrap bisector fits to the local FJR within selection boundaries, the hashed area indicates the bisector fits to the distant sample (within $\pm 1 \sigma$ ).

section 5.4.2). As for the latter no morphological information is available the Coma study by SBD93 was used as a local reference.

\subsection{The Faber-Jackson Rela- tion}

A first test of the formation and evolution of elliptical and lenticular galaxies allows the scaling relation between galaxy luminosity and velocity dispersion, the so-called Faber-Jackson relation. Fig. 6.1 illustrates the local Faber-Jackson relation (FJR) in Gunn $r$-band for 115 early-type galaxies in the Coma cluster by J99. The distant early-type galaxy samples in this study were all selected upon apparent magnitudes and are therefore limited in their absolute magnitudes. For this reason, the local FJR has to be restricted to the range in magnitudes and velocity dispersions covered by the distant samples.
The distant galaxies of the combined A 2218 and A 2390 clusters have velocity dispersions down to $105 \mathrm{~km} \mathrm{~s}^{-1}$ and the absolute magnitudes range down to $M_{r} \leq-20.42$. To match the selection boundaries for the distant sample represented by the combined A 2218 and A 2390 data, the Coma galaxies by J99 were restricted to $M_{r}<-20.42$ and $\log \sigma>2.02$, which reduced the number of early-type galaxies in the Coma cluster to 77. These selection boundaries are indicated as the dashed lines in Fig. 6.1. In case of the local Kormendy relation (KR), galaxies which do not meet these criteria are omitted and shown as open squares (see section 6.3). For the subsequent analysis of the FJR, both the distant clusters and the local sample are fitted only within the region shown by horizontal and/or vertical dashed lines.

A bootstrap bisector fit to the reduced Coma reference sample yields

$$
M_{r}=-(6.82 \pm 0.90) \log \sigma-(5.90 \pm 2.01) \text {. (6.1) }
$$

The observed $\pm 1 \sigma$ scatter is $\sigma_{r}=0.57^{m}$. The bisector fit together with the average $\pm 1 \sigma$ scatter of the local FJR are denoted in Fig. 6.1 as the dot-dashed line and the dotted lines, respectively. For purposes of comparison, a linear $\chi^{2}$-fit to the reduced Coma data set gives

$$
M_{r}=-(5.19 \pm 0.54) \log \sigma-(9.43 \pm 1.21)
$$

and a flatter slope than the bisector method.

The Faber-Jackson relation (FJR) in the Gunn $r$-band for 96 distant early-type galaxies in the clusters A 2218 and A 2390, compared to the local Coma sample of J99 is shown in Fig. 6.2. In order to consider the whole cluster data set for the Faber-Jackson scaling relation, the luminosities of the distant galaxies as derived from the ground-based photometry with SExtractor are applied. Very similar trends are found when the sample is restricted to the space-based magnitudes. For the construction of the Fundamental Plane, accurate measurements of the sizes of the galaxies are required which can only be achieved 
via the high-resolution images of the HST (cf. section 6.4). In the parameter space of the FJR diagram, the distant galaxies in the two rich clusters encompass a range in absolute magnitude of $-20.42 \geq M_{r} \geq-23.42$ and in velocity dispersion of $105 \mathrm{~km} \mathrm{~s}^{-1} \leq \sigma \leq 381 \mathrm{~km} \mathrm{~s}^{-1}$. In Fig. 6.2, the $\pm 1 \sigma$ scatter of the bisector fits to the local FJR is indicated as solid lines. Fits to the respective distant combined cluster sample (within $\pm 1 \sigma)$ are shown as the hashed area. Because of the younger mean ages of the stellar populations, the distant cluster galaxies are on average brighter than their local counterparts for a given velocity dispersion. If a formation redshift of $z_{\text {form }}=2$ for the cluster galaxies is assumed, the predictions of single-burst passive evolution models by BC96 (Salpeter IMF with $x=1.35$, mass range $0.10<M_{\odot}<125.0$ and burst duration of 1 Gyr) suggest an increase of their Gunn $r$ brightness by $0.21 \pm 0.05 \mathrm{mag}$ at $z=0.2$. Assuming that the local slope holds valid for the distant galaxies, the mean residuals from the local FJR are analysed, i.e. the difference in luminosity for each galaxy from the local FJR fit. The $\pm 1 \sigma$ scatter of these offsets for the distant galaxies is $\sigma_{r}=0.61^{m}$, which is only by $0.04^{m}$ or $7 \%$ larger than the dispersion observed locally.

Table 6.1 lists the derived mean and median luminosity evolution of the early-type cluster galaxies for various samples in the FJR. The final total error in the FJR evolution results from a linear sum of the total error in absolute magnitude $\delta M_{r}$ (cf. Eq. 4.14) and the error as introduced by the velocity dispersions as

$$
\sigma_{M_{r}}=\delta M_{r}+a \log \delta \sigma_{\mathrm{err}},
$$

where $\delta \sigma_{\text {err }}$ denotes the mean error in the velocity dispersion and $a$ the bisector slope of the A 2218 and A 2390 sub-samples. Typical uncertainties of the velocity dispersions can be found in Table 4.5. The error in the mean luminosity evolution $\sigma_{M_{r}}$ in Eq. 6.3 is given as the $\pm 1 \sigma$ uncertainty in the column $4\left(\sigma_{M_{r}}\right)$ of Table 6.1. This mean error in the measured evolution will be adopted in all subsequent derivations of the
Table 6.1: Evolution of the Faber-Jackson relation in Gunn $r$ derived for various cluster samples. $N$ is the number of galaxies and $\Delta \bar{M}_{r}$ indicates the mean luminosity evolution. The fourth column denotes the $\pm 1 \sigma$ deviation in the mean luminosity evolution and the last column $\Delta\left\langle M_{r}\right\rangle$ gives the median evolution.

\begin{tabular}{lrccc}
\hline Sample & $N$ & $\begin{array}{c}\Delta \bar{M}_{r} \\
{[\mathrm{mag}]}\end{array}$ & $\sigma_{M_{r}}$ & $\begin{array}{c}\Delta\left\langle M_{r}\right\rangle \\
{[\mathrm{mag}]}\end{array}$ \\
$(1)$ & $(2)$ & $(3)$ & $(4)$ & $(5)$ \\
\hline A 2218 & 48 & -0.31 & 0.15 & -0.29 \\
A 2390 & 48 & -0.32 & 0.29 & -0.35 \\
A 2218+A 2390 & 96 & -0.32 & 0.22 & -0.35 \\
Cl 0849 & 15 & -0.30 & 0.19 & -0.36 \\
Cl 1701 & 5 & -0.42 & 0.19 & -0.20 \\
Cl 1702 & 7 & -0.57 & 0.20 & -0.51 \\
Low $-L_{X}{ }^{a}$ & 26 & -0.39 & 0.19 & -0.36 \\
Low $-L_{X}{ }^{b}$ & 27 & -0.44 & 0.19 & -0.38
\end{tabular}

${ }^{a}$ as A 2218+A 2390: $\log \sigma>2.02, M_{r}<-20.42$.

${ }^{b}$ with \# 923: $\log \sigma>2.02, M_{r}<-20.08$.

amount of luminosity evolution and is listed in the Tables as $\sigma_{M_{r}}$. For the total sample of 96 galaxies in the FJR a luminosity evolution of $-0.32 \pm 0.22^{m}$ is detected. Similar offsets are deduced for each cluster separately. The earlytype galaxies in A 2218 show an evolution of $-0.31 \pm 0.15^{m}$, whereas the $\mathrm{E}+\mathrm{S} 0$ galaxies in A 2390 are brighter by $-0.32 \pm 0.29^{m}$. Both results agree with the BC96 model predictions.

Under assumption of a variable slope, Fig. 6.2 compares the FJR of the distant clusters A 2218 and A 2390 to the local Coma reference. To investigate the significance of a possible slope decrease a bootstrap bisector fit was performed, which are 100 iterations of repeated bisector fits with random rejection of data points between each iteration. In this method, the error can be derived from the scatter of the individual slopes of the fits. A bootstrap bisector fit to the distant cluster sample of A 2218 and A 2390 yields

$$
M_{r}=-(5.23 \pm 0.43) \log \sigma-(9.78 \pm 0.96),
$$




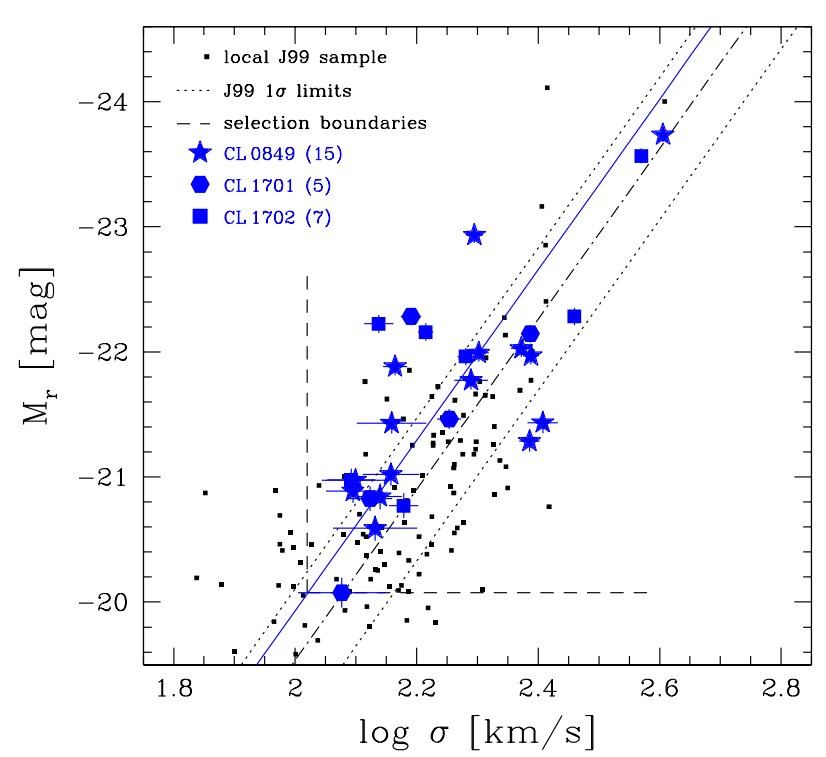

Figure 6.3: Faber-Jackson relation in Gunn $r$ for 27 early-type galaxies in the poor clusters $\mathrm{Cl} 0849$, $\mathrm{Cl} 1701$ and $\mathrm{Cl} 1702$, compared to the local Coma sample of J99. The linear bisector fit to the Coma galaxies was restricted to the range encompassed by the distant rich cluster galaxies $\left(M_{r}<-20.42\right.$ and $\log \sigma>2.02$ ). The area bounded by dotted lines indicates the mean $\pm 1 \sigma$ of the local FJR. The solid line shows the mean evolution of the poor cluster sample assuming the local slope.

which is illustrated as the hashed area in Fig. 6.2. In this first analysis, a slope change is significant on the $2 \sigma$ level based on the uncertainty of the local Coma slope (see Eq. 6.1).

To verify this result with a different fitting technique, a linear $\chi^{2}$-fit to the combined rich cluster sample was computed as

$$
M_{r}=-(3.76 \pm 0.45) \log \sigma-(13.09 \pm 1.00) .
$$

In this approach, a slope change would be significant on the $3 \sigma$ level again using the error estimate of the local Coma slope in Eq. 6.2. Both fitting methods give evidence that a massdependence evolution drives the change of the slope in the FJR. To quantify this in more detail, the rich cluster sample was splitted with respect to velocity dispersion into two equally large subsamples and analysed separately, which will be

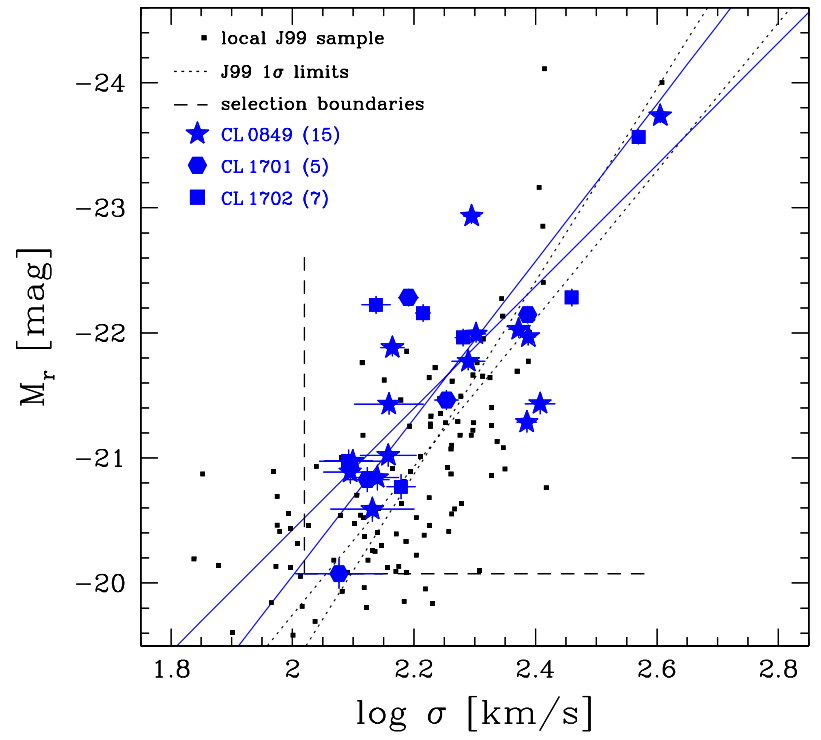

Figure 6.4: Faber-Jackson relation in Gunn $r$ for 27 early-type galaxies in the poor clusters $\mathrm{Cl} 0849$, Cl 1701 and Cl1702, compared to the local Coma sample of J99. The dotted lines show the $\pm 1 \sigma$ errors of the 100 iteration bootstrap bisector fits to the local FJR within selection boundaries, the solid lines indicate the bisector fits to the distant sample (within $\pm 1 \sigma)$.

addressed in section 7.1.2.

The Faber-Jackson Relation considers the total distant sample of 96 early-type cluster galaxies. For this reason, this scaling relation offers the possibility to perform a variety of tests in dividing the sample with respect to luminosity, velocity dispersion (which is a representative parameter of the underlying mass of a galaxy) and distance from the cluster centre. These extensive analyses will be discussed in the next chapter 7 . Fig. 6.3 displays the Faber-Jackson relation in Gunn $r$ for the three Low $-L_{X}$ clusters $\mathrm{Cl} 0849$, $\mathrm{Cl} 1701$ and $\mathrm{Cl} 1702$. The combined poor cluster sample comprises in total 27 early-type galaxies, divided into 15 galaxies in $\mathrm{Cl} 0849,5$ in $\mathrm{Cl} 1701$ and 7 in $\mathrm{Cl} 1702$. These numbers are also shown in brackets next to the cluster name in Fig. 6.3. Again, the linear bisector fit to the Coma galaxies was restricted to the range encompassed by the distant rich cluster galaxies $\left(M_{r}<-20.42\right.$ 
and $\log \sigma>2.02$ ). Under assumption of a nonchanging local slope with redshift, the mean residuals from the local FJR of the poor cluster sample can be investigated and their mean evolution measured. The offsets of the distant Low $-L_{X}$ galaxies have an observed $\pm 1 \sigma$ scatter of $\sigma_{r}=0.65^{m}$. The dispersion is $14 \%$ larger than the scatter of the local Coma cluster and similar to the dispersion as for the rich clusters. A difference in the scatter between the distant samples is therefore rather unlikely. Restricting the local Coma galaxies to $\log (\sigma)>2.02, M_{r}<-20.08$, for the total sample of 27 galaxies in the FJR an average luminosity evolution of $-0.44 \pm 0.19^{m}$ is derived. However, this result depends on the assumed selection boundaries. If the faintest galaxy (ID \# 923) is excluded and the Coma sample is restricted to $\log (\sigma)>2.02, M_{r}<-20.42$, an evolution of $-0.39 \pm 0.19^{m}$ for the Low $-L_{X}$ cluster galaxies is found. In Table 6.1 a summary of the measured mean and median luminosity evolution of the Low $-L_{X}$ clusters is given.

Accounting for the possibility that the distant poor cluster sample and the local reference have different slopes, the FJR is analysed using bisector fits in Fig. 6.4. To explore a change in the slope, a bootstrap bisector fit to the distant cluster sample gives

$$
M_{r}=-(5.58 \pm 0.71) \log \sigma-(9.08 \pm 1.61) .
$$

The $\pm 1 \sigma$ errors of this fit are denoted as the solid lines in Fig. 6.4. This approach implies that a slope change would be significant on the $2 \sigma$ level, which suggests again a stronger evolution for lower-mass galaxies. A further discussion on a possible mass-dependence will be the topic of section 7.1.2. In the following section the Kormendy relation will be discussed.

\subsection{The Kormendy Relation}

To study the change of surface brightness evolution for the FP sample at a fixed size, the magnitude-size relation was constructed. The

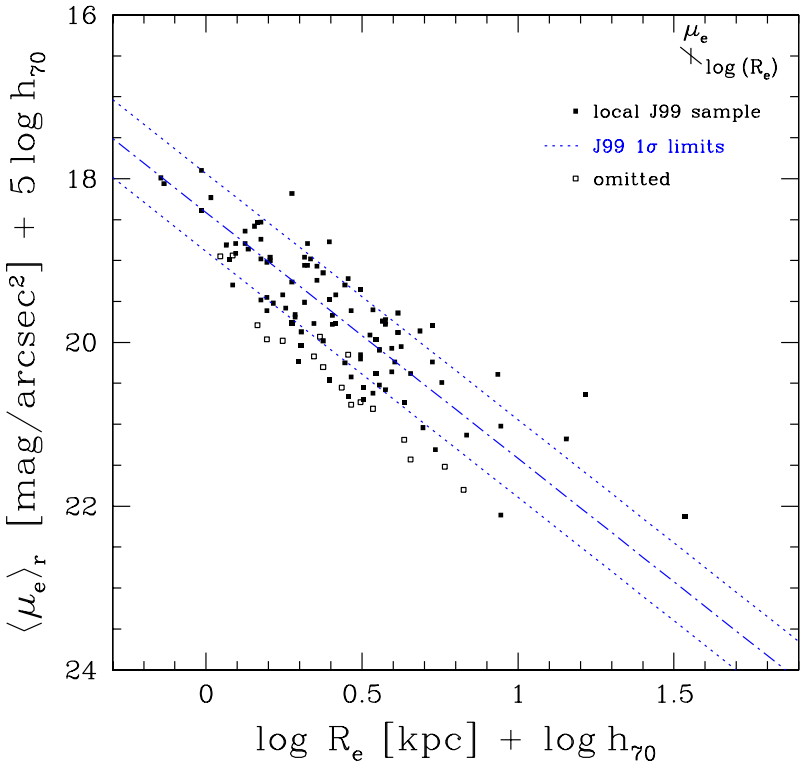

Figure 6.5: The local Kormendy relation in the Gunn $r$-band for 115 early-type galaxies in the Coma cluster from Jørgensen (1999, J99) and Jørgensen et al. 1995, J99). The individual objects of the J99 sample are denoted by the small solid squares and the open squares are excluded low-mass galaxies to match the distant cluster sample. The dot-dashed line is a bootstrap bisector fit to the local KR within the selection boundaries as defined by the distant cluster samples, together with the $1 \sigma$ errors (dashed lines). The error bar of $\mu_{\mathrm{e}}$ and $\log \left(R_{\mathrm{e}}\right)$ is indicated in the upper right corner.

Kormendy relation (KR) represents the projection of the Fundamental Plane along the velocity dispersion onto the photometric plane. As only the structural parameters are used, samples have larger scatter in the KR than in the FP but results should complement and endorse findings obtained with the Fundamental Plane.

Similar as for the FJR, the J99 Coma sample was restricted to the range in absolute magnitude and velocity dispersion encompassed by the combined rich cluster galaxies in A 2218 and A 2390 with lower limits of $M_{r}=-20.42$ and $\log \sigma=2.02$. Galaxies in the local Kormendy relation which do not meet these criteria are omitted and shown as open squares in Fig. 6.5. Only galaxies denoted as solid symbols are utilised for 


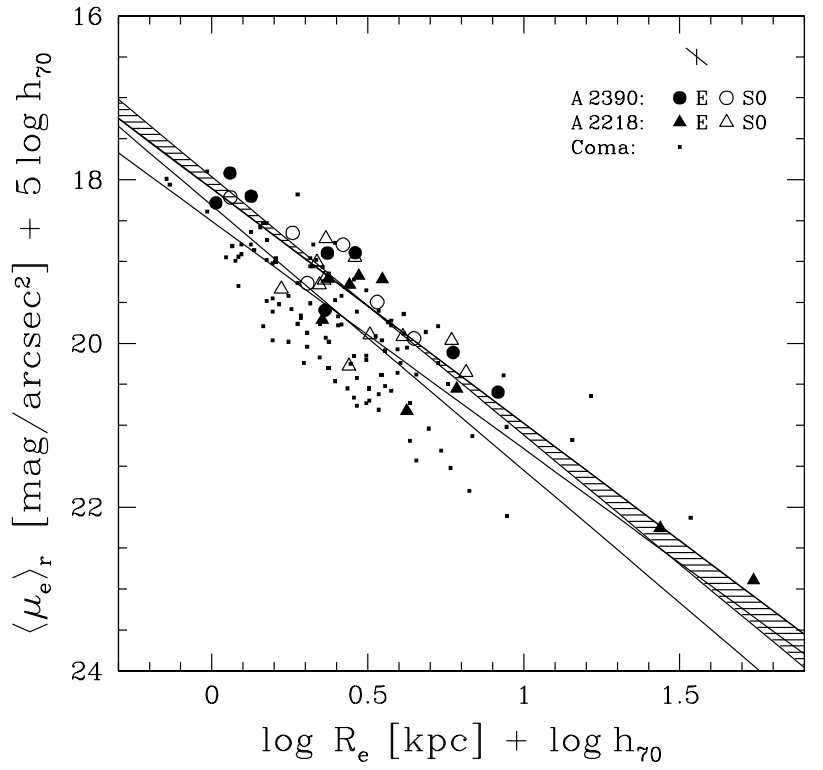

Figure 6.6: Kormendy relation in the Gunn $r$-band for A 2390 and A 2218, compared to the Coma sample of J99. The area bounded by solid lines indicates the $\pm 1 \sigma$ errors of the bisector fits to the local KR relation and the hashed region shows the bisector fits to the distant sample within $\pm 1 \sigma$. A typical error bar (mean error) is indicated in the upper right corner (see text for details).

the investigation of the KR. From Fig. 6.5 it can be seen that these neglected objects are predominantly low-surface brightness galaxies which are absent in the distant sample due to limitations in luminosity. If these faint galaxies were included for the KR analysis, the derived luminosity offset of the distant sample would be to large and therefore the evolution too strong.

The Kormendy relation in Gunn $r$ for the galaxies in A 2390 and A 2218 is shown in Fig. 6.6. The structural parameters of effective radius $R_{\mathrm{e}}$ and mean surface brightness within $R_{\mathrm{e}},\left\langle\mu_{\mathrm{e}}\right\rangle$, are based on curve of growth fits and derived using a combination of exponential disc plus $r^{1 / 4}$-law surface brightness models. The errors in $\log R_{\mathrm{e}}$ and $\left\langle\mu_{\mathrm{e}}\right\rangle$ are correlated. In the upper right corner an average error which enters the $\mathrm{KR}$ in the coupled form $\log R_{\mathrm{e}}-0.328\left\langle\mu_{\mathrm{e}}\right\rangle=0.08$ is displayed, assuming a typical uncertainty of

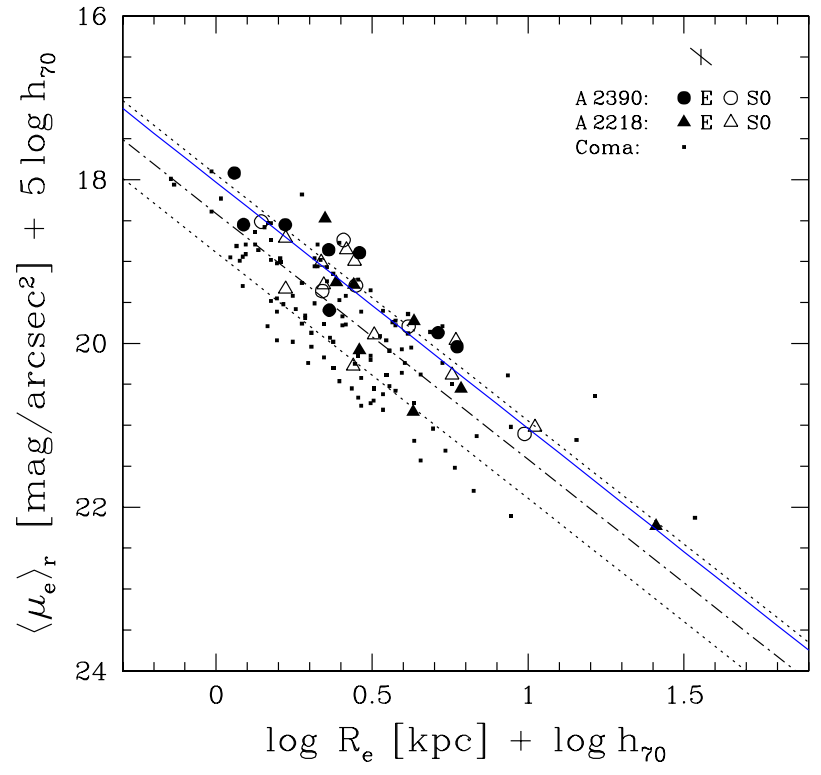

Figure 6.7: Kormendy relation in the Gunn $r$-band for A 2390 and A 2218, compared to the Coma sample of J99. The KR was constructed using solely $r^{1 / 4}$ law structural parameters. The dot-dashed line is a bisector fit to the local KR within the selection boundaries together with the mean $1 \sigma$ errors (dashed lines). The solid line shows the mean evolution of the rich cluster sample assuming a constant local slope.

$\Delta\left\langle\mu_{\mathrm{e}}\right\rangle=0.05$ and $\Delta \log R_{\mathrm{e}}=0.1$. In a similar manner as for the FJR, the luminosity evolution for the distant galaxies in the KR is computed. Within the selection boundaries, a bisector fit to the Coma sample gives

$$
\left\langle\mu_{\mathrm{e}}\right\rangle=(3.01 \pm 0.23) \log R_{\mathrm{e}}+(18.41 \pm 0.10),
$$

with an observed scatter of $\sigma_{\mathrm{KR}}=0.48^{m}$ in $\left\langle\mu_{\mathrm{e}}\right\rangle$. The $\pm 1 \sigma$ scatter of the bisector fits to the local KR is shown as solid lines in Fig. 6.6. For the distant clusters of of A 2218 and A 2390 the bisector method yields

$$
\left\langle\mu_{\mathrm{e}}\right\rangle=(3.03 \pm 0.17) \log R_{\mathrm{e}}+(18.01 \pm 0.09),
$$

which suggests that there is no significant slope change in comparison to the local reference. The average scatter of the bisector fits within $\pm 1 \sigma$ to the rich clusters are indicated as the hashed area in Fig. 6.6. 


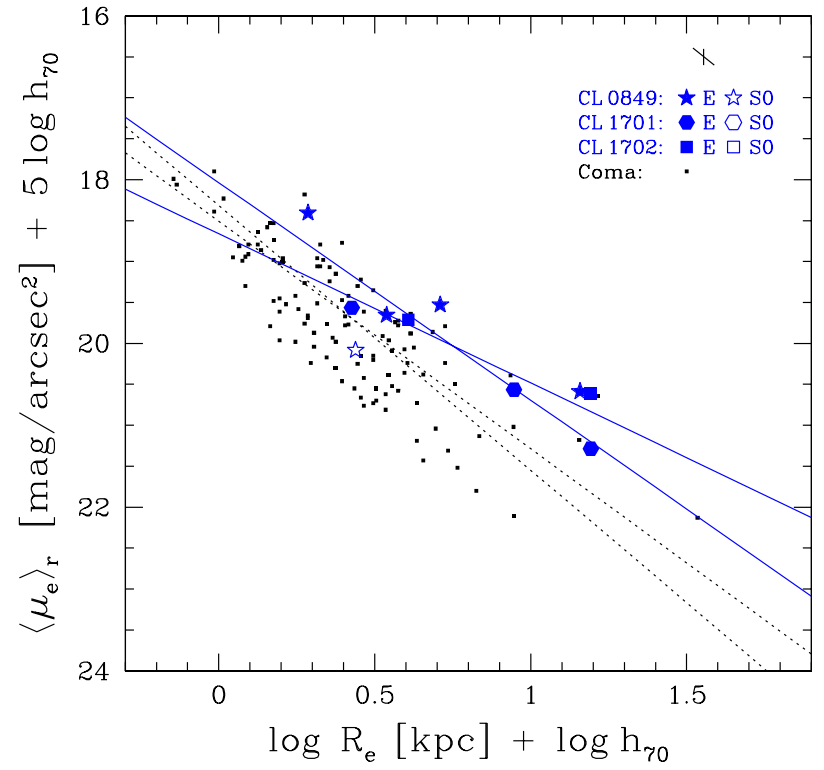

Figure 6.8: Kormendy relation in the Gunn $r$-band for the Low- $L_{X}$ clusters $\mathrm{Cl} 0849, \mathrm{Cl} 1701$ and $\mathrm{Cl} 1702$, compared to the Coma sample of J99. The area bounded by dotted lines indicates the $\pm 1 \sigma$ errors of the bisector fits to the local KR relation and the solid lines shows the bisector fits to the distant sample within $\pm 1 \sigma$. A typical error bar (mean error) is indicated in the upper right corner.

In order to examine the offsets of the distant cluster sample from the local KR again a constant slope with look-back time is assumed. The scatter of the observed offsets for the A 2218 and A 2390 galaxies is $\sigma_{\mathrm{KR}}=0.39^{m}$. Comparing the galaxies within their observed magnitude range $17.92 \leq\left\langle\mu_{\mathrm{e}}\right\rangle \leq 22.89$ with the reduced Coma KR, a larger luminosity evolution than with the FJR is found. In particular, in the restframe Gunn $r$ the 34 cluster galaxies are on average brighter by $-0.39 \pm 0.27^{m}$ (median value -0.46 ) compared to Coma. The two bright $\mathrm{cD}$ galaxies in $\mathrm{A} 2218$ at $\log R_{\mathrm{e}} \approx 1.45 \mathrm{kpc}$ and $\log R_{\mathrm{e}} \approx 1.75 \mathrm{kpc}$ follow the extension of the $\mathrm{KR}$ as defined by the distant cluster galaxies.

The KR depends on the assumed surface brightness profiles for the derivation of structural parameters of half-light radius $R_{\mathrm{e}}$ and mean surface brightness $\left\langle\mu_{\mathrm{e}}\right\rangle$, measured within the effective ra-

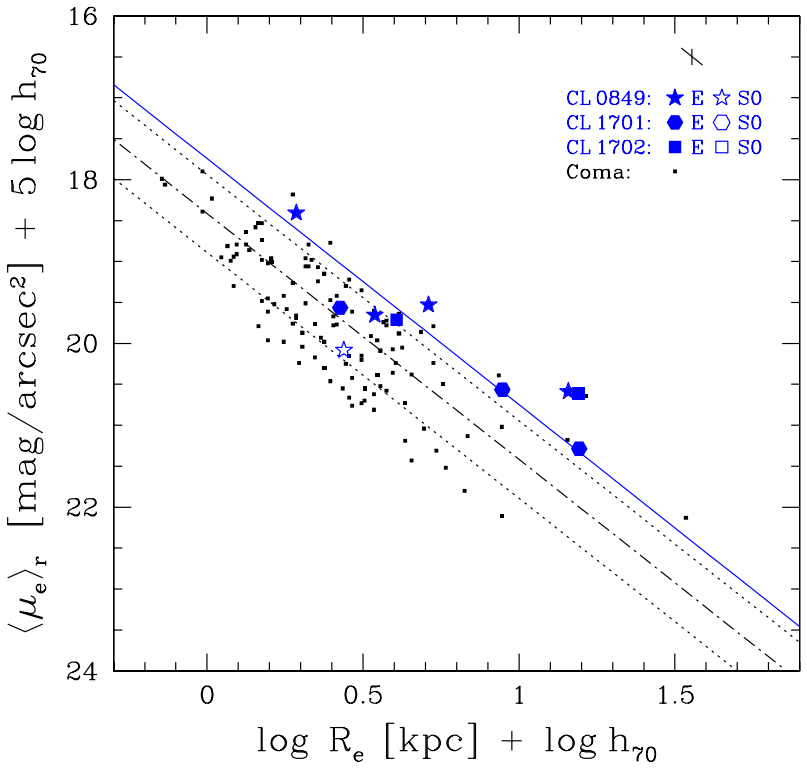

Figure 6.9: Kormendy relation in the Gunn $r$-band for the Low- $L_{X}$ clusters $\mathrm{Cl}$ 0849, $\mathrm{Cl} 1701$ and $\mathrm{Cl} 1702$, compared to the Coma sample of J99. The area bounded by dotted lines indicates the $\pm 1 \sigma$ errors of the bisector fit to the local KR relation and the solid lines shows the bisector fit to the distant sample within $\pm 1 \sigma$. A typical error bar (mean error) is indicated in the upper right corner.

dius. To test the effects on the KR, Fig. 6.7 illustrates the KR which was constructed using $\left\langle\mu_{\mathrm{e}}\right\rangle$ and $R_{\mathrm{e}}$ derived by a pure $r^{1 / 4}$ luminosity profile. The variations on the structural parameters are small and the galaxies move only along the average inclined error bar of $\log R_{\mathrm{e}}$ shown in the upper right corner in the KR figures. In general, the same conclusion can be drawn if the de Vaucouleurs structural parameters are used in the $\mathrm{KR}$. The $\mathrm{E}+\mathrm{S} 0$ galaxies of both clusters show an offset of $-0.38 \pm 0.27^{m}$, with a median of -0.42 . Note that, if $r^{1 / 4}$-law structural parameters are used to establish the KR, individual objects are differently distributed along the $K R$, whereas the derived average offsets from the local KR and the measured luminosity evolution are very similar. For purposes of comparison, a bisector fit to the distant KR of the rich clusters constructed with 
de Vaucouleurs structural parameters results in

$$
\left\langle\mu_{\mathrm{e}}\right\rangle=(3.09 \pm 0.17) \log R_{\mathrm{e}}+(17.99 \pm 0.09) .
$$

Neither a significant zero-point offset nor a slope change is found compared to the $\mathrm{KR}$ of the same sample but based on structural parameters which were deduced from a combination of bulge and exponential disc surface brightness profile fitting functions.

In Fig. 6.9, the KR for the three Low $-L_{X}$ clusters $\mathrm{Cl} 0849, \mathrm{Cl} 1701$ and $\mathrm{Cl} 1702$ is displayed. A total of ten spectroscopically confirmed earlytype cluster galaxies enters the KR and the FP, which are splitted into five, three and two galaxies for the clusters $\mathrm{Cl} 0849, \mathrm{Cl} 1701$ and $\mathrm{Cl} 1702$, respectively. A linear bisector fit to the Low $-L_{X}$ KR sample in Fig. 6.8 gives

$$
\left\langle\mu_{\mathrm{e}}\right\rangle=(2.24 \pm 0.42) \log R_{\mathrm{e}}+(18.35 \pm 0.31),
$$

which corresponds to a $2 \sigma$ flatter slope for the poor cluster galaxies. However, this result is at very low confidence level as the Low $-L_{X}$ sample lacks galaxies with small sizes of $\log R_{\mathrm{e}}<0.3$. As a result of this effect, the slope of the distant KR decreases.

To estimate the amount of luminosity evolution, the mean residuals from the local KR of the poor cluster sample by fixing the local slope are investigated. With respect to the Coma reference, the $\mathrm{E}+\mathrm{S} 0$ galaxies in poor clusters offer an observed scatter of $\sigma_{\mathrm{KR}}=0.53^{m}$ and brighter luminosities by $-0.67 \pm 0.23^{m}$. Furthermore, Figs. 6.8 and 6.9 show that the $\mathrm{cD}$ galaxies in Low $-L_{X}$ clusters are fainter and exhibit on average smaller sizes which implies that they are a less dominant galaxy population of the clusters. These galaxies are more comparable to their cluster member galaxies and not a distinct class of galaxies as their counterparts in rich galaxy clusters. This topic will be further addressed in the next section as the third parameter, the velocity dispersion, provides additional conclusions.

\subsection{The Fundamental Plane}

Via an investigation of the Fundamental Plane, tight constrains on the formation history and the dynamical status of early-type galaxies are possible. Three observables, characterising the size (effective radius $R_{\mathrm{e}}$ ), the luminosity (effective surface brightness within $\left.R_{\mathrm{e}},\left\langle\mu_{\mathrm{e}}\right\rangle\right)$ and the kinematics (velocity dispersion $\sigma$ ) of a galaxy form an empirical relationship, the Fundamental Plane, which provides information about the evolution and formation mechanisms of early-type galaxies. Fig. 6.10 shows two projections of the local Fundamental Plane relation in the Gunn $r$-band for 99 early-type galaxies in the Coma cluster from J99. The left hand panel shows the faceon view of the FP, the right hand panel the FP edge-on. The distribution of galaxies is affected by a selection effect, the completeness limit in the absolute magnitude of the Coma sample $\left(M_{r}=-20.02^{m}\right)$, which is indicated as the the dashed line in Fig. 6.10. The upper dotted line denotes the so-called 'exclusion zone' found for nearby galaxies by Bender et al. (1992), which is a region not occupied by dynamically hot stellar systems but not caused by selection effects. In terms of structural parameters, this limit implies $R_{\mathrm{e}} \sigma_{0}^{7.46}\langle I\rangle_{\mathrm{e}}^{-2.72} \leq$ constant, where $\sigma_{0}$ is the central velocity dispersion. As this borderline incorporates all three physical properties, both the density and the total mass of the progenitors of early-type galaxies are mutually constrained, which define an upper limit on the amount of involved dissipation for any given mass. In the edge-on view of Fig. 6.10, a principal component fit to the local FP relation is indicated by the thick solid line, which was derived via a principal component analysis (e.g., Ziegler et al. 2005). The correlation between $\langle I\rangle_{\mathrm{e}}$ (which is the mean surface brightness in units of $\mathrm{L}_{\odot} / \mathrm{pc}^{2}$, cf. Eq. 4.6 on page 69$), \sigma$ and $R_{\mathrm{e}}$ is displayed in both figures. Similar to the scaling relations presented before, the FP was restricted to the range in magnitudes and velocity dispersions covered by the distant samples. This is particularly impor- 

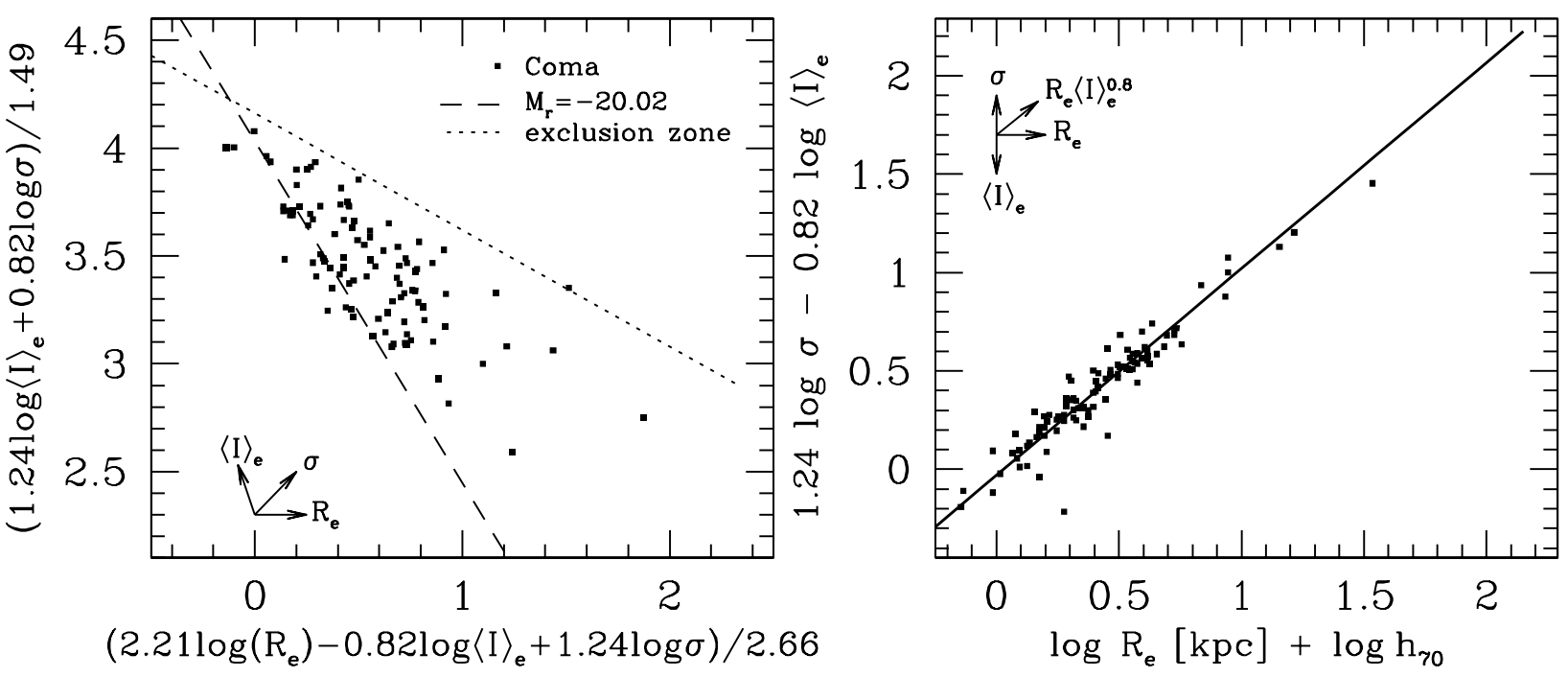

Figure 6.10: The local Fundamental Plane relation in the Gunn $r$-band for 99 early-type galaxies in the Coma cluster from Jørgensen (1999, J99) and Jørgensen et al. 1995, J99). Symbol notations as in Fig. 6.1. Left: FP face-on, Right: FP edge-on. The thick solid line is a principal component fit to the local FP relation. The arrows in both figures display the directions in which the basic parameters of $\langle I\rangle_{\mathrm{e}}, \sigma$ and $R_{\mathrm{e}}$ drive the FP.

tant when the amount of luminosity evolution of a distant sample with respect to a local reference is derived and will be further discussed in section 6.5 .

The Fundamental Plane in rest-frame Gunn $r$ for the distant clusters A $2218(z=0.175)$ and A $2390(z=0.228)$ is illustrated in Fig. 6.11. The figure also shows the FP for the local Coma samples of J99 $(z=0.024)$. All mean surface brightness magnitudes have been corrected for the dimming due to the expansion of the Universe. Errors are provided in the short edge-on FP projection (upper right panel of Fig. 6.11), with kinematic and photometric properties on separate axes. At a first glance, the following results can be deduced. Firstly, the FP for the individual galaxy populations as well as for the combined sample show a well-defined, tight relation with a small scatter. Both intermediate redshift clusters show a similar behaviour within and along all projections of the FP. There is no evidence for an increasing scatter with redshift. Bernardi et al. (2003) found for the SDSS sample an rms scatter of $\sigma=0.08$, measured as the rms scatter of the residuals in $\log R_{\mathrm{e}}$. The distant cluster galaxies have an rms scatter of $\sigma=0.113$, which is not significantly higher than the local value. This topic will be re-addressed in more detail for different sub-samples later on in this section. Secondly, only a moderate evolution of the FP and hence the stellar populations of the galaxies with redshift is seen. Assuming there is no evolution in the structure of the galaxies, i.e., at a fixed $R_{\mathrm{e}}$ and $\sigma$, the average brightening of the cluster galaxies can be determined.

In Table 6.2 the results for the FP in restframe Gunn $r$ are presented. The mean and median zero-point offsets from the local Coma $\mathrm{FP}$, their $1 \sigma$ scatter and the derived luminosity evolution are shown. For the combined sample of 34 early-type cluster galaxies an ZP offset of $\Delta \gamma=0.10 \pm 0.06$ compared to the local Coma reference is deduced, which corresponds, according to $\Delta \mu_{\mathrm{e}} \equiv \Delta \gamma / \beta$ with $\beta=-0.32$, to a modest luminosity evolution of $\Delta \mu_{\mathrm{e}}=-0.31 \pm 0.18^{m}$. The results are consistent with the picture of simple 

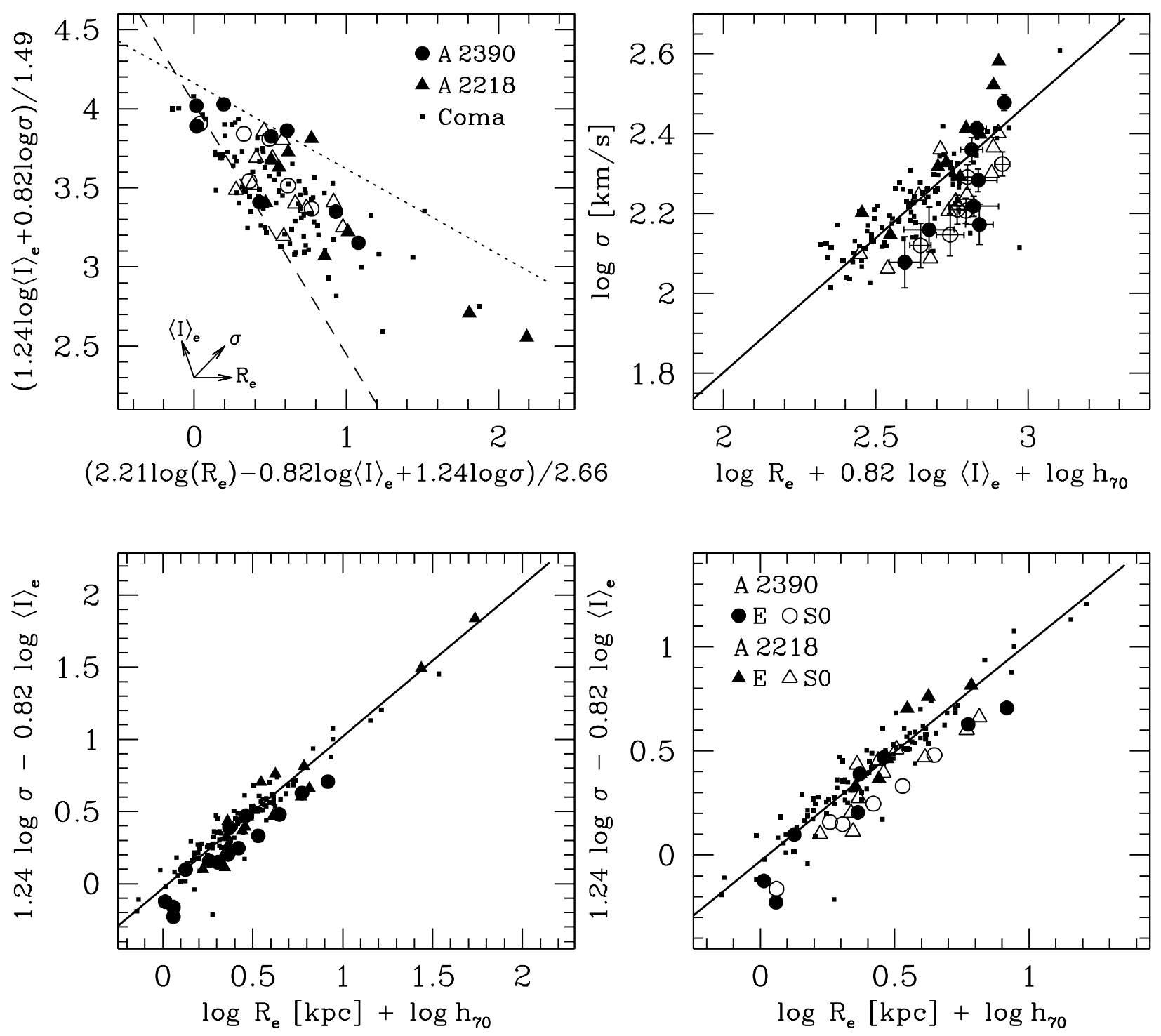

Figure 6.11: Fundamental Plane for A 2390 at $z=0.23$ (circles) and A 2218 at $z=0.18$ (triangles) in rest-frame Gunn $r$-band, compared to the Coma galaxies of J99 (small squares). Filled symbols denote ellipticals, open symbols S0 galaxies and Sa bulges. Upper panel, left: Face-on view of the FP. The dotted line indicates the so-called 'exclusion zone' (Bender et al. 1992), the dashed line the luminosity limit for the completeness of the Coma sample $M_{r \mathrm{~T}}=-20.02^{m}$. Upper panel, right: FP edge-on, along short axis. The solid line represents the principal component fit for the local Coma sample. Lower panel: Edge-on FP. On the right side a zoom of the edge-on FP with a separation into different morphologies is shown. 
Table 6.2: Evolution of the FP in Gunn $r$ as derived for various samples. $N$ shows the number of galaxies and $\Delta \bar{\gamma}$ indicates the mean FP zero-point offset. In the fourth and fifth column, the median FP zero-point evolution $\Delta\langle\gamma\rangle$ and the median evolution in the FP $\Delta\left\langle\mu_{\mathrm{e}}\right\rangle$ [in mag] are listed. The last column gives the $\pm 1 \sigma$ scatter of the mean offsets.

\begin{tabular}{lrrrrc}
\hline Sample & $N$ & $\Delta \bar{\gamma}$ & $\Delta\langle\gamma\rangle$ & $\Delta\left\langle\mu_{\mathrm{e}}\right\rangle$ & $\sigma_{\gamma}$ \\
\hline A 2218 & 20 & 0.032 & 0.026 & -0.079 & 0.108 \\
A 2390 & 14 & 0.140 & 0.158 & -0.482 & 0.088 \\
A 2218+A 2390 & 34 & 0.076 & 0.100 & -0.305 & 0.113 \\
\hline E $^{a}$ & 17 & 0.032 & 0.008 & -0.024 & 0.122 \\
S0 $^{a}$ & 17 & 0.120 & 0.144 & -0.439 & 0.085 \\
E (pure $\left.r^{1 / 4}\right)^{b}$ & 17 & 0.032 & -0.010 & 0.030 & 0.125 \\
S0 (pure $\left.r^{1 / 4}\right)^{b}$ & 17 & 0.115 & 0.134 & -0.409 & 0.079 \\
\hline low lum. $^{c}$ & 17 & 0.085 & 0.100 & -0.305 & 0.114 \\
E & 8 & 0.070 & 0.074 & -0.226 & 0.127 \\
S0 & 9 & 0.098 & 0.122 & -0.372 & 0.107 \\
high lum. & 17 & 0.068 & 0.093 & -0.284 & 0.114 \\
E & 9 & -0.001 & -0.020 & 0.061 & 0.114 \\
S0 & 8 & 0.145 & 0.168 & -0.512 & 0.045 \\
\hline low mass & 17 & 0.129 & 0.153 & -0.466 & 0.106 \\
E & 6 & 0.114 & 0.158 & -0.482 & 0.150 \\
S0 & 11 & 0.138 & 0.153 & -0.466 & 0.080 \\
high mass & 17 & 0.023 & 0.010 & -0.030 & 0.095 \\
E & 11 & -0.012 & -0.015 & 0.046 & 0.081 \\
S0 & 6 & 0.087 & 0.100 & -0.305 & 0.091 \\
\hline
\end{tabular}

${ }^{a}$ based on $r^{1 / 4}+$ exp. disc surface brightness profiles.

${ }^{b} r^{1 / 4}$ surface brightness profile only.

${ }^{c}$ lower-luminosity: $M_{r}>-21.493$, higher-luminosity: $M_{r}<-21.493$.

${ }^{d}$ less-massive: $\log \sigma<2.283$, more-massive: $\log \sigma>2.283$.

passive evolution models by BC96. Assuming a formation redshift of $z_{\text {form }}=2$ for all stars, these models predict a brightening by $\Delta m_{r} \approx 0.20^{m}$. For the individual clusters, different results are obtained. A zero-point offset of $0.16 \pm 0.06$ for A 2390 alone is found, meaning that the earlytype galaxies in A 2390 are on average more luminous by $\Delta \mu_{\mathrm{e}}=-0.49 \pm 0.18^{m}$ than the local Coma sample of J99. For the E+S0 in A 2218 an offset of $\Delta \gamma=0.03 \pm 0.06$ is detected, corresponding to a brightening of the stellar populations by $\Delta \mu_{\mathrm{e}}=-0.09 \pm 0.18^{m}$. This variation for the clusters may be caused by a combination of two effects: $(i)$ possible effect due to cosmic variance and (ii) sample selection. For example, Jørgensen et al. (1999) have analysed two different clusters (A 665 and A 2218) both at a redshift of $z=0.18$ and found a slight different evolution of $\Delta \mu_{\mathrm{e}} \sim-0.15^{m}$. This may give evidence that not all rich clusters have the same FP zero-point offset and that cosmic variance must be accounted for (see section 6.6 for further details on this topic). Another reason may arise from the sample selection. In case of A 2390 
particularly fainter galaxies have been selected in order to gain additional insights on the lowmass end of the FP at $z \sim 0.2$. For this reason, the A $2390 \mathrm{FP}$ sub-sample comprises more lowluminosity galaxies than that of A 2218, which results on average in a stronger luminosity evolution for the A 2390 cluster. Table 6.2 gives a comparison between the two sub-samples of less and more-massive galaxies and between faint and bright galaxies. If the total sample is divided into halves regarding to velocity dispersion at $\sim 192 \mathrm{~km} \mathrm{~s}^{-1}$ (corresponding to $\log \sigma<2.283$ ), a stronger evolution of $\Delta \mu_{\mathrm{e}}=-0.47 \pm 0.24^{\mathrm{m}}$ for the low-mass galaxies with respect to their more massive counterparts $\Delta \mu_{\mathrm{e}}=-0.03 \pm 0.21^{\mathrm{m}}$ is derived. Similar results are found when a cut in luminosity at $M_{r}=-21.493$ is made, although with a larger scatter. The difference between the lower-luminosity and higher-luminosity galaxies in the sample of the distant rich cluster is also visible in the size distribution of the two samples, see Fig. 5.28 on page 125 and in the Kormendy relation (cf. Fig. 6.6). The cluster A 2390 comprises more galaxies with small sizes and three galaxies even with $\log R_{\mathrm{e}} \leq 0.05$.

Looking at the thickness of the FP with respect to luminosity, an increasing scatter for the fainter galaxies within the rich cluster sample is not found. Both lower-luminosity galaxies with $M_{r}>-21.493$ and higher-luminosity ones exhibit a rms scatter of the residuals in $\log R_{\mathrm{e}}$ of 0.114. Jørgensen et al. (1996), divided their local sample with respect to luminosity into faint $\left(M_{r}>-23.16^{m}\right)$ and bright galaxies and reported on a larger rms scatter for the lower-luminosity galaxies of 0.086 , whereas the bright galaxies have 0.047 . These authors argued that the larger dispersion may be a result of the presence of discs or larger variations in the stellar populations of lower-luminosity galaxies since these objects showed a larger range in the $\mathrm{Mg}_{2}$ index. On the other hand, slight differences in the scatter between lower-mass and highermass cluster galaxies in A 2218 and A 2390 are found. Lower-mass galaxies with $\log \sigma<2.283$ feature a rms scatter of 0.106 , but higher-mass galaxies show only 0.095. The deviations are not significant, but nevertheless could be a hint that the lower-mass galaxies, which also exhibit a stronger luminosity evolution, have more diverse stellar populations or different star formation histories than their more-massive counterparts.

Fig. 6.12 displays the Fundamental Plane in restframe Gunn $r$ for the distant Low $-L_{X}$ clusters Cl $0849(z=0.234)$, Cl $1701(z=0.246)$ and $\mathrm{Cl} 1702(z=0.223)$. Again, the distant FP is compared to the local Coma FP of J99 $(z=0.024)$. The mean surface brightness magnitudes have been corrected for the dimming due to the expansion of the Universe and the errors are shown in the short edge-on FP projection (upper right panel of Fig. 6.12). Similar to the rich clusters, the FP for the combined poor cluster sample is a well-defined relation, with no significant differences or trend between the individual galaxy populations of a specific cluster. All three intermediate redshift clusters show a similar behaviour within and along all projections of the FP. The combined poor cluster sample has an rms scatter of $\sigma=0.161$, which is twice as large as the local Coma rms scatter. Dividing the sample according to luminosity, small differences between lower-luminosity and higherluminosity galaxies are detected. Fainter galaxies $\left(M_{r}>-21.855\right)$ offer a rms scatter of 0.194 , whereas brighter galaxies have 0.143. Similar to the rich cluster galaxies slight differences arise when the sample is separated with respect to mass. Lower-mass galaxies with $\log \sigma<2.295$ exhibit a rms scatter of 0.173 and higher-mass galaxies have 0.126 . This increasing scatter is also visible in the left panel of Fig. 6.20, where the $M / L$ ratios are plotted as a function of velocity dispersion. Table 6.3 lists the mean and median zero-point offsets from the local Coma FP, the measured luminosity evolution and the observed $1 \sigma$ scatter for various Low $-L_{X}$ clus- 

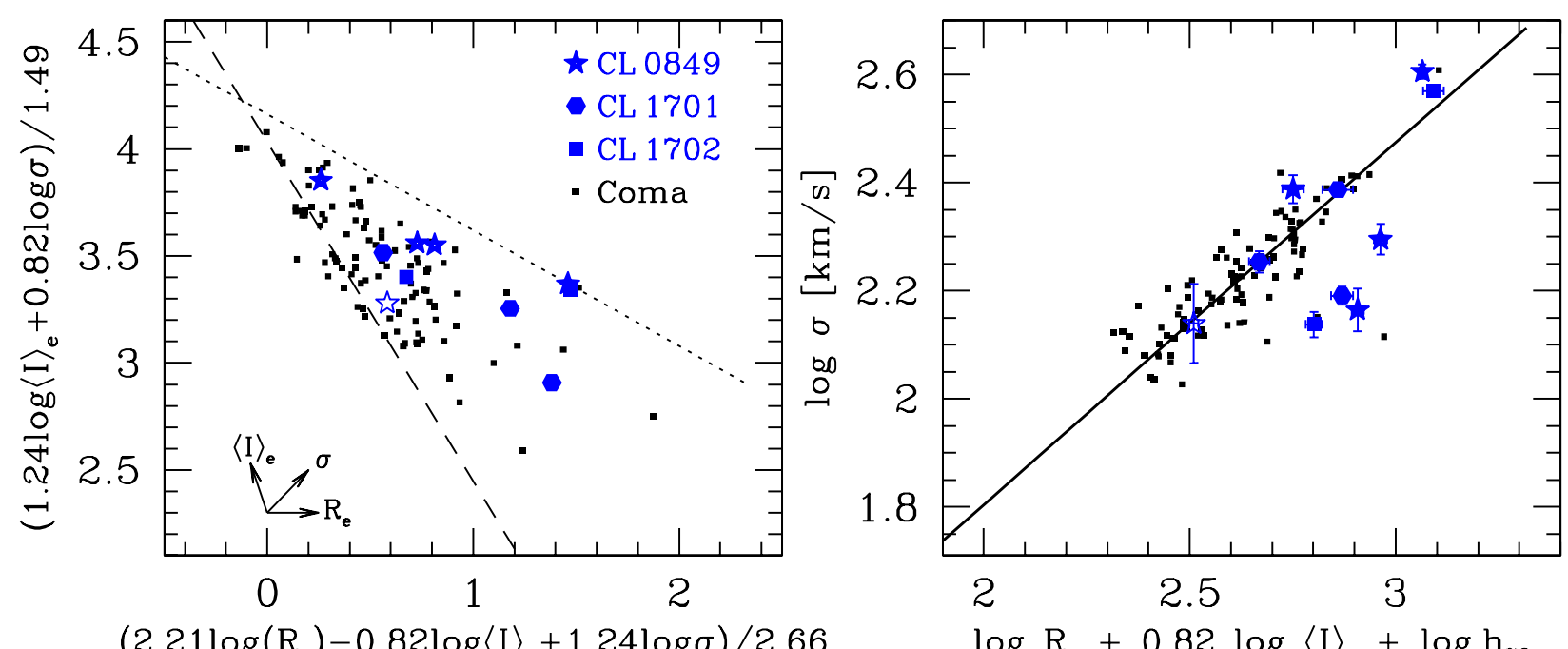

$\left(2.21 \log \left(R_{\mathbf{e}}\right)-0.82 \log \langle I\rangle_{\mathbf{e}}+1.24 \log \sigma\right) / 2.66$

$\log R_{e}+0.82 \log \langle I\rangle_{e}+\log h_{70}$
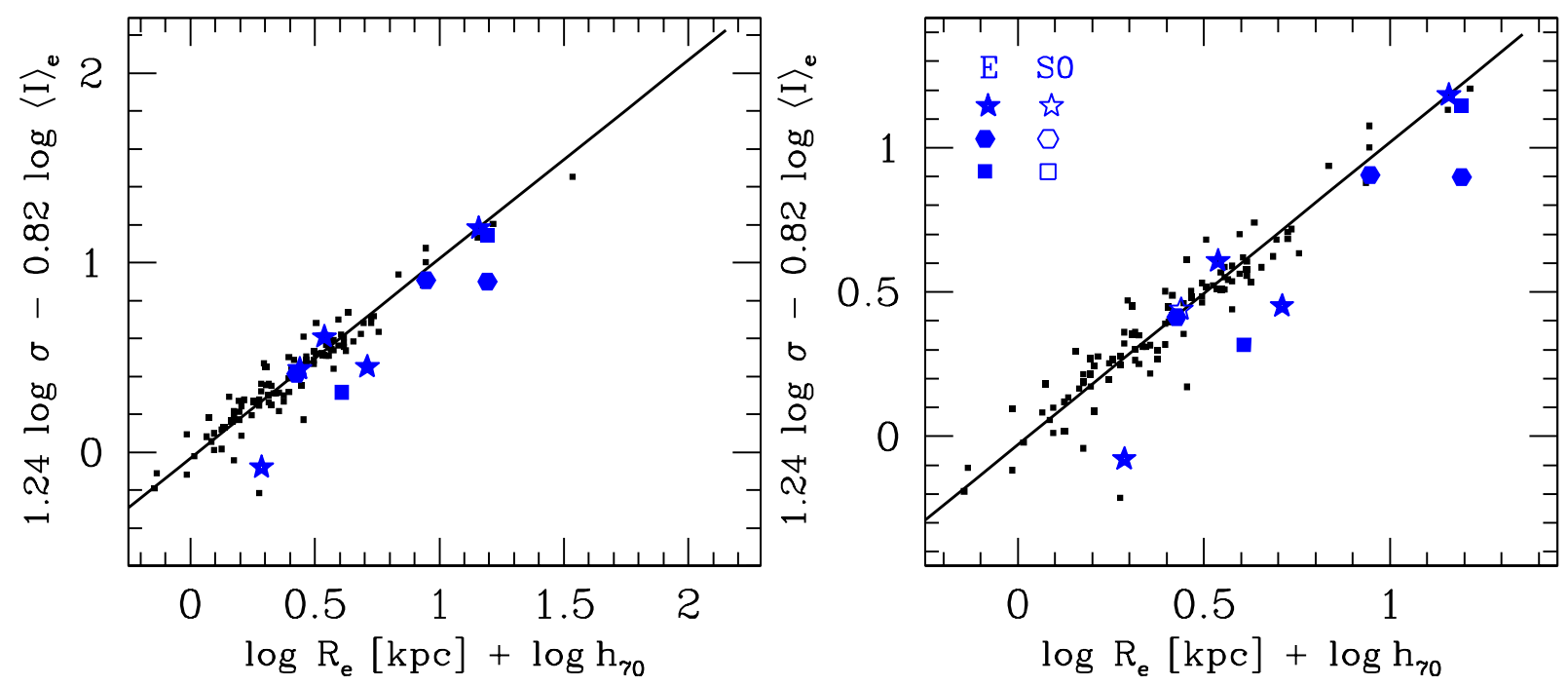

Figure 6.12: Fundamental Plane for the early-type galaxies in the poor clusters Cl 0849, Cl 1701 and $\mathrm{Cl} 1702$ (large symbols) in rest-frame Gunn $r$-band, compared to the Coma galaxies of J99 (small squares). Filled symbols denote ellipticals, open symbols S0 galaxies. Upper panel, left: Face-on view of the FP. The dotted line indicates the so-called 'exclusion zone' (Bender et al. 1992), the dashed line the luminosity limit for the completeness of the Coma sample $M_{r \mathrm{~T}}=-20.02^{m}$. Upper panel, right: FP edge-on, along short axis. The solid line represents the principal component fit for the local Coma sample. Lower panel: Edge-on FP. On the right side a zoom of the edge-on FP with a separation into different morphologies is shown. 
Table 6.3: Evolution of the FP in Gunn $r$ as derived for the Low $-L_{X}$ cluster samples in Cl 0849, Cl 1701 and $\mathrm{Cl}$ 1702. $N$ shows the number of galaxies and $\Delta \bar{\gamma}$ indicates the mean FP zero-point offset. In the fourth and fifth column, the median FP zero-point evolution $\Delta\langle\gamma\rangle$ and the median evolution in the FP $\Delta\left\langle\mu_{\mathrm{e}}\right\rangle$ [in mag] are listed. The last column gives the $\pm 1 \sigma$ scatter of the mean offsets.

\begin{tabular}{lrrrrc}
\hline Sample & $N$ & $\Delta \bar{\gamma}$ & $\Delta\langle\gamma\rangle$ & $\Delta\left\langle\mu_{\mathrm{e}}\right\rangle$ & $\sigma_{\gamma}$ \\
\hline Cl 0849 & 5 & 0.105 & -0.002 & 0.006 & 0.193 \\
Cl 1701 & 3 & 0.116 & 0.040 & -0.122 & 0.154 \\
Cl 1702 & 2 & 0.169 & 0.292 & -0.889 & 0.174 \\
${\text { Low }-L_{X}}$ & 10 & 0.121 & 0.046 & -0.141 & 0.161 \\
\hline low lum. $^{a}$ & 5 & 0.120 & 0.015 & -0.045 & 0.194 \\
high lum. & 5 & 0.123 & 0.046 & -0.141 & 0.143 \\
\hline low mass $^{b}$ & 5 & 0.193 & 0.292 & -0.889 & 0.173 \\
high mass & 5 & 0.050 & 0.040 & -0.122 & 0.126 \\
\hline
\end{tabular}

${ }^{a}$ lower-luminosity: $M_{r}>-21.855$, higher-luminosity: $M_{r}<-21.855$.

${ }^{b}$ less-massive: $\log \sigma<2.295$, more-massive: $\log \sigma>2.295$.

ter samples. The combined sample of 10 earlytype cluster galaxies exhibits an $\mathrm{ZP}$ offset of $\Delta \gamma=0.12 \pm 0.05$ compared to the local reference, which corresponds to a modest luminosity evolution of $\Delta \mu_{\mathrm{e}}=-0.37 \pm 0.15^{m}$. This mild evolution is in compliance with a passive evolution for the bulk of the stellar populations in these galaxies. Dividing the sample with respect to velocity dispersion, the lower-massive galaxies $(\log \sigma<2.295)$ show a stronger evolution with $\Delta \mu_{\mathrm{e}}=-0.59 \pm 0.49^{m}$ than the higher-mass ones with $\Delta \mu_{\mathrm{e}}=-0.15 \pm 0.13^{m}$. Although this comparison is based on low number statistics, it confirms the results of the FJR, with a larger offset and therefore larger average brightening for lower-mass galaxies with respect to the Coma reference. Splitting the data set according to luminosity at $M_{r}=-21.855$, the sub-samples indicate a similar scatter and evolution in luminosity but no significant differences. A continuation of this topic will be demonstrated in section 7.1.2 based on the whole poor cluster sample.

To complete the analysis of early-type galaxies in different environments, the Fundamental Plane for two field galaxy samples was constructed.
Fig. 6.13 shows the FP for the 21 early-type field galaxies in the FDF and WHDF in the rest-frame Johnson $B$-band, compared to the 39 early-type galaxies in the Coma cluster by SBD93. The local reference is indicated with small squares, whereas the distant field galaxies are shown as the large circles and stars. Filled symbols denote ellipticals, open symbols S0 galaxies and Sa bulges. The $21 \mathrm{FDF}$ and WHDF early-type field galaxies are morphologically classified into $9 \mathrm{E}$ and 12 S0. Five ellipticals and six S0s originate from the FDF and four Es and six S0s are located in the WHDF. Two galaxies are not visible on the ACS images and for one elliptical galaxy in the FDF no structural parameters could be derived. The distant field galaxies cover a redshift range of $0.21 \leq z_{\text {Field } \mathrm{E}} \leq 0.74$ with a median of $\left\langle z_{\text {Field } \mathrm{E}}\right\rangle=0.40$. The two outliers in the FP, \#6336 $\left(\log R_{\mathrm{e}}=0.46\right)$ and \# $508\left(\log R_{\mathrm{e}}=1.20\right)$, reveal on the ACS images a significant disc component and early spiral morphology. Galaxy \# 6336 has an extraordinary strong $\mathrm{H} \delta_{\mathrm{F}}$ absorption, which points to a young luminosity-weighted average model age under assumption of a single stellar population. 


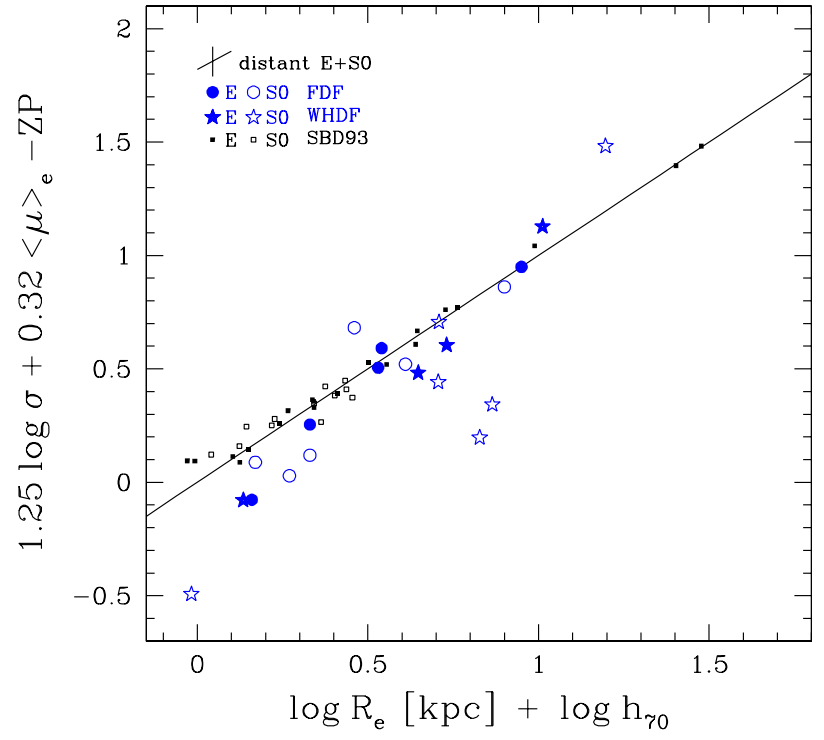

Figure 6.13: Edge-on view of the Fundamental Plane for the early-type field galaxies in the FDF and WHDF, compared to the Coma galaxies of SBD93 (small squares) in rest-frame Johnson $B$-band. Filled symbols denote ellipticals, open symbols $\mathrm{S} 0$ galaxies and Sa bulges. The distant field lenticular galaxies show a stronger evolution and larger scatter than the ellipticals.

On the ACS images, \# 508 shows a weird structure which might indicate that this object is an ongoing merger. Both galaxies were classified as Sa bulges hence these early-type spirals offer at least a low level of star formation which increases their total luminosity and displaces their position beyond the tight plane as established by early-type galaxies.

Rejecting the two outliers, for the total sample of 19 early-type field galaxies in the FDF and WHDF an average luminosity evolution of $\Delta M_{B}=-0.53 \pm 0.13^{m}$ with a median of $\Delta\left\langle M_{B}\right\rangle=-0.39$ is found. Similar results are measured for 17 cluster ellipticals at $\langle z\rangle \sim 0.4$ with similar look-back times ( $\sim 5$ Gyrs). These galaxies show an average brightening of their stellar populations of $\Delta M_{B}=-0.44 \pm 0.18^{m}$, compared to their local counterparts (Ziegler et al. 2005). Overall, the distribution along the FP and the measured luminosity evolution is similar for cluster and field galaxies at $\langle z\rangle \sim 0.4$ and within the cluster sample no differences between ellipticals and S0 galaxies were found. However, the sample of distant cluster galaxies lacks galaxies with small sizes. On the other hand, the field galaxy sample comprises no large ellipticals or $\mathrm{cD}$ galaxies. A comparison of the sizes of these two data sets is therefore not adequate and would cause misleading results. For this reason, the cluster galaxies at $\langle z\rangle \sim 0.4$ will not be compared to the field sample to test possible differences in their stellar populations. In Table 6.4 a summary of the results for the FP of the earlytype field galaxies in Johnson $B$-band is given. The thickness of the FP seems to vary between the individual field galaxy samples. For the FDF an average rms scatter of 0.136 is detected, whereas the WHDF show a larger $1 \sigma$ dispersion of 0.289 . This effect is partly caused by three high redshift objects $0.4 \leq z \leq 0.74$ in the WHDF, which are all discy galaxies. Dividing the field sample with respect to velocity dispersion at $\log \sigma=2.275$ or luminosity at $M_{B}=-21.404$, no strong variations in the amount of evolution between the sub-samples are detected. However, the comparisons rely on the assumed cutoffs in $\sigma$ and $M_{B}$ because outliers, such as early Sa spiral galaxies, can strongly influence the derived luminosity evolution. In the next section, possible differences between the populations of elliptical and lenticular galaxies will be addressed.

\subsubsection{Elliptical versus S0 galaxies}

Local investigations based on the Fundamental Plane have not revealed significant differences in the zero-point, slope and/or scatter between elliptical and lenticular galaxies (Bender, Burstein \& Faber 1992; Saglia et al. 1993b; Jørgensen et al. 1996). Moreover, they behave very similar as one single group of galaxies with respect to their $M / L$ ratios and within the FP. Going to higher redshifts, differences could be more significant if recent star formation activity plays a role for S0 
Table 6.4: Evolution of the FP in Johnson $B$-band as derived for the early-type field galaxies in the FDF and WHDF. $N$ shows the number of galaxies and $\Delta \bar{\gamma}$ indicates the mean FP zero-point offset. In the fourth and fifth column, the median FP zero-point evolution $\Delta\langle\gamma\rangle$ and the median evolution in the FP $\Delta\left\langle\mu_{\mathrm{e}}\right\rangle$ [in mag] are listed. The last column gives the $\pm 1 \sigma$ scatter of the mean offsets.

\begin{tabular}{lrcccc}
\hline Sample & $N$ & $\Delta \bar{\gamma}$ & $\Delta\langle\gamma\rangle$ & $\Delta\left\langle\mu_{\mathrm{e}}\right\rangle$ & $\sigma_{\gamma}$ \\
\hline FDF & 11 & 0.066 & 0.075 & -0.235 & 0.136 \\
WHDF & 10 & 0.199 & 0.214 & -0.667 & 0.289 \\
FDF+WHDF & 21 & 0.130 & 0.089 & -0.279 & 0.227 \\
FDF+WHDF $^{a}$ & 19 & 0.170 & 0.126 & -0.393 & 0.197 \\
\hline E & 9 & 0.075 & 0.075 & -0.230 & 0.121 \\
S0 $_{\text {S0 }^{a}}$ & 12 & 0.171 & 0.211 & -0.643 & 0.281 \\
S0 $^{b}$ & 10 & 0.255 & 0.241 & -0.735 & 0.219 \\
\hline low lum. $^{c}$ & 9 & 0.214 & 0.211 & -0.643 & 0.185 \\
high lum. $^{d}$ & 11 & 0.056 & 0.082 & -0.255 & 0.180 \\
high lum. $^{d}$ & 10 & 0.211 & 0.165 & -0.515 & 0.254 \\
low mass $^{e}$ & 9 & 0.164 & 0.126 & -0.393 & 0.219 \\
high mass & 11 & 0.099 & 0.089 & -0.279 & 0.221 \\
\hline
\end{tabular}

${ }^{a}$ Omitting \# 6336 and \# 508, both Sa bulges.

${ }^{b}$ Omitting \# 6336 and \# 508 and \# 111.

${ }^{c}$ lower-luminosity: $M_{B}>-21.404$, higher-luminosity: $M_{B}<-21.404$.

${ }^{d}$ Omitting \# 111 at $z=0.74$.

${ }^{e}$ less-massive: $\log \sigma<2.275$, more-massive: $\log \sigma>2.275$.

galaxies. However, previous studies of samples at higher redshift did not find any differences between elliptical and S0 galaxies (van Dokkum \& Franx 1996; Kelson et al. 2000b). For example, within the large sample of 30 early-type galaxies in CL $1358+62$ at $z=0.33$ of Kelson et al. (2000b), the 11 ellipticals displayed identical zero-points as the 13 (non-E+A) S0 galaxies with no hint for an offset between these groups at all. Moreover, the difference in the slope of $\sim 14 \%$ detected between the S0 and elliptical galaxies is not significant.

For the case of S0 galaxies, two main questions are still a matter of debate. How many are a priori S0 galaxies? Which and how many are the result of galaxy transformations and account for the dominant S0 population in rich clusters to- day? For scenarios which suggest a transformation of star-forming spiral galaxies into passive S0 systems (Dressler et al. 1997), these objects would only be classified as lenticulars after their morphology has been changed ( $\sim 5$ Gyrs). However, after this relatively long time-scale, their star formation (SF) could already have ceased which would make these galaxies hard to detect via their SF. E+A galaxies, which are characterised by strong $\mathrm{H} \delta$ absorption lines, could represent galaxies in an intermediate stage of such a transformation where the merging process of two individual galaxies just has stopped and the spheroidal remnant is indistinguishable from a regular E/S0 galaxy, but SF is still present due the result of a starburst which ended within the last 1.5-2 Gyr (Barger et al. 1996). Recently, 


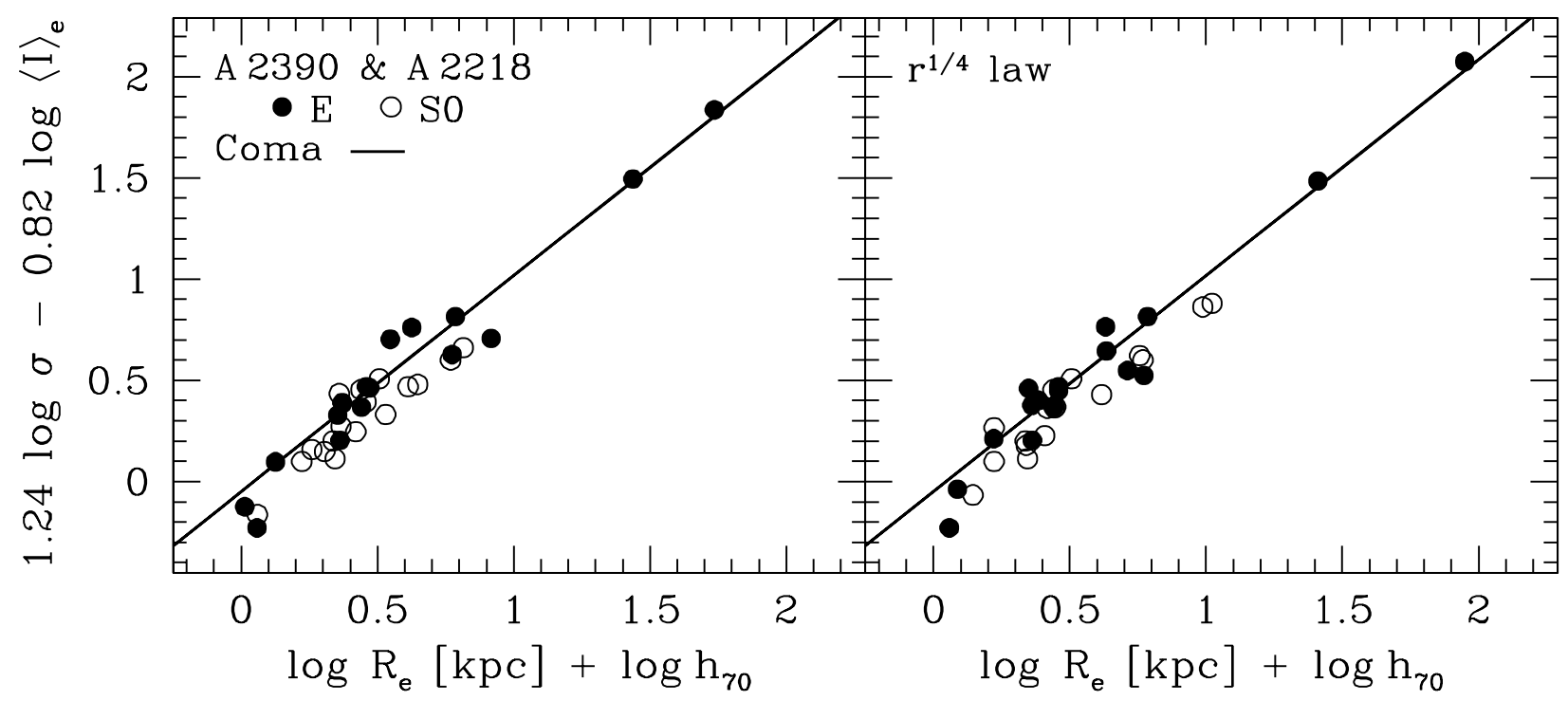

Figure 6.14: Edge-on view of the Fundamental Plane for A 2390 and A 2218 in rest-frame Gunn $r$. The distant FP is compared to the FP of the local Coma sample of J99, indicated by the principal component fit (thick solid line). Left: FP constructed using a combination of an $r^{1 / 4}$ and exponential disc profile. Right: Similar to the left plot except that $r^{1 / 4}$-law parameters were used.

Yang et al. (2004) performed a study of E+A galaxies in the local Universe and argued that these objects only show an offset in their surface brightnesses but not in their total magnitudes. If such galaxies, which contribute about $20 \%$ to the galaxy population in clusters at $z \sim 0.5$, are also within the galaxy samples investigated here, they would have no offset in their absolute luminosity and therefore would be indistinguishable from other non-E+A galaxies in the FJR which is based on total magnitude measurements. But $\mathrm{E}+\mathrm{A}$ galaxies could be identified within the FP due to their high surface brightness magnitudes which makes them stand apart from the FP of early-type galaxies.

Figure 6.14 illustrates the edge-on view of the $\mathrm{FP}$ for $34 \mathrm{E}+\mathrm{S} 0$ galaxies of the clusters A 2218 and A 2390 in rest-frame Gunn $r$-band. The FP constructed for the distant clusters at intermediate redshift is compared to the FP for the local Coma sample of J99. The combined sample represents the most extensive investigation of earlytype galaxies in clusters at $z \sim 0$.2. The Fig. 6.14 has been splitted in order to visualise the small variations if different luminosity profile fits are used for deriving structural parameters. The left figure displays the FP constructed using a combination of an $r^{1 / 4}$-law +exponential disc profile, the right figure is based on pure $r^{1 / 4}$-law fits. The variations in the structural parameters only affect the galaxies to move along the edge-on projection of the FP plane, thereby maintaining the tightness of the plane. Both the $r^{1 / 4} \mathrm{FP}$ and $r^{1 / n} \mathrm{FP}$ have the same scatter and slope within their errors. A second comparison is given by the FP parameter $R_{\mathrm{e}}\left\langle I_{\mathrm{e}}\right\rangle^{0.8}$ in Fig. 6.15, where the structural parameters of $R_{\mathrm{e}}$ and $\left\langle I_{\mathrm{e}}\right\rangle$ were derived from a combination of an $r^{1 / 4}+$ exponential disc profile and by a pure $r^{1 / 4}$-law model. As the errors in $R_{\mathrm{e}}$ and $\left\langle I_{\mathrm{e}}\right\rangle$ are correlated and this correlation is almost parallel to the FP, the product $R_{\mathrm{e}}\left\langle I_{\mathrm{e}}\right\rangle^{0.8}$ can be established with high accuracy. The individual measurements of Fig. 6.15 are listed in Table 4.5 and Table 4.6 (column 9). In general, the agreement is good for both elliptical and S0 galaxies. 


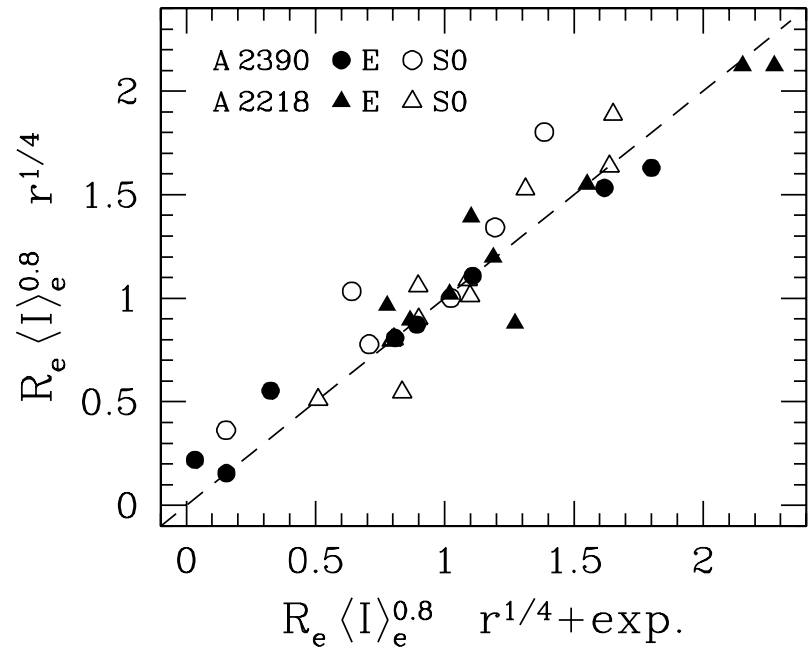

Figure 6.15: $\quad$ FP parameter $R_{\mathrm{e}}\left\langle I_{\mathrm{e}}\right\rangle^{0.8}$ derived from a pure $r^{1 / 4}$-law model compared to the $R_{\mathrm{e}}\left\langle I_{\mathrm{e}}\right\rangle^{0.8}$ constructed using a combination of an $r^{1 / 4_{-}}$ law+exponential disc profile. The product $R_{\mathrm{e}}\left\langle I_{\mathrm{e}}\right\rangle^{0.8}$ holds for both elliptical and S0 galaxies and displays a scatter of only $4 \%$.

In the following, possible differences between elliptical and lenticular galaxies will be considered in more detail. As a local reference the Coma FP coefficients by Jørgensen et al. (1996) for the Gunn $r$-band $\alpha=1.24 \pm 0.07$ and $\beta=-0.82 \pm 0.02$ were adopted. For the Johnson $B$-band $\alpha=1.25 \pm 0.06$ and $\beta=-0.80 \pm 0.02$ were assumed which are slightly different but still within the scatter found for the Coma FP parameters. The uncertainties in the coefficients of the local FP were assessed by the authors through a bootstrap fitting technique. Generally, the choice of the fitting technique, the selection criteria and the measurement errors which are correlated can lead to systematic uncertainties in the FP coefficients in the order of \pm 0.1 dex (Jørgensen et al. 1996). Nevertheless, as the FP coefficients $\alpha$ and $\beta$ depend only weakly on the wavelength and were fixed for the local and the distant sample, a significant change within a photometric band as a function of redshift can be ruled out.

A morphological analysis of the HST images re- vealed that the A 2390 sub-sample splits nearly equally into elliptical (8) and lenticular (S0) galaxies (6). Out of the 20 cluster members in the HST field of A 2218, nine and eleven were identified as ellipticals and S0s, respectively. The early-type sample of the Low- $L_{X}$ clusters was morphologically classified as nine ellipticals and one S0 galaxy. As a comparison of sub-classes cannot be performed with these numbers, the following analysis concentrates on the rich clusters only. Both ellipticals and lenticular galaxies are uniformly distributed along the surface of the FP plane. An edge-on projection can therefore be taken for a robust comparison of their stellar populations. Table 6.2 lists the derived evolution for the E and S0 galaxies. Fixing the slope of the local Coma reference, the zero-point offset for the ellipticals in the sample is

$$
\left\langle\Delta \gamma_{\mathrm{E}}^{\mathrm{c}}(z=0.2)\right\rangle=0.01 \pm 0.07 .
$$

Restricting the sample to the elliptical galaxies a negligible zero-point deviation with respect to the local FP is derived, which corresponds to an insignificant luminosity evolution of $\Delta M_{r}^{\mathrm{E}}=-0.02 \pm 0.21^{m}$ which is within their $1 \sigma$ scatter. On the other hand, the ZP offset for the $\mathrm{S} 0$ galaxies yields

$$
\left\langle\Delta \gamma_{\mathrm{S} 0}^{\mathrm{c}}(z=0.2)\right\rangle=0.14 \pm 0.06
$$

This zero-point offset for lenticular galaxies corresponds to an evolution $\Delta M_{r}^{\mathrm{S} 0}=-0.44 \pm 0.18^{m}$ with respect to the local counterparts. In comparison to the ellipticals, the difference in evolution between $\mathrm{E}$ and $\mathrm{S} 0$ galaxies is significant on the $2 \sigma$ level. For the combined sample of 34 $\mathrm{E}+\mathrm{S} 0$ cluster galaxies a median offset of

$$
\left\langle\Delta \gamma_{\mathrm{E}+\mathrm{S} 0}^{\mathrm{c}}(z=0.2)\right\rangle=0.10 \pm 0.06,
$$

is derived, which corresponds to a brightening of the stellar populations by $-0.31 \pm 0.18$ mag. The errors on the zero-points were individually derived as

$$
\delta \mathrm{ZP}^{2}=\delta \mathrm{FP}_{r}^{2}+\delta \mathrm{BS}^{2}
$$




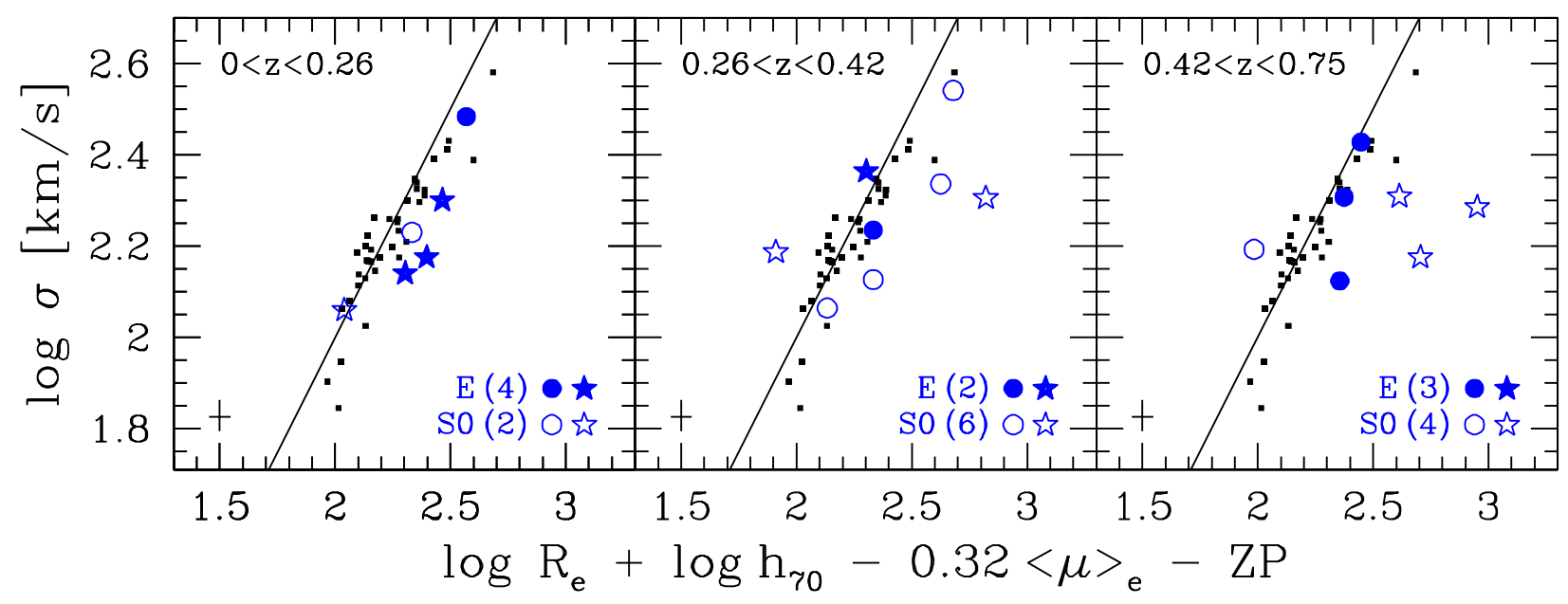

Figure 6.16: Evolution of the field FP in rest-frame Johnson $B$-band. The early-type field galaxies in the FDF and WHDF are binned into three different redshift slices, each compared to the Coma galaxies of SBD93 (small squares) and shown along the short axis of the edge-on view. Filled symbols denote ellipticals, open symbols S0 galaxies and Sa bulges and the brackets the number of the respective morphological class. For illustration reasons, only the mean error is shown in the left bottom. The offset of the distant field galaxies from the local FP increases with redshift, whereas the scatter appears to increase primarily for S0 galaxies with look-back time.

where $\delta \mathrm{FP}_{r}$ denotes the total error which enters the FP in the rest-frame Gunn $r$-band (cf. section 4.3.3) and $\delta \mathrm{BS}$ is the uncertainty computed through an iterative bootstrap re-sampling of the data points 100 times. On average, the lenticular galaxies in the sample show a larger luminosity evolution than the ellipticals. In both interpretations of Fig. 6.14 the S0 galaxies are predominantly located below the ellipticals and may indicate a different evolutionary trend between the stellar populations of elliptical and S0 galaxies. Dividing the sample with respect to velocity dispersion into a low mass $(\log (\sigma)<2.283)$ and a high mass subsample $(\log (\sigma)>2.283)$, a different evolution is found. The lower-mass galaxies are on average with $-0.47 \pm 0.24^{m}$ more luminous than their more massive counterparts with $-0.03 \pm 0.21^{m}$ at $z \sim 0.2$. This is also seen for the different subgroups of elliptical and S0 galaxies, but with less significance (see Table 6.2). To investigate differences between elliptical and lenticular galax- ies in clusters even further, the next section 6.5 concentrates on comparisons of $M / L$ ratios and masses of these galaxy types. Before this discussion, possible variations in the morphological sub-classes of field galaxies will be explored.

Looking at differences between the morphological types of field early-type galaxies, the S0 galaxies display a stronger evolution than the elliptical galaxies. Assuming that the slope of the local reference holds valid for the distant galaxies, the 9 field ellipticals show in edge-on projection of the FP a zero-point offset

$$
\left\langle\Delta \gamma_{\mathrm{E}}^{\mathrm{f}}(z=0.4)\right\rangle=0.08 \pm 0.06,
$$

which corresponds to a brightening in their stellar populations of $\Delta M_{B}^{\mathrm{E}}=-0.23 \pm 0.18^{m}$. By comparison, the 12 field lenticulars exhibit an offset in the zero-point with respect to the local Coma galaxies

$$
\left\langle\Delta \gamma_{\mathrm{S} 0}^{\mathrm{f}}(z=0.4)\right\rangle=0.21 \pm 0.09,
$$

which corresponds an average evolution of $\Delta M_{B}^{\mathrm{S} 0}=-0.64 \pm 0.27^{m}$. Errors in the zero- 
points of elliptical and S0 galaxies were analogously computed as in Eq. 6.14 with the only exception that the $\mathrm{FP}$ error in the $B$-band $\delta \mathrm{FP}_{B}$ was utilised. Limiting the S0 galaxies to redshifts $z<0.7$ and rejecting the two Sa bulges, the same results are derived (see Table 6.4). In addition, the field ellipticals obey a tight FP with a small $1 \sigma$ scatter of 0.121 , whereas the S0 types have a larger dispersion of 0.219 (omitting the two Sa bulges). This gives further evidence that the discy galaxies have a larger range of different stellar populations. Even more astonishing is the fact that three elliptical galaxies in the WHDF sample feature weak [O II] 3727 emission in their spectra. In particular, the galaxies \# 810, \# 437 and \# 810b exhibit [O II] $3727=-3.73 \pm 0.47 \AA$, $-0.35 \pm 0.37 \AA$ and $-6.62 \pm 1.87 \AA$. Generally, a line emission is evidence for star formation activity which increases the average luminosity and thereby causing the galaxy to be offset from the local FP relation. In contrast to what is expected, these ellipticals indicate no offset along the edge-on projection of the FP but a tight relation. One explanation could be that the star formation rates are too low to result in an visible effect within the FP. Although the evidence for the galaxy \# 437 is rather poor due to its measurement uncertainty, it could be that the low star formation rates of the galaxies are either the relicts of a former starburst or the weak imprints of galaxy-galaxy interactions which have taken place at an earlier epoch. This suggests that galaxy-galaxy interactions which trigger the star formation activity are a common phenomenon among field galaxies.

The evolution of the FP for the early-type FDF and WHDF field galaxies in the rest-frame Johnson $B$-band is illustrated in Fig. 6.16. Early-type galaxies were binned in redshift space to investigate their location within and along the edgeon FP as a function of redshift and to test the effects of possible outliers. Ellipticals are represented as filled, S0s and Sa bulges as open symbols. For each morphological type the number of galaxies within a redshift bin is indicated in the brackets. Small squares denote the Coma galaxies by SBD93 and the straight line is a principal component fit to the local sample (Ziegler et al. 2005). Fig. 6.16 clearly shows the evolution of the FP for the distant galaxies with respect to the local Coma FP. The offset of the field FP increases with redshift but the scatter appears to be mainly amplified for lenticular galaxies. The two galaxies with a "positive" evolution in the last two panels are the Sa bulges \# 6336 and \#508 and show a clear disc on the ACS images. These objects can mimic on average a weaker evolution in luminosity for the whole galaxy population (cf. Table 6.4). Regardless of the Sa bulges, the S0s exhibit a larger scatter at higher redshift $0.26<z<0.75$ than the ellipticals which suggests that the stellar populations of E and S0 types are different and that lenticulars are a more heterogeneous group. An explanation could be that these S0 galaxies resemble post-starburst galaxies as no strong emission lines were detected in their spectra.

\section{5 $M / L$ Evolution}

The evolution of the Fundamental Plane can be characterised in terms of its zero-point. In turn, the zero-point of the FP is related to the mean $M / L$ ratio. Thus, if the $\mathrm{FP}$ zero-point for a sample of early-type galaxies changes as a function of redshift $z$, this implies an evolution of the mean $M / L$ ratio and hence an evolution in the luminosity-weighted stellar population properties of the galaxies under consideration.

For the present-day zero-point of the FP a bootstrap bisector fit to the early-type Coma galaxies in rest-frame Gunn $r$-band was performed. This yielded a slope of $1.048 \pm 0.038$ and a zeropoint of $-0.029 \pm 0.010$. The early-type galaxies of A 2390 show a small shift with respect to the Coma galaxies (see Fig. 6.11). As the velocity dispersions and effective radii of the galaxies in A 2390 span similar ranges like their local 
counterparts (apart from the Coma $\mathrm{cD}$ galaxies with $R_{\mathrm{e}} \gtrsim 30 \mathrm{kpc}$ ) and the surface brightnesses are slightly increased with respect to the local sample, at least a fraction of the shift in the $M / L$ ratio is due to luminosity evolution. However, when deriving an exact amount of luminosity evolution, one has to be careful as additional combined effects also contribute to the total derived luminosity offset. Some studies found different FP slopes at intermediate redshift compared to the local slope, but they suffer from low number statistics (van Dokkum \& Franx 1996; Kelson et al. 1997; van Dokkum et al. 1998). However, more recent investigations based on larger samples reported similarly only a modest change in the FP slope (Kelson et al. 2000b; Treu et al. 2001b). Thus, the lack of a strong slope change gives evidence against the hypothesis of the FP being solely an age-mass relation. The Fundamental Plane is mainly a relation between the $M / L$ ratio and galaxy mass $M$. Therefore, when comparing small samples with significantly different ranges in galaxy mass, an offset in the $M / L$ ratio can heavily depend on the adopted FP slope. The $M / L$ offset will be a combination of three items: $(i)$ the difference in the slope of the FP, $(i i)$ the mean mass range of the sample and (iii) the 'true' offset. By reducing the differences in the mass distribution, the $M / L$ results become insensitive to the adopted slope of the FP and any derived evolution for the distant galaxies is valid for a given range of galaxy masses (Kelson et al. 2000b). For this reason, the local Coma sample was restricted to a similar mass function as for the galaxies in A 2218 and A 2390 and this reference was applied for the entire analyses within the FP and the $M / L$ evolution.

A comparison of the mass distributions of the distant early-type galaxies of A 2390 and A 2218 with the local Coma galaxies within the $\log \sigma-$ $\log R_{\mathrm{e}}$ plane is shown in Fig. 6.17. The distributions of galaxy masses for the Low $-L_{X} \mathrm{E}+\mathrm{S} 0$ galaxies are illustrated in Fig. 6.18. Regions

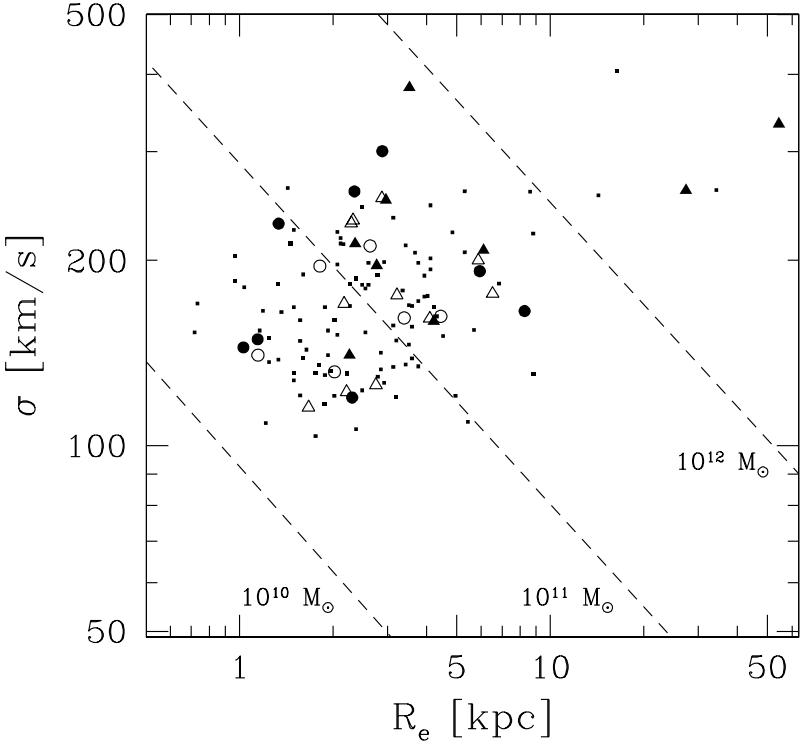

Figure 6.17: The $\log \sigma-\log R_{e}$ plane for the A 2390 and A 2218 early-type galaxies, compared to the Coma galaxies of J99. Symbol notations as in Fig. 6.11. Dashed lines indicate contours of constant mass $5 G^{-1} \sigma^{2} R_{\mathrm{e}}=10^{10}, 10^{11}$ and $10^{12} M_{\odot}$ (Bender et al. 1992). The early-type galaxy masses of A 2390 and A 2218 at $z \sim 0.2$ are similarly distributed as the early-type Coma sample.

of constant mass, ranging from $10^{12} M_{\odot}$ over $10^{11} M_{\odot}$ down to $10^{10} M_{\odot}$, derived with the relation $M=5 \sigma^{2} R_{\mathrm{e}} / G$ in units of $M_{\odot}$ (Bender, Burstein \& Faber 1992) where $G$ is the gravitational constant, are indicated as dashed lines. For the absolute magnitude in Gunn $r$ of the Sun, $M_{r, \odot}=4.87^{m}$ was adopted, which was derived from $M_{V, \odot}=5.72$ and $(V-R)_{\odot}=0.52$ (Schaifers et al. 1981) and the transformation $r=R+0.41+0.21(V-R)($ Kent 1985). Both samples exhibit similar ranges in mass $(\sigma)$ and size $\left(R_{\mathrm{e}}\right)$, assuring that a possible $M / L$ evolution will not be driven mainly by any differences between the galaxy mass ranges of the samples. The restrictions to the local Coma reference as defined for the rich cluster sample were also adopted for the early-type galaxies in the poor clusters (cf. section 6.2). To test the effects of different limits in velocity disper- 


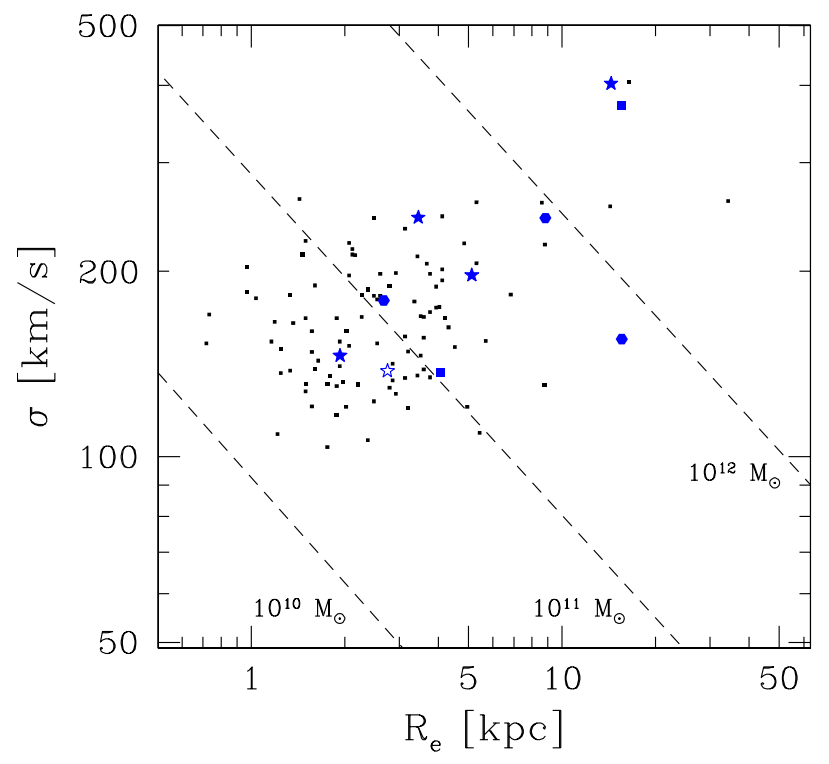

Figure 6.18: The $\log \sigma-\log R_{e}$ plane for the the $\mathrm{Cl}$ 0849, Cl 1701 and Cl 1702 early-type galaxies, compared to the Coma galaxies of J99. Symbol notations as in Fig. 6.12. Dashed lines indicate contours of constant mass $5 G^{-1} \sigma^{2} R_{\mathrm{e}}=10^{10}, 10^{11}$ and $10^{12} M_{\odot}$ (Bender et al. 1992). The early-type galaxy masses of poor cluster galaxies between $0.22 \leq z \leq 0.24$ are similarly distributed as the early-type Coma sample.

sion on the amount of derived luminosity evolution for the Low $-L_{X}$ galaxies, the selection boundaries of the Coma cluster were modified. The largest effect was detected for a limit of $\log \sigma_{\text {J99 }}>2.10$ (corresponding to a $\sim 22 \mathrm{~km} \mathrm{~s}^{-1}$ higher cutoff value), which yields a change in the amount of derived luminosity evolution of less than $0.0033^{m}$. This difference is negligible and assures that the assumption to use one definition of selection boundaries is also valid for the poor cluster sample.

Adopting a constant slope with redshift, the median zero-point offset for the combined sample of $34 \mathrm{E}+\mathrm{S} 0$ rich cluster galaxies yielded $\Delta \gamma_{\mathrm{E}+\mathrm{S} 0, \mathrm{z}=0.2}=0.10 \pm 0.06$. Alternatively using a variable slope in the FP for the distant cluster sample, negligible changes in the FP parameters $\alpha$ and $\beta$ compared to the locally defined parameters by the Coma data are found. In particular, a bootstrap bisector fit to all $34 \mathrm{E}+\mathrm{S} 0$ galaxies gives a slightly steeper slope of $1.152 \pm 0.047$ and a zero-point of $-0.153 \pm 0.018$, with a $3 \sigma$ confidence level for a slope change. This comparison and the results based on the $M / L$ ratios may give some evidence for a mass-dependent evolution which is stronger for low-mass galaxies.

Fig. 6.19 shows the observed mass-to-light $M / L$ ratio of the distant clusters A 2218 and A 2390 as a function of velocity dispersion $\sigma$ (left) and versus mass $M$ (right). The Coma sample was limited to galaxies with $\log \sigma>2.02$ (indicated by the vertical dotted lines in Fig. 6.19) in order to match the area of parameter space covered by the distant galaxies of A 2390 and A 2218. An analysis based on bootstrap bisector fits to $M / L=a \sigma^{m}$ revealed different $M / L$ slopes for the distant (hashed area) and the local (solid lines) samples. The slope difference between A 2218 and Coma is $\Delta m_{\mathrm{A} 2218}=0.27 \pm 0.17$ and an offset in the zero-point of $\Delta a_{\mathrm{A} 2218}=0.040 \pm 0.027$. For A $2390 \Delta m_{\mathrm{A} 2390}=0.11 \pm 0.21$ is detected and $\Delta a_{\mathrm{A} 2390}=0.157 \pm 0.019$. The combined sample of distant clusters has a slope difference of $\Delta m_{\mathrm{z}=0.2}=0.36 \pm 0.17$ and $\Delta a_{\mathrm{z}=0.2}=0.036 \pm 0.024$. With a $2 \sigma$ confidence different slopes for both are measured, the intermediate clusters and the Coma cluster (Coma slope value is $m=0.59 \pm 0.15$ ). However, a systematic zero-point offset of the distant $M / L$ relation is detected, which is not in agreement with the proposed change due to passive evolution. Adopting a formation redshift of $z_{\text {form }}=2$ for all stars in early-type galaxies, the BC96 models predict $\Delta a=0.12$. Ellipticals and S0 galaxies seem to have different $M / L$ slope changes, with a steeper slope for the elliptical galaxies. Ellipticals: $\Delta m_{\mathrm{E}}=0.54 \pm 0.30$ and $\Delta a_{\mathrm{E}}=0.041 \pm 0.037 ;$ S0s: $\Delta m_{\mathrm{S} 0}=0.06 \pm 0.23$ and $\Delta a_{\mathrm{S} 0}=0.141 \pm 0.026$.

The $M / L$ ratios as a function of mass $M$ are shown in the right plot of Fig. 6.19. In a similar manner as for the velocity disper- 


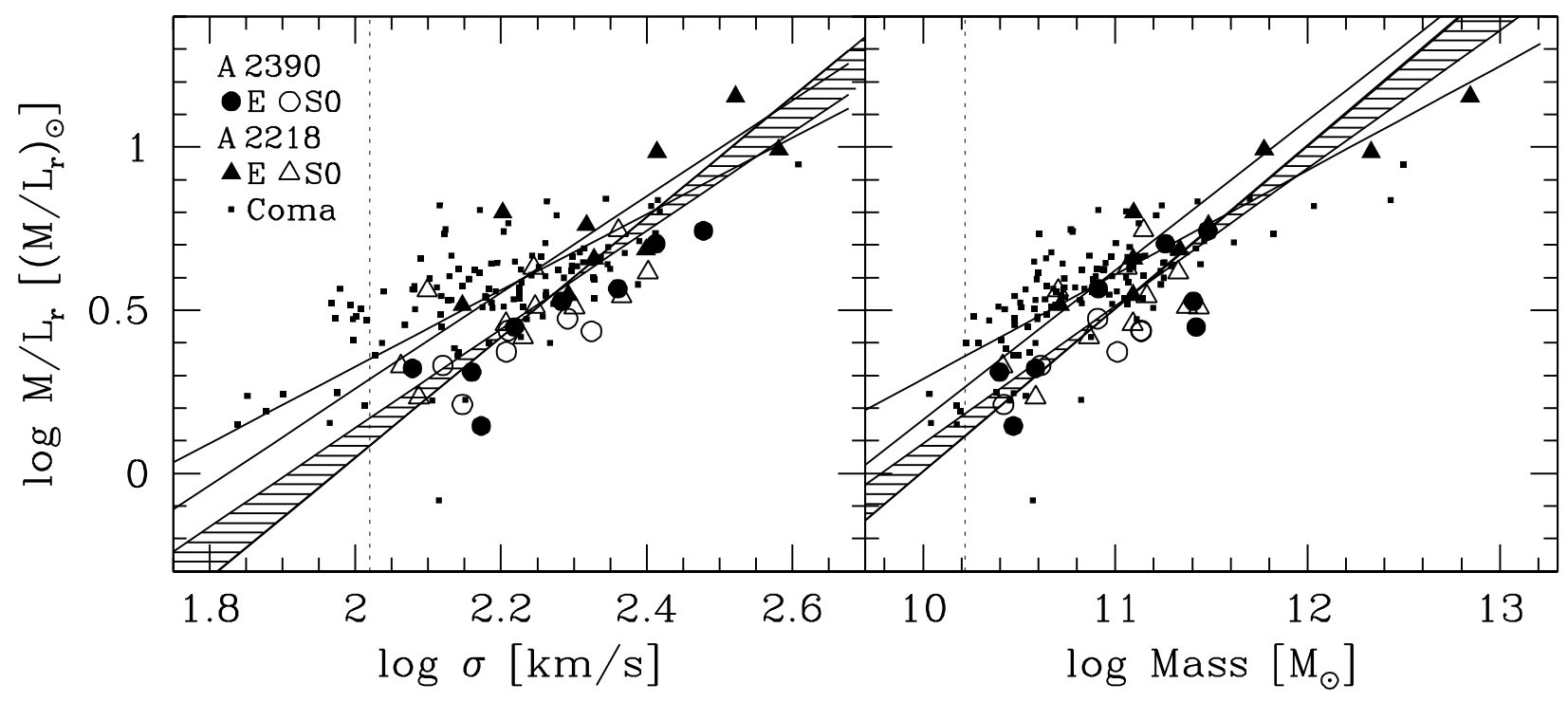

Figure 6.19: The $M / L$ ratio in Gunn $r$ for A 2390 and A 2218. Left: $M / L$ ratio as a function of velocity dispersion $\sigma$, Right: $M / L$ ratio as a function of mass in solar units. Symbol notations as in Fig. 6.11. Solid lines show the 100 iteration bootstrap bisector fits to the local Coma sample of J99 within selection boundaries of the distant samples ( $1 \sigma$ errors). The hashed area denotes the bisector fits within $1 \sigma$ errors to the combined distant sample of A 2390 and A 2218.

sions, the two samples are analysed in terms of bootstrap bisector fits. For the reduced Coma cluster alone $M / L \propto M^{0.59 \pm 0.07}$ is found. With respect to Coma, the slope difference for A 2218 is $\Delta m_{\mathrm{A} 2218}=0.025 \pm 0.060$ with a zero-point offset of $\Delta a_{\mathrm{A} 2218}=0.048 \pm 0.029$. For A 2390 a similar slope change is found $\Delta m_{\mathrm{A} 2390}=0.057 \pm 0.063$, but a larger offset of $\Delta a_{\mathrm{A} 2390}=0.165 \pm 0.028$. The two distant clusters as a whole have $\Delta m_{\mathrm{z}=0.2}=0.071 \pm 0.039$ and $\Delta a_{\mathrm{z}=0.2}=0.037 \pm 0.021$. Dividing the sample into elliptical and S0 galaxies gives no significant slope changes, for Es: $\Delta m_{\mathrm{E}}=0.049 \pm 0.056$ and for S0s: $\Delta m_{\mathrm{S} 0}=0.087 \pm 0.089$. However, the S0s exhibit a larger offset in the zero-point of $\Delta a_{\mathrm{S} 0}=0.138 \pm 0.030$, compared to the ellipticals $\Delta a_{\mathrm{E}}=0.050 \pm 0.034$.

Analogous to the cluster galaxies, the $M / L$ ratios for the poor clusters $\mathrm{Cl} 0849, \mathrm{Cl} 1701$ and $\mathrm{Cl} 1702$ were computed. Fig. 6.20 displays the $M / L$ ratios as a function of velocity dispersion $\sigma$ (left panel) and as a function of mass in solar units (right panel). Unfortunately, an analysis for the morphological types of ellipticals and lenticulars cannot be performed as the latter class comprises only one galaxy. Under assumption of a non-changing local slope with redshift, the mean residuals from the local $\log M / L-\log \sigma$ relation of the Low- $L_{X}$ cluster galaxies are investigated and their mean evolution in the $M / L$ ratio is derived. The $M / L$ ratios of the distant poor galaxies are on average offset by $\Delta M / L=0.138 \pm 0.049$ compared to the Coma $M / L$ ratios. In the right part of Fig. 6.20, the $M / L$ ratios are shown as a function of galaxy mass $M$. The low-mass cluster galaxies occupy a larger range in the $M / L$ ratios than the rich cluster galaxies in Fig. 6.19. In addition a larger offset to the local $\log M / L-\log M$ relation with $\Delta \log M / L=0.216 \pm 0.059$ for the poor sample than for the rich cluster sample with $\Delta \log M / L=0.109 \pm 0.021$ is found, where for the latter also the Coma slope was assumed.

In both illustrations of Fig. 6.20 the $M / L$ ratios of the Low $-L_{X} \mathrm{E}+\mathrm{S} 0$ galaxies feature on average a larger scatter with $\sigma_{\mathrm{M} / \mathrm{L} \text { Poor }}=0.29$ than their 


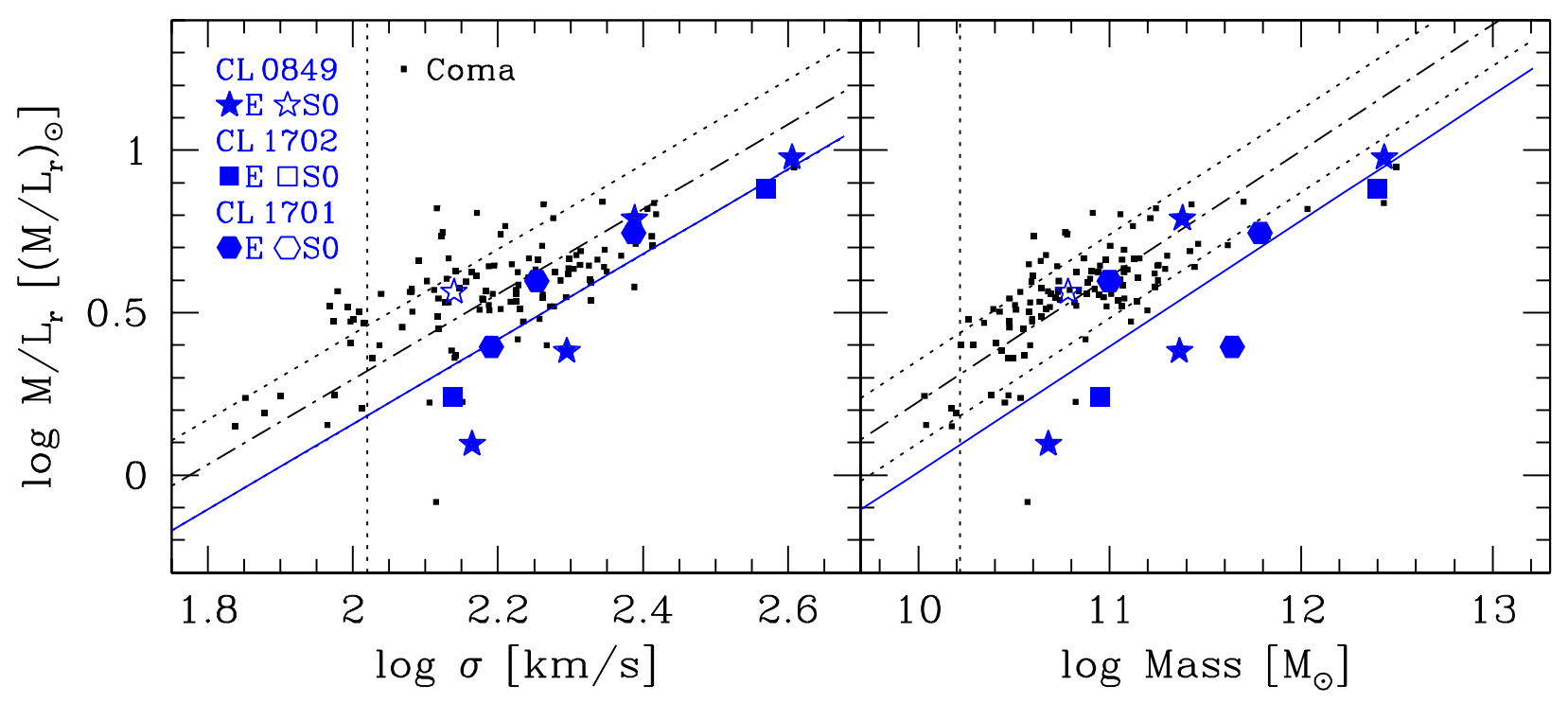

Figure 6.20: The $M / L$ ratio in Gunn $r$ for the poor clusters $\mathrm{Cl} 0849, \mathrm{Cl} 1701$ and $\mathrm{Cl} 1702$. Left: $M / L$ ratio as a function of velocity dispersion $\sigma$, Right: $M / L$ ratio as a function of mass in solar units. Symbol notations as in Fig. 6.12. The linear bisector fit to the Coma galaxies was restricted to the range encompassed by the distant rich cluster galaxies. The area bounded by dotted lines indicates the mean $\pm 1 \sigma$ of the local $M / L$ relation. The solid line shows the mean evolution of the poor cluster sample assuming the local slope.

counterparts in rich clusters $\sigma_{\mathrm{M} / \mathrm{L} \text { Rich }}=0.23$ and their $M / L$ are slightly more offset from the galaxies in Coma compared to the $M / L$ ratios in rich clusters. For a comparison, the reduced local reference sample has a dispersion in the $M / L$ ratios of $\sigma_{\mathrm{M} / \mathrm{L} \text { Coma }}=0.14$. In both, the FP (Fig. 6.12) and the $\log M / L-\log M$ diagram (Fig. 6.20), it seems that there are two different groups of cluster galaxies, one class which follows the local relation and another one which is clearly offset from the Coma reference. An explanation could be that the Low $-L_{X} \mathrm{E}+\mathrm{S} 0$ galaxies have larger variations in their stellar populations or that star formation processes work differently in these galaxies. Such effects should also be detectable in an analysis of the formation redshift for the galaxies which gives also clues on the global star formation histories in these systems (cf. section 7.2). Furthermore, in case of the bright $\mathrm{cD}$ galaxies, differences between the Low $-L_{X} \mathrm{E}+\mathrm{S} 0$ galaxies and the rich cluster members are found. Two of three central cluster galaxies in the poor clusters (\# 24 with $R_{\mathrm{e}} \approx 14 \mathrm{kpc}$ in CL 0849 and \# 81 with $R_{\mathrm{e}} \approx 16 \mathrm{kpc}$ in CL 1702) have higher velocity dispersions with $\Delta \bar{\sigma} \approx 118 \mathrm{~km} \mathrm{~s}^{-1}$, but at the same time smaller half-light radii of $\Delta \overline{R_{\mathrm{e}}} \approx 2.6 \mathrm{kpc}$ than their counterparts in the A 2218 cluster. The third cD galaxy of the cluster CL 1701 (\#123) at $\sigma=244 \mathrm{~km} \mathrm{~s}^{-1}$ and $R_{\mathrm{e}}=8.8 \mathrm{kpc}$ has a double nucleus and cannot be regarded as a typical $\mathrm{cD}$ galaxy as it is an ongoing merger of two galaxies. Another galaxy which shows an offset in the $\log \sigma-\log R_{\mathrm{e}}$ diagram is the object \#62 $\left(R_{\mathrm{e}}=15.5 \mathrm{kpc}\right)$ which is a large elliptical galaxy but not massive enough to be a central cluster galaxy as it has a low velocity dispersion of $\sim 155 \mathrm{~km} \mathrm{~s}^{-1}$. Together with the findings in the KR, the signs of interaction processes give further evidence, that the $\mathrm{cD}$ galaxies in lowmass clusters have longer formation timescales up to the recent past and therefore represent a different galaxy population compared to the $\mathrm{cD}$ galaxies in rich galaxy clusters.

Morphological transformation from late-type spiral to S0 galaxies as observed from redshift of 
$z=0.55$ to the present day Universe (Dressler et al. 1997) can have significant effects on the evolution of colours and luminosities of earlytype galaxies with redshift. If early-type galaxies were transformed from late-type galaxies in the recent past, the youngest progenitors of presentday early-type galaxies will drop out from an observed sample of early-type galaxies at high redshift. The samples at high and low redshift differ and the high- $z$ data set may be biased towards the oldest progenitors of present-day early-type galaxies. The correction for this socalled 'progenitor bias' (van Dokkum \& Franx 2001b) increases as a function of redshift and can be quoted in terms of the $M / L$ ratio as $\Delta \ln \left\langle M / L_{B}\right\rangle \approx 0.2 \times z$. It has a small $(\$ 20 \%)$ but non-negligible effect on the measured evolution of the mean the $M / L$ ratios. For the results in the $\mathrm{FP}$ and on the $M / L$ ratios at $z=0.2$ the effect is insignificant, the true evolution in the $M / L$ ratio is underestimated by $\Delta \ln \left\langle M / L_{B}\right\rangle \approx 0.04$ and was therefore not considered. This effect comes into play at a redshift of $z \gtrsim 0.4$ and has dramatic effect at high redshifts of about $z \geq 0.8$. In the analysis of the field early-type galaxies and the derivation of the formation epochs in the following chapter, the correction of the progenitor bias will be accounted for.

As a conclusion from the results of the scaling relations so far, for the whole sample of $34 \mathrm{E}+\mathrm{S} 0$ cluster galaxies in A 2218 and A 2390 and 10 early-type galaxies in the Low- $L_{X}$ clusters on average only a moderate amount of luminosity evolution is derived. From these findings it can be inferred that at a look-back time of $\sim 2.8$ Gyrs most early-type galaxies in the rich clusters consist of an old stellar population with the bulk of the stars formed at a high formation redshift of about $z_{\text {form }} \geq 2$. The elliptical galaxies in the poor clusters however, suggest a trend for a stronger evolution which favours a lower formation epoch. A detailed discussion on the stellar population ages will be presented in section 7.2.
In the following, the findings for the early-type cluster galaxies will be compared to previous results in the literature.

\subsection{Comparison with Previous Studies}

In the sections above it has been shown that the scaling relations of the Faber-Jackson and the Fundamental Plane show a stronger luminosity evolution for lower-mass systems. To verify these findings the results will now be compared to previous studies. The redshift interval of $0.05<z \lesssim 0.2$ is less well explored than the higher redshift range as the main focus of current interest is on redshifts of $z \geq 0.5$ and beyond. There is only one study by Jørgensen et al. (1999) which analysed two galaxy clusters at $z \sim 0.2$.

Jørgensen et al. (1999, hereafter JFHD) obtained ground-based photometry of 31 and 61 $\mathrm{E}+\mathrm{S} 0$ galaxies in the clusters A $665(z=0.181)$ and A $2218(z=0.176)$. Follow-up spectroscopy in A 665 and A 2218 was performed for 6 and 9 early-type galaxies, respectively. For a subset of objects also archival HST imaging is available to be able to construct the FP. In Fig. 6.21 the A 665 and A 2218 data of JFHD are shown in comparison to the FP sample of the rich (A 2390, A 2218, circles) and Low- $L_{X}$ clusters (Cl 0849, Cl 1701 and $\mathrm{Cl} 1702$, squares) for the edge-on projection of the FP. In order to be consistent with this work, the absolute magnitudes of JFHD have been re-computed to the concordance cosmology. Furthermore, two galaxies in the JFHD sample are based on ground-based imaging only but included in the FP. For this reason, for internal consistency between their data, JFHD calibrated their HST images to the ground-based $I_{C}$ photometry and therefore applied a zero-point offset to their HST photometry. For the analysis here, these offsets for A 665 of $0.065 \mathrm{mag}$ and for A 2218 of $0.016 \mathrm{mag}$ were taken back and the two galaxies of A 2218 with- 


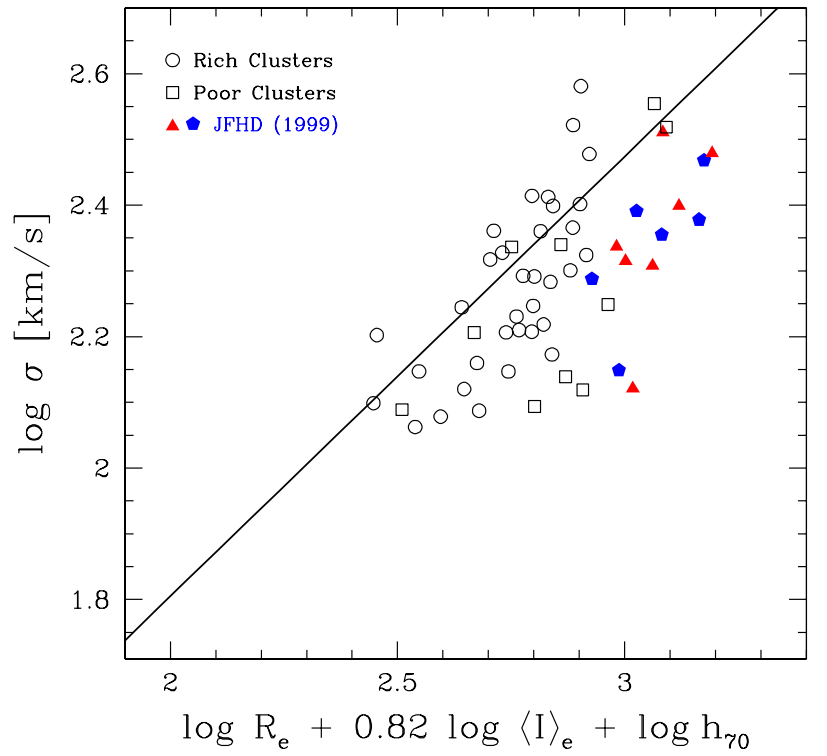

Figure 6.21: Comparison between the cluster samples and the distant FP samples of Jørgensen et al. (1999). The local Coma FP is indicated by the principal component fit. Open symbols denote the galaxies in A 2390, A 2218 and in $\mathrm{Cl} 0849, \mathrm{Cl} 1701$ and $\mathrm{Cl}$ 1702, solid symbols the galaxies in A 665 (pentagons) and A 2218 (triangles).

out HST photometry were discarded.

Both JFHD samples comprise on average more massive galaxies than the FP samples in this study and have a larger zero-point offset in the FP and a slightly stronger evolution. The six galaxies in A 665 show a median offset to Coma of $\left\langle\mu_{\mathrm{e}}\right\rangle=-0.49^{m}$ and the seven galaxies in A 2218 are offset by $\left\langle\mu_{\mathrm{e}}\right\rangle=-0.40^{m}$. However, the scatter is quite large for both clusters and there are only two objects which are less massive. Moreover, the massive galaxies in the JFHD samples are also more luminous $\left\langle\mu_{\mathrm{e}}\right\rangle=19.27^{\mathrm{m}}$ compared to our FP rich cluster sample $\left\langle\mu_{\mathrm{e}}\right\rangle=20.04^{\mathrm{m}}$. Both, the rich and poor cluster galaxies primarily populate the less-massive end of the FP. As JFHD targeted due to instrument limitations the brightest cluster galaxies their small sample is subject to a potential selection effect. A bias to more massive galaxies is detected which contain mainly old quiescent populations and thus a modest evolution is measured for the FP. Although JFHD favour a high formation redshift of $z_{\text {form }}>1.7$ for their sample, they argue that during the last 4 Gyrs possible star formation activity has taken place in their cluster galaxies. 


\section{Chapter 7}

\section{Environmental Effects on Galaxy Properties}

Most local studies of early-type galaxies did not reveal any dependence of their properties on a specific environment. Various approaches have been undertaken, such as the construction of scaling relations to deduce the luminosity evolution (Bender et al. 1992; Bernardi et al. 2003), the measurement of absorption linestrengths to determine the mean ages and metallicities (Kuntschner 2000; Thomas et al. 2005) or the analyses of luminosity functions for the field and cluster environments (De Propris et al. 2003). On the other hand, some evidence for young populations in local S0 galaxies was found by stellar population studies of the Coma cluster. More than $40 \%$ of the lower-luminous S0 galaxies $\left(M_{B} \gtrsim-19^{m}\right)$ have undergone star formation in their central regions during the last $\sim 5$ Gyrs, while such activity is absent in the ellipticals (Poggianti et al. 2001). In a recent study on the Coma cluster two classes of S0 galaxies were identified, one group is composed of old $\sim 10$ Gyrs stellar populations similar to ellipticals and a second one has very young average ages of $\sim 2$ Gyrs (Mehlert et al. 2003). However it is still unclear, whether these different galaxy types are specific to the Coma cluster environment or a more general property of all galaxy clusters. So far, there is only one investigation at higher redshift which concentrates on disentangling possible differences of early-type cluster galaxies at $z \sim 0.4$ (Moran et al. 2005). For the
FP of the rich cluster $\mathrm{Cl} 0024+1654$ an increased scatter and a radial trend of the $M / L$ ratios was found, with the oldest galaxies located in the cluster core and younger ones in the outer periphery regions. Discrepancies between the subpopulations of elliptical and S0 galaxies were not detected. However, this work is restricted to the special environment of rich galaxy clusters and up to now no studies have tested possible environmental trends of the properties of early-type galaxies by comparing a range of different environments at $z=0.2$. One reason for this lack of knowledge is the requirement of a large sample to explore the existence even of small differences. In section 7.1 the dependence of the cluster galaxy properties within the Faber-Jackson Relation is analysed. The rich and poor cluster samples are divided with respect to luminosity (section 7.1.1), velocity dispersion, which is representative for the mass of a galaxy, (section 7.1.2) and distance from the cluster centre (section 7.1.3). A possible connection between the colours of galaxies with their projected clustercentric radii will be established in section 7.1.4 and the colours will be compared to the luminosity evolution as derived from the Faber-Jackson relation. For the comparisons of the various properties, the two rich cluster samples and the three poor cluster samples have been combined to increase the statistics. As illustrated in section 5.6, a combination of the data sets is ade- 
quate and justified because the properties of the galaxies within the two rich clusters are very similarly distributed. The combined galaxy populations of the Low $-L_{X}$ clusters form also a homogeneous group with respect to their overall parameters such as luminosities, colours, velocity dispersions and sizes, which are comparable to those of a rich galaxy cluster. Afterwards differences in the stellar populations of early-type elliptical and S0 galaxies for the cluster and field environments are explored and constraints on their formation redshift presented (section 7.2).

\subsection{The Faber-Jackson Rela- tion}

The parameter space of the rich cluster sample of early-type galaxies covers the ranges $-20.42 \geq M_{r} \geq-23.42$ in absolute magnitude and $105 \mathrm{~km} \mathrm{~s}^{-1} \leq \sigma \leq 381 \mathrm{~km} \mathrm{~s}^{-1}$ in velocity dispersion with median values of $\left\langle M_{r}\right\rangle=-21.49$ and $\langle\sigma\rangle=170 \mathrm{~km} \mathrm{~s}^{-1}$. For a given galaxy with corresponding values for $M_{r}$ and $\sigma$, the mean residuals from the local FJR, i.e. the offsets from the local FJR fit can be calculated by the following

$$
\Delta M_{r}=6.82 \log \sigma+5.90+M_{r} .
$$

As previously mentioned (see also Eq. 6.1 on page 129), distant early-type galaxies are likely to comprise stellar populations which are on average younger than those of early-types in the local Universe. Therefore, the luminosities of the distant $\mathrm{E}+\mathrm{S} 0$ galaxies should be higher for a given mass (or a given $\sigma$ ). This was already demonstrated in Fig. 6.2 with an overall brightening of the distant cluster galaxies by $\sim 0.3 \mathrm{mag}-$ nitudes in the Gunn $r$-band.

\subsubsection{Luminosity Dependence}

Dividing our sample with respect to luminosity at $M_{r}=-21.49$ in two groups of equal number with each sub-sample containing 48 galaxies, the offsets of the FJR from the local FJR

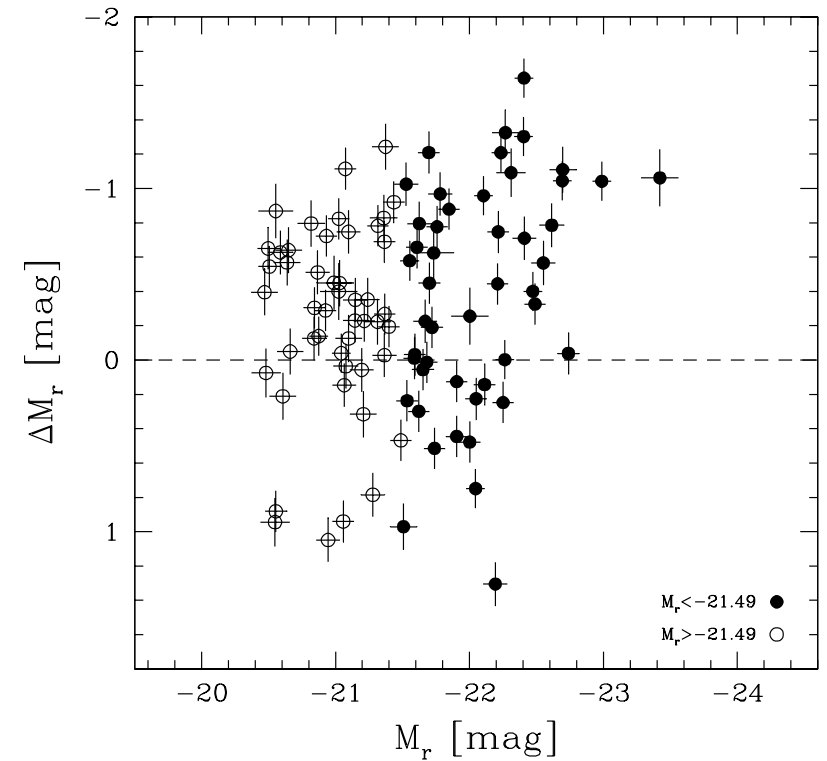

Figure 7.1: Offsets of the Faber-Jackson relation in Gunn $r$ for the early-type cluster galaxies in A 2390 and A 2218 from the local FJR by the J99 Coma sample as a function of luminosity $M_{r}$. Filled symbols denote brighter galaxies with $M_{r}<-21.49$, open symbols fainter galaxies with $M_{r}>-21.49$. The dashed line indicates a zero offset.

are explored. Fig. 7.1 shows the offsets of the FJR in Gunn $r$ for the early-type galaxies in the clusters A 2390 and A 2218 from the local FJR as defined by the J99 Coma sample as a function of luminosity $M_{r}$. Brighter galaxies with $M_{r}<-21.49$ are indicated as solid symbols, fainter objects with $M_{r}>-21.49$ as open symbols. Subdividing the total sample with respect to their luminosity $M_{r}$ at $M_{r}=-21.49$ (0.2 mag brighter than $\left.L_{r}^{*}\right)$ into two sub-samples of equal number, the brighter cluster galaxies $\left(M_{r}<-21.49\right)$ show on average an evolution with $\Delta \bar{M}_{r}=-0.39 \pm 0.13^{m}$. Fainter objects in the rich cluster sample $\left(M_{r}>-21.49\right)$ are on average more luminous by $\Delta \bar{M}_{r}=-0.25 \pm 0.18^{m}$. Table 7.1 gives a comparison between the two groups of early-type galaxies with different luminosities. The difference in mean luminosity between lower-luminous and higher-luminous galaxies is within their $1 \sigma$ error and therefore neg- 


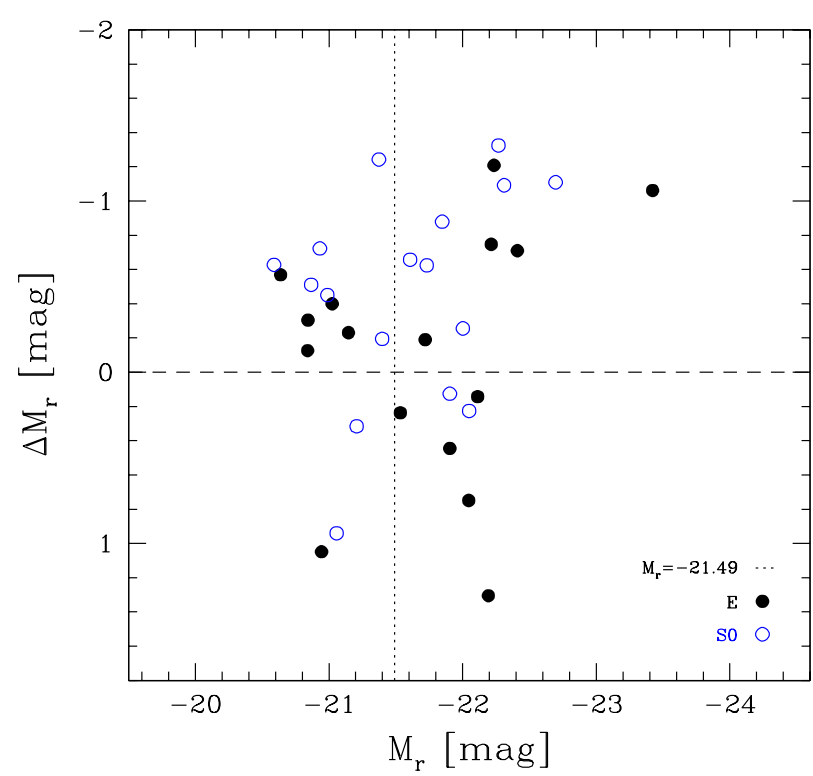

Figure 7.2: Offsets of the Faber-Jackson relation in Gunn $r$ for the morphologically classified elliptical and S0 galaxies in A 2390 and A 2218 from the local FJR by the J99 Coma sample as a function of $M_{r}$. Ellipticals are indicated as solid circles, S0s as open circles. The vertical line denotes the border between brighter and fainter galaxies at $M_{r}=-21.49$ and the dashed line a zero offset. Lenticulars show on average a stronger evolution than ellipticals.

ligible.

For the offsets from the local FJR in Fig. 7.1, the errors of the offsets on the absolute magnitudes were computed as

$$
\delta(\Delta \mathrm{FJR})^{2}=\delta M_{\mathrm{rclus}}^{2}+\delta M_{\mathrm{r} \text { J99 }}^{2} .
$$

The first term on the right hand side corresponds to the total error in absolute $r$ magnitude as given in Eq. 4.14 on page 91 and the second term are the errors in absolute $r$ magnitude for the Coma J99 data. Individual errors for the J99 data were adopted as published by the authors with mean errors of $\delta M_{\mathrm{r}}=0.09^{\mathrm{m}}$ on the photometry.

The galaxy samples with fainter and brighter luminosities exhibit different mean velocity dispersions $(\log \sigma=2.18$, and 2.31, respectively). For this reason, a two-dimensional
Table 7.1: Evolution of the Faber-Jackson relation in Gunn $r$ derived for various rich cluster samples. $N$ is the number of galaxies and $\Delta \bar{M}_{r}$ indicates the mean luminosity evolution. The fourth column denotes the $\pm 1 \sigma$ deviation in the mean luminosity evolution and the last column $\Delta\left\langle M_{r}\right\rangle$ gives the median evolution.

\begin{tabular}{|c|c|c|c|c|}
\hline $\begin{array}{l}\text { Sample } \\
(1)\end{array}$ & $\begin{array}{l}N \\
(2)\end{array}$ & $\begin{array}{c}\Delta \bar{M}_{r} \\
{[\mathrm{mag}]} \\
(3)\end{array}$ & $\begin{array}{r}\sigma_{M_{r}} \\
(4)\end{array}$ & $\begin{array}{c}\Delta\left\langle M_{r}\right\rangle \\
{[\mathrm{mag}]} \\
(5)\end{array}$ \\
\hline A 2218 & 48 & -0.31 & 0.15 & -0.29 \\
\hline A 2390 & 48 & -0.32 & 0.29 & -0.35 \\
\hline A $2218+$ A 2390 & 96 & -0.32 & 0.22 & -0.35 \\
\hline low $L^{a}$ & 48 & -0.25 & 0.18 & -0.29 \\
\hline high $L$ & 48 & -0.39 & 0.13 & -0.44 \\
\hline low $\operatorname{mass}^{b}$ & 48 & -0.62 & 0.34 & -0.63 \\
\hline high mass & 48 & -0.02 & 0.16 & -0.01 \\
\hline $\operatorname{in}^{c}$ & 48 & -0.26 & 0.20 & -0.24 \\
\hline out & 48 & -0.35 & 0.20 & -0.40 \\
\hline
\end{tabular}

${ }^{a}$ fainter: $M_{r}>-21.49$, brighter: $M_{r}<-21.49$.

$b$ less-massive: $\log \sigma<2.231$, more-massive: $\log \sigma>2.231$.

${ }^{c}$ in: only core sample $R<455.6 \mathrm{kpc}$, out: only outer region sample $R>455.6 \mathrm{kpc}$.

Kolmogorov-Smirnov (KS) test is not the appropriate statistical method for a comparison. Instead, bootstrap-bisector fits to the individual sub-samples of lower and higher luminosities are performed. A bootstrap bisector fit to the lower-luminosity cluster galaxies restricted to $M_{r}>-21.49$ gives

$$
M_{r}=-(2.65 \pm 0.46) \log \sigma-(15.21 \pm 1.00),
$$

whereas the same fitting method to the higherluminosity ones with $M_{r}<-21.49$ yields

$$
M_{r}=-(2.85 \pm 0.53) \log \sigma-(15.47 \pm 1.23) .
$$

Fainter and brighter galaxies have slightly different slopes but the difference is not statistically significant. An insignificant slope change 
Table 7.2: Evolution of the Faber-Jackson relation in Gunn $r$ derived for various Low- $L_{X}$ samples. $N$ is the number of galaxies and $\Delta \bar{M}_{r}$ indicates the mean luminosity evolution. (4) denotes the $\pm 1 \sigma$ deviation in the mean luminosity evolution and the last column $\Delta\left\langle M_{r}\right\rangle$ gives the median evolution.

\begin{tabular}{|c|c|c|c|c|}
\hline $\begin{array}{l}\text { Sample } \\
(1)\end{array}$ & $\begin{array}{l}N \\
(2)\end{array}$ & $\begin{array}{c}\Delta \bar{M}_{r} \\
{[\mathrm{mag}]} \\
(3)\end{array}$ & $\begin{array}{c}\sigma_{M_{r}} \\
(4)\end{array}$ & $\begin{array}{c}\Delta\left\langle M_{r}\right\rangle \\
{[\mathrm{mag}]} \\
(5)\end{array}$ \\
\hline $\mathrm{Cl} 0849$ & 15 & -0.30 & 0.19 & -0.36 \\
\hline Cl 1701 & 5 & -0.42 & 0.19 & -0.20 \\
\hline $\mathrm{Cl} 1702$ & 7 & -0.57 & 0.20 & -0.51 \\
\hline Low $-L_{X}$ & 27 & -0.44 & 0.19 & -0.38 \\
\hline low $L^{a}$ & 13 & -0.23 & 0.22 & -0.36 \\
\hline high $L$ & 14 & -0.55 & 0.13 & -0.26 \\
\hline low mass ${ }^{b}$ & 13 & -0.69 & 0.73 & -0.71 \\
\hline high mass & 14 & -0.12 & 0.15 & -0.07 \\
\hline in $^{c}$ & 13 & -0.30 & 0.17 & -0.15 \\
\hline out & 14 & -0.48 & 0.18 & -0.45 \\
\hline
\end{tabular}

${ }^{a}$ fainter: $M_{r}>-21.77$, brighter: $M_{r}<-21.77$.

${ }^{b}$ less-massive: $\log \sigma<2.215$,

more-massive: $\log \sigma>2.215$.

${ }^{c}$ in: only core sample $R<474.4 \mathrm{kpc}$,

out: only outer region sample $R>474.4 \mathrm{kpc}$.

of $\Delta a=0.2 \pm 0.5$ between the sub-samples is found.

An analysis of the FP for the rich cluster galaxies in the previous chapter 6 , revealed that the lenticular galaxies show a larger offset from the local FP relation and a stronger evolution than the elliptical galaxies. To verify this result with the FJR, the FJR offsets of early-type cluster galaxies in A 2390 and A 2218 from the local FJR are displayed in Fig. 7.2, similar to Fig. 7.1 but now divided into the morphological types of elliptical and S0 galaxies. For this analysis, the total rich cluster sample is restricted to the $33 \mathrm{E}+\mathrm{S} 0$ galaxies which enter the $\mathrm{FP}$ and where a morphological classification based upon HST images is available. The bright $\mathrm{cD}$ gal-

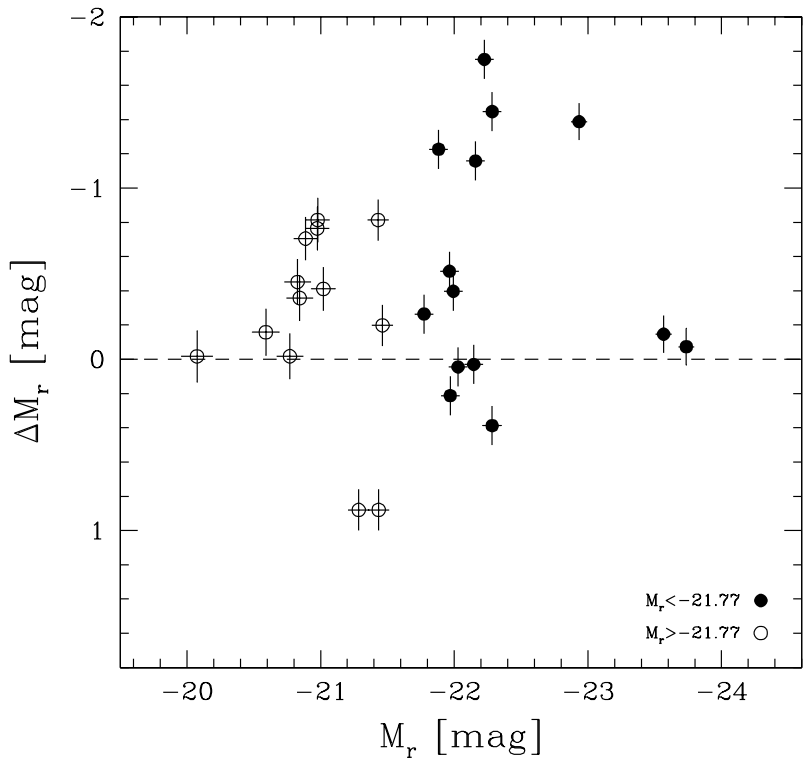

Figure 7.3: Offsets of the Faber-Jackson relation in Gunn $r$ for the early-type cluster galaxies in the Low$L_{X}$ clusters CL 0849, CL 1701 and CL 1702 from the local FJR by the J99 Coma sample as a function of luminosity $M_{r}$. Filled symbols denote brighter galaxies $M_{r}<-21.77$, open symbols fainter galaxies $M_{r}>-21.77$. The dashed line corresponds to a zero evolution.

axy of A 2218 was excluded as no counterpart of this galaxy type was observed in A 2390. In Fig. 7.2 the sample of ellipticals and S0 galaxies is shown as solid and open symbols. The higherluminosity Es $\left(M_{r}<-21.49\right)$ are brighter by $\left\langle\Delta \bar{M}_{r \mathrm{E}}\right\rangle=-0.14$ with a scatter $\sigma_{M_{r}}=0.11^{m}$, lower-luminosity Es $\left(M_{r}>-21.49\right)$ show an evolution of $\left\langle\Delta \bar{M}_{r \mathrm{E}}\right\rangle=-0.23$ with $\sigma_{M_{r}}=0.18^{m}$. The brighter S0s are offset to the local FJR relation by $\left\langle\Delta \bar{M}_{r}\right.$ So $\rangle=-0.66$ with $\sigma_{M_{r}}=0.12^{m}$, fainter S0s by $\left\langle\Delta \bar{M}_{r \text { so }}\right\rangle=-0.45$ with a scatter of $\sigma_{M_{r}}=0.13^{m}$. These results confirm the analysis of the FP where also the lenticular galaxies are more luminous than the ellipticals. Both sub-samples of brighter and fainter S0s show a stronger evolution than the Es. An interesting issue would be also if there is a difference between the sub-groups of faint and bright ellipticals and S0 galaxies. Unfortunately the sub-samples are 
too small to test this in greater detail.

In order to investigate the offsets of the FJR of the Low $-L_{X}$ clusters from the local FJR with respect to luminosity, the poor cluster sample is divided at $M_{r}=-21.77$ in two groups with the high-luminosity sub-sample containing 14 galaxies and the low-luminosity counterpart comprising 13 objects. Fig. 7.3 shows the offsets of the FJR in Gunn $r$ for the early-type galaxies in the Low $-L_{X}$ clusters CL 0849, CL 1701 and CL 1702 from the local FJR by the J99 Coma sample as a function of luminosity $M_{r}$. Brighter galaxies with $M_{r}<-21.77$ are represented as solid symbols, fainter galaxies with $M_{r}>-21.77$ are denoted as open symbols. Brighter cluster galaxies indicate on average an evolution of $\Delta \bar{M}_{r}=-0.55 \pm 0.13^{m}$, fainter objects in the poor cluster sample $\left(M_{r}>-21.77\right)$ are on average more luminous by $\Delta \bar{M}_{r}=-0.23 \pm 0.22^{m}$. At a first glance, these values suggest an impression of a larger offset for brighter galaxies. However, the scatter of the fainter galaxies is also larger than for the brighter ones and the median offsets are similar for both sub-classes. Table 7.2 compares the individual values for the two groups of early-type galaxies with different $M_{r}$. As the difference in median luminosity between lower-luminosity and higher-luminosity galaxies is within their $1 \sigma$ error, the differences in the sub-samples are negligible.

The Low $-L_{X}$ early-type cluster galaxies were visually classified as nine elliptical galaxies and one S0 galaxy (cf. section 4.4). As the number of galaxies in each sub-class is unbalanced but also too low, a reliable comparison and further analysis as for the rich cluster galaxies cannot be performed.

\subsubsection{Mass Dependence}

As the distant galaxies cover a broad range in velocity dispersions $(2.02 \leq \log \sigma \leq 2.58)$ down to $105 \mathrm{~km} \mathrm{~s}^{-1}$, it is also possible to explore an evolution in the slope of the FJR. The Coma sample has a steeper slope than that of the distant clusters, with a slope difference of $\Delta a=1.5 \pm 0.4$ (see Fig. 6.2). This gives some evidence for a difference between massive and less-massive $\mathrm{E}+\mathrm{S} 0$ galaxies. In Fig. 7.4 the offsets of the FJR for the early-type cluster galaxies in A 2390 and A 2218 from the local FJR were computed and plotted as a function of $\sigma$. As $\sigma$ is a measure for the stellar mass of a galaxy, a dependence on the galaxy mass can be tested. Subdividing the total sample with respect to their velocity dispersion at $\sigma<170 \mathrm{~km} \mathrm{~s}^{-1}(\log \sigma=2.231)$ into two subsamples of equal number, the lower-mass objects $(\log \sigma<2.231)$ show on average a stronger evolution with $\Delta \bar{M}_{r}=-0.62 \pm 0.34^{m}$. More massive galaxies $(\log \sigma>2.231)$ are on average brighter by $\Delta \bar{M}_{r}=-0.02 \pm 0.16^{m}$. This suggests that the luminosity evolution correlates with the galaxy mass rather than the luminosity of the galaxies. Table 7.1 gives a comparison between the two groups of early-type galaxies. A bisector fit as illustrated in Fig. 7.4 to the offsets for both FJR samples yields

$$
\Delta M_{r}=4.64 \pm 0.56 \log \sigma-10.74 \pm 1.33
$$

While galaxies with $\sigma>170 \mathrm{~km} \mathrm{~s}^{-1}$ show negligible offsets in Fig. 7.4, the less-massive earlytypes show a brightening by up to 1.4 magnitudes. For the offsets from the local FJR in Fig. 7.4, the errors in the FJR diagram were derived as

$$
\delta(\Delta \mathrm{FJR})^{2}=\delta M_{\mathrm{r}}^{2}+6.82^{2} \delta \log (\sigma)^{2} .
$$

Again, the first term on the right hand side corresponds to Eq. 4.14 on page 91 and the second term is the propagated error of $\sigma$ based on the J99 Coma slope.

Galaxies with lower and higher mass exhibit different mean velocity dispersions $(\log \sigma=2.15$, and 2.34, respectively). For this reason, a twodimensional KS test is not the appropriate statistical method for a comparison. Similar to the previous section, bootstrap-bisector fits to the sub-samples of different mass are computed. For the low-mass galaxies $(\log \sigma<2.231)$ this 


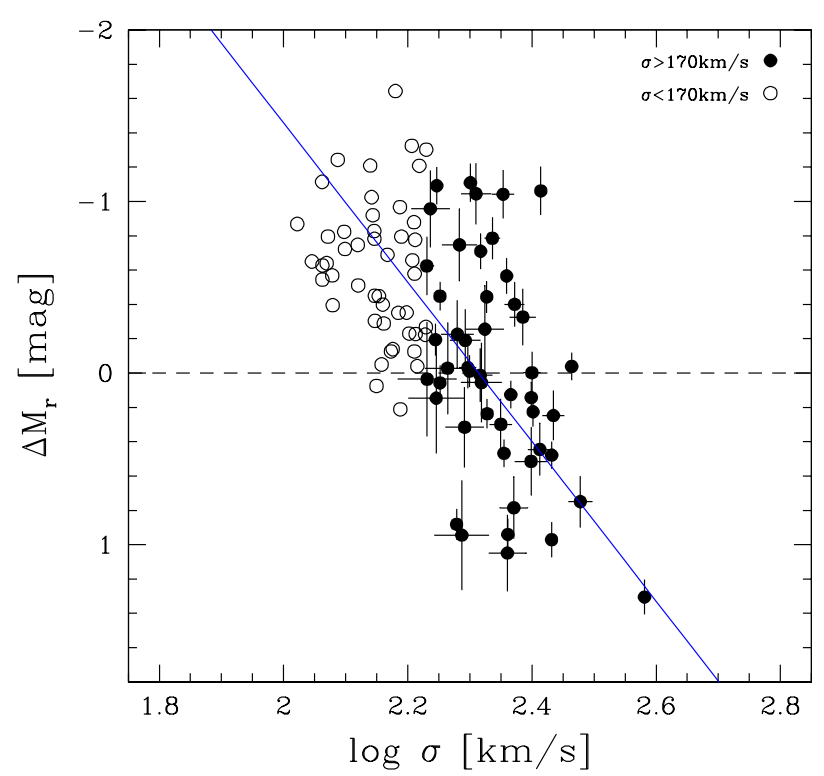

Figure 7.4: Offsets of the Faber-Jackson relation in Gunn $r$ for the early-type cluster galaxies in A 2390 and A 2218 from the local FJR by the J99 Coma sample as a function of $\sigma$ (mass). Filled symbols denote more-massive galaxies with $\log \sigma>170 \mathrm{~km} / \mathrm{s}$, open symbols less-massive galaxies with $\log \sigma<170 \mathrm{~km} / \mathrm{s}$. The dashed line corresponds to a zero evolution, the solid line is a bisector fit to all data. For matters of clarity, individual error bars are shown for the moremassive objects only.

method yields

$$
M_{r}=-(8.11 \pm 1.04) \log \sigma-(3.74 \pm 2.25),
$$

whereas for the more-massive $(\log \sigma>2.231)$ counterparts the bisector fitting routine results in

$$
M_{r}=-(5.57 \pm 1.53) \log \sigma-(8.83 \pm 3.58) .
$$

Low-mass galaxies and high-mass galaxies have different slopes with a slope difference of $\Delta a=2.5 \pm 1.5$, which is significant on the $2 \sigma$ level based on the uncertainty of the slope for the high-mass objects. Note that this results depends on the defined selection boundaries upon the Coma reference as the local slope and zeropoint were adopted for the derivation of the evolution of the distant sub-samples. Nevertheless,

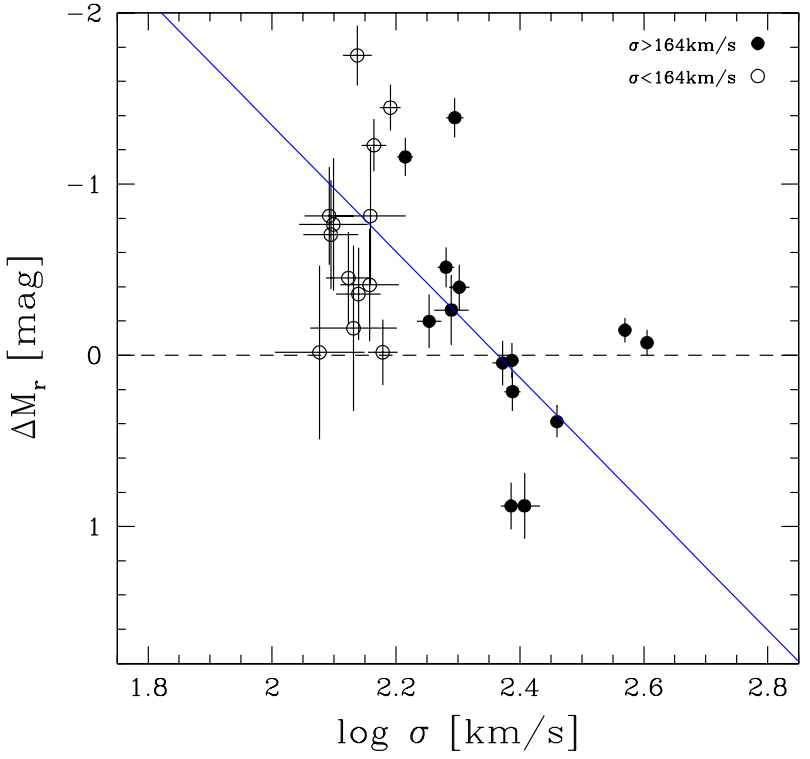

Figure 7.5: Offsets of the Faber-Jackson relation in Gunn $r$ for the early-type cluster galaxies in the Low$L_{X}$ clusters CL 0849, CL 1701 and CL 1702 from the local FJR by the J99 Coma sample as a function of $\sigma$ (mass). Filled symbols denote more-massive galaxies with $\log \sigma>164 \mathrm{~km} / \mathrm{s}$, open symbols less-massive galaxies with $\log \sigma<164 \mathrm{~km} / \mathrm{s}$. The dashed line denotes a zero offset, the solid line is a bisector fit to the whole sample.

it remains the trend that less-massive galaxies feature a larger luminosity offset which could be a possible hint for a faster evolution of the low-mass galaxies compared to the more massive counterparts. Another possibility is that the lower-mass galaxies have on average younger stellar populations or a broad range in star formation histories than galaxies with higher $\sigma$. Within the spectra, no signs of strong star formation activity was detected which argues against younger population ages. However, differences might also occur in the colours or in the metallicity of the systems with a broader distribution of metal sensitive absorption line indices in the galaxy spectra.

To explore if a mass-dependent luminosity evolution is also evident in the lower-mass galaxy clusters, the sample was divided into two sub-groups 
with respect to velocity dispersion at $164 \mathrm{~km} \mathrm{~s}^{-1}$ $(\log \sigma=2.215)$. Fig. 7.5 shows the FJR offsets for the 13 lower-mass and 14 more-massive $\mathrm{E}+\mathrm{S} 0$ galaxies in the Low $-L_{X}$ clusters as a function of velocity dispersion and Table 7.2 summarises the findings for both groups. The solid line in the Figure is a bisector to the offsets of the combined sample

$$
\Delta M_{r}=3.69 \pm 0.92 \log \sigma-8.72 \pm 2.07 .
$$

Galaxies with lower $\sigma$ exhibit a stronger brightening of $\Delta \bar{M}_{r}=-0.69 \pm 0.73^{m}$ than their high $\sigma$ counterparts with $\Delta \bar{M}_{r}=-0.12 \pm 0.15^{m}$. However, the scatter in the evolution, in particular for the less-massive objects, is quite large. The reason for this effect comes from a low number of galaxies and on average higher uncertainties in the velocity dispersions which enter the computation of the errors in combination, see Eq. 6.3. Regardless of the scatter, the difference between the two sub-samples is significant. $\mathrm{E}+\mathrm{S} 0$ galaxies with large $\sigma$ show a negligible evolution of $\left\langle\Delta M_{r}\right\rangle=-0.07^{m}$, all low- $\sigma$ objects feature a luminosity evolution with values up to $\approx 1.7 \mathrm{mag}$ nitudes and a median of $\left\langle\Delta M_{r}\right\rangle=-0.71^{m}$. Similar as for the rich cluster galaxies, bootstrapbisector fits are executed to assess the significance of a slope change for the sub-samples of different mass. A free bisector fit to the lessmassive $\mathrm{E}+\mathrm{S} 0$ galaxies in the low-mass clusters $(\log \sigma=2.215)$ yields

$$
M_{r}=-(16.01 \pm 4.63) \log \sigma+(13.02 \pm 9.88),
$$

whereas for the more-massive $(\log \sigma>2.215)$ ones

$$
M_{r}=-(5.94 \pm 3.04) \log \sigma-(8.10 \pm 7.22)
$$

is found. This relation is in agreement with the fit parameters in Eq. 7.8 as derived for the more massive sub-sample in rich clusters. The two relations of Eqs. 7.10 and 7.11 suggest a slope difference of $\Delta a=10.1 \pm 4.6$, which has a $2 \sigma$ level significance assuming the uncertainty of the slope for the less-massive galaxies.
In summary, the E+SO galaxies in the lowmass clusters show a similar trend with a massdependent luminosity evolution as measured for the early-type galaxies in more-massive clusters.

\subsubsection{Radial Dependence}

An adjuvant test of possible environmental effects on the properties of galaxies is looking at the radial distribution of the galaxies within a specific galaxy cluster. By combining the redshift measurements and the morphological type classification the distribution of cluster members as a function of projected cluster radius can be explored. Unfortunately, as the HST field is restricted to the central $\sim 0.6 \mathrm{Mpc}$ of a galaxy cluster the change of the morphological mix of galaxies cannot be investigated. In this central region of a cluster the gravitational potential of a cluster is steepest and most of the physical environmental mechanisms are simultaneous at work. Additionally projection effects with galaxies that are located in the outer regions play a role and have to be accounted for. Although processes such as tidal stripping and tidal triggering which are only relevant in the cores of clusters are expected to be most dominant, a distinction into the various mechanisms which are likely to operate is not possible.

The projected radial distribution of spectroscopic target galaxies for Abell 2390, CL 0849 CL 1701 and CL 1702 is shown in Fig. 7.6. Early-type cluster members which were verified through spectroscopy are indicated as open circles, non member galaxies as asterisks. Morphologically classified ellipticals and S0 galaxies (including Sa bulges) are represented as solid circles and stars, respectively. As expected, no radial trend for any morphological galaxy type is detectable within the central parts of the clusters. To analyse a possible change of the galaxies' properties as function of distance from the cluster centre, the MOSCA spectroscopy was designed and carried out to cover a comprehensive range of radial distances. However, this study is 

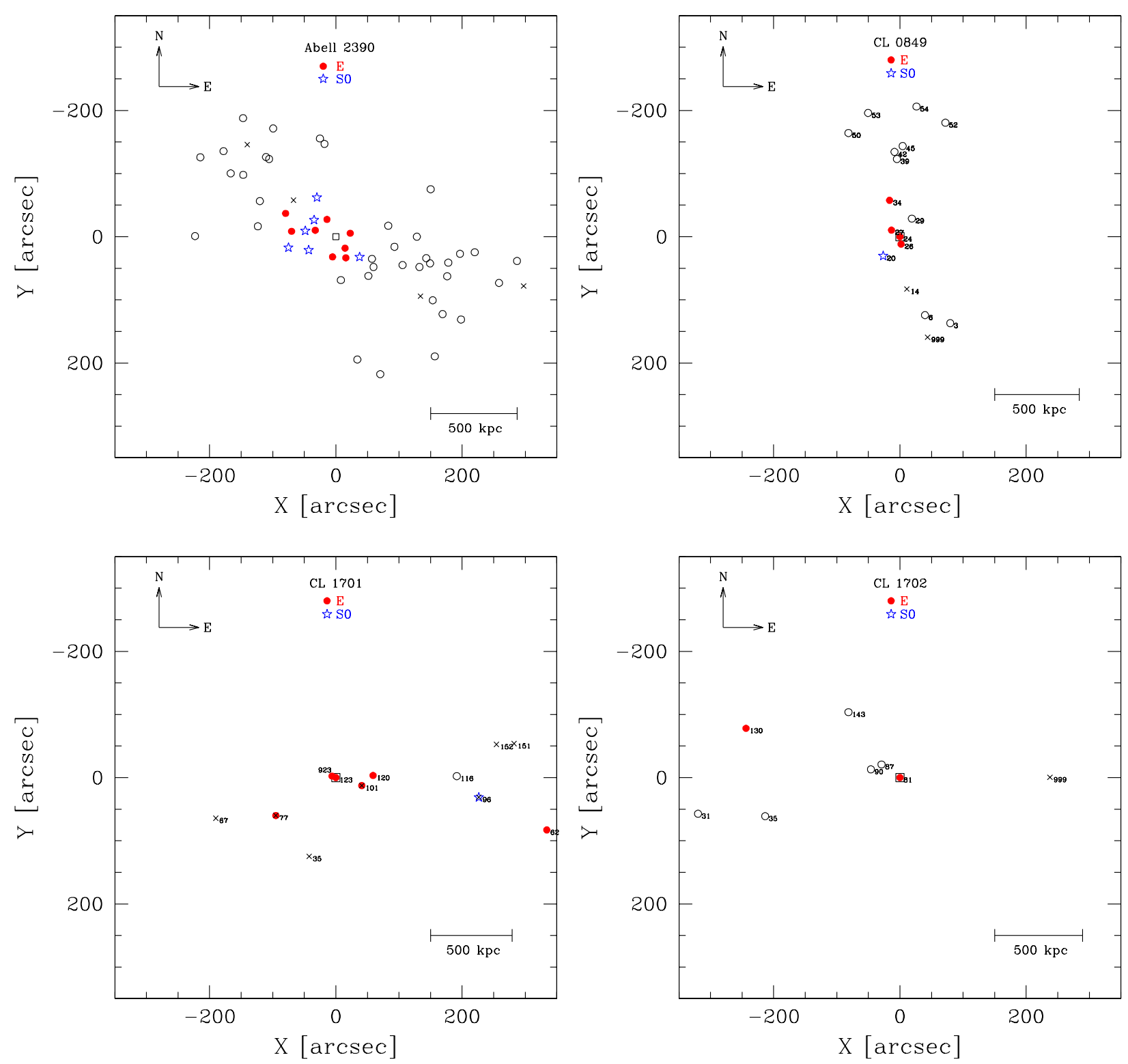

Figure 7.6: Distribution of early-type cluster galaxies. Spectroscopic confirmed cluster members are denoted as open circles, non member galaxies as asterisks. Morphologically classified ellipticals and S0 galaxies (including Sa bulges) are represented as solid circles and stars, respectively. Upper panel left: Abell 2390 at $z=0.228$, right: CL 0849 at $z=0.234$, Lower panel left: CL 1701 at $z=0.246$, right: CL 1702 at $z=0.223$. 
not comparable to wide field surveys out to large cluster radii of $\gtrsim 2$ virial radii (e.g., Gerken et al. 2004).

In order to investigate any possible dependence on clustercentric radius within the large data set, the projected distance of each galaxy from the respective brightest cluster galaxy (BCG) was measured and the samples subdivided into two radial bins of equal size so that they comprise equal numbers of cluster members.

The average projected radius for the rich cluster galaxies in the core region is $244 \mathrm{kpc}$ compared to $724 \mathrm{kpc}$ for galaxies in the outer bin. For the Low $-L_{X}$ clusters the average projected radii are $128 \mathrm{kpc}$ and $740 \mathrm{kpc}$ for galaxies in the central and outer districts, respectively. Based on the relation between the virial mass of a cluster and its projected velocity dispersion (Girardi et al. 1998), the virial radius $R_{v}$ can be derived as

$$
R_{v} \sim 0.0035(1+z)^{-1.5} \sigma_{p} h^{-1},
$$

where the $R_{v}$ is in units of $\mathrm{Mpc}$ and the projected velocity dispersion $\sigma_{p}$ is given in $\mathrm{km} \mathrm{s}^{-1}$. For A 2218, $\sigma_{p}=1370_{-120}^{+160} \mathrm{~km} \mathrm{~s}^{-1}$ is adopted (Le Borgne et al. 1992) and for A $2390 R_{v}=3.156 h_{100}^{-1} \mathrm{Mpc}$ is used (Carlberg et al. 1996). For our assumed cosmology, this yields to virial radii for the clusters A 2218 and $\mathrm{A} 2390$ of $R_{v}=3.765 \pm 0.440 \mathrm{Mpc}$ $\left(1268 \pm 148^{\prime \prime}\right)$ and $R_{v}=2.209 \pm 0.165 \mathrm{Mpc}$ $\left(605 \pm 45^{\prime \prime}\right)$, respectively. Adopting the cluster velocity dispersions as listed in Table 2.3 on page 31 from the low-resolution sample (Balogh et al. 2002b), virial radii for the Low $-L_{X}$ clusters were derived. This results in virial radii for the poor clusters CL 0849, CL 1701 and CL 1702 of $R_{v}=1.950 \pm 0.230 \mathrm{Mpc}\left(523 \pm 62^{\prime \prime}\right)$, $R_{v}=2.099 \pm 1.629 \mathrm{Mpc}\left(543 \pm 421^{\prime \prime}\right)$ and $R_{v}=0.999 \pm 1.102 \mathrm{Mpc}\left(278 \pm 307^{\prime \prime}\right)$, respectively.

Fig. 7.7 displays the FJR offsets for the earlytype rich cluster galaxies as a function of distance from the cluster centre $R$, with the average projected radius $R$ given in units of kpc. Each

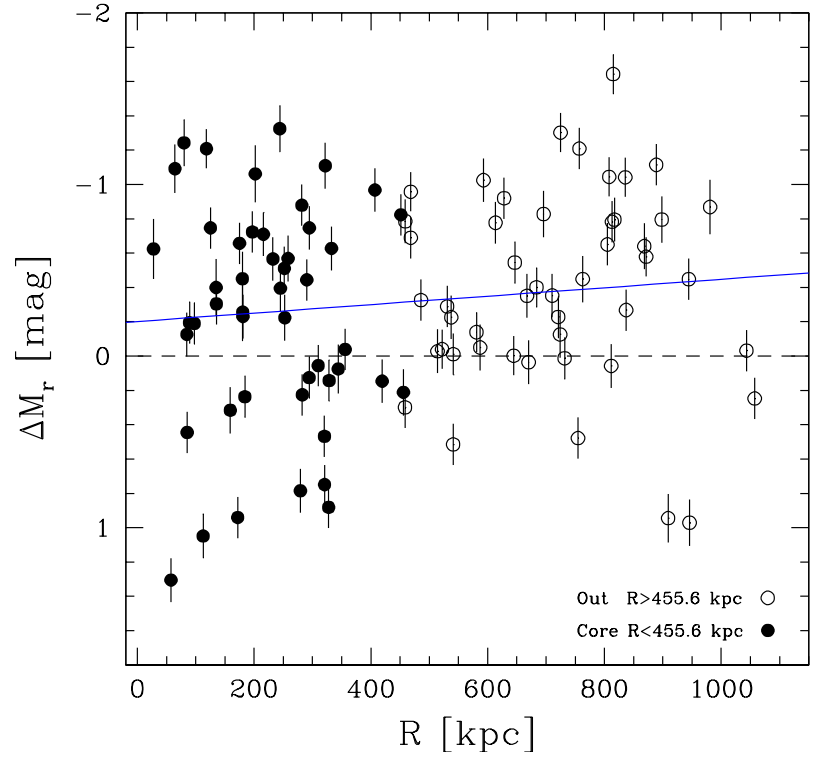

Figure 7.7: Offsets of the Faber-Jackson relation in Gunn $r$ for the early-type cluster galaxies in A 2390 and A 2218 from the local FJR by the J99 Coma sample as a function of distance from the cluster centre $R$ in units of kpc. The cluster centre is defined by the central cD galaxy (BCG). Filled symbols denote galaxies in the core region with $R<455.6 \mathrm{kpc}$, open symbols are galaxies in the outer districts with $R>455.6 \mathrm{kpc}$. The dashed line indicates a zero offset, the solid line is a $\chi^{2}$-fit to the complete sample.

cluster centre is defined by the brightest cluster galaxy (BCG), the central cD elliptical galaxy. The cluster galaxies were divided into two radial bins of the core region $(R<455.6 \mathrm{kpc})$ and the outer districts $(R>455.6 \mathrm{kpc})$, each comprising 48 galaxies. Applying a linear $\chi^{2}$-fit to the distant cluster sample in Fig. 7.7 of

$$
\begin{aligned}
\Delta M_{r}= & -(0.0003 \pm 0.0002)\left(\frac{R}{\mathrm{kpc}}\right) \\
& -0.2015 \pm 0.1214,
\end{aligned}
$$

a slight gradient of the FJR offsets with increasing clustercentric radius $R$ is found.

To test the significance of this gradient for the two sub-samples of inner and outer radial bin, the bootstrap bisector technique is performed. A bisector to the galaxies lying in the core region 


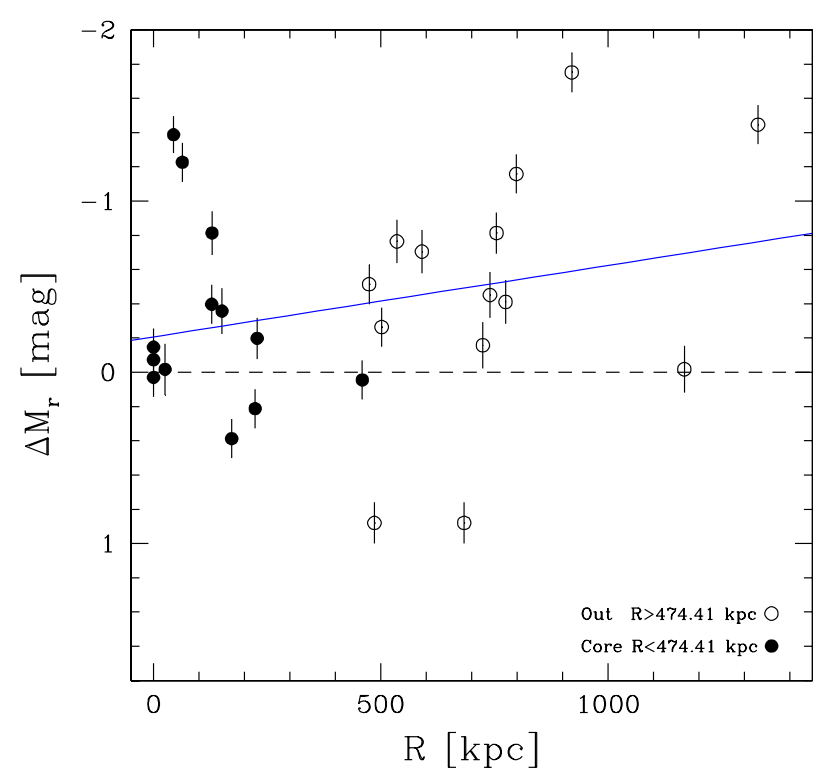

Figure 7.8: Offsets of the Faber-Jackson relation in Gunn $r$ for the early-type cluster galaxies in the Low $-L_{X}$ clusters CL 0849, CL 1701 and CL 1702 from the local FJR by the J99 Coma sample as a function of distance from the cluster centre $R$ in units of kpc. The cluster centre is defined by the central $\mathrm{cD}$ galaxy (BCG). Filled symbols denote galaxies in the core region with $R<474.4 \mathrm{kpc}$, open symbols are galaxies in the outer districts with $R>474.4 \mathrm{kpc}$. The dashed line indicates a zero offset, the solid line is a $\chi^{2}$-fit to the complete sample.

with $R<455.6$ kpc gives

$$
M_{r}=-(5.29 \pm 1.15) \log \sigma-(9.60 \pm 2.59),
$$

whereas for the galaxies in the outer districts $R>455.6 \mathrm{kpc}$ the method yields

$$
M_{r}=-(5.39 \pm 0.51) \log \sigma-(9.48 \pm 1.13) .
$$

The slope difference of $\Delta a=0.1 \pm 1.2$ between galaxies in the central and outer bins is statistically insignificant as the scatter is larger than the detected offset.

The mean and median evolution of the E+S0 galaxies for the central and the outer region are shown in Table 7.1. As the galaxies in the core and outer region feature similar mean velocity dispersions $(\log \sigma=2.26$, and 2.23, respectively), the two-dimensional KS test provides a good measure of consistency. Applying the KS test results in a probability of $P=0.46$ that the two distributions are similar. The slope for the galaxies in the core region is similar to the slope derived for the galaxies in the outskirts. Looking at the residuals from the local FJR, there is a slight trend with clustercentric radius, but not significantly (within $\pm 1 \sigma$ ). On average, galaxies in the outskirts of the cluster indicate a larger luminosity offset of $-0.35 \pm 0.20^{m}$ than in the core region $-0.26 \pm 0.20^{m}$. However, the offset has only a low significance and its not sure whether this gradient is real or maybe just a projection effect.

The $\Delta M_{r}-R$ distribution of the early-type galaxies in the low-massive clusters is shown in Fig. 7.8. Cluster members were divided into two radial bins of a central core $(R<474.4 \mathrm{kpc})$ and an outer region $(R>474.4 \mathrm{kpc})$, comprising 13 and 14 galaxies, respectively. The cluster centre was adopted to be the location of the BCG. A similar trend is seen as for the rich cluster sample, albeit with a larger scatter. A linear $\chi^{2}$-fit to the Low $-L_{X}$ members gives

$$
\begin{aligned}
\Delta M_{r}= & -(0.0004 \pm 0.0003)\left(\frac{R}{\mathrm{kpc}}\right) \\
& -(0.2071 \pm 0.1951),
\end{aligned}
$$

which is indicated as the solid line in Fig. 7.8. On average, the residuals from the local FJR indicate a slight trend with increasing projected radius, although it is within the $\pm 1 \sigma$ uncertainty. Table 7.2 gives a comparison between the core sample and the galaxies in the outskirts for the low massive clusters. Galaxies far away from the cluster centre have an offset of $-0.48 \pm 0.18^{\mathrm{m}}$, whereas in the central part only an brightening of $-0.30 \pm 0.17^{m}$ is measured. This difference is comparable to the result as derived for the environments of rich clusters. A further comparison involving the KS method is not possible as the sub-samples contain less than 20 galaxies where the KS test has no statistical significance.

Fig. 7.9 displays the offsets of the FJR of the early-type galaxies in the rich clusters from the 


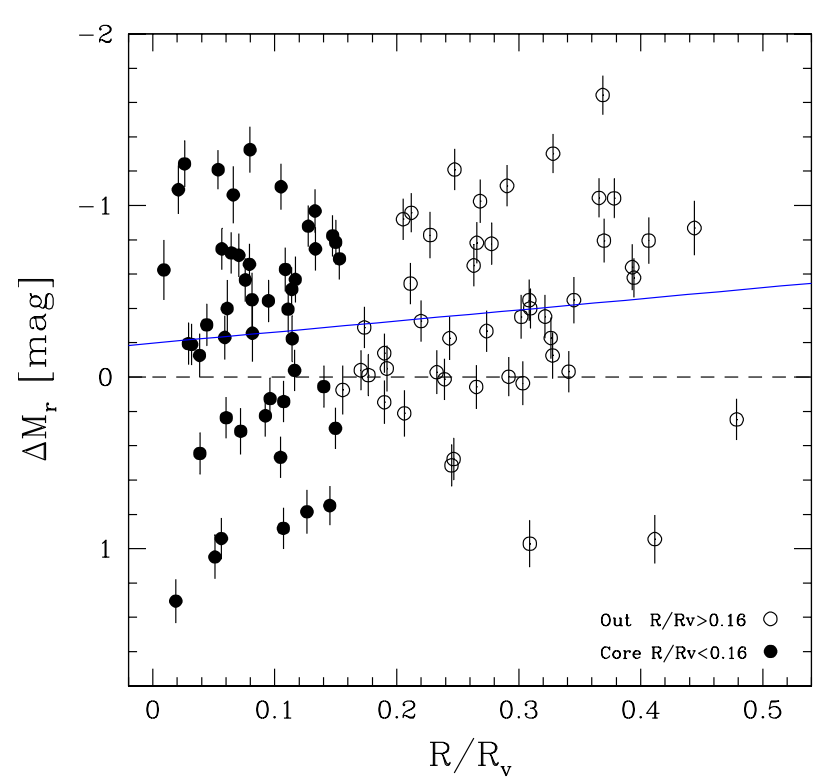

Figure 7.9: Offsets of the Faber-Jackson relation in Gunn $r$ for the early-type cluster galaxies in A 2390 and A 2218 from the local FJR by the J99 Coma sample as a function of distance from the cluster centre $R$, normalised by the virial radius $R_{v}$. The cluster centre is defined by the central cD galaxy (BCG). Filled symbols denote galaxies in the core region with $R / R_{v}<0.16$, open symbols are galaxies in the outer districts with $R / R_{v}>0.16$. The dashed line indicates a zero offset, the solid line is a $\chi^{2}$-fit to the complete sample.

local FJR as a function of distance from the cluster centre $R$, normalised to the virial radius $R_{v}$. Again, the central $\mathrm{cD}$ galaxy was assumed to be the cluster centre. Galaxies in the core region $\left(R / R_{v}<0.16\right)$ are represented as filled symbols, galaxies in the outer districts $\left(R / R_{v}>0.16\right)$ as open symbols. The linear $\chi^{2}$-fit to the distant galaxies in in Fig. 7.9 is

$$
\Delta M_{r}=-(0.65 \pm 0.53)\left(\frac{R}{R_{v}}\right)-(0.20 \pm 0.12) \text {. }
$$

In both Figs. 7.7 and 7.9, the errors of the offsets on the absolute magnitudes in the FJR diagrams were computed as given in Eq. 7.2. As a consequence of the normalisation with the virial radius, the trend of the FJR offsets with increas-

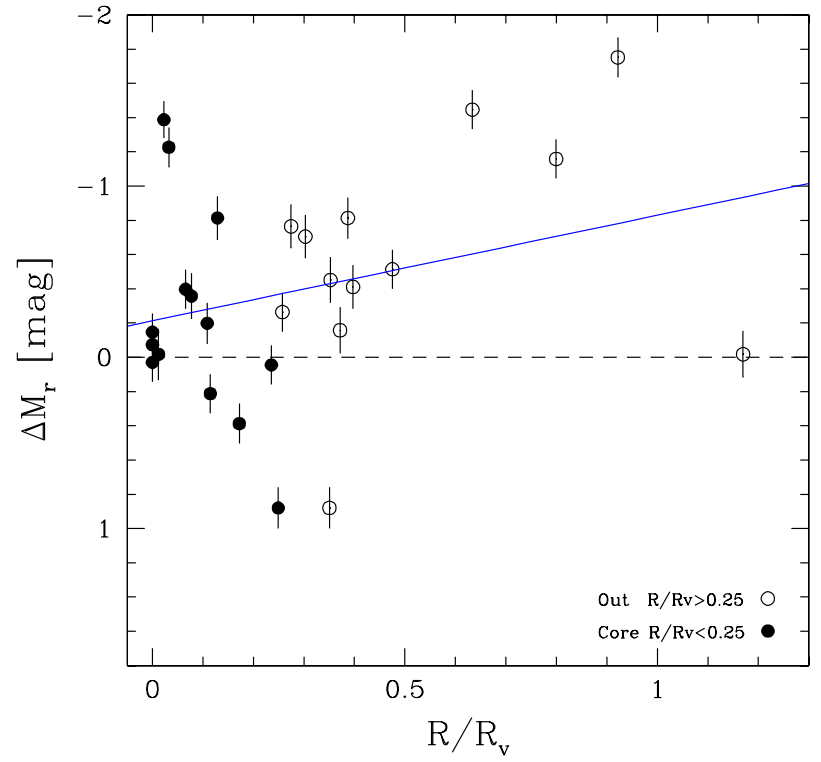

Figure 7.10: Offsets of the Faber-Jackson relation in Gunn $r$ for the early-type cluster galaxies in the Low- $L_{X}$ clusters CL 0849, CL 1701 and CL 1702 from the local FJR by the J99 Coma sample as a function of distance from the cluster centre $R$, normalised by the virial radius $R_{v}$. The cluster centre is defined by the central cD galaxy (BCG). Filled symbols denote galaxies in the core region with $R / R_{v}<0.25$, open symbols are galaxies in the outer districts with $R / R_{v}>0.25$. The dashed line indicates a zero offset, the solid line is a $\chi^{2}$-fit to the complete sample.

ing radius $R$ detected in Fig. 7.9 is even more dominant. In numbers, galaxies in the outskirts of the cluster shown in this projection a slight smaller luminosity offset of $\left\langle\Delta M_{r}\right\rangle=-0.34^{m}$ than their counterparts in the central region of $\left\langle\Delta M_{r}\right\rangle=-0.40^{m}$. However, both numbers are consistent within their $1 \sigma$ deviation. The cluster galaxies cover a radial range out to $\left(R / R_{v}\right) \sim 0.5$ $(1.1 \mathrm{Mpc})$, which is in case of A 2390 roughly the beginning of the transition region of the cluster that is far from the cluster centre but still within the virial radius of the cluster. Therefore, still many physical processes are simultaneously at work which could account for this slight observed trend. Star formation induced through tidal triggering or tidal halo stripping are proba- 
bly the most dominant mechanisms at work. In the transition region these two processes become less likely and effects such as ram pressure stripping, which is the removal of the galactic gas by pressure exerted by the intercluster medium, or a slow decrease of star formation activity ("strangulation") come into play. In addition, harassment (i.e. high speed interactions between galaxies in the gravitational potential of the cluster) is effective over almost all clustercentric regions, from the center to the outskirts of a galaxy cluster. The rich cluster sample does not reach the outer districts of the clusters such as the complete transition or periphery zone, where a possible radial gradient with luminosity would be more apparent. To test the origin of the radial trends in even greater detail, an analysis of the galaxy colours as a function of projected radii will be performed in section 7.1.4.

Looking at the offsets of the FJR for the poor cluster galaxies $\Delta M_{r}$ as a function of $R / R_{v}$ in Fig. 7.10, a steeper gradient with clustercentric radius compared to the rich counterparts is found. Galaxies in the outskirts $\left(R / R_{v}>0.25\right)$, and in the core region $\left(R / R_{v}<0.25\right)$ exhibit an evolution of $\left\langle\Delta M_{r}\right\rangle=-0.51^{m}$ and $\left\langle\Delta M_{r}\right\rangle=-0.07^{m}$, respectively. Assuming a linear $\chi^{2}$-fit to the whole sample of

$$
\Delta M_{r}=-(0.62 \pm 0.42)\left(\frac{R}{R_{v}}\right)-(0.21 \pm 0.17),
$$

gives a similar relation as for the rich cluster galaxies but a somewhat steeper slope. However, as the virial radii of the Low- $L_{X}$ clusters are smaller their members are distributed over a larger range of distances out to $\sim 1 R_{v}$. As a consequence, a stronger radial gradient is detected. Nevertheless, two issues have to be treated with caution. First, the low number of galaxies in the outer districts beyond $\sim 0.5 R / R_{v}$ used in the analysis of radial distribution can mimic a higher offset. Second, an explanation for a stronger radial trend may be the result of different processes which occur at larger clustercentric dis- tances than for the rich clusters. The detected net effect might also include mechanisms such as strangulation and ram pressure stripping. In addition, the truncation of star formation could also operate differently than in rich clusters as the intercluster medium for lower-mass clusters is less effective and exhibits lower densities that for more massive clusters.

\subsubsection{A Dependence on Galaxy Colours?}

In a previous study of the cluster A 2390, Abraham et al. (1996a) examined a wide field out to $\sim 5 \mathrm{Mpc}$ using moderate $S / N$ spectroscopy and ground-based imaging to study the infalling field galaxy populations. A radial gradient in the $(g-r)$ colour for galaxies in the outer districts (periphery) of 1.1 to $3.2 \mathrm{Mpc}$ was found, where the colours become predominantly bluer with increasing projected radius. In contrast, galaxies in the central regions $\lesssim 0.43 \mathrm{Mpc}$ show red, evolved populations with old ages of $\gtrsim 8$ Gyrs, assuming the Bruzual \& Charlot (1993; GISSEL93 version) stellar population models.

It is therefore interesting to test if a radial trend with the colours of the cluster galaxies exists also in this study. For this reason, the restframe $(B-I)$ colours for the two rich galaxy samples were used which show a very homogenous distribution, with galaxies in A 2390 encompassing a range $1.86<(B-I)<2.51$ and in A $22181.95<(B-I)<2.44$. The $\mathrm{E}+\mathrm{S} 0$ galaxies in A 2390 exhibit a median colour of $\langle(B-I)\rangle=2.29$, whereas for the galaxies in A 2218 a median of $\langle(B-I)\rangle=2.28$ is found. (cf. section 5.6 and Fig. 5.28 on page 125). Fig. 7.11 shows the rest-frame $(B-I)$ colour as a function of distance $R$ from the cluster centre for 95 early-type galaxies. One spiral (Sa) galaxy outlier with very blue colour of $(B-I)=-1.17$ is not displayed. The errors on the rest-frame colours were derived via the linear relation $\delta(B-I)=\delta B+\delta I+\delta k$, where $\delta B$ and $\delta I$ are the errors in the respective ground-based 


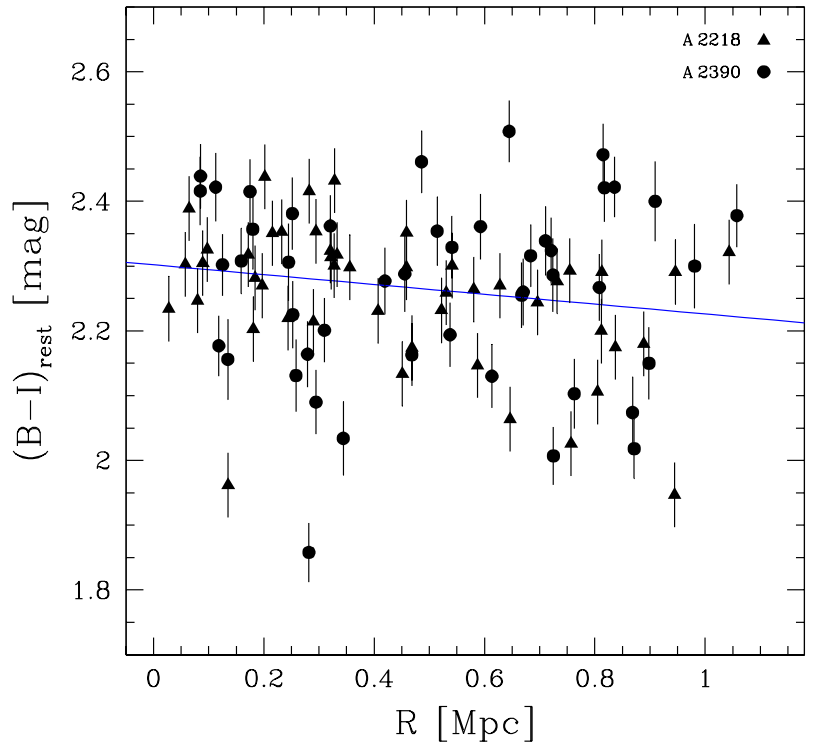

Figure 7.11: Rest-frame $(B-I)$ colour as a function of clustercentric distance (in units of $\mathrm{Mpc}$ ) for the early-type galaxies in A 2390 and A 2218. The central $\mathrm{cD}$ galaxy was assumed to define the cluster centre and the solid line is a $\chi^{2}$-fit to the combined data. A slight radial trend of $(B-I)$ galaxy colour with radius is seen.

filters and $\delta k$ the uncertainty in the $k$-correction. A linear $\chi^{2}$-fit to 94 galaxies with $(B-I)>1.9$ gives

$$
\begin{aligned}
(B-I)= & -(0.076 \pm 0.043)\left(\frac{R}{\mathrm{kpc}}\right) \\
& +(2.302 \pm 0.024),
\end{aligned}
$$

which is indicated as the solid line in Fig. 7.11. The slight colour gradient with increasing projected radius is significant on the $2 \sigma$ level and is also found within the sub-samples of A 2390 and A 2218. Overall, this trend of $(B-I)$ colour with $R$ is weak but already detected within the central 1.1 Mpc, which corresponds to half of the clusters virial radii.

Looking at the dispersion in the colours with increasing clustercentric distance, for the outer regions a larger scatter is found. Galaxies with $(B-I)>2.0$ located in central parts within $\left(R / R_{v}\right) \sim 0.2$, which corresponds to

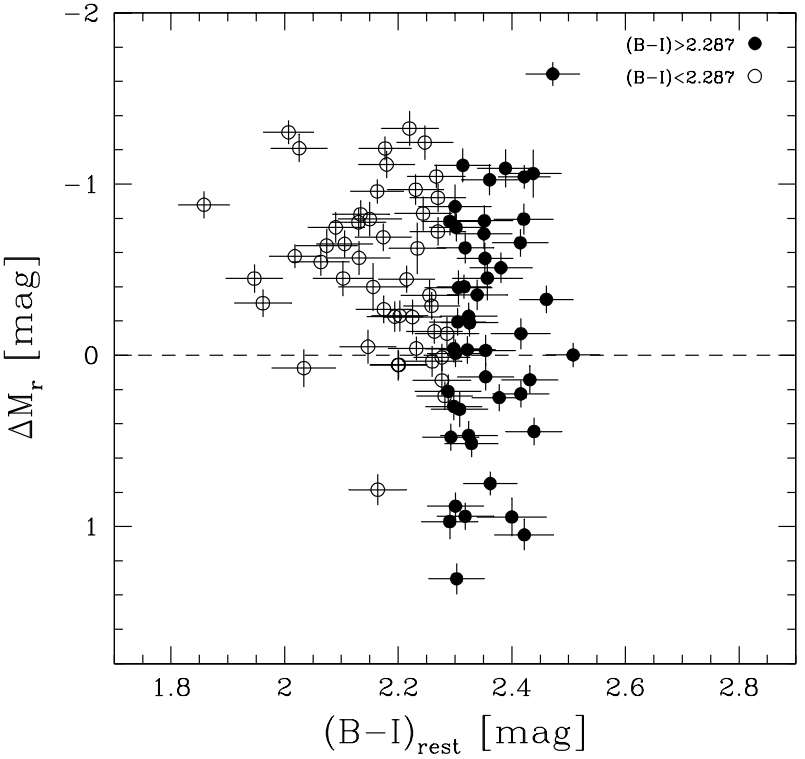

Figure 7.12: Offsets of the Faber-Jackson relation in Gunn $r$ for 95 early-type galaxies in A 2390 and A 2218 from the local FJR as a function of rest-frame $(B-I)$ colour. Filled symbols denote redder galaxies with $(B-I)>2.287$, open symbols indicate bluer galaxies with $(B-I)<2.287$. A zero evolution is shown by the dashed line.

a radial projected distance of $0.44 \mathrm{Mpc}$ from the cluster centre of A 2390 and $0.75 \mathrm{Mpc}$ in the case of A 2218, show a colour distribution in $(B-I)$ of $2.03<(B-I)<2.44$ (median of $\langle(B-I)\rangle=2.301)$ with a scatter of $\sigma(B-I)=0.095$. Cluster members far away from the centre are distributed over a slightly broader range in colours $2.00<(B-I)<2.51$ $(\langle(B-I)\rangle=2.286)$ with an approximately $35 \%$ larger scatter of $\sigma(B-I)=0.128$.

The $\Delta M_{r}-(B-I)$ diagram is displayed in Fig. 7.12. A clear trend of the FJR offsets with rest-frame $(B-I)$ colour is seen. Bluer galaxies defined as $(B-I)<2.287$ (open symbols) show an evolution of $-0.50 \pm 0.23^{m}$, whereas for the redder galaxies with $(B-I)>2.287$ (filled symbols) an insignificant offset of $-0.14 \pm 0.18^{\mathrm{m}}$ is found. In Table 7.3 a detailed comparison of the evolution as derived from the FJR for different sub-samples is given. In particular it is 
Table 7.3: Evolution of the Faber-Jackson relation in Gunn $r$ as a function of rest-frame $(B-I)$ colour for the rich clusters A 2218 and A 2390. $N$ is the number of galaxies and $\Delta \bar{M}_{r}$ indicates the mean luminosity evolution. The fourth column denotes the $\pm 1 \sigma$ deviation in the mean luminosity evolution and the last column $\Delta\left\langle M_{r}\right\rangle$ gives the median evolution.

\begin{tabular}{lrccc}
\hline Sample & $N$ & $\begin{array}{c}\Delta \bar{M}_{r} \\
{[\mathrm{mag}]}\end{array}$ & $\sigma_{M_{r}}$ & $\begin{array}{c}\Delta\left\langle M_{r}\right\rangle \\
{[\mathrm{mag}]}\end{array}$ \\
$(1)$ & $(2)$ & $(3)$ & $(4)$ & \multicolumn{1}{c}{$(5)$} \\
\hline$(B-I)>2.287$ & 48 & -0.14 & 0.18 & -0.13 \\
E & 10 & 0.09 & 0.17 & 0.14 \\
S0 & 11 & -0.28 & 0.12 & -0.45 \\
\hline$(B-I)<2.287$ & 48 & -0.50 & 0.23 & -0.45 \\
E & 6 & -0.41 & 0.23 & -0.30 \\
S0 & 6 & -0.84 & 0.24 & -0.72 \\
\hline
\end{tabular}

interesting, if the morphological types of elliptical and S0 galaxies show a trend with galaxy colour. Regardless of the colour cutoff, both groups of S0 galaxies exhibit a stronger evolution, which is at least by a factor of two larger than ellipticals. In case of the redder galaxies, the ellipticals even have on average an insignificant ("positive") evolution. These galaxies form most likely the evolved, passive stellar populations with old ages of $\gtrsim 8$ Gyrs that populate the red-sequence in the colour magnitude relation of galaxy clusters. Partly, this result is expected as bluer stellar populations are on average younger and therefore also show an excess in their luminosities. This analysis showed that the whole population of blue galaxies are the dominant contribution to the measured FJR offsets.

The results on the colour gradients give further evidence that the luminosity is the main driver for the evolution of the distant early-type galaxies in the FJR but the offsets are not caused due to differences in the kinematics of the local and distant scaling relations (see also the discussion on the $\sigma$ distributions in section 5.4 on page 114).

\subsection{Stellar Population Ages}

The observed evolution of the $M / L$ ratio as derived from the FP depends on the age of the galaxies' stellar population. In general, the luminosity of a young stellar population becomes rapidly fainter when the massive and bright stars which have a short lifetime disappear. For an old population comprising mainly low mass stars the dimming of the luminosity is on a more gradual evolutionary path. As a consequence of this, a stellar population formed at lower redshift will evolve faster than one generated at high redshift. In the following, the observed evolution of the $M / L$ ratio will be compared to simple stellar population models of a single burst formed at redshift $z_{\text {form }}$. The models have been generated following description of the analytic models by van Dokkum \& Franx (2001b).

The luminosity evolution of a single-age stellar population can be described by a power law as

$$
L \propto \frac{1}{\left(t-t_{\text {form }}\right)^{\kappa}},
$$

where $t_{\text {form }}$ is the stellar formation time which corresponds to a formation redshift of $z_{\text {form }}$ (e.g., Tinsley 1980). The coefficient $\kappa$ depends on the initial mass function (IMF), the metallicity and the chosen passband in which the luminosity is measured. In the models, a normal IMF with Salpeter (1955) slope, solar metallicity and $\kappa_{B}=0.96$ and $\kappa_{r}=0.79$ for the rest-frame $B$ and Gunn $r$-band was adopted (BC96). Model predictions are shown in Fig. 7.13 for the rich and poor clusters in the rest-frame Gunn $r$-band and in Fig. 7.14 for the field elliptical and S0 galaxies in the rest-frame $B$-band. Note that the predicted evolution of the models is independent of $H_{0}$ as the age dependence of the $M / L$ ratio is a power law. Two different model tracks for a single stellar population with formation redshift $z_{\text {form }}=1$ (lower line) and $z_{\text {form }}=2$ (upper line) are indicated as the dotted lines in Figs. 7.13, 7.14 and 7.15 .

To assess the evolution of the stellar populations 


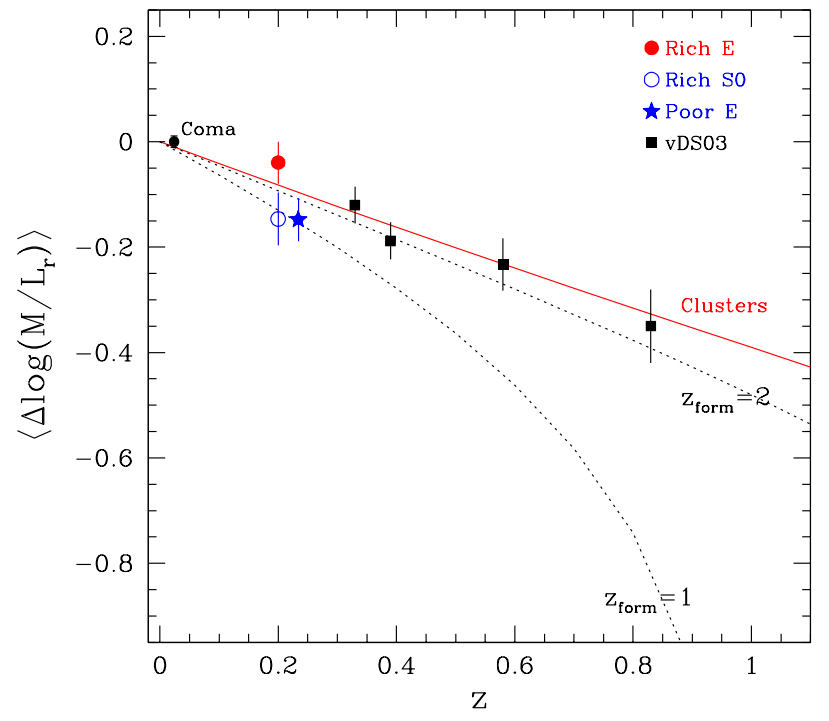

Figure 7.13: The average evolution of the $M / L$ ratio of the elliptical and S0 galaxies in the rich clusters, ellipticals in the poor clusters and cluster galaxies taken from van Dokkum \& Stanford (2003), in the rest-frame Gunn $r$-band. The small dot is the average $M / L$ ratio of the Coma cluster galaxies. Dotted lines are model tracks for a single stellar population with formation redshift 1 (lower line) and 2 (upper line). The solid line represents the single burst evolution of a stellar population formed at the mean cluster formation redshift of $z_{\text {form }}=3$ for masses $M>10^{11} M_{\odot}$. In high-mass clusters S0s evolve faster than ellipticals, whereas elliptical galaxies in low-mass clusters evolve as fast as SO galaxies in rich clusters.

in the early-type galaxies, the evolution of the FP zero-point $\gamma$ for the distant galaxies can be directly converted into an evolution of the average $M / L$ ratio as

$$
\langle\Delta \log (M / L)\rangle=-\frac{\langle\Delta \gamma\rangle}{2.5 \beta},
$$

where the average evolution of $\Delta \log (M / L)$ was derived via the average zero-point offset of the distant FP $\langle\Delta \gamma\rangle$ from the local Coma FP (cf. section 1.3 .3 on page 12 ).

Fig. 7.13 displays the evolution of the $M / L$ derived as the average offset from the local FP in the rest-frame Gunn $r$-band for the ellipticals and S0 galaxies in the rich clusters (large filled and open circles) and the ellipticals in the poor clusters (large stars). The cluster galaxies are compared to the cluster sample by van Dokkum \& Stanford (2003). These authors analysed a compilation of massive galaxies $\left(M>10^{11} M_{\odot}\right)$ in rich clusters taken from the literature and found an average formation redshift of $z_{\text {form }}=3$. The average $M / L$ ratio of the cluster galaxies in Coma is indicated as the small dot at $z=0.024$. Cluster galaxies are in compliance with a slow evolution of the bulk of their stars and a high formation redshift of $z_{\text {form }} \gtrsim 2.5$ which is denoted with the single burst evolution of a stellar population formed at $z_{\text {form }}=3$ (solid line). The average offset from the local FP $\langle\Delta \gamma\rangle$ was derived from the average stellar populations of one morphological type (elliptical and S0 galaxies) and the scatter is similar to the average uncertainty for the massive galaxies in a single cluster.

Lenticular galaxies in the rich clusters show a large evolution in their $M / L$ ratios and are in agreement with a low formation redshift $1 \leq z_{\text {form }} \leq 2$ and a fast evolution. In contrast, elliptical galaxies in rich cluster exhibit smaller offsets from the local FP which suggests a higher formation redshift $z_{\text {form }} \geq 2$. Elliptical galaxies in poor cluster indicate an opposite trend to what is found for the ellipticals in higher-mass clusters. These galaxies appear to evolve as fast as S0 galaxies in rich clusters.

In a recent study of 27 field early-type galaxies between $0.6<z<1.15$ a mass-dependent evolution was found (van der Wel 2005). This sample of high-redshift galaxies showed that the evolution of low-mass field galaxies with a characteristic masses of $M<2 \times 10^{11} M_{\odot}$ is faster than their more massive counterparts. The 21 field early-type galaxies in this thesis are located at a lower redshift range of $0.2<z<0.75$. But it is interesting, if already at a look-back time of $\sim 5$ Gyrs a possible evidence for a mass-dependent evolution or at least a trend with galaxy mass could be detected. For this 


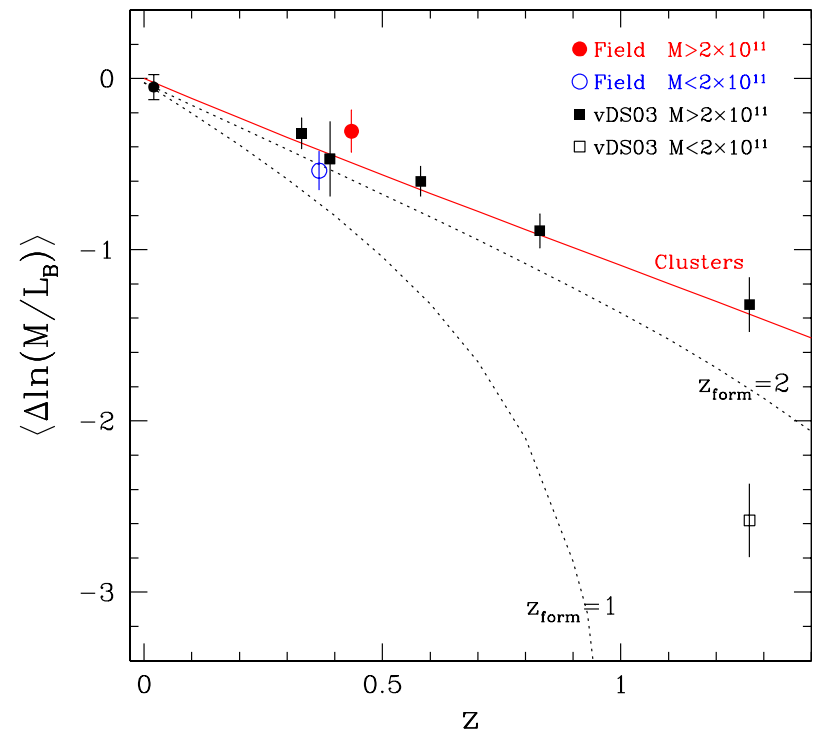

Figure 7.14: The average evolution of the $M / L$ ratio of the field elliptical and S0 galaxies in the FDF and WHDF and the early-type cluster galaxies taken from van Dokkum \& Stanford (2003), in the restframe $B$-band. Dotted lines are model tracks for a single stellar population with formation redshift 1 (lower line) and 2 (upper line). The model tracks are forced to go through the small dot which represents the average $M / L$ ratio of the field galaxies at $z=0.02$ by Faber et al. (1989). The solid line is the best fitting formation redshift for the evolution of massive cluster galaxies with masses $M>2 \times 10^{11} M_{\odot}$. Massive field galaxies with $M>2 \times 10^{11} M_{\odot}$ show a similar slow evolution as cluster galaxies, whereas less-massive field galaxies with $M<2 \times 10^{11} M_{\odot}$ evolve faster, similar to less-massive cluster galaxies.

reason, the average evolution of the $M / L$ ratio of the field elliptical and S0 galaxies in the FDF and WHDF was derived. Fig. 7.14 displays the average evolution of the $M / L$ ratios for the field galaxies in the rest-frame $B$-band compared to the early-type cluster galaxies taken from van Dokkum \& Stanford (2003). These authors deduced for a compilation of massive early-type galaxies with $M>2 \times 10^{11} M_{\odot}$ in rich galaxy clusters a formation redshift of $z_{\text {form }} \approx 3$, which is indicated as the solid line. All calculated model tracks are forced to go through the average $M / L$ ratio of the field galaxies at $z=0.02$

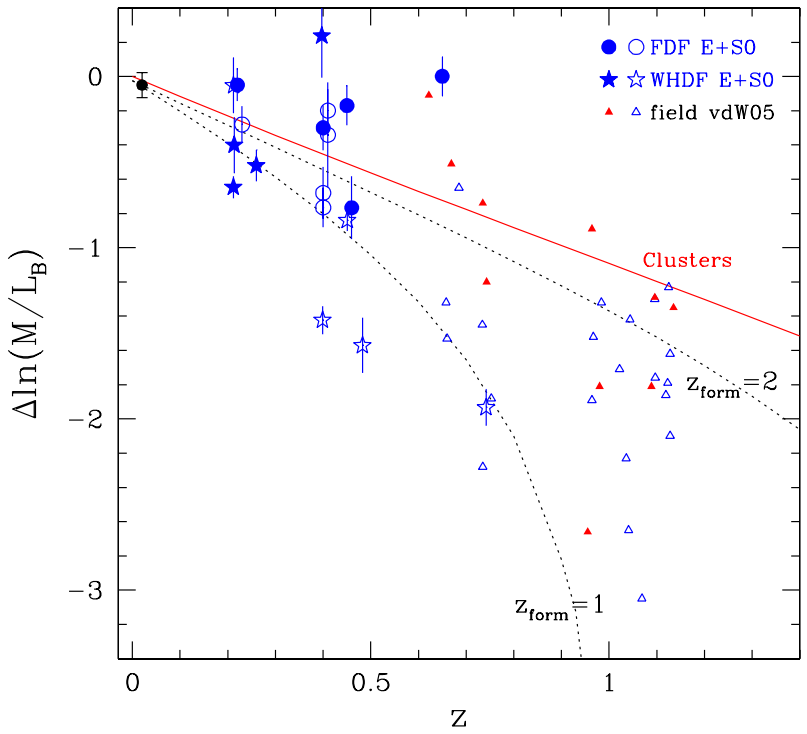

Figure 7.15: The evolution of the $M / L$ ratio of the field elliptical and S0 galaxies in the FDF and WHDF and the field early-type galaxies at $\langle z\rangle=1$ taken from van der Wel (2005), in the rest-frame $B$ band. Dotted lines are model tracks for a single stellar population with formation redshift 1 (lower line) and 2 (upper line). The model tracks are forced to go through the small dot which represents the average $M / L$ ratio of the field galaxies at $z=0.02$ by Faber et al. (1989). The solid line is the best fitting formation redshift for the evolution of cluster galaxies with masses $M>2 \times 10^{11} M_{\odot}$. The scatter in the $M / L$ ratios is large but there is a clear trend for field S0 galaxies to evolve faster. Field ellipticals appear to form two separate groups with respect to $M / L$ ratios.

by Faber et al. (1989), shown as the small dot in Figs. 7.14 and 7.15. The field FDF and WHDF sample was splitted according to the characteristic mass at $M=2 \times 10^{11} M_{\odot}$, which gives 13 lower-mass and 8 higher-mass galaxies. The average evolution of the higher-mass field galaxies is $\left\langle\Delta \ln \left(M / L_{B}\right)\right\rangle=-0.31 \pm 0.06$ at a redshift of $\langle z\rangle=0.44$. Lower-mass galaxies indicate on average a stronger evolution of $\left\langle\Delta \ln \left(M / L_{B}\right)\right\rangle=-0.54 \pm 0.07$ at $\langle z\rangle=0.38$. The slight deviation in the redshift is solely due to the not uniform number of galaxies in each sub-sample. Massive field galaxies are in compliance with a slow evolution as derived 
for the massive cluster galaxies and a high formation redshift for the bulk of the stars of $z_{\text {form }} \approx 3$. In contrast, less - massive field galaxies in the FDF and WHDF with $M<2 \times 10^{11} M_{\odot}$ evolve faster and follow an evolutionary track between $1 \lesssim z_{\text {form }} \lesssim 2$ which is similar to the less-massive cluster galaxies of van Dokkum \& Stanford (2003), which is indicated as the open square in Fig. 7.14. This result gives evidence that already at a look-back time of $\sim 5$ Gyrs differences in the measured $M / L$ evolution for the FDF and WHDF field galaxies can be revealed.

To translate the evolution in the $M / L$ ratios into a specific formation redshift $z_{\text {form }}$ for the field galaxies, the evolution of the $M / L$ ratios for the individual field early-type galaxies are investigated. Fig. 7.15 illustrates the the offset from the local FP in the rest-frame $B$-band for elliptical and S0 galaxies in the FDF and WHDF. The comparison sample of 27 field early-type galaxies at high redshift $0.6<z<1.15$ by van der Wel (2005) is also shown. Again, model tracks for a single-age stellar population with formation redshift 1 (lower line) and 2 (upper line) are displayed as dotted lines. All model tracks were constrained to cross on their way to zero redshift the average $M / L$ ratio of the field galaxies at $z=0.02$ by Faber et al. (1989), indicated as the small black dot. The evolution of cluster galaxies with masses $M>2 \times 10^{11} M_{\odot}$ can be best approximated assuming a formation redshift of $z_{\text {form }} \approx 3$, which is denoted as the solid line in Fig. 7.15. Although the scatter in the offsets from the local FP is large, a clear trend for a faster evolution of field S0 galaxies is found. On the other hand, field elliptical galaxies appear to form two separate groups with respect to $M / L$ ratios. To assess the formation redshift $z_{\text {form }}$, galaxies with $\Delta \ln \left(M / L_{B}\right)>0.2$ were neglected and the two field galaxy samples combined. An unrestricted linear $\chi^{2}$-fit to 31 low-mass early-type field galaxies with $M<2 \times 10^{11} M_{\odot}$ of this study and by van der Wel (2005) gives

$\Delta \ln \left(M / L_{B}\right)=-(1.69 \pm 0.26) z-(0.08 \pm 0.22)$.

This would correspond to a formation redshift of $z_{\text {form }}=1.7 \pm 0.9$ with an observed scatter in the $\Delta \ln \left(M / L_{B}\right)-z$ relation of of $\sigma\left(z_{\text {form }}\right)=1.4$. For the higher-mass sample of eleven galaxies with $M>2 \times 10^{11} M_{\odot}$ a linear $\chi^{2}$-fit yields

$$
\Delta \ln \left(M / L_{B}\right)=-(1.46 \pm 0.59) z-(0.03 \pm 0.51) .
$$

For the massive early-type field galaxies, the evolution of the $M / L$ predicts a mean star formation epoch of $z_{\text {form }}=3.2 \pm 1.0$. The $1 \sigma$ observed scatter of the $\Delta \ln \left(M / L_{B}\right)-z$ relation for the massive field galaxies is $\sigma\left(z_{\text {form }}\right)=1.9$. In comparison to the lower-mass counterparts, a higher formation redshift is significant on the $2 \sigma$ level based on the uncertainty of the highmass galaxies. The lack of field galaxies with masses of $M>2 \times 10^{11} M_{\odot}$ introduces a larger uncertainty on the $z_{\text {form }}$ value. By comparing the derived formation epoch of the high-mass cluster galaxies with numbers for cluster galaxies in the literature a good agreement is found. Based on a compilation of clusters out to redshift of $z=1.27$, van Dokkum \& Stanford (2003) deduce $z_{\text {form }}=2.6_{-0.4}^{+0.9}$. This study gives an upper limit for the formation redshift of massive cluster galaxies of $z_{\text {form }}=3.5(1 \sigma)$. The result obtained for the massive field galaxies here is within this upper limit, which suggests that these field galaxies have old passively evolving stellar populations similar to their massive counterparts in clusters. Besides the scatter of the individual galaxies, lower-mass field galaxies have a stronger evolution in their $M / L$ ratio. The mean stellar populations ages of the less-massive field galaxies are younger, with lower formation redshift of $1<z_{\text {form }} \leq 2$. This suggests a mass-dependent luminosity evolution with a stronger evolution for lower-mass galaxies with $M<2 \times 10^{11} M_{\odot}$. 


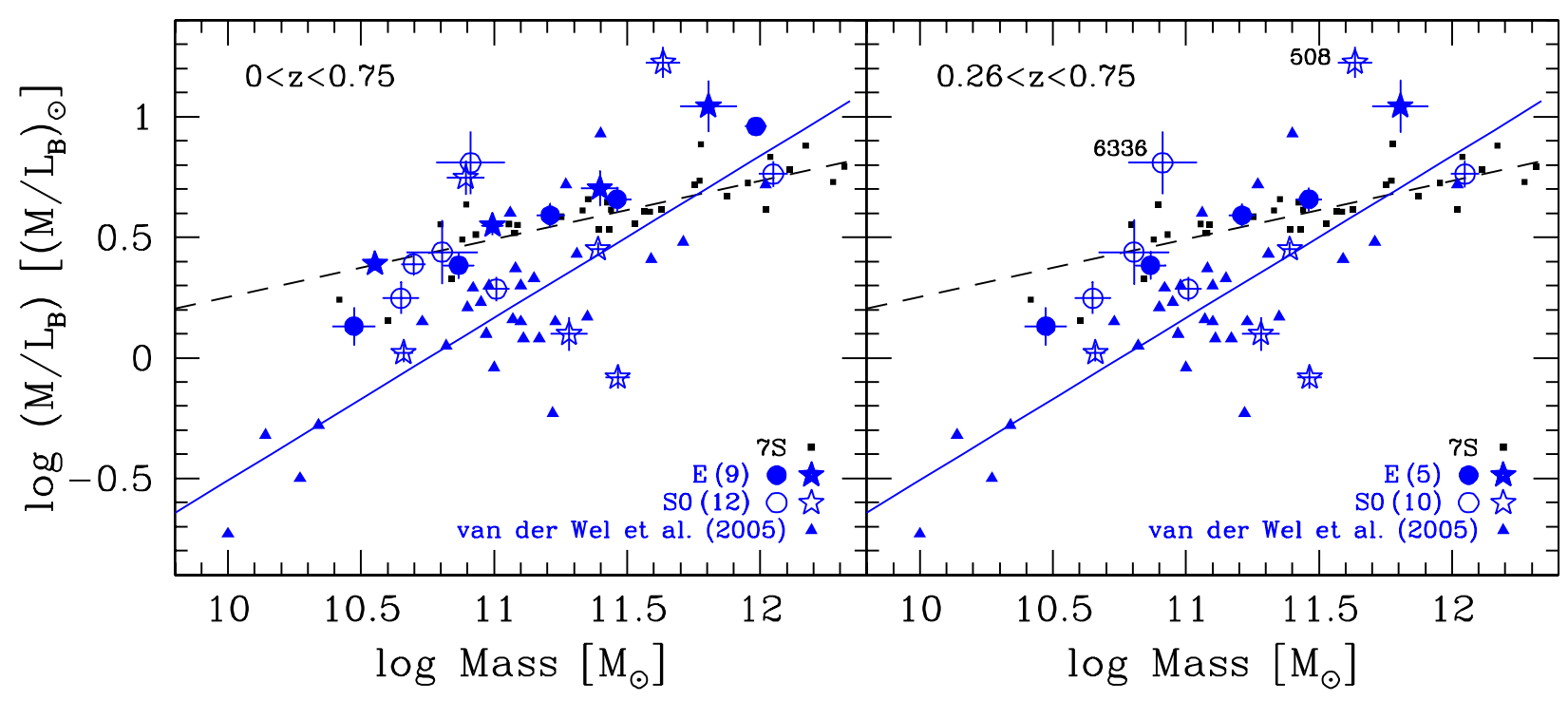

Figure 7.16: $M / L_{B}$ ratio as a function of mass $M$ in solar units as derived from the FP for the earlytype field galaxies in the FDF and WHDF, divided into two different redshift bins. Filled symbols denote ellipticals, open symbols S0 galaxies and Sa bulges. In brackets the respective number of a morphological class is listed. Errors for the distant sample are shown in both panels. The nearby 7S Coma cluster sample is indicated with small dots (dashed line is a $\chi^{2}$-fit to Coma) and the high-redshift field E+S0 galaxies at $z \sim 1$ by van der Wel et al. (2005) are shown as triangles (solid line is a $\chi^{2}$-fit). All data points have been corrected for maximum progenitor bias $\Delta \ln \left(M / L_{B}\right) \approx 0.2 z$. Left: $M / L_{B}-M$ for the whole sample of 21 field $\mathrm{E}+\mathrm{S} 0$ galaxies $0<z<0.75$. Right: $M / L_{B}-M$ for 15 higher-redshift field $\mathrm{E}+\mathrm{S} 0$ s with $0.26<z<0.75$. Ellipticals follow the local relation whereas the $\mathrm{S} 0$ galaxies have on average lower $M / L$ ratios, a larger scatter and match the intermediate-mass range of the $M / L_{B}-M$ relation of the $z \sim 1$ field E+S0 galaxies.

To test this mass-dependent evolution even further, in Fig. 7.16 the $M / L_{B}$ ratio of the earlytype field galaxies is shown as a function of mass $M$. The galaxies were divided according to their redshift into two different bins. The left panel shows the $M / L_{B}-M$ for all field galaxies, whereas in the right panel the field objects are restricted to a sub-sample of 15 high-redshift galaxies. As a comparison, the nearby $7 \mathrm{~S}$ Coma cluster sample (small dots) and the high-redshift field $\mathrm{E}+\mathrm{S} 0$ galaxies at $z \sim 1$ of van der Wel et al. (2005) (triangles) are indicated together with linear $\chi^{2}$-fits to both samples. All data points have been corrected for maximum progenitor bias $\Delta \ln \left(M / L_{B}\right) \approx 0.2 z$. Ellipticals, in particular below $z \lesssim 0.26$, follow the local relation whereas the $\mathrm{S} 0$ galaxies have on average lower $M / L$ ratios and match the intermediate- mass range of the $z \sim 1$ field $\mathrm{E}+\mathrm{S} 0$ galaxies. Lenticular galaxies show a larger scatter and the two outliers in the FP (Sa bulges \# 6336 and \# 508) are clearly offset from the rest of the sample. The difference between field elliptical and S0 galaxies found in the FP can be explained by a difference in their formation epochs. Ellipticals, especially massive ones, were generated at earlier times and follow rather moderate quiescent evolutionary tracks. On the contrary, lenticular and Sa bulges show more diverse stellar populations which are younger and have been formed at recent times with formation redshifts $1<z_{\text {form }} \leq 2$. 


\subsection{Further Discussion Conclusions}

The analysis of the distant scaling relations of the FJR, KR and FP for distant early-type galaxies at $z \sim 0.2$ is in several ways consistent with previous studies. Early-type galaxies in all environments obey tight relationships similar to the local Universe. Assuming the local slope holds valid with increasing redshift, on average a modest evolution in the Gunn $r$ and Johnson $B$-band luminosities of the galaxies is derived. There is no significant increase of scatter for the distant FJR. However, elliptical galaxies in poor clusters display a slightly larger scatter in the FP. In contrast to the local counterparts, the distant FJR have a shallower slope at the $2 \sigma$ significance level, which implies a massdependent luminosity evolution. Lower-mass galaxies in all cluster environments are on average brighter by $\sim 0.6$ mag in rest-frame Gunn $r$-band at $z \approx 0.2$ than locally, whereas the distant early-types with masses of $M \approx 3 \times 10^{11} M_{\odot}$ display only a mild evolution and have similar absolute magnitudes as their present-day counterparts.

The sub-classes of early-type galaxies, elliptical and S0 galaxies, appear not to represent a homogenous group but follow different evolutionary tracks. Lenticular galaxies in high-mass clusters evolve faster than ellipticals, whereas elliptical galaxies in low-mass clusters evolve as fast as S0 galaxies in rich clusters.

Assuming a typical galaxy in our sample with a size of $R_{\mathrm{e}}=0.34 \mathrm{kpc}$ (which corresponds to $0.6^{\prime \prime}$ ) and a measured velocity dispersion of $\sigma=200 \mathrm{~km} \mathrm{~s}^{-1}$ gives a galaxy mass of $M \approx 1.0 \times 10^{11} M_{\odot}$. This represents a typical mass of an early-type galaxy within the mass range covered by the cluster samples at $z \approx 0.2$. For galaxy masses lower than this limit, the $M / L$ evolution is faster than for galaxies with masses of $M>2 \times 10^{11} M_{\odot}$. This trend is clearly visible for all environments, from the highest mass densities in rich clusters over lower-mass densities in poor clusters down to the field environment. For the less-massive field galaxies a faster evolution of the $M / L$ ratios of $\Delta \ln \left(M / L_{B}\right)=-(1.69 \pm 0.26) z$ is found, whereas the more-massive ones evolve slower as $\Delta \ln \left(M / L_{B}\right)=-(1.46 \pm 0.59) z$. This evolution in $M / L$ ratios can be translated in to a formation redshift for the lower-mass galaxies $\left(M<2 \times 10^{11} M_{\odot}\right)$ of $z_{\text {form }}=1.7 \pm 0.9$, whereas for massive galaxies $\left(M>2 \times 10^{11} M_{\odot}\right)$ $z_{\text {form }}=3.2 \pm 1.0$ is derived. The FDF and WHDF field early-type galaxies are consistent with the mass dependence of the $M / L$ ratios as found in a recent study of E and S0 field galaxies up to redshifts of $z=1$ (van der Wel 2005). These authors derive from a comparison with low-redshift data for the whole field galaxy sample $\Delta \ln \left(M / L_{B}\right)=-(1.75 \pm 0.16) z$ whereas galaxies with masses $M>2 \times 10^{11} M_{\odot}$ show a $M / L$ evolution of $\Delta \ln \left(M / L_{B}\right)=-(1.20 \pm 0.17) z$.

These results suggests that the evolution correlates with the galaxy mass rather than the luminosity of the galaxies. The lack of age difference found for field and cluster early-type galaxies and the dependence of the evolution on galaxy mass suggests that environmental effects play a rather minor role in the formation of early-type galaxies. More important to the evolutionary history of a galaxy are its internal properties, such as mass, size, velocity dispersion or chemical composition.

The evolution of the $M / L$ ratio in all environments favours a down-sizing formation scenario (Kodama et al. 2004). In this picture, massive galaxies are predominantly dominated by red old stellar populations which evolve on longer timescales and passively. Less-massive systems, however, exhibit more extended star formation histories. In the down-sizing approach, the mass of galaxies hosting star formation processes decreases with increasing age of the Universe. Both, the mass assembly on the one hand and the star formation on the other hand are 
accelerated in massive stellar systems located in the high density environments. In contrast, in less-massive (smaller) systems, these physical processes work on longer timescales. Support for this formation picture is endorsed by several other observational studies, such as the lack of star formation in massive galaxies at lower redshifts $z \leq 1$ (De Lucia et al. 2004), a fossil record of star formation in early-type galaxies detected in the local Universe (Thomas et al. 2005) or the evidence for a mass-dependent evolution in the Tully-Fisher scaling relation of spiral galaxies up to $z=1$ (Ziegler et al. 2002; Böhm et al. 2004).

It is difficult to anticipate on specific improvements of our knowledge of galaxy formation and evolution for the upcoming future. More observational evidence is necessary to strengthen the results of this thesis and other studies. With the continuous rapid increase of computational power, a detailed numerical modelling on higherspatial resolution scales will be possible to put tighter constraints on the involved physical processes as well as the stellar populations properties and chemical compositions of galaxies.

In the near future, the project described in this thesis is going to be continued and the size of the sample will be enlarged by additional high quality spectra of a further $\sim 50$ distant early-type galaxies. Thanks to the intermediate-resolution of the observed galaxy spectra, absorption linestrengths can be measured. By comparing ages, metallicities and chemical element abundance ratios to the calibrated library of local early-type galaxies of the Lick/IDS index system, deeper insights on the involved physical mechanisms and stellar population properties of early-type galaxies will be provided. A short outline of this plan will be presented in the outlook at the end of the following summary. 


\section{Chapter 8}

\section{Summary and Outlook}

Using high signal-to-noise intermediate-resolution spectra taken with the ESO Very Large Telescope (VLT) and Calar Alto (CAHA) $3.5 \mathrm{~m}$ telescope complemented by deep ground-based and Hubble Space Telescope (HST) imaging, a study of early-type galaxies at redshifts $0.2 \leq z \leq 0.4$ has been performed. An extensive sample of 121 early-type galaxies in various densities ranging from galaxy clusters with highest richness class, rich clusters, over poor richness class, poor Low- $L_{X}$ clusters, down to the isolated field population was constructed to investigate the evolution over the last $\sim 3$ Gyrs, corresponding to $20 \%$ of the age of the Universe. The motivation of the project was to look how the physical properties of early-type galaxies depend on their environment. The evolution of galaxies in luminosity, size, mass and their stellar populations were intercompared to test possible environmental effects and to constrain the theoretical formation and evolution model predictions.

Target objects were selected based on multiband ground-based imaging data. For the earlytype galaxies in the rich cluster Abell 2390, UBI broad-band colours were used, whereas earlytype candidates for three poor clusters were selected by a combination of a spectroscopic redshift catalog and ground-based $B V R I$ photometry. These Low $-L_{X}$ clusters display $1.0 \mathrm{dex}$ lower X-ray luminosities than typical rich clusters and are, as a difference in $L_{X}$ corresponds directly to a difference in mass via the correlation
$L_{X} \propto M^{2}$, therefore considered to be low-mass galaxy clusters. Early-type field galaxies were selected over a redshift range of $0.2<z<0.75$ from the FORS Deep Field (FDF) and William Herschel Deep Field (WHDF), two multi-band imaging studies located in the southern and northern hemisphere respectively, with optical limiting magnitudes comparable to the Hubble Deep Fields and each covering a field-of-view of $\sim 7^{\prime} \times 7^{\prime}$.

Spectroscopic candidates were selected according to their elongated structureless appearance, their luminosity, photometric redshift and an additional constraint of spectrophotometric type for the field targets. Main criterion was the total apparent $I$ or $R$-band brightness with a faint limit of $I \leq 20 \mathrm{mag}$ for the cluster galaxies and $R \leq 22$ mag in case of the early-type field galaxies. To study the evolution of early-type galaxies out to large clustercentric distances of $\sim 1$ virial radii, the spectroscopic targets of the cluster environments were distributed over a wide field-ofview of $\sim 10^{\prime} \times 10^{\prime}$, which corresponds to a physical field-of-view of $\sim 1.4$ Abell radii $(\sim 2.7 \mathrm{Mpc})$ at $z=0.23$.

In total, 142 high signal-to-noise spectra of 121 different early-type galaxies and additional 28 stellar template spectra were taken between Sept. 1999 to Oct. 2002 in Multi Object Spectroscopy mode with the MOSCA spectrograph at the CAHA $3.5 \mathrm{~m}$ telescope and the FORS $1+2$ instruments mounted on the VLT. The seeing conditions ranged between 1.2 to 1.7 arcseconds 
for the CAHA observations and 0.4 to 1.0 arcseconds for the VLT Paranal observations.

Spectroscopic redshifts could be derived for 121 early-type galaxies and 9 spiral galaxies and 12 secondary objects. No galaxy spectra of the targets had too low signal-to-noise to determine a redshift.

The multi-band photometry data provided the possibility to measure accurate luminosities of the galaxies. Apparent brightnesses for the cluster galaxies were transformed to the rest-frame Gunn $r$-band. For the field galaxies in the FDF and WHDF apparent magnitudes were derived in either of the $B, g, R$ or $I$ passband which, depending on the redshift of the galaxy, best matched the rest-frame $B$-band. $k$-corrections were computed via synthetic photometry with an observed spectral template and a spectral template from chemically consistent evolutionary synthesis models. Both independent approaches yielded within their uncertainties very similar results. For the cluster galaxies the uncertainties in the $k$-corrections were $\delta k_{r} \leq 0.05 \mathrm{mag}$ and in case of the field objects $\delta k_{B} \leq 0.1$ mag over the complete redshift range.

Structural parameters for $1 / 4$ of all early-type galaxies were analysed on the HST/WFPC2 (Wide Field and Planetary Camera 2) and HST/ACS (Advanced Camera for Surveys) images using the surface brightness profile fitting algorithm developed by Saglia et al. (1997a), which searchs for the best combination of seeingconvolved, sky-corrected $r^{1 / 4}$ and exponential laws. The total HST/F702W or F814W-band magnitude, effective (half-light) radius, central surface brightness and the mean surface brightness within the effective radius, were derived for the entire galaxy as well as for its bulge and disc component separately. This approach accounts for various types of observed surface brightness profiles, such as elliptical galaxies with a flat core, discy S0 galaxies or the extended profiles of central cD galaxies. The two-dimensional surface brightness distribution of the early-type field galaxies was fitted with a combination of $r^{1 / n}$ plus exponential disc profiles using the GALFIT package. Both algorithms gave within their errors consistent results. The complete set of structural parameters was subject to a robust and careful error treatment.

Elliptical and lenticular (S0) galaxies were morphologically classified in three independent methods using the high-resolution HST/WFPC2 and HST/ACS images. A visual inspection was executed for a sub-set of elliptical and S0 galaxies. Results from the surface brightness profile fitting provided a second verification and a third quantitative analysis was conducted using the bulge-to-total $(B / T)$ fractions of the galaxies. A weak correlation of the measured bulge fraction with visual morphological Hubble type was found.

Radial velocities and velocity dispersions $(\sigma)$ of the early-type galaxies were computed from the G4300-band, $\mathrm{H} \beta$ or $\mathrm{Mg}_{b}$ absorption lines using the Fourier Correlation Quotient (FCQ) method (Bender 1990). To derive the kinematic properties of the distant galaxies several modifications on the parameter settings were implemented, such as the subtraction of the continuum level in the spectra or the instrumental resolution. To ensure a reliable error treatment for the velocity dispersions and radial velocity measurements, Monte Carlo simulations for a set of different stellar templates over a range of input velocity dispersions and $S / N$ ratios were performed. Synthetic galaxy spectra with artificial noise and for a specific instrumental setup and resolution were generated and the velocity dispersions recovered by the FCQ software.

Velocity dispersions based on high signal-tonoise $(\langle S / N\rangle \gtrsim 30$ per $\AA)$ spectra could be derived for 110 early-type galaxies regardless of their environment. For six galaxies with very low signal $(S / N \lesssim 8$ per $\AA$ ), no $\sigma$ measurement was possible. A visual inspection of the $\sigma$ values was conducted for each galaxy spectrum to assess the overall quality and reliability of 
the measurement. Extensive consistency checks were performed by comparing $\sigma$ determinations from different absorption lines, repeat observations, different extraction procedures and stellar libraries.

The total sample analysed in this study comprises 147 early-type galaxies. 48 early-type galaxies are members of the rich cluster A 2390 $(z=0.23), 27$ galaxies are associated to one of the three Low $-L_{X}$ cluster CL 0849, CL 1701 and CL $1702(\langle z\rangle=0.23)$, and 24 are field galaxies in the FDF and WHDF $(\langle z\rangle=0.4)$. In addition, 48 early-type galaxies of a previous study of the rich A 2218 cluster were combined with the A 2390 data to study a total of 96 early-type galaxies in the densest environments.

In the further analysis, the scaling relations of the Faber-Jackson (FJR) and Kormendy relation (KR) as well as the Fundamental Plane (FP) for distant early-type galaxies at $z \sim 0.2$ obey tight relationships similar to the local Universe. Assuming the local slope holds valid with increasing redshift, on average a modest evolution in the Gunn $r$ and Johnson $B$-band luminosities of the galaxies is derived, regardless of their environment locus. There is no significant increase of scatter for the distant FJR, whereas elliptical galaxies in poor clusters indicate a slightly larger scatter in the FP. In contrast to the local counterparts, the distant FJR have shallower slopes at the $2 \sigma$ significance level, which implies a mass-dependent luminosity evolution. Lower-mass galaxies in all cluster environments are on average brighter by $\sim 0.6$ mag in rest-frame Gunn $r$-band at $z \approx 0.2$ than locally, whereas the distant early-types with masses of $M \approx 3 \times 10^{11} M_{\odot}$ display only a mild evolution and have similar absolute magnitudes as their present-day counterparts. Evidence for this result is even found for the early-type field galaxies. Their mass-to-light ratios $(M / L)$ are consistent with the trend detected within a recent study of $\mathrm{E}$ and $\mathrm{S} 0$ field galaxies in the literature. For early-type cluster galaxies, a slight radial de- pendence is found. Galaxies in the outer districts of clusters at $\sim 0.25$ virial radii $(\sim 0.46 \mathrm{Mpc})$ show a stronger brightening by $\sim 0.2$ mag than their counterparts in the central cluster regions.

A difference between the morphological subclasses of early-type galaxies, elliptical and S0 galaxies, was revealed. Lenticular galaxies in rich clusters exhibit a stronger luminosity evolution compared to the local Coma FP than the ellipticals. The stellar populations of these systems could be more diverse or comprise more complex star formation histories. Support of this assumption is accumulated by an analysis of the rest-frame galaxy colours. The bluer galaxy population $((B-I)<2.287)$ shows larger FJR residuals from the local FJR, whereas the redder galaxies of the red-sequence of early-type cluster galaxies are less offset. Interestingly, the $S 0$ galaxies in both colour ranges show a stronger luminosity evolution (brighter by $\sim 0.7$ mag for the bluer and $\sim 0.45$ mag for the redder group), but the blue ellipticals have on average only a mild brightening of $\sim 0.3 \mathrm{mag}$ and the redder even a "positive" evolution, i.e. they are fainter than the local reference.

The lack of an age difference found for field and cluster early-type galaxies and the dependence of the evolution on galaxy mass suggests that environmental effects are not the dominant factors which drive the formation of early-type galaxies. Internal properties of the galaxies, such as mass, size, velocity dispersion or chemical composition, are the main contributors to the evolutionary history of elliptical and lenticular galaxies.

The evolution of the $M / L$ ratio in all environments favours a down-sizing formation scenario (Kodama et al. 2004). Massive galaxies are predominantly dominated by red old passively evolving stellar populations, whereas less massive systems have more extended star formation histories. The mass of galaxies hosting star formation processes thereby decreases with the age of the Universe. Both mass assembly and star 
formation are accelerated in massive stellar systems located in the high density environments, whereas the processes work on longer timescales in less-massive (smaller) systems.

The results of this thesis therefore strengthen the need for more realistic implementations of stellar population properties into the numerical codes of $N$-body simulations. In particular, the fundamental physics of the star formation processes and their involved timescales still are not well understood. Progress in the near future seems promising, looking at the most recent analytical approaches and their improvements. Recently a detailed simulation of the formation of individual early-type galaxies in a fully cosmological context was achieved (Meza et al. 2003). Nevertheless, crucial mechanisms such as star formation processes were still treated based on simplified assumptions.

It is hard to predict whether a more realistic modelling of the stellar content of the galaxies will lead to a consistency between theory and observations. Some modifications on shorter scales of the hierarchical scenario would probably be needed to reach a better consensus. Further research in the upcoming years is going to be very important for our understanding of the formation and evolution of early-type galaxies to achieve a complete picture of the fundamental processes of structure growth from the early universe up to the present.

The project on distant early-type galaxies described in this thesis will be continued in the next years by the author. Spectroscopy of the integrated stellar light of a galaxy is a powerful tool for deriving the age, metallicity and gravity or temperature of their stellar population. To quantify these intrinsic properties, spectral indices have been introduced which characterise the strength of a specific absorption or emission line. Thanks to the intermediate-resolution of the observed early-type galaxy spectra, absorp- tion line-strengths can be measured and compared to the Lick/IDS index system. Lightaveraged ages, metallicities and chemical element abundance ratios can be derived and directly confronted to the predictions of stellar population models. In combination with the luminosity evolution measured via the scaling relations, results will give strong constraints on the formation redshift and the involved physical mechanisms of early-type galaxies.

To extent the sample even more, observations of $\sim 50$ early-type galaxies in three Low $-L_{X}$ clusters at $0.2<z<3.0$ have been acquired using MOSCA spectra. This data will allow to enlarge the statistical significance of the results for the poor clusters presented in this thesis.

Furthermore, a joint research project together with the MPE in Munich to study the colour gradients in cluster galaxies for different environments was already initiated by the author. A detailed analysis of HST/WFPC2 and HST/ACS structural parameters in different filter passbands and with independent algorithms for modelling the galaxy surface brightness profiles will reveal how the colour gradients and the shape of galaxies depend on the computed structural parameters.

A combination of all spectroscopic data and the HST images will make up one of the largest kinematic samples of early-type galaxies at redshift $z=0.2$. Together with the analysis of the stellar populations and chemical abundances of these galaxies deeper observational insights will be achieved into how early-type galaxies, in particular S0 galaxies, form and evolve with redshift. 


\section{Appendix A}

\section{Stellar Templates}

During the observing runs to acquire intermediate-resolution MOSCA spectra for the early-type galaxies in the rich and poor clusters, a total of 28 stellar spectra of 15 different Lick/IDS index standard stars with the same instrument configuration have been observed. The stars were taken from the Lick/IDS star library list by Worthey et al. (1994). Table A.1 shows the observations for all 28 Lick/IDS index standard stars from CAHA splitted into different observing runs. In total, 12 Lick/IDS standards were observed in September 1999, 7 in July 2000, 6 in April 2001, and finally 3 in February 2002. The reduction of these stellar templates is discussed in section 3.7 on page 44 . A set of nine kinematic templates which exhibit the highest instrumental resolution were utilised for the derivation of the galaxies' velocity dispersions. To test possible systematic effects within the measurements and/or systematic offsets arising from different observing runs, a comparison between repeat observations of Lick/IDS stars acquired at CAHA was performed.

Absorption line-strengths for 15 different kinematic template stars were measured and calibrated to the Lick/IDS system following the method as described in Faber et al. (1985). In order to test whether possible offsets exist between the measurements of Lick/IDS absorption line indices for different observing runs, the results of the line-strengths for 10 stars which are included in both runs of Sept. 1999 and July 2000 are compared to each other. Fig-
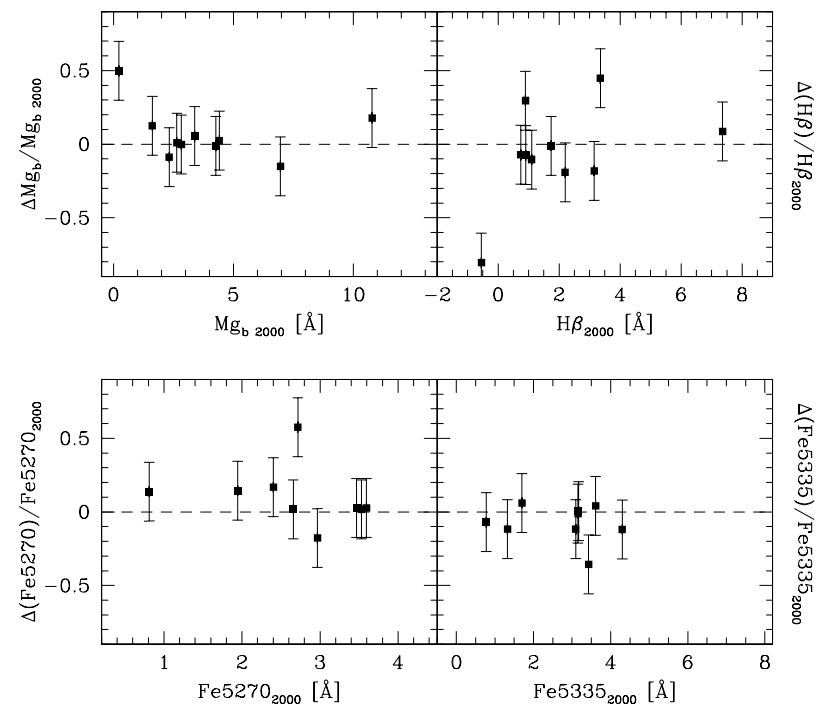

Figure A.1: Relative differences of the Lick/IDS absorption line indices of $\mathrm{Mg}_{b}, \mathrm{H} \beta, \mathrm{Fe} 5270$ and Fe5335 for 10 kinematic stellar templates with spectral types from A0V to M6III of the CAHA observing runs 1999 and 2000. See also Table A.2.

ure A.1 shows a comparison of the relative differences between the Lick/IDS line indices of $\mathrm{Mg}_{b}$, $\mathrm{H} \beta$, Fe5270 and Fe5335 for 10 stars with different spectral types (A0V to M6III). The relative difference of line-strength indices from 1999 and $2000 \Delta\left(\right.$ Index $_{1999}-$ Index $\left._{2000}\right) /$ Index $_{2000}$ are plotted as a function of the derived line-strength index of the run in 2000 Index 2000 . A typical Lick/IDS error of $0.2 \AA$ was adopted. The agreement between all templates is good, only negligible deviations are detected. The mean 
and median (in brackets) values of the relative differences are $\Delta\left(\mathrm{Mg}_{b}\right) / \mathrm{Mg}_{b 2000}=0.06 \pm 0.18$ (0.02), $\Delta(\mathrm{H} \beta) / \mathrm{H} \beta_{2000}=-0.06 \pm 0.33(-0.07)$, $\Delta(\mathrm{Fe} 5270) / \mathrm{Fe} 5270_{2000}=0.10 \pm 0.21(0.03)$ and $\Delta(\mathrm{Fe} 5335) / \mathrm{Fe} 5335_{2000}=-0.08 \pm 0.13(-0.07)$. There is no systematic offset neither a change with spectral type found. Table A.2 gives a summary of the derived relative differences for the Lick/IDS indices of Figure A.1. For the star SAO 004401 (A0V) no Fe5270 and Fe5335 indices could be measured for the 2000 run and therefore this star is not included in the comparison of the Fe-index diagrams. For purposes of comparison, the published Lick/IDS index measurements by Worthey et al. (1994) are listed in the Tables A.3, A.4 and A.5. In general, the agreement between all indices is good and only negligible deviations are found. In addition, this tests demonstrates the accuracy and reliability of the data reduction and the wavelength calibration for the stellar template spectra as no systematic errors originating from possible errors in flat-fielding, sky background subtraction or wavelength calibration were detected. 
Table A.1: Observed stellar templates at Calar Alto. The exposure times were $T_{\text {tot }}=60$ sec.

\begin{tabular}{|c|c|c|c|c|c|c|c|c|c|}
\hline \multirow[t]{2}{*}{ Name } & \multirow[t]{2}{*}{ ID } & \multirow[t]{2}{*}{ spec type } & \multirow[t]{2}{*}{ run } & \multirow{2}{*}{$\begin{array}{l}\text { slit width } \\
\text { [arcsec] }\end{array}$} & \multirow{2}{*}{$\begin{array}{r}\mathrm{v}_{\mathrm{rad}} \\
{\left[\mathrm{km} \mathrm{s}^{-1}\right]}\end{array}$} & \multicolumn{4}{|c|}{$\sigma_{\text {inst }}$ FWHM } \\
\hline & & & & & & \multicolumn{2}{|c|}{$\mathrm{H} \beta \& \mathrm{Mg}$} & \multicolumn{2}{|c|}{$\begin{array}{c}\text { G-band } \\
[\AA]\rceil\left[\mathrm{km} \mathrm{s}^{-1}\right]\end{array}$} \\
\hline SAO 004401 & 048 & $\mathrm{~A} 0 \mathrm{~V}$ & 09/99 & 1.5 & +74.5 & 5.0 & 126 & 4.6 & 139 \\
\hline SAO 005056 & 052 & $\mathrm{~K} 4 \mathrm{~V}$ & 09/99 & 1.5 & +07.2 & 5.2 & 131 & 4.6 & 139 \\
\hline SAO 038597 & 100 & G0V & 09/99 & 1.5 & +27.4 & 5.2 & 131 & 4.7 & 142 \\
\hline SAO 055946 & 050 & K7III & 09/99 & 1.5 & +41.8 & 5.1 & 129 & 4.5 & 136 \\
\hline SAO 056928 & 054 & G5 & 09/99 & 1.5 & -91.1 & - & - & - & - \\
\hline SAO 077592 & 022 & G8IIIv & 09/99 & 1.5 & +02.8 & 4.9 & 124 & 4.4 & 133 \\
\hline SAO 093189 & 098 & M6III & 09/99 & 1.5 & +67.3 & 5.2 & 131 & 4.6 & 139 \\
\hline SAO 110065 & 011 & K3IIIbBa0 & 09/99 & 1.5 & +02.3 & 5.2 & 131 & 4.5 & 136 \\
\hline SAO 112958 & 055 & K0IIIbCN-2 & 09/99 & 1.5 & +104.4 & 5.0 & 126 & 4.5 & 136 \\
\hline SAO 123140 & 116 & G8III & 09/99 & 1.5 & +43.0 & 5.3 & 134 & 4.6 & 139 \\
\hline SAO 124799 & 087 & K3IIIb & 09/99 & 1.5 & +25.1 & 5.3 & 134 & 4.7 & 142 \\
\hline SAO 130649 & 024 & F9V & 09/99 & 1.5 & +89.8 & 4.9 & 124 & 4.2 & 127 \\
\hline \multicolumn{6}{|c|}{ mean CAsep99: } & 5.1 & 129 & 4.5 & 136 \\
\hline SAO 055946 & 072 & K7III & $07 / 00$ & 1.5 & -12.1 & 5.2 & 131 & 4.5 & 136 \\
\hline SAO 093189 & 077 & M6III & $07 / 00$ & 1.5 & - & 5.0 & 126 & 4.6 & 140 \\
\hline SAO 110065 & 070 & K3IIIbBa0 & $07 / 00$ & 1.5 & -28.5 & 5.3 & 134 & 4.6 & 140 \\
\hline SAO 112958 & 118 & K0IIIbCN-2 & $07 / 00$ & 1.5 & +62.3 & 5.1 & 129 & 4.7 & 143 \\
\hline SAO 123140 & 102 & G8III & $07 / 00$ & 1.5 & -85.6 & 5.2 & 131 & 4.4 & 133 \\
\hline SAO 124799 & 141 & K3IIIb & $07 / 00$ & 1.5 & -28.8 & 5.3 & 134 & 4.7 & 143 \\
\hline SAO 130649 & 164 & F9V & $07 / 00$ & 1.5 & +84.3 & 5.3 & 134 & 4.7 & 143 \\
\hline \multicolumn{6}{|c|}{ mean CAjul00: } & 5.2 & 131 & 4.6 & 140 \\
\hline SAO 032042 & 024 & K3III & 04/01 & 1.0 & -06.2 & 3.5 & 88 & 3.0 & 91 \\
\hline SAO 032042 & 019 & K3III & $04 / 01$ & 1.0 & -06.5 & 3.6 & 91 & 3.1 & 94 \\
\hline SAO 098087 & 006 & K0III & $04 / 01$ & 1.0 & +63.7 & 3.4 & 86 & 3.2 & 97 \\
\hline SAO 123140 & 032 & G8III & $04 / 01$ & 1.0 & +07.5 & 3.5 & 88 & 3.3 & 100 \\
\hline SAO 123140 & 020 & G8III & 04/01 & 1.0 & - & 3.4 & 86 & 3.2 & 97 \\
\hline SAO 124799 & 022 & K3IIIb & $04 / 01$ & 1.0 & -22.6 & 3.5 & 88 & 3.1 & 94 \\
\hline \multicolumn{6}{|c|}{ mean CAapr01: } & 3.5 & 88 & 3.2 & 97 \\
\hline SAO 032042 & 116 & K3III & $02 / 02$ & 0.5 & +32.4 & 2.2 & 55 & 2.3 & 70 \\
\hline SAO 080333 & 084 & K0III & 02/02 & 0.5 & +35.3 & 2.2 & 55 & 2.5 & 76 \\
\hline SAO 098087 & 157 & K0III & $02 / 02$ & 0.5 & -24.1 & 2.5 & 64 & 2.2 & 67 \\
\hline \multicolumn{6}{|c|}{ mean CAfeb02: } & 2.3 & 58 & 2.3 & 71 \\
\hline
\end{tabular}


Table A.2: Relative differences of Lick/IDS indices for the stellar templates of the observations in 1999 and 2000 as show in Fig. A.1.

\begin{tabular}{lllrrrrrrrr}
\hline Name & ID & spec type & $\begin{array}{r}\mathrm{Mg}_{b} \\
{[\AA]}\end{array}$ & $\begin{array}{r}\Delta \sigma\left(\mathrm{Mg}_{b}\right) \\
{[\AA]}\end{array}$ & $\begin{array}{r}\mathrm{H} \beta \\
{[\AA]}\end{array}$ & $\begin{array}{r}\Delta \sigma(\mathrm{H} \beta) \\
{[\AA]}\end{array}$ & $\begin{array}{r}\mathrm{Fe} 5270 \\
{[\AA]}\end{array}$ & $\begin{array}{r}\Delta \sigma(\mathrm{Fe} 5270) \\
{[\AA]}\end{array}$ & $\begin{array}{r}\mathrm{Fe} 5335 \\
{[\AA]}\end{array}$ & $\begin{array}{r}\Delta \sigma(\mathrm{Fe} 5335) \\
{[\AA]}\end{array}$ \\
\hline SAO 004401 & 048 & A0V & 0.23 & 0.50 & 7.36 & 0.09 & - & - & - & - \\
SAO 005056 & 052 & K4V & 6.97 & -0.15 & -0.55 & -0.81 & 2.72 & 0.58 & 4.30 & -0.12 \\
SAO 055946 & 050 & K7III & 4.27 & -0.01 & 0.74 & -0.07 & 3.59 & 0.03 & 3.61 & 0.04 \\
SAO 077592 & 022 & G8IIIV & 2.65 & 0.01 & 2.20 & -0.19 & 2.97 & -0.18 & 3.10 & -0.12 \\
SAO 093189 & 098 & M6III & 10.79 & 0.18 & 3.35 & 0.45 & 2.66 & 0.02 & 1.33 & -0.12 \\
SAO 110065 & 011 & K3IIIbBa0 & 3.39 & 0.05 & 0.91 & -0.07 & 3.47 & 0.03 & 3.17 & 0.00 \\
SAO 112958 & 055 & K0IIIbCN-2 & 2.83 & -0.00 & 0.90 & 0.30 & 1.95 & 0.14 & 1.70 & 0.06 \\
SAO 123140 & 116 & G8III & 2.33 & -0.09 & 1.74 & -0.01 & 2.40 & 0.17 & 3.43 & -0.36 \\
SAO 124799 & 087 & K3IIIb & 4.42 & 0.02 & 1.10 & -0.10 & 3.53 & 0.02 & 3.15 & -0.01 \\
SAO 130649 & 024 & F9V & 1.62 & 0.13 & 3.15 & -0.18 & 0.81 & 0.14 & 0.78 & -0.07 \\
\hline
\end{tabular}

Table A.3: Lick/IDS standards stars for the indices $\mathrm{CN}_{1}, \mathrm{CN}_{2}$, Ca4227, G4300, Fe4383, Ca4455 and Fe4531 (Worthey et al. 1994).

\begin{tabular}{|c|c|c|c|c|c|c|c|c|c|c|c|}
\hline $\mathrm{SAO}$ & R.A. & DEC. & $\begin{array}{c}\mathrm{V} \\
{[\mathrm{mag}]}\end{array}$ & Spec Type & $\begin{array}{r}\mathrm{CN}_{1} \\
{[\mathrm{mag}]}\end{array}$ & $\begin{array}{r}\mathrm{CN}_{2} \\
{[\mathrm{mag}]}\end{array}$ & $\begin{array}{r}\mathrm{Ca} 4227 \\
{[\AA]}\end{array}$ & $\begin{array}{r}\mathrm{G} 4300 \\
[\AA]]\end{array}$ & $\begin{array}{r}\mathrm{Fe} 4383 \\
{[\AA]}\end{array}$ & $\begin{array}{r}\mathrm{Ca} 4455 \\
{[\AA]}\end{array}$ & $\begin{array}{r}\mathrm{Fe} 4531 \\
{[\AA]}\end{array}$ \\
\hline 110065 & 013849.559 & +051407.13 & 4.44 & K3 IIIbBa01 & 0.208 & 0.260 & 2.660 & 6.590 & 8.060 & 2.530 & 4.590 \\
\hline 055946 & 024825.535 & +345119.20 & 4.53 & K7 III & 0.114 & 0.192 & 4.380 & 6.020 & 8.815 & 3.140 & 5.885 \\
\hline 005056 & 035448.885 & +760133.96 & 9.99 & $\mathrm{~K} 4 \mathrm{~V}$ & -0.056 & -0.008 & 4.595 & 5.280 & 8.775 & 3.115 & 5.190 \\
\hline 093189 & 025259.608 & +180748.82 & 5.91 & M6 III & -0.134 & -0.108 & 6.030 & 3.590 & -0.105 & 4.175 & 4.065 \\
\hline 004401 & 012203.732 & +704313.05 & 6.49 & A0 Vnn & -0.231 & -0.142 & -0.090 & -2.583 & -0.998 & -0.201 & 0.337 \\
\hline 123140 & 180454.790 & +084333.79 & 4.64 & G8 III & 0.170 & 0.197 & 0.510 & 6.430 & 5.470 & 1.690 & 3.530 \\
\hline 112958 & 053409.415 & +091554.81 & 4.09 & K0 IIIbCN2 & 0.027 & 0.037 & 0.720 & 6.690 & 4.770 & 1.455 & 3.150 \\
\hline 077592 & 054556.744 & +243309.24 & 4.86 & G8 IIIv & 0.204 & 0.243 & 0.840 & 5.760 & 6.510 & 1.920 & 3.750 \\
\hline 124799 & 193138.752 & +071616.89 & 4.45 & K3 IIIb & 0.244 & 0.302 & 1.890 & 6.550 & 8.010 & 2.300 & 4.260 \\
\hline 130649 & 033749.156 & -032229.10 & 6.68 & F9 V & -0.069 & -0.044 & 0.477 & 3.270 & 1.827 & 0.417 & 1.387 \\
\hline 056928 & 035953.188 & +350917.28 & 8.50 & G5 ZZZ & -0.046 & -0.004 & 1.830 & 5.570 & 4.260 & 0.750 & 1.810 \\
\hline 038597 & 030526.709 & +492526.63 & 4.05 & G0 V & -0.060 & -0.042 & 0.625 & 4.265 & 2.950 & 1.050 & 2.400 \\
\hline 110065 & 013849.559 & +051407.13 & 4.44 & K3 IIIbBa01 & 0.208 & 0.260 & 2.660 & 6.590 & 8.060 & 2.530 & 4.590 \\
\hline 055946 & 024825.535 & +345119.20 & 4.53 & K7 III & 0.114 & 0.192 & 4.380 & 6.020 & 8.815 & 3.140 & 5.885 \\
\hline 004401 & 012203.732 & +704313.05 & 6.49 & Ao Vnn & -0.231 & -0.142 & -0.090 & -2.583 & -0.998 & -0.201 & 0.337 \\
\hline 112958 & 053409.415 & +091554.81 & 4.09 & K0 IIIbCN2 & 0.027 & 0.037 & 0.720 & 6.690 & 4.770 & 1.455 & 3.150 \\
\hline 077592 & 054556.744 & +243309.24 & 4.86 & G8 IIIv & 0.204 & 0.243 & 0.840 & 5.760 & 6.510 & 1.920 & 3.750 \\
\hline 124799 & 193138.752 & +071616.89 & 4.45 & K3 IIIb & 0.244 & 0.302 & 1.890 & 6.550 & 8.010 & 2.300 & 4.260 \\
\hline 130649 & 033749.156 & -032229.10 & 6.68 & F9 V & -0.069 & -0.044 & 0.477 & 3.270 & 1.827 & 0.417 & 1.387 \\
\hline 080333 & 083713.995 & +201108.03 & 6.39 & K0 III & 0.256 & 0.283 & 0.645 & 6.700 & 6.112 & 1.772 & 2.840 \\
\hline 098087 & 084150.747 & +182021.83 & 3.94 & K0 III-IIIb & 0.209 & 0.230 & 1.046 & 7.020 & 6.806 & 1.948 & 3.218 \\
\hline 032042 & 194922.412 & +525137.60 & 5.03 & K3 III-CN1 & 0.387 & 0.456 & 2.300 & 6.700 & 9.110 & 2.640 & 5.090 \\
\hline
\end{tabular}


Table A.4: Same as A.3, but for the Lick/IDS indices Fe4668, H $\beta$, Fe5015, $\mathrm{Mg}_{1}, \mathrm{Mg}_{2}, \mathrm{Mg}_{b}$, Fe5270, Fe5335, Fe5406, Fe5709, Fe5782 and Na5895 (Worthey et al. 1994).

\begin{tabular}{|c|c|c|c|c|c|c|c|c|c|c|c|c|}
\hline $\mathrm{SAO}$ & $\begin{array}{r}\mathrm{Fe} 4668 \\
{[\AA]}\end{array}$ & $\begin{array}{l}\mathrm{H} \beta \\
{[\AA]}\end{array}$ & $\begin{array}{r}\mathrm{Fe} 5015 \\
{[\AA]}\end{array}$ & $\begin{array}{r}\mathrm{Mg}_{1} \\
{[\mathrm{mag}]}\end{array}$ & $\begin{array}{r}\mathrm{Mg}_{2} \\
{[\mathrm{mag}]}\end{array}$ & $\begin{array}{r}\mathrm{Mg}_{b} \\
{[\AA]}\end{array}$ & $\begin{array}{r}\mathrm{Fe} 5270 \\
{[\AA]}\end{array}$ & $\begin{array}{r}\mathrm{Fe} 5335 \\
{[\AA]}\end{array}$ & $\begin{array}{r}\mathrm{Fe} 5406 \\
{[\AA]}\end{array}$ & $\begin{array}{r}\mathrm{Fe} 5709 \\
{[\AA]}\end{array}$ & $\begin{array}{r}\mathrm{Fe} 5782 \\
{[\AA]}\end{array}$ & $\begin{array}{r}\mathrm{Na} 5895 \\
{[\AA]}\end{array}$ \\
\hline 110065 & 5.880 & 0.870 & 6.510 & 0.172 & 0.315 & 3.730 & 3.880 & 3.530 & 2.590 & 1.450 & 1.310 & 3.820 \\
\hline 055946 & 7.655 & 0.600 & 7.205 & 0.237 & 0.436 & 4.050 & 4.140 & 4.110 & 3.350 & 1.290 & 1.370 & 4.290 \\
\hline 005056 & 2.145 & -0.009 & 5.365 & 0.296 & 0.504 & 6.440 & 4.770 & 4.260 & 2.565 & 0.830 & 0.975 & 7.160 \\
\hline 093189 & 24.705 & 5.375 & 28.775 & -0.108 & 0.408 & 15.235 & 4.740 & 1.630 & 0.680 & -0.750 & -1.360 & 4.970 \\
\hline 004401 & -0.786 & 8.118 & 0.179 & -0.008 & 0.008 & 0.258 & 0.190 & 0.181 & 0.123 & -0.016 & -0.031 & 0.816 \\
\hline 123140 & 5.820 & 1.810 & 6.260 & 0.037 & 0.139 & 2.310 & 3.030 & 2.410 & 1.620 & 1.250 & 0.760 & 2.060 \\
\hline 112958 & 3.505 & 1.000 & 4.140 & 0.072 & 0.174 & 2.810 & 2.300 & 1.740 & 1.135 & 0.955 & 0.470 & 1.430 \\
\hline 077592 & 7.500 & 1.860 & 6.520 & 0.089 & 0.216 & 3.030 & 3.380 & 2.920 & 1.990 & 1.280 & 1.030 & 2.820 \\
\hline 124799 & 8.720 & 1.020 & 6.440 & 0.184 & 0.346 & 4.800 & 3.810 & 3.500 & 2.480 & 1.400 & 1.180 & 3.610 \\
\hline 130649 & -0.133 & 2.100 & 2.717 & 0.002 & 0.082 & 1.663 & 1.128 & 0.988 & 0.353 & 0.207 & 0.143 & 1.118 \\
\hline 056928 & -0.245 & 0.550 & 1.900 & 0.080 & 0.247 & 4.625 & 2.200 & 1.685 & 0.895 & 0.325 & -0.155 & 2.255 \\
\hline 038597 & 2.650 & 3.125 & 4.545 & 0.017 & 0.115 & 2.165 & 1.605 & 1.345 & 0.850 & 0.480 & 0.220 & 1.370 \\
\hline 110065 & 5.880 & 0.870 & 6.510 & 0.172 & 0.315 & 3.730 & 3.880 & 3.530 & 2.590 & 1.450 & 1.310 & 3.820 \\
\hline 055946 & 7.655 & 0.600 & 7.205 & 0.237 & 0.436 & 4.050 & 4.140 & 4.110 & 3.350 & 1.290 & 1.370 & 4.290 \\
\hline 004401 & -0.786 & 8.118 & 0.179 & -0.008 & 0.008 & 0.258 & 0.190 & 0.181 & 0.123 & -0.016 & -0.031 & 0.816 \\
\hline 112958 & 3.505 & 1.000 & 4.140 & 0.072 & 0.174 & 2.810 & 2.300 & 1.740 & 1.135 & 0.955 & 0.470 & 1.430 \\
\hline 077592 & 7.500 & 1.860 & 6.520 & 0.089 & 0.216 & 3.030 & 3.380 & 2.920 & 1.990 & 1.280 & 1.030 & 2.820 \\
\hline 124799 & 8.720 & 1.020 & 6.440 & 0.184 & 0.346 & 4.800 & 3.810 & 3.500 & 2.480 & 1.400 & 1.180 & 3.610 \\
\hline 130649 & -0.133 & 2.100 & 2.717 & 0.002 & 0.082 & 1.663 & 1.128 & 0.988 & 0.353 & 0.207 & 0.143 & 1.118 \\
\hline 080333 & 6.584 & 1.770 & 6.650 & 0.041 & 0.168 & 2.550 & 3.190 & 2.730 & 1.962 & 1.014 & 1.138 & 2.620 \\
\hline 098087 & 7.220 & 1.330 & 5.986 & 0.088 & 0.211 & 3.160 & 3.380 & 2.610 & 1.834 & 1.148 & 1.008 & 2.180 \\
\hline 032042 & 11.520 & 1.170 & 7.860 & 0.215 & 0.382 & 4.620 & 4.350 & 4.100 & 2.880 & 1.540 & 1.560 & 5.500 \\
\hline
\end{tabular}

Table A.5: Same as A.3, but for the Lick/IDS indices TiO1, TiO2, $\mathrm{H} \delta_{A}, \mathrm{H} \gamma_{A}, \mathrm{H} \delta_{F}$ and $\mathrm{H} \gamma_{F}$. Values for effective temperature $\mathrm{T}_{\text {eff }}$, gravity $\log (\mathrm{g})$, the metallicity $[\mathrm{Fe} / \mathrm{H}]$ and a note if the star is used as a Lick Standard (LS) are also displayed (Worthey et al. 1994).

\begin{tabular}{|c|c|c|c|c|c|c|c|c|c|c|}
\hline $\mathrm{SAO}$ & $\begin{array}{l}\mathrm{TiO} 1 \\
{[\mathrm{mag}]}\end{array}$ & $\begin{array}{l}\mathrm{TiO} 2 \\
{[\mathrm{mag}]}\end{array}$ & $\begin{array}{r}\mathrm{H} \delta_{A} \\
{[\AA]}\end{array}$ & $\begin{array}{r}\mathrm{H} \gamma_{A} \\
{[\AA]}\end{array}$ & $\begin{array}{r}\mathrm{H} \delta_{F} \\
{[\AA]}\end{array}$ & $\begin{array}{r}\mathrm{H} \gamma_{F} \\
{[\AA]}\end{array}$ & $\begin{array}{c}\mathrm{T}_{\text {eff }} \\
\mathrm{K}\end{array}$ & $\begin{array}{c}\log (\mathrm{g}) \\
{\left[\mathrm{cm} / \mathrm{s}^{2}\right]}\end{array}$ & {$[\mathrm{Fe} / \mathrm{H}]$} & com \\
\hline 110065 & 0.027 & 0.055 & -6.153 & -10.051 & -1.558 & -2.930 & 4133 & 1.20 & -0.11 & LS \\
\hline 055946 & 0.104 & 0.250 & -5.844 & -10.124 & -1.325 & -2.605 & 3905 & 1.10 & -0.18 & \\
\hline 005056 & 0.014 & 0.029 & -5.090 & -11.303 & -0.775 & -3.700 & 4357 & 4.61 & 9.99 & \\
\hline 093189 & 0.480 & 0.883 & -1.677 & -5.633 & -1.300 & -1.860 & 3250 & 0.30 & 9.99 & \\
\hline 004401 & 0.010 & -0.003 & 10.678 & 10.866 & 7.790 & 8.130 & 8455 & 4.10 & 9.99 & \\
\hline 123140 & 0.013 & 0.011 & -4.688 & -7.274 & -1.040 & -1.893 & 4957 & 2.00 & -0.05 & LS \\
\hline 112958 & 0.020 & 0.005 & -3.059 & -6.807 & -0.490 & -2.190 & 4751 & 2.90 & -0.55 & \\
\hline 077592 & 0.020 & 0.016 & -4.096 & -7.298 & -0.518 & -1.883 & 4751 & 2.20 & 0.03 & LS \\
\hline 124799 & 0.018 & 0.032 & -7.114 & -9.705 & -1.568 & -3.290 & 4428 & 2.45 & 0.22 & LS \\
\hline 130649 & 0.005 & 0.002 & 1.642 & -0.629 & 1.630 & 0.980 & 5780 & 4.27 & -0.85 & \\
\hline 056928 & 0.012 & -0.009 & -2.168 & -6.597 & 0.455 & -2.460 & 4862 & 4.77 & -1.61 & \\
\hline 038597 & 0.015 & -0.002 & 0.760 & -1.872 & 1.540 & 0.750 & 5984 & 4.10 & 0.09 & \\
\hline 110065 & 0.027 & 0.055 & -6.153 & -10.051 & -1.558 & -2.930 & 4133 & 1.20 & -0.11 & LS \\
\hline 055946 & 0.104 & 0.250 & -5.844 & -10.124 & -1.325 & -2.605 & 3905 & 1.10 & -0.18 & \\
\hline 004401 & 0.010 & -0.003 & 10.678 & 10.866 & 7.790 & 8.130 & 8455 & 4.10 & 9.99 & \\
\hline 112958 & 0.020 & 0.005 & -3.059 & -6.807 & -0.490 & -2.190 & 4751 & 2.90 & -0.55 & \\
\hline 077592 & 0.020 & 0.016 & -4.096 & -7.298 & -0.518 & -1.883 & 4751 & 2.20 & 0.03 & LS \\
\hline 124799 & 0.018 & 0.032 & -7.114 & -9.705 & -1.568 & -3.290 & 4428 & 2.45 & 0.22 & LS \\
\hline 130649 & 0.005 & 0.002 & 1.642 & -0.629 & 1.630 & 0.980 & 5780 & 4.27 & -0.85 & \\
\hline 080333 & 0.009 & 0.007 & -4.672 & -8.752 & -1.250 & -2.380 & 4965 & 2.35 & 0.16 & \\
\hline 098087 & 0.014 & 0.019 & -5.643 & -9.092 & -1.380 & -3.070 & 4651 & 2.30 & -0.04 & \\
\hline 032042 & 0.028 & 0.052 & -8.338 & -11.455 & -2.044 & -3.790 & 4355 & 2.15 & 0.42 & LS \\
\hline
\end{tabular}




\section{Bibliography}

Abraham, R. G., Smecker-Hane, T. A., Hutchings, J. B., et al. 1996a, ApJ, 471, 694

Abraham, R. G.; van den Bergh, S., Glazebrook, K., et al. 1996b, ApJS, 107, 1

Allen, C. W. 1973, "Astrophysical Quantities", 3rd Edition, London

Andredakis, Y. C., Peletier, R. F., Balcells, M. 1995, MNRAS, 275, 874

Appenzeller, I., Fricke, K. J., Fürtig, W., et al. 1998, The Messenger, 94, 1

Appenzeller, I., Bender, R., Böhm, A., et al., 2000, The Messenger, 100, 44

Arimoto, A., \& Yoshii, Y. 1987, A\&A, 173, 23

Babul, A., Rees, M. J. 1992, MNRAS, 255, 346

Balogh, M. L., Smail, I., Bower, R. G., Ziegler, B. L., Smith, G. P., Davies, R. L., Gaztelu, A., Kneib, J.-P., \& Ebeling, H. 2002a, ApJ, 566, 123

Balogh M., Bower, R. G., Smail, I., Ziegler, B. L., Davies, R. L., Gaztelu, A., \& Fritz, A. 2002b, MNRAS, 337, 256

Barger, A. J., Aragón-Salamanca, A., Ellis, R. S., Couch, W. J., Smail, I., \& Sharples, R. M. 1996, MNRAS, 279, 1

Barnes, J. E., \& Hernquist, L. 1992, ARA\&A 30, 705

Baugh, C. M., Cole, S., \& Frenk, C. S. 1996, MNRAS, 283, 1361

Bell, E. F., Wolf, C., Meisenheimer, K., et al. 2004, ApJ, 608, 752
Bender, R., \& Möllenhoff, C. 1987, A\&A, 177, 71

Bender, R. 1988, A\&A, 193, L7

Bender, R. 1990, A\&A, 229, 441

Bender, R., Burstein, D., \& Faber, S. M. 1992, ApJ, 399, 462

Bender, R., Burstein, D., \& Faber, S. M. 1993, ApJ, 411, 153

Bender, R., Appenzeller, I., Böhm, A., et al. 2001, in "Deep Fields", Cristiani, S., Renzini, A., \& Williams, R. E. (eds.), ESO astrophysics symposia, Springer, p. 96

Bernardi, M., Renzini, A., da Costa, L. N., et al. 1998, ApJ, 508, L143

Bernardi, M., Sheth, R. K., Annis, J., et al. 2003, AJ, 125, 1866

Bertin, E., \& Arnouts, S. 1996, A\&AS, 117, 393

Blanton, M. R., Dalcanton, J., Eisenstein, D., et al. 2001, AJ, 121, 2358

Bower, R. G., Lucey, J. R., \& Ellis, R. S. 1992, MNRAS, 254, 601

Bower, R. G., Terlevich, A., Kodama, T., \& Caldwell, N. 1999, in "Star Formation in Early Type Galaxies", Carral, P., \& Cepa, J. (eds.), ASP Conference Series, Vol. 163, p. 211

Böhm, A. 2003, PhD thesis, Universität Göttingen

Böhm, A., Ziegler, B. L., Saglia, R. P., et al. 2004, A\&A, 420, 9 
Böhm, A., \& Ziegler, B. L. 2006, ApJL, sub.

Brault, J. W., \& White, O. R. 1971, A\&A, 13, 169

Bruzual, G. A., \& Charlot, S. 1993, ApJ, 405, 538 (GISSEL96 version, BC96)

Bunker, A. J., Moustakas, L. A., \& Davis, M. 2000, ApJ, 531, 95

Burstein, D., \& Heiles, C. 1982, AJ, 87, 1165 $(\mathrm{BH})$

Butcher, H., \& Oemler, A., Jr. 1984, ApJ, 285, 426

Caon, N., Capaccioli, M., \& D'Onofrio, M. 1993, MNRAS, 265, 1013

Capaccioli, M., Caon, N., \& D'Onofrio, M. 1992, MNRAS, 259, 323

Cardelli, J. A., Clayton, G. C., \& Mathis, J. S. 1989, ApJ, 345, 245

Carlberg, R. G., Yee, H. K. C., Ellingson, E., et al. 1996, ApJ, 462, 32

Carter, D. 1987, ApJ, 312, 514

Cole, S., Lacey, C. G., Baugh, C. M., \& Frenk, C. S. 2000, MNRAS, 319, 168

Contardo, G., Steinmetz, M., \& Fritze-v. Alvensleben, U. 1998, ApJ, 507, 497

Davies, R. L., Efstathiou, G., Fall, S. M., Illingworth, G., \& Schechter, P. L. 1983, ApJ, 266, 41

Davies, R. L., Burstein, D., Dressler, A., et al. 1987 ApJS, 64, 581

de Carvalho, R. R., \& Djorgovski, S. 1992, ApJ, 389, L49

De Lucia, G., Poggianti, B. M., AragónSalamanca, A., et al. 2004, ApJ, 610, L77
De Propris, R., Colless, M., Driver, S. P., et al. 2003, MNRAS, 342, 725

de Vaucouleurs, G. 1948, Annales d'Astrophysique, 11, 247

de Vaucouleurs, G. 1959, Handbuch der Physik, 53,275

Djorgovski, S., \& Davis, M. 1987, ApJ, 313, 59

Dressler, A. 1980, ApJ, 236, 351

Dressler, A., Lynden-Bell, D., Burstein, D., Davies, R. L., Faber, S. M., Terlevich, R., \& Wegner, G. 1987, ApJ, 313, 42 (7S)

Dressler, A., Oemler Jr., A., Couch, W. J., Smail, I., Ellis, R. S., Barger, A., Butcher, H., Poggianti, B. M., \& Sharples, R. M. 1997, ApJ, 490, 577

Eggen, O. J., Lynden-Bell, D., \& Sandage, A. R. 1962, ApJ, 136, 748

Ellis, R. S., Smail, I., Dressler, A., et al. 1997, ApJ, 483, 582

Faber, S. M., \& Jackson, R. E. 1976, ApJ, 204, 668

Faber, S. M., Friel, E. D., Burstein, D., \& Gaskell, C. M. 1985, ApJS, 57, 711

Faber, S. M., Wegner, G., Burstein, D., Davies, R. L., Dressler, A., Lynden-Bell, D., \& Terlevich, R. J. 1989, ApJS, 69, 763 (7S)

Faber, S. M., Tremaine, S., Ajhar, E. A., et al. 1997, AJ, 114, 1771

Franx, M., \& Illingworth, G. D. 1988, ApJ, 327, L55

Frei, Z., \& Gunn, J. E. 1994, AJ, 108, 1476

Fish, R. A. 1964, ApJ, 139, 284

Fritz, A., Ziegler, B. L., Bower, R. G., Smail, I., \& Davies, R. L. 2005a, MNRAS, 358, 233 
Fritz, A., Ziegler, B. L., Böhm, A., Balogh, M. L., Bower, R. G., Smail, I., \& Davies, R. L. 2005b, in "The Fabulous Destiny of Galaxies: Bridging Past and Present", Marseille, in press

Fritze-v. Alvensleben, U. 1989, PhD thesis, Universität Göttingen

Fukugita, M., Shimasaku, K., \& Ichikawa, T. 1995, PASP, 107, 945

Graham, A. W., \& Guzmán, R. 2003, AJ, 125, 2936

Gaztelu, A. 2000, Master of Science Thesis, University of Durham

Gebhardt, K., Faber, S. M., Koo, D. C., et al. 2003, ApJ, 597, 239

Geller, M. J., \& Huchra J. P. 1989, Science, 246, 879

Gerhard, O. E. 1993, MNRAS, 265, 213

Gerken, B., Ziegler, B., Balogh, M., Gilbank, D., Fritz, A., \& Jäger, K. 2004, A\&A, 421, 59

Giovanelli, R., Haynes, M. P., Rubin, V. C., \& Ford, W. K., Jr. 1986, ApJ, 301, L7

Girardi, M., Giuricin, G., Mardirossian, F., Mezzetti, M., \& Boschin, W. 1998, ApJ, 505, 74

González, J. J. 1993, PhD thesis, University of California, Santa Cruz

Heidt, J., Appenzeller, I., Gabasch, A., et al. 2003, A\&A, 398, 49

Hill, R. J., Ferrarese, L., Stetson, P. B., et al., 1998, ApJ, 496, 648

Hoessel, J. G., Oegerle, W. R., \& Schneider, D. P. 1987, AJ, 94, 1111

Holden, B. P., Stanford, S. A., Eisenhardt, P., \& Dickinson, M. 2004, AJ, 127, 2484

Holden, B. P., Blakeslee, J. P., Postman, M., et al. 2005, ApJ, 626, 809
Holtzman, J. A., Burrows, C. J., Casertano, S., et al. 1995, PASP, 107, 1065

Horne, K. 1986, PASP, 98, 609

Hubble, E. 1926, ApJ, 64, 321

James, P. A., \& Mobasher, B. 1999, MNRAS, 306,199

Jørgensen, I. 1994, PASP, 106, 967

Jørgensen, I., Franx, M., \& Kjærgaard, P. 1995, MNRAS, 273, 1097 (J99)

Jørgensen, I., Franx, M., \& Kjærgaard, P. 1996, MNRAS, 280, 167

Jørgensen, I. 1999, MNRAS, 306, 607 (J99)

Jørgensen, I., Franx, M., Hjorth, J., van Dokkum, P. G. 1999, MNRAS, 308, 833 (JFHD)

Kauffmann, G., 1996, MNRAS, 281, 487

Kauffmann, G., \& Charlot, S. 1998, MNRAS, 297L, 23

Kelson, D. D., van Dokkum, P. G., Franx, M., Illingworth, G. D., Fabricant, D. 1997, ApJ, 478, L13

Kelson, D. D., Illingworth, G. D., van Dokkum, P. G., \& Franx, M., 2000a, ApJ, 531, 137

Kelson, D. D., Illingworth, G. D., van Dokkum, P. G., \& Franx, M., 2000b, ApJ, 531, 184

Kennicutt, R. C. 1992, ApJS, 79, 255

Kent, S. M. 1985, PASP, 97, 165

Kinney, A. L., Calzetti, D., Bohlin, R. C., McQuade, K., Storchi-Bergmann, T., \& Schmitt, H. R. 1996, ApJ, 467, 38

Kodama, T., \& Arimoto, N. 1997, A\&A, 320, 41 (KA97)

Kodama, T., Yamada, T., Akiyama, M., et al. 2004, MNRAS, 350, 1005 
Kormendy, J., 1977, ApJ, 218, 333

Kormendy, J., \& Djorgovski, S. 1989, ARA\&A, 27,235

Kormendy, J., \& Bender, R. 1996, ApJ, 464, L119

Kron, R. G. 1980, ApJS, 43, 305

Kuijken, K., \& Merrifield, M. R. 1993, MNRAS, 264,712

Kuntschner, H. 2000, MNRAS, 315, 184

Kuntschner, H., Smith, R. J., Colless, M., Davies, R. L., Kaldare, R., \& Vazdekis, A. 2002 MNRAS, 337, 172

La Barbera, F., Busarello, G., Merluzzi, P., Massarotti, M., \& Capaccioli, M. 2002, ApJ, 571, 790

La Barbera, F., Busarello, G., Merluzzi, P., Massarotti, M., \& Capaccioli, M. 2003, ApJ, 595, 127

Laird, J. B., \& Levison, H. F. 1985, AJ, 90, 2652

Larson, R. B. 1974, MNRAS, 166, 585

Larson, R. B., 1975, MNRAS, 173, 671

Larson, R. B., \& Tinsley, B. M. 1978, ApJ, 219, 46

Le Borgne, J.-F., Mathez, G., Mellier, Y., et al. 1991, A\&AS, 88, 133

Le Borgne, J.-F., Pelló R., \& Sanahuja, B. 1992, A\&AS, 95, 87

Le Borgne, J.-F., Bruzual, G., Pelló R., et al. 2003, A\&A 402, 433

Lidman, C., Rosati, P., Demarco, R., Nonino, M., Mainieri, V., Stanford, S. A., \& Toft, S. 2004, A\&A 416, 829

Lubin, L. M., \& Sandage, A. 2001, AJ, 122, 1084

Lucey, J. R., Guzman, R., Carter, D., \& Terlevich, R. J. 1991, MNRAS, 253, 584,
Lynden-Bell, D. 1967, MNRAS, 136, 101

Malmquist, K. G. 1920, Medd. Lund Astron. Obs., Ser. II, No. 22

Marleau, F. R., \& Simard, L. 1998, ApJ, 507, 585

Mehlert, D., Noll, S., Appenzeller, I., et al. 2002, A\&A, 393, 809

Mehlert, D., Thomas, D., Saglia, R. P., Bender, R., \& Wegner, G. 2003, A\&A, 407, 423

Menanteau, F., Abraham, R. G., \& Ellis, R. S. 2001, MNRAS, 322, 1

Metcalfe, N., Shanks, T., Campos, A., McCracken, H. J., \& Fong, R. 2001, MNRAS, 323, 795

Meza, A., Navarro, J. F., Steinmetz, M., \& Eke, V. R. 2003, ApJ, 590, 619

Moore, B., Katz, N., Lake, G., Dressler, A., \& Oemler, A. 1996, Nature 379, 613

Möller, C. S., Fritze-v.Alvensleben, U., Fricke, K. J., \& Calzetti, D. 2001, Ap\&SS, 276, 799

Moran, S. M., Ellis, R. S., Treu, T., Smail, I., Dressler, A., Coil, A. L., \& Smith, G. P. 2005, ApJ, 634, 977

Naab, T., \& Burkert, A. 2003, ApJ, 597, 893

Noll, S., Mehlert, D., Appenzeller, I., et al. 2004, A\&A, 418, 885

Osterbrock, D. E. 1989, in "Astrophysics of Gaseous Nebulae and Active Galactic Nuclei" (Mill Valley, CA, University Science Books)

Pahre, M. A., Djorgovski, S. G., \& de Carvalho, R. R. 1998, AJ, 116, 1591

Pelló, R., Kneib, J. P., Le Borgne, J.-F., et al. 1999, A\&A, 346, 359

Peng, C. Y., Ho, L. C., Impey, C. D., \& Rix, H.-W. 2002, AJ, 124, 266 
Pickles, A. J. 1998, PASP, 110, 863

Pipino, A., \& Matteucci, F. 2004 MNRAS, 347, 968

Poggianti, B. M., Smail, I., Dressler, A., et al. 1999, ApJ, 518, 576

Poggianti, B. M., Bridges, T. J., Mobasher, B., et al. 2001, ApJ, 562, 689

Press, W. H., Teukolsky, S. A., Vetterling, W. T., \& Flannery, B. P. 1992, "Numerical Recipes in Fortran 77", Cambridge University Press

Rix, H.-W., \& White, S. D. M. 1990, ApJ, 362, 52

Rix, H.-W., \& White, S. D. M. 1992, MNRAS, 254,389

Rusin, D., \& Kochanek, C. S. 2005, ApJ, 623, 666

Saglia R. P., Bertin G., Bertola F., et al. 1993a, ApJ 567, 572

Saglia, R. P., Bender, R., \& Dressler, A. 1993b, A\&A, 279, 75 (SBD93)

Saglia, R. P., Bertschinger, E., Baggley, G., et al. 1997a, ApJS, 109, 79

Saglia, R. P., Burstein, D., Baggley, G., et al. 1997b, MNRAS, 292, 499

Saglia, R. P., Maraston, C., Greggio, L., Bender, R., \& Ziegler, B. 2000, A\&A, 360, 911

Salpeter, E. E. 1955, ApJ, 121, 161

Sánchez-Blázquez, P., Gorgas, J., Cardiel, N., Cenarro, J., \& González, J. J. 2003, ApJ, 590, L91

Sandage, A., \& Visvanathan, N. 1978, ApJ, 225, 742

Sandage, A., \& Perelmuter, J.-M. 1990, ApJ, 350,481
Sargent, W. L. W., Schechter, P. L., Boksenberg, A., \& Shortridge, K. 1977, ApJ, 212, 326

Schade, D., Lilly, S. J., Crampton, D., Hammer, F., Le Fevre, O., \& Tresse, L. 1995, ApJ, 451, L1

Schade, D., Lilly, S. J., Crampton, D., et al. 1999, ApJ, 525, 31

Schaifers, K., Vogt, H. H. (eds.) 1981, LandoltBörnstein series, Vol. VI/2a, Springer

Schlegel, D. J., Finkbeiner, D. P., \& Davis, M. 1998, ApJ, 500, 525 (SFD)

Sérsic, J. L. 1968, Atlas de Galaxias Australes (Córdoba, Argentina: Observatorio Astronomico, Univ. Nac. Córdoba)

Silk, J. 2000, MNRAS, 324, 313

Simard, L., Willmer, C. N., Vogt, N. P., et al. 2002, ApJS, 142, 1

Simkin, S. M. 1974, A\&A, 31, 129

Sinachopoulos, D., \& van Dessel, E. 1998, A\&AS, 130, 290

Smail, I., Dressler, A., Couch, W. J., et al. 1997, ApJS, 110, 213

Smail, I., Edge, A. C., Ellis, R. S., \& Blandford, R. D. 1998, MNRAS, 293, 124

Spergel, D. N., Verde, L., Peiris, H. V., et al. 2003, ApJS, 148, 175

Teerikorpi, P. 1997, ARA\&A, 35, 101

Thomas, D., Maraston, C., \& Bender, R. 2002, RvMA, 15, 219

Thomas, D., Maraston, C., Bender, R., de Oliveira, C. M. 2005, ApJ, 621, 673

Thuan, T. X., \& Gunn, J. E. 1976 PASP, 88, 543

Tinsley, B. M. 1980, Fundamentals of Cosmic Physics, Vol. 5, p. 287 
Tonry, J., \& Davis, M. 1979, AJ, 84, 1511

Toomre, A., \& Toomre, J. 1972, ApJ, 178, 623

Tran, K. H., Simard, L., Illingworth, G., \& Franx, M. 2003, ApJ, 590, 238

Treu, T., Stiavelli, M., Møller, P., Casertano, S., \& Bertin, G. 2001a MNRAS, 326, 221

Treu, T., Stiavelli, M., Bertin, G., Casertano, S., \& Møller, P. 2001b MNRAS, 326, 237

Treu, T., Stiavelli, M., Casertano, S., Møller, P., \& Bertin, G. 2002, ApJ, 564, L13

van der Marel, R. P., \& Franx, M. 1993, ApJ, 407,525

van der Wel, A., Franx, M., van Dokkum, P. G., Rix, H.-W., Illingworth, G. D., \& Rosati, P. 2005, ApJ, 631, 145

van Dokkum, P. G., \& Franx, M. 1996, MNRAS, 281, 985

van Dokkum, P. G., Franx, M., Kelson, D. D., \& Illingworth, G. D. 1998, ApJ, 504, L17

van Dokkum, P. G., Franx, M., Fabricant, D., Kelson, D. D., \& Illingworth, G. D. 1999, ApJ, 520, L95

van Dokkum, P. G., Franx, M., Fabricant, D., Illingworth, G. D., \& Kelson, D. D. 2000, ApJ, 541,95

van Dokkum, P. G., Stanford, S. A., Holden, B. P., Eisenhardt, P. R., Dickinson, M., \& Elston, R. 2001a, ApJ 552, L101.

van Dokkum, P. G., \& Franx, M. 2001b, ApJ, 553,90

van Dokkum, P. G., Franx, M., Kelson, D. D. \& Illingworth, G. D. 2001c, ApJ, 553, L39

van Dokkum, P. G., \& Stanford, S. A. 2003, ApJ, 585,78
Vikhlinin, A., McNamara, B. R., Forman, W., Jones, C., Quintana, H., \& Hornstrup, A. 1998, ApJ, 502, 558

Wagner, R. M., 1992, in "Astronomical CCD observing and reduction techniques", ASP Conf. Series Vol. 23, Howell., S. B. (ed.), Astronomical Society of the Pacific, p. 160

White, S. D. M., \& Rees, M. J. 1978, MNRAS, 183,341

Williams, R. E., Blacker, B., Dickinson, M., et al. 1996, AJ, 112, 1335

Williams, R. E., Baum, S., Bergeron, L., et al. 2000, AJ, 120, 2735

Worthey, G., Faber, S. M., Gonzalez, J. J., \& Burstein, D. 1994, ApJS, 94, 687

Wuyts, S., van Dokkum, P. G., Kelson, D. D., Franx, M., \& Illingworth, G. D. 2004, ApJ, 605, 677

Yang, Y., Zabludoff, A. I., Zaritsky, D., Lauer, T. R., \& Mihos, J. C. 2004, ApJ, 607, 258

Yee, H. K. C., Ellingson, E., Abraham, R. G., et al. 1996, ApJS, 102, 289

Ziegler, B. L., \& Bender, R. 1997, MNRAS, 291, 527

Ziegler, B. L., Saglia, R. P., Bender, R., Belloni, P., Greggio, L., \& Seitz, S. 1999, A\&A, 346, 13

Ziegler, B. L., Bower, R. G., Smail, I., Davies, R. L., \& Lee, D., 2001a, MNRAS, 325, 1571

Ziegler, B. L., Fricke, K. J., Balogh, M. L., Bower, R. G., Gaztelu, A., Smail, I., \& Davies, R. L. 2001b, in "Gas and Galaxy Evolution", Hibbard, J. E., Rupen, M., \& van Gorkom, J. H. (eds.), ASP Conf. Proceedings, Vol. 240, p. 619

Ziegler, B. L., Böhm, A., Fricke, K. J., et al. 2002, ApJ, 564, L69 
Ziegler, B. L., Thomas, D., Böhm, A., Bender, R., Fritz, A., \& Maraston, C. 2005, A\&A, 433, 519 


\section{Acknowledgements}

First of all I want to thank Prof. Dr. K. J. Fricke for his permanent interest in my work, his continuous support and for giving me the opportunity to acquire the doctoral level in one of the most spellbinding fields of modern extragalactic astronomy by using spectroscopic data from the state-of-the-art telescopes, the VLT and CA $3.5 \mathrm{~m}$. My further thanks go to Prof. Dr. W. Lauterborn, who kindly agreed to become the secondary referee of my thesis. In particular, I am deeply grateful to Dr. B. L. Ziegler, the leader of our Junior Research Group (sponsored by the Volkswagen Foundation) for his support and encouragement, many helpful and interesting discussions, and also for the thorough proof-reading of the draft giving many useful comments on it.

Furthermore, I wish to thank the other members of the disputation committee, Prof. Dr. K. Bahr, Prof. Dr. U. Christensen, Prof. Dr. F. Kneer and Prof. Dr. W. Kollatschny.

Thanks also to Dr. A. Böhm, Dr. C. Halliday and Dr. P. Papaderos for their proof-reading of some chapters.

I am indebted a lot to my main collaborators, Prof. Dr. M. L. Balogh (Waterloo), Prof. Dr. R. G. Bower (Durham), Prof. Dr. R. L. Davies (Oxford), Prof. Dr. I. R. Smail (Durham), and Dr. D. Thomas (Oxford) for their support and stimulating discussions on various topics.

I am also grateful to the P.I. of the FORS Deep Field project, Prof. Dr. I. Appenzeller, for his continuous support of the field elliptical galaxy project. Prof. Dr. R. Bender and Priv. Doz. Dr. R. P. Saglia (München) are thanked for kindly providing the FCQ program and the surface brightness fitting routine, respectively.

Thanks a lot to the (former) members of our Junior Research Group, in particular to Drs. A. Böhm, K. Jäger and I. Berentzen, for all the helpful and interesting discussions, helps in various aspects and the nice working atmosphere.
I am also grateful to Priv. Doz. Dr. J. Heidt, Drs. D. Mehlert and S. Noll (all Heidelberg), who performed the main parts of the spectroscopy for the FDF project during the Guaranteed Time Observations in 2000 and 2001. I also thank Drs. K. Reinsch and C. Möllenhoff (Heidelberg) for the pilot observations in Dec. 1999.

Several other people from foreign institutes have contributed to my understanding of the project in various ways and thus I want to thank are Drs. A. Diaferio (Torino), A. W. Graham (RSAA), H. Kuntschner (ESO), S. Mieske (ESO), U. Thiele (CAHA), and T. Treu (UCLA).

A continuous strong assistance during the course of this period has been the support of my parents, Susanne and Karl, my grandmother Agnes, my aunt Eva, uncle Willi as well as my cousin Thomas. Without their big endeavours, their love and understanding it would have been a lot harder and therefore I am deeply thankful to all of them. I am also indebted to my parents for giving me the possibility to carry out my studies. Last but not least, thanks a lot to Andi, Asmus, Gregor and Markus, for enriching my life with their great friendship and for having cheered me up so many times.

Financial support by the Volkswagen Foundation (I/76 520) and the Deutsche Forschungsgemeinschaft (ZI 663/1-1, ZI 663/2-1, ZI 663/3-1, ZI 663/5-1) are gratefully acknowledged. I am furthermore grateful for travel founds to the JENAM 2002 conference in Porto and several AG annual meetings (München, Berlin, Freiburg and Prague). The Calar Alto and ESO VLT staff are thanked for efficient observational support. This research has made use of the NASA's Astrophysics Data System and NASA/IPAC Extragalactic Database (NED). This thesis has been created on computers of the UniversitätsSternwarte Göttingen, Institut für Astrophysik and the Gesellschaft für Wissenschaftliche Datenverarbeitung Göttingen (GWDG). 


\title{
Curriculum Vitae
}

\author{
PERSÖNLICHE DATEN: \\ Name: $\quad$ Alexander Fritz \\ Adresse: $\quad$ Christophorusweg 12/102 \\ 37075 Göttingen \\ Geburtsdatum: 23. August 1976 \\ Geburtsort: Wien, Österreich \\ Nationalität: österreichisch \\ Familienstand: ledig
}

\section{Ausbildung:}

Jan. 2001 Beginn der Doktorarbeit an der Universitäts-Sternwarte Göttingen The Dependence of the Evolution of Early-Type Galaxies on their Environment Betreuer: Prof. Dr. K. J. Fricke

Sep. $2000 \quad$ Verleihung des akademischen Grades „Magister Rerum Naturalium“

Sep. 2000 Diplomarbeit am Institut für Astronomie, Universität Wien, Österreich

Bestimmung Extragalaktischer Entfernungen mit Flächenhelligkeitsfluktuationen

Betreuer: Prof. Dr. W. W. Zeilinger

Sep. 2000 2. Diplomprüfung Astronomie mit Auszeichnung (Note: „sehr gut“)

Dez. 1997 1. Diplomprüfung Astronomie

$1994-2000 \quad$ Studium Astronomie \& Geschichte an der Universität Wien, Österreich

Juni 1994 Matura mit Auszeichnung am Realgymnasium, 1150 Wien

1986 - $1994 \quad$ Privates Realgymnasium, 1150 Wien, Österreich

1982 - 1986 Öffentliche Volksschule, 1230 Wien, Österreich

\section{Beruflicher Werdegang:}

Seit 2001 Wissenschaftlicher Mitarbeiter an der Universitäts-Sternwarte Göttingen und Institut für Astrophysik Göttingen;

Kollaborationen: München, Potsdam, Oxford, Durham (UK), Waterloo (CA); Mehrere Beobachtungsreisen zum Calar Alto Observatorium (Spanien); Vorträge auf internationalen und nationalen Tagungen:

Okt. 2005 Alpbach (Österreich), München, Porto (PT), Heidelberg, Freiburg, Prag (CZ)

Sep. 2004 Öffentliche Führungen am Tag der offenen Tür des Physik-Neubaus, Göttingen

Sep. 2004

2002

Mai 2000

$1998-2000$ Öffentliche Führungen am 2. internationalen Tag der Astronomie „Lange Nacht der Sterne“ an der Universitäts-Sternwarte Göttingen Betreuung der Praktikantin Sarah Bühler Teilnahme an der „Science Week @ Austria“ für das Inst. für Astronomie, Betreuer für Sonnenteleskop, zwei Spektrographen sowie öffentliche Führungen Tutor im Astronomischen Praktikum für Fortgeschrittene 